KEY WORDS: PORFLOW code Sum-of-fractions

\title{
SPECIAL ANALYSIS: \\ REVISION OF INTERMEDIATE LEVEL VAULT DISPOSAL \\ LIMITS (U)
}

JULY 20, 2004

PREPARED BY:

Gregory P. Flach

Robert A. Hiergesell

Westinghouse Savannah River Company LLC

Westinghouse Savannah River Company LLC Savannah River Site Aiken, SC 29808

Prepared for the U.S. Department of Energy under Contract No. DE-AC09.

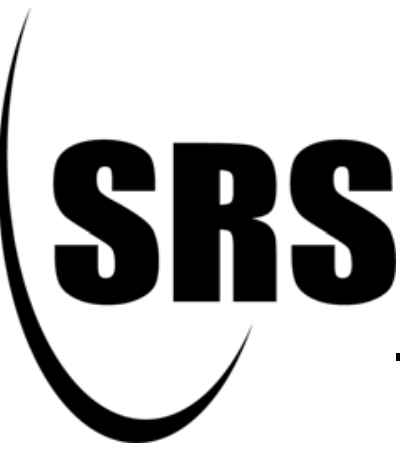


This document was prepared in conjunction with work accomplished under Contract No. DE-AC09-96SR18500 with the U. S. Department of Energy.

\section{DISCLAIMER}

This report was prepared as an account of work sponsored by an agency of the United States Government. Neither the United States Government nor any agency thereof, nor any of their employees, makes any warranty, express or implied, or assumes any legal liability or responsibility for the accuracy, completeness, or usefulness of any information, apparatus, product or process disclosed, or represents that its use would not infringe privately owned rights. Reference herein to any specific commercial product, process or service by trade name, trademark, manufacturer, or otherwise does not necessarily constitute or imply its endorsement, recommendation, or favoring by the United States Government or any agency thereof. The views and opinions of authors expressed herein do not necessarily state or reflect those of the United States Government or any agency thereof.

This report has been reproduced directly from the best available copy.

Available for sale to the public, in paper, from: U.S. Department of Commerce, National Technical Information Service, 5285 Port Royal Road, Springfield, VA 22161, phone: (800) 553-6847, fax: (703) 605-6900

email: orders@ntis.fedworld.gov

online ordering: http://www.ntis.gov/help/index.asp

Available electronically at http://www.osti.gov/bridge

Available for a processing fee to U.S. Department of Energy and its contractors, in paper, from: U.S. Department of Energy, Office of Scientific and Technical Information, P.O. Box 62, Oak Ridge, TN 37831-0062,

phone: (865)576-8401,

fax: (865)576-5728

email: $\underline{\text { reports@ adonis.osti.gov }}$ 
This page was intentionally left blank 
THIS PAGE INTENTIONALLY LEFT BLANK 


\section{EXECUTIVE SUMMARY}

New disposal limits have been computed for the IL vaults based on several revisions to the PA. The most important changes are implementation of a 1,000 year time for compliance, rather than 10,000 years, and consideration of additional radon precursors. Other revisions include refinement of the aquifer mesh to more accurately model the footprint of two IL vaults, a new $\mathrm{Pu}$ chemistry model accounting for the different transport properties of oxidation states III/IV and V/VI, and implementation of a timed sum-of-fractions approach to setting limits. A significant decrease in the groundwater pathway limits for I-129 was speculated in the FY2003 interim measures assessment, in response to refinement of the aquifer mesh and source node definition. In fact, the new limits for these nuclides are only slightly lower. Based on the IL vault inventory as of 7/2/04 and disposal limits developed herein, the largest inventory fractions are $30 \%$ for Ra-226 and the radon analysis, $11 \%$ for I-129 (generic) and the groundwater pathway, and 9\% for C-14 and the air pathway. For comparison the volume-filled fraction is at about $36 \%$. Continued operation of the IL vault should not challenge performance objectives, assuming future disposal patterns are similar to historic use of the facility. 
THIS PAGE INTENTIONALLY LEFT BLANK 


\section{TABLE OF CONTENTS}

EXECUTIVE SUMMARY

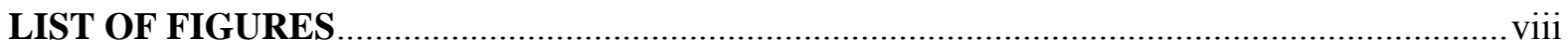

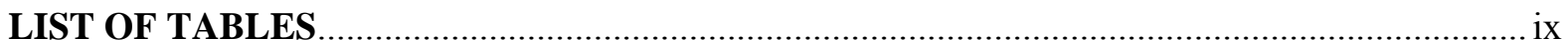

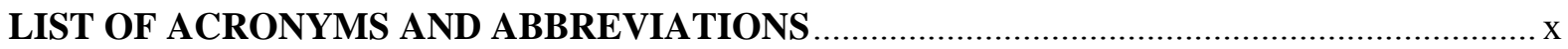

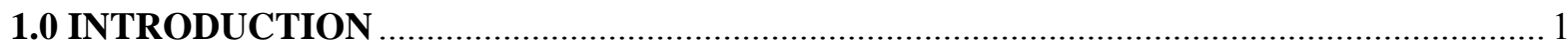

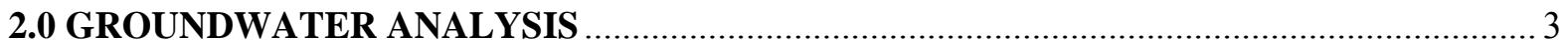

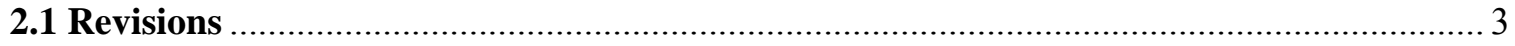

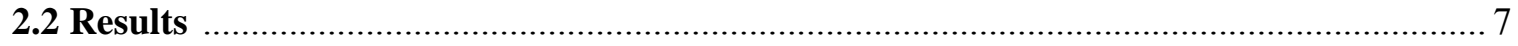

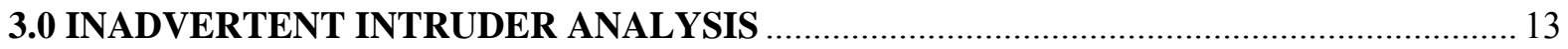

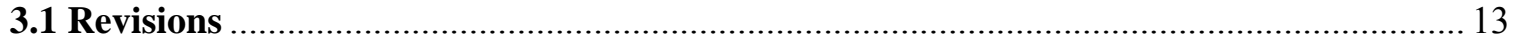

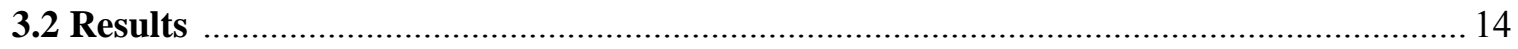

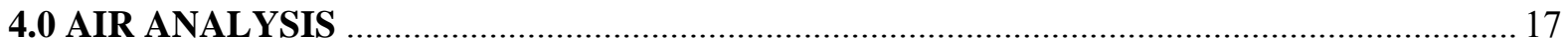

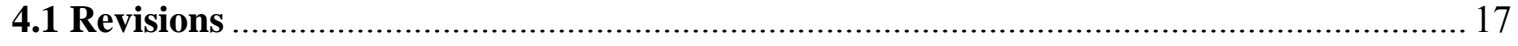

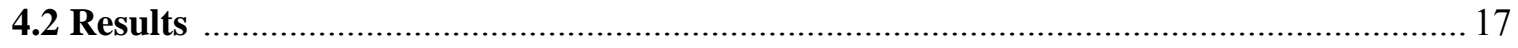

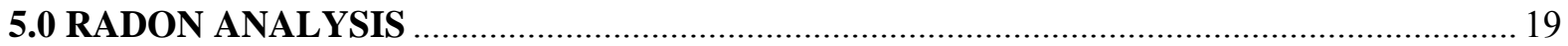

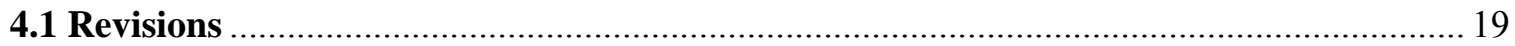

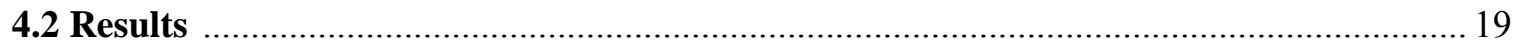

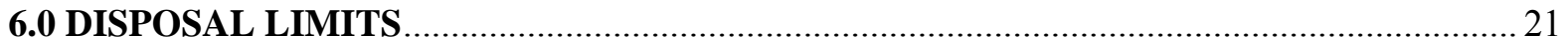

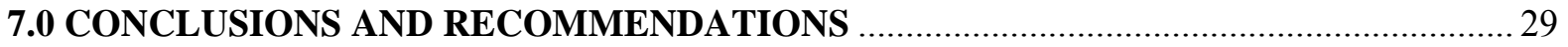

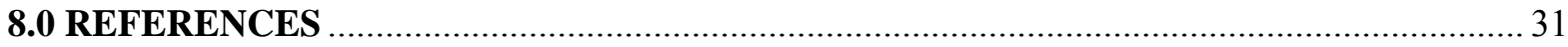

APPENDIX A - ADDITIONAL INFORMATION ON GROUNDWATER ANAL YSIS ......... A-1

APPENDIX B - ADDITIONAL INFORMATION ON INTRUDER ANALYSIS …................ B-1

APPENDIX C - ADDITIONAL INFORMATION ON AIR ANAL YSIS …......................... C-1

APPENDIX D - ADDITIONAL INFORMATI ON RADON ANALYSIS ............................ D-1

APPENDIX E - ADDITIONAL INFORMATION ON LIMITS CALCULATIONS................ E-1

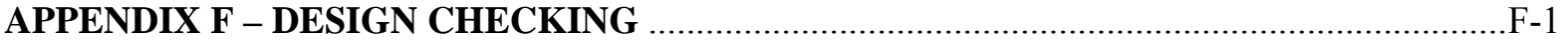




\section{LIST OF FIGURES}

Figure 2-1 Infiltration estimate for IL vault cover system ................................................. 5

Figure 2-2 Computational mesh and material zones for PORFLOW vadose flow and

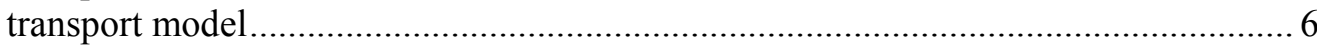

Figure 2-3 Plan view of computational mesh used for aquifer transport simulations, with

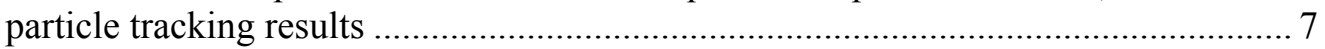

Figure 5-1 Decay chain of parent radionuclides leading to $\mathrm{Rn}-222$ formation........................... 20 


\section{LIST OF TABLES}

Table 2-1 Initial $\mathrm{U}$ and $\mathrm{Pu}$ concentrations in the waste zone for various inventories.................. 6

Table 2-2 Sum-of-fraction results for Pu-239 and four inventories in the 1,000-10,000 year

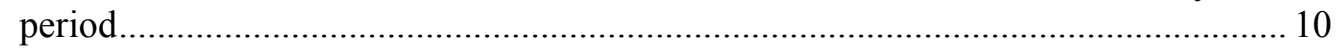

Table 2-3 Inventory limits (Ci) for groundwater pathway for three time periods (current time of compliance is 1,350 years for this pathway)

Table 3-1 Inventory limits for the intruder resident scenario; transient calculation for 1,000

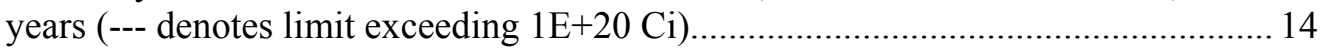

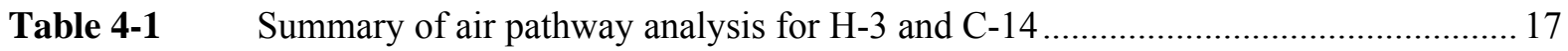

Table 5-1 Simulated peak instantaneous Rn-222 flux over 1,000-years at the land surface and associated disposal limits for parent isotopes....

Table 6-1 Inventory limits from all pathways compared to the IL vault inventory as of $7 / 2 / 04$

Table 6-2 Disposal limits for IL vaults. 26 


\section{LIST OF ACRONYMS AND ABBREVIATIONS}

\section{ACRONYMS}

DOE Department of Energy

EPA Environmental Protection Agency

FACT Subsurface Flow and Contaminant Transport code

GSA General Separations Area

HELP Hydrologic Evaluation of Landfill Performance code

IL Intermediate Level

ILV Intermediate Level Vault

ILNTV Intermediate Level Non-Tritium Vault

ILTV Intermediate Level Tritium Vault

LLC Limited Liability Company

MCL Maximum Contaminant Level

PA Performance Assessment

SOF Sum-Of-Fractions

SRS Savannah River Site

\begin{tabular}{ll}
\multicolumn{2}{l}{ ABBREVIATIONS } \\
$\mathrm{Ci}$ & Curie \\
$\mathrm{cm}$ & centimeter \\
$\mathrm{d}$ & day \\
$\mathrm{ft}$ & feet \\
$\mathrm{g}$ & gram \\
$\mathrm{in}$ & inch \\
$\mathrm{m}$ & meter \\
$\mathrm{mL}$ & milliliter \\
$\mathrm{mrem}$ & millirem \\
$\mathrm{s}$ & second \\
$\mathrm{yr}$ & year
\end{tabular}




\subsection{INTRODUCTION}

This Special Analysis report describes a general revision to disposal limits for the Intermediate Level Vault in E-Area, that supersedes limits defined by the E-Area Performance Assessment (McDowell-Boyer et al. 2000) and subsequent Special Analyses cited below. Relative to the PA, the analysis incorporates the following revisions

- implementation of a 1,000 year time for compliance (Wilhite 2003) and timed sum-offractions approach (Collard 2003)

- additional radionuclides based on a recent updated screening analysis (Cook 2004)

- special waste forms: high-concentration activated-carbon I-129 vessels (Collard 2000), and $\mathrm{K}$ and $\mathrm{L}$ Basin resins containing radionuclides of interest C-14, Tc-99 and I-129 (Collard 2003)

- a revised treatment of $\mathrm{Pu}$ chemistry in the groundwater pathway, in which $\mathrm{Pu}(\mathrm{III} / \mathrm{IV})$ and $\mathrm{Pu}(\mathrm{V} / \mathrm{VI}$ ) are modeled separately with differing geochemical properties (Cook 2002b, Kaplan 2004)

- updated soil-solute distribution coefficients ( $K_{d}$ 's) for various elements and soil/waste types (Kaplan 2004)

- a more extensive investigation of solubility controls on $\mathrm{U}$ and $\mathrm{Pu}$ groundwater migration

- a revised infiltration transient to reflect the present E-Area closure plan and a cover degradation analysis (Phifer 2004a, b)

- refinement of the aquifer model computational mesh to allow a more accurate representation of the IL vault footprint (Wilhite and Butcher 2003)

- optimal selection of aquifer source cells receiving the flux computed by the vadose zone transport model (Flach and Collard 2003)

- groundwater flow field results from a new GSA/PORFLOW model that replaces the previous GSA/FACT model (Flach 2004)

- an expanded radon analysis considering Ra-226, Th-230, U-238 and Pu-238 as ancestors of Rn-222 in addition to U-234, which was the only precursor considered in the PA

- updated meteorology parameters and dose factors (Simpkins 2004) in the air pathway analysis

- a consideration of decay-only in a new automated intruder analysis that uses updated Federal Guidance Report 11 and 12 dose conversion factors (Koffman 2004).

Disposal limits are computed based on analyses of groundwater, inadvertent intruder, radon, and air pathways for potential exposure. Each pathway analysis is discussed in subsequent sections of the report, followed by presentation of disposal limits based on all pathways, and conclusions and recommendations. 
THIS PAGE INTENTIONALLY LEFT BLANK 


\subsection{GROUNDWATER ANALYSIS}

The groundwater pathway analysis for each radionuclide involves a vadose zone flow and transport simulation to estimate flux to the water table for a disposed parent and any subsequent progeny, followed by saturated zone flow and transport modeling to estimate the groundwater concentration(s) at a hypothetical well placed 100 meters down-gradient from the disposal unit.

\subsection{Revisions}

The vadose zone flow model was revised to reflect the current E-Area closure plan (Phifer 2004a), which calls for a geosynthetic cover system instead of a kaolin cap as assumed in the PA. An initial analysis of cover degradation and performance over time (Phifer 2004b) indicates infiltration through the vault roof will be nearly zero during a 25 year operational period and 100 years of institutional control (Figure 2-1). Infiltration over the adjacent native soil and backfill is about $40 \mathrm{~cm} / \mathrm{yr}$ during the same period. After institutional control and placement of the cover system, infiltration is predicted to gradually increase over time due to cover degradation, such as intrusion of deep-rooted plants (e.g. trees) and silting of drainage layers. At 1,050 years after operational closure, the vault roof is assumed to fail and infiltration is predicted to abruptly rise to about $40 \mathrm{~cm} / \mathrm{yr}$ (McDowell-Boyer et al. 2000, Section C.1.1.2).

In the PA a cap comprised of low-permeability kaolin and high-permeability drainage layers was explicitly included in the PORFLOW computational model. For the present analysis, the upper layers of the model representing the now obsolete cap were removed, in favor of direct specification of flux through the bottom of an implied cover system (Figure 2-2). Pre-closure (0$125 \mathrm{yrs}$ ), the infiltration flux is effectively applied to the vault roof and adjacent soil at the same elevation. Post-closure, infiltration is specified at the top of structural backfill (included in the model) at the bottom of the geosynthetic clay liner (omitted).

Preliminary flow simulations using the infiltration estimate from Phifer (2004b) indicated that the low-permeability vault roof would backup water into overlying layers between about 300 years and roof/cap failure. The preliminary infiltration analysis using HELP (USEPA 1994a, b) did not consider potential effects of a low permeability layer underlying the cover system. Infiltration over the ILV was thus reduced to a sufficiently low level to avoid ponding, in recognition of the fact that the drainage layer overlying the ILV roof would remove excess water. No change to infiltration over the backfill and native soil portion of the model was required. Figure 2-1 illustrates the infiltration rates used in the final PORFLOW model. The vadose zone flow simulation was performed as a sequence of steady-state runs approximating average conditions during the time intervals of $0-25,25-125,125-325,325-575,575-1075$, and 1075+ years, as indicated by the stair-step variation shown in Figure 2-1. Time zero is the start of disposal unit operation, assumed to precede operational closure by 25 years. Material properties used in the PA were retained. The material property variations include a "degraded" vault condition from $575-$ 1,075 years, and a "failed" state after year 1,075 (1,050 years after roof construction and operational closure). The saturation and velocity fields produced in each time period are plotted in Appendix A.

In the vadose transport model, the concentrations of $\mathrm{U}$ and Pu isotopes may be solubility-limited in the waste zone depending on the inventory disposed. In the PA, a single transport simulation was performed for each parent. Generally, the modeled inventory was sufficiently large to create solubility-limited conditions. For the present analysis, multiple simulations using much lower inventories in the range of 0.001 to $1 \mathrm{Ci}$ per 2 disposal units (vaults) were performed $(0.001-10$ $\mathrm{Ci}$ for $\mathrm{Pu}-238)$. This range was chosen to bracket realistic disposal quantities of $\mathrm{U}$ and $\mathrm{Pu}$ 
isotopes. Table 2-1 shows the waste zone concentration that would be produced for a uniform distribution of parent and no solubility limit. Partitioning between the solid and liquid phases is controlled by the soil-solute distribution coefficient $K_{d}(\mathrm{~mL} / \mathrm{g})$, or equivalently the retardation factor $R=1+\rho_{b} K_{d} / \theta$ (unitless) (cf. Fetter 1993). The highlighted entries exceed the solubility limit. Within this range of inventories, U-232, U-233, U-234 and Pu-238 are never solubilitylimited, while $\mathrm{Pu}-244$ is always solubility-limited. For the remaining nuclides, the inventory range straddles the point of initial solubility control. Solubility limits can complicate interpretation of inventory limits as presently calculated for any chain containing $\mathrm{U}$ or $\mathrm{Pu}$, as discussed later in the report.

The new plutonium chemistry implemented for the trench disposal units (Cook 2002b, Kaplan $2004)$ has been included in the present special analysis. The $\mathrm{Pu}(\mathrm{III} / \mathrm{IV})$ oxidation state is far more abundant than $\mathrm{Pu}(\mathrm{V} / \mathrm{VI})$, but the latter is significantly more mobile in sediments: a soil-solute distribution coefficient of $K_{d}=370 \mathrm{~mL} / \mathrm{g}$ is assumed for $\mathrm{Pu}(\mathrm{III} / \mathrm{IV})$ versus $K_{d}=15 \mathrm{~mL} / \mathrm{g}$ for $\mathrm{Pu}(\mathrm{V} / \mathrm{VI})$. Although present in trace amounts, the relatively high mobility of $\mathrm{Pu}(\mathrm{V} / \mathrm{VI})$ could potentially lead to a significant contribution to the sum-of-fractions at the 100 meter well. The two pairs of oxidation states are tracked separately in the vadose zone transport simulations to accommodate the difference in mobility. Implementation of the new Pu chemistry in terms of decay and regeneration rates is presented in Appendix A.

In addition to the geochemistry modifications described above, some distribution coefficients were updated to reflect current knowledge. Of particular note, the $K_{d}$ for $\mathrm{U}$ in soil was raised from $35 \mathrm{~mL} / \mathrm{g}$ to $800 \mathrm{~mL} / \mathrm{g}$ (Cook 2000, Kaplan 2004). Appendix A provides a complete listing of $K_{d}$ values used in the groundwater analysis and other key input data such as, parents analyzed, half-lives, atomic mass, concentration limits, solubility limits, and assumed decay chains. Based on an updated screening analysis (Cook 2004), K-40, Zr-95, Nb-94, Nb-95, Nb95m, Bi-210, Ra-226, Th-230, Cm-242 were added to the groundwater pathway analysis.

The FACT code model of the General Separations Area was recently superseded by an equivalent model using the PORFLOW code, in order to consolidate PA subsurface flow and transport modeling to a single software product (Flach 2004). The flow field computed by GSA/PORFLOW is used in the present study. GSA/PORFLOW is a regional scale model with a mesh resolution in the horizontal plane of $200 \mathrm{ft}$, compared to a width of about $50 \mathrm{ft}$ for the IL vault. In order to more accurately represent the physical width of an IL vault, higher resolution velocity and saturation fields in the vicinity of the IL vaults were created by interpolating values from GSA/FACT onto a $50 \mathrm{ft}$ by $50 \mathrm{ft}$ grid. Interpolation onto the finer-scale grid was done in a mass-conserving manner.

Figure 2-3 illustrates locations of the two IL vaults being modeled and aquifer model mesh. Under current plans a second IL vault will be constructed at footprint $\# 5,150 \mathrm{ft}$ away from the existing vault (cf. drawing W2017860 and Appendix B). However, adjoining IL footprints are modeled as a conservatism. Figure 2-3 also shows the extent of the aquifer flow and transport model (blue border) and the mesh resolution in the horizontal plane (light gray dashes). Particle tracking results starting from the four corners of the combined facility indicate the groundwater flow direction. Time markers (red dots) are shown every 10 years of travel. At the $50 \mathrm{ft}$ square grid resolution, the physical area of an IL vault $(50 \mathrm{ft} \times 279 \mathrm{ft})$ can be well approximated by 6 grid blocks. The depth of aquifer source cells was optimally selected following Flach and Collard (2003). 


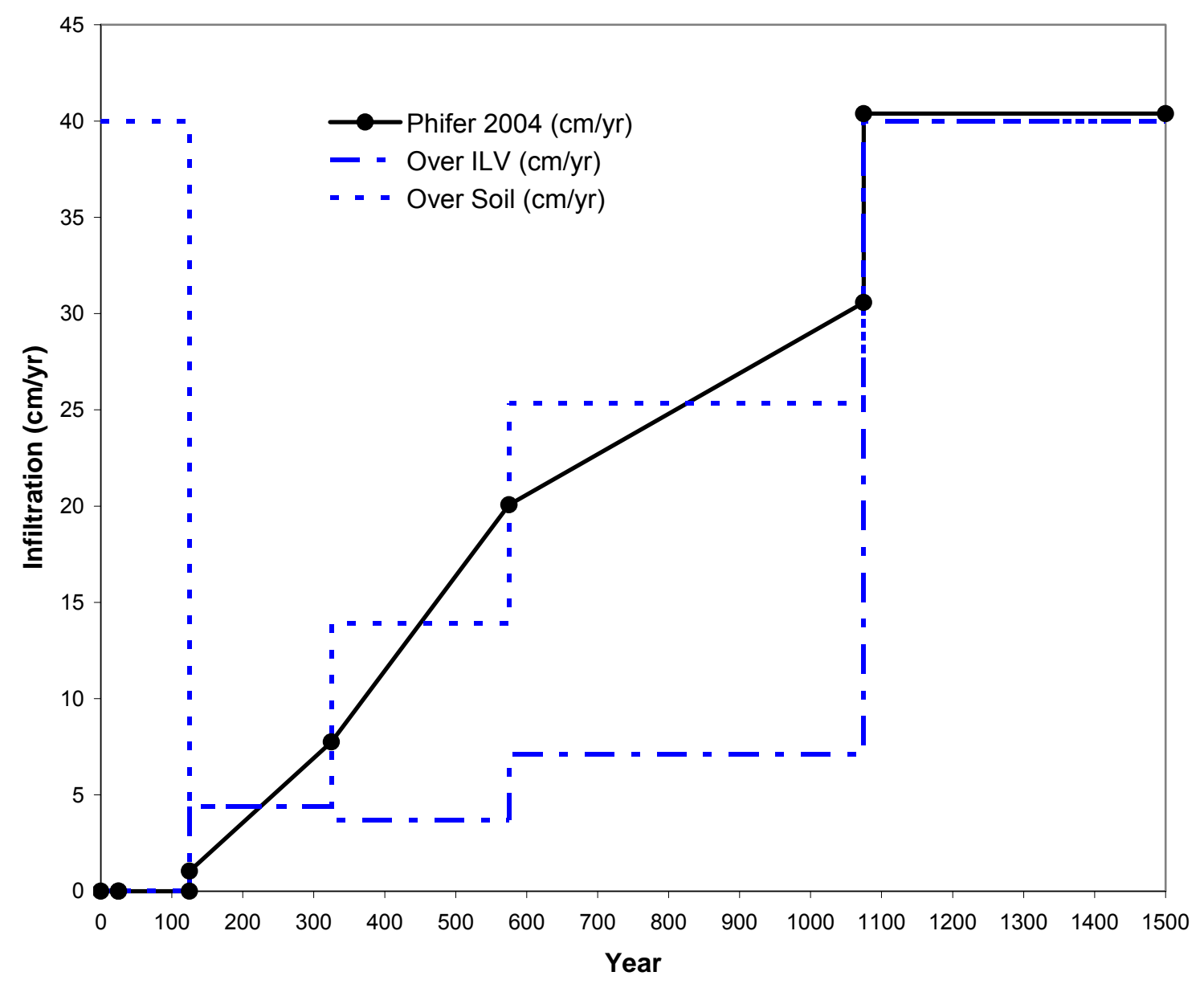

Figure 2-1. Infiltration estimate for IL vault cover system. 


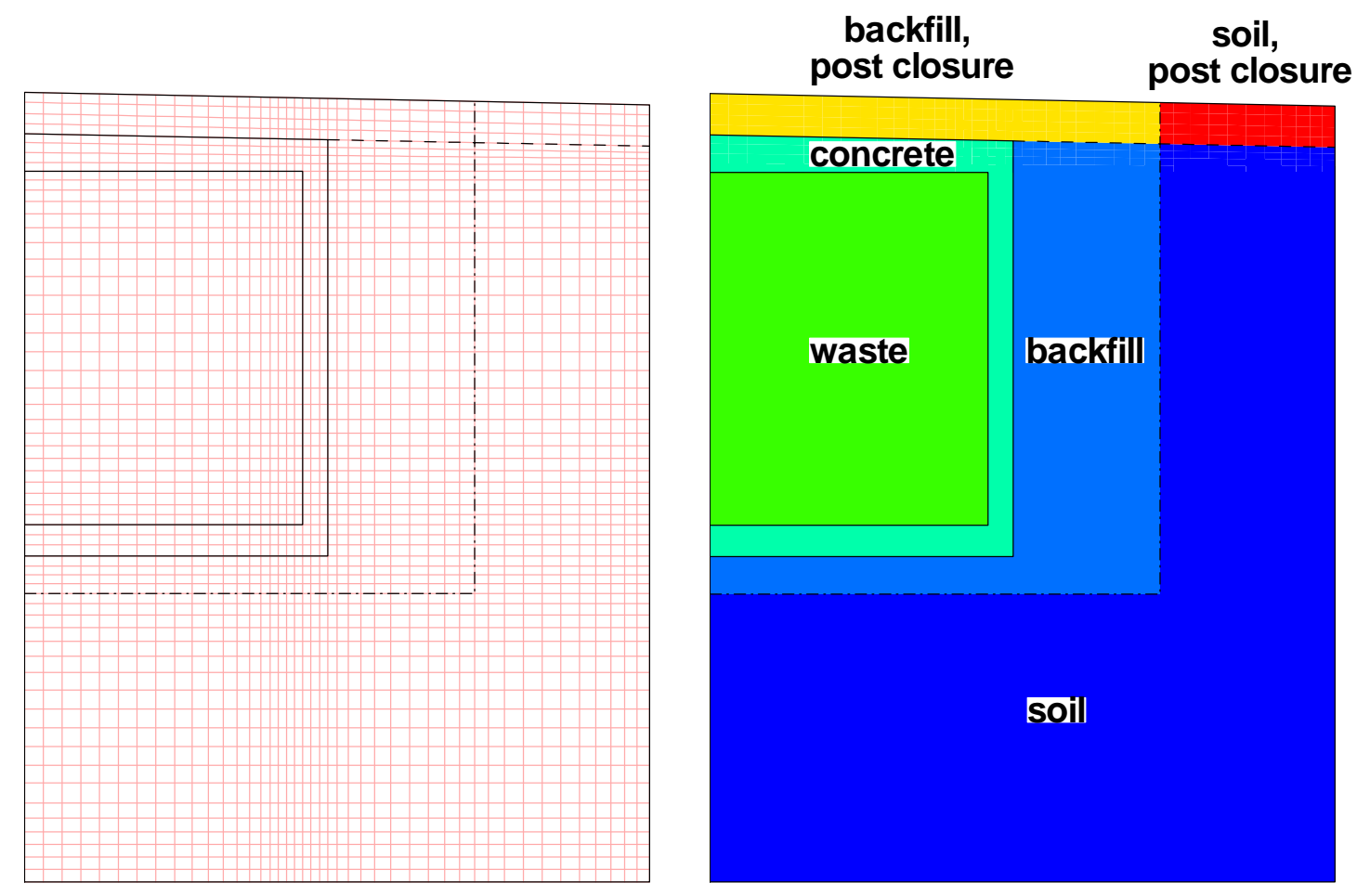

Figure 2-2. Computational mesh and material zones for PORFLOW vadose flow and transport model.

Table 2-1. Initial $U$ and $P u$ concentrations in the waste zone for various inventories.

\begin{tabular}{|c|c|c|c|c|c|c|c|}
\hline \multicolumn{4}{|c|}{$\begin{array}{l}\text { inventory for } 2 \text { disposal units: activity }(\mathrm{Ci}) \\
\text { activity per unit length per half width }(\mathrm{pCi} / \mathrm{cm})\end{array}$} & $\begin{array}{c}0.001 \\
2.94 \mathrm{E}+04\end{array}$ & $\begin{array}{c}0.01 \\
2.94 \mathrm{E}+05\end{array}$ & $\begin{array}{c}0.1 \\
2.94 \mathrm{E}+06\end{array}$ & $\begin{array}{c}1 \\
2.94 \mathrm{E}+07\end{array}$ \\
\hline parent & $\begin{array}{l}\text { sol limit } \\
(\mathrm{pCi} / \mathrm{mL})\end{array}$ & $\mathrm{Kd}(\mathrm{mL} / \mathrm{g})$ & $\mathrm{R}$ & $\begin{array}{c}\text { conc } \\
(\mathrm{pCi} / \mathrm{mL})\end{array}$ & $\begin{array}{c}\text { conc } \\
(\mathrm{pCi} / \mathrm{mL})\end{array}$ & $\begin{array}{c}\text { conc } \\
(\mathrm{pCi} / \mathrm{mL})\end{array}$ & $\begin{array}{c}\text { conc } \\
(\mathrm{pCi} / \mathrm{mL})\end{array}$ \\
\hline$U-232$ & $1.56 \mathrm{E}+03$ & 2000 & 24145 & $1.16 \mathrm{E}-05$ & $1.16 \mathrm{E}-04$ & $1.16 \mathrm{E}-03$ & $1.16 \mathrm{E}-02$ \\
\hline U-233 & 6.74E-01 & 2000 & 24145 & 1.16E-05 & 1.16E-04 & 1.16E-03 & 1.16E-02 \\
\hline U-234 & 4.37E-01 & 2000 & 24145 & 1.16E-05 & 1.16E-04 & 1.16E-03 & 1.16E-02 \\
\hline U-235 & 1.52E-04 & 2000 & 24145 & 1.16E-05 & 1.16E-04 & 1.16E-03 & 1.16E-02 \\
\hline U-236 & 4.58E-03 & 2000 & 24145 & 1.16E-05 & 1.16E-04 & 1.16E-03 & 1.16E-02 \\
\hline U-238 & 2.40E-05 & 2000 & 24145 & 1.16E-05 & 1.16E-04 & 1.16E-03 & 1.16E-02 \\
\hline Pu-238 & $1.79 E+00$ & 100 & 1208 & 2.32E-04 & 2.32E-03 & 2.32E-02 & 2.32E-01 \\
\hline Pu-239 & 6.52E-03 & 100 & 1208 & 2.32E-04 & 2.32E-03 & 2.32E-02 & 2.32E-01 \\
\hline Pu-240 & 2.40E-02 & 100 & 1208 & 2.32E-04 & 2.32E-03 & 2.32E-02 & 2.32E-01 \\
\hline Pu-241 & $1.10 E+01$ & 100 & 1208 & 2.32E-04 & $2.32 \mathrm{E}-03$ & 2.32E-02 & 2.32E-01 \\
\hline Pu-242 & 4.21E-04 & 100 & 1208 & 2.32E-04 & 2.32E-03 & 2.32E-02 & 2.32E-01 \\
\hline Pu-244 & 1.97E-06 & 100 & 1208 & 2.32E-04 & 2.32E-03 & 2.32E-02 & 2.32E-01 \\
\hline
\end{tabular}




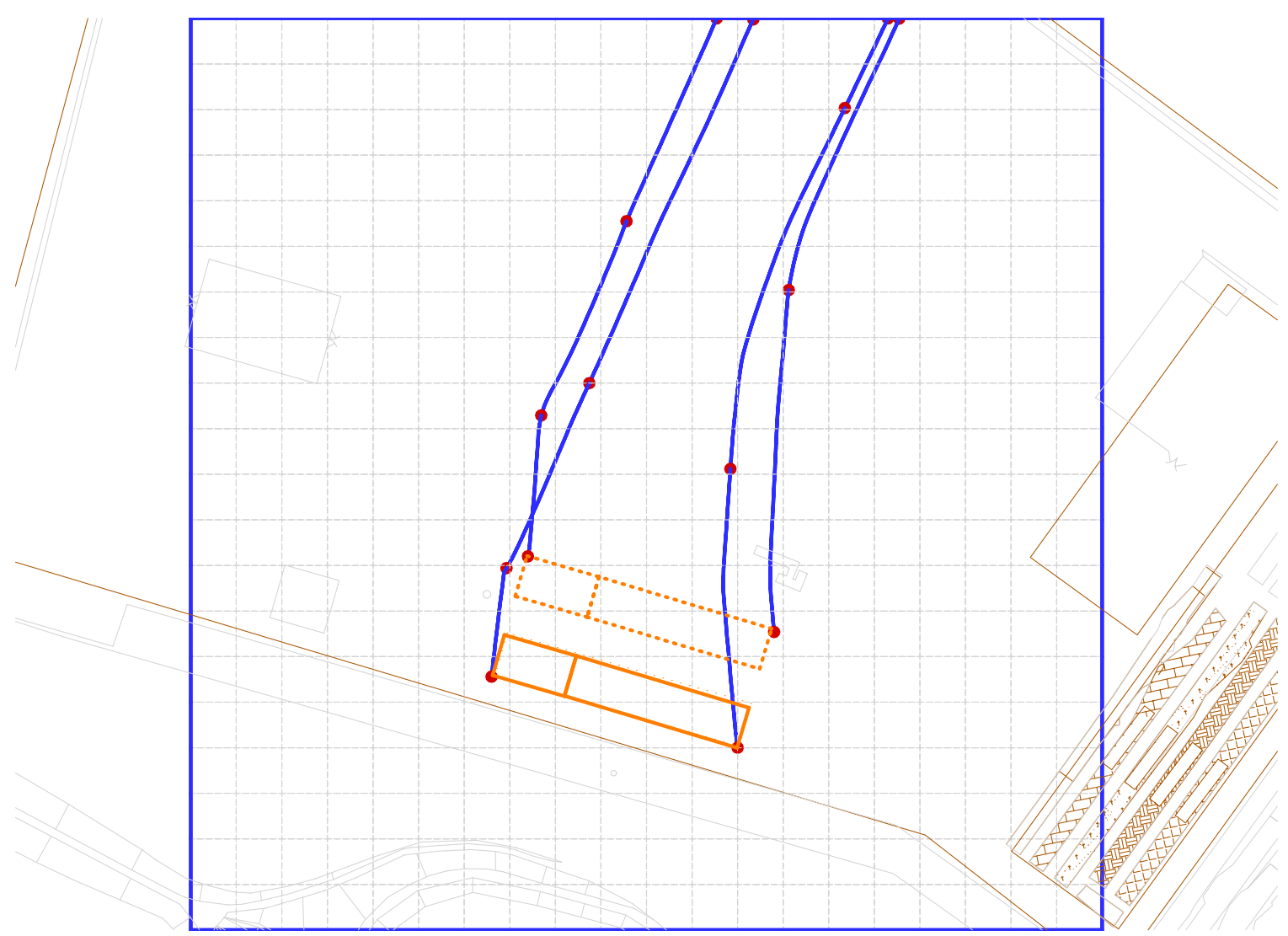

Figure 2-3. Plan view of computational mesh used for aquifer transport simulations, with particle tracking results.

\subsection{Results}

The EPA and DOE have defined limits on radionuclide concentrations required to safeguard human health. The lower of the EPA MCL and the concentration corresponding to a $25 \mathrm{mrem} / \mathrm{yr}$ dose is taken as the concentration limit for the groundwater pathway in this analysis (Appendix A). Disposal limits are based on a sum-of-fractions concept defined by

$$
S O F=\sum_{j}^{\text {parents }} \sum_{i}^{\text {chain }} \frac{c_{i j}\left(A_{j}\right)}{c_{M C L, i}} \leq 1
$$

where $c_{i j}\left(A_{j}\right)$ is groundwater concentration computed for the $i^{t h}$ member of a chain starting with the ${ }_{j}$ th parent for a disposal unit inventory of $A_{j}$, and $c_{M C L, i}$ is the concentration limit for the $i^{\text {th }}$ nuclide set by either an MCL or the $25 \mathrm{mrem} / \mathrm{yr}$ dose limit. For reference, the sum-offractions approach is used by the EPA to assess compliance with the MCL for beta and photon radioactivity (10 CFR 141.66(d)) and the NRC for classification of waste for near surface disposal when mixtures are involved (10 CFR 61.55). The groundwater concentration $c_{i j}$ is chosen as the concentration occurring at the time and location where the sum-of-fractions computed for the $j^{\text {th }}$ chain is maximized. Equation (2-1) can be abbreviated as

$$
S O F=\sum_{j}^{\text {parents }} \sum_{i}^{\text {chain }} f_{i j}\left(A_{j}\right) \leq 1
$$


where $f_{i j}$ denotes the concentration ratio (unitless). Equation (2-2) is more conveniently written as

$$
S O F=\sum_{j}^{\text {parents }} A_{j} \sum_{i}^{\text {chain }} \frac{f_{i j}\left(A_{j}\right)}{A_{j}}=\sum_{j}^{\text {parents }} A_{j} \sum_{i}^{\text {chain }} F_{i j}\left(A_{j}\right) \leq 1
$$

where $F_{i j}$ is the concentration ratio or fraction per unit inventory of parent $(1 / \mathrm{Ci})$. For many radionuclides, specifically those with constant $K_{d}$ and no solubility limits, concentration and concentration ratio are proportional to the inventory of parent. That is, $F_{i j}$ is a constant that can be computed from a single PORFLOW transport simulation. In the present analysis, $\mathrm{U}$ and $\mathrm{Pu}$ have solubility limits and the fraction per unit inventory varies with the amount disposed.

An example involving Pu-239 in the 1,000-10,000 year time frame is shown in Table 2-2, where "Pu-239" denotes Pu-239(III/IV) and "Pu-239_56" denotes Pu-239(V/VI). As indicated in Table 2-1, the initial concentration of Pu-239 is below the solubility limit for disposal unit inventories of 0.0005 and $0.005 \mathrm{Ci}$. At the higher inventories of 0.05 and $0.5 \mathrm{Ci}$, concentration is limited by solubility in the waste zone to $6.52 \mathrm{pCi} / \mathrm{L}=4.4 \mathrm{e}-13 \mathrm{~mol} / \mathrm{L}$ (McDowell-Boyer et al. 2000, Table 4.1-5). Progeny U-235 is well below its solubility limit in all cases. At the two lower inventories, the peak $\mathrm{Pu}$ concentrations and concentration ratios are proportional to the initial inventory, and the $\mathrm{Pu}$ fractions on a per unit inventory basis are the same. The last column of Table 2-2 gives the limit based on the sum-of-fractions for the chain on the line denoted "SOF-chain". The other values in the column indicate the percentage contribution to the sum-of-fractions from each member of the chain.

$\mathrm{Pu}-239$ (III/IV) is observed to be the dominant contributor to the sum-of-fractions on a per unit inventory basis (99.96\%), and thus the inventory "limit" (1/SOF) is the same for each case. At the two higher inventories, the peak $\mathrm{Pu}$ concentrations and ratios are fixed, and the fractions per unit inventory are inversely proportional to inventory. The inventory "limit" is proportional to inventory. For this time frame, none of the inventory "limits" have the conventional meaning as the maximum inventory that can be disposed without exceeding a groundwater concentration limit. Were U-235 not present in the chain, the inventory limit would effectively be infinite because the sum of concentration ratios for the chain has leveled off at $1.5 \times 10^{-5}$.

From an implementation perspective, a disposal limit can be conservatively defined by $1 / \mathrm{SOF}$ from a simulation with an assumed inventory below the actual inventory. Solubility controls can potentially be used to increase disposal limits, depending on the relationship between concentration and solubility limits.

In general, the time for compliance in this study is taken to be 1,000 years after disposal unit closure, following Wilhite (2003). An operational period of 25 years followed by 100 years of institutional control and interim closure is assumed to precede the 1,000 year time for compliance (Phifer 2004a). The groundwater analysis should therefore extend at least 1,125 years from the start of operations, for the purpose of defining limits. In this study, groundwater results through year 1,350 are used to define limits, to account for uncertainty in the vault failure time. A 1,000 year time for compliance is retained for the other pathways. Vault failure is assumed to occur at year 1,075 , or 50 years before the end of the compliance period. The longer time for compliance allows ample time for relatively mobile nuclides to migrate from the IL vault to the 100 meter well following vault failure, and ensures that limits will not be highly sensitive to the assumed time of vault failure. Although the present limits are based on a 1,350 year window, PORFLOW simulations for most nuclides are run to 10,000 years for comparison to previous limits. 
Plots of fractional flux, concentration, fractions and sum-of-fractions for each radionuclide and inventory modeled with PORFLOW are presented in Appendix A. Table 2-3 summarizes $1 /$ SOF "limits" from the groundwater pathway analysis. Results are segregated into three time intervals for input to a timed sum-of-fractions approach to setting disposal limits. The time for compliance for the groundwater pathway is chosen as 1,350 years, so only the first two periods impact the present limits. Results from the 1,350-10,000 year period are included for comparison to previous limits, which are often based on results past 1,350 years. For chains containing $U$ and/or $\mathrm{Pu}$, four simulations were run at disposal unit inventories of $0.0005,0.005,0.05$ and $0.5 \mathrm{Ci}$ (0.001, 0.01, 0.1 and $1 \mathrm{Ci}$ for two disposal units in the model). For Pu-238, a $5 \mathrm{Ci}$ inventory was also run because the current inventory for the nuclide on 7/2/04 was $1.34 \mathrm{Ci}$. Otherwise, a single simulation corresponding to $0.5 \mathrm{Ci}$ in a single disposal unit was run. The limits are generally based directly on a PORFLOW simulation starting from the parent. In the case of a relatively short-lived parent, namely Nb-95m, Bi-210, Pu-241, Am-241, Cm-242, Cm-244, Cf-252, the parent is instantly decayed to a sufficiently long-lived daughter. The limit for the parent is computed from

$$
A_{p}=\frac{\lambda_{p}}{\lambda_{d}} A_{d}=\frac{\tau_{d}}{\tau_{p}} A_{d}
$$

where $A$ denotes activity (Ci), $\lambda$ decay rate (1/yr), $\tau$ half-life (yr), and the subscripts "parent" and "daughter". More comprehensive groundwater pathway results are included in Appendix A.

A significant decrease in the limits for I-129 (generic) and I-129_ETF-Carbon was speculated in the FY2003 interim measures assessment, in response to refinement of the aquifer mesh and source node definition (Wilhite and Butcher 2003). The limits for these nuclides are only slightly lower (Table E-1). Because several changes were made to the groundwater analysis, a rigorous explanation for the present outcome is not possible without performing a detailed sensitivity analysis. Apparently the impact of mesh and source node refinement was less than speculated and/or other model changes compensated. Mitigating factors probably include a decrease in vertical mesh resolution and slower groundwater travel introduced in switching from the GSA/FACT model to the GSA/PORFLOW model.

For the IL vault inventory and volume-filled (36\%) as of $7 / 2 / 04$, only I-129 (generic) and I-129_ETF-Carbon produce a fraction of the groundwater pathway limit exceeding $1 \%$. Thus the conservative approach to defining limits as the minimum 1/SOF value among inventories modeled in the PORFLOW transport simulations for solubility-limited radionuclides should be adequate, assuming future disposal patterns are similar to historic use of the facility. Fractions for the groundwater pathway and current inventory are presented in Chapter 6.0, where this topic is further discussed. 
Table 2-2. Sum-of-fraction results for Pu-239 and four inventories in the 1,000-10,000 year period.

\begin{tabular}{|c|c|c|c|c|c|c|c|}
\hline $\begin{array}{l}\frac{0}{\frac{0}{0}} \\
\frac{0}{2} \\
\underline{\underline{e}}\end{array}$ & 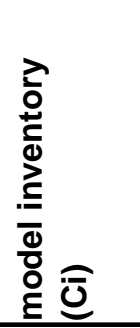 & 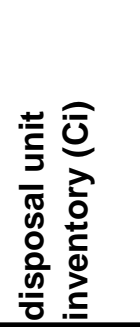 & 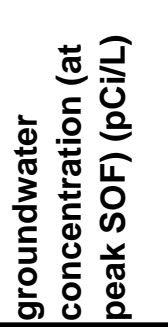 & 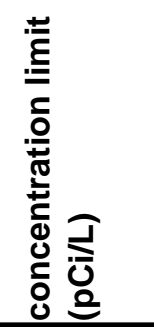 & 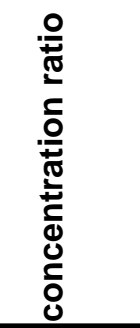 & 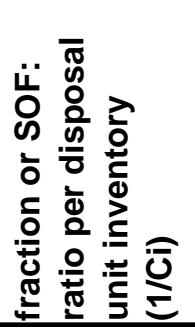 & 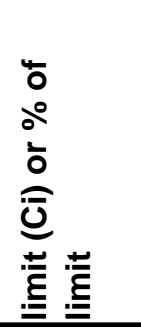 \\
\hline \multicolumn{8}{|c|}{$1000-10000$ yrs } \\
\hline Pu-239 & 0.001 & 0.0005 & $6.5 \mathrm{E}-06$ & $8.1 \mathrm{E}+00$ & 8.0E-07 & 1.6E-03 & 99.963 \\
\hline Pu-239_56 & & & 2.4E-09 & $8.1 \mathrm{E}+00$ & $2.9 \mathrm{E}-10$ & $5.8 \mathrm{E}-07$ & 0.036 \\
\hline U-235 & & & $3.2 \mathrm{E}-12$ & $6.5 E+01$ & 5.0E-14 & 9.9E-11 & 0 \\
\hline SOF-chain & & & & & & 1.6E-03 & $6.3 E+02$ \\
\hline Pu-239 & 0.01 & 0.005 & $6.5 E-05$ & $8.1 \mathrm{E}+00$ & 8.0E-06 & 1.6E-03 & 99.963 \\
\hline Pu-239_56 & & & $2.4 \mathrm{E}-08$ & $8.1 \mathrm{E}+00$ & 2.9E-09 & 5.8E-07 & 0.036 \\
\hline U-235 & & & $3.2 \mathrm{E}-11$ & $6.5 \mathrm{E}+01$ & $5.0 \mathrm{E}-13$ & $9.9 \mathrm{E}-11$ & 0 \\
\hline SOF-chain & & & & & & 1.6E-03 & $6.3 E+02$ \\
\hline Pu-239 & 0.1 & 0.05 & $1.2 \mathrm{E}-04$ & $8.1 \mathrm{E}+00$ & $1.5 \mathrm{E}-05$ & 3.0E-04 & 99.963 \\
\hline Pu-239_56 & & & 4.4E-08 & $8.1 \mathrm{E}+00$ & 5.5E-09 & 1.1E-07 & 0.036 \\
\hline U-235 & & & $6.1 \mathrm{E}-11$ & $6.5 \mathrm{E}+01$ & $9.3 \mathrm{E}-13$ & $1.9 \mathrm{E}-11$ & 0 \\
\hline SOF-chain & & & & & & 3.0E-04 & $3.3 E+03$ \\
\hline Pu-239 & 1 & 0.5 & $1.2 \mathrm{E}-04$ & $8.1 \mathrm{E}+00$ & $1.5 \mathrm{E}-05$ & 3.0E-05 & 99.963 \\
\hline Pu-239_56 & & & 4.4E-08 & $8.1 \mathrm{E}+00$ & 5.5E-09 & 1.1E-08 & 0.036 \\
\hline U-235 & & & $6.1 \mathrm{E}-11$ & $6.5 E+01$ & $9.3 \mathrm{E}-13$ & 1.9E-12 & 0 \\
\hline SOF-chain & & & & & $1.5 \mathrm{E}-05$ & 3.0E-05 & $3.3 E+04$ \\
\hline
\end{tabular}

notes: Pu concentration limit is based on DOE 25 mrem/yr dose limit (Cook 2002a)

$U$ concentration limit is based on EPA MCL (Cook 2002a)

Table 2-3. Inventory limits (Ci) for groundwater pathway for three time periods (current time of compliance is 1,350 years for this pathway).

\begin{tabular}{|l|c|c|c|c|}
\hline \multicolumn{1}{|c|}{ Nuclide } & Activity (Ci/unit) & $\begin{array}{c}\text { Limit (Ci) } \\
\mathbf{0 - 1 0 0} \text { yrs }\end{array}$ & $\begin{array}{c}\text { Limit (Ci) } \\
\mathbf{1 0 0 - 1 , 3 5 0} \text { yrs }\end{array}$ & $\begin{array}{c}\text { Limit (Ci) } \\
\mathbf{1 , 3 5 0 - 1 0 , 0 0 0} \text { yrs }\end{array}$ \\
\hline H-3 & 0.5 & $5.32 \mathrm{E}+09$ & $5.48 \mathrm{E}+09$ & $6.50 \mathrm{E}+39$ \\
\hline C-14 & 0.5 & $2.06 \mathrm{E}+31$ & $9.07 \mathrm{E}+07$ & $1.69 \mathrm{E}+04$ \\
\hline C-14_KB & 0.5 & $5.77 \mathrm{E}+29$ & $2.55 \mathrm{E}+06$ & $6.72 \mathrm{E}+02$ \\
\hline K-40 & 0.5 & $2.34 \mathrm{E}+19$ & $1.98 \mathrm{E}-01$ & $2.33 \mathrm{E}+01$ \\
\hline Ni-59 & 0.5 & $7.17 \mathrm{E}+82$ & $3.87 \mathrm{E}+39$ & $1.64 \mathrm{E}+02$ \\
\hline Se-79 & 0.5 & $6.18 \mathrm{E}+40$ & $1.17 \mathrm{E}+06$ & $3.29 \mathrm{E}+00$ \\
\hline Sr-90 & 0.5 & $1.60 \mathrm{E}+28$ & $6.12 \mathrm{E}+11$ & $1.83 \mathrm{E}+12$ \\
\hline Zr-93 & 0.5 & $6.09 \mathrm{E}+76$ & $7.30 \mathrm{E}+37$ & $5.69 \mathrm{E}+08$ \\
\hline Zr-95 & 0.5 & $1.00 \mathrm{E}+99$ & $1.00 \mathrm{E}+99$ & $1.00 \mathrm{E}+99$ \\
\hline Nb-94 & 0.5 & $9.51 \mathrm{E}+73$ & $1.40 \mathrm{E}+31$ & $1.51 \mathrm{E}+02$ \\
\hline Nb-95m & 0.5 & $9.70 \mathrm{E}+99$ & $9.70 \mathrm{E}+99$ & $9.70 \mathrm{E}+99$ \\
\hline Tc-99 & 0.5 & $4.04 \mathrm{E}+19$ & $1.32 \mathrm{E}+02$ & $1.87 \mathrm{E}+01$ \\
\hline Tc-99_KB & 0.5 & $3.02 \mathrm{E}+22$ & $2.41 \mathrm{E}+04$ & $1.30 \mathrm{E}+02$ \\
\hline Sn-126 & 0.5 & $8.25 \mathrm{E}+71$ & $1.74 \mathrm{E}+29$ & $7.26 \mathrm{E}+01$ \\
\hline I-129 & 0.5 & $4.33 \mathrm{E}+10$ & $4.55 \mathrm{E}-04$ & $3.54 \mathrm{E}+01$ \\
\hline I-129_ETF-Carbon & 0.5 & $1.25 \mathrm{E}+13$ & $9.67 \mathrm{E}-02$ & $1.03 \mathrm{E}-01$ \\
\hline I-129_KB & 0.5 & $4.28 \mathrm{E}+23$ & $6.24 \mathrm{E}+03$ & $1.20 \mathrm{E}+00$ \\
\hline Cs-135 & 0.5 & $9.38 \mathrm{E}+77$ & $1.20 \mathrm{E}+35$ & $4.60 \mathrm{E}+01$ \\
\hline Bi-210 & 0.5 & $2.76 \mathrm{E}+100$ & $2.76 \mathrm{E}+100$ & $2.76 \mathrm{E}+100$ \\
\hline
\end{tabular}




\begin{tabular}{|c|c|c|c|c|}
\hline Nuclide & Activity (Ci/unit) & $\begin{array}{l}\text { Limit (Ci) } \\
0-100 \text { yrs }\end{array}$ & $\begin{array}{c}\text { Limit (Ci) } \\
100-1,350 \text { yrs }\end{array}$ & $\begin{array}{c}\text { Limit (Ci) } \\
1,350-10,000 \text { yrs }\end{array}$ \\
\hline Ra-226 & 0.5 & $5.76 \mathrm{E}+75$ & $7.88 \mathrm{E}+34$ & $9.60 \mathrm{E}+01$ \\
\hline Th-228 & 0.5 & $1.00 \mathrm{E}+99$ & $1.00 \mathrm{E}+99$ & $1.00 \mathrm{E}+99$ \\
\hline Th-230 & 0.5 & $4.10 \mathrm{E}+78$ & $2.18 \mathrm{E}+35$ & $8.81 \mathrm{E}+01$ \\
\hline Th-232 & 0.5 & $4.62 \mathrm{E}+88$ & $1.84 \mathrm{E}+55$ & $2.11 \mathrm{E}+23$ \\
\hline U-232 & 0.0005 & $1.89 \mathrm{E}+96$ & $1.29 \mathrm{E}+58$ & $3.65 E+38$ \\
\hline $\mathrm{U}-232$ & 0.005 & $1.89 \mathrm{E}+96$ & $1.29 \mathrm{E}+58$ & $2.87 \mathrm{E}+38$ \\
\hline U-232 & 0.05 & $1.89 \mathrm{E}+96$ & $1.29 \mathrm{E}+58$ & $2.82 \mathrm{E}+38$ \\
\hline $\mathrm{U}-232$ & 0.5 & $1.89 \mathrm{E}+96$ & $1.29 \mathrm{E}+58$ & $2.82 \mathrm{E}+38$ \\
\hline $\mathrm{U}-233$ & 0.0005 & $9.54 \mathrm{E}+96$ & $2.77 \mathrm{E}+53$ & $1.68 \mathrm{E}+09$ \\
\hline U-233 & 0.005 & $9.55 \mathrm{E}+96$ & $2.77 \mathrm{E}+53$ & $1.68 \mathrm{E}+09$ \\
\hline $\mathrm{U}-233$ & 0.05 & $9.56 \mathrm{E}+96$ & $2.77 \mathrm{E}+53$ & $1.68 \mathrm{E}+09$ \\
\hline U-233 & 0.5 & $9.57 \mathrm{E}+96$ & $2.77 \mathrm{E}+53$ & $1.68 \mathrm{E}+09$ \\
\hline $\mathrm{U}-234$ & 0.0005 & $1.40 \mathrm{E}+83$ & $6.06 \mathrm{E}+37$ & $8.53 E+03$ \\
\hline U-234 & 0.005 & $1.40 \mathrm{E}+83$ & $6.06 \mathrm{E}+37$ & $8.53 E+03$ \\
\hline U-234 & 0.05 & $1.40 \mathrm{E}+83$ & $6.06 \mathrm{E}+37$ & $8.53 E+03$ \\
\hline U-234 & 0.5 & $1.40 \mathrm{E}+83$ & $6.06 \mathrm{E}+37$ & $8.53 E+03$ \\
\hline U-235 & 0.0005 & $1.26 \mathrm{E}+92$ & $1.86 \mathrm{E}+48$ & $4.68 \mathrm{E}+05$ \\
\hline U-235 & 0.005 & $1.26 \mathrm{E}+92$ & $1.86 \mathrm{E}+48$ & $5.60 \mathrm{E}+05$ \\
\hline U-235 & 0.05 & $9.03 E+92$ & $9.48 \mathrm{E}+48$ & $4.22 \mathrm{E}+06$ \\
\hline U-235 & 0.5 & $5.46 \mathrm{E}+93$ & $1.70 \mathrm{E}+49$ & $1.23 \mathrm{E}+07$ \\
\hline U-236 & 0.0005 & $1.03 \mathrm{E}+97$ & $3.03 E+53$ & $2.82 \mathrm{E}+09$ \\
\hline U-236 & 0.005 & $1.03 \mathrm{E}+97$ & $3.03 E+53$ & $2.81 \mathrm{E}+09$ \\
\hline U-236 & 0.05 & $1.03 \mathrm{E}+97$ & $3.02 \mathrm{E}+53$ & $2.81 \mathrm{E}+09$ \\
\hline U-236 & 0.5 & $2.60 \mathrm{E}+97$ & $9.40 \mathrm{E}+53$ & $1.12 \mathrm{E}+10$ \\
\hline U-238 & 0.0005 & $7.42 \mathrm{E}+95$ & $2.17 \mathrm{E}+52$ & $2.02 \mathrm{E}+08$ \\
\hline $\mathrm{U}-238$ & 0.005 & $3.56 \mathrm{E}+96$ & $1.29 \mathrm{E}+53$ & $1.53 \mathrm{E}+09$ \\
\hline $\mathrm{U}-238$ & 0.05 & $3.56 \mathrm{E}+97$ & $1.28 \mathrm{E}+54$ & $1.50 \mathrm{E}+10$ \\
\hline U-238 & 0.5 & $3.55 \mathrm{E}+98$ & $1.19 \mathrm{E}+55$ & $1.25 \mathrm{E}+11$ \\
\hline $\mathrm{Np}-237$ & 0.5 & $1.83 \mathrm{E}+35$ & $1.53 \mathrm{E}+06$ & $2.30 \mathrm{E}+01$ \\
\hline Pu-238 & 0.0005 & $5.56 \mathrm{E}+85$ & $5.08 \mathrm{E}+46$ & $7.48 \mathrm{E}+12$ \\
\hline Pu-238 & 0.005 & $5.56 \mathrm{E}+85$ & $5.08 \mathrm{E}+46$ & $7.49 \mathrm{E}+12$ \\
\hline Pu-238 & 0.05 & $5.56 \mathrm{E}+85$ & $5.08 \mathrm{E}+46$ & $7.50 \mathrm{E}+12$ \\
\hline Pu-238 & 0.5 & $5.56 \mathrm{E}+85$ & $5.08 \mathrm{E}+46$ & $7.51 \mathrm{E}+12$ \\
\hline Pu-238 & 5 & $7.00 \mathrm{E}+85$ & $5.08 \mathrm{E}+46$ & $7.51 \mathrm{E}+12$ \\
\hline Pu-239 & 0.0005 & $2.14 \mathrm{E}+85$ & $1.04 \mathrm{E}+42$ & $6.26 \mathrm{E}+02$ \\
\hline Pu-239 & 0.005 & $2.12 \mathrm{E}+85$ & $1.04 \mathrm{E}+42$ & $6.26 \mathrm{E}+02$ \\
\hline Pu-239 & 0.05 & $7.52 \mathrm{E}+85$ & $4.43 \mathrm{E}+42$ & $3.34 \mathrm{E}+03$ \\
\hline Pu-239 & 0.5 & $7.52 \mathrm{E}+86$ & $4.43 E+43$ & $3.34 \mathrm{E}+04$ \\
\hline Pu-240 & 0.0005 & $2.15 \mathrm{E}+85$ & $1.16 \mathrm{E}+42$ & $1.35 \mathrm{E}+03$ \\
\hline Pu-240 & 0.005 & $2.15 \mathrm{E}+85$ & $1.15 \mathrm{E}+42$ & $1.35 \mathrm{E}+03$ \\
\hline Pu-240 & 0.05 & $2.15 \mathrm{E}+85$ & $1.28 \mathrm{E}+42$ & $1.74 \mathrm{E}+03$ \\
\hline Pu-240 & 0.5 & $2.07 \mathrm{E}+86$ & $1.28 \mathrm{E}+43$ & $1.73 \mathrm{E}+04$ \\
\hline Pu-241 & 0.5 & $2.74 \mathrm{E}+40$ & $2.29 \mathrm{E}+11$ & $3.46 \mathrm{E}+06$ \\
\hline Pu-242 & 0.0005 & $2.18 \mathrm{E}+85$ & $1.03 \mathrm{E}+42$ & $4.89 \mathrm{E}+02$ \\
\hline Pu-242 & 0.005 & $1.19 \mathrm{E}+86$ & $6.90 \mathrm{E}+42$ & $4.22 \mathrm{E}+03$ \\
\hline Pu-242 & 0.05 & $1.19 \mathrm{E}+87$ & $6.89 \mathrm{E}+43$ & $4.22 \mathrm{E}+04$ \\
\hline Pu-242 & 0.5 & $1.18 \mathrm{E}+88$ & $6.83 \mathrm{E}+44$ & $4.17 \mathrm{E}+05$ \\
\hline $\mathrm{Pu}-244$ & 0.0005 & $2.64 \mathrm{E}+87$ & $1.53 \mathrm{E}+44$ & $9.23 \mathrm{E}+04$ \\
\hline Pu-244 & 0.005 & $2.61 \mathrm{E}+88$ & $1.50 \mathrm{E}+45$ & $9.00 \mathrm{E}+05$ \\
\hline Pu-244 & 0.05 & $2.27 \mathrm{E}+89$ & $1.26 \mathrm{E}+46$ & $7.22 \mathrm{E}+06$ \\
\hline Pu-244 & 0.5 & $1.35 \mathrm{E}+90$ & $7.67 \mathrm{E}+46$ & $4.62 \mathrm{E}+07$ \\
\hline Am-241 & 0.5 & $9.05 E+38$ & $7.57 \mathrm{E}+09$ & $1.14 \mathrm{E}+05$ \\
\hline Am-243 & 0.0005 & $1.03 E+89$ & $5.55 \mathrm{E}+43$ & $1.40 \mathrm{E}+04$ \\
\hline Am-243 & 0.005 & $1.03 \mathrm{E}+89$ & $5.55 \mathrm{E}+43$ & $1.40 \mathrm{E}+04$ \\
\hline Am-243 & 0.05 & $1.03 \mathrm{E}+89$ & $5.55 \mathrm{E}+43$ & $1.40 \mathrm{E}+04$ \\
\hline Am-243 & 0.5 & $1.03 \mathrm{E}+89$ & $5.63 \mathrm{E}+43$ & $3.17 \mathrm{E}+04$ \\
\hline $\mathrm{Cm}-242$ & 0.0005 & $1.09 \mathrm{E}+88$ & $9.99 \mathrm{E}+48$ & $1.47 \mathrm{E}+15$ \\
\hline
\end{tabular}




\begin{tabular}{|l|c|c|c|c|}
\hline Nuclide & Activity (Ci/unit) & $\begin{array}{c}\text { Limit (Ci) } \\
\mathbf{0 - 1 0 0} \text { yrs }\end{array}$ & $\begin{array}{c}\text { Limit (Ci) } \\
\mathbf{1 0 0 - 1 , 3 5 0} \text { yrs }\end{array}$ & $\begin{array}{c}\text { Limit (Ci) } \\
\mathbf{1 , 3 5 0 - 1 0 , 0 0 0} \text { yrs }\end{array}$ \\
\hline Cm-242 & 0.005 & $1.09 \mathrm{E}+88$ & $9.99 \mathrm{E}+48$ & $1.47 \mathrm{E}+15$ \\
\hline Cm-242 & 0.05 & $1.09 \mathrm{E}+88$ & $9.99 \mathrm{E}+48$ & $1.48 \mathrm{E}+15$ \\
\hline Cm-242 & 0.5 & $1.09 \mathrm{E}+88$ & $9.99 \mathrm{E}+48$ & $1.48 \mathrm{E}+15$ \\
\hline Cm-242 & 5 & $1.38 \mathrm{E}+88$ & $9.99 \mathrm{E}+48$ & $1.48 \mathrm{E}+15$ \\
\hline Cm-244 & 0.0005 & $7.79 \mathrm{E}+87$ & $4.19 \mathrm{E}+44$ & $4.90 \mathrm{E}+05$ \\
\hline Cm-244 & 0.005 & $7.80 \mathrm{E}+87$ & $4.19 \mathrm{E}+44$ & $4.90 \mathrm{E}+05$ \\
\hline Cm-244 & 0.05 & $7.81 \mathrm{E}+87$ & $4.63 \mathrm{E}+44$ & $6.30 \mathrm{E}+05$ \\
\hline Cm-244 & 0.5 & $7.50 \mathrm{E}+88$ & $4.62 \mathrm{E}+45$ & $6.29 \mathrm{E}+06$ \\
\hline Cm-245 & 0.0005 & $1.88 \mathrm{E}+41$ & $4.84 \mathrm{E}+09$ & $8.28 \mathrm{E}+03$ \\
\hline Cm-245 & 0.005 & $1.88 \mathrm{E}+41$ & $4.84 \mathrm{E}+09$ & $8.28 \mathrm{E}+03$ \\
\hline Cm-245 & 0.05 & $1.88 \mathrm{E}+41$ & $4.84 \mathrm{E}+09$ & $8.28 \mathrm{E}+03$ \\
\hline Cm-245 & 0.5 & $3.46 \mathrm{E}+41$ & $4.84 \mathrm{E}+09$ & $8.28 \mathrm{E}+03$ \\
\hline Cm-246 & 0.5 & $1.00 \mathrm{E}+99$ & $2.40 \mathrm{E}+75$ & $8.32 \mathrm{E}+27$ \\
\hline Cm-247 & 0.0005 & $1.85 \mathrm{E}+48$ & $6.81 \mathrm{E}+18$ & $1.50 \mathrm{E}+05$ \\
\hline Cm-247 & 0.005 & $1.85 \mathrm{E}+48$ & $6.81 \mathrm{E}+18$ & $1.50 \mathrm{E}+05$ \\
\hline Cm-247 & 0.05 & $1.85 \mathrm{E}+48$ & $6.81 \mathrm{E}+18$ & $1.50 \mathrm{E}+05$ \\
\hline Cm-247 & 0.5 & $1.85 \mathrm{E}+48$ & $6.81 \mathrm{E}+18$ & $1.50 \mathrm{E}+05$ \\
\hline Cm-248 & 0.0005 & $3.63 \mathrm{E}+92$ & $1.84 \mathrm{E}+47$ & $3.49 \mathrm{E}+07$ \\
\hline Cm-248 & 0.005 & $3.63 \mathrm{E}+92$ & $1.84 \mathrm{E}+47$ & $3.49 \mathrm{E}+07$ \\
\hline Cm-248 & 0.05 & $3.63 \mathrm{E}+92$ & $1.84 \mathrm{E}+47$ & $3.49 \mathrm{E}+07$ \\
\hline Cm-248 & 0.5 & $3.63 \mathrm{E}+92$ & $1.88 \mathrm{E}+47$ & $8.68 \mathrm{E}+07$ \\
\hline Cf-249 & 0.0005 & $1.03 \mathrm{E}+45$ & $1.88 \mathrm{E}+11$ & $2.05 \mathrm{E}+05$ \\
\hline Cf-249 & 0.005 & $1.03 \mathrm{E}+45$ & $1.88 \mathrm{E}+11$ & $2.05 \mathrm{E}+05$ \\
\hline Cf-249 & 0.05 & $1.03 \mathrm{E}+45$ & $1.88 \mathrm{E}+11$ & $2.05 \mathrm{E}+05$ \\
\hline Cf-249 & 0.5 & $1.03 \mathrm{E}+45$ & $1.88 \mathrm{E}+11$ & $2.05 \mathrm{E}+05$ \\
\hline Cf-252 & 0.0005 & $4.77 \mathrm{E}+97$ & $2.42 \mathrm{E}+52$ & $4.60 \mathrm{E}+12$ \\
\hline Cf-252 & 0.005 & $4.77 \mathrm{E}+97$ & $2.42 \mathrm{E}+52$ & $4.60 \mathrm{E}+12$ \\
\hline Cf-252 & 0.05 & $4.77 \mathrm{E}+97$ & $2.42 \mathrm{E}+52$ & $4.60 \mathrm{E}+12$ \\
\hline Cf-252 & 0.5 & $4.77 \mathrm{E}+97$ & $2.47 \mathrm{E}+52$ & $1.14 \mathrm{E}+13$ \\
\hline
\end{tabular}




\subsection{INADVERTENT INTRUDER ANALYSIS}

The inadvertent intruder analysis considers the radiological impacts to hypothetical persons who are assumed to intrude into the E-Area LLWF after institutional control ceases 100 years after facility closure.

\subsection{Revisions}

The intruder analysis was performed with a newly completed software tool for automated analyses (Koffman 2004). The tool eliminates the historical use of extensive spreadsheets that require extensive design checks.

The first major change in the method of analysis was to decouple the intruder analyses from the groundwater pathway analysis by neglecting leaching and only considering decay for the amount of contaminant remaining at the time of intrusion. The groundwater pathway typically used a distribution coefficient $\left(K_{d}\right)$ that was conservative for its own pathway by enhancing the release slightly. However, that value was typically slightly non-conservative for the intruder pathways, because too much release meant that less contaminant remained for the intruder to encounter. This non-conservatism has been removed with the revised method.

The second major change in the method of analysis was to introduce a transient analysis for each type of intrusion, rather than selecting a fixed time. The decay process continually changes the amount of contaminant present in the waste zone that the intruder can encounter. While the amount of parent monotonically decreases, the amount of each progeny initially increases and ultimately decreases. At the same time as the decay process, sediments and engineered materials can erode and degrade. Determining the time when the most conservative impact on the intruder will occur is impossible, unless a rigorous examination is conducted with calculus or a transient analysis is performed. The new method selected a transient analysis that is valid across the spectrum of disposal units and does not require extensive calculations by the analyst; rather it requires the analyst to define geometry and process inputs, and then relies on the computer model to perform pathways calculations at a specified time increment that is nominally 10 years.

The first change in the implementation was selection of a new geometry that includes a 12 inch thick erosion barrier near the top of the cap (Phifer 2004a). Because the erosion barrier is assumed to never erode and all the layers between the waste and the erosion barrier always remain in place at their design thickness, about $10.4 \mathrm{ft}$ of material always exists above the waste. That thickness is greater than the depth of a typical basement $(3 \mathrm{~m}$ or $9.8 \mathrm{ft}$ ) and the agriculture scenario can never occur, because the agriculture scenario relies on a basement extending into the waste zone. Additionally, the concrete roof of the vault will prevent excavation through it for more than 1,000 years.

For the resident scenario, the erosion barrier greatly increases the amount of material above the waste that serves to shield the residential intruder. For the post-drilling scenario, the erosion barrier does not prevent drilling through the waste. However, the grout was assumed to prevent drilling through the waste, thus the post-drilling scenario was not considered for the ILV.

The second change in the implementation was selection of a consistent volume for the ILV. The ILV consists of 9 cells, where 2 were designed to contain tritiated waste, while the other 7 were designed for non-tritium waste. The PA intruder analysis was based on the volume of the nontritium, "ILNTV", portion of the facility, or 5,700 $\mathrm{m}^{3}$ (McDowell-Boyer et al. 2000, Section 3.3.2.3). Presently, no distinction is made between the ILNTV and tritium (ILTV) portions of the facility in setting limits, and a second facility will not have separate tritium and non-tritium compartments. Therefore, in the present analysis considering two disposal units, an IL vault 
volume of 7,300 $\mathrm{m}^{3}$ is assumed in the intruder analysis. The larger facility volume includes 1,600 $\mathrm{m}^{3}$ reported in the PA for the ILTV (McDowell-Boyer et al. 2000, Section 3.3.2.3).

The PA states "the geometrical correction factor, G, in Eq. 6.3-1 takes into account that a largescale excavation into disposal units, as assumed in the agriculture and resident scenarios, would involve exposure to uncontaminated material between individual disposal units as well as disposed waste itself." "Therefore, the geometrical correction factor is given by the fraction of the land area encompassed by the disposal units of a particular type that contains waste." (McDowellBoyer et al. 2000, page 6-20). The PA provides a geometrical reduction factor, G, in Table 6-3-4 of McDowell-Boyer et al. (2000) for the ILV of 0.4 for IL vaults. This setting is consistent with current plans to locate a second IL vault at footprint \#5 (cf. drawing W2017860). The resulting spacing between IL vaults would be $150 \mathrm{ft}$.

Soil/cover layers overlying the IL vault roof were adjusted to be consistent with the current closure plan (Phifer 2004a, b). Appendix B provides additional information on disposal unit specific inputs to the automated intruder analysis.

\subsection{Results}

Because the agriculture scenario was eliminated based on using an erosion barrier, the PA agriculture scenario table (Table 6.3-5) was eliminated. Results of the resident intruder analyses are provided in Table 3-1 (that replaces Table 6.3-11 and Table 6.3-12 in the PA). Additional detail is provided in Appendix B. The PA tables were for 100 years and 10,000 years. Because the new method applies a transient analysis, it provides the lowest inventory for the entire time period, regardless of when it occurs. The new analysis stopped at 1,000 years, which is the new time for compliance.

The vault roof and upper layer of clean grout provide more than a meter of shielding throughout the resident scenario transient. Consequently, exposure levels are relatively low compared to other disposal units (e.g. slit and engineered trenches). Limits are correspondingly high. For the IL vault inventory and volume-filled (36\%) as of $7 / 2 / 04$, none of the radionuclides produce a significantly large fraction of the intruder limit, as discussed later in Chapter 6.0.

Table 3-1. Inventory limits for the intruder resident scenario; transient calculation for $1,000$ years (--- denotes limit exceeding $1 \mathrm{E}+20 \mathrm{Ci})$.

$\begin{array}{ccc}\begin{array}{c}\text { Time of Maximum Dose } \\ \text { (Years) }\end{array} & \begin{array}{c}\text { Concentration Limit } \\ \left(\mathbf{m C i}^{3} \mathbf{m}^{3}\right.\end{array} & \begin{array}{c}\text { Inventory Limit } \\ \text { (Ci/unit) }\end{array} \\ --- & --- & --- \\ --- & --- & -- \\ 100 & 1.48 \mathrm{E}+17 & 1.08 \mathrm{E}+15 \\ 100 & 9.08 \mathrm{E}+04 & 6.62 \mathrm{E}+02 \\ --- & --- & --- \\ --- & -- & -- \\ --- & --- & -- \\ 100 & 2.10 \mathrm{E}+06 & 1.53 \mathrm{E}+04 \\ --- & -- & -- \\ 100 & --- & --- \\ 100 & 1.09 \mathrm{E}+11 & 7.98 \mathrm{E}+08 \\ --- & --- & --- \\ --- & -- & --- \\ --- & --- & -- \\ 100 & 5.20 \mathrm{E}+12 & 3.79 \mathrm{E}+10 \\ --- & -- & --\end{array}$

Rev. 0 
Time of Maximum Dose Concentration Limit Inventory Limit

\begin{tabular}{|c|c|c|c|}
\hline Radionuclide & (Years) & $\left(\mathrm{mCi} / \mathrm{m}^{3}\right)$ & (Cilunit) \\
\hline Sr-90 & --- & --- & --- \\
\hline Zr-93 & --- & --- & --- \\
\hline $\mathrm{Nb}-93 \mathrm{~m}$ & --- & --- & --- \\
\hline $\mathrm{Nb}-94$ & 100 & $1.81 \mathrm{E}+06$ & $1.32 \mathrm{E}+04$ \\
\hline Mo-93 & --- & --- & --- \\
\hline Tc-99 & 100 & --- & $7.66 \mathrm{E}+17$ \\
\hline $\mathrm{Pd}-107$ & --- & --- & --- \\
\hline $\mathrm{Ag}-108 \mathrm{~m}$ & 100 & $5.35 \mathrm{E}+06$ & $3.91 \mathrm{E}+04$ \\
\hline Cd-113m & --- & --- & --- \\
\hline Sn-121m & --- & --- & --- \\
\hline Sn-126 & 100 & $2.92 \mathrm{E}+06$ & $2.13 \mathrm{E}+04$ \\
\hline Sb-125 & 100 & $2.69 \mathrm{E}+18$ & $1.96 \mathrm{E}+16$ \\
\hline $\mathrm{I}-129$ & 100 & --- & --- \\
\hline Cs-134 & 100 & --- & $5.72 \mathrm{E}+18$ \\
\hline Cs-135 & --- & --- & --- \\
\hline Cs-137 & 100 & $1.14 \mathrm{E}+08$ & $8.33 E+05$ \\
\hline Ba-133 & 100 & $2.29 \mathrm{E}+11$ & $1.68 \mathrm{E}+09$ \\
\hline Sm-151 & --- & --- & --- \\
\hline Eu-152 & 100 & $1.22 \mathrm{E}+08$ & $8.92 \mathrm{E}+05$ \\
\hline Eu-154 & 100 & $2.19 \mathrm{E}+09$ & $1.60 \mathrm{E}+07$ \\
\hline Eu-155 & 100 & --- & $1.55 \mathrm{E}+18$ \\
\hline W-181 & 100 & --- & --- \\
\hline W-185 & 100 & --- & --- \\
\hline W-188 & 100 & --- & --- \\
\hline $\mathrm{Pb}-210$ & 100 & $7.49 \mathrm{E}+12$ & $5.47 \mathrm{E}+10$ \\
\hline Bi-207 & 100 & $5.87 \mathrm{E}+06$ & $4.28 \mathrm{E}+04$ \\
\hline Ra-226 & 100 & $1.94 \mathrm{E}+05$ & $1.42 \mathrm{E}+03$ \\
\hline Ra-228 & 100 & 7.07E+09 & $5.16 \mathrm{E}+07$ \\
\hline Ac-227 & 100 & $1.67 \mathrm{E}+09$ & $1.22 \mathrm{E}+07$ \\
\hline Th-228 & 100 & --- & $2.61 \mathrm{E}+18$ \\
\hline Th-229 & 100 & $7.20 \mathrm{E}+06$ & $5.25 \mathrm{E}+04$ \\
\hline Th-230 & 1000 & $5.32 \mathrm{E}+05$ & $3.88 \mathrm{E}+03$ \\
\hline Th-232 & 180 & $6.05 \mathrm{E}+04$ & $4.42 \mathrm{E}+02$ \\
\hline Pa-231 & 230 & $6.93 \mathrm{E}+07$ & $5.06 \mathrm{E}+05$ \\
\hline U-232 & 100 & $1.71 \mathrm{E}+05$ & $1.25 \mathrm{E}+03$ \\
\hline U-233 & 1000 & $7.93 \mathrm{E}+07$ & $5.79 \mathrm{E}+05$ \\
\hline U-234 & 1000 & $1.08 \mathrm{E}+08$ & 7.87E+05 \\
\hline U-235 & 1000 & $3.30 \mathrm{E}+09$ & $2.41 \mathrm{E}+07$ \\
\hline U-236 & 1000 & $1.24 \mathrm{E}+12$ & $9.05 E+09$ \\
\hline U-238 & 1000 & $1.06 \mathrm{E}+08$ & 7.74E+05 \\
\hline Np-237 & 1000 & $8.82 \mathrm{E}+08$ & $6.44 \mathrm{E}+06$ \\
\hline Pu-238 & 1000 & $3.82 E+11$ & $2.79 \mathrm{E}+09$ \\
\hline Pu-239 & 1000 & $4.55 \mathrm{E}+15$ & $3.32 E+13$ \\
\hline Pu-240 & 1000 & $8.77 \mathrm{E}+16$ & $6.40 \mathrm{E}+14$ \\
\hline Pu-241 & 1000 & 1.69E+14 & $1.24 \mathrm{E}+12$ \\
\hline Pu-242 & 1000 & $6.84 \mathrm{E}+14$ & $5.00 \mathrm{E}+12$ \\
\hline Pu-244 & 100 & $5.01 \mathrm{E}+06$ & $3.66 \mathrm{E}+04$ \\
\hline Am-241 & 1000 & $5.55 \mathrm{E}+12$ & $4.05 E+10$ \\
\hline Am-242m & 100 & $7.84 \mathrm{E}+08$ & $5.72 \mathrm{E}+06$ \\
\hline Am-243 & 100 & $6.02 \mathrm{E}+09$ & $4.39 \mathrm{E}+07$ \\
\hline $\mathrm{Cm}-242$ & 1000 & $7.53 \mathrm{E}+13$ & $5.49 \mathrm{E}+11$ \\
\hline $\mathrm{Cm}-243$ & 100 & $1.33 \mathrm{E}+11$ & $9.73 \mathrm{E}+08$ \\
\hline $\mathrm{Cm}-244$ & 1000 & $3.35 E+19$ & $2.45 E+17$ \\
\hline $\mathrm{Cm}-245$ & 1000 & $1.13 \mathrm{E}+12$ & $8.24 \mathrm{E}+09$ \\
\hline
\end{tabular}

Rev. 0 


\begin{tabular}{cccc} 
Radionuclide & $\begin{array}{c}\text { Time of Maximum Dose } \\
\text { (Years) }\end{array}$ & $\begin{array}{c}\text { Concentration Limit } \\
\left(\mathbf{m C i}^{\mathbf{3}}\right)\end{array}$ & $\begin{array}{c}\text { Inventory Limit } \\
\text { (Ci/unit) }\end{array}$ \\
\hline Cm-246 & 1000 & $7.74 \mathrm{E}+17$ & $5.65 \mathrm{E}+15$ \\
Cm-247 & 1000 & $1.96 \mathrm{E}+08$ & $1.43 \mathrm{E}+06$ \\
Cm-248 & 1000 & $6.31 \mathrm{E}+11$ & $4.60 \mathrm{E}+09$ \\
Bk-249 & 100 & $1.10 \mathrm{E}+11$ & $8.06 \mathrm{E}+08$ \\
Cf-249 & 100 & $2.85 \mathrm{E}+08$ & $2.08 \mathrm{E}+06$ \\
Cf-250 & 1000 & --- & $2.13 \mathrm{E}+18$ \\
Cf-251 & 100 & $5.10 \mathrm{E}+10$ & $3.72 \mathrm{E}+08$ \\
Cf-252 & 1000 & $8.60 \mathrm{E}+16$ & $6.27 \mathrm{E}+14$
\end{tabular}




\subsection{AIR ANALYSIS}

The air analysis considers diffusion of volatile radionuclides from the waste zone through overlying soil layers to the ground surface. The specific radionuclides analyzed are $\mathrm{H}-3$ and C-14; radon is assessed separately in Chapter 5.0.

\subsection{Revisions}

The present analysis uses updated dose factors based on average meterological conditions for the period 1997-2001 measured near H-Area (Simpkins 2004), but retains the calculation method used in the PA. In computing dose-release factors, an atmospheric plume was assumed to result from a point release at ground level, and be depleted by radioactive decay and deposition. Dose to a maximally exposed individual was calculated for six pathways: plume shine, ground shine, inhalation, vegetable consumption, milk consumption, and meat consumption. Parameters were chosen to maximize the dose. The release fractions ( $\mathrm{Ci} / \mathrm{yr}$ annual release per $\mathrm{Ci}$ of inventory) computed in the PA were retained in the present analysis. Among the waste forms considered in the PA for the IL vault, job control waste is most limiting and the focus of the present analysis.

\subsection{Results}

Table 4-1 summarizes inputs and results for the air pathway analysis, following the calculation method described in the PA (McDowell-Boyer et al. 2000, Sections 4.1.2, 4.2.2 and 5.2). Appendix C provides additional detail. The current inventory of C-14 in the IL vaults corresponds to about $9 \%$ of the air pathway limit, while the contribution of $\mathrm{H}-3$ to the sum-of-fractions is negligible.

Table 4-1. Summary of air pathway analysis for H-3 and C-14.

\begin{tabular}{|cc|cc|l|}
\hline $\mathbf{H}-3$ & & $\mathbf{C}-14$ & & ILV \\
$100 m$ & Boundary & $100 m$ & Boundary & Parameter \\
\hline 10 & 10 & 10 & 10 & Dose limit (mrem/yr) \\
$6.6 \mathrm{E}-03$ & $2.4 \mathrm{E}-06$ & 2.4 & $1.1 \mathrm{E}-03$ & Dose factor (mrem/Ci) \\
$3.2 \mathrm{E}-04$ & $3.2 \mathrm{E}-04$ & 1 & 1 & Release fraction $(\mathrm{Ci} / \mathrm{yr}$ per Ci inventory) \\
$2.1 \mathrm{E}-06$ & $7.7 \mathrm{E}-10$ & $2.4 \mathrm{E}+00$ & $1.1 \mathrm{E}-03$ & Dose fraction (mrem/yr per Ci inventory) \\
100 & 0 & 100 & 0 & Lag time (yrs) \\
12.33 & 12.33 & 5730 & 5730 & Halflife (yrs) \\
276 & 1 & 1 & 1 & Decay factor \\
$1.3 \mathrm{E}+09$ & $1.3 \mathrm{E}+10$ & 4.2 & $9.1 \mathrm{E}+03$ & Limit (Ci inventory) \\
\hline
\end{tabular}


THIS PAGE INTENTIONALLY LEFT BLANK 


\subsection{RADON ANALYSIS}

DOE Order 435.1 sets a performance objective of $20 \mathrm{pCi} / \mathrm{m}^{2} / \mathrm{s}$ or less for the average surface flux of radon gas emanating from a disposal facility (USDOE 1999a). Disposal unit performance against this objective is assessed through a separate analysis for radon.

\subsection{Revisions}

In the PA, radon is assumed to be released through the ground surface from decay of disposed U-234. In the present analysis, additional ancesters of Rn-222 are considered, namely Ra-226, Th-230, U-238 and Pu-238. Radionuclides that create Rn-222 are illustrated in Figure 5-1. The diagram shows the specific decay chains that lead to the formation of Rn-222, as well as the halflives for each nuclide. The extremely long half-lives of Pu-242 and U-238 (375,500 and $4,500,000$ years respectively) cause the other radioisotopes higher up in the chain of parents to be of little concern with regard to their potential to contribute significantly to the Rn-222 flux at the land surface over the period of interest.

A conservative approach to estimating radon flux from the waste zone to ground surface was taken in the PA. In the present Special Analysis, a more realistic conceptual model of radon transport was developed, as described in detail in Appendix D. The revised analysis incorporates a radon emanation coefficient, and waste zone layering and properties (e.g. porosity) more consistent with anticipated conditions.

\subsection{Results}

Table 5-1 summarizes the limits resulting from the radon analysis. The inventory of Ra-226 in the IL vaults as $7 / 2 / 04$ corresponds to about $30 \%$ of the radon limit, while the contribution of other nuclides in Table 5-1 to the sum-of-fractions is negligible. For comparison the volume-filled is approximately $36 \%$.

Table 5-1 Simulated peak instantaneous Rn-222 flux over 1,000-years at the land surface and associated disposal limits for parent isotopes.

\begin{tabular}{cccc}
$\begin{array}{c}\text { Parent Source } \\
(1 \mathrm{Ci})\end{array}$ & $\begin{array}{c}\text { Peak Instantaneous Rn- } \\
\text { 222 Flux at land surface } \\
\left(\mathrm{pCi} / \mathrm{m}^{2}-\mathrm{s}\right)\end{array}$ & $\begin{array}{c}\text { Unit Disposal Limit } \\
\text { of parent isotope } \\
\left(\mathrm{Ci} / \mathrm{m}^{2}\right)\end{array}$ & $\begin{array}{c}\text { Disposal Limit of parent } \\
\text { per ILV }\end{array}$ \\
\hline Pu-238 & $5.38 \mathrm{E}-03$ & $3.72 \mathrm{E}+03$ & $\begin{array}{c}\left(75 \mathrm{~m} \times 15 \mathrm{~m}=1125 \mathrm{~m}^{2}\right) \\
(\mathrm{Ci} / / \mathrm{LV})\end{array}$ \\
U-238 & $2.05 \mathrm{E}-02$ & $9.74 \mathrm{E}+02$ & $4.18 \mathrm{E}+06$ \\
U-234 & $1.86 \mathrm{E}+01$ & $1.08 \mathrm{E}+00$ & $1.10 \mathrm{E}+06$ \\
Th-230 & $3.31 \mathrm{E}+03$ & $6.04 \mathrm{E}-03$ & $1.21 \mathrm{E}+03$ \\
Ra-226 & $8.72 \mathrm{E}+03$ & $2.29 \mathrm{E}-03$ & $6.79 \mathrm{E}+00$ \\
& & & $2.58 \mathrm{E}+00$
\end{tabular}




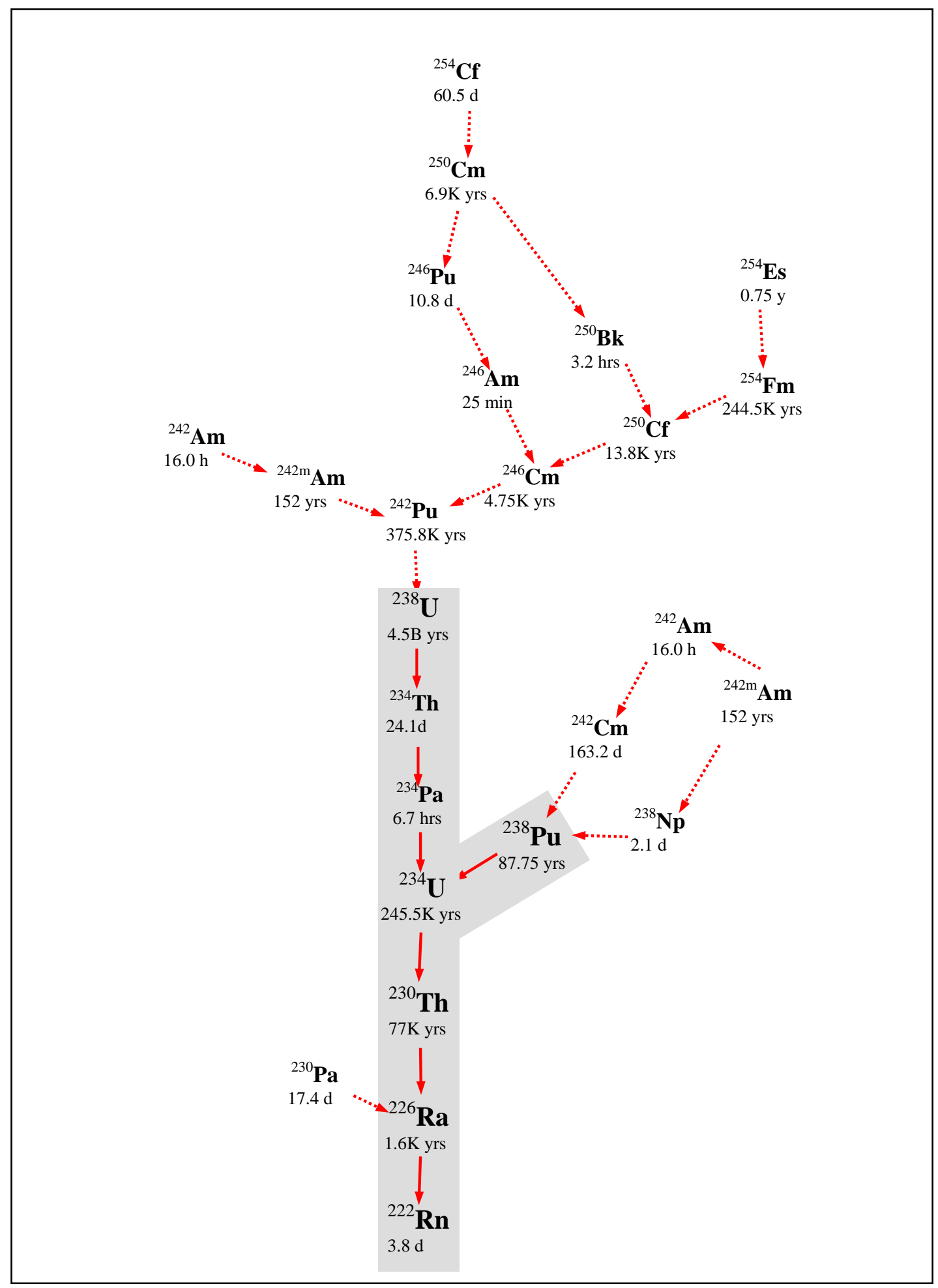

Figure 5-1. Decay chain of parent radionuclides leading to Rn-222 formation. 


\subsection{DISPOSAL LIMITS}

Table 6-1 compares the disposal limits computed for each pathway to the IL vault inventory as of $7 / 2 / 04$. No limit is shown for nuclides that were previously screened (e.g. Cook (2004) for the intruder and groundwater pathways). Also shown in the table are inventory fractions and the sumof-fractions for each pathway. GW1 refers to the 0-100 year period, and GW2 to the $100-1,350$ year time frame for the groundwater pathway.

Present limits are based on a time of compliance of 1,000 years $(1,350$ for the groundwater pathway to account for uncertainty in the vault failure time), compared to 10,000 years in the PA. As a consequence, many of the limits are substantially higher than those in Cook (2002b). Limits for $\mathrm{U}$ isotopes in the groundwater pathway increased significantly due to a change of $K_{d}$ in soil from $35 \mathrm{~mL} / \mathrm{g}$ to $800 \mathrm{~mL} / \mathrm{g}$. The revised infiltration scenario in the vadose zone analysis, refinement of the aquifer model, and expanded consideration of solubility limits, among other revisions listed in the Introduction, produced smaller differences.

The largest fraction is $30 \%$ for Ra-226 and the radon analysis, followed by $11 \%$ for I-129 in the groundwater pathway, and 9\% for $\mathrm{C}-14$ and the air pathway. For comparison, the volume-filled fraction is about $36 \%$. Considering that essentially all the radon fraction is due to a single container, continued operation of the IL vault should not challenge performance objectives, assuming future disposal patterns are similar to historic use of the facility.

Table 6-2 summarizes the disposal limits recommended for the IL vault. 
Table 6-1 Inventory limits from all pathways compared to the IL vault inventory as of 7/2/04.

\begin{tabular}{|c|c|c|c|c|c|c|c|c|c|c|c|}
\hline nuclide & $\begin{array}{l}\text { resident } \\
\text { limit (Ci) }\end{array}$ & $\begin{array}{c}\text { air limit } \\
\text { (Ci) }\end{array}$ & $\begin{array}{c}\text { radon } \\
\text { limit }(\mathrm{Ci})\end{array}$ & $\begin{array}{l}\text { GW1 limit } \\
\text { (Ci) }\end{array}$ & $\begin{array}{l}\text { GW2 limit } \\
\text { (Ci) }\end{array}$ & $\begin{array}{l}\text { inventory } \\
\text { (Ci) }\end{array}$ & $\begin{array}{l}\text { resident } \\
\text { fraction }\end{array}$ & $\begin{array}{c}\text { air } \\
\text { fraction }\end{array}$ & $\begin{array}{c}\text { radon } \\
\text { fraction }\end{array}$ & $\begin{array}{c}\text { GW1 } \\
\text { fraction }\end{array}$ & $\begin{array}{c}\text { GW2 } \\
\text { fraction }\end{array}$ \\
\hline SOF & & & & & & & 0.001 & 0.101 & 0.295 & 0.000 & 0.132 \\
\hline Ac-227 & $1.2 \mathrm{E}+07$ & & & & & & $0.0 \mathrm{E}+00$ & & & & \\
\hline Ac-228 & & & & & & $1.8 \mathrm{E}-05$ & & & & & \\
\hline $\mathrm{Ag}-108 \mathrm{~m}$ & $3.9 \mathrm{E}+04$ & & & & & & $0.0 \mathrm{E}+00$ & & & & \\
\hline $\mathrm{Ag}-110 \mathrm{~m}$ & & & & & & $3.0 \mathrm{E}-09$ & & & & & \\
\hline Al-26 & $6.6 \mathrm{E}+02$ & & & & & & $0.0 \mathrm{E}+00$ & & & & \\
\hline $\mathrm{Am}-241$ & $4.1 \mathrm{E}+10$ & & & $\geq 1.0 \mathrm{E}+20$ & $7.6 \mathrm{E}+09$ & $3.5 \mathrm{E}-01$ & $8.6 \mathrm{E}-12$ & & & $3.5 \mathrm{E}-21$ & $4.6 \mathrm{E}-11$ \\
\hline Am-242m & $5.7 \mathrm{E}+06$ & & & & & $3.2 \mathrm{E}-04$ & $5.5 \mathrm{E}-11$ & & & & \\
\hline Am-243 & $4.4 \mathrm{E}+07$ & & & $\geq 1.0 \mathrm{E}+20$ & $\geq 1.0 \mathrm{E}+20$ & 4.3E-04 & $9.8 \mathrm{E}-12$ & & & $4.3 \mathrm{E}-24$ & $4.3 \mathrm{E}-24$ \\
\hline Ba-133 & $1.7 \mathrm{E}+09$ & & & & & & $0.0 \mathrm{E}+00$ & & & & \\
\hline Ba-137m & & & & & & $1.5 \mathrm{E}+02$ & & & & & \\
\hline $\mathrm{Bi}-207$ & $4.3 \mathrm{E}+04$ & & & & & & $0.0 \mathrm{E}+00$ & & & & \\
\hline Bi-210 & & & & $\geq 1.0 \mathrm{E}+20$ & $\geq 1.0 \mathrm{E}+20$ & $6.1 \mathrm{E}-07$ & & & & $6.1 \mathrm{E}-27$ & $6.1 \mathrm{E}-27$ \\
\hline $\mathrm{Bi}-211$ & & & & & & $3.2 \mathrm{E}-07$ & & & & & \\
\hline Bi-212 & & & & & & 1.3E-04 & & & & & \\
\hline Bi-214 & & & & & & 7.7E-01 & & & & & \\
\hline Bk-249 & $8.1 \mathrm{E}+08$ & & & & & & $0.0 \mathrm{E}+00$ & & & & \\
\hline C-14 & $\geq 1.0 \mathrm{E}+20$ & $4.2 \mathrm{E}+00$ & & $\geq 1.0 \mathrm{E}+20$ & $9.1 \mathrm{E}+07$ & 3.6E-01 & 3.6E-21 & 8.7E-02 & & $3.6 \mathrm{E}-21$ & $4.0 \mathrm{E}-09$ \\
\hline C-14 KB & & $4.2 \mathrm{E}+00$ & & $\geq 1.0 \mathrm{E}+20$ & $2.6 \mathrm{E}+06$ & $6.1 \mathrm{E}-02$ & & $1.4 \mathrm{E}-02$ & & $6.1 \mathrm{E}-22$ & $2.4 \mathrm{E}-08$ \\
\hline $\mathrm{Ca}-41$ & $\geq 1.0 \mathrm{E}+20$ & & & & & & $0.0 \mathrm{E}+00$ & & & & \\
\hline $\mathrm{Cd}-113 \mathrm{~m}$ & $\geq 1.0 \mathrm{E}+20$ & & & & & & $0.0 \mathrm{E}+00$ & & & & \\
\hline Ce-144 & & & & & & $4.2 \mathrm{E}-01$ & & & & & \\
\hline Cf-249 & $2.1 \mathrm{E}+06$ & & & $\geq 1.0 \mathrm{E}+20$ & $1.9 \mathrm{E}+11$ & $5.6 \mathrm{E}-07$ & $2.7 \mathrm{E}-13$ & & & $5.6 \mathrm{E}-27$ & $3.0 \mathrm{E}-18$ \\
\hline Cf-250 & $2.1 \mathrm{E}+18$ & & & & & & $0.0 \mathrm{E}+00$ & & & & \\
\hline Cf-251 & $3.7 \mathrm{E}+08$ & & & & & 1.7E-06 & 4.6E-15 & & & & \\
\hline Cf-252 & $6.3 \mathrm{E}+14$ & & & $\geq 1.0 \mathrm{E}+20$ & $\geq 1.0 \mathrm{E}+20$ & & $0.0 \mathrm{E}+00$ & & & $0.0 \mathrm{E}+00$ & $0.0 \mathrm{E}+0 \mathrm{C}$ \\
\hline $\mathrm{Cl}-36$ & $\geq 1.0 \mathrm{E}+20$ & & & & & & $0.0 \mathrm{E}+00$ & & & & \\
\hline $\mathrm{Cm}-242$ & $5.5 \mathrm{E}+11$ & & & $\geq 1.0 \mathrm{E}+20$ & $\geq 1.0 \mathrm{E}+20$ & $1.4 \mathrm{E}-05$ & $2.6 \mathrm{E}-17$ & & & $1.4 \mathrm{E}-25$ & $1.4 \mathrm{E}-25$ \\
\hline $\mathrm{Cm}-243$ & $9.7 \mathrm{E}+08$ & & & & & $7.1 \mathrm{E}-03$ & $7.3 \mathrm{E}-12$ & & & & \\
\hline $\mathrm{Cm}-244$ & $2.5 \mathrm{E}+17$ & & & $\geq 1.0 \mathrm{E}+20$ & $\geq 1.0 \mathrm{E}+20$ & 5.9E-01 & $2.4 \mathrm{E}-18$ & & & $5.9 \mathrm{E}-21$ & $5.9 \mathrm{E}-21$ \\
\hline $\mathrm{Cm}-245$ & $8.2 \mathrm{E}+09$ & & & $\geq 1.0 \mathrm{E}+20$ & $4.8 \mathrm{E}+09$ & $7.0 \mathrm{E}-06$ & $8.5 \mathrm{E}-16$ & & & $7.0 \mathrm{E}-26$ & $1.4 \mathrm{E}-15$ \\
\hline $\mathrm{Cm}-246$ & $5.7 \mathrm{E}+15$ & & & $\geq 1.0 \mathrm{E}+20$ & $\geq 1.0 \mathrm{E}+20$ & $8.5 \mathrm{E}-06$ & $1.5 \mathrm{E}-21$ & & & $8.5 \mathrm{E}-26$ & $8.5 \mathrm{E}-26$ \\
\hline $\mathrm{Cm}-247$ & $1.4 \mathrm{E}+06$ & & & $\geq 1.0 \mathrm{E}+20$ & $6.8 \mathrm{E}+18$ & $2.0 \mathrm{E}-10$ & $1.4 \mathrm{E}-16$ & & & $2.0 \mathrm{E}-30$ & $2.9 \mathrm{E}-29$ \\
\hline $\mathrm{Cm}-248$ & $4.6 \mathrm{E}+09$ & & & $\geq 1.0 \mathrm{E}+20$ & $\geq 1.0 \mathrm{E}+20$ & $3.1 \mathrm{E}-15$ & $6.7 \mathrm{E}-25$ & & & $3.1 \mathrm{E}-35$ & $3.1 \mathrm{E}-35$ \\
\hline Co-57 & & & & & & $5.1 \mathrm{E}-05$ & & & & & \\
\hline Co-58 & & & & & & $2.3 \mathrm{E}+00$ & & & & & \\
\hline Co-60 & $8.0 \mathrm{E}+08$ & & & & & $7.5 \mathrm{E}+01$ & $9.4 \mathrm{E}-08$ & & & & \\
\hline Cr-51 & & & & & & $8.4 \mathrm{E}-03$ & & & & & \\
\hline Cs-134 & & & & & & 7.7E-01 & & & & & \\
\hline
\end{tabular}




\begin{tabular}{|c|c|c|c|c|c|c|c|c|c|c|c|}
\hline nuclide & $\begin{array}{l}\text { resident } \\
\text { limit (Ci) }\end{array}$ & $\begin{array}{l}\text { air limit } \\
\text { (Ci) }\end{array}$ & $\begin{array}{c}\text { radon } \\
\text { limit (Ci) }\end{array}$ & $\begin{array}{l}\text { GW1 limit } \\
\text { (Ci) }\end{array}$ & $\begin{array}{l}\text { GW2 limit } \\
\text { (Ci) }\end{array}$ & $\begin{array}{l}\text { inventory } \\
\text { (Ci) }\end{array}$ & $\begin{array}{l}\text { resident } \\
\text { fraction }\end{array}$ & $\begin{array}{c}\text { air } \\
\text { fraction }\end{array}$ & $\begin{array}{c}\text { radon } \\
\text { fraction }\end{array}$ & $\begin{array}{c}\text { GW1 } \\
\text { fraction }\end{array}$ & $\begin{array}{c}\text { GW2 } \\
\text { fraction }\end{array}$ \\
\hline Cs-135 & $\geq 1.0 \mathrm{E}+20$ & & & $\geq 1.0 \mathrm{E}+20$ & $\geq 1.0 \mathrm{E}+20$ & & $0.0 \mathrm{E}+00$ & & & $0.0 \mathrm{E}+00$ & $0.0 \mathrm{E}+00$ \\
\hline Cs-137 & $8.3 \mathrm{E}+05$ & & & & & $2.2 \mathrm{E}+02$ & $2.6 \mathrm{E}-04$ & & & & \\
\hline Eu-152 & $8.9 \mathrm{E}+05$ & & & & & 4.1E-05 & $4.6 \mathrm{E}-11$ & & & & \\
\hline Eu-154 & $1.6 \mathrm{E}+07$ & & & & & 1.8E-01 & 1.1E-08 & & & & \\
\hline Eu-155 & $1.6 \mathrm{E}+18$ & & & & & $1.2 \mathrm{E}-02$ & $8.0 \mathrm{E}-21$ & & & & \\
\hline Fe-55 & & & & & & $8.4 \mathrm{E}+01$ & & & & & \\
\hline Fe-59 & & & & & & 1.6E-02 & & & & & \\
\hline $\mathrm{H}-3$ & $\geq 1.0 \mathrm{E}+20$ & $1.3 \mathrm{E}+09$ & & $5.3 E+09$ & $5.5 \mathrm{E}+09$ & $5.3 \mathrm{E}+05$ & $5.3 \mathrm{E}-15$ & 4.0E-04 & & 9.9E-05 & 9.6E-05 \\
\hline $\mathrm{Hf}-175$ & & & & & & $1.9 \mathrm{E}-05$ & & & & & \\
\hline Hf-181 & & & & & & $6.1 \mathrm{E}-03$ & & & & & \\
\hline $\mid-129$ & $\geq 1.0 \mathrm{E}+20$ & & & $4.3 \mathrm{E}+10$ & $4.6 \mathrm{E}-04$ & $5.1 \mathrm{E}-05$ & $5.1 \mathrm{E}-25$ & & & $1.2 \mathrm{E}-15$ & 1.1E-01 \\
\hline $\begin{array}{l}\text { I- } \\
129 \text { ETF- } \\
\text { Carbon }\end{array}$ & & & & $1.2 \mathrm{E}+13$ & 9.7E-02 & $2.0 \mathrm{E}-03$ & & & & $1.6 \mathrm{E}-16$ & $2.1 \mathrm{E}-02$ \\
\hline I-129_KB & & & & $\geq 1.0 \mathrm{E}+20$ & $6.2 \mathrm{E}+03$ & $6.0 \mathrm{E}-06$ & & & & $6.0 \mathrm{E}-26$ & $9.7 \mathrm{E}-10$ \\
\hline $\ln -113 \mathrm{~m}$ & & & & & & 5.0E-06 & & & & & \\
\hline $\mathrm{K}-40$ & $1.5 \mathrm{E}+04$ & & & $2.3 E+19$ & $2.0 \mathrm{E}-01$ & $7.8 \mathrm{E}-07$ & $5.1 \mathrm{E}-11$ & & & $3.3 \mathrm{E}-26$ & 3.9E-06 \\
\hline $\mathrm{Kr}-85$ & $3.8 \mathrm{E}+10$ & & & & & $2.0 \mathrm{E}+01$ & 5.3E-10 & & & & \\
\hline $\mathrm{Mn}-54$ & & & & & & $4.2 \mathrm{E}-01$ & & & & & \\
\hline Mo-93 & $\geq 1.0 \mathrm{E}+20$ & & & & & & $0.0 \mathrm{E}+00$ & & & & \\
\hline $\mathrm{Na}-22$ & $1.1 \mathrm{E}+15$ & & & & & & $0.0 \mathrm{E}+00$ & & & & \\
\hline $\mathrm{Nb}-93 \mathrm{~m}$ & $\geq 1.0 \mathrm{E}+20$ & & & & & $2.7 \mathrm{E}-03$ & 2.7E-23 & & & & \\
\hline $\mathrm{Nb}-94$ & $1.3 \mathrm{E}+04$ & & & $\geq 1.0 \mathrm{E}+20$ & $\geq 1.0 \mathrm{E}+20$ & 1.1E-03 & 8.0E-08 & & & 1.1E-23 & 1.1E-23 \\
\hline $\mathrm{Nb}-95$ & & & & & & $2.5 \mathrm{E}-02$ & & & & & \\
\hline $\mathrm{Nb}-95 \mathrm{~m}$ & & & & $\geq 1.0 \mathrm{E}+20$ & $\geq 1.0 \mathrm{E}+20$ & 9.6E-05 & & & & $9.6 \mathrm{E}-25$ & 9.6E-25 \\
\hline $\mathrm{Ni}-59$ & $\geq 1.0 \mathrm{E}+20$ & & & $\geq 1.0 \mathrm{E}+20$ & $\geq 1.0 \mathrm{E}+20$ & $5.4 \mathrm{E}-02$ & $5.4 \mathrm{E}-22$ & & & $5.4 \mathrm{E}-22$ & $5.4 \mathrm{E}-22$ \\
\hline $\mathrm{Ni}-63$ & $\geq 1.0 \mathrm{E}+20$ & & & & & $1.2 \mathrm{E}+01$ & $1.2 \mathrm{E}-19$ & & & & \\
\hline $\mathrm{Np}-237$ & $6.4 \mathrm{E}+06$ & & & $\geq 1.0 \mathrm{E}+20$ & $1.5 \mathrm{E}+06$ & 1.9E-03 & $3.0 \mathrm{E}-10$ & & & 1.9E-23 & 1.3E-09 \\
\hline Pa-231 & $5.1 \mathrm{E}+05$ & & & & & & $0.0 \mathrm{E}+00$ & & & & \\
\hline Pa-234 & & & & & & 1.3E-01 & & & & & \\
\hline $\mathrm{Pa}-234 \mathrm{~m}$ & & & & & & $1.8 \mathrm{E}-01$ & & & & & \\
\hline $\mathrm{Pb}-210$ & $5.5 \mathrm{E}+10$ & & & & & 7.7E-01 & $1.4 \mathrm{E}-11$ & & & & \\
\hline $\mathrm{Pb}-212$ & & & & & & 1.3E-04 & & & & & \\
\hline $\mathrm{Pb}-214$ & & & & & & 7.7E-01 & & & & & \\
\hline Pd-107 & $\geq 1.0 \mathrm{E}+20$ & & & & & & $0.0 \mathrm{E}+00$ & & & & \\
\hline $\mathrm{Pm}-147$ & & & & & & $2.8 \mathrm{E}+00$ & & & & & \\
\hline Po-210 & & & & & & $6.1 \mathrm{E}-07$ & & & & & \\
\hline Po-212 & & & & & & $5.3 \mathrm{E}-07$ & & & & & \\
\hline Po-214 & & & & & & 7.7E-01 & & & & & \\
\hline Po-216 & & & & & & 1.3E-04 & & & & & \\
\hline Po-218 & & & & & & 7.7E-01 & & & & & \\
\hline
\end{tabular}




\begin{tabular}{|c|c|c|c|c|c|c|c|c|c|c|c|}
\hline nuclide & $\begin{array}{l}\text { resident } \\
\text { limit (Ci) }\end{array}$ & $\begin{array}{l}\text { air limit } \\
\text { (Ci) }\end{array}$ & $\begin{array}{c}\text { radon } \\
\text { limit (Ci) }\end{array}$ & $\begin{array}{l}\text { GW1 limit } \\
\text { (Ci) }\end{array}$ & $\begin{array}{l}\text { GW2 limit } \\
\text { (Ci) }\end{array}$ & $\begin{array}{l}\text { inventory } \\
\text { (Ci) }\end{array}$ & $\begin{array}{l}\text { resident } \\
\text { fraction }\end{array}$ & $\begin{array}{c}\text { air } \\
\text { fraction }\end{array}$ & $\begin{array}{c}\text { radon } \\
\text { fraction }\end{array}$ & $\begin{array}{c}\text { GW1 } \\
\text { fraction }\end{array}$ & $\begin{array}{c}\text { GW2 } \\
\text { fraction }\end{array}$ \\
\hline Pr-144 & & & & & & 3.3E-01 & & & & & \\
\hline Pr-144m & & & & & & $3.1 \mathrm{E}-03$ & & & & & \\
\hline Pu-238 & $2.8 \mathrm{E}+09$ & & $4.2 \mathrm{E}+06$ & $\geq 1.0 \mathrm{E}+20$ & $\geq 1.0 \mathrm{E}+20$ & $1.3 E+00$ & $4.8 \mathrm{E}-10$ & & 3.2E-07 & 1.3E-20 & 1.3E-20 \\
\hline Pu-239 & $3.3 E+13$ & & & $\geq 1.0 \mathrm{E}+20$ & $\geq 1.0 \mathrm{E}+20$ & 3.6E-01 & 1.1E-14 & & & 3.6E-21 & $3.6 \mathrm{E}-21$ \\
\hline Pu-240 & $6.4 \mathrm{E}+14$ & & & $\geq 1.0 \mathrm{E}+20$ & $\geq 1.0 \mathrm{E}+20$ & $4.5 \mathrm{E}-02$ & $7.0 \mathrm{E}-17$ & & & $4.5 \mathrm{E}-22$ & $4.5 \mathrm{E}-22$ \\
\hline Pu-241 & $1.2 \mathrm{E}+12$ & & & $\geq 1.0 \mathrm{E}+20$ & $2.3 \mathrm{E}+11$ & $1.5 \mathrm{E}+00$ & $1.2 \mathrm{E}-12$ & & & 1.5E-20 & $6.4 \mathrm{E}-12$ \\
\hline Pu-242 & $5.0 \mathrm{E}+12$ & & & $\geq 1.0 \mathrm{E}+20$ & $\geq 1.0 \mathrm{E}+20$ & 3.0E-03 & $6.0 \mathrm{E}-16$ & & & $3.0 \mathrm{E}-23$ & $3.0 \mathrm{E}-23$ \\
\hline Pu-244 & $3.7 \mathrm{E}+04$ & & & $\geq 1.0 \mathrm{E}+20$ & $\geq 1.0 \mathrm{E}+20$ & $1.2 \mathrm{E}-02$ & 3.2E-07 & & & $1.2 \mathrm{E}-22$ & 1.2E-22 \\
\hline Ra-224 & & & & & & 1.3E-04 & & & & & \\
\hline Ra-226 & $1.4 \mathrm{E}+03$ & & $2.6 \mathrm{E}+00$ & $\geq 1.0 \mathrm{E}+20$ & $\geq 1.0 \mathrm{E}+20$ & 7.7E-01 & 5.4E-04 & & 3.0E-01 & 7.7E-21 & 7.7E-21 \\
\hline Ra-228 & $5.2 \mathrm{E}+07$ & & & & & 1.8E-05 & $3.5 \mathrm{E}-13$ & & & & \\
\hline $\mathrm{Rb}-87$ & $\geq 1.0 \mathrm{E}+20$ & & & & & & $0.0 \mathrm{E}+00$ & & & & \\
\hline Rh-103m & & & & & & 6.9E-05 & & & & & \\
\hline Rh-106 & & & & & & 4.1E-02 & & & & & \\
\hline Rn-220 & & & & & & 1.3E-04 & & & & & \\
\hline $\mathrm{Rn}-222$ & & & & & & 7.7E-01 & & & & & \\
\hline Ru-103 & & & & & & $6.9 \mathrm{E}-05$ & & & & & \\
\hline Ru-106 & & & & & & 1.1E-01 & & & & & \\
\hline Sb-125 & $2.0 \mathrm{E}+16$ & & & & & 1.0E-01 & 5.1E-18 & & & & \\
\hline Se-79 & $\geq 1.0 \mathrm{E}+20$ & & & $\geq 1.0 \mathrm{E}+20$ & $1.2 \mathrm{E}+06$ & 1.0E-04 & $1.0 \mathrm{E}-24$ & & & 1.0E-24 & 8.7E-11 \\
\hline Sm-151 & $\geq 1.0 \mathrm{E}+20$ & & & & & & $0.0 \mathrm{E}+00$ & & & & \\
\hline Sn-113 & & & & & & 5.0E-06 & & & & & \\
\hline $\mathrm{Sn}-121 \mathrm{~m}$ & $\geq 1.0 \mathrm{E}+20$ & & & & & & $0.0 \mathrm{E}+00$ & & & & \\
\hline Sn-126 & $2.1 \mathrm{E}+04$ & & & $\geq 1.0 \mathrm{E}+20$ & $\geq 1.0 \mathrm{E}+20$ & 4.9E-04 & 2.3E-08 & & & 4.9E-24 & 4.9E-24 \\
\hline Sr-90 & $\geq 1.0 \mathrm{E}+20$ & & & $\geq 1.0 \mathrm{E}+20$ & $6.1 \mathrm{E}+11$ & $2.1 \mathrm{E}+01$ & 2.1E-19 & & & 2.1E-19 & $3.4 \mathrm{E}-11$ \\
\hline Ta-182 & & & & & & 3.4E-05 & & & & & \\
\hline Tc-99 & $7.7 \mathrm{E}+17$ & & & $4.0 \mathrm{E}+19$ & $1.3 \mathrm{E}+02$ & $6.1 \mathrm{E}-02$ & 8.0E-20 & & & $1.5 \mathrm{E}-21$ & 4.7E-04 \\
\hline TC-99_KB & $7.7 \mathrm{E}+17$ & & & $\geq 1.0 \mathrm{E}+20$ & $2.4 \mathrm{E}+04$ & $2.0 \mathrm{E}-02$ & 2.7E-20 & & & $2.0 \mathrm{E}-22$ & 8.5E-07 \\
\hline $\mathrm{Te}-125 \mathrm{~m}$ & & & & & & 3.0E-03 & & & & & \\
\hline Th-228 & $2.6 \mathrm{E}+18$ & & & $\geq 1.0 \mathrm{E}+20$ & $\geq 1.0 \mathrm{E}+20$ & $1.3 \mathrm{E}-04$ & 4.9E-23 & & & $1.3 \mathrm{E}-24$ & 1.3E-24 \\
\hline Th-229 & $5.3 \mathrm{E}+04$ & & & & & & $0.0 \mathrm{E}+00$ & & & & \\
\hline Th-230 & $3.9 \mathrm{E}+03$ & & $6.8 \mathrm{E}+00$ & $\geq 1.0 \mathrm{E}+20$ & $\geq 1.0 \mathrm{E}+20$ & 1.8E-05 & 4.7E-09 & & 2.7E-06 & $1.8 \mathrm{E}-25$ & 1.8E-25 \\
\hline Th-231 & & & & & & 1.9E-03 & & & & & \\
\hline Th-232 & $4.4 \mathrm{E}+02$ & & & $\geq 1.0 \mathrm{E}+20$ & $\geq 1.0 \mathrm{E}+20$ & 4.9E-05 & 1.1E-07 & & & $4.9 \mathrm{E}-25$ & 4.9E-25 \\
\hline Th-234 & & & & & & 3.1E-01 & & & & & \\
\hline TI-208 & & & & & & $1.2 \mathrm{E}-07$ & & & & & \\
\hline U-232 & $1.3 \mathrm{E}+03$ & & & $\geq 1.0 \mathrm{E}+20$ & $\geq 1.0 \mathrm{E}+20$ & 1.1E-04 & 8.5E-08 & & & 1.1E-24 & 1.1E-24 \\
\hline $\mathrm{U}-233$ & $5.8 \mathrm{E}+05$ & & & $\geq 1.0 \mathrm{E}+20$ & $\geq 1.0 \mathrm{E}+20$ & $1.2 \mathrm{E}-01$ & $2.1 \mathrm{E}-07$ & & & $1.2 \mathrm{E}-21$ & $1.2 \mathrm{E}-21$ \\
\hline $\mathrm{U}-234$ & $7.9 \mathrm{E}+05$ & & $1.2 \mathrm{E}+03$ & $\geq 1.0 \mathrm{E}+20$ & $\geq 1.0 \mathrm{E}+20$ & $2.4 \mathrm{E}-01$ & 3.1E-07 & & $2.0 \mathrm{E}-04$ & $2.4 \mathrm{E}-21$ & $2.4 \mathrm{E}-21$ \\
\hline U-235 & $2.4 \mathrm{E}+07$ & & & $\geq 1.0 \mathrm{E}+20$ & $\geq 1.0 \mathrm{E}+20$ & 8.5E-03 & $3.5 \mathrm{E}-10$ & & & 8.5E-23 & 8.5E-23 \\
\hline $\mathrm{U}-236$ & $9.1 \mathrm{E}+09$ & & & $\geq 1.0 \mathrm{E}+20$ & $\geq 1.0 \mathrm{E}+20$ & 1.7E-03 & $1.9 \mathrm{E}-13$ & & & 1.7E-23 & $1.7 \mathrm{E}-23$ \\
\hline
\end{tabular}




\begin{tabular}{|c|c|c|c|c|c|c|c|c|c|c|c|}
\hline nuclide & $\begin{array}{l}\text { resident } \\
\text { limit (Ci) }\end{array}$ & $\begin{array}{l}\text { air limit } \\
\text { (Ci) }\end{array}$ & $\begin{array}{c}\text { radon } \\
\text { limit (Ci) }\end{array}$ & $\begin{array}{l}\text { GW1 limit } \\
\text { (Ci) }\end{array}$ & $\begin{array}{l}\text { GW2 limit } \\
\text { (Ci) }\end{array}$ & $\begin{array}{l}\text { inventory } \\
\text { (Ci) }\end{array}$ & $\begin{array}{l}\text { resident } \\
\text { fraction }\end{array}$ & $\begin{array}{c}\text { air } \\
\text { fraction }\end{array}$ & $\begin{array}{c}\text { radon } \\
\text { fraction }\end{array}$ & $\begin{array}{c}\text { GW1 } \\
\text { fraction }\end{array}$ & $\begin{array}{c}\text { GW2 } \\
\text { fraction }\end{array}$ \\
\hline $\mathrm{U}-238$ & $7.7 \mathrm{E}+05$ & & $1.1 \mathrm{E}+06$ & $\geq 1.0 \mathrm{E}+20$ & $\geq 1.0 \mathrm{E}+20$ & 5.8E-01 & 7.4E-07 & & 5.2E-07 & $5.8 \mathrm{E}-21$ & $5.8 \mathrm{E}-21$ \\
\hline W-188 & $\geq 1.0 \mathrm{E}+20$ & & & & & & $0.0 \mathrm{E}+00$ & & & & \\
\hline Y-90 & & & & & & $1.6 \mathrm{E}+01$ & & & & & \\
\hline Zn-65 & & & & & & 3.6E-01 & & & & & \\
\hline Zr-93 & $\geq 1.0 \mathrm{E}+20$ & & & $\geq 1.0 \mathrm{E}+20$ & $\geq 1.0 \mathrm{E}+20$ & $6.4 \mathrm{E}-06$ & $6.4 \mathrm{E}-26$ & & & $6.4 \mathrm{E}-26$ & $6.4 \mathrm{E}-26$ \\
\hline Zr-95 & & & & $\geq 1.0 \mathrm{E}+20$ & $\geq 1.0 \mathrm{E}+20$ & 1.1E-02 & & & & 1.1E-22 & 1.1E-22 \\
\hline
\end{tabular}


Table 6-2 Disposal limits for IL vaults.

\begin{tabular}{|c|c|c|c|c|c|}
\hline nuclide & $\begin{array}{c}\text { resident limit } \\
\text { (Ci) }\end{array}$ & $\begin{array}{c}\text { air limit } \\
(\mathrm{Ci})\end{array}$ & $\begin{array}{c}\text { radon limit } \\
\text { (Ci) }\end{array}$ & $\begin{array}{l}\text { GW1 limit } \\
\text { (Ci) }\end{array}$ & $\begin{array}{l}\text { GW2 limit } \\
\text { (Ci) }\end{array}$ \\
\hline Ac-227 & $1.2 \mathrm{E}+07$ & & & & \\
\hline $\mathrm{Ag}-108 \mathrm{~m}$ & $3.9 \mathrm{E}+04$ & & & & \\
\hline Al-26 & $6.6 \mathrm{E}+02$ & & & & \\
\hline Am-241 & $4.1 \mathrm{E}+10$ & & & & $7.6 \mathrm{E}+09$ \\
\hline Am-242m & $5.7 \mathrm{E}+06$ & & & & \\
\hline Am-243 & $4.4 \mathrm{E}+07$ & & & & \\
\hline Ba-133 & $1.7 \mathrm{E}+09$ & & & & \\
\hline Bi-207 & $4.3 \mathrm{E}+04$ & & & & \\
\hline Bk-249 & $8.1 \mathrm{E}+08$ & & & & \\
\hline C-14 & & $4.2 \mathrm{E}+00$ & & & $9.1 \mathrm{E}+07$ \\
\hline C-14_KB & & $4.2 \mathrm{E}+00$ & & & $2.6 \mathrm{E}+06$ \\
\hline Cf-249 & $2.1 \mathrm{E}+06$ & & & & $1.9 \mathrm{E}+11$ \\
\hline Cf-250 & $2.1 \mathrm{E}+18$ & & & & \\
\hline Cf-251 & $3.7 \mathrm{E}+08$ & & & & \\
\hline Cf-252 & $6.3 \mathrm{E}+14$ & & & & \\
\hline $\mathrm{Cm}-242$ & $5.5 \mathrm{E}+11$ & & & & \\
\hline $\mathrm{Cm}-243$ & $9.7 \mathrm{E}+08$ & & & & \\
\hline $\mathrm{Cm}-244$ & $2.5 \mathrm{E}+17$ & & & & \\
\hline $\mathrm{Cm}-245$ & $8.2 \mathrm{E}+09$ & & & & $4.8 \mathrm{E}+09$ \\
\hline $\mathrm{Cm}-246$ & $5.7 \mathrm{E}+15$ & & & & \\
\hline $\mathrm{Cm}-247$ & $1.4 \mathrm{E}+06$ & & & & $6.8 \mathrm{E}+18$ \\
\hline $\mathrm{Cm}-248$ & $4.6 \mathrm{E}+09$ & & & & \\
\hline Co-60 & $8.0 \mathrm{E}+08$ & & & & \\
\hline Cs-137 & $8.3 \mathrm{E}+05$ & & & & \\
\hline Eu-152 & $8.9 \mathrm{E}+05$ & & & & \\
\hline Eu-154 & $1.6 \mathrm{E}+07$ & & & & \\
\hline Eu-155 & $1.6 \mathrm{E}+18$ & & & & \\
\hline $\mathrm{H}-3$ & & $1.3 \mathrm{E}+09$ & & $5.3 \mathrm{E}+09$ & $5.5 \mathrm{E}+09$ \\
\hline $1-129$ & & & & $4.3 \mathrm{E}+10$ & $4.6 \mathrm{E}-04$ \\
\hline $\begin{array}{l}\text { I-129_ETF- } \\
\text { Carbon }\end{array}$ & & & & $1.2 \mathrm{E}+13$ & 9.7E-02 \\
\hline $\mathrm{l}-129 \mathrm{~KB}$ & & & & & $6.2 \mathrm{E}+03$ \\
\hline $\mathrm{K}-40$ & $1.5 \mathrm{E}+04$ & & & $2.3 \mathrm{E}+19$ & $2.0 \mathrm{E}-01$ \\
\hline Kr-85 & $3.8 \mathrm{E}+10$ & & & & \\
\hline $\mathrm{Na}-22$ & $1.1 \mathrm{E}+15$ & & & & \\
\hline $\mathrm{Nb}-94$ & $1.3 \mathrm{E}+04$ & & & & \\
\hline Np-237 & $6.4 \mathrm{E}+06$ & & & & $1.5 \mathrm{E}+06$ \\
\hline $\mathrm{Pa}-231$ & $5.1 \mathrm{E}+05$ & & & & \\
\hline $\mathrm{Pb}-210$ & $5.5 \mathrm{E}+10$ & & & & \\
\hline Pu-238 & $2.8 \mathrm{E}+09$ & & $4.2 \mathrm{E}+06$ & & \\
\hline Pu-239 & $3.3 \mathrm{E}+13$ & & & & \\
\hline Pu-240 & $6.4 \mathrm{E}+14$ & & & & \\
\hline Pu-241 & $1.2 \mathrm{E}+12$ & & & & $2.3 \mathrm{E}+11$ \\
\hline Pu-242 & $5.0 \mathrm{E}+12$ & & & & \\
\hline Pu-244 & $3.7 \mathrm{E}+04$ & & & & \\
\hline Ra-226 & $1.4 \mathrm{E}+03$ & & $2.6 \mathrm{E}+00$ & & \\
\hline Ra-228 & $5.2 \mathrm{E}+07$ & & & & \\
\hline Sb-125 & $2.0 \mathrm{E}+16$ & & & & \\
\hline Se-79 & & & & & $1.2 \mathrm{E}+06$ \\
\hline Sn-126 & $2.1 \mathrm{E}+04$ & & & & \\
\hline Sr-90 & & & & & $6.1 \mathrm{E}+11$ \\
\hline TC-99 & $7.7 \mathrm{E}+17$ & & & $4.0 \mathrm{E}+19$ & $1.3 \mathrm{E}+02$ \\
\hline TC-99 KB & $7.7 \mathrm{E}+17$ & & & & $2.4 \mathrm{E}+04$ \\
\hline Th-228 & $2.6 \mathrm{E}+18$ & & & & \\
\hline Th-229 & $5.3 \mathrm{E}+04$ & & & & \\
\hline Th-230 & $3.9 \mathrm{E}+03$ & & $6.8 \mathrm{E}+00$ & & \\
\hline
\end{tabular}




\begin{tabular}{|l|c|c|c|c|c|}
\hline nuclide & $\begin{array}{c}\text { resident limit } \\
(\mathbf{C i})\end{array}$ & $\begin{array}{c}\text { air limit } \\
(\mathbf{C i})\end{array}$ & $\begin{array}{c}\text { radon limit } \\
(\mathbf{C i})\end{array}$ & $\begin{array}{c}\text { GW1 limit } \\
(\mathbf{C i})\end{array}$ & $\begin{array}{c}\text { GW2 limit } \\
\text { (Ci) }\end{array}$ \\
\hline $\mathrm{Th}-232$ & $4.4 \mathrm{E}+02$ & & & & \\
\hline $\mathrm{U}-232$ & $1.3 \mathrm{E}+03$ & & & & \\
\hline $\mathrm{U}-233$ & $5.8 \mathrm{E}+05$ & & & & \\
\hline$U-234$ & $7.9 \mathrm{E}+05$ & & $1.2 \mathrm{E}+03$ & & \\
\hline$U-235$ & $2.4 \mathrm{E}+07$ & & & & \\
\hline$U-236$ & $9.1 \mathrm{E}+09$ & & & & \\
\hline$U-238$ & $7.7 \mathrm{E}+05$ & & $1.1 \mathrm{E}+06$ & & \\
\hline
\end{tabular}

Rev. 0 
THIS PAGE INTENTIONALLY LEFT BLANK 


\subsection{CONCLUSIONS AND RECOMMENDATIONS}

New disposal limits have been computed for the IL vaults based on several revisions to the PA, as noted in the Introduction. The most important changes were implementation of a 1,000 year time of compliance $(1,350$ for the groundwater pathway to account for uncertainty in the vault failure time), rather than 10,000 years, and consideration of additional radon precursors. Based on the IL vault inventory as of 7/2/04 and inventory limits developed herein, the largest inventory fractions are $30 \%$ for Ra-226 and the radon analysis, $11 \%$ for I-129 and the groundwater pathway, and 9\% for $\mathrm{C}-14$ and the air pathway. Considering that $36 \%$ of the volume is currently filled and nearly all the radon fraction is due to a single container, continued operation of the IL vault should not challenge performance objectives, assuming future disposal patterns are similar to historic use of the facility.

A significant decrease in the limits for I-129 (generic) and I-129_ETF-Carbon was speculated in the FY2003 interim measures assessment, in response to refinement of the aquifer mesh and source node definition (Wilhite and Butcher 2003). In fact, the new limits for these nuclides are only slightly lower. Because several changes were made to the groundwater analysis, a rigorous explanation for the present outcome is not possible without performing a detailed sensitivity analysis. Apparently the impact of mesh and source node refinement was less than speculated and/or other model changes compensated. Mitigating factors probably include a decrease in vertical mesh resolution and slower groundwater travel introduced in switching from the GSA/FACT model to the GSA/PORFLOW model.

Phifer (2004b) developed a preliminary estimate of infiltration through the new IL vault cover system described in the recently revised E-Area closure plan (Phifer 2004a). For expediency the HELP code analysis did not consider the potential influence of the very-low permeability IL vault on cover performance. The resulting infiltration estimate produced ponding of water above the cap in PORFLOW simulations. This behavior indicates that a more rigorous analysis of cap infiltration, explicitly incorporating the IL vault into the HELP model, is needed. A follow-up analysis of this nature is recommended as part of the PA revision scheduled for FY05. 
THIS PAGE INTENTIONALLY LEFT BLANK 


\subsection{REFERENCES}

Collard, L. B., 2000, Special Analysis for Disposal of High-Concentration I-129 Waste in the Intermediate-Level Vaults at the E-Area Low-Level Waste Facility, WSRC-RP-99-01070, Westinghouse Savannah River Company, Aiken, South Carolina.

Collard, L. B., 2003, Special Analysis: Disposal of $K$ and L Basin Resins in the Intermediate Level Vault at the E-Area Low-Level Waste Facility, WSRC-TR-2003-00375, Revision 0, Westinghouse Savannah River Company, Aiken, South Carolina.

Collard, L. B., 2003, Special Analysis: Implementation of Sum-of-Fractions for Multiple Pathways Including Select Groundwater Pathway Time Intervals, WSRC-TR-2003-00438, Revision 0, Westinghouse Savannah River Company, Aiken, South Carolina.

Cook, J. R., 2000, Special Analysis: Updated Analysis of the Effect of Wood Products on Trench Disposal Limits at the E-Area Low-Level Waste Facility, WSRC-RP-2000-00523, Revision 0, Westinghouse Savannah River Company, Aiken, South Carolina.

Cook, J. R., 2002a, Special Analysis: Correction and Update of E-Area Disposal Limits, WSRCTR-2002-00047, Revision 2, Westinghouse Savannah River Company, Aiken, South Carolina.

Cook, J. R., 2002b, Special Analysis: Effect of New Plutonium Chemistry on SRS Trench Disposal Limits, WSRC-TR-2002-00154, Revision 0, Westinghouse Savannah River Company, Aiken, South Carolina.

Cook, J. R., 2004, Special Analysis: Radionuclide Screening Analysis for E Area, WSRC-TR2004-00294, Revision 0, Westinghouse Savannah River Company, Aiken, South Carolina.

Fetter, C. W., 1993, Contaminant Hydrogeology, MacMillan, New York, 458 p.

Flach, G. P., 2004, Groundwater Flow Model of the General Separations Area Using PORFLOW, WSRC-TR-2004-00106, Revision 0, Westinghouse Savannah River Company, Aiken, South Carolina.

Flach, G. P., and Collard, L. B., 2003, Evaluation of Aquifer Source Node Location Alternatives for E-Area Slit Trench Performance Assessment, WSRC-TR-2003-00123, Revision 1, Westinghouse Savannah River Company, Aiken, South Carolina.

Kaplan, D. I., 2004, Recommended Geochemical Input Values for the Special Analysis of the Slit/Engineered Trenches and Intermediate Level Vault, WSRC-RP-2004-00267, Revision 0, Westinghouse Savannah River Company, Aiken, South Carolina.

Koffman, L. D., 2004, An Automated Inadvertent Intruder Analysis Application, WSRC-TR2004-00293, Revision 0, Westinghouse Savannah River Company, Aiken, South Carolina.

McDowell-Boyer, L., Yu, A.D., Cook, J.R., Kocher, D.C., Wilhite, E.L., Holmes-Burns, H., and Young, K.E. 2000. Radiological Performance Assessment for the E-Area Low-Level Waste Facility, WSRC-RP-94-218, Revision 1, Westinghouse Savannah River Company, Aiken, South Carolina.

Phifer, M. A., 2004a, Preliminary E-Area Trench Closure Cap Closure Sequence, Infiltration, and Waste Thickness (U), WSRC-TR-2004-00119, Revision 0, Westinghouse Savannah River Company, Aiken, South Carolina.

Phifer, M. A., 2004b, Preliminary E-Area Intermediate Level Vault Closure Cap Infiltration, WSRC-TR-2004-00280, Revision 0, Westinghouse Savannah River Company, Aiken, South Carolina. 
Simpkins, A. A., 2004, Modeling of Releases from the Intermediate Level Vault in E Area, Rev. 1, SRNL-EST-2004-00001, Rev. 1, Westinghouse Savannah River Company, Aiken, South Carolina.

USDOE, 1999a, Radioactive Waste Management, USDOE Order 435.1, Office of Environmental Management, United States Department of Energy, Washington, D.C.

USEPA, 1994a, The Hydrologic Evaluation of Landfill Performance (HELP) Model User's Guide for Version 3, EPA/600/R-94/168a, Office of Research and Development, United States Environmental Protection Agency, Washington, DC.

USEPA, 1994b, The Hydrologic Evaluation of Landfill Performance (HELP) Engineering Documentation for Version 3, EPA/600/R-94/168b, Office of Research and Development, United States Environmental Protection Agency, Washington, DC.

Wilhite, E. L., 2003, Performance Assessment Position Paper: Time for Compliance, WSRC-RP2003-00390, Westinghouse Savannah River Company, Aiken, South Carolina.

Wilhite, E. L., and Butcher, B. T., 2003, E-Area Performance Assessment Interim Measures Assessment FY2003, WSRC-RP-2003-00436, Westinghouse Savannah River Company, Aiken, South Carolina. 


\section{APPENDIX A - ADDITIONAL INFORMATION ON GROUNDWATER ANALYSIS}

This appendix provides additional detail about the groundwater pathway on assorted topics, primarily for design checking and diagnostic purposes. The topics addressed are

- Vadose zone flow field results

- Implementation of new Pu chemistry

- Input parameters to transport simulations

- Method for refining the GSA/PORFLOW saturation and velocity fields

- Implementation of solubility limits for $\mathrm{Pu}$ and $\mathrm{U}$ isotopes

- Recommendations for groundwater analysis improvements

- Peak flux to the water table from PORFLOW vadose zone transport simulations

- Peak concentration and SOF results from PORFLOW aquifer transport simulations

- Plots of peak water table flux and 100 meter well concentration from PORFLOW aquifer transport simulations

\section{Vadose model flow field results}

Saturation and Darcy velocity from the vadose zone flow simulation are used as inputs to the transport calculation. Figures A-1 through A-6 below show the steady-state saturation and flow fields computed for each of 6 stages to the infiltration transient depicted in Figure 2-1: 0-25, 25$125,125-325,325-575,575-1075$, and $1075+$ years. The left image shows flow direction and magnitudes (Darcy velocity vectors), while the right image depicts flow direction only using constant length arrows. 


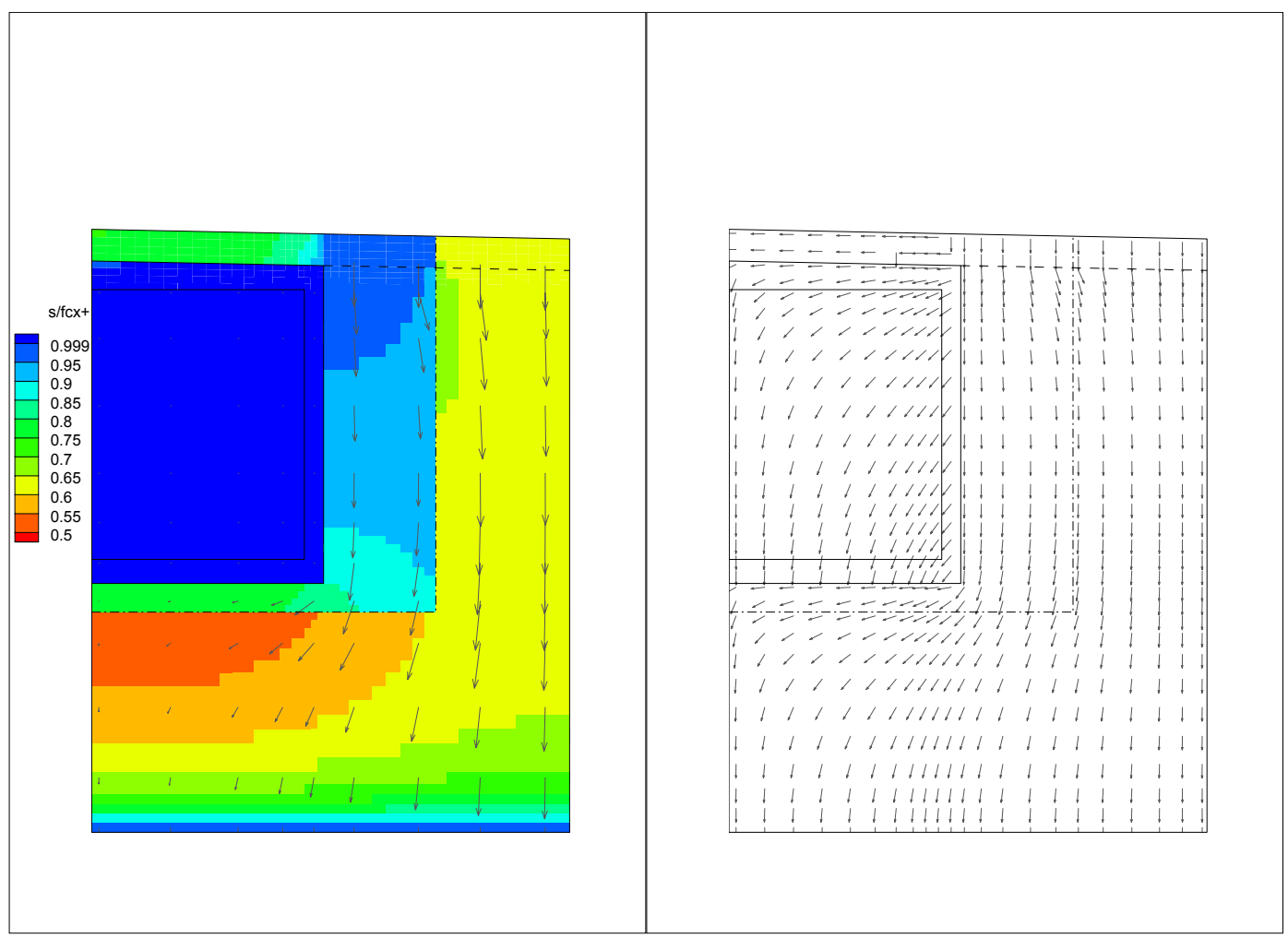

Figure A-1 Saturation and velocity fields for 0-25 years.

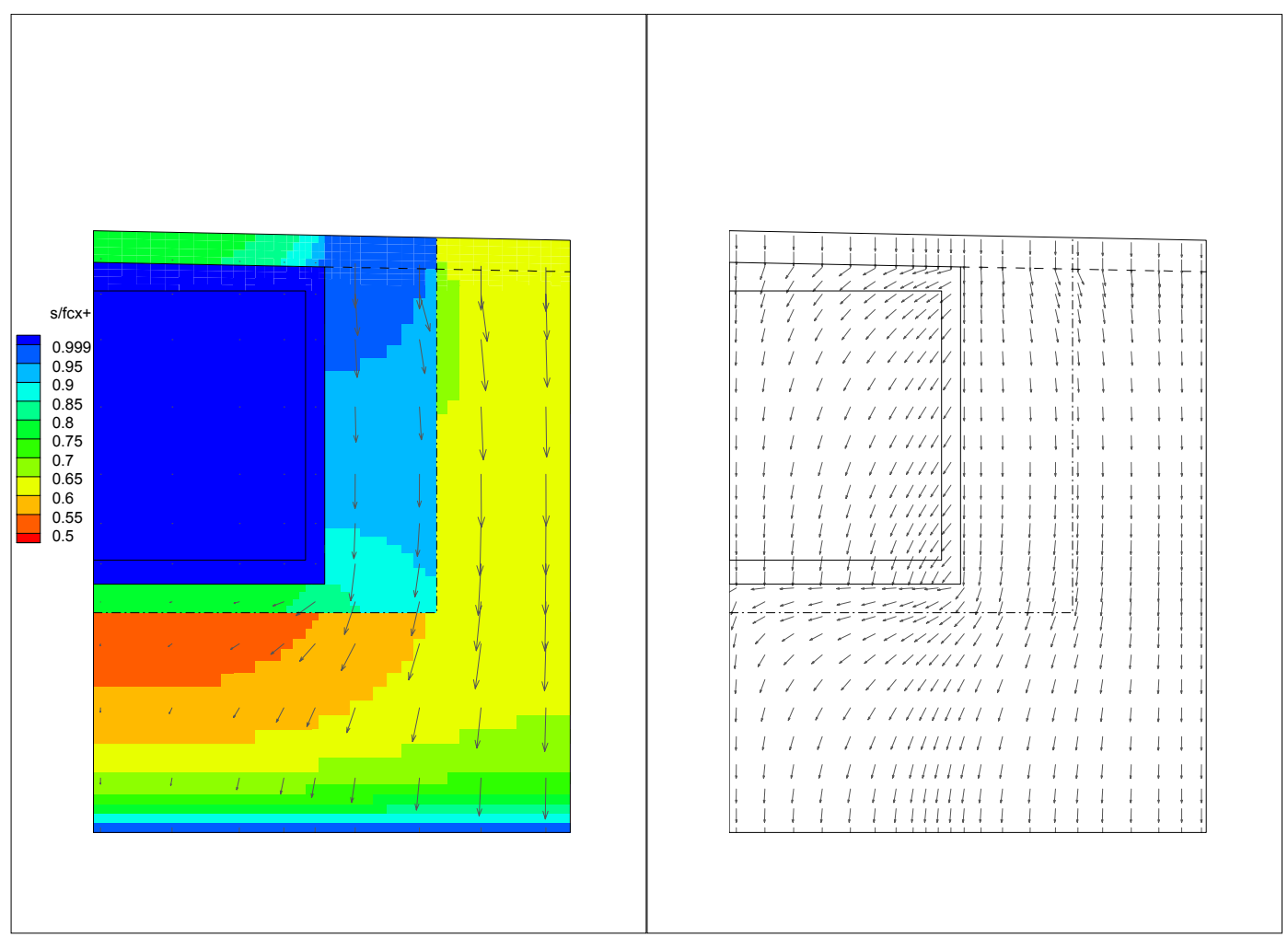

Figure A-2 Saturation and velocity fields for 25-125 years. 


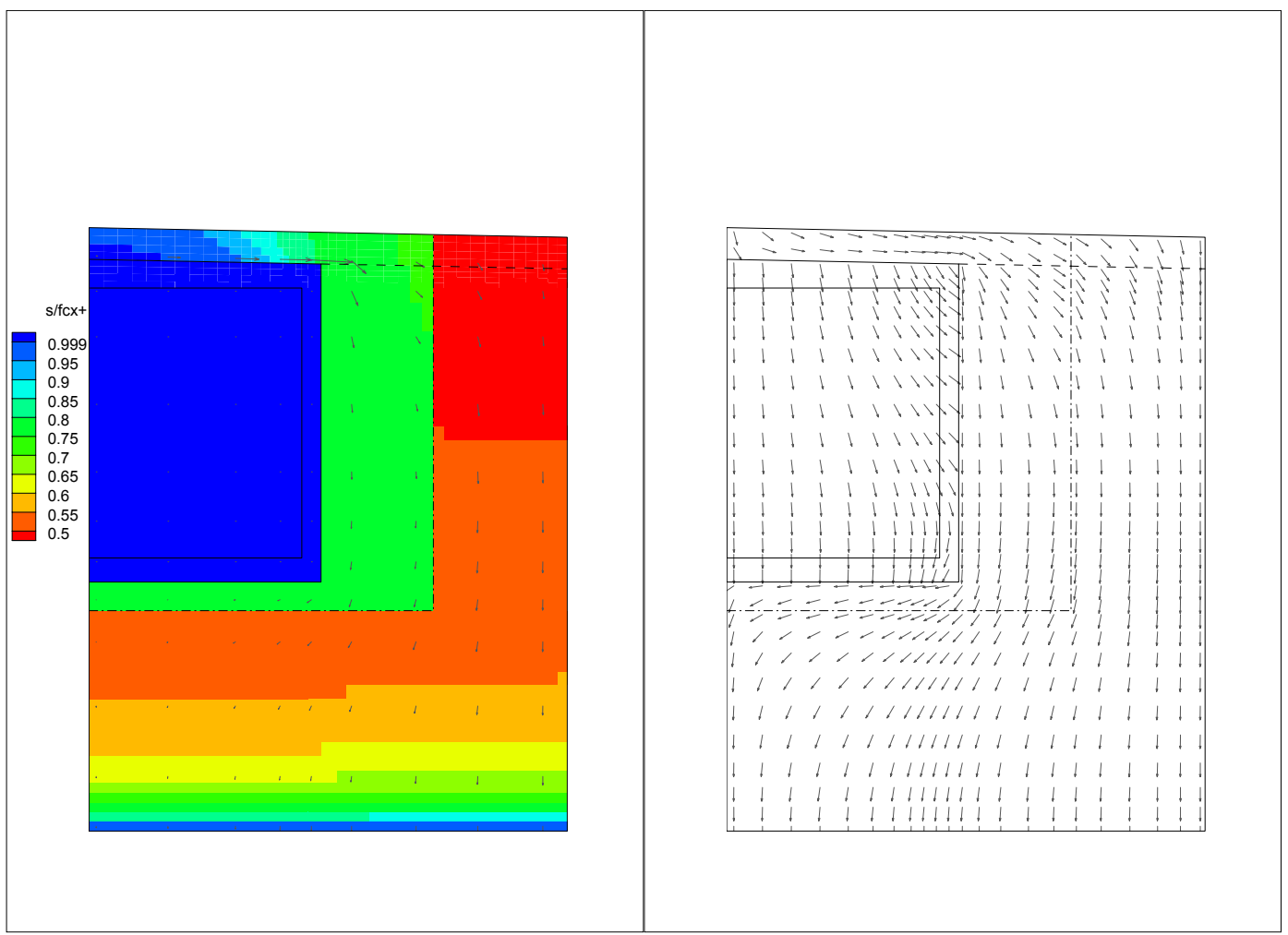

Figure A-3 Saturation and velocity fields for 125-325 years.

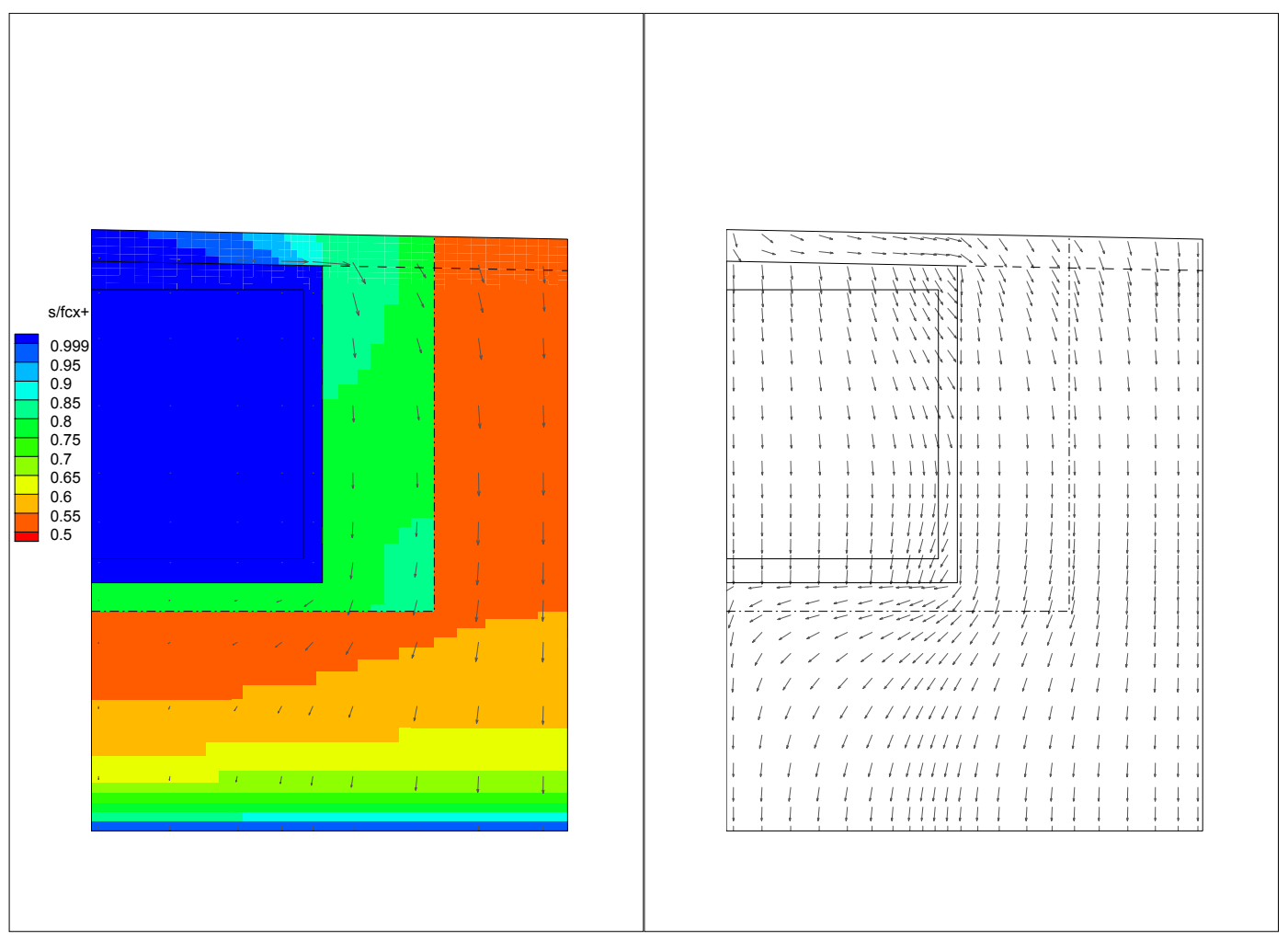

Figure A-4 Saturation and velocity fields for 325-575 years. 


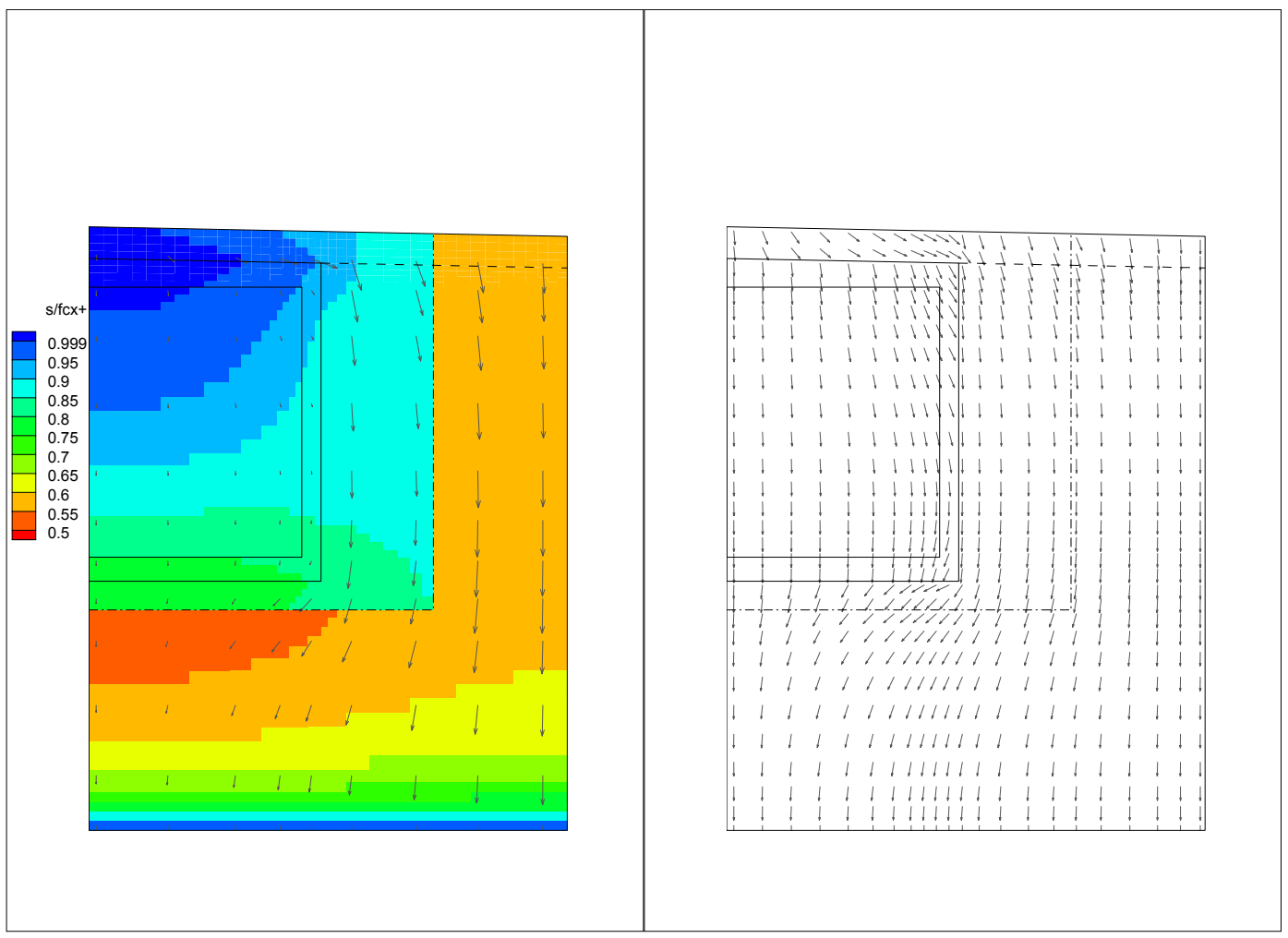

Figure A-5 Saturation and velocity fields for 575-1075 years.

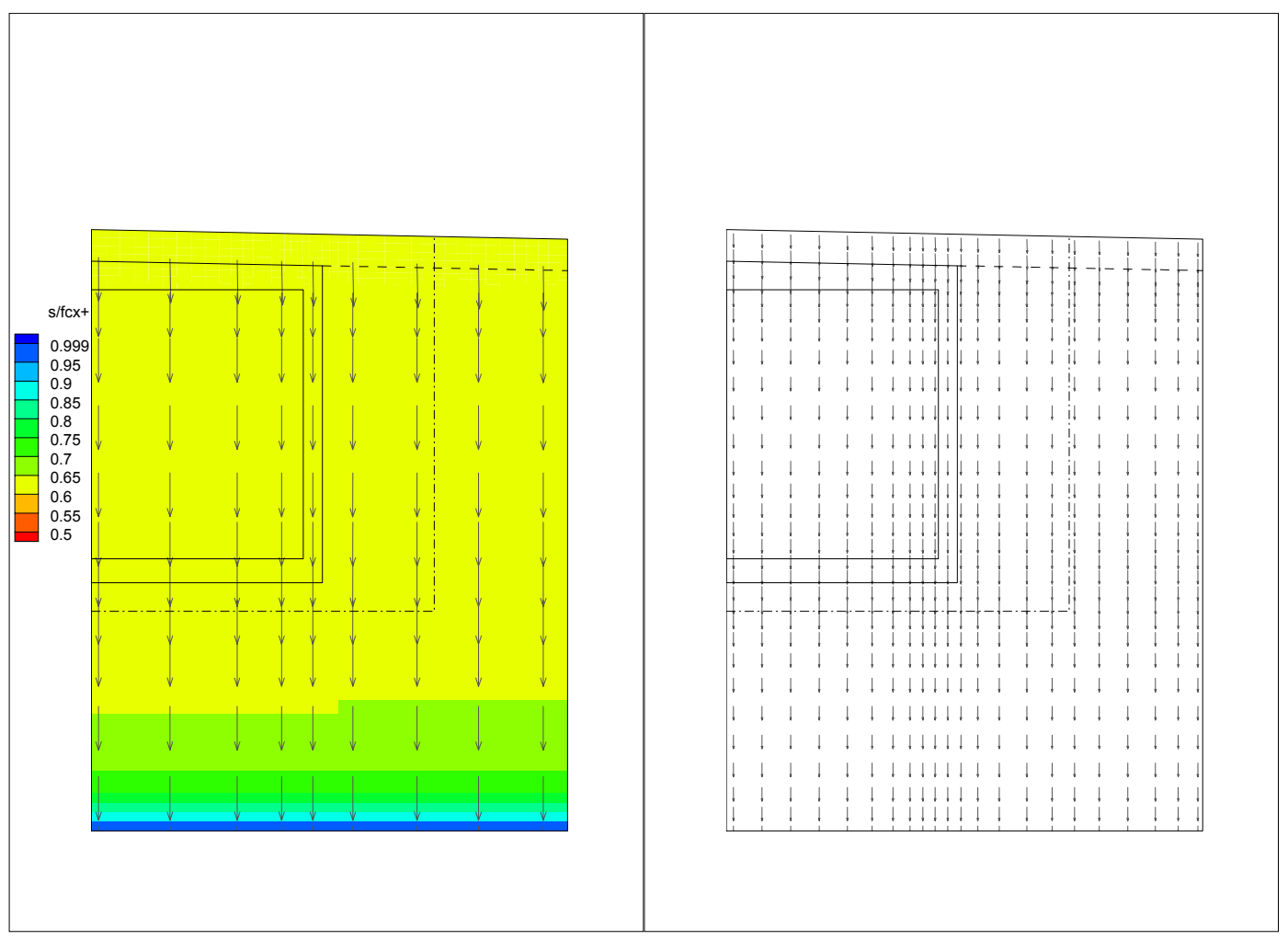

Figure A-6 Saturation and velocity fields for 1075+ years. 


\section{Implementation of new Pu chemistry}

Cook (2002b) describes a new approach to modeling transport of $\mathrm{Pu}$ isotopes in performance assessments that accounts for the differing geochemical behavior of $\mathrm{Pu}$ oxidation states (III/IV) and (V/VI). Pu(V/VI) is significantly more mobile than Pu(III/IV). Kaplan (2004) subsequently updated the kinetic rates assigned to $\mathrm{Pu}(\mathrm{V} / \mathrm{VI}) \rightarrow \mathrm{Pu}(\mathrm{III} / \mathrm{IV})$ reduction and $\mathrm{Pu}(\mathrm{III} / \mathrm{IV}) \rightarrow$ $\mathrm{Pu}(\mathrm{V} / \mathrm{VI})$ oxidation as $1 \times 10^{-3} \mathrm{hr}^{-1}\left(8.77 \mathrm{yr}^{-1}\right)$ and $1.5 \times 10^{-8} \mathrm{hr}^{-1}\left(1.31 \times 10^{-4} \mathrm{yr}^{-1}\right)$, respectively. Cook (2002b) viewed the reduction/oxidation processes as mathematically equivalent to first-order radioactive decay/regeneration. In this section, parameters required by the PORFLOW DECAy and REGEneration keywords are derived for an arbitrary n-member system of species.

For the ith species, a mass balance in terms of moles can be written as

$$
\begin{aligned}
\frac{d}{d t} N_{i} & =-\sum_{j \neq i} \lambda_{i j} \times N_{i}+\sum_{j \neq i} \lambda_{j i} N_{j} \\
& =-\lambda_{i} N_{i}+\sum_{j \neq i} \lambda_{j i} N_{j}
\end{aligned}
$$

where $\lambda_{i j}$ is the rate of conversion from species $i$ to species $j$ and

$$
\lambda_{i}=\sum_{j \neq i} \lambda_{i j}
$$

is the total decay rate for species $i$. Activity is defined by

$$
A=\lambda N
$$

where $N$ is the number of nuclei. Equation (A-1) can be rewritten in terms of activity using Equation (A-3) as follows

$$
\begin{gathered}
\frac{d}{d t}\left(\frac{A_{i}}{\lambda_{i}}\right)=-A_{i}+\sum_{j \neq i} \lambda_{j i}\left(\frac{A_{j}}{\lambda_{j}}\right) \\
\frac{d}{d t} A_{i}=-\lambda_{i} A_{i}+\lambda_{i} \sum_{j \neq i}\left(\frac{\lambda_{j i}}{\lambda_{j}}\right) A_{j} \\
\frac{d}{d t} A_{i}=-\lambda_{i} A_{i}+\sum_{j \neq i}\left(\frac{\lambda_{j i}}{\lambda_{j}}\right)\left(\frac{\lambda_{i}}{\lambda_{j}}\right) \lambda_{j} A_{j} \\
\frac{d}{d t} A_{i}=-\lambda_{i} A_{i}+\sum_{j \neq i} f_{j i}\left(\frac{\lambda_{i}}{\lambda_{j}}\right) \times \lambda_{j} A_{j}
\end{gathered}
$$

In Equation (A-7), $f_{j i}$ is recognized as a "branching fraction", the fraction of decaying species $j$ that converts to species $i$.

In PORFLOW parlance, the DECAy rate for the $i^{\text {th }}$ species is

$$
\lambda_{i}=\sum_{j \neq i} \lambda_{i j}
$$

Rev. 0 
The REGEneration rate is described as the "factor to convert the decay rate of $\Psi$ to the source for the decay product $\Phi "$ ". With respect to Equation (A-7), $\lambda_{j} A_{j}$ is the decay rate of species $j$. Thus

$$
R_{j i}=f_{j i}\left(\frac{\lambda_{i}}{\lambda_{j}}\right)
$$

is the REGEneration factor to convert the decay rate of species $j$ to the source for decay product $i$. Example PORFLOW input for the chain Pu-239(III/IV), Pu-239(V/VI) and U-235 is shown below:

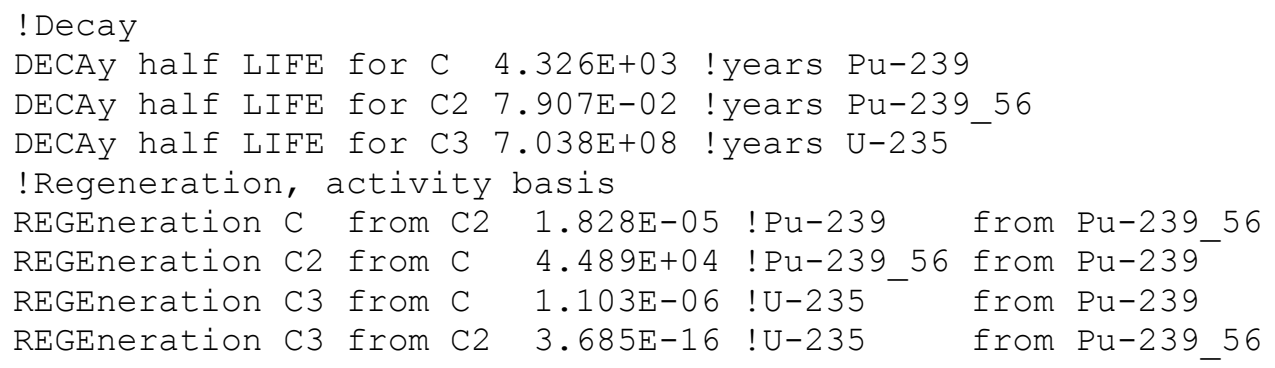

The above values generated by a pre-processing program can be confirmed by hand-calculation as follows:

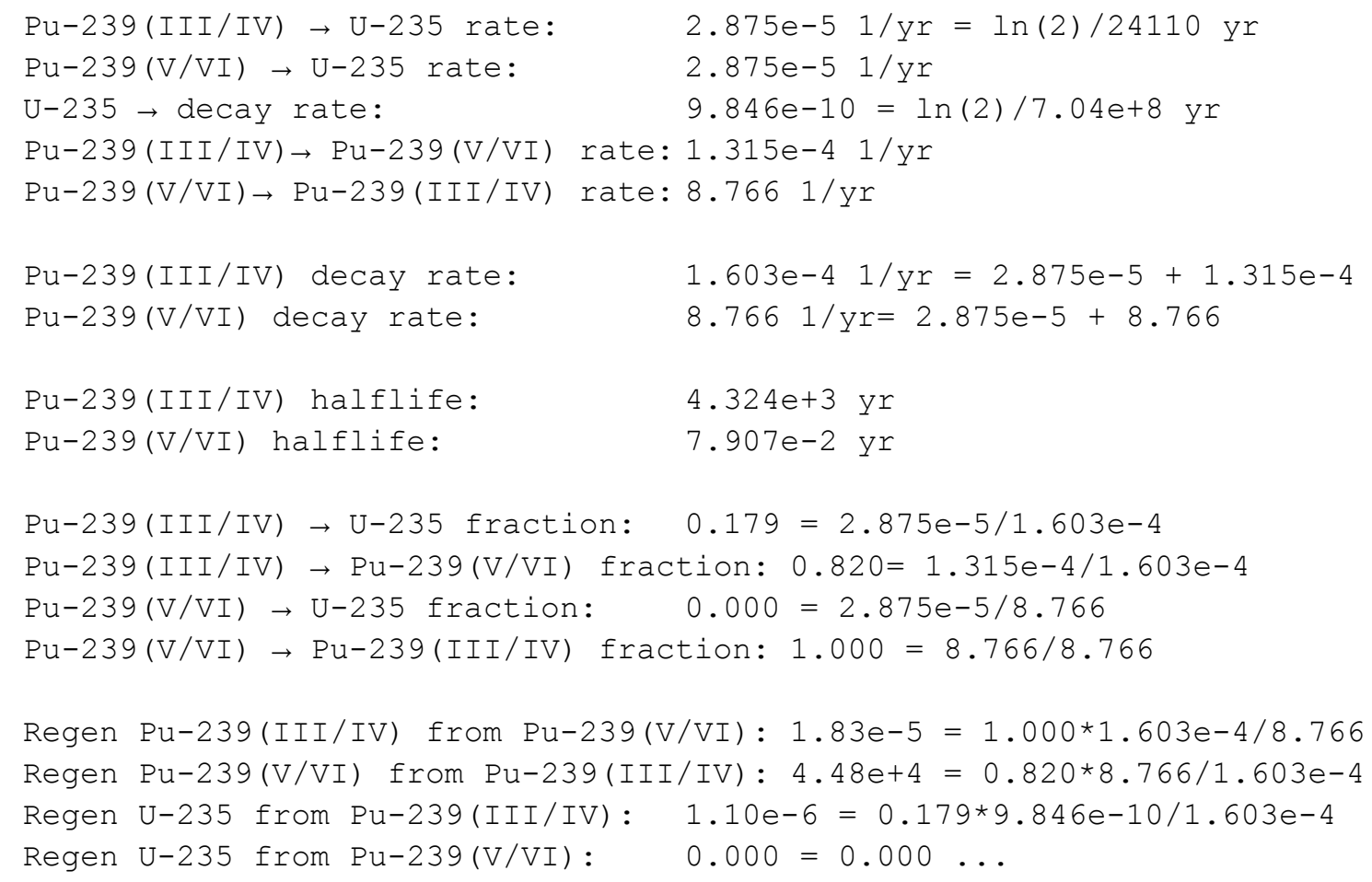

For $\mathrm{Pu}$ isotopes, activity as defined by equation (A-3) includes both radiological and chemical transformations, unlike the conventional definition which considers only radioactive decay. Letting $\lambda_{r}$ denote the first-order rate of radiological decay, and $\lambda_{c}$ the first-order rate of oxidation or reduction, equation (A-3) becomes for $\mathrm{Pu}$ isotopes

$$
A_{r+c}=\left(\lambda_{r}+\lambda_{c}\right) N
$$

compared to the conventional radiological definition of 


$$
A_{r}=\lambda_{r} N
$$

The two definitions of activity can be related by eliminating $N$ between the two equations

$$
\frac{A_{r+c}}{\lambda_{r}+\lambda_{c}}=\frac{A_{r}}{\lambda_{r}}
$$

Upon input to PORFLOW, radiological activity $A_{r}$ must be converted to combined radiological and chemical activity $A_{r+c}$ to be consistent with the new Pu chemistry model. PORFLOW outputs will be in terms of combined radiological and chemical activity, which can be reverted to the conventional radiological activity using equation (A-11). 
Input parameters to transport simulations

Table A-1 lists key inputs to transport simulations, including parents analyzed, half-lives, atomic mass, concentration limits, solubility limits, $K_{d}$ values and assumed decay chains.

Table A-1. Key inputs to transport calculations.

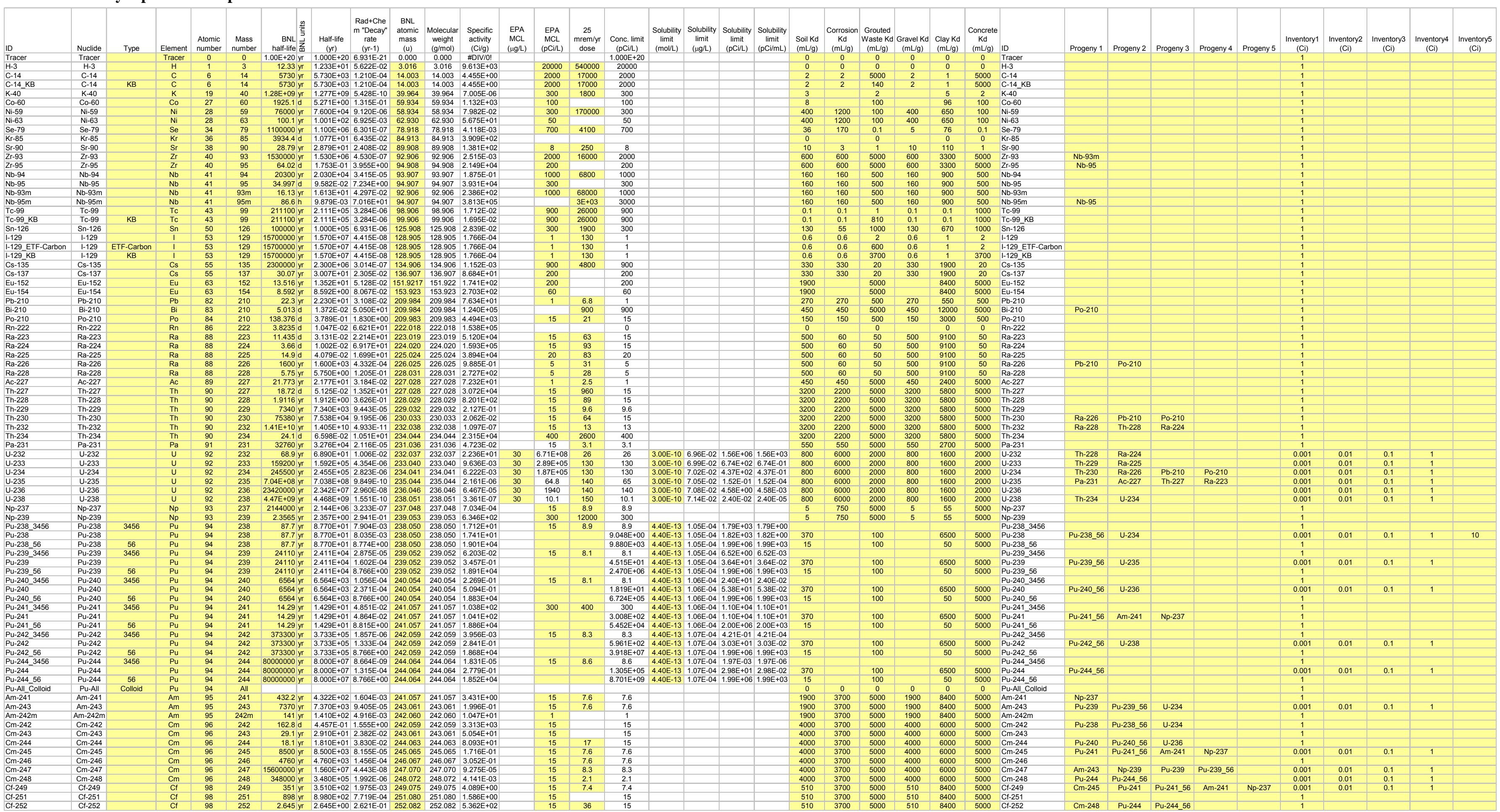




\section{Method for refining the GSA/PORFLOW saturation and velocity fields}

Inputs to the PORFLOW saturated zone solute transport model include water saturation and Darcy velocity fields. Rather than develop a fine-scale flow model of the IL vault area, we chose to refine the steady-state saturation and velocity fields previously computed for the entire General Separations Area using PORFLOW (Flach 2004). Mesh refinement was performed by defining a sub-zone of grid blocks surrounding the IL vaults, and uniformly dividing these into an integer number of smaller blocks. The refined mesh has a footprint as indicated in Figure A-7. The resolution is $50 \mathrm{ft}$ in the horizontal plane. No refinement was made to the vertical mesh. Under current plans a second IL vault will be constructed at footprint \#5, $150 \mathrm{ft}$ away from the existing vault (cf. drawing W2017860 and Appendix B). However, adjoining IL footprints are modeled as a conservatism.

Cells in the refined mesh are assigned the saturation value of the parent coarse-mesh cell in which they reside. The resulting saturation field retains the coarseness of that computed from the GSA/PORFLOW model, but this attribute is of little consequence because plume migration occurs below the water table where cells are $100 \%$ saturated.

The Darcy velocity field computed from the GSA/PORFLOW model is in the form of volumetric flowrates defined at faces between adjoining cells ("FC" variable). For steady-state and constant properties, flowrates across the six faces of a cell satisfy the mass balance

$$
F C_{X-}-F C_{X+}+F C_{Y_{-}}-F C_{Y+}+F C_{Z_{-}}-F C_{Z_{+}}=0
$$

where flow in the positive coordinate direction is positive. In developing an interpolation scheme to transfer flowrates from the coarse-mesh to the refined-mesh, each face flow is assumed to be uniformly distributed across its face. The flowrate across an arbitrary plane perpendicular to the $\mathrm{X}$-axis and within the cell of interest can be written

$$
F C_{X}=F C_{X-}+\xi\left(F C_{Y_{-}}-F C_{Y+}+F C_{Z_{-}}-F C_{Z_{+}}\right)
$$

where

$$
\xi=\frac{x-x^{-}}{x^{+}-x^{-}}
$$

ranges between 0 and 1 . Letting $n y$ and $n z$ denote the number of subdivisions in the $\mathrm{Y}$ and $\mathrm{Z}$ directions, a coarse-mesh $X$ face is comprised of $n y \cdot n z$ faces in the refined mesh. The flowrate across each of these smaller faces at position $\xi$ becomes

$$
f c_{X}=\frac{F C_{X}}{n y \cdot n z}
$$

Similar expressions can be written for the other two coordinate directions. 


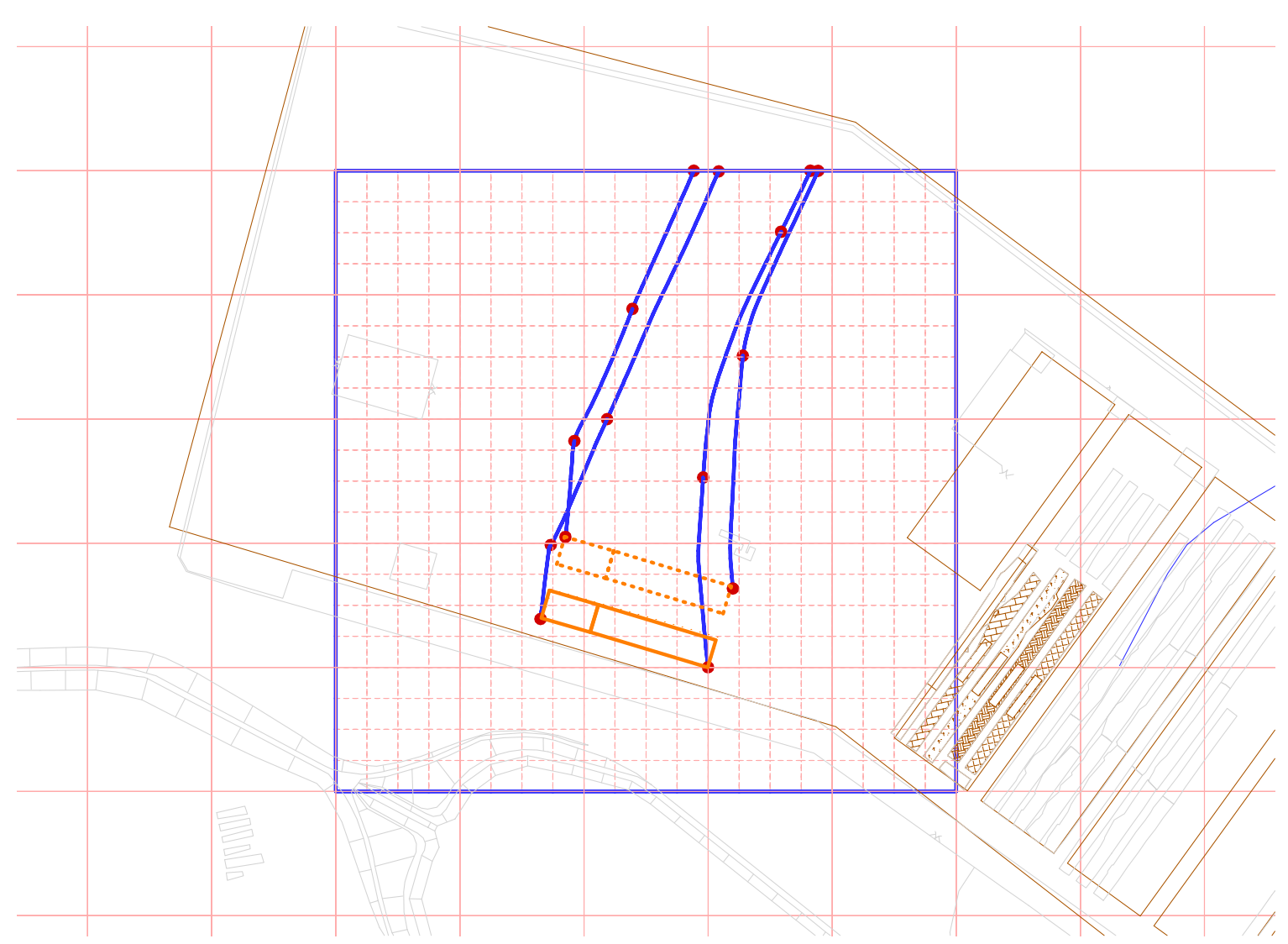

Figure A-7 Mesh refinement for IL vault Special Analysis.

\section{Implementation of solubility limits for Pu and $\mathrm{U}$ isotopes in PORFLOW}

Solubility controls are implemented in the IL vault waste zone using the DISTribution keyword in PORFLOW. Mode 3 of the command allows the user to specify a table of solid and liquid concentration pairs, which defines the radionuclide distribution between the two phases. Typically PORFLOW uses linear interpolation of TABLe values, in which case 3 pairs would be sufficient to describe a linear soil-solute distribution with a solubility cutoff:

$$
\begin{aligned}
& \left(K_{d} C_{\text {sol }}, C_{\text {sol }}\right) \\
& \left(\infty, C_{\text {sol }}\right)
\end{aligned}
$$

where $K_{d}$ is the soil-solute distribution coefficient and $C_{s o l}$ is the liquid solubility limit. However, PORFLOW apparently uses step-wise interpolation in connection with this mode. To mimic the desired variation below the solubility limit across several orders of magnitude, a large number (2000-3000) of additional points are added between the first two points listed above. Adjacent points in the series differ by $1 \%$.

Inspection of the results for $\mathrm{Pu}-244$ revealed that significant numerical inaccuracies occur when the initial inventory exceeds that required to produce the solubility limit by four or more orders of magnitude. For Pu-244, the four inventories considered in PORFLOW simulations range from 2 to 5 times that required to achieve the solubility limit. All four runs should produce the same groundwater concentration, yet the latter two runs produced noticeably higher values. A 
diagnostic run was performed using the SOLUbility limited SOURce keyword and omitting the DISTribution command. The results from the diagnostic case were close to those from the baseline runs at the lower initial inventories.

The inaccuracy described above is much less significant for the other Pu nuclides and $\mathrm{U}$ isotopes. In all cases, the limits derived from peak groundwater concentration results are not impacted. The inaccuracy produces a higher $1 / S O F$ value and the minimum value is used to define the limit.

\section{Recommendations for groundwater analysis improvements}

A number of modifications to the groundwater analysis should be considered for implementation in the upcoming PA revision

- if numerically feasible, combine the vadose zone and aquifer zone simulations to avoid having to perform and design check two PORFLOW analyses and the handshaking between the vadose zone flux and aquifer source node placement

- simplify implementation of the $\mathrm{Pu}$ chemistry model by performing PORFLOW simulations in terms of moles and instead of activity; this avoids the overhead and potential confusion in converting between radiological activity and combined radiological and chemical activity

- use the solubility-limited source keyword command in PORFLOW to implement solubility limits for parents; this avoids apparent numerical inaccuracies discussed above

\section{Peak flux to the water table from PORFLOW vadose zone transport simulations}

For each parent and inventory modeled with PORFLOW, Table A-2 provides the peak flux $(\mathrm{Ci} / \mathrm{yr})$ to the water table for each member of the modeled chain. Also included in the table are the time the peak occurred, and the fractional flux (flux divided by the disposal unit inventory). 
Table A-2. Peak water table flux results.

\begin{tabular}{|c|c|c|c|c|c|}
\hline Nuclide chains & $\begin{array}{c}\text { Inventory for } 2 \\
\text { disposal units (Ci) }\end{array}$ & $\begin{array}{c}\text { Inventory per disposal } \\
\text { unit (Ci) }\end{array}$ & $\begin{array}{l}\text { Time peak flux } \\
\text { occurred (yr) }\end{array}$ & $\begin{array}{c}\text { Peak flux from } 2 \\
\text { disposal units (Ci/yr) }\end{array}$ & Fractional flux (1/yr) \\
\hline Tracer & 1 & 0.5 & 627 & $2.51 \mathrm{E}-02$ & $2.51 \mathrm{E}-02$ \\
\hline $\mathrm{H}-3$ & 1 & 0.5 & 96 & $2.98 \mathrm{E}-11$ & $2.98 \mathrm{E}-11$ \\
\hline C-14 & 1 & 0.5 & 10000 & $7.11 \mathrm{E}-07$ & $7.11 \mathrm{E}-07$ \\
\hline C-14_KB & 1 & 0.5 & 8897 & 1.79E-05 & 1.79E-05 \\
\hline $\mathrm{K}-40$ & 1 & 0.5 & 1152 & $1.22 \mathrm{E}-02$ & $1.22 \mathrm{E}-02$ \\
\hline $\mathrm{Ni}-59$ & 1 & 0.5 & 10000 & 1.71E-04 & 1.71E-04 \\
\hline Se-79 & 1 & 0.5 & 1745 & $2.41 \mathrm{E}-03$ & $2.41 \mathrm{E}-03$ \\
\hline Sr-90 & 1 & 0.5 & 1203 & $1.13 \mathrm{E}-15$ & $1.13 \mathrm{E}-15$ \\
\hline Zr-93 & 1 & 0.5 & 10000 & $2.58 \mathrm{E}-09$ & $2.58 \mathrm{E}-09$ \\
\hline $\mathrm{Nb}-93 \mathrm{~m}$ & & & 10000 & $1.06 \mathrm{E}-08$ & $1.06 \mathrm{E}-08$ \\
\hline Zr-95 & 1 & 0.5 & 7 & $6.96 \mathrm{E}-123$ & $6.96 \mathrm{E}-123$ \\
\hline $\mathrm{Nb}-95$ & & & 4 & $3.17 \mathrm{E}-112$ & $3.17 \mathrm{E}-112$ \\
\hline $\mathrm{Nb}-94$ & 1 & 0.5 & 8067 & $5.15 \mathrm{E}-05$ & $5.15 \mathrm{E}-05$ \\
\hline $\mathrm{Nb}-95$ & 1 & 0.5 & 4 & $1.36 \mathrm{E}-112$ & $1.36 \mathrm{E}-112$ \\
\hline Tc-99 & 1 & 0.5 & 2773 & $2.88 \mathrm{E}-04$ & $2.88 \mathrm{E}-04$ \\
\hline Tc-99_KB & 1 & 0.5 & 9645 & $4.14 \mathrm{E}-05$ & $4.14 \mathrm{E}-05$ \\
\hline Sn-126 & 1 & 0.5 & 10000 & $3.14 \mathrm{E}-05$ & $3.14 \mathrm{E}-05$ \\
\hline $\mathrm{I}-129$ & 1 & 0.5 & 1105 & $1.46 \mathrm{E}-02$ & $1.46 \mathrm{E}-02$ \\
\hline I-129_ET & 1 & 0.5 & 1127 & $6.22 \mathrm{E}-05$ & $6.22 \mathrm{E}-05$ \\
\hline $\mathrm{I}-129 \mathrm{~KB}$ & 1 & 0.5 & 10000 & 4.99E-06 & 4.99E-06 \\
\hline Cs-135 & 1 & 0.5 & 7865 & $2.73 \mathrm{E}-04$ & $2.73 \mathrm{E}-04$ \\
\hline Po-210 & 1 & 0.5 & 14 & $2.59 \mathrm{E}-96$ & $2.59 \mathrm{E}-96$ \\
\hline Ra-226 & 1 & 0.5 & 9965 & $1.66 \mathrm{E}-06$ & $1.66 \mathrm{E}-06$ \\
\hline $\mathrm{Pb}-210$ & & & 9937 & $3.15 \mathrm{E}-06$ & $3.15 \mathrm{E}-06$ \\
\hline Po-210 & & & 9936 & $5.68 \mathrm{E}-06$ & $5.68 \mathrm{E}-06$ \\
\hline Th-228 & 1 & 0.5 & 72 & $1.54 \mathrm{E}-112$ & $1.54 \mathrm{E}-112$ \\
\hline Th-230 & 1 & 0.5 & 10000 & $4.86 \mathrm{E}-21$ & $4.86 \mathrm{E}-21$ \\
\hline Ra-226 & & & 10000 & $3.30 \mathrm{E}-06$ & $3.30 \mathrm{E}-06$ \\
\hline $\mathrm{Pb}-210$ & & & 10000 & $6.32 \mathrm{E}-06$ & $6.32 \mathrm{E}-06$ \\
\hline Po-210 & & & 10000 & 1.14E-05 & $1.14 \mathrm{E}-05$ \\
\hline Th-232 & 1 & 0.5 & 10000 & $5.33 \mathrm{E}-21$ & $5.33 \mathrm{E}-21$ \\
\hline Ra-228 & & & 10000 & $3.85 \mathrm{E}-20$ & $3.85 E-20$ \\
\hline Th-228 & & & 10000 & $6.01 \mathrm{E}-21$ & $6.01 \mathrm{E}-21$ \\
\hline Ra-224 & & & 10000 & $3.85 \mathrm{E}-20$ & $3.85 E-20$ \\
\hline U-232 & 0.001 & 0.0005 & 3273 & $1.25 \mathrm{E}-38$ & $1.25 \mathrm{E}-35$ \\
\hline Th-228 & & & 3275 & $3.15 \mathrm{E}-39$ & $3.15 E-36$ \\
\hline
\end{tabular}




\begin{tabular}{|c|c|c|c|c|c|}
\hline Nuclide chains & $\begin{array}{c}\text { Inventory for } 2 \\
\text { disposal units (Ci) }\end{array}$ & $\begin{array}{c}\text { Inventory per disposal } \\
\text { unit (Ci) }\end{array}$ & $\begin{array}{l}\text { Time peak flux } \\
\text { occurred (yr) }\end{array}$ & $\begin{array}{c}\text { Peak flux from } 2 \\
\text { disposal units (Ci/yr) }\end{array}$ & Fractional flux (1/yr) \\
\hline Ra-224 & & & 3275 & $2.02 \mathrm{E}-38$ & $2.02 \mathrm{E}-35$ \\
\hline $\mathrm{U}-232$ & 0.01 & 0.005 & 3310 & $1.59 \mathrm{E}-37$ & $1.59 \mathrm{E}-35$ \\
\hline Th-228 & & & 3312 & $4.00 \mathrm{E}-38$ & $4.00 \mathrm{E}-36$ \\
\hline Ra-224 & & & 3312 & $2.56 \mathrm{E}-37$ & $2.56 \mathrm{E}-35$ \\
\hline $\mathrm{U}-232$ & 0.1 & 0.05 & 3316 & $1.61 \mathrm{E}-36$ & 1.61E-35 \\
\hline Th-228 & & & 3318 & $4.06 \mathrm{E}-37$ & $4.06 \mathrm{E}-36$ \\
\hline Ra-224 & & & 3318 & $2.60 \mathrm{E}-36$ & $2.60 \mathrm{E}-35$ \\
\hline U-232 & 1 & 0.5 & 3317 & $1.61 \mathrm{E}-35$ & $1.61 \mathrm{E}-35$ \\
\hline Th-228 & & & 3319 & $4.06 \mathrm{E}-36$ & $4.06 \mathrm{E}-36$ \\
\hline Ra-224 & & & 3319 & $2.60 \mathrm{E}-35$ & $2.60 \mathrm{E}-35$ \\
\hline $\mathrm{U}-233$ & 0.001 & 0.0005 & 10000 & $2.28 \mathrm{E}-12$ & $2.28 \mathrm{E}-09$ \\
\hline Th-229 & & & 10000 & $3.73 \mathrm{E}-14$ & $3.73 \mathrm{E}-11$ \\
\hline Ra-225 & & & 10000 & $2.39 \mathrm{E}-13$ & $2.39 \mathrm{E}-10$ \\
\hline U-233 & 0.01 & 0.005 & 10000 & $2.28 \mathrm{E}-11$ & $2.28 \mathrm{E}-09$ \\
\hline Th-229 & & & 10000 & $3.73 \mathrm{E}-13$ & $3.73 \mathrm{E}-11$ \\
\hline Ra-225 & & & 10000 & $2.38 \mathrm{E}-12$ & $2.38 \mathrm{E}-10$ \\
\hline U-233 & 0.1 & 0.05 & 10000 & $2.28 \mathrm{E}-10$ & 2.28E-09 \\
\hline Th-229 & & & 10000 & $3.73 \mathrm{E}-12$ & $3.73 \mathrm{E}-11$ \\
\hline Ra-225 & & & 10000 & $2.38 \mathrm{E}-11$ & $2.38 \mathrm{E}-10$ \\
\hline U-233 & 1 & 0.5 & 10000 & 2.28E-09 & $2.28 \mathrm{E}-09$ \\
\hline Th-229 & & & 10000 & 3.73E-11 & $3.73 \mathrm{E}-11$ \\
\hline Ra-225 & & & 10000 & $2.39 \mathrm{E}-10$ & $2.39 \mathrm{E}-10$ \\
\hline U-234 & 0.001 & 0.0005 & 10000 & $2.32 \mathrm{E}-12$ & 2.32E-09 \\
\hline Th-230 & & & 10000 & $3.90 \mathrm{E}-15$ & $3.90 \mathrm{E}-12$ \\
\hline Ra-226 & & & 10000 & $5.66 \mathrm{E}-11$ & 5.66E-08 \\
\hline $\mathrm{Pb}-210$ & & & 10000 & $1.09 \mathrm{E}-10$ & 1.09E-07 \\
\hline Po-210 & & & 10000 & $1.97 \mathrm{E}-10$ & 1.97E-07 \\
\hline U-234 & 0.01 & 0.005 & 10000 & $2.32 \mathrm{E}-11$ & 2.32E-09 \\
\hline Th-230 & & & 10000 & 3.91E-14 & 3.91E-12 \\
\hline Ra-226 & & & 10000 & $5.66 \mathrm{E}-10$ & 5.66E-08 \\
\hline $\mathrm{Pb}-210$ & & & 10000 & 1.09E-09 & $1.09 \mathrm{E}-07$ \\
\hline Po-210 & & & 10000 & 1.97E-09 & 1.97E-07 \\
\hline U-234 & 0.1 & 0.05 & 10000 & $2.32 \mathrm{E}-10$ & 2.32E-09 \\
\hline Th-230 & & & 10000 & $3.91 \mathrm{E}-13$ & $3.91 \mathrm{E}-12$ \\
\hline Ra-226 & & & 10000 & 5.66E-09 & $5.66 \mathrm{E}-08$ \\
\hline $\mathrm{Pb}-210$ & & & 10000 & $1.09 \mathrm{E}-08$ & $1.09 \mathrm{E}-07$ \\
\hline Po-210 & & & 10000 & 1.97E-08 & 1.97E-07 \\
\hline
\end{tabular}




\begin{tabular}{|c|c|c|c|c|c|}
\hline Nuclide chains & $\begin{array}{c}\text { Inventory for } 2 \\
\text { disposal units (Ci) }\end{array}$ & $\begin{array}{c}\text { Inventory per disposal } \\
\text { unit (Ci) }\end{array}$ & $\begin{array}{l}\text { Time peak flux } \\
\text { occurred (yr) }\end{array}$ & $\begin{array}{c}\text { Peak flux from } 2 \\
\text { disposal units (Ci/yr) }\end{array}$ & Fractional flux (1/yr) \\
\hline U-234 & 1 & 0.5 & 10000 & $2.32 \mathrm{E}-09$ & $2.32 \mathrm{E}-09$ \\
\hline Th-230 & & & 10000 & $3.91 \mathrm{E}-12$ & $3.91 \mathrm{E}-12$ \\
\hline Ra-226 & & & 10000 & $5.66 \mathrm{E}-08$ & $5.66 \mathrm{E}-08$ \\
\hline $\mathrm{Pb}-210$ & & & 10000 & $1.09 \mathrm{E}-07$ & $1.09 \mathrm{E}-07$ \\
\hline Po-210 & & & 10000 & $1.97 \mathrm{E}-07$ & $1.97 \mathrm{E}-07$ \\
\hline $\mathrm{U}-235$ & 0.001 & 0.0005 & 10000 & $2.39 \mathrm{E}-12$ & 2.39E-09 \\
\hline Pa-231 & & & 10000 & $6.66 \mathrm{E}-12$ & $6.66 \mathrm{E}-09$ \\
\hline Ac-227 & & & 10000 & $8.23 E-12$ & 8.23E-09 \\
\hline Th-227 & & & 10000 & $1.16 \mathrm{E}-12$ & $1.16 \mathrm{E}-09$ \\
\hline Ra-223 & & & 10000 & $7.41 \mathrm{E}-12$ & 7.41E-09 \\
\hline $\mathrm{U}-235$ & 0.01 & 0.005 & 10000 & 1.97E-11 & 1.97E-09 \\
\hline Pa-231 & & & 10000 & $5.52 \mathrm{E}-11$ & 5.52E-09 \\
\hline Ac-227 & & & 10000 & $6.82 \mathrm{E}-11$ & 6.82E-09 \\
\hline Th-227 & & & 10000 & $9.59 \mathrm{E}-12$ & $9.59 \mathrm{E}-10$ \\
\hline Ra-223 & & & 10000 & $6.14 \mathrm{E}-11$ & $6.14 \mathrm{E}-09$ \\
\hline U-235 & 0.1 & 0.05 & 10000 & $1.98 \mathrm{E}-11$ & $1.98 \mathrm{E}-10$ \\
\hline $\mathrm{Pa}-231$ & & & 10000 & $7.13 \mathrm{E}-11$ & $7.13 \mathrm{E}-10$ \\
\hline Ac-227 & & & 10000 & $8.80 \mathrm{E}-11$ & $8.80 \mathrm{E}-10$ \\
\hline Th-227 & & & 10000 & $1.24 \mathrm{E}-11$ & $1.24 \mathrm{E}-10$ \\
\hline Ra-223 & & & 10000 & $7.92 \mathrm{E}-11$ & $7.92 \mathrm{E}-10$ \\
\hline U-235 & 1 & 0.5 & 10000 & $1.98 \mathrm{E}-11$ & $1.98 \mathrm{E}-11$ \\
\hline $\mathrm{Pa}-231$ & & & 10000 & $2.30 \mathrm{E}-10$ & $2.30 \mathrm{E}-10$ \\
\hline Ac-227 & & & 10000 & $2.84 \mathrm{E}-10$ & $2.84 \mathrm{E}-10$ \\
\hline Th-227 & & & 10000 & $4.00 \mathrm{E}-11$ & $4.00 \mathrm{E}-11$ \\
\hline Ra-223 & & & 10000 & $2.56 \mathrm{E}-10$ & $2.56 \mathrm{E}-10$ \\
\hline U-236 & 0.001 & 0.0005 & 10000 & $2.39 \mathrm{E}-12$ & $2.39 \mathrm{E}-09$ \\
\hline$U-236$ & 0.01 & 0.005 & 10000 & $2.39 \mathrm{E}-11$ & 2.39E-09 \\
\hline U-236 & 0.1 & 0.05 & 10000 & $2.39 \mathrm{E}-10$ & $2.39 \mathrm{E}-09$ \\
\hline$U-236$ & 1 & 0.5 & 10000 & $5.96 \mathrm{E}-10$ & $5.96 \mathrm{E}-10$ \\
\hline U-238 & 0.001 & 0.0005 & 10000 & $2.39 \mathrm{E}-12$ & 2.39E-09 \\
\hline Th-234 & & & 10000 & $5.97 \mathrm{E}-13$ & $5.97 \mathrm{E}-10$ \\
\hline U-234 & & & 10000 & $6.66 \mathrm{E}-14$ & $6.66 \mathrm{E}-11$ \\
\hline U-238 & 0.01 & 0.005 & 10000 & $3.12 \mathrm{E}-12$ & $3.12 \mathrm{E}-10$ \\
\hline Th-234 & & & 10000 & $7.81 \mathrm{E}-13$ & $7.81 \mathrm{E}-11$ \\
\hline U-234 & & & 10000 & $1.77 \mathrm{E}-13$ & $1.77 \mathrm{E}-11$ \\
\hline U-238 & 0.1 & 0.05 & 10000 & $3.12 \mathrm{E}-12$ & $3.12 \mathrm{E}-11$ \\
\hline Th-234 & & & 10000 & $7.81 \mathrm{E}-13$ & $7.81 \mathrm{E}-12$ \\
\hline
\end{tabular}




\begin{tabular}{|c|c|c|c|c|c|}
\hline Nuclide chains & $\begin{array}{c}\text { Inventory for } 2 \\
\text { disposal units (Ci) }\end{array}$ & $\begin{array}{c}\text { Inventory per disposal } \\
\text { unit (Ci) }\end{array}$ & $\begin{array}{l}\text { Time peak flux } \\
\text { occurred (yr) }\end{array}$ & $\begin{array}{c}\text { Peak flux from } 2 \\
\text { disposal units (Ci/yr) }\end{array}$ & Fractional flux (1/yr) \\
\hline $\mathrm{U}-234$ & & & 10000 & 1.11E-12 & $1.11 \mathrm{E}-11$ \\
\hline $\mathrm{U}-238$ & 1 & 0.5 & 10000 & $3.12 \mathrm{E}-12$ & $3.12 \mathrm{E}-12$ \\
\hline Th-234 & & & 10000 & $7.81 \mathrm{E}-13$ & $7.81 \mathrm{E}-13$ \\
\hline U-234 & & & 10000 & $1.04 \mathrm{E}-11$ & $1.04 \mathrm{E}-11$ \\
\hline $\mathrm{Np}-237$ & 1 & 0.5 & 10000 & $2.35 \mathrm{E}-06$ & $2.35 \mathrm{E}-06$ \\
\hline Pu-238 & 0.001 & 0.0005 & 3499 & $5.61 \mathrm{E}-29$ & $5.61 \mathrm{E}-26$ \\
\hline Pu-238_5 & & & 3497 & $2.09 \mathrm{E}-32$ & $2.09 E-29$ \\
\hline U-234 & & & 10000 & 8.34E-16 & 8.34E-13 \\
\hline Pu-238 & 0.01 & 0.005 & 3499 & $5.61 \mathrm{E}-28$ & $5.61 \mathrm{E}-26$ \\
\hline Pu-238_5 & & & 3497 & $2.09 E-31$ & $2.09 E-29$ \\
\hline U-234 & & & 10000 & 8.33E-15 & 8.33E-13 \\
\hline $\mathrm{Pu}-238$ & 0.1 & 0.05 & 3499 & $5.61 \mathrm{E}-27$ & $5.61 \mathrm{E}-26$ \\
\hline Pu-238_5 & & & 3497 & $2.09 \mathrm{E}-30$ & $2.09 E-29$ \\
\hline U-234 & & & 10000 & $8.32 \mathrm{E}-14$ & $8.32 E-13$ \\
\hline $\mathrm{Pu}-238$ & 1 & 0.5 & 3499 & $5.61 \mathrm{E}-26$ & $5.61 \mathrm{E}-26$ \\
\hline Pu-238_5 & & & 3497 & $2.09 E-29$ & 2.09E-29 \\
\hline U-234 & & & 10000 & $8.32 \mathrm{E}-13$ & 8.32E-13 \\
\hline Pu-238 & 10 & 5 & 3499 & $5.61 \mathrm{E}-25$ & $5.61 \mathrm{E}-26$ \\
\hline Pu-238_5 & & & 3497 & $2.09 E-28$ & $2.09 E-29$ \\
\hline U-234 & & & 10000 & 8.31E-12 & $8.31 \mathrm{E}-13$ \\
\hline Pu-239 & 0.001 & 0.0005 & 10000 & 3.75E-09 & 3.75E-06 \\
\hline Pu-239_5 & & & 10000 & $1.37 \mathrm{E}-12$ & 1.37E-09 \\
\hline U-235 & & & 10000 & $3.13 E-15$ & $3.13 E-12$ \\
\hline Pu-239 & 0.01 & 0.005 & 10000 & $3.75 \mathrm{E}-08$ & $3.75 \mathrm{E}-06$ \\
\hline Pu-239_5 & & & 10000 & $1.37 \mathrm{E}-11$ & 1.37E-09 \\
\hline U-235 & & & 10000 & $3.13 \mathrm{E}-14$ & $3.13 \mathrm{E}-12$ \\
\hline Pu-239 & 0.1 & 0.05 & 10000 & 7.26E-08 & 7.26E-07 \\
\hline Pu-239_5 & & & 10000 & $2.65 \mathrm{E}-11$ & $2.65 \mathrm{E}-10$ \\
\hline U-235 & & & 10000 & $5.98 \mathrm{E}-14$ & $5.98 \mathrm{E}-13$ \\
\hline Pu-239 & 1 & 0.5 & 10000 & 7.26E-08 & 7.26E-08 \\
\hline Pu-239_5 & & & 10000 & $2.65 \mathrm{E}-11$ & $2.65 \mathrm{E}-11$ \\
\hline U-235 & & & 10000 & $6.31 \mathrm{E}-14$ & $6.31 \mathrm{E}-14$ \\
\hline Pu-240 & 0.001 & 0.0005 & 10000 & 1.74E-09 & 1.74E-06 \\
\hline Pu-240_5 & & & 10000 & $6.34 \mathrm{E}-13$ & $6.34 \mathrm{E}-10$ \\
\hline U-236 & & & 10000 & $4.95 \mathrm{E}-14$ & $4.95 \mathrm{E}-11$ \\
\hline Pu-240 & 0.01 & 0.005 & 10000 & 1.74E-08 & $1.74 \mathrm{E}-06$ \\
\hline Pu-240_5 & & & 10000 & $6.34 \mathrm{E}-12$ & $6.34 \mathrm{E}-10$ \\
\hline
\end{tabular}




\begin{tabular}{|c|c|c|c|c|c|}
\hline Nuclide chains & $\begin{array}{c}\text { Inventory for } 2 \\
\text { disposal units (Ci) }\end{array}$ & $\begin{array}{c}\text { Inventory per disposal } \\
\text { unit (Ci) }\end{array}$ & $\begin{array}{l}\text { Time peak flux } \\
\text { occurred (yr) }\end{array}$ & $\begin{array}{c}\text { Peak flux from } 2 \\
\text { disposal units (Ci/yr) }\end{array}$ & Fractional flux (1/yr) \\
\hline U-236 & & & 10000 & $4.95 \mathrm{E}-13$ & $4.95 \mathrm{E}-11$ \\
\hline Pu-240 & 0.1 & 0.05 & 10000 & $1.42 \mathrm{E}-07$ & $1.42 \mathrm{E}-06$ \\
\hline Pu-240_5 & & & 10000 & $5.17 \mathrm{E}-11$ & $5.17 \mathrm{E}-10$ \\
\hline U-236 & & & 10000 & $3.95 \mathrm{E}-12$ & $3.95 \mathrm{E}-11$ \\
\hline Pu-240 & 1 & 0.5 & 10000 & 1.44E-07 & $1.44 \mathrm{E}-07$ \\
\hline Pu-240_5 & & & 10000 & $5.24 \mathrm{E}-11$ & $5.24 \mathrm{E}-11$ \\
\hline $\mathrm{U}-236$ & & & 10000 & $4.06 \mathrm{E}-12$ & $4.06 \mathrm{E}-12$ \\
\hline Pu-242 & 0.001 & 0.0005 & 10000 & 4.91E-09 & 4.91E-06 \\
\hline Pu-242_5 & & & 10000 & 1.79E-12 & 1.79E-09 \\
\hline $\mathrm{U}-238$ & & & 10000 & $6.19 \mathrm{E}-16$ & $6.19 \mathrm{E}-13$ \\
\hline Pu-242 & 0.01 & 0.005 & 10000 & 5.82E-09 & $5.82 \mathrm{E}-07$ \\
\hline Pu-242_5 & & & 10000 & $2.12 \mathrm{E}-12$ & $2.12 \mathrm{E}-10$ \\
\hline U-238 & & & 10000 & $7.30 \mathrm{E}-16$ & $7.30 \mathrm{E}-14$ \\
\hline Pu-242 & 0.1 & 0.05 & 10000 & 5.83E-09 & 5.83E-08 \\
\hline Pu-242_5 & & & 10000 & 2.12E-12 & $2.12 \mathrm{E}-11$ \\
\hline $\mathrm{U}-238$ & & & 10000 & $7.83 \mathrm{E}-16$ & $7.83 \mathrm{E}-15$ \\
\hline Pu-242 & 1 & 0.5 & 10000 & 5.90E-09 & 5.90E-09 \\
\hline Pu-242_5 & & & 10000 & $2.15 \mathrm{E}-12$ & $2.15 \mathrm{E}-12$ \\
\hline $\mathrm{U}-238$ & & & 10000 & $1.32 \mathrm{E}-15$ & $1.32 \mathrm{E}-15$ \\
\hline Pu-244 & 0.001 & 0.0005 & 10000 & $2.76 \mathrm{E}-11$ & $2.76 \mathrm{E}-08$ \\
\hline Pu-244_5 & & & 10000 & $1.01 \mathrm{E}-14$ & 1.01E-11 \\
\hline $\mathrm{Pu}-244$ & 0.01 & 0.005 & 10000 & $2.83 \mathrm{E}-11$ & 2.83E-09 \\
\hline Pu-244_5 & & & 10000 & 1.03E-14 & $1.03 E-12$ \\
\hline Pu-244 & 0.1 & 0.05 & 10000 & $3.53 E-11$ & $3.53 E-10$ \\
\hline Pu-244_5 & & & 10000 & $1.29 \mathrm{E}-14$ & 1.29E-13 \\
\hline Pu-244 & 1 & 0.5 & 10000 & $5.50 \mathrm{E}-11$ & $5.50 \mathrm{E}-11$ \\
\hline Pu-244_5 & & & 10000 & $2.01 \mathrm{E}-14$ & $2.01 \mathrm{E}-14$ \\
\hline Am-243 & 0.001 & 0.0005 & 10000 & 4.69E-20 & $4.69 \mathrm{E}-17$ \\
\hline Pu-239 & & & 10000 & $2.04 \mathrm{E}-10$ & 2.04E-07 \\
\hline Pu-239_5 & & & 10000 & $7.42 \mathrm{E}-14$ & $7.42 \mathrm{E}-11$ \\
\hline U-234 & & & 10000 & $4.47 \mathrm{E}-13$ & 4.47E-10 \\
\hline Am-243 & 0.01 & 0.005 & 10000 & 4.69E-19 & 4.69E-17 \\
\hline Pu-239 & & & 10000 & 2.04E-09 & 2.04E-07 \\
\hline Pu-239_5 & & & 10000 & $7.42 \mathrm{E}-13$ & $7.42 \mathrm{E}-11$ \\
\hline U-234 & & & 10000 & $4.47 \mathrm{E}-12$ & $4.47 \mathrm{E}-10$ \\
\hline Am-243 & 0.1 & 0.05 & 10000 & 4.69E-18 & 4.69E-17 \\
\hline Pu-239 & & & 10000 & $2.04 \mathrm{E}-08$ & $2.04 \mathrm{E}-07$ \\
\hline
\end{tabular}




\begin{tabular}{|c|c|c|c|c|c|}
\hline Nuclide chains & $\begin{array}{c}\text { Inventory for } 2 \\
\text { disposal units (Ci) }\end{array}$ & $\begin{array}{c}\text { Inventory per disposal } \\
\text { unit (Ci) }\end{array}$ & $\begin{array}{l}\text { Time peak flux } \\
\text { occurred (yr) }\end{array}$ & $\begin{array}{c}\text { Peak flux from } 2 \\
\text { disposal units (Ci/yr) }\end{array}$ & Fractional flux (1/yr) \\
\hline Pu-239_5 & & & 10000 & 7.43E-12 & $7.43 \mathrm{E}-11$ \\
\hline U-234 & & & 10000 & 4.47E-11 & 4.47E-10 \\
\hline Am-243 & 1 & 0.5 & 10000 & 4.69E-17 & $4.69 \mathrm{E}-17$ \\
\hline Pu-239 & & & 10000 & $7.85 \mathrm{E}-08$ & $7.85 \mathrm{E}-08$ \\
\hline Pu-239_5 & & & 10000 & $2.86 \mathrm{E}-11$ & $2.86 \mathrm{E}-11$ \\
\hline $\mathrm{U}-234$ & & & 10000 & $1.82 \mathrm{E}-10$ & $1.82 \mathrm{E}-10$ \\
\hline $\mathrm{Cm}-245$ & 0.001 & 0.0005 & 10000 & $2.49 \mathrm{E}-26$ & $2.49 \mathrm{E}-23$ \\
\hline Pu-241 & & & 10000 & $5.46 \mathrm{E}-25$ & $5.46 \mathrm{E}-22$ \\
\hline Pu-241_5 & & & 10000 & $3.86 \mathrm{E}-26$ & $3.86 \mathrm{E}-23$ \\
\hline Am-241 & & & 10000 & $1.40 \mathrm{E}-23$ & $1.40 \mathrm{E}-20$ \\
\hline $\mathrm{Np}-237$ & & & 10000 & $6.57 \mathrm{E}-12$ & 6.57E-09 \\
\hline $\mathrm{Cm}-245$ & 0.01 & 0.005 & 10000 & $2.49 \mathrm{E}-25$ & $2.49 \mathrm{E}-23$ \\
\hline $\mathrm{Pu}-241$ & & & 10000 & $5.46 \mathrm{E}-24$ & $5.46 \mathrm{E}-22$ \\
\hline Pu-241_5 & & & 10000 & $3.86 \mathrm{E}-25$ & $3.86 \mathrm{E}-23$ \\
\hline Am-241 & & & 10000 & $1.40 \mathrm{E}-22$ & $1.40 \mathrm{E}-20$ \\
\hline $\mathrm{Np}-237$ & & & 10000 & $6.57 \mathrm{E}-11$ & 6.57E-09 \\
\hline $\mathrm{Cm}-245$ & 0.1 & 0.05 & 10000 & $2.49 \mathrm{E}-24$ & $2.49 \mathrm{E}-23$ \\
\hline Pu-241 & & & 10000 & $5.46 \mathrm{E}-23$ & $5.46 \mathrm{E}-22$ \\
\hline Pu-241_5 & & & 10000 & 3.86E-24 & $3.86 \mathrm{E}-23$ \\
\hline Am-241 & & & 10000 & $1.40 \mathrm{E}-21$ & $1.40 \mathrm{E}-20$ \\
\hline $\mathrm{Np}-237$ & & & 10000 & $6.57 \mathrm{E}-10$ & 6.57E-09 \\
\hline $\mathrm{Cm}-245$ & 1 & 0.5 & 10000 & $2.49 \mathrm{E}-23$ & $2.49 \mathrm{E}-23$ \\
\hline Pu-241 & & & 10000 & 5.46E-22 & $5.46 \mathrm{E}-22$ \\
\hline Pu-241_5 & & & 10000 & $3.86 \mathrm{E}-23$ & $3.86 \mathrm{E}-23$ \\
\hline Am-241 & & & 10000 & $1.40 \mathrm{E}-20$ & $1.40 \mathrm{E}-20$ \\
\hline $\mathrm{Np}-237$ & & & 10000 & 6.57E-09 & 6.57E-09 \\
\hline $\mathrm{Cm}-246$ & 1 & 0.5 & 10000 & $1.31 \mathrm{E}-23$ & $1.31 \mathrm{E}-23$ \\
\hline $\mathrm{Cm}-247$ & 0.001 & 0.0005 & 10000 & 5.64E-26 & 5.64E-23 \\
\hline Am-243 & & & 10000 & $1.75 \mathrm{E}-20$ & $1.75 \mathrm{E}-17$ \\
\hline $\mathrm{Np}-239$ & & & 10000 & $4.20 \mathrm{E}-14$ & $4.20 \mathrm{E}-11$ \\
\hline Pu-239 & & & 10000 & $2.49 \mathrm{E}-11$ & $2.49 \mathrm{E}-08$ \\
\hline Pu-239_5 & & & 10000 & 9.07E-15 & 9.07E-12 \\
\hline $\mathrm{Cm}-247$ & 0.01 & 0.005 & 10000 & $5.64 \mathrm{E}-25$ & $5.64 \mathrm{E}-23$ \\
\hline Am-243 & & & 10000 & $1.75 \mathrm{E}-19$ & $1.75 \mathrm{E}-17$ \\
\hline $\mathrm{Np}-239$ & & & 10000 & $4.20 \mathrm{E}-13$ & $4.20 \mathrm{E}-11$ \\
\hline Pu-239 & & & 10000 & $2.49 \mathrm{E}-10$ & $2.49 \mathrm{E}-08$ \\
\hline Pu-239_5 & & & 10000 & $9.07 \mathrm{E}-14$ & $9.07 \mathrm{E}-12$ \\
\hline
\end{tabular}




\begin{tabular}{|c|c|c|c|c|c|}
\hline Nuclide chains & $\begin{array}{c}\text { Inventory for } 2 \\
\text { disposal units (Ci) }\end{array}$ & $\begin{array}{c}\text { Inventory per disposal } \\
\text { unit (Ci) }\end{array}$ & $\begin{array}{l}\text { Time peak flux } \\
\text { occurred (yr) }\end{array}$ & $\begin{array}{c}\text { Peak flux from } 2 \\
\text { disposal units (Ci/yr) }\end{array}$ & Fractional flux (1/yr) \\
\hline $\mathrm{Cm}-247$ & 0.1 & 0.05 & 10000 & 5.64E-24 & $5.64 \mathrm{E}-23$ \\
\hline Am-243 & & & 10000 & $1.75 \mathrm{E}-18$ & $1.75 \mathrm{E}-17$ \\
\hline $\mathrm{Np}-239$ & & & 10000 & $4.20 \mathrm{E}-12$ & $4.20 \mathrm{E}-11$ \\
\hline Pu-239 & & & 10000 & $2.49 \mathrm{E}-09$ & $2.49 \mathrm{E}-08$ \\
\hline Pu-239_5 & & & 10000 & 9.07E-13 & 9.07E-12 \\
\hline $\mathrm{Cm}-247$ & 1 & 0.5 & 10000 & $5.64 \mathrm{E}-23$ & $5.64 \mathrm{E}-23$ \\
\hline Am-243 & & & 10000 & $1.75 \mathrm{E}-17$ & $1.75 \mathrm{E}-17$ \\
\hline $\mathrm{Np}-239$ & & & 10000 & $4.20 \mathrm{E}-11$ & $4.20 \mathrm{E}-11$ \\
\hline Pu-239 & & & 10000 & $2.47 \mathrm{E}-08$ & $2.47 \mathrm{E}-08$ \\
\hline Pu-239_5 & & & 10000 & $9.01 \mathrm{E}-12$ & $9.01 \mathrm{E}-12$ \\
\hline $\mathrm{Cm}-248$ & 0.001 & 0.0005 & 10000 & $5.53 \mathrm{E}-26$ & $5.53 \mathrm{E}-23$ \\
\hline $\mathrm{Pu}-244$ & & & 10000 & $8.81 \mathrm{E}-14$ & $8.81 \mathrm{E}-11$ \\
\hline Pu-244_5 & & & 10000 & $3.21 \mathrm{E}-17$ & $3.21 \mathrm{E}-14$ \\
\hline $\mathrm{Cm}-248$ & 0.01 & 0.005 & 10000 & $5.53 E-25$ & $5.53 \mathrm{E}-23$ \\
\hline $\mathrm{Pu}-244$ & & & 10000 & $8.81 \mathrm{E}-13$ & $8.81 \mathrm{E}-11$ \\
\hline Pu-244_5 & & & 10000 & $3.21 \mathrm{E}-16$ & $3.21 \mathrm{E}-14$ \\
\hline $\mathrm{Cm}-248$ & 0.1 & 0.05 & 10000 & $5.53 E-24$ & $5.53 \mathrm{E}-23$ \\
\hline $\mathrm{Pu}-244$ & & & 10000 & $8.81 \mathrm{E}-12$ & $8.81 \mathrm{E}-11$ \\
\hline Pu-244_5 & & & 10000 & $3.21 \mathrm{E}-15$ & $3.21 \mathrm{E}-14$ \\
\hline $\mathrm{Cm}-248$ & 1 & 0.5 & 10000 & $5.53 \mathrm{E}-23$ & $5.53 \mathrm{E}-23$ \\
\hline $\mathrm{Pu}-244$ & & & 10000 & 3.04E-11 & 3.04E-11 \\
\hline Pu-244_5 & & & 10000 & $1.11 \mathrm{E}-14$ & $1.11 \mathrm{E}-14$ \\
\hline Cf-249 & 0.001 & 0.0005 & 7972 & 7.01E-20 & $7.01 \mathrm{E}-17$ \\
\hline $\mathrm{Cm}-245$ & & & 10000 & $4.05 E-21$ & $4.05 \mathrm{E}-18$ \\
\hline Pu-241 & & & 10000 & $4.66 \mathrm{E}-20$ & $4.66 \mathrm{E}-17$ \\
\hline Pu-241_5 & & & 10000 & $3.08 \mathrm{E}-21$ & $3.08 \mathrm{E}-18$ \\
\hline Am-241 & & & 10000 & $1.09 \mathrm{E}-20$ & $1.09 \mathrm{E}-17$ \\
\hline $\mathrm{Np}-237$ & & & 10000 & $2.66 \mathrm{E}-13$ & $2.66 \mathrm{E}-10$ \\
\hline Cf-249 & 0.01 & 0.005 & 7972 & $7.01 \mathrm{E}-19$ & $7.01 \mathrm{E}-17$ \\
\hline $\mathrm{Cm}-245$ & & & 10000 & 4.05E-20 & $4.05 \mathrm{E}-18$ \\
\hline Pu-241 & & & 10000 & $4.66 \mathrm{E}-19$ & $4.66 \mathrm{E}-17$ \\
\hline Pu-241_5 & & & 10000 & $3.08 \mathrm{E}-20$ & $3.08 \mathrm{E}-18$ \\
\hline Am-241 & & & 10000 & $1.09 \mathrm{E}-19$ & $1.09 \mathrm{E}-17$ \\
\hline $\mathrm{Np}-237$ & & & 10000 & $2.66 \mathrm{E}-12$ & $2.66 \mathrm{E}-10$ \\
\hline Cf-249 & 0.1 & 0.05 & 7972 & $7.01 \mathrm{E}-18$ & $7.01 \mathrm{E}-17$ \\
\hline $\mathrm{Cm}-245$ & & & 10000 & $4.05 \mathrm{E}-19$ & $4.05 \mathrm{E}-18$ \\
\hline Pu-241 & & & 10000 & $4.66 \mathrm{E}-18$ & $4.66 \mathrm{E}-17$ \\
\hline
\end{tabular}




\begin{tabular}{|c|c|c|c|c|c|}
\hline Nuclide chains & $\begin{array}{c}\text { Inventory for } 2 \\
\text { disposal units (Ci) }\end{array}$ & $\begin{array}{c}\text { Inventory per disposal } \\
\text { unit (Ci) }\end{array}$ & $\begin{array}{l}\text { Time peak flux } \\
\text { occurred (yr) }\end{array}$ & $\begin{array}{c}\text { Peak flux from } 2 \\
\text { disposal units (Ci/yr) }\end{array}$ & Fractional flux (1/yr) \\
\hline Pu-241_5 & & & 10000 & $3.08 \mathrm{E}-19$ & $3.08 \mathrm{E}-18$ \\
\hline Am-241 & & & 10000 & 1.09E-18 & $1.09 \mathrm{E}-17$ \\
\hline $\mathrm{Np}-237$ & & & 10000 & $2.66 \mathrm{E}-11$ & $2.66 \mathrm{E}-10$ \\
\hline Cf-249 & 1 & 0.5 & 7972 & $7.01 \mathrm{E}-17$ & $7.01 \mathrm{E}-17$ \\
\hline $\mathrm{Cm}-245$ & & & 10000 & $4.05 \mathrm{E}-18$ & $4.05 \mathrm{E}-18$ \\
\hline Pu-241 & & & 10000 & $4.66 \mathrm{E}-17$ & $4.66 \mathrm{E}-17$ \\
\hline Pu-241_5 & & & 10000 & $3.08 \mathrm{E}-18$ & $3.08 \mathrm{E}-18$ \\
\hline Am-241 & & & 10000 & $1.09 \mathrm{E}-17$ & 1.09E-17 \\
\hline $\mathrm{Np}-237$ & & & 10000 & $2.66 \mathrm{E}-10$ & $2.66 \mathrm{E}-10$ \\
\hline
\end{tabular}




\section{Peak concentration and SOF results from PORFLOW aquifer transport simulations}

For each parent and inventory modeled with PORFLOW, Table A-3 provides the peak sum-offractions for the periods $0-100,100-1350$, and 1350-10,000 years. Also shown are the location and time of the peak, groundwater concentrations at the peak SOF, the 1/SOF "limit", and the percentage contribution of each member of the chain to the limit.

Each decay chain was simulated over a 10,000 year period, with the exception of H-3, Zr-95 and Nb-95. H-3 is unretarded $\left(K_{d}=0\right)$ and rapidly migrates out of the model domain after vault failure at 1075 years. Zr-95 and $\mathrm{Nb}-95$ have short half-lives and rapidly decay to negligible amounts. For these three nuclides, PORFLOW simulations were performed to 1500 years. 
Table A-3. Peak groundwater concentration and SOF results.

\begin{tabular}{|c|c|c|c|c|c|c|c|c|c|c|c|c|c|c|}
\hline 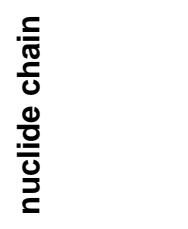 & 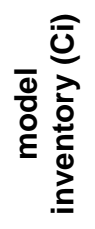 & 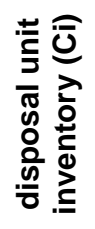 & 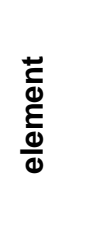 & 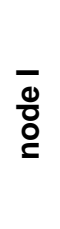 & $\begin{array}{l}\mathfrak{0} \\
\stackrel{0}{0} \\
\stackrel{0}{\complement}\end{array}$ & 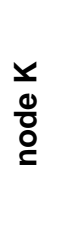 & 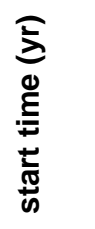 & 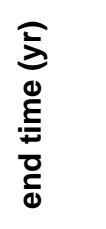 & 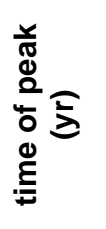 & 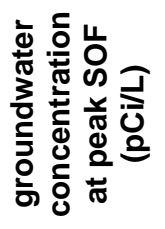 & 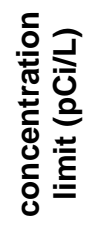 & 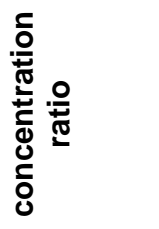 & 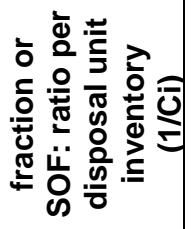 & 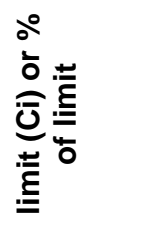 \\
\hline Tracer & 1 & 0.5 & 3871 & 12 & 15 & 11 & 0 & 100 & 100 & $5.20 \mathrm{E}-04$ & $1 \mathrm{E}+20$ & $5.20 \mathrm{E}-24$ & $1.04 \mathrm{E}-23$ & 100 \\
\hline SOF-chain & & & & & & & & & & & & & 1.04E-23 & $9.61 \mathrm{E}+22$ \\
\hline Tracer & 1 & 0.5 & 3871 & 12 & 15 & 11 & 100 & 1350 & 630 & $2.04 \mathrm{E}+03$ & $1 \mathrm{E}+20$ & $2.04 \mathrm{E}-17$ & $4.08 \mathrm{E}-17$ & 100 \\
\hline SOF-chain & & & & & & & & & & & & & $4.08 \mathrm{E}-17$ & $2.45 E+16$ \\
\hline Tracer & 1 & 0.5 & 1475 & 16 & 15 & 5 & 1350 & 10000 & 1360 & 5.68E-04 & $1 \mathrm{E}+20$ & 5.68E-24 & 1.14E-23 & 100 \\
\hline SOF-chain & & & & & & & & & & & & & 1.14E-23 & $8.80 E+22$ \\
\hline $\mathrm{H}-3$ & 1 & 0.5 & 3871 & 12 & 15 & 11 & 0 & 100 & 100 & $1.88 \mathrm{E}-06$ & 20000 & $9.40 \mathrm{E}-11$ & $1.88 \mathrm{E}-10$ & 100 \\
\hline SOF-chain & & & & & & & & & & & & & $1.88 \mathrm{E}-10$ & $5.32 E+09$ \\
\hline $\mathrm{H}-3$ & 1 & 0.5 & 3871 & 12 & 15 & 11 & 100 & 1350 & 110 & 1.83E-06 & 20000 & $9.12 \mathrm{E}-11$ & $1.83 \mathrm{E}-10$ & 100 \\
\hline SOF-chain & & & & & & & & & & & & & $1.83 \mathrm{E}-10$ & $5.48 E+09$ \\
\hline $\mathrm{H}-3$ & 1 & 0.5 & 1475 & 16 & 15 & 5 & 1350 & 1500 & 1360 & 1.54E-36 & 20000 & $7.70 \mathrm{E}-41$ & $1.54 \mathrm{E}-40$ & 100 \\
\hline SOF-chain & & & & & & & & & & & & & $1.54 \mathrm{E}-40$ & $6.50 \mathrm{E}+39$ \\
\hline SOF-chain & & & & & & & & & & & & & $4.86 \mathrm{E}-32$ & $2.06 \mathrm{E}+31$ \\
\hline C-14 & 1 & 0.5 & 3871 & 12 & 15 & 11 & 100 & 1350 & 1350 & 1.10E-05 & 2000 & 5.51E-09 & 1.10E-08 & 100 \\
\hline SOF-chain & & & & & & & & & & & & & 1.10E-08 & $9.07 E+07$ \\
\hline C-14 & 1 & 0.5 & 3871 & 12 & 15 & 11 & 1350 & 10000 & 10000 & $5.91 \mathrm{E}-02$ & 2000 & $2.96 \mathrm{E}-05$ & 5.91E-05 & 100 \\
\hline SOF-chain & & & & & & & & & & & & & 5.91E-05 & $1.69 \mathrm{E}+04$ \\
\hline C-14_KB & 1 & 0.5 & 4671 & 12 & 15 & 13 & 0 & 100 & 100 & 1.73E-27 & 2000 & $8.67 \mathrm{E}-31$ & $1.73 \mathrm{E}-30$ & 100 \\
\hline SOF-chain & & & & & & & & & & & & & $1.73 E-30$ & $5.77 E+29$ \\
\hline C-14_KB & 1 & 0.5 & 3871 & 12 & 15 & 11 & 100 & 1350 & 1350 & 3.92E-04 & 2000 & 1.96E-07 & $3.92 \mathrm{E}-07$ & 100 \\
\hline SOF-chain & & & & & & & & & & & & & $3.92 \mathrm{E}-07$ & $2.55 \mathrm{E}+06$ \\
\hline C-14_KB & 1 & 0.5 & 3871 & 12 & 15 & 11 & 1350 & 10000 & 8950 & $1.49 \mathrm{E}+00$ & 2000 & $7.45 \mathrm{E}-04$ & 1.49E-03 & 100 \\
\hline SOF-chain & & & & & & & & & & & & & 1.49E-03 & $6.72 E+02$ \\
\hline $\mathrm{K}-40$ & 1 & 0.5 & 4671 & 12 & 15 & 13 & 0 & 100 & 100 & $6.40 \mathrm{E}-18$ & 300 & $2.13 \mathrm{E}-20$ & $4.27 \mathrm{E}-20$ & 100 \\
\hline SOF-chain & & & & & & & & & & & & & $4.27 \mathrm{E}-20$ & $2.34 \mathrm{E}+19$ \\
\hline $\mathrm{K}-40$ & 1 & 0.5 & 3871 & 12 & 15 & 11 & 100 & 1350 & 1200 & $7.59 \mathrm{E}+02$ & 300 & $2.53 \mathrm{E}+00$ & $5.06 \mathrm{E}+00$ & 100 \\
\hline SOF-chain & & & & & & & & & & & & & $5.06 \mathrm{E}+00$ & $1.98 \mathrm{E}-01$ \\
\hline $\mathrm{K}-40$ & 1 & 0.5 & 3871 & 12 & 15 & 11 & 1350 & 10000 & 1360 & $6.43 \mathrm{E}+00$ & 300 & $2.14 \mathrm{E}-02$ & 4.29E-02 & 100 \\
\hline SOF-chain & & & & & & & & & & & & & 4.29E-02 & $2.33 E+01$ \\
\hline
\end{tabular}




\begin{tabular}{|c|c|c|c|c|c|c|c|c|c|c|c|c|c|c|}
\hline 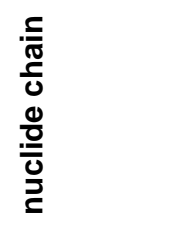 & 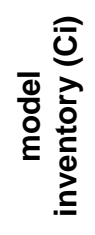 & 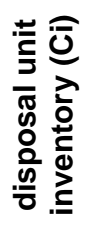 & 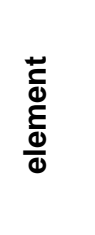 & 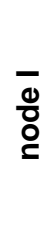 & $\begin{array}{l}0 \\
0 \\
0 \\
0\end{array}$ & $\begin{array}{l}\searrow \\
\frac{0}{0} \\
0\end{array}$ & 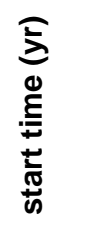 & 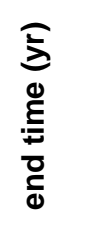 & 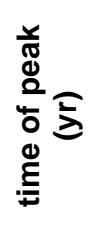 & 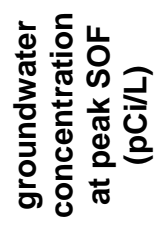 & 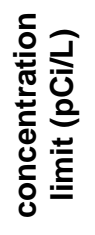 & 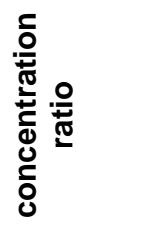 & 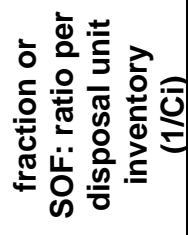 & 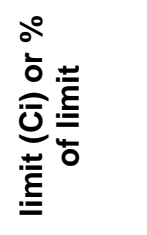 \\
\hline $\mathrm{Ni}-59$ & 1 & 0.5 & 4673 & 14 & 15 & 13 & 0 & 100 & 100 & $2.09 E-81$ & 300 & $6.98 \mathrm{E}-84$ & $1.40 \mathrm{E}-83$ & 100 \\
\hline SOF-chain & & & & & & & & & & & & & $1.40 \mathrm{E}-83$ & $7.17 \mathrm{E}+82$ \\
\hline $\mathrm{Ni}-59$ & 1 & 0.5 & 4673 & 14 & 15 & 13 & 100 & 1350 & 1350 & 3.87E-38 & 300 & $1.29 \mathrm{E}-40$ & $2.58 \mathrm{E}-40$ & 100 \\
\hline SOF-chain & & & & & & & & & & & & & $2.58 \mathrm{E}-40$ & $3.87 \mathrm{E}+39$ \\
\hline $\mathrm{Ni}-59$ & 1 & 0.5 & 4671 & 12 & 15 & 13 & 1350 & 10000 & 10000 & 9.16E-01 & 300 & 3.05E-03 & $6.11 \mathrm{E}-03$ & 100 \\
\hline SOF-chain & & & & & & & & & & & & & 6.11E-03 & $1.64 \mathrm{E}+02$ \\
\hline Se-79 & 1 & 0.5 & 4673 & 14 & 15 & 13 & 0 & 100 & 100 & $5.66 \mathrm{E}-39$ & 700 & 8.09E-42 & 1.62E-41 & 100 \\
\hline SOF-chain & & & & & & & & & & & & & 1.62E-41 & $6.18 \mathrm{E}+40$ \\
\hline Se-79 & 1 & 0.5 & 4673 & 14 & 15 & 13 & 100 & 1350 & 1350 & 3.00E-04 & 700 & 4.29E-07 & 8.57E-07 & 100 \\
\hline SOF-chain & & & & & & & & & & & & & $8.57 \mathrm{E}-07$ & $1.17 \mathrm{E}+06$ \\
\hline Se-79 & 1 & 0.5 & 3871 & 12 & 15 & 11 & 1350 & 10000 & 2310 & $1.07 \mathrm{E}+02$ & 700 & $1.52 \mathrm{E}-01$ & 3.04E-01 & 100 \\
\hline SOF-chain & & & & & & & & & & & & & 3.04E-01 & $3.29 E+00$ \\
\hline SOF-chain & & & & & & & & & & & & & $6.26 \mathrm{E}-29$ & $1.60 \mathrm{E}+28$ \\
\hline Sr-90 & 1 & 0.5 & 4671 & 12 & 15 & 13 & 100 & 1350 & 1280 & $6.54 \mathrm{E}-12$ & 8 & 8.17E-13 & 1.63E-12 & 100 \\
\hline SOF-chain & & & & & & & & & & & & & 1.63E-12 & $6.12 \mathrm{E}+11$ \\
\hline Sr-90 & 1 & 0.5 & 4671 & 12 & 15 & 13 & 1350 & 10000 & 1360 & $2.19 \mathrm{E}-12$ & 8 & $2.74 \mathrm{E}-13$ & $5.47 \mathrm{E}-13$ & 100 \\
\hline SOF-chain & & & & & & & & & & & & & $5.47 \mathrm{E}-13$ & $1.83 \mathrm{E}+12$ \\
\hline Zr-93 & 1 & 0.5 & 4673 & 14 & 15 & 13 & 0 & 100 & 100 & $1.26 \mathrm{E}-93$ & 2000 & $6.28 \mathrm{E}-97$ & $1.26 \mathrm{E}-96$ & 0 \\
\hline $\mathrm{Nb}-93 \mathrm{~m}$ & & & & & & & & & & $8.21 \mathrm{E}-75$ & 1000 & $8.21 \mathrm{E}-78$ & 1.64E-77 & 100 \\
\hline SOF-chain & & & & & & & & & & & & & 1.64E-77 & $6.09 \mathrm{E}+76$ \\
\hline $\mathrm{Zr}-93$ & 1 & 0.5 & 4673 & 14 & 15 & 13 & 100 & 1350 & 1350 & $3.78 \mathrm{E}-50$ & 2000 & 1.89E-53 & $3.78 \mathrm{E}-53$ & 0 \\
\hline $\mathrm{Nb}-93 \mathrm{~m}$ & & & & & & & & & & $6.85 \mathrm{E}-36$ & 1000 & $6.85 E-39$ & 1.37E-38 & 100 \\
\hline SOF-chain & & & & & & & & & & & & & 1.37E-38 & $7.30 \mathrm{E}+37$ \\
\hline Zr-93 & 1 & 0.5 & 4673 & 14 & 15 & 13 & 1350 & 10000 & 10000 & 1.82E-07 & 2000 & 9.07E-11 & $1.82 \mathrm{E}-10$ & 10.325 \\
\hline $\mathrm{Nb}-93 \mathrm{~m}$ & & & & & & & & & & $7.88 \mathrm{E}-07$ & 1000 & $7.88 \mathrm{E}-10$ & 1.58E-09 & 89.675 \\
\hline SOF-chain & & & & & & & & & & & & & 1.76E-09 & $5.69 \mathrm{E}+08$ \\
\hline Zr-95 & 1 & 0.5 & 4673 & 14 & 15 & 13 & 0 & 100 & 5.08 & $2.01 \mathrm{E}-146$ & 200 & 1.00E-148 & $2.01 \mathrm{E}-148$ & 0 \\
\hline $\mathrm{Nb}-95$ & & & & & & & & & & $3.20 \mathrm{E}-132$ & 300 & 1.07E-134 & $2.13 \mathrm{E}-134$ & 0 \\
\hline SOF-chain & & & & & & & & & & & & & 2.13E-134 & $1.00 \mathrm{E}+99$ \\
\hline Zr-95 & 1 & 0.5 & 4673 & 14 & 15 & 13 & 100 & 1350 & 100.1 & $1.53 \mathrm{E}-243$ & 200 & $7.64 \mathrm{E}-246$ & $1.53 \mathrm{E}-245$ & 0 \\
\hline
\end{tabular}




\begin{tabular}{|c|c|c|c|c|c|c|c|c|c|c|c|c|c|c|}
\hline $\begin{array}{l}\frac{.}{\pi} \\
\frac{\pi}{0} \\
\frac{0}{0} \\
\frac{0}{0} \\
\frac{0}{2}\end{array}$ & 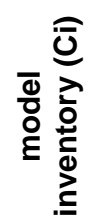 & 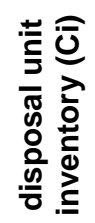 & 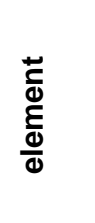 & $\begin{array}{l}\overline{0} \\
\frac{0}{0}\end{array}$ & 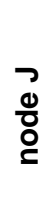 & $\begin{array}{l}\searrow \\
\frac{0}{0} \\
0\end{array}$ & 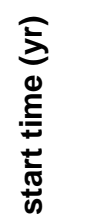 & 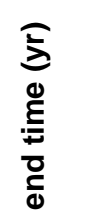 & 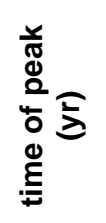 & 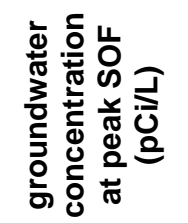 & 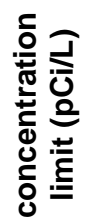 & 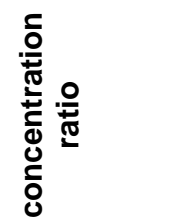 & 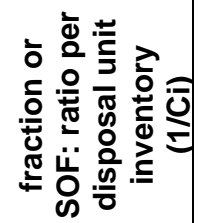 & 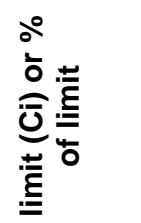 \\
\hline Zr-95 & 1 & 0.5 & 1488 & 9 & 16 & 5 & 1350 & 1500 & 1350 & $0.00 \mathrm{E}+00$ & 200 & $0.00 \mathrm{E}+00$ & $0.00 \mathrm{E}+00$ & 0 \\
\hline $\mathrm{Nb}-95$ & & & & & & & & & & $0.00 \mathrm{E}+00$ & 300 & $0.00 \mathrm{E}+00$ & $0.00 \mathrm{E}+00$ & 0 \\
\hline SOF-chain & & & & & & & & & & & & & $0.00 \mathrm{E}+00$ & $1.00 \mathrm{E}+99$ \\
\hline $\mathrm{Nb}-94$ & 1 & 0.5 & 4673 & 14 & 15 & 13 & 0 & 100 & 100 & $5.26 \mathrm{E}-72$ & 1000 & $5.26 \mathrm{E}-75$ & $1.05 \mathrm{E}-74$ & 100 \\
\hline SOF-chain & & & & & & & & & & & & & 1.05E-74 & $9.51 \mathrm{E}+73$ \\
\hline $\mathrm{Nb}-94$ & 1 & 0.5 & 4673 & 14 & 15 & 13 & 100 & 1350 & 1350 & $3.58 \mathrm{E}-29$ & 1000 & $3.58 \mathrm{E}-32$ & $7.16 \mathrm{E}-32$ & 100 \\
\hline SOF-chain & & & & & & & & & & & & & $7.16 \mathrm{E}-32$ & $1.40 \mathrm{E}+31$ \\
\hline $\mathrm{Nb}-94$ & 1 & 0.5 & 3871 & 12 & 15 & 11 & 1350 & 10000 & 10000 & $3.31 \mathrm{E}+00$ & 1000 & 3.31E-03 & 6.62E-03 & 100 \\
\hline SOF-chain & & & & & & & & & & & & & $6.62 \mathrm{E}-03$ & $1.51 \mathrm{E}+02$ \\
\hline $\mathrm{Nb}-95$ & 1 & 0.5 & 4673 & 14 & 15 & 13 & 0 & 100 & 5.08 & $1.42 \mathrm{E}-132$ & 300 & $4.72 \mathrm{E}-135$ & $9.45 \mathrm{E}-135$ & 0 \\
\hline SOF-chain & & & & & & & & & & & & & $9.45 \mathrm{E}-135$ & $1.00 \mathrm{E}+9 \mathrm{~g}$ \\
\hline $\mathrm{Nb}-95$ & 1 & 0.5 & 4673 & 14 & 15 & 13 & 100 & 1350 & 100.1 & 2.899E-316 & 300 & 9.663E-319 & 1.933E-318 & 0 \\
\hline $\mathrm{Nb}-95$ & 1 & 0.5 & 1488 & 9 & 16 & 5 & 1350 & 1500 & 1350 & $0.00 \mathrm{E}+00$ & 300 & $0.00 \mathrm{E}+00$ & $0.00 \mathrm{E}+00$ & 0 \\
\hline SOF-chain & & & & & & & & & & & & & $0.00 E+00$ & $1.00 \mathrm{E}+9 \mathrm{~g}$ \\
\hline Tc-99 & 1 & 0.5 & 3871 & 12 & 15 & 11 & 0 & 100 & 100 & $1.12 \mathrm{E}-17$ & 900 & $1.24 \mathrm{E}-20$ & $2.48 \mathrm{E}-20$ & 100 \\
\hline SOF-chain & & & & & & & & & & & & & $2.48 \mathrm{E}-20$ & $4.04 \mathrm{E}+19$ \\
\hline Tc-99 & 1 & 0.5 & 3871 & 12 & 15 & 11 & 100 & 1350 & 1350 & $3.42 E+00$ & 900 & $3.80 \mathrm{E}-03$ & $7.60 \mathrm{E}-03$ & 100 \\
\hline SOF-chain & & & & & & & & & & & & & $7.60 \mathrm{E}-03$ & $1.32 \mathrm{E}+02$ \\
\hline Tc-99 & 1 & 0.5 & 3871 & 12 & 15 & 11 & 1350 & 10000 & 2780 & $2.41 \mathrm{E}+01$ & 900 & $2.68 \mathrm{E}-02$ & $5.36 \mathrm{E}-02$ & 100 \\
\hline SOF-chain & & & & & & & & & & & & & $5.36 \mathrm{E}-02$ & $1.87 \mathrm{E}+01$ \\
\hline Tc-99 KB & 1 & 0.5 & 3871 & 12 & 15 & 11 & 0 & 100 & 100 & $1.49 \mathrm{E}-20$ & 900 & $1.66 \mathrm{E}-23$ & $3.31 \mathrm{E}-23$ & 100 \\
\hline SOF-chain & & & & & & & & & & & & & $3.31 \mathrm{E}-23$ & $3.02 \mathrm{E}+22$ \\
\hline Tc-99_KB & 1 & 0.5 & 3871 & 12 & 15 & 11 & 100 & 1350 & 1350 & 1.87E-02 & 900 & $2.08 \mathrm{E}-05$ & 4.16E-05 & 100 \\
\hline SOF-chain & & & & & & & & & & & & & 4.16E-05 & $2.41 \mathrm{E}+04$ \\
\hline Tc-99_KB & 1 & 0.5 & 3871 & 12 & 15 & 11 & 1350 & 10000 & 9410 & $3.47 E+00$ & 900 & $3.85 E-03$ & 7.71E-03 & 100 \\
\hline SOF-chain & & & & & & & & & & & & & 7.71E-03 & $1.30 \mathrm{E}+02$ \\
\hline Sn-126 & 1 & 0.5 & 4673 & 14 & 15 & 13 & 0 & 100 & 100 & 1.82E-70 & 300 & $6.06 \mathrm{E}-73$ & $1.21 \mathrm{E}-72$ & 100 \\
\hline SOF-chain & & & & & & & & & & & & & $1.21 \mathrm{E}-72$ & $8.25 \mathrm{E}+71$ \\
\hline Sn-126 & 1 & 0.5 & 4673 & 14 & 15 & 13 & 100 & 1350 & 1350 & 8.60E-28 & 300 & $2.87 \mathrm{E}-30$ & $5.73 \mathrm{E}-30$ & 100 \\
\hline SOF-chain & & & & & & & & & & & & & $5.73 \mathrm{E}-30$ & $1.74 \mathrm{E}+2 \mathrm{~S}$ \\
\hline
\end{tabular}




\begin{tabular}{|c|c|c|c|c|c|c|c|c|c|c|c|c|c|c|}
\hline 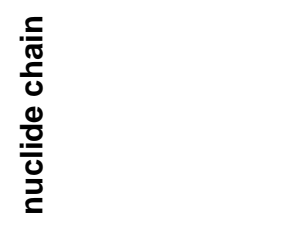 & 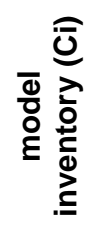 & 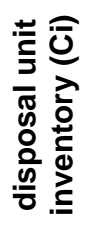 & 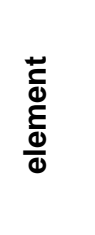 & $\begin{array}{l}\bar{\Phi} \\
\frac{0}{\varrho}\end{array}$ & $\begin{array}{l}0 \\
\frac{0}{0} \\
0\end{array}$ & $\begin{array}{l}\searrow \\
\frac{0}{0} \\
0\end{array}$ & 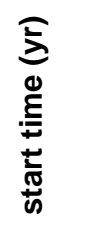 & 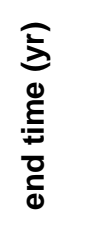 & 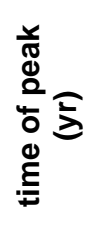 & 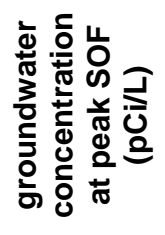 & 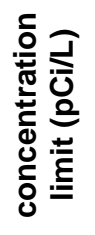 & 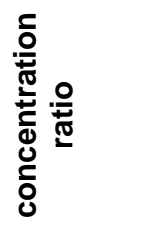 & 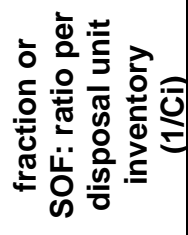 & 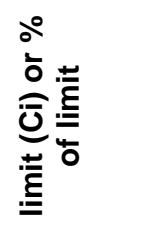 \\
\hline $\mathrm{I}-129$ & 1 & 0.5 & 4671 & 12 & 15 & 13 & 0 & 100 & 100 & $1.15 \mathrm{E}-11$ & 1 & $1.15 \mathrm{E}-11$ & $2.31 \mathrm{E}-11$ & 100 \\
\hline SOF-chain & & & & & & & & & & & & & $2.31 \mathrm{E}-11$ & $4.33 E+10$ \\
\hline $\mathrm{I}-129$ & 1 & 0.5 & 3871 & 12 & 15 & 11 & 100 & 1350 & 1120 & $1.10 \mathrm{E}+03$ & 1 & $1.10 \mathrm{E}+03$ & $2.20 \mathrm{E}+03$ & 100 \\
\hline SOF-chain & & & & & & & & & & & & & $2.20 \mathrm{E}+03$ & $4.55 \mathrm{E}-04$ \\
\hline $\mathrm{I}-129$ & 1 & 0.5 & 1475 & 16 & 15 & 5 & 1350 & 10000 & 1360 & $1.42 \mathrm{E}-02$ & 1 & 1.42E-02 & 2.83E-02 & 100 \\
\hline SOF-chain & & & & & & & & & & & & & 2.83E-02 & $3.54 \mathrm{E}+01$ \\
\hline I-129_ETF-Carbon & 1 & 0.5 & 4671 & 12 & 15 & 13 & 0 & 100 & 100 & $4.01 \mathrm{E}-14$ & 1 & $4.01 \mathrm{E}-14$ & $8.01 \mathrm{E}-14$ & 100 \\
\hline SOF-chain & & & & & & & & & & & & & $8.01 \mathrm{E}-14$ & $1.25 \mathrm{E}+13$ \\
\hline I-129_ETF-Carbon & 1 & 0.5 & 3871 & 12 & 15 & 11 & 100 & 1350 & 1150 & $5.17 \mathrm{E}+00$ & 1 & $5.17 \mathrm{E}+00$ & $1.03 \mathrm{E}+01$ & 100 \\
\hline SOF-chain & & & & & & & & & & & & & $1.03 \mathrm{E}+01$ & 9.67E-02 \\
\hline I-129_ETF-Carbon & 1 & 0.5 & 3871 & 12 & 15 & 11 & 1350 & 10000 & 8030 & $4.85 \mathrm{E}+00$ & 1 & $4.85 \mathrm{E}+00$ & $9.71 \mathrm{E}+00$ & 100 \\
\hline SOF-chain & & & & & & & & & & & & & $9.71 \mathrm{E}+00$ & 1.03E-01 \\
\hline SOF-chain & & & & & & & & & & & & & 2.34E-24 & $4.28 \mathrm{E}+23$ \\
\hline I-129_KB & 1 & 0.5 & 3871 & 12 & 15 & 11 & 100 & 1350 & 1350 & 8.02E-05 & 1 & 8.02E-05 & 1.60E-04 & 100 \\
\hline SOF-chain & & & & & & & & & & & & & 1.60E-04 & $6.24 \mathrm{E}+03$ \\
\hline $\mathrm{I}-129 \_\mathrm{KB}$ & 1 & 0.5 & 3871 & 12 & 15 & 11 & 1350 & 10000 & 10000 & $4.18 \mathrm{E}-01$ & 1 & $4.18 \mathrm{E}-01$ & $8.35 \mathrm{E}-01$ & 100 \\
\hline SOF-chain & & & & & & & & & & & & & 8.35E-01 & $1.20 \mathrm{E}+00$ \\
\hline Cs-135 & 1 & 0.5 & 4673 & 14 & 15 & 13 & 0 & 100 & 100 & 4.80E-76 & 900 & 5.33E-79 & $1.07 \mathrm{E}-78$ & 100 \\
\hline SOF-chain & & & & & & & & & & & & & 1.07E-78 & $9.38 \mathrm{E}+77$ \\
\hline Cs-135 & 1 & 0.5 & 4673 & 14 & 15 & 13 & 100 & 1350 & 1350 & $3.75 E-33$ & 900 & $4.17 E-36$ & 8.34E-36 & 100 \\
\hline SOF-chain & & & & & & & & & & & & & $8.34 \mathrm{E}-36$ & $1.20 \mathrm{E}+35$ \\
\hline Cs-135 & 1 & 0.5 & 4671 & 12 & 15 & 13 & 1350 & 10000 & 10000 & $9.77 \mathrm{E}+00$ & 900 & 1.09E-02 & $2.17 \mathrm{E}-02$ & 100 \\
\hline SOF-chain & & & & & & & & & & & & & 2.17E-02 & $4.60 \mathrm{E}+01$ \\
\hline Po-210 & 1 & 0.5 & 4673 & 14 & 15 & 13 & 0 & 100 & 20 & $2.20 \mathrm{E}-111$ & 15 & 1.47E-112 & 2.93E-112 & 0 \\
\hline SOF-chain & & & & & & & & & & & & & $2.93 \mathrm{E}-112$ & $1.00 \mathrm{E}+99$ \\
\hline Po-210 & 1 & 0.5 & 4673 & 14 & 15 & 13 & 100 & 1350 & 110 & $2.95 \mathrm{E}-141$ & 15 & 1.96E-142 & 3.93E-142 & 0 \\
\hline SOF-chain & & & & & & & & & & & & & $3.93 \mathrm{E}-142$ & $1.00 \mathrm{E}+99$ \\
\hline Po-210 & 1 & 0.5 & 1488 & 9 & 16 & 5 & 1350 & 10000 & 1360 & $0.00 \mathrm{E}+00$ & 15 & $0.00 \mathrm{E}+00$ & $0.00 \mathrm{E}+00$ & 0 \\
\hline SOF-chain & & & & & & & & & & & & & $0.00 \mathrm{E}+00$ & $1.00 \mathrm{E}+99$ \\
\hline Ra-226 & 1 & 0.5 & 4673 & 14 & 15 & 13 & 0 & 100 & 100 & 3.29E-83 & 5 & $6.59 \mathrm{E}-84$ & 1.32E-83 & 0 \\
\hline
\end{tabular}




\begin{tabular}{|c|c|c|c|c|c|c|c|c|c|c|c|c|c|c|}
\hline 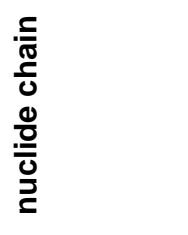 & 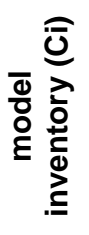 & 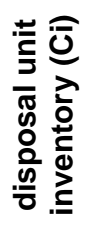 & 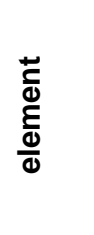 & $\begin{array}{l}\overline{0} \\
\frac{0}{0} \\
\stackrel{0}{\varrho}\end{array}$ & $\begin{array}{l}0 \\
\frac{0}{0} \\
\stackrel{0}{0}\end{array}$ & 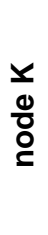 & 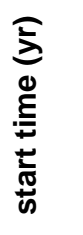 & 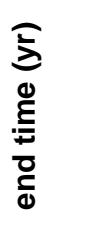 & 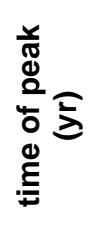 & 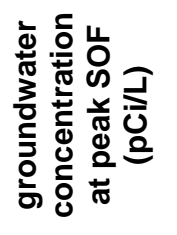 & 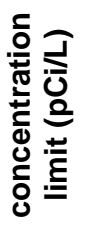 & 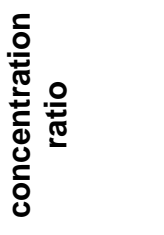 & 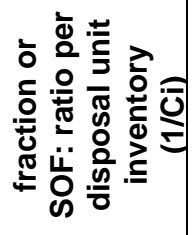 & 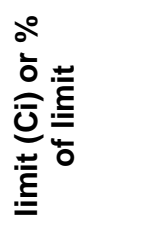 \\
\hline SOF-chain & & & & & & & & & & & & & 1.74E-76 & $5.76 \mathrm{E}+75$ \\
\hline Ra-226 & 1 & 0.5 & 4673 & 14 & 15 & 13 & 100 & 1350 & 1350 & $3.46 \mathrm{E}-40$ & 5 & $6.92 \mathrm{E}-41$ & $1.38 \mathrm{E}-40$ & 0.001 \\
\hline $\mathrm{Pb}-210$ & & & & & & & & & & $5.62 \mathrm{E}-36$ & 1 & 5.62E-36 & 1.13E-35 & 88.65 \\
\hline Po-210 & & & & & & & & & & $1.08 \mathrm{E}-35$ & 15 & $7.20 \mathrm{E}-37$ & $1.44 \mathrm{E}-36$ & 11.348 \\
\hline SOF-chain & & & & & & & & & & & & & $1.27 \mathrm{E}-35$ & $7.88 \mathrm{E}+34$ \\
\hline Ra-226 & 1 & 0.5 & 4671 & 12 & 15 & 13 & 1350 & 10000 & 10000 & $2.18 \mathrm{E}-03$ & 5 & 4.36E-04 & 8.72E-04 & 8.37 \\
\hline $\mathrm{Pb}-210$ & & & & & & & & & & $4.26 \mathrm{E}-03$ & 1 & $4.26 \mathrm{E}-03$ & $8.52 \mathrm{E}-03$ & 81.803 \\
\hline Po-210 & & & & & & & & & & $7.68 \mathrm{E}-03$ & 15 & $5.12 \mathrm{E}-04$ & $1.02 \mathrm{E}-03$ & 9.828 \\
\hline SOF-chain & & & & & & & & & & & & & 1.04E-02 & $9.60 \mathrm{E}+01$ \\
\hline Th-228 & 1 & 0.5 & 4673 & 14 & 15 & 13 & 0 & 100 & 90 & $1.50 \mathrm{E}-132$ & 15 & $1.00 \mathrm{E}-133$ & $2.00 \mathrm{E}-133$ & 0 \\
\hline SOF-chain & & & & & & & & & & & & & $2.00 \mathrm{E}-133$ & $1.00 E+99$ \\
\hline Th-228 & 1 & 0.5 & 4673 & 14 & 15 & 13 & 100 & 1350 & 110 & 1.04E-132 & 15 & $6.91 \mathrm{E}-134$ & $1.38 \mathrm{E}-133$ & 0 \\
\hline Th-228 & 1 & 0.5 & 4673 & 14 & 15 & 13 & 1350 & 10000 & 1360 & 1.77E-281 & 15 & $1.18 \mathrm{E}-282$ & $2.36 \mathrm{E}-282$ & 0 \\
\hline SOF-chain & & & & & & & & & & & & & 2.36E-282 & $1.00 \mathrm{E}+99$ \\
\hline Th-230 & 1 & 0.5 & 4673 & 14 & 15 & 13 & 0 & 100 & 100 & $4.10 \mathrm{E}-116$ & 15 & $2.73 \mathrm{E}-117$ & 5.47E-117 & 0 \\
\hline Ra-226 & & & & & & & & & & $4.41 \mathrm{E}-86$ & 5 & $8.82 \mathrm{E}-87$ & $1.76 \mathrm{E}-86$ & 0 \\
\hline $\mathrm{Pb}-210$ & & & & & & & & & & 1.07E-79 & 1 & 1.07E-79 & 2.14E-79 & 87.648 \\
\hline Po-210 & & & & & & & & & & $2.26 \mathrm{E}-79$ & 15 & $1.51 \mathrm{E}-80$ & $3.01 \mathrm{E}-80$ & 12.352 \\
\hline SOF-chain & & & & & & & & & & & & & $2.44 \mathrm{E}-79$ & $4.10 \mathrm{E}+78$ \\
\hline Th-230 & 1 & 0.5 & 4673 & 14 & 15 & 13 & 100 & 1350 & 1350 & 1.88E-72 & 15 & 1.25E-73 & $2.50 \mathrm{E}-73$ & 0 \\
\hline Ra-226 & & & & & & & & & & 1.13E-40 & 5 & $2.26 \mathrm{E}-41$ & $4.53 \mathrm{E}-41$ & 0.001 \\
\hline $\mathrm{Pb}-210$ & & & & & & & & & & $2.03 E-36$ & 1 & $2.03 \mathrm{E}-36$ & $4.07 E-36$ & 88.646 \\
\hline Po-210 & & & & & & & & & & $3.91 \mathrm{E}-36$ & 15 & $2.61 \mathrm{E}-37$ & $5.21 \mathrm{E}-37$ & 11.353 \\
\hline SOF-chain & & & & & & & & & & & & & $4.59 \mathrm{E}-36$ & $2.18 \mathrm{E}+35$ \\
\hline Th-230 & 1 & 0.5 & 4671 & 12 & 15 & 13 & 1350 & 10000 & 10000 & $5.26 \mathrm{E}-25$ & 15 & $3.51 \mathrm{E}-26$ & $7.02 E-26$ & 0 \\
\hline Ra-226 & & & & & & & & & & 2.37E-03 & 5 & 4.73E-04 & 9.46E-04 & 8.338 \\
\hline $\mathrm{Pb}-210$ & & & & & & & & & & 4.64E-03 & 1 & 4.64E-03 & $9.28 \mathrm{E}-03$ & 81.829 \\
\hline Po-210 & & & & & & & & & & 8.37E-03 & 15 & $5.58 \mathrm{E}-04$ & 1.12E-03 & 9.833 \\
\hline SOF-chain & & & & & & & & & & & & & 1.14E-02 & $8.81 \mathrm{E}+01$ \\
\hline Th-232 & 1 & 0.5 & 4673 & 14 & 15 & 13 & 0 & 100 & 100 & $4.11 \mathrm{E}-116$ & 13 & $3.16 \mathrm{E}-117$ & $6.32 \mathrm{E}-117$ & 0 \\
\hline
\end{tabular}




\begin{tabular}{|c|c|c|c|c|c|c|c|c|c|c|c|c|c|c|}
\hline $\begin{array}{l}\frac{5}{\pi} \\
\frac{5}{0} \\
\frac{0}{0} \\
\frac{0}{0} \\
\frac{0}{2}\end{array}$ & 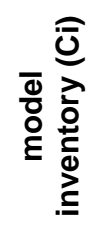 & 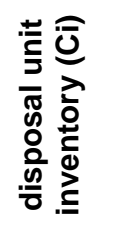 & 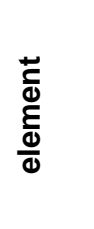 & $\begin{array}{l}\overline{0} \\
\frac{0}{0} \\
\text { 인 }\end{array}$ & $\begin{array}{l}\mathfrak{0} \\
\stackrel{0}{0} \\
\stackrel{0}{0}\end{array}$ & $\begin{array}{l}\stackrel{y}{0} \\
\stackrel{0}{0} \\
\stackrel{0}{\varrho}\end{array}$ & 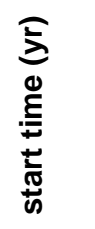 & 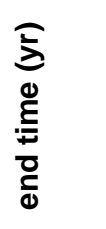 & 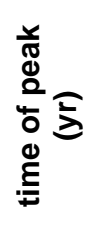 & 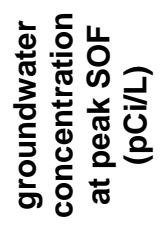 & 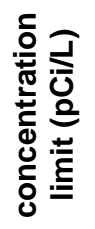 & 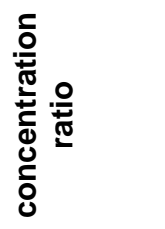 & 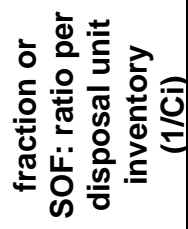 & 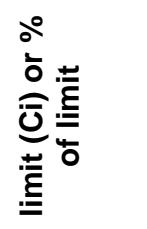 \\
\hline Ra-224 & & & & & & & & & & $3.01 \mathrm{E}-89$ & 15 & $2.00 \mathrm{E}-90$ & 4.01E-90 & 18.511 \\
\hline SOF-chain & & & & & & & & & & & & & $2.16 \mathrm{E}-89$ & $4.62 \mathrm{E}+88$ \\
\hline Th-232 & 1 & 0.5 & 4673 & 14 & 15 & 13 & 100 & 1350 & 1350 & 1.90E-72 & 13 & 1.46E-73 & 2.92E-73 & 0 \\
\hline Ra-228 & & & & & & & & & & $9.75 \mathrm{E}-56$ & 5 & $1.95 \mathrm{E}-56$ & $3.90 \mathrm{E}-56$ & 71.874 \\
\hline Th-228 & & & & & & & & & & $1.55 \mathrm{E}-56$ & 15 & $1.03 \mathrm{E}-57$ & $2.06 \mathrm{E}-57$ & 3.797 \\
\hline Ra-224 & & & & & & & & & & $9.90 \mathrm{E}-56$ & 15 & $6.60 \mathrm{E}-57$ & 1.32E-56 & 24.329 \\
\hline SOF-chain & & & & & & & & & & & & & 5.43E-56 & $1.84 \mathrm{E}+55$ \\
\hline Th-232 & 1 & 0.5 & 4673 & 14 & 15 & 13 & 1350 & 10000 & 10000 & 1.09E-24 & 13 & 8.39E-26 & 1.68E-25 & 3.548 \\
\hline Ra-228 & & & & & & & & & & $8.23 \mathrm{E}-24$ & 5 & $1.65 \mathrm{E}-24$ & $3.29 \mathrm{E}-24$ & 69.618 \\
\hline Th-228 & & & & & & & & & & $1.29 \mathrm{E}-24$ & 15 & $8.58 \mathrm{E}-26$ & $1.72 \mathrm{E}-25$ & 3.627 \\
\hline Ra-224 & & & & & & & & & & $8.24 \mathrm{E}-24$ & 15 & $5.49 \mathrm{E}-25$ & $1.10 \mathrm{E}-24$ & 23.207 \\
\hline SOF-chain & & & & & & & & & & & & & $4.73 \mathrm{E}-24$ & $2.11 \mathrm{E}+23$ \\
\hline Th-228 & & & & & & & & & & $3.60 \mathrm{E}-100$ & 15 & $2.40 \mathrm{E}-101$ & $4.80 \mathrm{E}-98$ & 9.086 \\
\hline Ra-224 & & & & & & & & & & 2.31E-99 & 15 & $1.54 \mathrm{E}-100$ & 3.08E-97 & 58.272 \\
\hline SOF-chain & & & & & & & & & & & & & $5.28 \mathrm{E}-97$ & $1.89 \mathrm{E}+96$ \\
\hline $\mathrm{U}-232$ & 0.001 & 0.0005 & 4673 & 14 & 15 & 13 & 100 & 1350 & 1350 & $2.67 \mathrm{E}-61$ & 26 & $1.03 \mathrm{E}-62$ & $2.06 \mathrm{E}-59$ & 26.514 \\
\hline Th-228 & & & & & & & & & & $5.77 \mathrm{E}-62$ & 15 & $3.85 \mathrm{E}-63$ & $7.69 \mathrm{E}-60$ & 9.927 \\
\hline Ra-224 & & & & & & & & & & $3.69 \mathrm{E}-61$ & 15 & $2.46 \mathrm{E}-62$ & $4.93 E-59$ & 63.559 \\
\hline SOF-chain & & & & & & & & & & & & & $7.75 E-59$ & $1.29 \mathrm{E}+58$ \\
\hline U-232 & 0.001 & 0.0005 & 4673 & 14 & 15 & 13 & 1350 & 10000 & 3970 & $8.42 \mathrm{E}-42$ & 26 & $3.24 \mathrm{E}-43$ & $6.48 \mathrm{E}-40$ & 23.645 \\
\hline Th-228 & & & & & & & & & & $2.12 \mathrm{E}-42$ & 15 & $1.41 \mathrm{E}-43$ & $2.83 \mathrm{E}-40$ & 10.321 \\
\hline Ra-224 & & & & & & & & & & $1.36 \mathrm{E}-41$ & 15 & $9.04 \mathrm{E}-43$ & $1.81 \mathrm{E}-39$ & 66.034 \\
\hline SOF-chain & & & & & & & & & & & & & $2.74 \mathrm{E}-39$ & $3.65 E+38$ \\
\hline$U-232$ & 0.01 & 0.005 & 4673 & 14 & 15 & 13 & 0 & 100 & 100 & $2.24 \mathrm{E}-98$ & 26 & $8.62 \mathrm{E}-100$ & $1.72 \mathrm{E}-97$ & 32.641 \\
\hline Th-228 & & & & & & & & & & $3.60 \mathrm{E}-99$ & 15 & $2.40 \mathrm{E}-100$ & 4.80E-98 & 9.086 \\
\hline Ra-224 & & & & & & & & & & $2.31 \mathrm{E}-98$ & 15 & 1.54E-99 & $3.08 E-97$ & 58.272 \\
\hline SOF-chain & & & & & & & & & & & & & $5.28 \mathrm{E}-97$ & $1.89 \mathrm{E}+96$ \\
\hline U-232 & 0.01 & 0.005 & 4673 & 14 & 15 & 13 & 100 & 1350 & 1350 & $2.67 \mathrm{E}-60$ & 26 & $1.03 \mathrm{E}-61$ & $2.06 \mathrm{E}-59$ & 26.514 \\
\hline Th-228 & & & & & & & & & & $5.77 \mathrm{E}-61$ & 15 & $3.85 \mathrm{E}-62$ & $7.69 \mathrm{E}-60$ & 9.927 \\
\hline Ra-224 & & & & & & & & & & $3.69 \mathrm{E}-60$ & 15 & $2.46 \mathrm{E}-61$ & 4.93E-59 & 63.559 \\
\hline
\end{tabular}




\begin{tabular}{|c|c|c|c|c|c|c|c|c|c|c|c|c|c|c|}
\hline $\begin{array}{l}\frac{5}{\pi} \\
\frac{0}{0} \\
\frac{0}{0} \\
\frac{0}{0} \\
\underline{x}\end{array}$ & 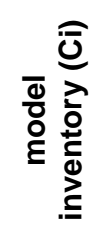 & 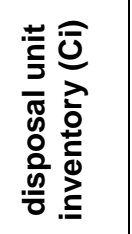 & 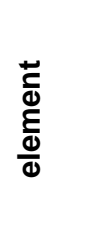 & $\begin{array}{l}\bar{\Xi} \\
\stackrel{0}{\varrho}\end{array}$ & $\begin{array}{l}\overparen{0} \\
\stackrel{0}{0} \\
\stackrel{0}{0}\end{array}$ & 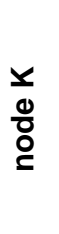 & 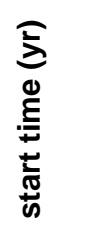 & 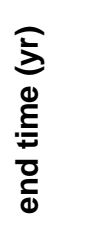 & 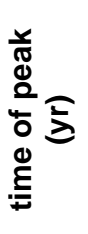 & 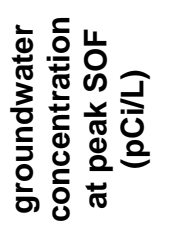 & 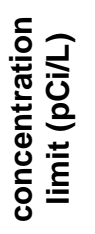 & 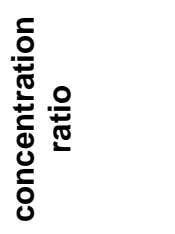 & 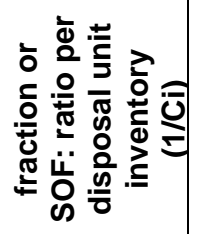 & 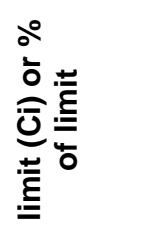 \\
\hline Th-228 & & & & & & & & & & $2.70 \mathrm{E}-41$ & 15 & $1.80 \mathrm{E}-42$ & $3.60 \mathrm{E}-40$ & 10.321 \\
\hline Ra-224 & & & & & & & & & & $1.73 \mathrm{E}-40$ & 15 & $1.15 \mathrm{E}-41$ & $2.30 \mathrm{E}-39$ & 66.035 \\
\hline SOF-chain & & & & & & & & & & & & & $3.48 \mathrm{E}-39$ & $2.87 \mathrm{E}+38$ \\
\hline $\mathrm{U}-232$ & 0.1 & 0.05 & 4673 & 14 & 15 & 13 & 0 & 100 & 100 & $2.24 \mathrm{E}-97$ & 26 & $8.62 \mathrm{E}-99$ & $1.72 \mathrm{E}-97$ & 32.641 \\
\hline Th-228 & & & & & & & & & & $3.60 \mathrm{E}-98$ & 15 & $2.40 \mathrm{E}-99$ & $4.80 \mathrm{E}-98$ & 9.086 \\
\hline Ra-224 & & & & & & & & & & 2.31E-97 & 15 & $1.54 \mathrm{E}-98$ & $3.08 \mathrm{E}-97$ & 58.272 \\
\hline SOF-chain & & & & & & & & & & & & & $5.28 \mathrm{E}-97$ & $1.89 \mathrm{E}+96$ \\
\hline $\mathrm{U}-232$ & 0.1 & 0.05 & 4673 & 14 & 15 & 13 & 100 & 1350 & 1350 & $2.67 \mathrm{E}-59$ & 26 & $1.03 \mathrm{E}-60$ & $2.06 \mathrm{E}-59$ & 26.514 \\
\hline Th-228 & & & & & & & & & & $5.77 \mathrm{E}-60$ & 15 & $3.85 \mathrm{E}-61$ & $7.69 \mathrm{E}-60$ & 9.927 \\
\hline Ra-224 & & & & & & & & & & $3.69 \mathrm{E}-59$ & 15 & $2.46 \mathrm{E}-60$ & $4.93 \mathrm{E}-59$ & 63.559 \\
\hline SOF-chain & & & & & & & & & & & & & $7.75 \mathrm{E}-59$ & $1.29 \mathrm{E}+58$ \\
\hline U-232 & 0.1 & 0.05 & 4673 & 14 & 15 & 13 & 1350 & 10000 & 4020 & 1.09E-39 & 26 & $4.19 \mathrm{E}-41$ & $8.38 \mathrm{E}-40$ & 23.644 \\
\hline Th-228 & & & & & & & & & & $2.74 \mathrm{E}-40$ & 15 & $1.83 \mathrm{E}-41$ & $3.66 \mathrm{E}-40$ & 10.321 \\
\hline Ra-224 & & & & & & & & & & $1.76 \mathrm{E}-39$ & 15 & $1.17 \mathrm{E}-40$ & $2.34 \mathrm{E}-39$ & 66.035 \\
\hline SOF-chain & & & & & & & & & & & & & $3.54 \mathrm{E}-39$ & $2.82 \mathrm{E}+38$ \\
\hline $\mathrm{U}-232$ & 1 & 0.5 & 4673 & 14 & 15 & 13 & 0 & 100 & 100 & $2.24 \mathrm{E}-96$ & 26 & $8.62 \mathrm{E}-98$ & $1.72 \mathrm{E}-97$ & 32.641 \\
\hline Th-228 & & & & & & & & & & $3.60 \mathrm{E}-97$ & 15 & $2.40 \mathrm{E}-98$ & $4.80 \mathrm{E}-98$ & 9.086 \\
\hline Ra-224 & & & & & & & & & & 2.31E-96 & 15 & $1.54 \mathrm{E}-97$ & $3.08 \mathrm{E}-97$ & 58.272 \\
\hline SOF-chain & & & & & & & & & & & & & $5.28 \mathrm{E}-97$ & $1.89 \mathrm{E}+96$ \\
\hline $\mathrm{U}-232$ & 1 & 0.5 & 4673 & 14 & 15 & 13 & 100 & 1350 & 1350 & $2.67 \mathrm{E}-58$ & 26 & $1.03 E-59$ & $2.06 \mathrm{E}-59$ & 26.514 \\
\hline Th-228 & & & & & & & & & & $5.77 \mathrm{E}-59$ & 15 & $3.85 \mathrm{E}-60$ & $7.69 \mathrm{E}-60$ & 9.927 \\
\hline Ra-224 & & & & & & & & & & $3.69 \mathrm{E}-58$ & 15 & $2.46 \mathrm{E}-59$ & $4.93 \mathrm{E}-59$ & 63.559 \\
\hline SOF-chain & & & & & & & & & & & & & $7.75 \mathrm{E}-59$ & $1.29 \mathrm{E}+58$ \\
\hline $\mathrm{U}-232$ & 1 & 0.5 & 4673 & 14 & 15 & 13 & 1350 & 10000 & 4020 & $1.09 \mathrm{E}-38$ & 26 & $4.19 \mathrm{E}-40$ & $8.39 \mathrm{E}-40$ & 23.644 \\
\hline Th-228 & & & & & & & & & & $2.75 \mathrm{E}-39$ & 15 & $1.83 \mathrm{E}-40$ & $3.66 \mathrm{E}-40$ & 10.321 \\
\hline Ra-224 & & & & & & & & & & $1.76 \mathrm{E}-38$ & 15 & $1.17 \mathrm{E}-39$ & $2.34 \mathrm{E}-39$ & 66.035 \\
\hline SOF-chain & & & & & & & & & & & & & $3.55 \mathrm{E}-39$ & $2.82 \mathrm{E}+38$ \\
\hline $\mathrm{U}-233$ & 0.001 & 0.0005 & 4673 & 14 & 15 & 13 & 0 & 100 & 100 & $6.77 \mathrm{E}-99$ & 130 & $5.21 \mathrm{E}-101$ & $1.04 \mathrm{E}-97$ & 99.387 \\
\hline Th-229 & & & & & & & & & & $7.52 \mathrm{E}-103$ & 9.6 & $7.83 \mathrm{E}-104$ & $1.57 \mathrm{E}-100$ & 0.149 \\
\hline Ra-225 & & & & & & & & & & $4.86 \mathrm{E}-102$ & 20 & $2.43 \mathrm{E}-103$ & $4.86 \mathrm{E}-100$ & 0.463 \\
\hline SOF-chain & & & & & & & & & & & & & 1.05E-97 & $9.54 \mathrm{E}+96$ \\
\hline $\mathrm{U}-233$ & 0.001 & 0.0005 & 4673 & 14 & 15 & 13 & 100 & 1350 & 1350 & $2.30 \mathrm{E}-55$ & 130 & $1.77 \mathrm{E}-57$ & $3.54 \mathrm{E}-54$ & 98.085 \\
\hline Th-229 & & & & & & & & & & $8.12 \mathrm{E}-59$ & 9.6 & $8.46 \mathrm{E}-60$ & 1.69E-56 & 0.469 \\
\hline
\end{tabular}




\begin{tabular}{|c|c|c|c|c|c|c|c|c|c|c|c|c|c|c|}
\hline $\begin{array}{l}\frac{5}{\pi} \\
\frac{0}{0} \\
\frac{0}{0} \\
\frac{0}{0} \\
\underline{x}\end{array}$ & 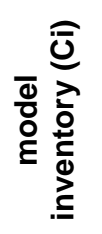 & 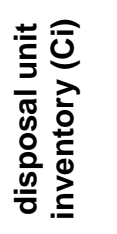 & 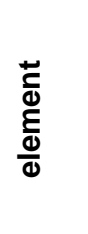 & $\begin{array}{l}\bar{\Xi} \\
\stackrel{0}{\varrho}\end{array}$ & $\begin{array}{l}\overparen{0} \\
\stackrel{0}{0} \\
\stackrel{0}{0}\end{array}$ & 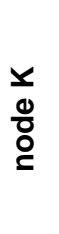 & 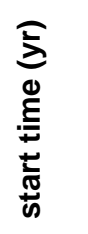 & 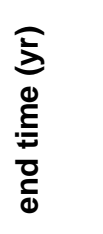 & 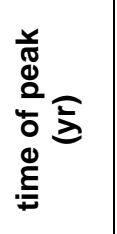 & 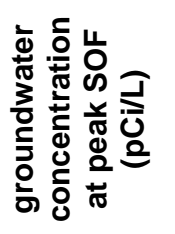 & 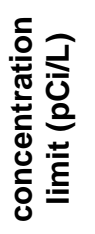 & 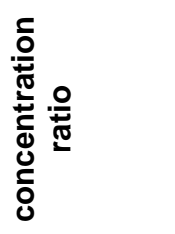 & 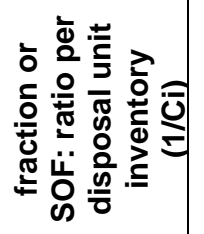 & 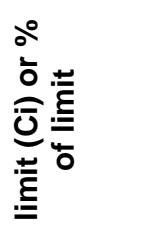 \\
\hline Ra-225 & & & & & & & & & & $5.21 \mathrm{E}-58$ & 20 & $2.61 \mathrm{E}-59$ & $5.21 \mathrm{E}-56$ & 1.446 \\
\hline SOF-chain & & & & & & & & & & & & & $3.60 \mathrm{E}-54$ & $2.77 \mathrm{E}+53$ \\
\hline $\mathrm{U}-233$ & 0.001 & 0.0005 & 4673 & 14 & 15 & 13 & 1350 & 10000 & 10000 & $2.37 \mathrm{E}-11$ & 130 & $1.83 \mathrm{E}-13$ & $3.65 \mathrm{E}-10$ & 61.359 \\
\hline Th-229 & & & & & & & & & & $2.71 \mathrm{E}-13$ & 9.6 & $2.82 \mathrm{E}-14$ & $5.65 \mathrm{E}-11$ & 9.494 \\
\hline Ra-225 & & & & & & & & & & $1.73 \mathrm{E}-12$ & 20 & $8.67 \mathrm{E}-14$ & $1.73 \mathrm{E}-10$ & 29.147 \\
\hline SOF-chain & & & & & & & & & & & & & $5.95 \mathrm{E}-10$ & $1.68 \mathrm{E}+09$ \\
\hline $\mathrm{U}-233$ & 0.01 & 0.005 & 4673 & 14 & 15 & 13 & 0 & 100 & 100 & $6.77 \mathrm{E}-98$ & 130 & $5.20 \mathrm{E}-100$ & $1.04 \mathrm{E}-97$ & 99.387 \\
\hline Th-229 & & & & & & & & & & $7.51 \mathrm{E}-102$ & 9.6 & $7.82 \mathrm{E}-103$ & $1.56 \mathrm{E}-100$ & 0.149 \\
\hline Ra-225 & & & & & & & & & & $4.85 \mathrm{E}-101$ & 20 & $2.43 \mathrm{E}-102$ & $4.85 \mathrm{E}-100$ & 0.463 \\
\hline SOF-chain & & & & & & & & & & & & & 1.05E-97 & $9.55 \mathrm{E}+96$ \\
\hline $\mathrm{U}-233$ & 0.01 & 0.005 & 4673 & 14 & 15 & 13 & 100 & 1350 & 1350 & $2.30 \mathrm{E}-54$ & 130 & $1.77 \mathrm{E}-56$ & $3.54 \mathrm{E}-54$ & 98.085 \\
\hline Th-229 & & & & & & & & & & $8.13 \mathrm{E}-58$ & 9.6 & $8.47 \mathrm{E}-59$ & 1.69E-56 & 0.469 \\
\hline Ra-225 & & & & & & & & & & $5.22 \mathrm{E}-57$ & 20 & $2.61 \mathrm{E}-58$ & $5.22 \mathrm{E}-56$ & 1.445 \\
\hline SOF-chain & & & & & & & & & & & & & $3.61 \mathrm{E}-54$ & $2.77 \mathrm{E}+53$ \\
\hline $\mathrm{U}-233$ & 0.01 & 0.005 & 4673 & 14 & 15 & 13 & 1350 & 10000 & 10000 & $2.37 \mathrm{E}-10$ & 130 & $1.82 \mathrm{E}-12$ & $3.65 \mathrm{E}-10$ & 61.359 \\
\hline Th-229 & & & & & & & & & & $2.71 \mathrm{E}-12$ & 9.6 & $2.82 \mathrm{E}-13$ & $5.64 \mathrm{E}-11$ & 9.494 \\
\hline Ra-225 & & & & & & & & & & $1.73 \mathrm{E}-11$ & 20 & $8.66 \mathrm{E}-13$ & $1.73 \mathrm{E}-10$ & 29.147 \\
\hline SOF-chain & & & & & & & & & & & & & $5.94 \mathrm{E}-10$ & $1.68 \mathrm{E}+09$ \\
\hline $\mathrm{U}-233$ & 0.1 & 0.05 & 4673 & 14 & 15 & 13 & 0 & 100 & 100 & $6.76 \mathrm{E}-97$ & 130 & $5.20 \mathrm{E}-99$ & $1.04 \mathrm{E}-97$ & 99.387 \\
\hline Th-229 & & & & & & & & & & $7.50 \mathrm{E}-101$ & 9.6 & $7.82 \mathrm{E}-102$ & $1.56 \mathrm{E}-100$ & 0.149 \\
\hline Ra-225 & & & & & & & & & & $4.85 \mathrm{E}-100$ & 20 & $2.42 \mathrm{E}-101$ & $4.85 \mathrm{E}-100$ & 0.463 \\
\hline SOF-chain & & & & & & & & & & & & & $1.05 \mathrm{E}-97$ & $9.56 \mathrm{E}+96$ \\
\hline U-233 & 0.1 & 0.05 & 4673 & 14 & 15 & 13 & 100 & 1350 & 1350 & $2.30 \mathrm{E}-53$ & 130 & $1.77 \mathrm{E}-55$ & $3.54 \mathrm{E}-54$ & 98.086 \\
\hline Th-229 & & & & & & & & & & $8.13 \mathrm{E}-57$ & 9.6 & $8.46 \mathrm{E}-58$ & 1.69E-56 & 0.469 \\
\hline Ra-225 & & & & & & & & & & $5.21 \mathrm{E}-56$ & 20 & $2.61 \mathrm{E}-57$ & $5.21 \mathrm{E}-56$ & 1.445 \\
\hline SOF-chain & & & & & & & & & & & & & $3.61 \mathrm{E}-54$ & $2.77 \mathrm{E}+53$ \\
\hline $\mathrm{U}-233$ & 0.1 & 0.05 & 4673 & 14 & 15 & 13 & 1350 & 10000 & 10000 & 2.37E-09 & 130 & $1.82 \mathrm{E}-11$ & $3.64 \mathrm{E}-10$ & 61.359 \\
\hline Th-229 & & & & & & & & & & $2.71 \mathrm{E}-11$ & 9.6 & $2.82 \mathrm{E}-12$ & $5.64 \mathrm{E}-11$ & 9.494 \\
\hline Ra-225 & & & & & & & & & & $1.73 \mathrm{E}-10$ & 20 & $8.66 \mathrm{E}-12$ & $1.73 \mathrm{E}-10$ & 29.147 \\
\hline SOF-chain & & & & & & & & & & & & & $5.94 \mathrm{E}-10$ & $1.68 \mathrm{E}+09$ \\
\hline $\mathrm{U}-233$ & 1 & 0.5 & 4673 & 14 & 15 & 13 & 0 & 100 & 100 & $\begin{array}{l}6.75 \mathrm{E}-96 \\
\end{array}$ & 130 & $5.20 \mathrm{E}-98$ & 1.04E-97 & 99.387 \\
\hline Th-229 & & & & & & & & & & $7.50 \mathrm{E}-100$ & 9.6 & $7.81 \mathrm{E}-101$ & $1.56 \mathrm{E}-100$ & 0.149 \\
\hline Ra-225 & & & & & & & & & & 4.84E-99 & 20 & $2.42 \mathrm{E}-100$ & $4.84 \mathrm{E}-100$ & 0.463 \\
\hline
\end{tabular}




\begin{tabular}{|c|c|c|c|c|c|c|c|c|c|c|c|c|c|c|}
\hline $\begin{array}{l}. \frac{5}{\pi} \\
\frac{c}{0} \\
\frac{0}{0} \\
\frac{0}{0} \\
\frac{0}{2}\end{array}$ & 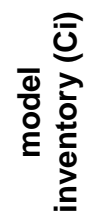 & 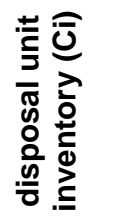 & 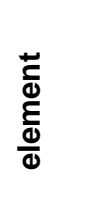 & $\begin{array}{l}\overline{0} \\
\frac{0}{0} \\
\stackrel{\varrho}{\varrho}\end{array}$ & 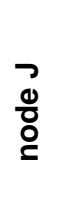 & $\begin{array}{l}\searrow \\
\frac{1}{0} \\
\stackrel{0}{\complement}\end{array}$ & 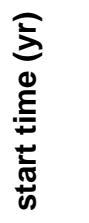 & 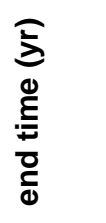 & 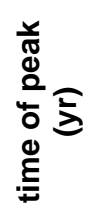 & 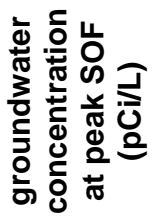 & 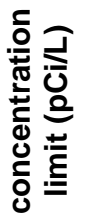 & 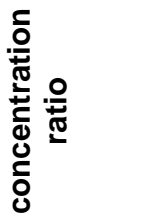 & 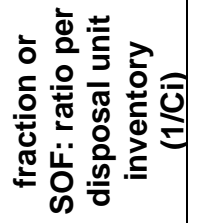 & 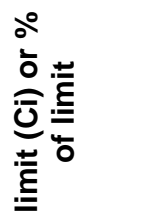 \\
\hline SOF-chain & & & & & & & & & & & & & 1.05E-97 & $9.57 E+96$ \\
\hline U-233 & 1 & 0.5 & 4673 & 14 & 15 & 13 & 100 & 1350 & 1350 & $2.30 \mathrm{E}-52$ & 130 & 1.77E-54 & $3.54 \mathrm{E}-54$ & 98.086 \\
\hline Th-229 & & & & & & & & & & $8.12 \mathrm{E}-56$ & 9.6 & $8.46 \mathrm{E}-57$ & 1.69E-56 & 0.469 \\
\hline Ra-225 & & & & & & & & & & $5.21 \mathrm{E}-55$ & 20 & $2.61 \mathrm{E}-56$ & $5.21 \mathrm{E}-56$ & 1.445 \\
\hline SOF-chain & & & & & & & & & & & & & $3.61 \mathrm{E}-54$ & $2.77 E+53$ \\
\hline U-233 & 1 & 0.5 & 4673 & 14 & 15 & 13 & 1350 & 10000 & 10000 & $2.38 \mathrm{E}-08$ & 130 & 1.83E-10 & $3.66 \mathrm{E}-10$ & 61.355 \\
\hline Th-229 & & & & & & & & & & $2.72 \mathrm{E}-10$ & 9.6 & $2.83 \mathrm{E}-11$ & $5.66 \mathrm{E}-11$ & 9.495 \\
\hline Ra-225 & & & & & & & & & & 1.74E-09 & 20 & $8.68 \mathrm{E}-11$ & 1.74E-10 & 29.15 \\
\hline SOF-chain & & & & & & & & & & & & & $5.96 \mathrm{E}-10$ & $1.68 \mathrm{E}+09$ \\
\hline U-234 & 0.001 & 0.0005 & 4673 & 14 & 15 & 13 & 0 & 100 & 100 & 6.77E-99 & 130 & $5.21 \mathrm{E}-101$ & 1.04E-97 & 0 \\
\hline Th-230 & & & & & & & & & & 7.32E-104 & 15 & $4.88 \mathrm{E}-105$ & $9.75 \mathrm{E}-102$ & 0 \\
\hline Ra-226 & & & & & & & & & & 1.24E-93 & 5 & 2.47E-94 & 4.95E-91 & 0 \\
\hline Po-210 & & & & & & & & & & $6.65 \mathrm{E}-87$ & 15 & $4.43 \mathrm{E}-88$ & $8.87 \mathrm{E}-85$ & 12.393 \\
\hline SOF-chain & & & & & & & & & & & & & $7.15 E-84$ & $1.40 \mathrm{E}+83$ \\
\hline U-234 & 0.001 & 0.0005 & 4673 & 14 & 15 & 13 & 100 & 1350 & 1350 & $2.31 \mathrm{E}-55$ & 130 & 1.77E-57 & $3.55 \mathrm{E}-54$ & 0 \\
\hline Th-230 & & & & & & & & & & 7.94E-60 & 15 & 5.29E-61 & $1.06 \mathrm{E}-57$ & 0 \\
\hline Ra-226 & & & & & & & & & & 3.67E-46 & 5 & 7.35E-47 & $1.47 \mathrm{E}-43$ & 0.001 \\
\hline $\mathrm{Pb}-210$ & & & & & & & & & & $7.31 \mathrm{E}-42$ & 1 & $7.31 \mathrm{E}-42$ & $1.46 \mathrm{E}-38$ & 88.641 \\
\hline Po-210 & & & & & & & & & & $1.41 \mathrm{E}-41$ & 15 & $9.37 \mathrm{E}-43$ & 1.87E-39 & 11.358 \\
\hline SOF-chain & & & & & & & & & & & & & 1.65E-38 & $6.06 \mathrm{E}+37$ \\
\hline U-234 & 0.001 & 0.0005 & 4671 & 12 & 15 & 13 & 1350 & 10000 & 10000 & 1.90E-11 & 130 & $1.46 \mathrm{E}-13$ & $2.92 \mathrm{E}-10$ & 0 \\
\hline Th-230 & & & & & & & & & & $2.15 \mathrm{E}-14$ & 15 & $1.43 \mathrm{E}-15$ & $2.87 \mathrm{E}-12$ & 0 \\
\hline Ra-226 & & & & & & & & & & $2.43 \mathrm{E}-08$ & 5 & 4.87E-09 & 9.73E-06 & 8.305 \\
\hline $\mathrm{Pb}-210$ & & & & & & & & & & $4.80 \mathrm{E}-08$ & 1 & $4.80 \mathrm{E}-08$ & 9.59E-05 & 81.857 \\
\hline Po-210 & & & & & & & & & & $8.65 \mathrm{E}-08$ & 15 & 5.77E-09 & 1.15E-05 & 9.838 \\
\hline SOF-chain & & & & & & & & & & & & & 1.17E-04 & $8.53 E+03$ \\
\hline U-234 & 0.01 & 0.005 & 4673 & 14 & 15 & 13 & 0 & 100 & 100 & $6.76 \mathrm{E}-98$ & 130 & $5.20 \mathrm{E}-100$ & 1.04E-97 & 0 \\
\hline Th-230 & & & & & & & & & & 7.31E-103 & 15 & $4.87 \mathrm{E}-104$ & $9.75 \mathrm{E}-102$ & 0 \\
\hline Ra-226 & & & & & & & & & & 1.24E-92 & 5 & $2.47 \mathrm{E}-93$ & 4.95E-91 & 0 \\
\hline $\mathrm{Pb}-210$ & & & & & & & & & & $3.13 \mathrm{E}-86$ & 1 & $3.13 \mathrm{E}-86$ & $6.27 \mathrm{E}-84$ & 87.607 \\
\hline
\end{tabular}




\begin{tabular}{|c|c|c|c|c|c|c|c|c|c|c|c|c|c|c|}
\hline 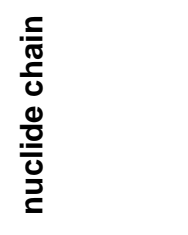 & 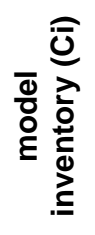 & 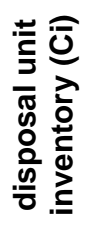 & 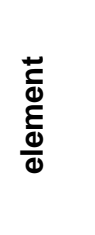 & $\begin{array}{l}\bar{\varrho} \\
\text { O }\end{array}$ & $\begin{array}{l}0 \\
\frac{0}{0} \\
0\end{array}$ & $\begin{array}{l}\text { Y } \\
\frac{0}{0} \\
\stackrel{0}{\complement}\end{array}$ & 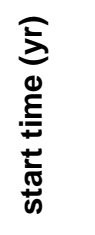 & 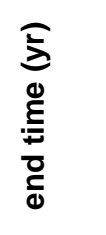 & 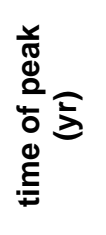 & 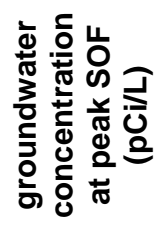 & 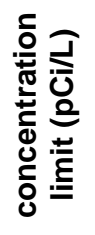 & 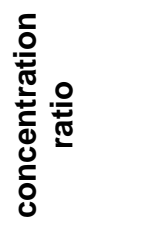 & 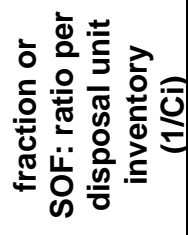 & 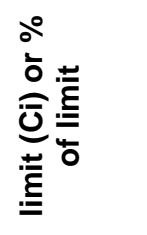 \\
\hline U-234 & 0.01 & 0.005 & 4673 & 14 & 15 & 13 & 100 & 1350 & 1350 & $2.31 \mathrm{E}-54$ & 130 & 1.77E-56 & $3.55 \mathrm{E}-54$ & 0 \\
\hline Th-230 & & & & & & & & & & 7.94E-59 & 15 & 5.29E-60 & 1.06E-57 & 0 \\
\hline Ra-226 & & & & & & & & & & 3.67E-45 & 5 & 7.35E-46 & 1.47E-43 & 0.001 \\
\hline $\mathrm{Pb}-210$ & & & & & & & & & & 7.31E-41 & 1 & 7.31E-41 & $1.46 \mathrm{E}-38$ & 88.641 \\
\hline Po-210 & & & & & & & & & & $1.41 \mathrm{E}-40$ & 15 & $9.37 E-42$ & 1.87E-39 & 11.358 \\
\hline SOF-chain & & & & & & & & & & & & & 1.65E-38 & $6.06 \mathrm{E}+37$ \\
\hline U-234 & 0.01 & 0.005 & 4671 & 12 & 15 & 13 & 1350 & 10000 & 10000 & 1.90E-10 & 130 & $1.46 \mathrm{E}-12$ & $2.92 \mathrm{E}-10$ & 0 \\
\hline Th-230 & & & & & & & & & & $2.15 \mathrm{E}-13$ & 15 & $1.44 \mathrm{E}-14$ & $2.87 \mathrm{E}-12$ & 0 \\
\hline Ra-226 & & & & & & & & & & 2.43E-07 & 5 & 4.87E-08 & 9.73E-06 & 8.305 \\
\hline $\mathrm{Pb}-210$ & & & & & & & & & & $4.80 \mathrm{E}-07$ & 1 & $4.80 \mathrm{E}-07$ & $9.59 \mathrm{E}-05$ & 81.857 \\
\hline Po-210 & & & & & & & & & & 8.65E-07 & 15 & 5.77E-08 & 1.15E-05 & 9.838 \\
\hline SOF-chain & & & & & & & & & & & & & 1.17E-04 & $8.53 E+03$ \\
\hline Th-230 & & & & & & & & & & $7.30 \mathrm{E}-102$ & 15 & 4.87E-103 & 9.73E-102 & 0 \\
\hline Ra-226 & & & & & & & & & & 1.24E-91 & 5 & 2.47E-92 & 4.95E-91 & 0 \\
\hline $\mathrm{Pb}-210$ & & & & & & & & & & 3.13E-85 & 1 & 3.13E-85 & 6.27E-84 & 87.607 \\
\hline Po-210 & & & & & & & & & & $6.65 \mathrm{E}-85$ & 15 & $4.43 \mathrm{E}-86$ & $8.86 \mathrm{E}-85$ & 12.393 \\
\hline SOF-chain & & & & & & & & & & & & & $7.15 \mathrm{E}-84$ & $1.40 \mathrm{E}+83$ \\
\hline U-234 & 0.1 & 0.05 & 4673 & 14 & 15 & 13 & 100 & 1350 & 1350 & $2.31 \mathrm{E}-53$ & 130 & 1.77E-55 & $3.55 \mathrm{E}-54$ & 0 \\
\hline Th-230 & & & & & & & & & & 7.94E-58 & 15 & 5.30E-59 & 1.06E-57 & 0 \\
\hline Ra-226 & & & & & & & & & & 3.67E-44 & 5 & 7.35E-45 & 1.47E-43 & 0.001 \\
\hline $\mathrm{Pb}-210$ & & & & & & & & & & $7.31 \mathrm{E}-40$ & 1 & $7.31 \mathrm{E}-40$ & 1.46E-38 & 88.641 \\
\hline Po-210 & & & & & & & & & & 1.41E-39 & 15 & $9.37 \mathrm{E}-41$ & 1.87E-39 & 11.358 \\
\hline SOF-chain & & & & & & & & & & & & & 1.65E-38 & $6.06 \mathrm{E}+37$ \\
\hline U-234 & 0.1 & 0.05 & 4671 & 12 & 15 & 13 & 1350 & 10000 & 10000 & 1.90E-09 & 130 & $1.46 \mathrm{E}-11$ & $2.92 \mathrm{E}-10$ & 0 \\
\hline Th-230 & & & & & & & & & & $2.15 \mathrm{E}-12$ & 15 & $1.44 \mathrm{E}-13$ & $2.87 \mathrm{E}-12$ & 0 \\
\hline Ra-226 & & & & & & & & & & 2.43E-06 & 5 & 4.87E-07 & 9.73E-06 & 8.305 \\
\hline $\mathrm{Pb}-210$ & & & & & & & & & & $4.80 \mathrm{E}-06$ & 1 & $4.80 \mathrm{E}-06$ & 9.59E-05 & 81.857 \\
\hline Po-210 & & & & & & & & & & $8.65 \mathrm{E}-06$ & 15 & $5.77 \mathrm{E}-07$ & 1.15E-05 & 9.838 \\
\hline SOF-chain & & & & & & & & & & & & & 1.17E-04 & $8.53 E+03$ \\
\hline U-234 & 1 & 0.5 & 4673 & 14 & 15 & 13 & 0 & 100 & 100 & 6.81E-96 & 130 & 5.24E-98 & $1.05 \mathrm{E}-97$ & 0 \\
\hline
\end{tabular}




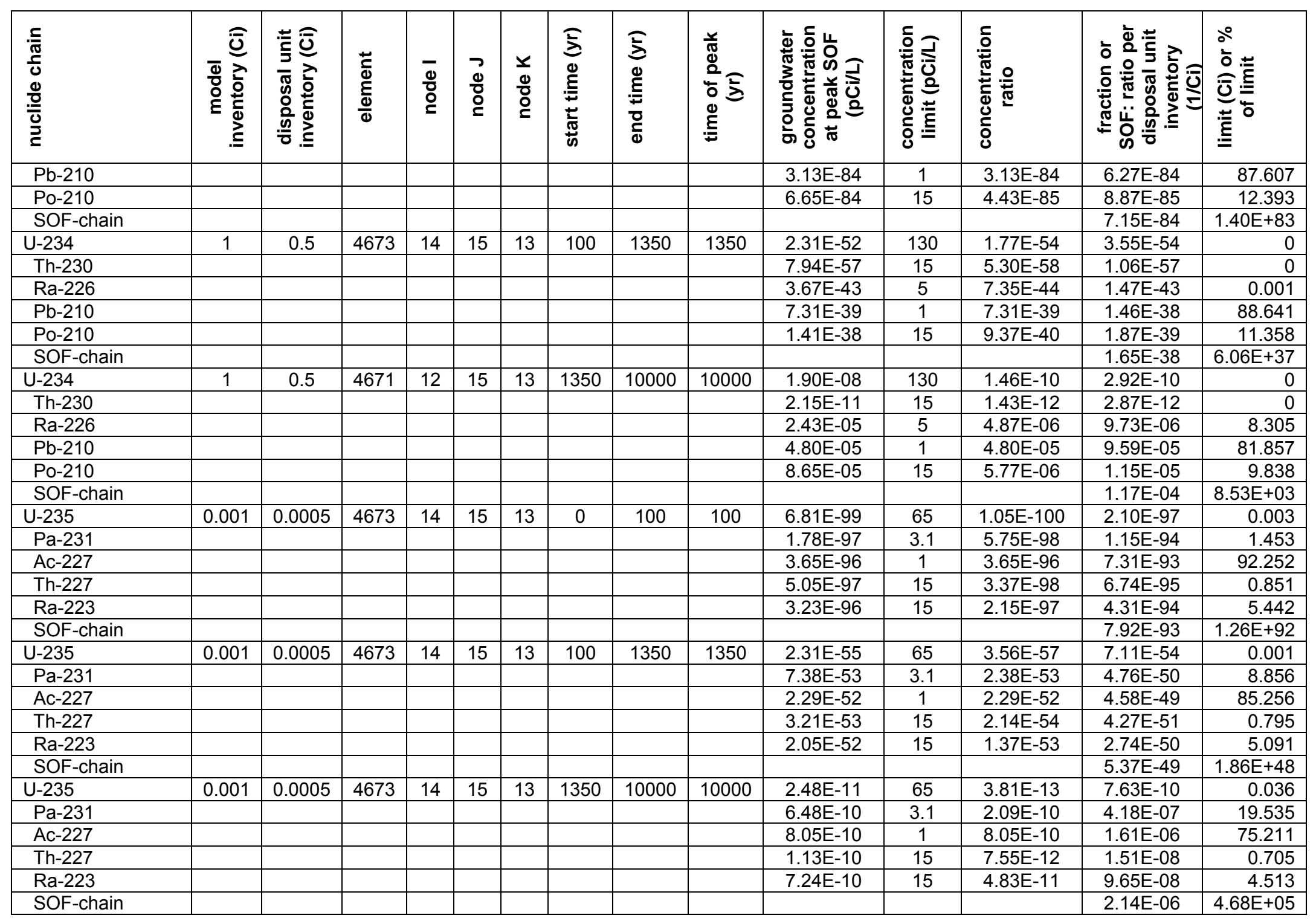




\begin{tabular}{|c|c|c|c|c|c|c|c|c|c|c|c|c|c|c|}
\hline 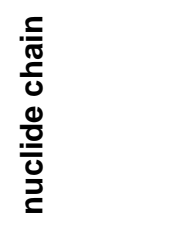 & 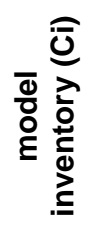 & 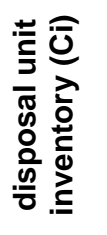 & 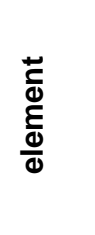 & $\begin{array}{l}\bar{\varrho} \\
\text { O }\end{array}$ & $\begin{array}{l}0 \\
\frac{0}{0} \\
0\end{array}$ & $\begin{array}{l}\text { Y } \\
\frac{0}{0} \\
\stackrel{0}{\complement}\end{array}$ & 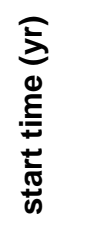 & 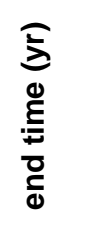 & 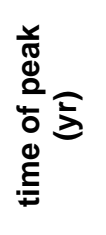 & 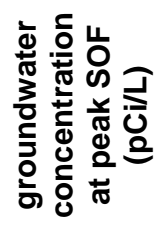 & 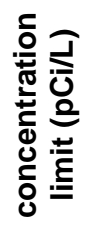 & 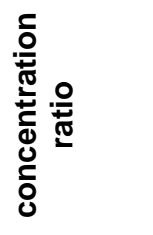 & 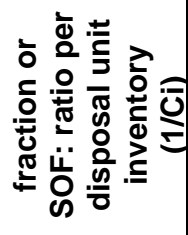 & 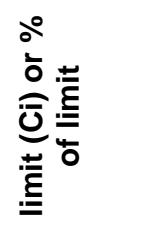 \\
\hline U-235 & 0.01 & 0.005 & 4673 & 14 & 15 & 13 & 0 & 100 & 100 & $6.80 \mathrm{E}-98$ & 65 & 1.05E-99 & 2.09E-97 & 0.003 \\
\hline Pa-231 & & & & & & & & & & $1.78 \mathrm{E}-96$ & 3.1 & 5.75E-97 & 1.15E-94 & 1.453 \\
\hline Ac-227 & & & & & & & & & & 3.65E-95 & 1 & 3.65E-95 & 7.30E-93 & 92.252 \\
\hline Th-227 & & & & & & & & & & $5.05 E-96$ & 15 & 3.37E-97 & $6.73 \mathrm{E}-95$ & 0.851 \\
\hline Ra-223 & & & & & & & & & & 3.23E-95 & 15 & $2.15 \mathrm{E}-96$ & 4.31E-94 & 5.442 \\
\hline SOF-chain & & & & & & & & & & & & & 7.91E-93 & $1.26 \mathrm{E}+92$ \\
\hline U-235 & 0.01 & 0.005 & 4673 & 14 & 15 & 13 & 100 & 1350 & 1350 & 2.31E-54 & 65 & $3.55 \mathrm{E}-56$ & $7.10 \mathrm{E}-54$ & 0.001 \\
\hline $\mathrm{Pa}-231$ & & & & & & & & & & $7.37 \mathrm{E}-52$ & 3.1 & $2.38 \mathrm{E}-52$ & $4.75 \mathrm{E}-50$ & 8.857 \\
\hline Ac-227 & & & & & & & & & & 2.29E-51 & 1 & 2.29E-51 & $4.58 \mathrm{E}-49$ & 85.255 \\
\hline Th-227 & & & & & & & & & & $3.20 \mathrm{E}-52$ & 15 & $2.14 \mathrm{E}-53$ & $4.27 \mathrm{E}-51$ & 0.795 \\
\hline Ra-223 & & & & & & & & & & $2.05 E-51$ & 15 & 1.37E-52 & $2.73 \mathrm{E}-50$ & 5.091 \\
\hline SOF-chain & & & & & & & & & & & & & 5.37E-49 & $1.86 \mathrm{E}+48$ \\
\hline $\mathrm{Pa}-231$ & & & & & & & & & & 5.41E-09 & 3.1 & 1.74E-09 & 3.49E-07 & 19.535 \\
\hline Ac-227 & & & & & & & & & & 6.72E-09 & 1 & 6.72E-09 & 1.34E-06 & 75.211 \\
\hline Th-227 & & & & & & & & & & $9.45 \mathrm{E}-10$ & 15 & $6.30 \mathrm{E}-11$ & 1.26E-08 & 0.705 \\
\hline Ra-223 & & & & & & & & & & 6.04E-09 & 15 & $4.03 \mathrm{E}-10$ & $8.06 \mathrm{E}-08$ & 4.513 \\
\hline SOF-chain & & & & & & & & & & & & & 1.79E-06 & $5.60 \mathrm{E}+05$ \\
\hline U-235 & 0.1 & 0.05 & 4673 & 14 & 15 & 13 & 0 & 100 & 100 & $8.92 E-98$ & 65 & 1.37E-99 & $2.75 \mathrm{E}-98$ & 0.002 \\
\hline $\mathrm{Pa}-231$ & & & & & & & & & & $2.44 \mathrm{E}-96$ & 3.1 & 7.87E-97 & 1.57E-95 & 1.421 \\
\hline Ac-227 & & & & & & & & & & 5.11E-95 & 1 & 5.11E-95 & 1.02E-93 & 92.282 \\
\hline Th-227 & & & & & & & & & & 7.07E-96 & 15 & $4.71 \mathrm{E}-97$ & 9.42E-96 & 0.851 \\
\hline Ra-223 & & & & & & & & & & $4.52 \mathrm{E}-95$ & 15 & $3.01 \mathrm{E}-96$ & $6.03 E-95$ & 5.443 \\
\hline SOF-chain & & & & & & & & & & & & & 1.11E-93 & $9.03 E+92$ \\
\hline U-235 & 0.1 & 0.05 & 4673 & 14 & 15 & 13 & 100 & 1350 & 1350 & $2.47 \mathrm{E}-54$ & 65 & $3.80 \mathrm{E}-56$ & 7.61E-55 & 0.001 \\
\hline $\mathrm{Pa}-231$ & & & & & & & & & & $1.43 \mathrm{E}-51$ & 3.1 & $4.62 \mathrm{E}-52$ & $9.24 \mathrm{E}-51$ & 8.761 \\
\hline Ac-227 & & & & & & & & & & 4.50E-51 & 1 & 4.50E-51 & $9.00 \mathrm{E}-50$ & 85.346 \\
\hline Th-227 & & & & & & & & & & $6.30 \mathrm{E}-52$ & 15 & $4.20 \mathrm{E}-53$ & $8.40 \mathrm{E}-52$ & 0.796 \\
\hline Ra-223 & & & & & & & & & & $4.03 E-51$ & 15 & 2.69E-52 & $5.38 \mathrm{E}-51$ & 5.096 \\
\hline SOF-chain & & & & & & & & & & & & & 1.06E-49 & $9.48 \mathrm{E}+48$ \\
\hline U-235 & 0.1 & 0.05 & 4673 & 14 & 15 & 13 & 1350 & 10000 & 10000 & $2.07 \mathrm{E}-10$ & 65 & $3.19 \mathrm{E}-12$ & $6.38 \mathrm{E}-11$ & 0.027 \\
\hline
\end{tabular}




\begin{tabular}{|c|c|c|c|c|c|c|c|c|c|c|c|c|c|c|}
\hline $\begin{array}{l}. \frac{5}{\pi} \\
\frac{0}{0} \\
\frac{0}{0} \\
\frac{0}{0} \\
\frac{0}{2}\end{array}$ & 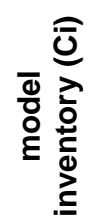 & 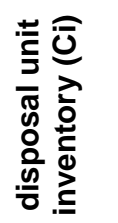 & 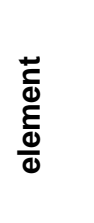 & $\begin{array}{l}\overline{0} \\
\frac{0}{0} \\
\stackrel{\varrho}{\varrho}\end{array}$ & $\begin{array}{l}\mathfrak{0} \\
\stackrel{0}{0} \\
\stackrel{0}{0}\end{array}$ & 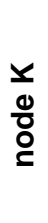 & 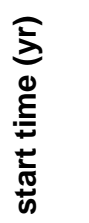 & 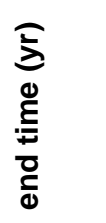 & 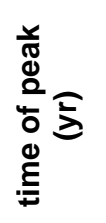 & 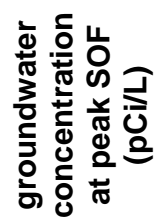 & 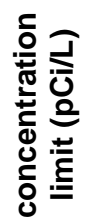 & 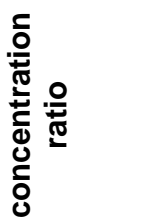 & 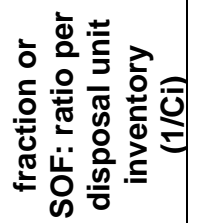 & 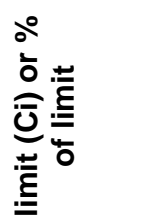 \\
\hline Th-227 & & & & & & & & & & 1.25E-09 & 15 & $8.36 \mathrm{E}-11$ & 1.67E-09 & 0.705 \\
\hline Ra-223 & & & & & & & & & & $8.02 \mathrm{E}-09$ & 15 & $5.35 \mathrm{E}-10$ & $1.07 \mathrm{E}-08$ & 4.513 \\
\hline SOF-chain & & & & & & & & & & & & & $2.37 \mathrm{E}-07$ & $4.22 E+06$ \\
\hline U-235 & 1 & 0.5 & 4673 & 14 & 15 & 13 & 0 & 100 & 100 & 8.92E-98 & 65 & 1.37E-99 & $2.75 E-99$ & 0.001 \\
\hline Pa-231 & & & & & & & & & & $3.51 \mathrm{E}-96$ & 3.1 & 1.13E-96 & $2.26 \mathrm{E}-96$ & 1.235 \\
\hline Ac-227 & & & & & & & & & & $8.47 \mathrm{E}-95$ & 1 & 8.47E-95 & 1.69E-94 & 92.457 \\
\hline Th-227 & & & & & & & & & & 1.17E-95 & 15 & 7.81E-97 & 1.56E-96 & 0.853 \\
\hline Ra-223 & & & & & & & & & & $7.50 \mathrm{E}-95$ & 15 & 5.00E-96 & 9.99E-96 & 5.454 \\
\hline SOF-chain & & & & & & & & & & & & & $1.83 \mathrm{E}-94$ & $5.46 \mathrm{E}+93$ \\
\hline U-235 & 1 & 0.5 & 4673 & 14 & 15 & 13 & 100 & 1350 & 1350 & $2.47 \mathrm{E}-54$ & 65 & $3.80 \mathrm{E}-56$ & $7.61 \mathrm{E}-56$ & 0 \\
\hline Pa-231 & & & & & & & & & & $7.91 \mathrm{E}-51$ & 3.1 & $2.55 \mathrm{E}-51$ & $5.10 \mathrm{E}-51$ & 8.656 \\
\hline Ac-227 & & & & & & & & & & $2.52 \mathrm{E}-50$ & 1 & $2.52 \mathrm{E}-50$ & 5.04E-50 & 85.444 \\
\hline Ra-223 & & & & & & & & & & $2.26 \mathrm{E}-50$ & 15 & $1.50 \mathrm{E}-51$ & $3.01 \mathrm{E}-51$ & 5.102 \\
\hline SOF-chain & & & & & & & & & & & & & $5.89 \mathrm{E}-50$ & $1.70 \mathrm{E}+49$ \\
\hline U-235 & 1 & 0.5 & 4673 & 14 & 15 & 13 & 1350 & 10000 & 10000 & $2.07 \mathrm{E}-10$ & 65 & $3.19 \mathrm{E}-12$ & $6.38 \mathrm{E}-12$ & 0.008 \\
\hline Pa-231 & & & & & & & & & & $2.46 \mathrm{E}-08$ & 3.1 & 7.92E-09 & 1.58E-08 & 19.546 \\
\hline Ac-227 & & & & & & & & & & $3.05 \mathrm{E}-08$ & 1 & $3.05 \mathrm{E}-08$ & $6.10 \mathrm{E}-08$ & 75.227 \\
\hline Th-227 & & & & & & & & & & $4.29 \mathrm{E}-09$ & 15 & $2.86 \mathrm{E}-10$ & $5.72 \mathrm{E}-10$ & 0.706 \\
\hline Ra-223 & & & & & & & & & & $2.74 \mathrm{E}-08$ & 15 & $1.83 \mathrm{E}-09$ & $3.66 \mathrm{E}-09$ & 4.514 \\
\hline SOF-chain & & & & & & & & & & & & & $8.11 \mathrm{E}-08$ & $1.23 E+07$ \\
\hline U-236 & 0.001 & 0.0005 & 4673 & 14 & 15 & 13 & 0 & 100 & 100 & 6.80E-99 & 140 & $4.86 \mathrm{E}-101$ & $9.71 \mathrm{E}-98$ & 100 \\
\hline SOF-chain & & & & & & & & & & & & & $9.71 \mathrm{E}-98$ & $1.03 E+97$ \\
\hline $\mathrm{U}-236$ & 0.001 & 0.0005 & 4673 & 14 & 15 & 13 & 100 & 1350 & 1350 & $2.31 \mathrm{E}-55$ & 140 & 1.65E-57 & $3.30 \mathrm{E}-54$ & 100 \\
\hline SOF-chain & & & & & & & & & & & & & $3.30 \mathrm{E}-54$ & $3.03 E+53$ \\
\hline U-236 & 0.001 & 0.0005 & 4673 & 14 & 15 & 13 & 1350 & 10000 & 10000 & $2.48 \mathrm{E}-11$ & 140 & 1.77E-13 & $3.55 \mathrm{E}-10$ & 100 \\
\hline SOF-chain & & & & & & & & & & & & & $3.55 \mathrm{E}-10$ & $2.82 E+09$ \\
\hline U-236 & 0.01 & 0.005 & 4673 & 14 & 15 & 13 & 0 & 100 & 100 & $6.79 \mathrm{E}-98$ & 140 & $4.85 \mathrm{E}-100$ & $9.70 \mathrm{E}-98$ & 100 \\
\hline SOF-chain & & & & & & & & & & & & & $9.70 \mathrm{E}-98$ & $1.03 E+97$ \\
\hline U-236 & 0.01 & 0.005 & 4673 & 14 & 15 & 13 & 100 & 1350 & 1350 & $2.31 \mathrm{E}-54$ & 140 & $1.65 \mathrm{E}-56$ & $3.31 \mathrm{E}-54$ & 100 \\
\hline SOF-chain & & & & & & & & & & & & & $3.31 \mathrm{E}-54$ & $3.03 E+53$ \\
\hline
\end{tabular}




\begin{tabular}{|c|c|c|c|c|c|c|c|c|c|c|c|c|c|c|}
\hline 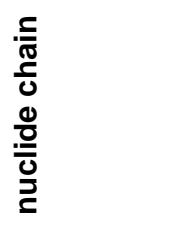 & 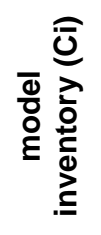 & 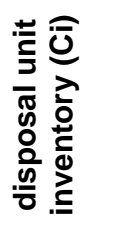 & 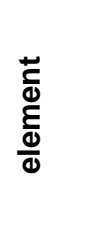 & $\begin{array}{l}\overline{0} \\
\frac{0}{0} \\
\stackrel{0}{\varrho}\end{array}$ & $\begin{array}{l}\overparen{0} \\
\stackrel{0}{0} \\
\stackrel{0}{\varrho}\end{array}$ & $\begin{array}{l}\searrow \\
\frac{1}{0} \\
\stackrel{0}{\complement}\end{array}$ & 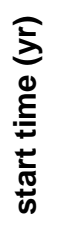 & 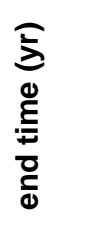 & 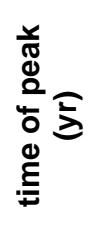 & 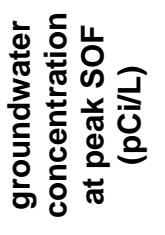 & 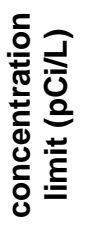 & 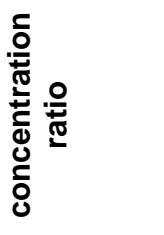 & 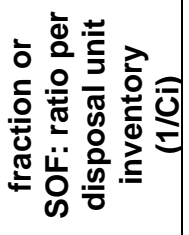 & 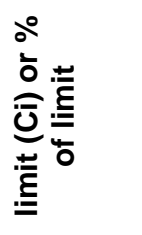 \\
\hline U-236 & 0.1 & 0.05 & 4673 & 14 & 15 & 13 & 0 & 100 & 100 & $6.79 \mathrm{E}-97$ & 140 & 4.85E-99 & $9.70 \mathrm{E}-98$ & 100 \\
\hline SOF-chain & & & & & & & & & & & & & 9.70E-98 & $1.03 E+97$ \\
\hline U-236 & 0.1 & 0.05 & 4673 & 14 & 15 & 13 & 100 & 1350 & 1350 & $2.32 \mathrm{E}-53$ & 140 & 1.65E-55 & $3.31 \mathrm{E}-54$ & 100 \\
\hline SOF-chain & & & & & & & & & & & & & $3.31 \mathrm{E}-54$ & $3.02 E+53$ \\
\hline U-236 & 0.1 & 0.05 & 4673 & 14 & 15 & 13 & 1350 & 10000 & 10000 & 2.49E-09 & 140 & $1.78 \mathrm{E}-11$ & $3.56 \mathrm{E}-10$ & 100 \\
\hline SOF-chain & & & & & & & & & & & & & $3.56 \mathrm{E}-10$ & $2.81 \mathrm{E}+09$ \\
\hline U-236 & 1 & 0.5 & 4673 & 14 & 15 & 13 & 0 & 100 & 100 & $2.69 \mathrm{E}-96$ & 140 & 1.92E-98 & 3.84E-98 & 100 \\
\hline SOF-chain & & & & & & & & & & & & & $3.84 \mathrm{E}-98$ & $2.60 \mathrm{E}+97$ \\
\hline U-236 & 1 & 0.5 & 4673 & 14 & 15 & 13 & 100 & 1350 & 1350 & 7.45E-53 & 140 & 5.32E-55 & 1.06E-54 & 100 \\
\hline SOF-chain & & & & & & & & & & & & & $1.06 \mathrm{E}-54$ & $9.40 \mathrm{E}+53$ \\
\hline U-236 & 1 & 0.5 & 4673 & 14 & 15 & 13 & 1350 & 10000 & 10000 & $6.25 \mathrm{E}-09$ & 140 & $4.46 \mathrm{E}-11$ & $8.93 \mathrm{E}-11$ & 100 \\
\hline SOF-chain & & & & & & & & & & & & & 8.93E-11 & $1.12 \mathrm{E}+10$ \\
\hline Th-234 & & & & & & & & & & 1.66E-99 & 400 & $4.15 \mathrm{E}-102$ & 8.29E-99 & 0.615 \\
\hline U-234 & & & & & & & & & & $2.82 \mathrm{E}-102$ & 130 & $2.17 \mathrm{E}-104$ & 4.34E-101 & 0.003 \\
\hline SOF-chain & & & & & & & & & & & & & 1.35E-96 & $7.42 \mathrm{E}+95$ \\
\hline $\mathrm{U}-238$ & 0.001 & 0.0005 & 4673 & 14 & 15 & 13 & 100 & 1350 & 1350 & $2.31 \mathrm{E}-55$ & 10.1 & $2.29 \mathrm{E}-56$ & $4.58 \mathrm{E}-53$ & 99.347 \\
\hline Th-234 & & & & & & & & & & $5.75 \mathrm{E}-56$ & 400 & 1.44E-58 & 2.87E-55 & 0.623 \\
\hline U-234 & & & & & & & & & & $8.85 \mathrm{E}-58$ & 130 & $6.81 \mathrm{E}-60$ & $1.36 \mathrm{E}-56$ & 0.03 \\
\hline SOF-chain & & & & & & & & & & & & & $4.61 \mathrm{E}-53$ & $2.17 E+52$ \\
\hline U-238 & 0.001 & 0.0005 & 4673 & 14 & 15 & 13 & 1350 & 10000 & 10000 & $2.48 \mathrm{E}-11$ & 10.1 & $2.46 \mathrm{E}-12$ & 4.92E-09 & 99.159 \\
\hline Th-234 & & & & & & & & & & $6.21 \mathrm{E}-12$ & 400 & $1.55 \mathrm{E}-14$ & $3.11 \mathrm{E}-11$ & 0.626 \\
\hline U-234 & & & & & & & & & & $6.93 \mathrm{E}-13$ & 130 & $5.33 \mathrm{E}-15$ & $1.07 \mathrm{E}-11$ & 0.215 \\
\hline SOF-chain & & & & & & & & & & & & & 4.96E-09 & $2.02 \mathrm{E}+08$ \\
\hline U-238 & 0.01 & 0.005 & 4673 & 14 & 15 & 13 & 0 & 100 & 100 & 1.41E-98 & 10.1 & 1.40E-99 & $2.79 \mathrm{E}-97$ & 99.378 \\
\hline Th-234 & & & & & & & & & & 3.46E-99 & 400 & $8.64 \mathrm{E}-102$ & 1.73E-99 & 0.615 \\
\hline U-234 & & & & & & & & & & $1.23 \mathrm{E}-101$ & 130 & $9.49 \mathrm{E}-104$ & $1.90 \mathrm{E}-101$ & 0.007 \\
\hline SOF-chain & & & & & & & & & & & & & $2.81 \mathrm{E}-97$ & $3.56 \mathrm{E}+96$ \\
\hline U-238 & 0.01 & 0.005 & 4673 & 14 & 15 & 13 & 100 & 1350 & 1350 & $3.90 \mathrm{E}-55$ & 10.1 & 3.86E-56 & 7.73E-54 & 99.277 \\
\hline Th-234 & & & & & & & & & & $9.70 \mathrm{E}-56$ & 400 & $2.43 \mathrm{E}-58$ & $4.85 E-56$ & 0.623 \\
\hline U-234 & & & & & & & & & & $5.05 \mathrm{E}-57$ & 130 & 3.88E-59 & $7.76 \mathrm{E}-57$ & 0.1 \\
\hline
\end{tabular}




\begin{tabular}{|c|c|c|c|c|c|c|c|c|c|c|c|c|c|c|}
\hline $\begin{array}{l}\frac{5}{\pi} \\
\frac{0}{0} \\
\frac{0}{0} \\
\frac{0}{0} \\
\end{array}$ & 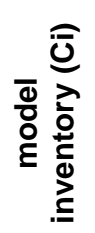 & 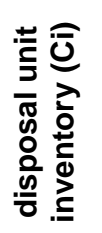 & 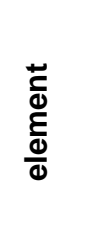 & $\begin{array}{l}\bar{\Phi} \\
\stackrel{0}{\circ}\end{array}$ & $\begin{array}{l}7 \\
\stackrel{0}{0} \\
\stackrel{0}{0}\end{array}$ & $\begin{array}{l}\underline{y} \\
\stackrel{0}{0} \\
\stackrel{0}{\varrho}\end{array}$ & 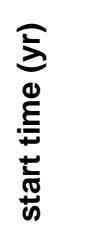 & 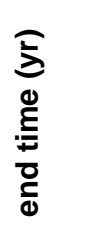 & 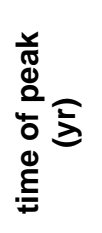 & 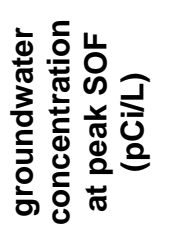 & 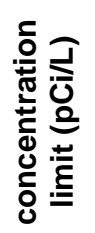 & 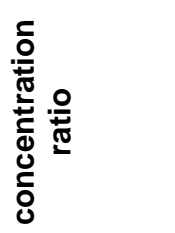 & 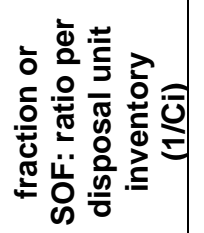 & 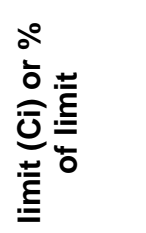 \\
\hline Th-234 & & & & & & & & & & $8.19 \mathrm{E}-12$ & 400 & $2.05 \mathrm{E}-14$ & $4.09 \mathrm{E}-12$ & 0.625 \\
\hline $\mathrm{U}-234$ & & & & & & & & & & $1.74 \mathrm{E}-12$ & 130 & $1.34 \mathrm{E}-14$ & $2.68 \mathrm{E}-12$ & 0.409 \\
\hline SOF-chain & & & & & & & & & & & & & $6.55 \mathrm{E}-10$ & $1.53 \mathrm{E}+09$ \\
\hline $\mathrm{U}-238$ & 0.1 & 0.05 & 4673 & 14 & 15 & 13 & 0 & 100 & 100 & $1.41 \mathrm{E}-98$ & 10.1 & $1.40 \mathrm{E}-99$ & $2.79 \mathrm{E}-98$ & 99.338 \\
\hline Th-234 & & & & & & & & & & $3.46 \mathrm{E}-99$ & 400 & 8.64E-102 & $1.73 \mathrm{E}-100$ & 0.615 \\
\hline $\mathrm{U}-234$ & & & & & & & & & & $8.58 \mathrm{E}-101$ & 130 & $6.60 \mathrm{E}-103$ & 1.32E-101 & 0.047 \\
\hline SOF-chain & & & & & & & & & & & & & $2.81 \mathrm{E}-98$ & $3.56 \mathrm{E}+97$ \\
\hline $\mathrm{U}-238$ & 0.1 & 0.05 & 4673 & 14 & 15 & 13 & 100 & 1350 & 1350 & $3.90 \mathrm{E}-55$ & 10.1 & $3.86 \mathrm{E}-56$ & $7.73 \mathrm{E}-55$ & 98.529 \\
\hline Th-234 & & & & & & & & & & $9.70 \mathrm{E}-56$ & 400 & $2.43 \mathrm{E}-58$ & $4.85 \mathrm{E}-57$ & 0.618 \\
\hline U-234 & & & & & & & & & & $4.35 \mathrm{E}-56$ & 130 & $3.35 \mathrm{E}-58$ & $6.69 \mathrm{E}-57$ & 0.853 \\
\hline SOF-chain & & & & & & & & & & & & & $7.84 \mathrm{E}-55$ & $1.28 \mathrm{E}+54$ \\
\hline $\mathrm{U}-238$ & 0.1 & 0.05 & 4673 & 14 & 15 & 13 & 1350 & 10000 & 10000 & $3.28 \mathrm{E}-11$ & 10.1 & $3.24 \mathrm{E}-12$ & $6.49 \mathrm{E}-11$ & 97.008 \\
\hline Th-234 & & & & & & & & & & $8.19 \mathrm{E}-12$ & 400 & $2.05 \mathrm{E}-14$ & $4.09 \mathrm{E}-13$ & 0.612 \\
\hline $\mathrm{U}-234$ & & & & & & & & & & $1.03 \mathrm{E}-11$ & 130 & $7.95 \mathrm{E}-14$ & $1.59 \mathrm{E}-12$ & 2.379 \\
\hline SOF-chain & & & & & & & & & & & & & $6.69 \mathrm{E}-11$ & $1.50 \mathrm{E}+10$ \\
\hline $\mathrm{U}-238$ & 1 & 0.5 & 4673 & 14 & 15 & 13 & 0 & 100 & 100 & $1.41 \mathrm{E}-98$ & 10.1 & $1.40 \mathrm{E}-99$ & $2.79 \mathrm{E}-99$ & 98.94 \\
\hline Th-234 & & & & & & & & & & $3.46 \mathrm{E}-99$ & 400 & $8.64 \mathrm{E}-102$ & $1.73 \mathrm{E}-101$ & 0.613 \\
\hline U-234 & & & & & & & & & & $8.20 \mathrm{E}-100$ & 130 & $6.31 \mathrm{E}-102$ & $1.26 \mathrm{E}-101$ & 0.447 \\
\hline SOF-chain & & & & & & & & & & & & & $2.82 \mathrm{E}-99$ & $3.55 \mathrm{E}+98$ \\
\hline $\mathrm{U}-238$ & 1 & 0.5 & 4673 & 14 & 15 & 13 & 100 & 1350 & 1350 & $3.90 \mathrm{E}-55$ & 10.1 & $3.86 \mathrm{E}-56$ & $7.73 \mathrm{E}-56$ & 91.621 \\
\hline Th-234 & & & & & & & & & & 9.70E-56 & 400 & $2.43 \mathrm{E}-58$ & $4.85 \mathrm{E}-58$ & 0.575 \\
\hline U-234 & & & & & & & & & & $4.28 \mathrm{E}-55$ & 130 & $3.29 \mathrm{E}-57$ & $6.58 \mathrm{E}-57$ & 7.804 \\
\hline SOF-chain & & & & & & & & & & & & & $8.44 \mathrm{E}-56$ & $1.19 \mathrm{E}+55$ \\
\hline $\mathrm{U}-238$ & 1 & 0.5 & 4673 & 14 & 15 & 13 & 1350 & 10000 & 10000 & $3.28 \mathrm{E}-11$ & 10.1 & $3.24 \mathrm{E}-12$ & $6.49 \mathrm{E}-12$ & 80.988 \\
\hline Th-234 & & & & & & & & & & $8.19 \mathrm{E}-12$ & 400 & $2.05 \mathrm{E}-14$ & $4.09 \mathrm{E}-14$ & 0.511 \\
\hline U-234 & & & & & & & & & & $9.63 \mathrm{E}-11$ & 130 & $7.41 \mathrm{E}-13$ & $1.48 \mathrm{E}-12$ & 18.501 \\
\hline SOF-chain & & & & & & & & & & & & & $8.01 \mathrm{E}-12$ & $1.25 \mathrm{E}+11$ \\
\hline $\mathrm{Np}-237$ & 1 & 0.5 & 4673 & 14 & 15 & 13 & 0 & 100 & 100 & $2.44 \mathrm{E}-35$ & 8.9 & $2.74 \mathrm{E}-36$ & $5.48 \mathrm{E}-36$ & 100 \\
\hline SOF-chain & & & & & & & & & & & & & $5.48 \mathrm{E}-36$ & $1.83 \mathrm{E}+35$ \\
\hline $\mathrm{Np}-237$ & 1 & 0.5 & 4671 & 12 & 15 & 13 & 100 & 1350 & 1350 & $2.92 \mathrm{E}-06$ & 8.9 & $3.28 \mathrm{E}-07$ & $6.56 \mathrm{E}-07$ & 100 \\
\hline SOF-chain & & & & & & & & & & & & & $6.56 \mathrm{E}-07$ & $1.53 \mathrm{E}+06$ \\
\hline Np-237 & 1 & 0.5 & 3871 & 12 & 15 & 11 & 1350 & 10000 & 10000 & $1.93 \mathrm{E}-01$ & 8.9 & $2.17 \mathrm{E}-02$ & $4.34 \mathrm{E}-02$ & 100 \\
\hline SOF-chain & & & & & & & & & & & & & $4.34 \mathrm{E}-02$ & $2.30 \mathrm{E}+01$ \\
\hline
\end{tabular}




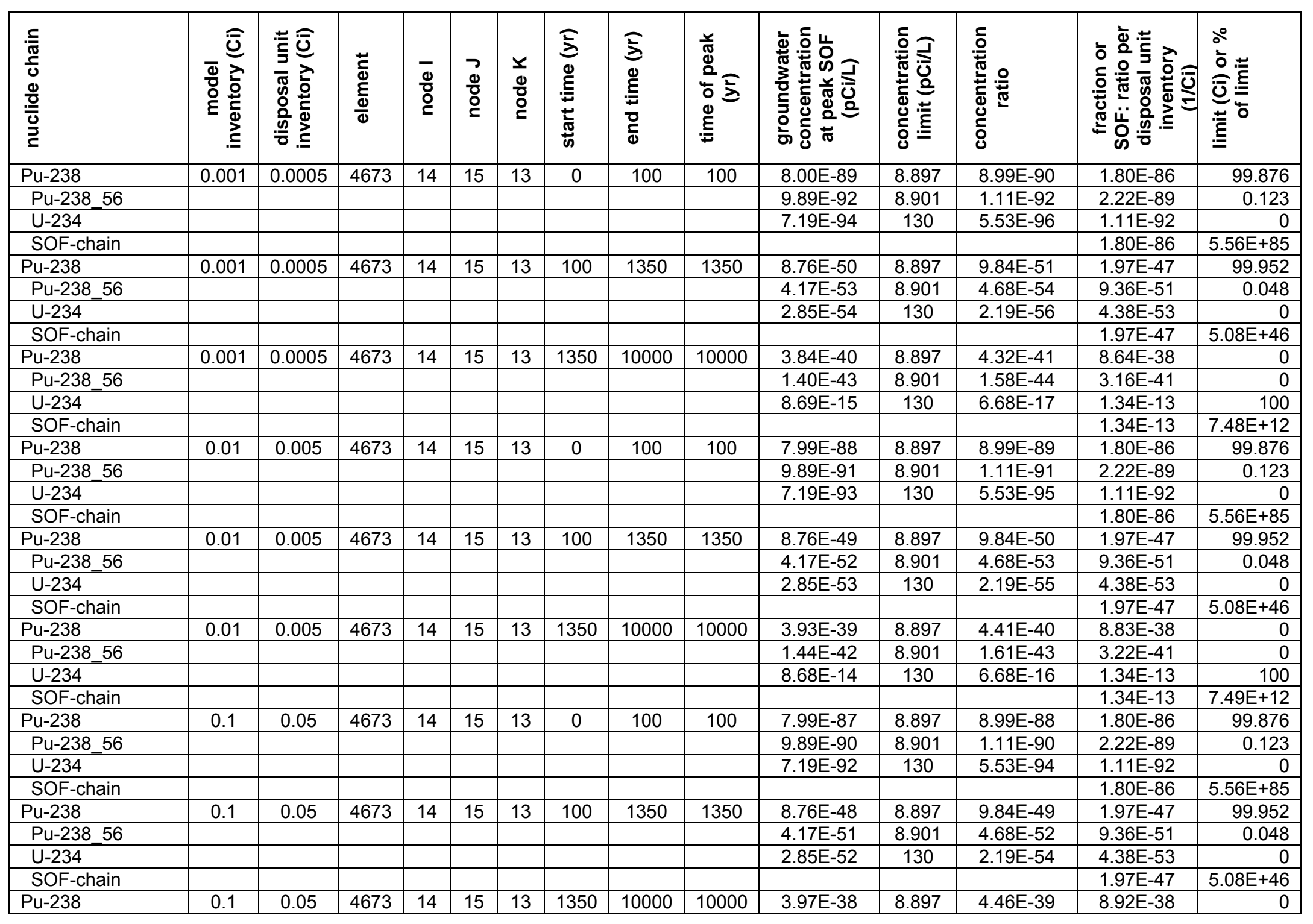




\begin{tabular}{|c|c|c|c|c|c|c|c|c|c|c|c|c|c|c|}
\hline 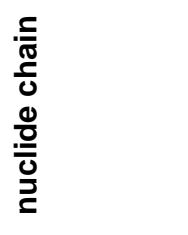 & 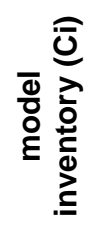 & 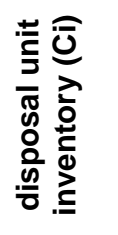 & 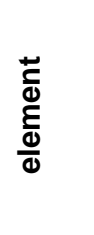 & $\begin{array}{l}\overline{0} \\
\frac{0}{0} \\
\stackrel{0}{\varrho}\end{array}$ & 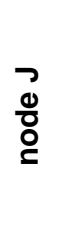 & 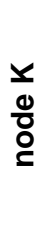 & 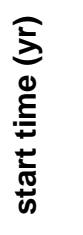 & 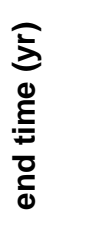 & 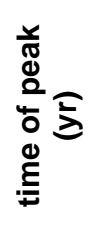 & 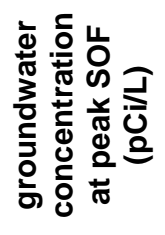 & 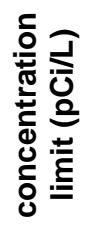 & 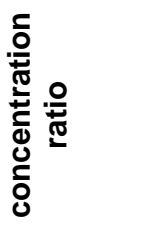 & 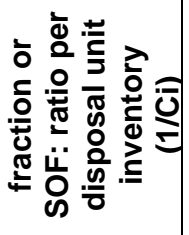 & 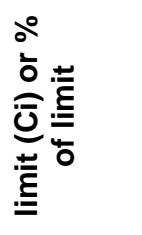 \\
\hline Pu-238_56 & & & & & & & & & & $1.45 \mathrm{E}-41$ & 8.901 & $1.63 E-42$ & $3.26 \mathrm{E}-41$ & 0 \\
\hline U-234 & & & & & & & & & & 8.67E-13 & 130 & $6.67 \mathrm{E}-15$ & $1.33 \mathrm{E}-13$ & 100 \\
\hline SOF-chain & & & & & & & & & & & & & 1.33E-13 & $7.50 \mathrm{E}+12$ \\
\hline $\mathrm{Pu}-238$ & 1 & 0.5 & 4673 & 14 & 15 & 13 & 0 & 100 & 100 & $7.99 \mathrm{E}-86$ & 8.897 & $8.98 \mathrm{E}-87$ & 1.80E-86 & 99.876 \\
\hline Pu-238_56 & & & & & & & & & & 9.89E-89 & 8.901 & 1.11E-89 & $2.22 \mathrm{E}-89$ & 0.123 \\
\hline U-234 & & & & & & & & & & 7.19E-91 & 130 & 5.53E-93 & 1.11E-92 & 0 \\
\hline SOF-chain & & & & & & & & & & & & & $1.80 \mathrm{E}-86$ & $5.56 \mathrm{E}+85$ \\
\hline $\mathrm{Pu}-238$ & 1 & 0.5 & 4673 & 14 & 15 & 13 & 100 & 1350 & 1350 & $8.76 \mathrm{E}-47$ & 8.897 & $9.84 \mathrm{E}-48$ & $1.97 \mathrm{E}-47$ & 99.952 \\
\hline Pu-238_56 & & & & & & & & & & 4.17E-50 & 8.901 & 4.68E-51 & 9.36E-51 & 0.048 \\
\hline U-234 & & & & & & & & & & $2.85 \mathrm{E}-51$ & 130 & $2.19 \mathrm{E}-53$ & $4.38 \mathrm{E}-53$ & 0 \\
\hline SOF-chain & & & & & & & & & & & & & $1.97 \mathrm{E}-47$ & $5.08 \mathrm{E}+46$ \\
\hline Pu-238 & 1 & 0.5 & 4673 & 14 & 15 & 13 & 1350 & 10000 & 10000 & 3.99E-37 & 8.897 & $4.48 \mathrm{E}-38$ & 8.97E-38 & 0 \\
\hline $\mathrm{U}-234$ & & & & & & & & & & $8.66 \mathrm{E}-12$ & 130 & $6.66 \mathrm{E}-14$ & $1.33 \mathrm{E}-13$ & 100 \\
\hline SOF-chain & & & & & & & & & & & & & 1.33E-13 & $7.51 \mathrm{E}+12$ \\
\hline Pu-238 & 10 & 5 & 4673 & 14 & 15 & 13 & 0 & 100 & 100 & $6.34 \mathrm{E}-85$ & 8.897 & $7.13 E-86$ & $1.43 E-86$ & 99.876 \\
\hline Pu-238_56 & & & & & & & & & & $7.86 \mathrm{E}-88$ & 8.901 & $8.83 E-89$ & 1.77E-89 & 0.124 \\
\hline U-234 & & & & & & & & & & $5.70 \mathrm{E}-90$ & 130 & 4.39E-92 & 8.77E-93 & 0 \\
\hline SOF-chain & & & & & & & & & & & & & $1.43 \mathrm{E}-86$ & $7.00 \mathrm{E}+85$ \\
\hline Pu-238 & 10 & 5 & 4673 & 14 & 15 & 13 & 100 & 1350 & 1350 & $8.75 \mathrm{E}-46$ & 8.897 & $9.84 \mathrm{E}-47$ & $1.97 \mathrm{E}-47$ & 99.952 \\
\hline Pu-238_56 & & & & & & & & & & $4.17 \mathrm{E}-49$ & 8.901 & $4.68 \mathrm{E}-50$ & $9.36 \mathrm{E}-51$ & 0.048 \\
\hline U-234 & & & & & & & & & & $2.85 \mathrm{E}-50$ & 130 & $2.19 \mathrm{E}-52$ & $4.38 \mathrm{E}-53$ & 0 \\
\hline SOF-chain & & & & & & & & & & & & & $1.97 \mathrm{E}-47$ & $5.08 \mathrm{E}+46$ \\
\hline Pu-238 & 10 & 5 & 4673 & 14 & 15 & 13 & 1350 & 10000 & 10000 & $4.00 \mathrm{E}-36$ & 8.897 & $4.49 \mathrm{E}-37$ & 8.98E-38 & 0 \\
\hline Pu-238_56 & & & & & & & & & & 1.46E-39 & 8.901 & 1.64E-40 & $3.28 \mathrm{E}-41$ & 0 \\
\hline U-234 & & & & & & & & & & $8.65 \mathrm{E}-11$ & 130 & $6.66 \mathrm{E}-13$ & $1.33 \mathrm{E}-13$ & 100 \\
\hline SOF-chain & & & & & & & & & & & & & 1.33E-13 & $7.51 \mathrm{E}+12$ \\
\hline Pu-239 & 0.001 & 0.0005 & 4673 & 14 & 15 & 13 & 0 & 100 & 100 & $1.89 \mathrm{E}-88$ & 8.1 & $2.34 \mathrm{E}-89$ & $4.67 \mathrm{E}-86$ & 99.878 \\
\hline Pu-239_56 & & & & & & & & & & $2.30 \mathrm{E}-91$ & 8.101 & $2.84 \mathrm{E}-92$ & $5.68 \mathrm{E}-89$ & 0.122 \\
\hline U-235 & & & & & & & & & & 5.68E-97 & 65 & 8.74E-99 & 1.75E-95 & 0 \\
\hline SOF-chain & & & & & & & & & & & & & $4.68 \mathrm{E}-86$ & $2.14 \mathrm{E}+85$ \\
\hline
\end{tabular}




\begin{tabular}{|c|c|c|c|c|c|c|c|c|c|c|c|c|c|c|}
\hline $\begin{array}{l}\frac{5}{0} \\
\frac{0}{0} \\
\frac{0}{0} \\
\frac{0}{0} \\
\end{array}$ & 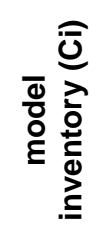 & 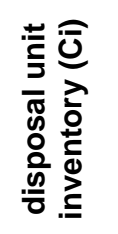 & 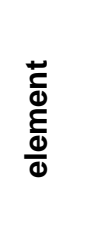 & 임 & 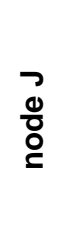 & 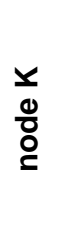 & 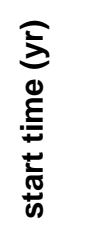 & 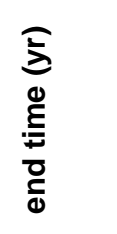 & 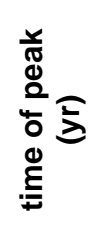 & 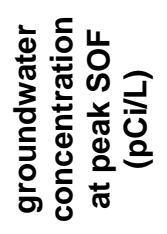 & 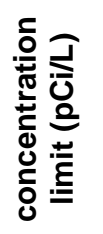 & 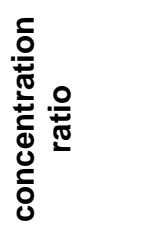 & 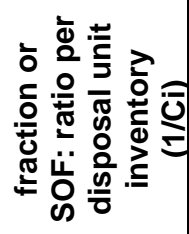 & 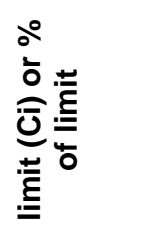 \\
\hline U-235 & & & & & & & & & & $3.72 \mathrm{E}-53$ & 65 & $5.72 \mathrm{E}-55$ & $1.14 \mathrm{E}-51$ & 0 \\
\hline SOF-chain & & & & & & & & & & & & & $9.61 \mathrm{E}-43$ & $1.04 \mathrm{E}+42$ \\
\hline Pu-239 & 0.001 & 0.0005 & 4671 & 12 & 15 & 13 & 1350 & 10000 & 10000 & $6.47 \mathrm{E}-06$ & 8.1 & 7.99E-07 & $1.60 \mathrm{E}-03$ & 99.963 \\
\hline Pu-239_56 & & & & & & & & & & $2.36 \mathrm{E}-09$ & 8.101 & $2.92 \mathrm{E}-10$ & $5.83 \mathrm{E}-07$ & 0.036 \\
\hline $\mathrm{U}-235$ & & & & & & & & & & $3.23 \mathrm{E}-12$ & 65 & $4.97 \mathrm{E}-14$ & $9.94 \mathrm{E}-11$ & 0 \\
\hline SOF-chain & & & & & & & & & & & & & $1.60 \mathrm{E}-03$ & $6.26 \mathrm{E}+02$ \\
\hline Pu-239 & 0.01 & 0.005 & 4673 & 14 & 15 & 13 & 0 & 100 & 100 & 1.91E-87 & 8.1 & $2.35 \mathrm{E}-88$ & $4.71 \mathrm{E}-86$ & 99.879 \\
\hline Pu-239_56 & & & & & & & & & & $2.32 \mathrm{E}-90$ & 8.101 & $2.86 \mathrm{E}-91$ & $5.72 \mathrm{E}-89$ & 0.121 \\
\hline U-235 & & & & & & & & & & 5.72E-96 & 65 & $8.80 \mathrm{E}-98$ & 1.76E-95 & 0 \\
\hline SOF-chain & & & & & & & & & & & & & $4.71 \mathrm{E}-86$ & $2.12 \mathrm{E}+85$ \\
\hline Pu-239 & 0.01 & 0.005 & 4673 & 14 & 15 & 13 & 100 & 1350 & 1350 & $3.89 \mathrm{E}-44$ & 8.1 & $4.80 \mathrm{E}-45$ & $9.61 \mathrm{E}-43$ & 99.953 \\
\hline Pu-239_56 & & & & & & & & & & 1.85E-47 & 8.101 & $2.28 \mathrm{E}-48$ & $4.56 \mathrm{E}-46$ & 0.047 \\
\hline $\mathrm{U}-235$ & & & & & & & & & & $3.72 \mathrm{E}-52$ & 65 & $5.72 \mathrm{E}-54$ & 1.14E-51 & 0 \\
\hline SOF-chain & & & & & & & & & & & & & $9.61 \mathrm{E}-43$ & $1.04 \mathrm{E}+42$ \\
\hline Pu-239 & 0.01 & 0.005 & 4671 & 12 & 15 & 13 & 1350 & 10000 & 10000 & $6.47 \mathrm{E}-05$ & 8.1 & 7.99E-06 & $1.60 \mathrm{E}-03$ & 99.963 \\
\hline Pu-239_56 & & & & & & & & & & $2.36 \mathrm{E}-08$ & 8.101 & $2.92 \mathrm{E}-09$ & $5.83 \mathrm{E}-07$ & 0.036 \\
\hline $\mathrm{U}-235$ & & & & & & & & & & $3.23 \mathrm{E}-11$ & 65 & $4.97 \mathrm{E}-13$ & $9.94 \mathrm{E}-11$ & 0 \\
\hline SOF-chain & & & & & & & & & & & & & $1.60 \mathrm{E}-03$ & $6.26 \mathrm{E}+02$ \\
\hline Pu-239 & 0.1 & 0.05 & 4673 & 14 & 15 & 13 & 0 & 100 & 100 & $5.38 \mathrm{E}-87$ & 8.1 & $6.64 \mathrm{E}-88$ & $1.33 \mathrm{E}-86$ & 99.878 \\
\hline Pu-239_56 & & & & & & & & & & $6.54 \mathrm{E}-90$ & 8.101 & $8.08 \mathrm{E}-91$ & $1.62 \mathrm{E}-89$ & 0.122 \\
\hline U-235 & & & & & & & & & & 1.61E-95 & 65 & $2.48 \mathrm{E}-97$ & 4.97E-96 & 0 \\
\hline SOF-chain & & & & & & & & & & & & & $1.33 \mathrm{E}-86$ & $7.52 \mathrm{E}+85$ \\
\hline Pu-239 & 0.1 & 0.05 & 4673 & 14 & 15 & 13 & 100 & 1350 & 1350 & $9.13 \mathrm{E}-44$ & 8.1 & $1.13 \mathrm{E}-44$ & $2.26 \mathrm{E}-43$ & 99.953 \\
\hline Pu-239_56 & & & & & & & & & & $4.33 \mathrm{E}-47$ & 8.101 & $5.35 \mathrm{E}-48$ & $1.07 \mathrm{E}-46$ & 0.047 \\
\hline U-235 & & & & & & & & & & $8.74 \mathrm{E}-52$ & 65 & $1.35 \mathrm{E}-53$ & $2.69 \mathrm{E}-52$ & 0 \\
\hline SOF-chain & & & & & & & & & & & & & $2.26 \mathrm{E}-43$ & $4.43 \mathrm{E}+42$ \\
\hline Pu-239 & 0.1 & 0.05 & 4671 & 12 & 15 & 13 & 1350 & 10000 & 10000 & $1.21 \mathrm{E}-04$ & 8.1 & $1.50 \mathrm{E}-05$ & $3.00 \mathrm{E}-04$ & 99.963 \\
\hline Pu-239_56 & & & & & & & & & & $4.43 \mathrm{E}-08$ & 8.101 & 5.47E-09 & 1.09E-07 & 0.036 \\
\hline U-235 & & & & & & & & & & $6.05 \mathrm{E}-11$ & 65 & $9.31 \mathrm{E}-13$ & $1.86 \mathrm{E}-11$ & 0 \\
\hline SOF-chain & & & & & & & & & & & & & $3.00 \mathrm{E}-04$ & $3.34 \mathrm{E}+03$ \\
\hline Pu-239 & 1 & 0.5 & 4673 & 14 & 15 & 13 & 0 & 100 & 100 & $5.38 \mathrm{E}-87$ & 8.1 & $6.64 \mathrm{E}-88$ & $1.33 \mathrm{E}-87$ & 99.878 \\
\hline Pu-239_56 & & & & & & & & & & $6.54 \mathrm{E}-90$ & 8.101 & $8.08 \mathrm{E}-91$ & $1.62 \mathrm{E}-90$ & 0.122 \\
\hline U-235 & & & & & & & & & & 1.62E-95 & 65 & $2.49 \mathrm{E}-97$ & $4.97 \mathrm{E}-97$ & 0 \\
\hline
\end{tabular}




\begin{tabular}{|c|c|c|c|c|c|c|c|c|c|c|c|c|c|c|}
\hline $\begin{array}{l}\frac{5}{0} \\
\frac{0}{0} \\
\frac{0}{0} \\
\frac{0}{0} \\
\frac{0}{2}\end{array}$ & 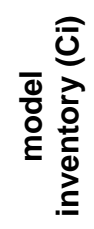 & 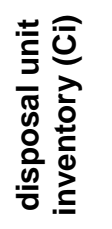 & 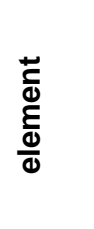 & 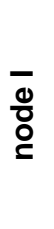 & $\begin{array}{l}7 \\
\stackrel{0}{0} \\
0\end{array}$ & 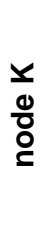 & 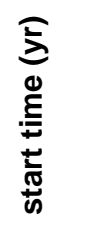 & 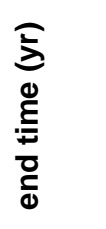 & 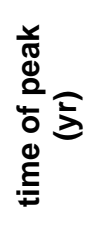 & 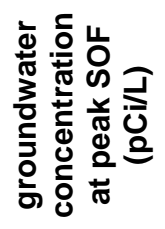 & 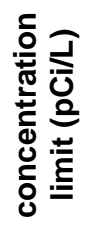 & 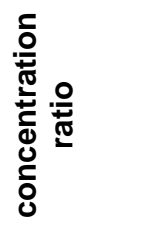 & 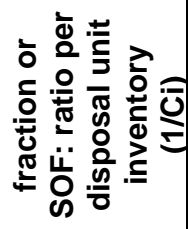 & 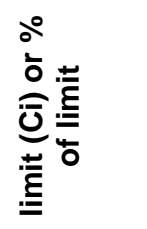 \\
\hline SOF-chain & & & & & & & & & & & & & 1.33E-87 & $7.52 \mathrm{E}+86$ \\
\hline Pu-239 & 1 & 0.5 & 4673 & 14 & 15 & 13 & 100 & 1350 & 1350 & $9.14 \mathrm{E}-44$ & 8.1 & $1.13 \mathrm{E}-44$ & $2.26 \mathrm{E}-44$ & 99.953 \\
\hline Pu-239_56 & & & & & & & & & & $4.33 \mathrm{E}-47$ & 8.101 & $5.35 \mathrm{E}-48$ & $1.07 \mathrm{E}-47$ & 0.047 \\
\hline U-235 & & & & & & & & & & $8.75 E-52$ & 65 & $1.35 \mathrm{E}-53$ & $2.69 \mathrm{E}-53$ & 0 \\
\hline SOF-chain & & & & & & & & & & & & & $2.26 \mathrm{E}-44$ & $4.43 \mathrm{E}+43$ \\
\hline Pu-239 & 1 & 0.5 & 4671 & 12 & 15 & 13 & 1350 & 10000 & 10000 & $1.21 \mathrm{E}-04$ & 8.1 & 1.50E-05 & $3.00 \mathrm{E}-05$ & 99.963 \\
\hline Pu-239_56 & & & & & & & & & & 4.43E-08 & 8.101 & 5.47E-09 & 1.09E-08 & 0.036 \\
\hline U-235 & & & & & & & & & & $6.06 \mathrm{E}-11$ & 65 & $9.32 \mathrm{E}-13$ & $1.86 \mathrm{E}-12$ & 0 \\
\hline SOF-chain & & & & & & & & & & & & & 3.00E-05 & $3.34 \mathrm{E}+04$ \\
\hline Pu-240 & 0.001 & 0.0005 & 4673 & 14 & 15 & 13 & 0 & 100 & 100 & $1.88 \mathrm{E}-88$ & 8.102 & $2.33 \mathrm{E}-89$ & $4.65 \mathrm{E}-86$ & 99.878 \\
\hline Pu-240_56 & & & & & & & & & & $2.29 \mathrm{E}-91$ & 8.1 & $2.83 \mathrm{E}-92$ & $5.66 \mathrm{E}-89$ & 0.122 \\
\hline U-236 & & & & & & & & & & 1.70E-95 & 140 & $1.21 \mathrm{E}-97$ & 2.43E-94 & 0 \\
\hline Pu-240 & 0.001 & 0.0005 & 4673 & 14 & 15 & 13 & 100 & 1350 & 1350 & $3.51 \mathrm{E}-45$ & 8.102 & $4.33 \mathrm{E}-46$ & $8.66 \mathrm{E}-43$ & 99.953 \\
\hline Pu-240_56 & & & & & & & & & & $1.66 \mathrm{E}-48$ & 8.1 & $2.06 \mathrm{E}-49$ & $4.11 \mathrm{E}-46$ & 0.047 \\
\hline $\mathrm{U}-236$ & & & & & & & & & & $1.01 \mathrm{E}-51$ & 140 & $7.20 \mathrm{E}-54$ & $1.44 \mathrm{E}-50$ & 0 \\
\hline SOF-chain & & & & & & & & & & & & & $8.66 \mathrm{E}-43$ & $1.16 \mathrm{E}+42$ \\
\hline $\mathrm{Pu}-240$ & 0.001 & 0.0005 & 4671 & 12 & 15 & 13 & 1350 & 10000 & 10000 & $3.00 \mathrm{E}-06$ & 8.102 & $3.70 \mathrm{E}-07$ & $7.41 \mathrm{E}-04$ & 99.963 \\
\hline Pu-240_56 & & & & & & & & & & $1.10 \mathrm{E}-09$ & 8.1 & $1.35 \mathrm{E}-10$ & $2.71 \mathrm{E}-07$ & 0.037 \\
\hline$U-236$ & & & & & & & & & & $4.86 \mathrm{E}-11$ & 140 & $3.47 \mathrm{E}-13$ & $6.95 \mathrm{E}-10$ & 0 \\
\hline SOF-chain & & & & & & & & & & & & & $7.41 \mathrm{E}-04$ & $1.35 \mathrm{E}+03$ \\
\hline Pu-240 & 0.01 & 0.005 & 4673 & 14 & 15 & 13 & 0 & 100 & 100 & $1.88 \mathrm{E}-87$ & 8.102 & $2.32 \mathrm{E}-88$ & $4.64 \mathrm{E}-86$ & 99.878 \\
\hline Pu-240 56 & & & & & & & & & & $2.29 \mathrm{E}-90$ & 8.1 & $2.83 E-91$ & $5.65 E-89$ & 0.122 \\
\hline U-236 & & & & & & & & & & $1.70 \mathrm{E}-94$ & 140 & $1.21 \mathrm{E}-96$ & $2.43 E-94$ & 0 \\
\hline SOF-chain & & & & & & & & & & & & & $4.65 \mathrm{E}-86$ & $2.15 \mathrm{E}+85$ \\
\hline Pu-240 & 0.01 & 0.005 & 4673 & 14 & 15 & 13 & 100 & 1350 & 1350 & $3.51 \mathrm{E}-44$ & 8.102 & $4.33 \mathrm{E}-45$ & $8.66 \mathrm{E}-43$ & 99.953 \\
\hline Pu-240_56 & & & & & & & & & & $1.66 \mathrm{E}-47$ & 8.1 & $2.06 \mathrm{E}-48$ & $4.11 \mathrm{E}-46$ & 0.047 \\
\hline U-236 & & & & & & & & & & $1.01 \mathrm{E}-50$ & 140 & $7.20 \mathrm{E}-53$ & $1.44 \mathrm{E}-50$ & 0 \\
\hline SOF-chain & & & & & & & & & & & & & $8.66 \mathrm{E}-43$ & $1.15 \mathrm{E}+42$ \\
\hline Pu-240 & 0.01 & 0.005 & 4671 & 12 & 15 & 13 & 1350 & 10000 & 10000 & 3.00E-05 & 8.102 & 3.70E-06 & 7.41E-04 & 99.963 \\
\hline Pu-240_56 & & & & & & & & & & 1.10E-08 & 8.1 & 1.35E-09 & $2.71 \mathrm{E}-07$ & 0.037 \\
\hline
\end{tabular}




\begin{tabular}{|c|c|c|c|c|c|c|c|c|c|c|c|c|c|c|}
\hline 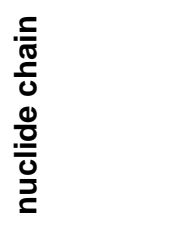 & 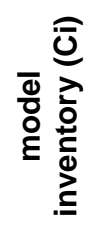 & 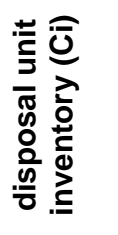 & 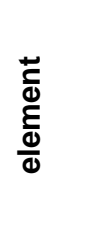 & $\begin{array}{l}\overline{0} \\
\frac{0}{0} \\
\stackrel{0}{\varrho}\end{array}$ & $\begin{array}{l}\mathfrak{0} \\
\frac{0}{0} \\
\stackrel{0}{c}\end{array}$ & 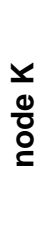 & 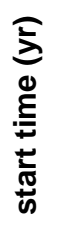 & 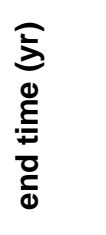 & 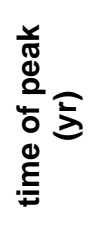 & 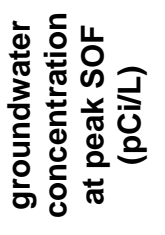 & 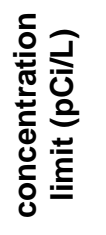 & 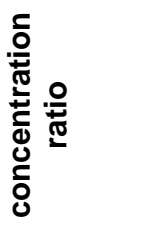 & 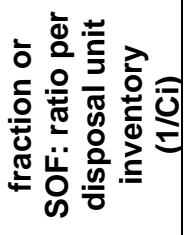 & 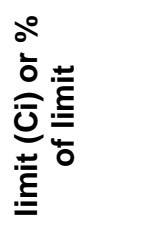 \\
\hline Pu-240 & 0.1 & 0.05 & 4673 & 14 & 15 & 13 & 0 & 100 & 100 & $1.88 \mathrm{E}-86$ & 8.102 & $2.32 \mathrm{E}-87$ & $4.64 \mathrm{E}-86$ & 99.878 \\
\hline Pu-240_56 & & & & & & & & & & $2.29 \mathrm{E}-89$ & 8.1 & $2.82 \mathrm{E}-90$ & $5.65 E-89$ & 0.122 \\
\hline U-236 & & & & & & & & & & 1.70E-93 & 140 & 1.21E-95 & $2.42 E-94$ & 0 \\
\hline SOF-chain & & & & & & & & & & & & & $4.65 \mathrm{E}-86$ & $2.15 \mathrm{E}+85$ \\
\hline Pu-240 & 0.1 & 0.05 & 4673 & 14 & 15 & 13 & 100 & 1350 & 1350 & $3.17 \mathrm{E}-43$ & 8.102 & $3.92 \mathrm{E}-44$ & $7.83 \mathrm{E}-43$ & 99.953 \\
\hline Pu-240_56 & & & & & & & & & & $1.51 \mathrm{E}-46$ & 8.1 & 1.86E-47 & $3.72 E-46$ & 0.047 \\
\hline U-236 & & & & & & & & & & $9.13 \mathrm{E}-50$ & 140 & $6.52 \mathrm{E}-52$ & 1.31E-50 & 0 \\
\hline SOF-chain & & & & & & & & & & & & & $7.84 \mathrm{E}-43$ & $1.28 \mathrm{E}+42$ \\
\hline Pu-240 & 0.1 & 0.05 & 4671 & 12 & 15 & 13 & 1350 & 10000 & 10000 & 2.33E-04 & 8.102 & 2.88E-05 & 5.76E-04 & 99.963 \\
\hline Pu-240_56 & & & & & & & & & & $8.52 \mathrm{E}-08$ & 8.1 & $1.05 E-08$ & $2.10 \mathrm{E}-07$ & 0.037 \\
\hline U-236 & & & & & & & & & & 3.76E-09 & 140 & $2.68 \mathrm{E}-11$ & 5.37E-10 & 0 \\
\hline SOF-chain & & & & & & & & & & & & & 5.76E-04 & $1.74 \mathrm{E}+03$ \\
\hline Pu-240_56 & & & & & & & & & & $2.38 \mathrm{E}-89$ & 8.1 & $2.94 \mathrm{E}-90$ & $5.88 \mathrm{E}-90$ & 0.122 \\
\hline U-236 & & & & & & & & & & 1.77E-93 & 140 & 1.26E-95 & $2.52 E-95$ & 0 \\
\hline SOF-chain & & & & & & & & & & & & & $4.84 \mathrm{E}-87$ & $2.07 E+86$ \\
\hline Pu-240 & 1 & 0.5 & 4673 & 14 & 15 & 13 & 100 & 1350 & 1350 & $3.18 \mathrm{E}-43$ & 8.102 & $3.92 \mathrm{E}-44$ & $7.84 \mathrm{E}-44$ & 99.953 \\
\hline Pu-240_56 & & & & & & & & & & $1.51 \mathrm{E}-46$ & 8.1 & 1.86E-47 & $3.72 \mathrm{E}-47$ & 0.047 \\
\hline $\mathrm{U}-236$ & & & & & & & & & & $9.14 \mathrm{E}-50$ & 140 & $6.53 E-52$ & $1.31 \mathrm{E}-51$ & 0 \\
\hline SOF-chain & & & & & & & & & & & & & $7.84 \mathrm{E}-44$ & $1.28 \mathrm{E}+43$ \\
\hline Pu-240 & 1 & 0.5 & 4671 & 12 & 15 & 13 & 1350 & 10000 & 10000 & 2.34E-04 & 8.102 & 2.88E-05 & 5.76E-05 & 99.963 \\
\hline Pu-240_56 & & & & & & & & & & $8.53 \mathrm{E}-08$ & 8.1 & 1.05E-08 & $2.11 \mathrm{E}-08$ & 0.037 \\
\hline U-236 & & & & & & & & & & 3.76E-09 & 140 & $2.69 \mathrm{E}-11$ & $5.37 \mathrm{E}-11$ & 0 \\
\hline SOF-chain & & & & & & & & & & & & & 5.77E-05 & $1.73 E+04$ \\
\hline Pu-242 & 0.001 & 0.0005 & 4673 & 14 & 15 & 13 & 0 & 100 & 100 & 1.91E-88 & 8.301 & 2.30E-89 & 4.59E-86 & 99.878 \\
\hline Pu-242_56 & & & & & & & & & & $2.32 \mathrm{E}-91$ & 8.299 & $2.79 \mathrm{E}-92$ & 5.59E-89 & 0.122 \\
\hline U-238 & & & & & & & & & & 9.01E-98 & 10.1 & 8.92E-99 & 1.79E-95 & 0 \\
\hline SOF-chain & & & & & & & & & & & & & $4.60 \mathrm{E}-86$ & $2.18 \mathrm{E}+85$ \\
\hline Pu-242 & 0.001 & 0.0005 & 4673 & 14 & 15 & 13 & 100 & 1350 & 1350 & $4.04 \mathrm{E}-45$ & 8.301 & $4.86 \mathrm{E}-46$ & $9.73 \mathrm{E}-43$ & 99.953 \\
\hline $\mathrm{Pu}-242 \_56$ & & & & & & & & & & $1.92 \mathrm{E}-48$ & 8.299 & $2.31 \mathrm{E}-49$ & $4.62 \mathrm{E}-46$ & 0.047 \\
\hline U-238 & & & & & & & & & & $6.07 E-54$ & 10.1 & 6.01E-55 & 1.20E-51 & 0 \\
\hline
\end{tabular}




\begin{tabular}{|c|c|c|c|c|c|c|c|c|c|c|c|c|c|c|}
\hline $\begin{array}{l}\frac{5}{\pi} \\
\frac{c}{0} \\
\frac{0}{0} \\
\frac{0}{0} \\
\frac{0}{2}\end{array}$ & 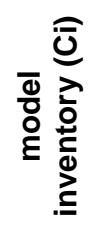 & 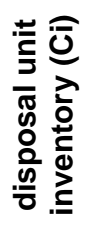 & 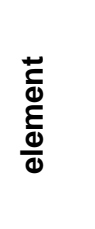 & $\begin{array}{l}\bar{\varrho} \\
\text { O }\end{array}$ & $\begin{array}{l}\mathfrak{0} \\
\stackrel{0}{0} \\
\stackrel{0}{\varrho}\end{array}$ & $\begin{array}{l}\searrow \\
\stackrel{0}{0} \\
\stackrel{0}{\varrho}\end{array}$ & 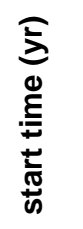 & 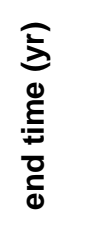 & 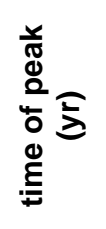 & 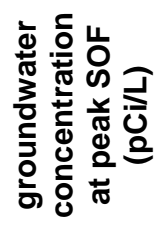 & 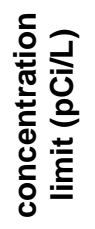 & 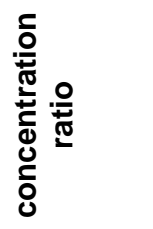 & 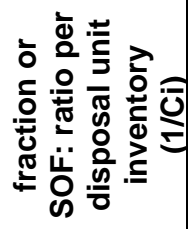 & 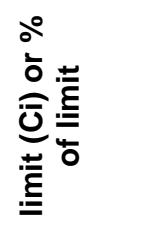 \\
\hline Pu-242_56 & & & & & & & & & & 3.10E-09 & 8.299 & $3.73 \mathrm{E}-10$ & 7.46E-07 & 0.037 \\
\hline $\mathrm{U}-238$ & & & & & & & & & & $6.50 \mathrm{E}-13$ & 10.1 & 6.43E-14 & $1.29 \mathrm{E}-10$ & 0 \\
\hline SOF-chain & & & & & & & & & & & & & 2.04E-03 & $4.89 \mathrm{E}+02$ \\
\hline Pu-242 & 0.01 & 0.005 & 4673 & 14 & 15 & 13 & 0 & 100 & 100 & $3.48 \mathrm{E}-88$ & 8.301 & $4.20 \mathrm{E}-89$ & 8.39E-87 & 99.878 \\
\hline Pu-242_56 & & & & & & & & & & 4.24E-91 & 8.299 & $5.11 \mathrm{E}-92$ & 1.02E-89 & 0.122 \\
\hline U-238 & & & & & & & & & & 1.65E-97 & 10.1 & 1.63E-98 & $3.26 E-96$ & 0 \\
\hline SOF-chain & & & & & & & & & & & & & $8.41 \mathrm{E}-87$ & $1.19 \mathrm{E}+86$ \\
\hline Pu-242 & 0.01 & 0.005 & 4673 & 14 & 15 & 13 & 100 & 1350 & 1350 & $6.01 \mathrm{E}-45$ & 8.301 & $7.24 \mathrm{E}-46$ & $1.45 \mathrm{E}-43$ & 99.953 \\
\hline Pu-242_56 & & & & & & & & & & $2.85 \mathrm{E}-48$ & 8.299 & $3.44 \mathrm{E}-49$ & $6.87 \mathrm{E}-47$ & 0.047 \\
\hline $\mathrm{U}-238$ & & & & & & & & & & $9.06 \mathrm{E}-54$ & 10.1 & $8.97 \mathrm{E}-55$ & $1.80 \mathrm{E}-52$ & 0 \\
\hline SOF-chain & & & & & & & & & & & & & $1.45 E-43$ & $6.90 \mathrm{E}+42$ \\
\hline $\mathrm{Pu}-242$ & 0.01 & 0.005 & 4671 & 12 & 15 & 13 & 1350 & 10000 & 10000 & 9.83E-06 & 8.301 & 1.18E-06 & 2.37E-04 & 99.963 \\
\hline $\mathrm{U}-238$ & & & & & & & & & & $7.53 \mathrm{E}-13$ & 10.1 & $7.46 \mathrm{E}-14$ & 1.49E-11 & 0 \\
\hline SOF-chain & & & & & & & & & & & & & 2.37E-04 & $4.22 \mathrm{E}+03$ \\
\hline $\mathrm{Pu}-242$ & 0.1 & 0.05 & 4673 & 14 & 15 & 13 & 0 & 100 & 100 & $3.49 \mathrm{E}-88$ & 8.301 & 4.20E-89 & $8.40 \mathrm{E}-88$ & 99.878 \\
\hline Pu-242_56 & & & & & & & & & & 4.24E-91 & 8.299 & $5.11 \mathrm{E}-92$ & $1.02 E-90$ & 0.122 \\
\hline $\mathrm{U}-238$ & & & & & & & & & & 1.65E-97 & 10.1 & 1.63E-98 & $3.26 \mathrm{E}-97$ & 0 \\
\hline SOF-chain & & & & & & & & & & & & & $8.41 \mathrm{E}-88$ & $1.19 \mathrm{E}+87$ \\
\hline Pu-242 & 0.1 & 0.05 & 4673 & 14 & 15 & 13 & 100 & 1350 & 1350 & $6.02 \mathrm{E}-45$ & 8.301 & $7.25 \mathrm{E}-46$ & $1.45 \mathrm{E}-44$ & 99.953 \\
\hline Pu-242_56 & & & & & & & & & & $2.85 \mathrm{E}-48$ & 8.299 & $3.44 \mathrm{E}-49$ & $6.88 \mathrm{E}-48$ & 0.047 \\
\hline $\mathrm{U}-238$ & & & & & & & & & & 9.07E-54 & 10.1 & $8.98 \mathrm{E}-55$ & $1.80 \mathrm{E}-53$ & 0 \\
\hline SOF-chain & & & & & & & & & & & & & 1.45E-44 & $6.89 \mathrm{E}+43$ \\
\hline $\mathrm{Pu}-242$ & 0.1 & 0.05 & 4671 & 12 & 15 & 13 & 1350 & 10000 & 10000 & 9.84E-06 & 8.301 & 1.19E-06 & 2.37E-05 & 99.963 \\
\hline Pu-242_56 & & & & & & & & & & 3.59E-09 & 8.299 & $4.33 \mathrm{E}-10$ & 8.66E-09 & 0.037 \\
\hline $\mathrm{U}-238$ & & & & & & & & & & $7.55 \mathrm{E}-13$ & 10.1 & 7.47E-14 & $1.49 \mathrm{E}-12$ & 0 \\
\hline SOF-chain & & & & & & & & & & & & & 2.37E-05 & $4.22 \mathrm{E}+04$ \\
\hline Pu-242 & 1 & 0.5 & 4673 & 14 & 15 & 13 & 0 & 100 & 100 & $3.51 \mathrm{E}-88$ & 8.301 & $4.23 \mathrm{E}-89$ & $8.46 \mathrm{E}-89$ & 99.878 \\
\hline Pu-242_56 & & & & & & & & & & 4.27E-91 & 8.299 & $5.15 \mathrm{E}-92$ & 1.03E-91 & 0.122 \\
\hline $\mathrm{U}-238$ & & & & & & & & & & 1.66E-97 & 10.1 & 1.64E-98 & $3.29 E-98$ & 0 \\
\hline SOF-chain & & & & & & & & & & & & & $8.47 \mathrm{E}-89$ & $1.18 \mathrm{E}+88$ \\
\hline
\end{tabular}




\begin{tabular}{|c|c|c|c|c|c|c|c|c|c|c|c|c|c|c|}
\hline $\begin{array}{l}\frac{5}{\pi} \\
\frac{0}{0} \\
\frac{0}{0} \\
\frac{0}{0} \\
\frac{0}{2}\end{array}$ & 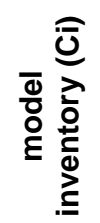 & 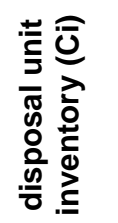 & 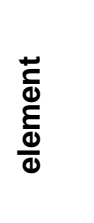 & $\begin{array}{l}\overline{0} \\
\frac{0}{0} \\
\stackrel{0}{c}\end{array}$ & $\begin{array}{l}\mathfrak{0} \\
\stackrel{0}{0} \\
\stackrel{0}{0}\end{array}$ & $\begin{array}{l}\stackrel{y}{0} \\
\stackrel{0}{0} \\
\stackrel{0}{\varrho}\end{array}$ & 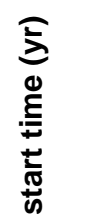 & 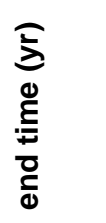 & 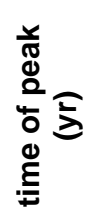 & 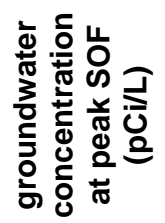 & 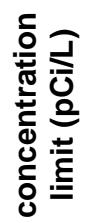 & 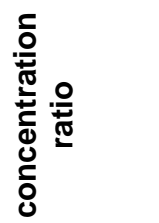 & 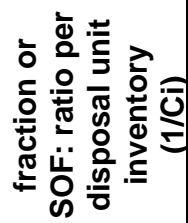 & 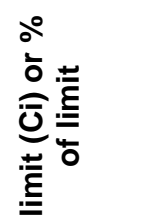 \\
\hline U-238 & & & & & & & & & & $9.15 \mathrm{E}-54$ & 10.1 & 9.06E-55 & $1.81 \mathrm{E}-54$ & 0 \\
\hline SOF-chain & & & & & & & & & & & & & $1.46 \mathrm{E}-45$ & $6.83 E+44$ \\
\hline Pu-242 & 1 & 0.5 & 4671 & 12 & 15 & 13 & 1350 & 10000 & 10000 & 9.96E-06 & 8.301 & $1.20 \mathrm{E}-06$ & $2.40 \mathrm{E}-06$ & 99.963 \\
\hline Pu-242_56 & & & & & & & & & & $3.64 \mathrm{E}-09$ & 8.299 & $4.38 \mathrm{E}-10$ & $8.76 \mathrm{E}-10$ & 0.037 \\
\hline U-238 & & & & & & & & & & $7.67 \mathrm{E}-13$ & 10.1 & $7.60 \mathrm{E}-14$ & $1.52 \mathrm{E}-13$ & 0 \\
\hline SOF-chain & & & & & & & & & & & & & $2.40 \mathrm{E}-06$ & $4.17 E+05$ \\
\hline Pu-244 & 0.001 & 0.0005 & 4673 & 14 & 15 & 13 & 0 & 100 & 100 & 1.62E-90 & 8.597 & $1.89 E-91$ & $3.78 \mathrm{E}-88$ & 99.878 \\
\hline Pu-244_56 & & & & & & & & & & 1.98E-93 & 8.598 & $2.30 \mathrm{E}-94$ & $4.60 \mathrm{E}-91$ & 0.122 \\
\hline SOF-chain & & & & & & & & & & & & & $3.78 \mathrm{E}-88$ & $2.64 \mathrm{E}+87$ \\
\hline $\mathrm{Pu}-244$ & 0.001 & 0.0005 & 4673 & 14 & 15 & 13 & 100 & 1350 & 1350 & $2.81 \mathrm{E}-47$ & 8.597 & $3.27 \mathrm{E}-48$ & $6.53 \mathrm{E}-45$ & 99.953 \\
\hline Pu-244_56 & & & & & & & & & & $1.33 \mathrm{E}-50$ & 8.598 & $1.55 \mathrm{E}-51$ & $3.10 \mathrm{E}-48$ & 0.047 \\
\hline SOF-chain & & & & & & & & & & & & & $6.53 E-45$ & $1.53 E+44$ \\
\hline Pu-244_56 & & & & & & & & & & $1.70 \mathrm{E}-11$ & 8.598 & $1.98 \mathrm{E}-12$ & 3.96E-09 & 0.036 \\
\hline SOF-chain & & & & & & & & & & & & & 1.08E-05 & $9.23 E+04$ \\
\hline $\mathrm{Pu}-244$ & 0.01 & 0.005 & 4673 & 14 & 15 & 13 & 0 & 100 & 100 & 1.65E-90 & 8.597 & $1.92 \mathrm{E}-91$ & 3.83E-89 & 99.878 \\
\hline Pu-244_56 & & & & & & & & & & $2.01 \mathrm{E}-93$ & 8.598 & $2.33 E-94$ & $4.66 \mathrm{E}-92$ & 0.122 \\
\hline SOF-chain & & & & & & & & & & & & & $3.84 \mathrm{E}-89$ & $2.61 \mathrm{E}+88$ \\
\hline $\mathrm{Pu}-244$ & 0.01 & 0.005 & 4673 & 14 & 15 & 13 & 100 & 1350 & 1350 & $2.86 \mathrm{E}-47$ & 8.597 & $3.33 E-48$ & $6.66 \mathrm{E}-46$ & 99.953 \\
\hline Pu-244_56 & & & & & & & & & & $1.36 \mathrm{E}-50$ & 8.598 & $1.58 \mathrm{E}-51$ & $3.16 \mathrm{E}-49$ & 0.047 \\
\hline SOF-chain & & & & & & & & & & & & & $6.66 \mathrm{E}-46$ & $1.50 \mathrm{E}+45$ \\
\hline Pu-244 & 0.01 & 0.005 & 4671 & 12 & 15 & 13 & 1350 & 10000 & 10000 & $4.78 \mathrm{E}-08$ & 8.597 & 5.56E-09 & $1.11 \mathrm{E}-06$ & 99.964 \\
\hline Pu-244_56 & & & & & & & & & & $1.74 \mathrm{E}-11$ & 8.598 & $2.03 E-12$ & $4.06 \mathrm{E}-10$ & 0.036 \\
\hline SOF-chain & & & & & & & & & & & & & 1.11E-06 & $9.00 \mathrm{E}+05$ \\
\hline Pu-244 & 0.1 & 0.05 & 4673 & 14 & 15 & 13 & 0 & 100 & 100 & $1.89 \mathrm{E}-90$ & 8.597 & $2.20 \mathrm{E}-91$ & $4.39 E-90$ & 99.878 \\
\hline Pu-244_56 & & & & & & & & & & $2.30 \mathrm{E}-93$ & 8.598 & 2.67E-94 & $5.35 E-93$ & 0.122 \\
\hline SOF-chain & & & & & & & & & & & & & $4.40 \mathrm{E}-90$ & $2.27 E+89$ \\
\hline $\mathrm{Pu}-244$ & 0.1 & 0.05 & 4673 & 14 & 15 & 13 & 100 & 1350 & 1350 & $3.41 \mathrm{E}-47$ & 8.597 & 3.97E-48 & $7.93 \mathrm{E}-47$ & 99.953 \\
\hline Pu-244_56 & & & & & & & & & & $1.62 \mathrm{E}-50$ & 8.598 & $1.88 \mathrm{E}-51$ & $3.76 \mathrm{E}-50$ & 0.047 \\
\hline SOF-chain & & & & & & & & & & & & & $7.94 \mathrm{E}-47$ & $1.26 \mathrm{E}+46$ \\
\hline Pu-244 & 0.1 & 0.05 & 4671 & 12 & 15 & 13 & 1350 & 10000 & 10000 & 5.95E-08 & 8.597 & 6.92E-09 & $1.38 \mathrm{E}-07$ & 99.964 \\
\hline
\end{tabular}




\begin{tabular}{|c|c|c|c|c|c|c|c|c|c|c|c|c|c|c|}
\hline 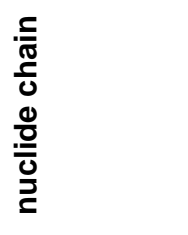 & 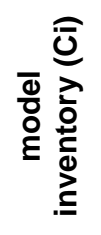 & 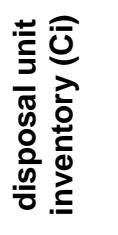 & 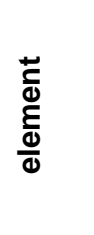 & $\begin{array}{l}\overline{0} \\
\frac{0}{0} \\
\stackrel{0}{\varrho}\end{array}$ & $\begin{array}{l}\overparen{0} \\
\stackrel{0}{0} \\
\stackrel{0}{\varrho}\end{array}$ & 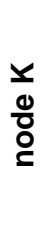 & 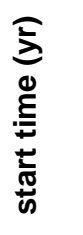 & 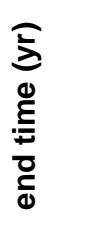 & 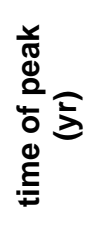 & 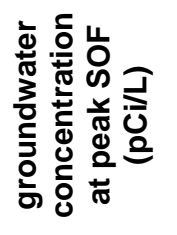 & 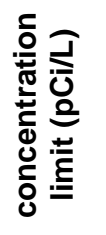 & 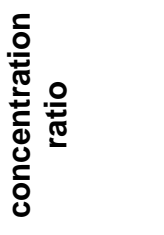 & 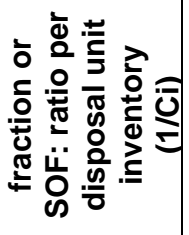 & 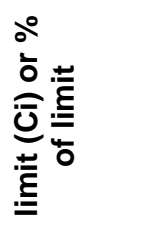 \\
\hline $\mathrm{Pu}-244$ & 1 & 0.5 & 4673 & 14 & 15 & 13 & 0 & 100 & 100 & $3.19 \mathrm{E}-90$ & 8.597 & $3.71 \mathrm{E}-91$ & $7.41 \mathrm{E}-91$ & 99.878 \\
\hline Pu-244_56 & & & & & & & & & & $3.88 \mathrm{E}-93$ & 8.598 & $4.51 \mathrm{E}-94$ & 9.02E-94 & 0.122 \\
\hline SOF-chain & & & & & & & & & & & & & 7.42E-91 & $1.35 \mathrm{E}+90$ \\
\hline Pu-244 & 1 & 0.5 & 4673 & 14 & 15 & 13 & 100 & 1350 & 1350 & $5.60 \mathrm{E}-47$ & 8.597 & $6.52 \mathrm{E}-48$ & $1.30 \mathrm{E}-47$ & 99.953 \\
\hline Pu-244_56 & & & & & & & & & & $2.66 \mathrm{E}-50$ & 8.598 & 3.09E-51 & $6.18 \mathrm{E}-51$ & 0.047 \\
\hline SOF-chain & & & & & & & & & & & & & $1.30 \mathrm{E}-47$ & $7.67 E+46$ \\
\hline Pu-244 & 1 & 0.5 & 4671 & 12 & 15 & 13 & 1350 & 10000 & 10000 & 9.30E-08 & 8.597 & 1.08E-08 & $2.16 \mathrm{E}-08$ & 99.964 \\
\hline Pu-244_56 & & & & & & & & & & $3.40 \mathrm{E}-11$ & 8.598 & $3.95 \mathrm{E}-12$ & $7.90 \mathrm{E}-12$ & 0.036 \\
\hline SOF-chain & & & & & & & & & & & & & 2.16E-08 & $4.62 E+07$ \\
\hline Am-243 & 0.001 & 0.0005 & 4673 & 14 & 15 & 13 & 0 & 100 & 100 & $4.16 \mathrm{E}-112$ & 7.6 & $5.47 \mathrm{E}-113$ & $1.10 \mathrm{E}-109$ & 0 \\
\hline Pu-239 & & & & & & & & & & $3.92 \mathrm{E}-92$ & 8.1 & $4.84 \mathrm{E}-93$ & $9.68 \mathrm{E}-90$ & 99.879 \\
\hline Pu-239_56 & & & & & & & & & & 4.76E-95 & 8.101 & 5.88E-96 & 1.18E-92 & 0.121 \\
\hline SOF-chain & & & & & & & & & & & & & $9.69 \mathrm{E}-90$ & $1.03 E+89$ \\
\hline Am-243 & 0.001 & 0.0005 & 4673 & 14 & 15 & 13 & 100 & 1350 & 1350 & 1.60E-68 & 7.6 & 2.10E-69 & 4.20E-66 & 0 \\
\hline Pu-239 & & & & & & & & & & $7.29 \mathrm{E}-47$ & 8.1 & $9.00 \mathrm{E}-48$ & $1.80 \mathrm{E}-44$ & 99.952 \\
\hline Pu-239_56 & & & & & & & & & & $3.47 \mathrm{E}-50$ & 8.101 & $4.29 \mathrm{E}-51$ & $8.57 \mathrm{E}-48$ & 0.048 \\
\hline U-234 & & & & & & & & & & 1.97E-51 & 130 & 1.52E-53 & 3.03E-50 & 0 \\
\hline SOF-chain & & & & & & & & & & & & & $1.80 \mathrm{E}-44$ & $5.55 \mathrm{E}+43$ \\
\hline Am-243 & 0.001 & 0.0005 & 4671 & 12 & 15 & 13 & 1350 & 10000 & 10000 & $3.39 \mathrm{E}-22$ & 7.6 & $4.46 \mathrm{E}-23$ & $8.91 \mathrm{E}-20$ & 0 \\
\hline Pu-239 & & & & & & & & & & 2.89E-07 & 8.1 & 3.57E-08 & 7.14E-05 & 99.955 \\
\hline Pu-239_56 & & & & & & & & & & $1.06 \mathrm{E}-10$ & 8.101 & $1.30 \mathrm{E}-11$ & $2.61 \mathrm{E}-08$ & 0.036 \\
\hline U-234 & & & & & & & & & & $3.97 \mathrm{E}-10$ & 130 & $3.06 \mathrm{E}-12$ & $6.11 \mathrm{E}-09$ & 0.009 \\
\hline SOF-chain & & & & & & & & & & & & & 7.14E-05 & $1.40 \mathrm{E}+04$ \\
\hline Am-243 & 0.01 & 0.005 & 4673 & 14 & 15 & 13 & 0 & 100 & 100 & $4.16 \mathrm{E}-111$ & 7.6 & 5.47E-112 & 1.10E-109 & 0 \\
\hline Pu-239 & & & & & & & & & & 3.92E-91 & 8.1 & 4.84E-92 & $9.68 \mathrm{E}-90$ & 99.879 \\
\hline Pu-239_56 & & & & & & & & & & 4.76E-94 & 8.101 & 5.88E-95 & 1.18E-92 & 0.121 \\
\hline U-234 & & & & & & & & & & $3.38 \mathrm{E}-96$ & 130 & $2.60 \mathrm{E}-98$ & $5.20 \mathrm{E}-96$ & 0 \\
\hline SOF-chain & & & & & & & & & & & & & $9.69 \mathrm{E}-90$ & $1.03 E+89$ \\
\hline Am-243 & 0.01 & 0.005 & 4673 & 14 & 15 & 13 & 100 & 1350 & 1350 & 1.60E-67 & 7.6 & $2.10 \mathrm{E}-68$ & $4.20 \mathrm{E}-66$ & 0 \\
\hline Pu-239 & & & & & & & & & & $7.29 \mathrm{E}-46$ & 8.1 & $9.00 \mathrm{E}-47$ & $1.80 \mathrm{E}-44$ & 99.952 \\
\hline
\end{tabular}




\begin{tabular}{|c|c|c|c|c|c|c|c|c|c|c|c|c|c|c|}
\hline $\begin{array}{l}\frac{.}{\pi} \\
\frac{\pi}{0} \\
\frac{0}{0} \\
\frac{0}{0} \\
\frac{0}{2}\end{array}$ & 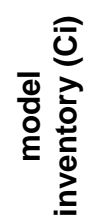 & 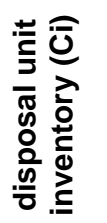 & 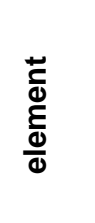 & $\begin{array}{l}\overline{0} \\
\frac{0}{0}\end{array}$ & $\begin{array}{l}7 \\
\stackrel{0}{0} \\
0\end{array}$ & $\begin{array}{l}\searrow \\
\frac{0}{0} \\
0\end{array}$ & 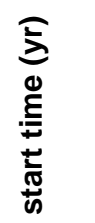 & 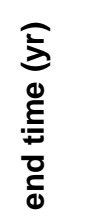 & 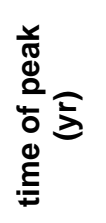 & 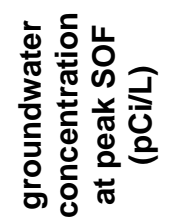 & 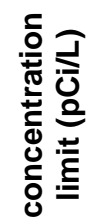 & 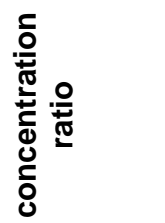 & 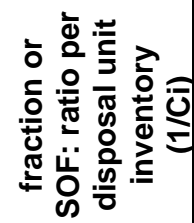 & 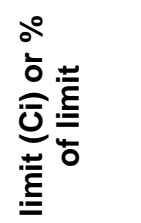 \\
\hline SOF-chain & & & & & & & & & & & & & 1.80E-44 & $5.55 \mathrm{E}+43$ \\
\hline Am-243 & 0.01 & 0.005 & 4671 & 12 & 15 & 13 & 1350 & 10000 & 10000 & $3.39 \mathrm{E}-21$ & 7.6 & $4.46 \mathrm{E}-22$ & $8.91 \mathrm{E}-20$ & 0 \\
\hline Pu-239 & & & & & & & & & & 2.89E-06 & 8.1 & 3.57E-07 & 7.14E-05 & 99.955 \\
\hline Pu-239 56 & & & & & & & & & & 1.06E-09 & 8.101 & $1.30 \mathrm{E}-10$ & $2.61 \mathrm{E}-08$ & 0.036 \\
\hline U-234 & & & & & & & & & & 3.97E-09 & 130 & $3.06 \mathrm{E}-11$ & $6.11 \mathrm{E}-09$ & 0.009 \\
\hline SOF-chain & & & & & & & & & & & & & 7.14E-05 & $1.40 \mathrm{E}+04$ \\
\hline Am-243 & 0.1 & 0.05 & 4673 & 14 & 15 & 13 & 0 & 100 & 100 & $4.16 \mathrm{E}-110$ & 7.6 & $5.47 \mathrm{E}-111$ & 1.10E-109 & 0 \\
\hline Pu-239 & & & & & & & & & & 3.92E-90 & 8.1 & 4.84E-91 & 9.68E-90 & 99.879 \\
\hline Pu-239_56 & & & & & & & & & & $4.76 \mathrm{E}-93$ & 8.101 & $5.88 \mathrm{E}-94$ & $1.18 \mathrm{E}-92$ & 0.121 \\
\hline U-234 & & & & & & & & & & $3.38 \mathrm{E}-95$ & 130 & $2.60 \mathrm{E}-97$ & $5.20 \mathrm{E}-96$ & 0 \\
\hline SOF-chain & & & & & & & & & & & & & $9.69 \mathrm{E}-90$ & $1.03 \mathrm{E}+89$ \\
\hline Am-243 & 0.1 & 0.05 & 4673 & 14 & 15 & 13 & 100 & 1350 & 1350 & 1.60E-66 & 7.6 & $2.10 \mathrm{E}-67$ & $4.20 \mathrm{E}-66$ & 0 \\
\hline Pu-239_56 & & & & & & & & & & $3.47 \mathrm{E}-48$ & 8.101 & $4.29 \mathrm{E}-49$ & $8.57 \mathrm{E}-48$ & 0.048 \\
\hline $\mathrm{U}-234$ & & & & & & & & & & 1.97E-49 & 130 & $1.52 \mathrm{E}-51$ & $3.03 E-50$ & 0 \\
\hline SOF-chain & & & & & & & & & & & & & 1.80E-44 & $5.55 \mathrm{E}+43$ \\
\hline Am-243 & 0.1 & 0.05 & 4671 & 12 & 15 & 13 & 1350 & 10000 & 10000 & $3.39 \mathrm{E}-20$ & 7.6 & $4.46 \mathrm{E}-21$ & $8.91 \mathrm{E}-20$ & 0 \\
\hline Pu-239 & & & & & & & & & & $2.89 \mathrm{E}-05$ & 8.1 & $3.57 \mathrm{E}-06$ & $7.14 \mathrm{E}-05$ & 99.955 \\
\hline Pu-239_56 & & & & & & & & & & $1.06 \mathrm{E}-08$ & 8.101 & $1.30 \mathrm{E}-09$ & $2.61 \mathrm{E}-08$ & 0.036 \\
\hline $\mathrm{U}-234$ & & & & & & & & & & $3.97 \mathrm{E}-08$ & 130 & $3.06 \mathrm{E}-10$ & $6.11 \mathrm{E}-09$ & 0.009 \\
\hline SOF-chain & & & & & & & & & & & & & 7.14E-05 & $1.40 \mathrm{E}+04$ \\
\hline Am-243 & 1 & 0.5 & 4673 & 14 & 15 & 13 & 0 & 100 & 100 & $4.16 \mathrm{E}-109$ & 7.6 & $5.47 \mathrm{E}-110$ & 1.10E-109 & 0 \\
\hline Pu-239 & & & & & & & & & & 3.92E-89 & 8.1 & $4.84 \mathrm{E}-90$ & $9.68 \mathrm{E}-90$ & 99.879 \\
\hline Pu-239_56 & & & & & & & & & & 4.76E-92 & 8.101 & $5.88 E-93$ & 1.18E-92 & 0.121 \\
\hline$U-234$ & & & & & & & & & & $3.38 \mathrm{E}-94$ & 130 & $2.60 \mathrm{E}-96$ & $5.20 \mathrm{E}-96$ & 0 \\
\hline SOF-chain & & & & & & & & & & & & & $9.69 \mathrm{E}-90$ & $1.03 \mathrm{E}+8 \mathrm{~g}$ \\
\hline $\mathrm{Am}-243$ & 1 & 0.5 & 4673 & 14 & 15 & 13 & 100 & 1350 & 1350 & $1.60 \mathrm{E}-65$ & 7.6 & $2.10 \mathrm{E}-66$ & $4.20 \mathrm{E}-66$ & 0 \\
\hline Pu-239 & & & & & & & & & & $7.20 \mathrm{E}-44$ & 8.1 & $8.89 \mathrm{E}-45$ & $1.78 \mathrm{E}-44$ & 99.952 \\
\hline Pu-239_56 & & & & & & & & & & $3.43 \mathrm{E}-47$ & 8.101 & $4.23 \mathrm{E}-48$ & $8.46 \mathrm{E}-48$ & 0.048 \\
\hline U-234 & & & & & & & & & & $1.95 \mathrm{E}-48$ & 130 & $1.50 \mathrm{E}-50$ & $3.00 \mathrm{E}-50$ & 0 \\
\hline SOF-chain & & & & & & & & & & & & & $1.78 \mathrm{E}-44$ & $5.63 \mathrm{E}+43$ \\
\hline
\end{tabular}




\begin{tabular}{|c|c|c|c|c|c|c|c|c|c|c|c|c|c|c|}
\hline 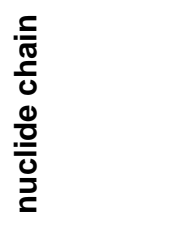 & 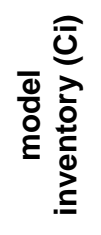 & 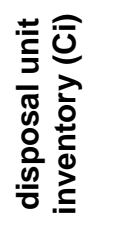 & 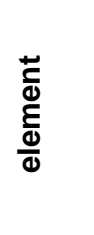 & $\begin{array}{l}\overline{0} \\
\frac{0}{0} \\
\stackrel{0}{\varrho}\end{array}$ & $\begin{array}{l}\mathfrak{0} \\
\frac{0}{0} \\
\stackrel{0}{c}\end{array}$ & $\begin{array}{l}\text { Y } \\
\frac{0}{0} \\
\stackrel{0}{\complement}\end{array}$ & $\begin{array}{l}\bar{\Sigma} \\
0 \\
\vdots \\
\vdots \\
\frac{ \pm}{\pi} \\
0 \\
0\end{array}$ & 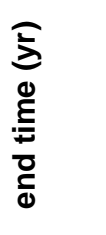 & 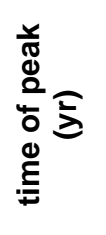 & 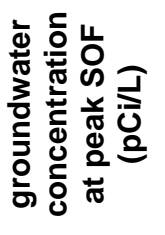 & 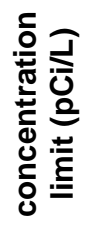 & 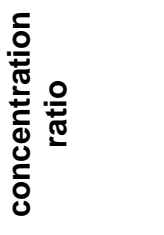 & 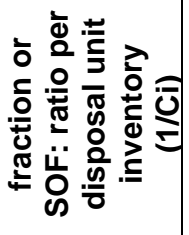 & 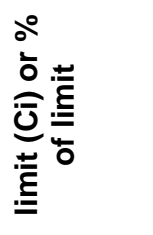 \\
\hline Pu-239_56 & & & & & & & & & & 4.66E-08 & 8.101 & 5.76E-09 & 1.15E-08 & 0.036 \\
\hline U-234 & & & & & & & & & & 1.81E-07 & 130 & 1.39E-09 & 2.79E-09 & 0.009 \\
\hline SOF-chain & & & & & & & & & & & & & 3.16E-05 & $3.17 \mathrm{E}+04$ \\
\hline Cm-245 & 0.001 & 0.0005 & 4673 & 14 & 15 & 13 & 0 & 100 & 100 & $4.05 \mathrm{E}-122$ & 7.6 & $5.33 \mathrm{E}-123$ & $1.07 \mathrm{E}-119$ & 0 \\
\hline Pu-241 & & & & & & & & & & $3.63 E-91$ & 300.8 & $1.21 \mathrm{E}-93$ & $2.41 \mathrm{E}-90$ & 0 \\
\hline Pu-241_56 & & & & & & & & & & 8.93E-92 & 54520 & 1.64E-96 & $3.28 \mathrm{E}-93$ & 0 \\
\hline Am-241 & & & & & & & & & & 5.87E-94 & 7.6 & 7.72E-95 & 1.55E-91 & 0 \\
\hline $\mathrm{Np}-237$ & & & & & & & & & & $2.37 \mathrm{E}-44$ & 8.9 & $2.66 \mathrm{E}-45$ & $5.33 \mathrm{E}-42$ & 100 \\
\hline SOF-chain & & & & & & & & & & & & & $5.33 \mathrm{E}-42$ & $1.88 \mathrm{E}+41$ \\
\hline $\mathrm{Cm}-245$ & 0.001 & 0.0005 & 4671 & 12 & 15 & 13 & 100 & 1350 & 1350 & 7.99E-79 & 7.6 & 1.05E-79 & $2.10 \mathrm{E}-76$ & 0 \\
\hline Pu-241 & & & & & & & & & & $9.11 \mathrm{E}-53$ & 300.8 & 3.03E-55 & $6.06 \mathrm{E}-52$ & 0 \\
\hline Pu-241_56 & & & & & & & & & & 8.65E-54 & 54520 & 1.59E-58 & 3.17E-55 & 0 \\
\hline Np-237 & & & & & & & & & & $9.20 \mathrm{E}-13$ & 8.9 & 1.03E-13 & $2.07 \mathrm{E}-10$ & 100 \\
\hline SOF-chain & & & & & & & & & & & & & $2.07 \mathrm{E}-10$ & $4.84 \mathrm{E}+09$ \\
\hline $\mathrm{Cm}-245$ & 0.001 & 0.0005 & 3871 & 12 & 15 & 11 & 1350 & 10000 & 10000 & $3.18 \mathrm{E}-34$ & 7.6 & $4.19 \mathrm{E}-35$ & $8.38 \mathrm{E}-32$ & 0 \\
\hline Pu-241 & & & & & & & & & & $1.18 \mathrm{E}-32$ & 300.8 & 3.93E-35 & $7.86 \mathrm{E}-32$ & 0 \\
\hline Pu-241_56 & & & & & & & & & & 8.64E-34 & 54520 & $1.59 \mathrm{E}-38$ & $3.17 \mathrm{E}-35$ & 0 \\
\hline Am-241 & & & & & & & & & & 5.92E-29 & 7.6 & 7.79E-30 & $1.56 \mathrm{E}-26$ & 0 \\
\hline $\mathrm{Np}-237$ & & & & & & & & & & 5.37E-07 & 8.9 & 6.04E-08 & $1.21 \mathrm{E}-04$ & 100 \\
\hline SOF-chain & & & & & & & & & & & & & $1.21 \mathrm{E}-04$ & $8.28 \mathrm{E}+03$ \\
\hline $\mathrm{Cm}-245$ & 0.01 & 0.005 & 4673 & 14 & 15 & 13 & 0 & 100 & 100 & $4.05 \mathrm{E}-121$ & 7.6 & $5.33 \mathrm{E}-122$ & $1.07 \mathrm{E}-119$ & 0 \\
\hline Pu-241 & & & & & & & & & & $3.63 E-90$ & 300.8 & $1.21 \mathrm{E}-92$ & $2.41 \mathrm{E}-90$ & 0 \\
\hline Pu-241_56 & & & & & & & & & & $8.93 E-91$ & 54520 & 1.64E-95 & $3.28 \mathrm{E}-93$ & 0 \\
\hline Am-241 & & & & & & & & & & 5.87E-93 & 7.6 & 7.72E-94 & 1.55E-91 & 0 \\
\hline Np-237 & & & & & & & & & & $2.37 \mathrm{E}-43$ & 8.9 & $2.66 \mathrm{E}-44$ & 5.33E-42 & 100 \\
\hline SOF-chain & & & & & & & & & & & & & $5.33 \mathrm{E}-42$ & $1.88 \mathrm{E}+41$ \\
\hline $\mathrm{Cm}-245$ & 0.01 & 0.005 & 4671 & 12 & 15 & 13 & 100 & 1350 & 1350 & 7.99E-78 & 7.6 & 1.05E-78 & $2.10 \mathrm{E}-76$ & 0 \\
\hline Pu-241 & & & & & & & & & & $9.12 \mathrm{E}-52$ & 300.8 & 3.03E-54 & $6.06 \mathrm{E}-52$ & 0 \\
\hline Pu-241_56 & & & & & & & & & & 8.65E-53 & 54520 & 1.59E-57 & 3.17E-55 & 0 \\
\hline $\mathrm{Am}-241$ & & & & & & & & & & $5.58 \mathrm{E}-54$ & 7.6 & 7.34E-55 & $1.47 \mathrm{E}-52$ & 0 \\
\hline
\end{tabular}




\begin{tabular}{|c|c|c|c|c|c|c|c|c|c|c|c|c|c|c|}
\hline $\begin{array}{l}. \frac{5}{\pi} \\
\frac{0}{0} \\
\frac{0}{0} \\
\frac{0}{0} \\
\frac{0}{2}\end{array}$ & 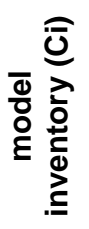 & 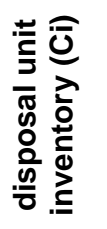 & 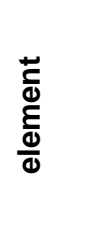 & $\begin{array}{l}\overline{0} \\
\frac{0}{0} \\
\stackrel{\varrho}{\varrho}\end{array}$ & $\begin{array}{l}\mathfrak{0} \\
\frac{0}{0} \\
\stackrel{0}{c}\end{array}$ & 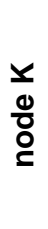 & 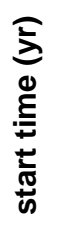 & 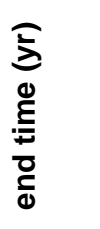 & 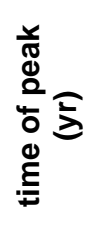 & 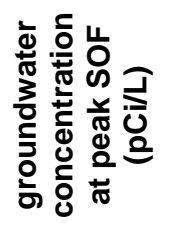 & 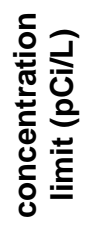 & 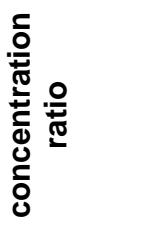 & 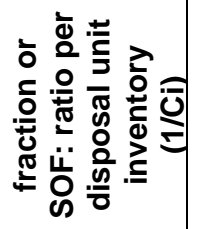 & 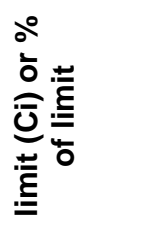 \\
\hline $\mathrm{Cm}-245$ & 0.01 & 0.005 & 3871 & 12 & 15 & 11 & 1350 & 10000 & 10000 & $3.18 \mathrm{E}-33$ & 7.6 & $4.19 \mathrm{E}-34$ & $8.38 \mathrm{E}-32$ & 0 \\
\hline Pu-241 & & & & & & & & & & $1.18 \mathrm{E}-31$ & 300.8 & 3.93E-34 & $7.86 \mathrm{E}-32$ & 0 \\
\hline Pu-241_56 & & & & & & & & & & $8.64 \mathrm{E}-33$ & 54520 & $1.59 \mathrm{E}-37$ & $3.17 \mathrm{E}-35$ & 0 \\
\hline Am-241 & & & & & & & & & & $5.92 \mathrm{E}-28$ & 7.6 & $7.79 \mathrm{E}-29$ & $1.56 \mathrm{E}-26$ & 0 \\
\hline $\mathrm{Np}-237$ & & & & & & & & & & 5.37E-06 & 8.9 & 6.04E-07 & $1.21 \mathrm{E}-04$ & 100 \\
\hline SOF-chain & & & & & & & & & & & & & 1.21E-04 & $8.28 \mathrm{E}+03$ \\
\hline $\mathrm{Cm}-245$ & 0.1 & 0.05 & 4673 & 14 & 15 & 13 & 0 & 100 & 100 & $4.05 E-120$ & 7.6 & $5.33 \mathrm{E}-121$ & 1.07E-119 & 0 \\
\hline $\mathrm{Pu}-241$ & & & & & & & & & & 3.63E-89 & 300.8 & $1.21 \mathrm{E}-91$ & $2.41 \mathrm{E}-90$ & 0 \\
\hline Pu-241_56 & & & & & & & & & & 8.93E-90 & 54520 & 1.64E-94 & $3.28 \mathrm{E}-93$ & 0 \\
\hline $\mathrm{Am}-241$ & & & & & & & & & & $5.87 \mathrm{E}-92$ & 7.6 & 7.73E-93 & $1.55 \mathrm{E}-91$ & 0 \\
\hline Np-237 & & & & & & & & & & $2.37 \mathrm{E}-42$ & 8.9 & $2.66 \mathrm{E}-43$ & $5.33 \mathrm{E}-42$ & 100 \\
\hline SOF-chain & & & & & & & & & & & & & $5.33 E-42$ & $1.88 \mathrm{E}+41$ \\
\hline Pu-241 & & & & & & & & & & $9.12 \mathrm{E}-51$ & 300.8 & 3.03E-53 & $6.06 \mathrm{E}-52$ & 0 \\
\hline Pu-241_56 & & & & & & & & & & $8.65 E-52$ & 54520 & $1.59 \mathrm{E}-56$ & $3.17 \mathrm{E}-55$ & 0 \\
\hline Am-241 & & & & & & & & & & $5.57 E-53$ & 7.6 & $7.33 \mathrm{E}-54$ & 1.47E-52 & 0 \\
\hline Np-237 & & & & & & & & & & $9.20 \mathrm{E}-11$ & 8.9 & $1.03 \mathrm{E}-11$ & $2.07 \mathrm{E}-10$ & 100 \\
\hline SOF-chain & & & & & & & & & & & & & $2.07 \mathrm{E}-10$ & $4.84 \mathrm{E}+09$ \\
\hline $\mathrm{Cm}-245$ & 0.1 & 0.05 & 3871 & 12 & 15 & 11 & 1350 & 10000 & 10000 & $3.18 \mathrm{E}-32$ & 7.6 & $4.19 \mathrm{E}-33$ & $8.38 \mathrm{E}-32$ & 0 \\
\hline Pu-241 & & & & & & & & & & $1.18 \mathrm{E}-30$ & 300.8 & 3.93E-33 & $7.86 \mathrm{E}-32$ & 0 \\
\hline Pu-241_56 & & & & & & & & & & 8.64E-32 & 54520 & 1.59E-36 & $3.17 E-35$ & 0 \\
\hline Am-241 & & & & & & & & & & $5.92 \mathrm{E}-27$ & 7.6 & 7.79E-28 & $1.56 \mathrm{E}-26$ & 0 \\
\hline $\mathrm{Np}-237$ & & & & & & & & & & 5.37E-05 & 8.9 & $6.04 \mathrm{E}-06$ & $1.21 \mathrm{E}-04$ & 100 \\
\hline SOF-chain & & & & & & & & & & & & & 1.21E-04 & $8.28 \mathrm{E}+03$ \\
\hline $\mathrm{Cm}-245$ & 1 & 0.5 & 4673 & 14 & 15 & 13 & 0 & 100 & 100 & $4.05 \mathrm{E}-119$ & 7.6 & $5.33 \mathrm{E}-120$ & 1.07E-119 & 0 \\
\hline $\mathrm{Pu}-241$ & & & & & & & & & & $1.85 \mathrm{E}-88$ & 300.8 & $6.15 \mathrm{E}-91$ & $1.23 \mathrm{E}-90$ & 0 \\
\hline Pu-241_56 & & & & & & & & & & 4.86E-89 & 54520 & 8.92E-94 & 1.78E-93 & 0 \\
\hline Am-241 & & & & & & & & & & $2.90 \mathrm{E}-91$ & 7.6 & $3.81 \mathrm{E}-92$ & $7.63 \mathrm{E}-92$ & 0 \\
\hline Np-237 & & & & & & & & & & 1.29E-41 & 8.9 & $1.44 \mathrm{E}-42$ & $2.89 \mathrm{E}-42$ & 100 \\
\hline SOF-chain & & & & & & & & & & & & & 2.89E-42 & $3.46 \mathrm{E}+41$ \\
\hline $\mathrm{Cm}-245$ & 1 & 0.5 & 4671 & 12 & 15 & 13 & 100 & 1350 & 1350 & 7.99E-76 & 7.6 & $1.05 \mathrm{E}-76$ & $2.10 \mathrm{E}-76$ & 0 \\
\hline
\end{tabular}




\begin{tabular}{|c|c|c|c|c|c|c|c|c|c|c|c|c|c|c|}
\hline $\begin{array}{l}\frac{5}{\pi} \\
\frac{c}{0} \\
\frac{0}{0} \\
\frac{0}{0} \\
\frac{0}{2}\end{array}$ & 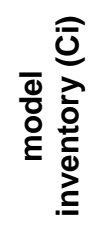 & 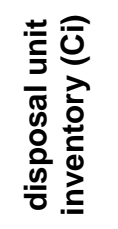 & 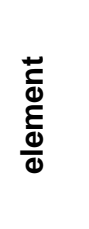 & $\begin{array}{l}\overline{0} \\
\frac{0}{0}\end{array}$ & 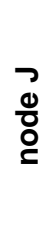 & $\begin{array}{l}\searrow \\
\stackrel{0}{0} \\
\stackrel{0}{\varrho}\end{array}$ & 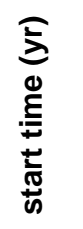 & 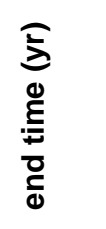 & 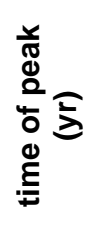 & 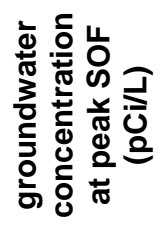 & 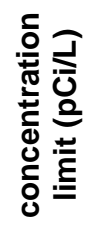 & 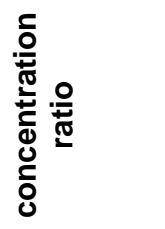 & 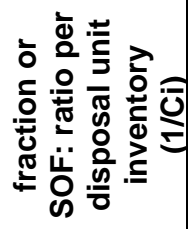 & 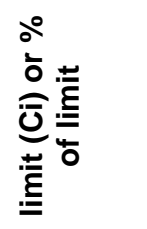 \\
\hline Am-241 & & & & & & & & & & $5.57 \mathrm{E}-52$ & 7.6 & $7.33 E-53$ & 1.47E-52 & 0 \\
\hline $\mathrm{Np}-237$ & & & & & & & & & & $9.20 \mathrm{E}-10$ & 8.9 & $1.03 \mathrm{E}-10$ & $2.07 \mathrm{E}-10$ & 100 \\
\hline SOF-chain & & & & & & & & & & & & & $2.07 \mathrm{E}-10$ & $4.84 \mathrm{E}+09$ \\
\hline $\mathrm{Cm}-245$ & 1 & 0.5 & 3871 & 12 & 15 & 11 & 1350 & 10000 & 10000 & $3.18 \mathrm{E}-31$ & 7.6 & $4.19 E-32$ & $8.38 \mathrm{E}-32$ & 0 \\
\hline Pu-241 & & & & & & & & & & 1.18E-29 & 300.8 & 3.93E-32 & $7.86 \mathrm{E}-32$ & 0 \\
\hline Pu-241_56 & & & & & & & & & & $8.64 \mathrm{E}-31$ & 54520 & $1.59 \mathrm{E}-35$ & $3.17 \mathrm{E}-35$ & 0 \\
\hline Am-241 & & & & & & & & & & 5.92E-26 & 7.6 & 7.79E-27 & 1.56E-26 & 0 \\
\hline $\mathrm{Np}-237$ & & & & & & & & & & 5.37E-04 & 8.9 & 6.04E-05 & $1.21 \mathrm{E}-04$ & 100 \\
\hline SOF-chain & & & & & & & & & & & & & $1.21 \mathrm{E}-04$ & $8.28 \mathrm{E}+03$ \\
\hline $\mathrm{Cm}-246$ & 1 & 0.5 & 4673 & 14 & 15 & 13 & 0 & 100 & 100 & 4.02E-119 & 7.6 & $5.29 \mathrm{E}-120$ & 1.06E-119 & 0 \\
\hline SOF-chain & & & & & & & & & & & & & $1.06 \mathrm{E}-119$ & $1.00 \mathrm{E}+99$ \\
\hline $\mathrm{Cm}-246$ & 1 & 0.5 & 4673 & 14 & 15 & 13 & 100 & 1350 & 1350 & $1.58 \mathrm{E}-75$ & 7.6 & $2.08 \mathrm{E}-76$ & 4.16E-76 & 100 \\
\hline $\mathrm{Cm}-246$ & 1 & 0.5 & 4673 & 14 & 15 & 13 & 1350 & 10000 & 10000 & $4.57 \mathrm{E}-28$ & 7.6 & $6.01 \mathrm{E}-29$ & $1.20 \mathrm{E}-28$ & 100 \\
\hline SOF-chain & & & & & & & & & & & & & $1.20 \mathrm{E}-28$ & $8.32 E+27$ \\
\hline Cm-247 & 0.001 & 0.0005 & 4673 & 14 & 15 & 13 & 0 & 100 & 100 & $4.09 \mathrm{E}-122$ & 8.3 & $4.93 \mathrm{E}-123$ & $9.85 \mathrm{E}-120$ & 0 \\
\hline Am-243 & & & & & & & & & & $7.04 \mathrm{E}-115$ & 7.6 & $9.27 \mathrm{E}-116$ & $1.85 \mathrm{E}-112$ & 0 \\
\hline $\mathrm{Np}-239$ & & & & & & & & & & $8.12 \mathrm{E}-50$ & 300 & $2.71 \mathrm{E}-52$ & $5.42 \mathrm{E}-49$ & 99.987 \\
\hline Pu-239 & & & & & & & & & & $2.84 \mathrm{E}-55$ & 8.1 & $3.51 \mathrm{E}-56$ & $7.01 \mathrm{E}-53$ & 0.013 \\
\hline Pu-239_56 & & & & & & & & & & $1.04 \mathrm{E}-58$ & 8.101 & $1.28 \mathrm{E}-59$ & $2.56 \mathrm{E}-56$ & 0 \\
\hline SOF-chain & & & & & & & & & & & & & 5.42E-49 & $1.85 \mathrm{E}+48$ \\
\hline Cm-247 & 0.001 & 0.0005 & 4673 & 14 & 15 & 13 & 100 & 1350 & 1350 & 1.93E-78 & 8.3 & $2.32 \mathrm{E}-79$ & $4.65 \mathrm{E}-76$ & 0 \\
\hline $\mathrm{Am}-243$ & & & & & & & & & & $1.49 \mathrm{E}-69$ & 7.6 & $1.96 \mathrm{E}-70$ & $3.92 \mathrm{E}-67$ & 0 \\
\hline Np-239 & & & & & & & & & & $2.20 \mathrm{E}-20$ & 300 & 7.34E-23 & $1.47 \mathrm{E}-19$ & 99.907 \\
\hline Pu-239 & & & & & & & & & & $5.51 \mathrm{E}-25$ & 8.1 & $6.81 \mathrm{E}-26$ & $1.36 \mathrm{E}-22$ & 0.093 \\
\hline Pu-239_56 & & & & & & & & & & $2.03 E-28$ & 8.101 & $2.50 \mathrm{E}-29$ & $5.00 \mathrm{E}-26$ & 0 \\
\hline SOF-chain & & & & & & & & & & & & & $1.47 \mathrm{E}-19$ & $6.81 \mathrm{E}+18$ \\
\hline Cm-247 & 0.001 & 0.0005 & 4671 & 12 & 15 & 13 & 1350 & 10000 & 10000 & $1.01 \mathrm{E}-30$ & 8.3 & $1.22 \mathrm{E}-31$ & $2.44 \mathrm{E}-28$ & 0 \\
\hline Am-243 & & & & & & & & & & $1.01 \mathrm{E}-22$ & 7.6 & $1.32 \mathrm{E}-23$ & $2.65 \mathrm{E}-20$ & 0 \\
\hline $\mathrm{Np}-239$ & & & & & & & & & & $5.59 \mathrm{E}-13$ & 300 & $1.86 \mathrm{E}-15$ & $3.73 \mathrm{E}-12$ & 0 \\
\hline Pu-239 & & & & & & & & & & $2.70 \mathrm{E}-08$ & 8.1 & 3.33E-09 & 6.66E-06 & 99.963 \\
\hline
\end{tabular}




\begin{tabular}{|c|c|c|c|c|c|c|c|c|c|c|c|c|c|c|}
\hline 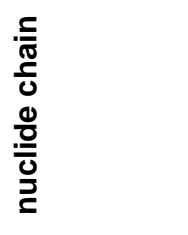 & 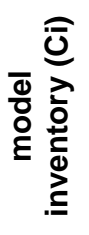 & 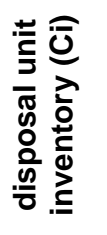 & 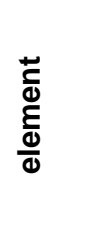 & $\begin{array}{l}\overline{0} \\
\frac{0}{0} \\
\stackrel{\varrho}{\varrho}\end{array}$ & $\begin{array}{l}\mathfrak{0} \\
\frac{0}{0} \\
\stackrel{0}{c}\end{array}$ & 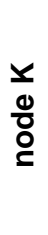 & 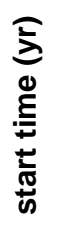 & 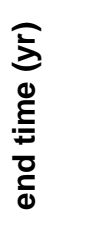 & 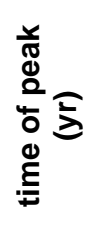 & 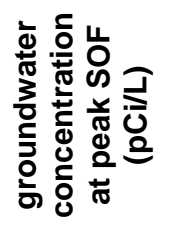 & 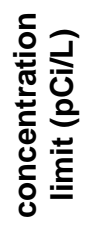 & 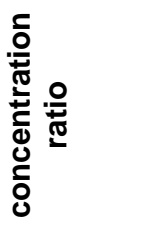 & 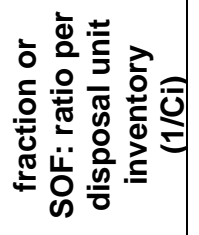 & 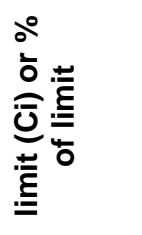 \\
\hline $\mathrm{Cm}-247$ & 0.01 & 0.005 & 4673 & 14 & 15 & 13 & 0 & 100 & 100 & 4.09E-121 & 8.3 & $4.93 \mathrm{E}-122$ & $9.85 \mathrm{E}-120$ & 0 \\
\hline Am-243 & & & & & & & & & & $7.04 \mathrm{E}-114$ & 7.6 & $9.27 \mathrm{E}-115$ & $1.85 \mathrm{E}-112$ & 0 \\
\hline Np-239 & & & & & & & & & & $8.12 \mathrm{E}-49$ & 300 & 2.71E-51 & 5.42E-49 & 99.987 \\
\hline Pu-239 & & & & & & & & & & $2.84 \mathrm{E}-54$ & 8.1 & $3.51 \mathrm{E}-55$ & $7.01 \mathrm{E}-53$ & 0.013 \\
\hline Pu-239_56 & & & & & & & & & & 1.04E-57 & 8.101 & $1.28 \mathrm{E}-58$ & $2.56 \mathrm{E}-56$ & 0 \\
\hline SOF-chain & & & & & & & & & & & & & $5.42 E-49$ & $1.85 \mathrm{E}+48$ \\
\hline $\mathrm{Cm}-247$ & 0.01 & 0.005 & 4673 & 14 & 15 & 13 & 100 & 1350 & 1350 & 1.93E-77 & 8.3 & $2.32 \mathrm{E}-78$ & $4.65 E-76$ & 0 \\
\hline Am-243 & & & & & & & & & & 1.49E-68 & 7.6 & 1.96E-69 & $3.92 \mathrm{E}-67$ & 0 \\
\hline Np-239 & & & & & & & & & & 2.20E-19 & 300 & 7.34E-22 & 1.47E-19 & 99.907 \\
\hline Pu-239 & & & & & & & & & & $5.51 \mathrm{E}-24$ & 8.1 & $6.81 \mathrm{E}-25$ & 1.36E-22 & 0.093 \\
\hline Pu-239_56 & & & & & & & & & & $2.03 \mathrm{E}-27$ & 8.101 & $2.50 \mathrm{E}-28$ & $5.00 \mathrm{E}-26$ & 0 \\
\hline SOF-chain & & & & & & & & & & & & & $1.47 \mathrm{E}-19$ & $6.81 \mathrm{E}+18$ \\
\hline Am-243 & & & & & & & & & & $1.01 \mathrm{E}-21$ & 7.6 & $1.32 \mathrm{E}-22$ & $2.65 \mathrm{E}-20$ & 0 \\
\hline Np-239 & & & & & & & & & & 5.59E-12 & 300 & 1.86E-14 & 3.73E-12 & 0 \\
\hline Pu-239 & & & & & & & & & & $2.70 \mathrm{E}-07$ & 8.1 & 3.33E-08 & 6.66E-06 & 99.963 \\
\hline Pu-239_56 & & & & & & & & & & $9.85 \mathrm{E}-11$ & 8.101 & $1.22 \mathrm{E}-11$ & $2.43 E-09$ & 0.037 \\
\hline SOF-chain & & & & & & & & & & & & & 6.66E-06 & $1.50 \mathrm{E}+05$ \\
\hline $\mathrm{Cm}-247$ & 0.1 & 0.05 & 4673 & 14 & 15 & 13 & 0 & 100 & 100 & $4.09 \mathrm{E}-120$ & 8.3 & $4.93 \mathrm{E}-121$ & $9.85 \mathrm{E}-120$ & 0 \\
\hline Am-243 & & & & & & & & & & $7.04 \mathrm{E}-113$ & 7.6 & $9.27 \mathrm{E}-114$ & $1.85 \mathrm{E}-112$ & 0 \\
\hline Np-239 & & & & & & & & & & $8.12 E-48$ & 300 & $2.71 \mathrm{E}-50$ & 5.42E-49 & 99.987 \\
\hline Pu-239 & & & & & & & & & & $2.84 \mathrm{E}-53$ & 8.1 & $3.51 \mathrm{E}-54$ & $7.01 \mathrm{E}-53$ & 0.013 \\
\hline Pu-239_56 & & & & & & & & & & $1.04 \mathrm{E}-56$ & 8.101 & $1.28 \mathrm{E}-57$ & $2.56 \mathrm{E}-56$ & 0 \\
\hline SOF-chain & & & & & & & & & & & & & $5.42 E-49$ & $1.85 \mathrm{E}+48$ \\
\hline $\mathrm{Cm}-247$ & 0.1 & 0.05 & 4673 & 14 & 15 & 13 & 100 & 1350 & 1350 & 1.93E-76 & 8.3 & $2.32 \mathrm{E}-77$ & 4.65E-76 & 0 \\
\hline Am-243 & & & & & & & & & & 1.49E-67 & 7.6 & $1.96 \mathrm{E}-68$ & $3.92 \mathrm{E}-67$ & 0 \\
\hline Np-239 & & & & & & & & & & $2.20 \mathrm{E}-18$ & 300 & 7.34E-21 & 1.47E-19 & 99.907 \\
\hline Pu-239 & & & & & & & & & & $5.51 \mathrm{E}-23$ & 8.1 & $6.81 \mathrm{E}-24$ & $1.36 \mathrm{E}-22$ & 0.093 \\
\hline Pu-239_56 & & & & & & & & & & $2.03 \mathrm{E}-26$ & 8.101 & $2.50 \mathrm{E}-27$ & $5.00 \mathrm{E}-26$ & 0 \\
\hline SOF-chain & & & & & & & & & & & & & $1.47 \mathrm{E}-19$ & $6.81 \mathrm{E}+18$ \\
\hline $\mathrm{Cm}-247$ & 0.1 & 0.05 & 4671 & 12 & 15 & 13 & 1350 & 10000 & 10000 & $1.01 \mathrm{E}-28$ & 8.3 & $1.22 \mathrm{E}-29$ & $2.44 \mathrm{E}-28$ & 0 \\
\hline
\end{tabular}




\begin{tabular}{|c|c|c|c|c|c|c|c|c|c|c|c|c|c|c|}
\hline $\begin{array}{l}\frac{5}{0} \\
\frac{0}{0} \\
\frac{0}{0} \\
\frac{0}{0} \\
\frac{0}{2}\end{array}$ & 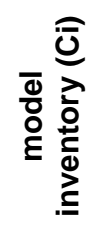 & 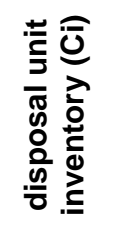 & 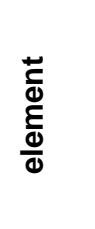 & $\begin{array}{l}\overline{0} \\
\frac{0}{0}\end{array}$ & 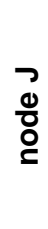 & $\begin{array}{l}\searrow \\
\frac{0}{0} \\
0\end{array}$ & 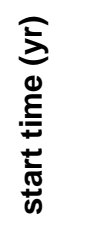 & 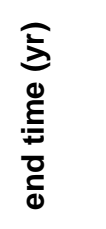 & 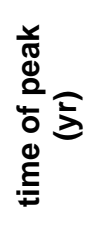 & 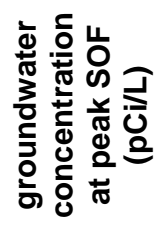 & 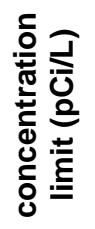 & 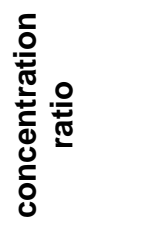 & 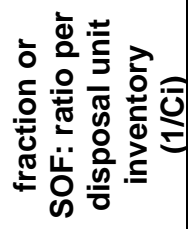 & 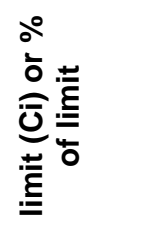 \\
\hline Pu-239 & & & & & & & & & & $2.70 \mathrm{E}-06$ & 8.1 & 3.33E-07 & $6.66 \mathrm{E}-06$ & 99.963 \\
\hline Pu-239_56 & & & & & & & & & & $9.85 \mathrm{E}-10$ & 8.101 & $1.22 \mathrm{E}-10$ & $2.43 \mathrm{E}-09$ & 0.037 \\
\hline SOF-chain & & & & & & & & & & & & & 6.66E-06 & $1.50 \mathrm{E}+05$ \\
\hline Cm-247 & 1 & 0.5 & 4673 & 14 & 15 & 13 & 0 & 100 & 100 & $4.09 \mathrm{E}-119$ & 8.3 & $4.93 \mathrm{E}-120$ & $9.85 \mathrm{E}-120$ & 0 \\
\hline Am-243 & & & & & & & & & & 7.04E-112 & 7.6 & $9.27 \mathrm{E}-113$ & $1.85 \mathrm{E}-112$ & 0 \\
\hline Np-239 & & & & & & & & & & $8.12 \mathrm{E}-47$ & 300 & $2.71 \mathrm{E}-49$ & $5.42 \mathrm{E}-49$ & 99.987 \\
\hline Pu-239 & & & & & & & & & & 2.84E-52 & 8.1 & $3.51 \mathrm{E}-53$ & 7.01E-53 & 0.013 \\
\hline Pu-239_56 & & & & & & & & & & 1.04E-55 & 8.101 & $1.28 \mathrm{E}-56$ & $2.56 \mathrm{E}-56$ & 0 \\
\hline SOF-chain & & & & & & & & & & & & & $5.42 \mathrm{E}-49$ & $1.85 E+48$ \\
\hline $\mathrm{Cm}-247$ & 1 & 0.5 & 4673 & 14 & 15 & 13 & 100 & 1350 & 1350 & 1.93E-75 & 8.3 & $2.32 \mathrm{E}-76$ & 4.65E-76 & 0 \\
\hline Am-243 & & & & & & & & & & $1.49 \mathrm{E}-66$ & 7.6 & $1.96 \mathrm{E}-67$ & $3.92 \mathrm{E}-67$ & 0 \\
\hline $\mathrm{Np}-239$ & & & & & & & & & & $2.20 \mathrm{E}-17$ & 300 & $7.34 \mathrm{E}-20$ & $1.47 \mathrm{E}-19$ & 99.907 \\
\hline Pu-239_56 & & & & & & & & & & $2.03 E-25$ & 8.101 & $2.50 \mathrm{E}-26$ & $5.00 \mathrm{E}-26$ & 0 \\
\hline SOF-chain & & & & & & & & & & & & & $1.47 \mathrm{E}-19$ & $6.81 \mathrm{E}+18$ \\
\hline Cm-247 & 1 & 0.5 & 4671 & 12 & 15 & 13 & 1350 & 10000 & 10000 & $1.01 \mathrm{E}-27$ & 8.3 & $1.22 \mathrm{E}-28$ & $2.44 \mathrm{E}-28$ & 0 \\
\hline Am-243 & & & & & & & & & & $1.01 \mathrm{E}-19$ & 7.6 & $1.32 \mathrm{E}-20$ & $2.65 \mathrm{E}-20$ & 0 \\
\hline $\mathrm{Np}-239$ & & & & & & & & & & $5.59 \mathrm{E}-10$ & 300 & $1.86 \mathrm{E}-12$ & $3.73 \mathrm{E}-12$ & 0 \\
\hline Pu-239 & & & & & & & & & & $2.70 \mathrm{E}-05$ & 8.1 & $3.33 \mathrm{E}-06$ & $6.66 \mathrm{E}-06$ & 99.963 \\
\hline Pu-239_56 & & & & & & & & & & $9.85 \mathrm{E}-09$ & 8.101 & $1.22 \mathrm{E}-09$ & $2.43 \mathrm{E}-09$ & 0.037 \\
\hline SOF-chain & & & & & & & & & & & & & $6.66 \mathrm{E}-06$ & $1.50 \mathrm{E}+05$ \\
\hline Cm-248 & 0.001 & 0.0005 & 4673 & 14 & 15 & 13 & 0 & 100 & 100 & $4.09 \mathrm{E}-122$ & 2.1 & $1.95 \mathrm{E}-122$ & $3.89 \mathrm{E}-119$ & 0 \\
\hline Pu-244 & & & & & & & & & & $1.18 \mathrm{E}-95$ & 8.597 & $1.38 \mathrm{E}-96$ & $2.75 \mathrm{E}-93$ & 99.879 \\
\hline Pu-244_56 & & & & & & & & & & 1.44E-98 & 8.598 & 1.67E-99 & $3.34 \mathrm{E}-96$ & 0.121 \\
\hline SOF-chain & & & & & & & & & & & & & $2.76 \mathrm{E}-93$ & $3.63 E+92$ \\
\hline $\mathrm{Cm}-248$ & 0.001 & 0.0005 & 4673 & 14 & 15 & 13 & 100 & 1350 & 1350 & $1.92 \mathrm{E}-78$ & 2.1 & $9.16 \mathrm{E}-79$ & $1.83 \mathrm{E}-75$ & 0 \\
\hline $\mathrm{Pu}-244$ & & & & & & & & & & $2.33 \mathrm{E}-50$ & 8.597 & $2.71 \mathrm{E}-51$ & $5.43 \mathrm{E}-48$ & 99.952 \\
\hline Pu-244_56 & & & & & & & & & & $1.11 \mathrm{E}-53$ & 8.598 & $1.29 \mathrm{E}-54$ & $2.59 \mathrm{E}-51$ & 0.048 \\
\hline SOF-chain & & & & & & & & & & & & & $5.43 \mathrm{E}-48$ & $1.84 \mathrm{E}+47$ \\
\hline Cm-248 & 0.001 & 0.0005 & 4671 & 12 & 15 & 13 & 1350 & 10000 & 10000 & $9.92 \mathrm{E}-31$ & 2.1 & $4.72 \mathrm{E}-31$ & $9.45 \mathrm{E}-28$ & 0 \\
\hline Pu-244 & & & & & & & & & & $1.23 \mathrm{E}-10$ & 8.597 & $1.43 \mathrm{E}-11$ & $2.86 \mathrm{E}-08$ & 99.963 \\
\hline
\end{tabular}




\begin{tabular}{|c|c|c|c|c|c|c|c|c|c|c|c|c|c|c|}
\hline 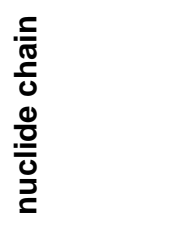 & 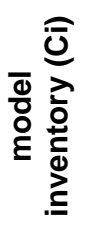 & 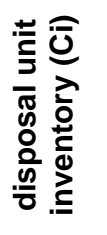 & 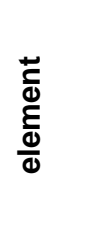 & $\begin{array}{l}\overline{0} \\
\frac{0}{0} \\
\stackrel{0}{\varrho}\end{array}$ & $\begin{array}{l}\mathfrak{0} \\
\frac{0}{0} \\
\stackrel{0}{c}\end{array}$ & 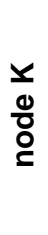 & 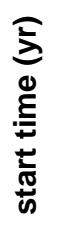 & 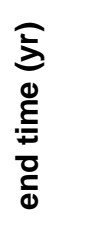 & 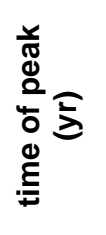 & 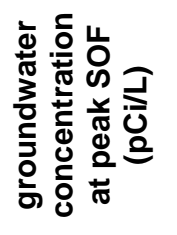 & 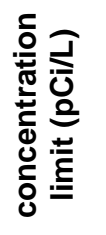 & 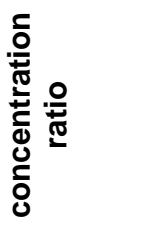 & 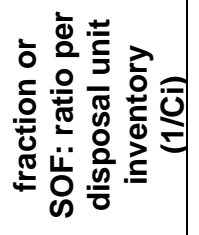 & 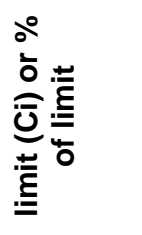 \\
\hline $\mathrm{Cm}-248$ & 0.01 & 0.005 & 4673 & 14 & 15 & 13 & 0 & 100 & 100 & 4.09E-121 & 2.1 & $1.95 \mathrm{E}-121$ & 3.89E-119 & 0 \\
\hline Pu-244 & & & & & & & & & & 1.18E-94 & 8.597 & $1.38 \mathrm{E}-95$ & $2.75 E-93$ & 99.879 \\
\hline Pu-244_56 & & & & & & & & & & 1.44E-97 & 8.598 & 1.67E-98 & 3.34E-96 & 0.121 \\
\hline SOF-chain & & & & & & & & & & & & & $2.76 \mathrm{E}-93$ & $3.63 E+92$ \\
\hline $\mathrm{Cm}-248$ & 0.01 & 0.005 & 4673 & 14 & 15 & 13 & 100 & 1350 & 1350 & 1.92E-77 & 2.1 & $9.16 \mathrm{E}-78$ & 1.83E-75 & 0 \\
\hline $\mathrm{Pu}-244$ & & & & & & & & & & $2.33 E-49$ & 8.597 & $2.71 \mathrm{E}-50$ & $5.43 E-48$ & 99.952 \\
\hline Pu-244_56 & & & & & & & & & & 1.11E-52 & 8.598 & 1.29E-53 & $2.59 \mathrm{E}-51$ & 0.048 \\
\hline SOF-chain & & & & & & & & & & & & & $5.43 E-48$ & $1.84 \mathrm{E}+47$ \\
\hline $\mathrm{Cm}-248$ & 0.01 & 0.005 & 4671 & 12 & 15 & 13 & 1350 & 10000 & 10000 & 9.92E-30 & 2.1 & $4.72 \mathrm{E}-30$ & $9.45 \mathrm{E}-28$ & 0 \\
\hline $\mathrm{Pu}-244$ & & & & & & & & & & 1.23E-09 & 8.597 & $1.43 \mathrm{E}-10$ & $2.86 \mathrm{E}-08$ & 99.963 \\
\hline Pu-244_56 & & & & & & & & & & $4.49 \mathrm{E}-13$ & 8.598 & $5.22 \mathrm{E}-14$ & $1.05 \mathrm{E}-11$ & 0.037 \\
\hline SOF-chain & & & & & & & & & & & & & $2.86 \mathrm{E}-08$ & $3.49 E+07$ \\
\hline Pu-244 & & & & & & & & & & $1.18 \mathrm{E}-93$ & 8.597 & $1.38 \mathrm{E}-94$ & $2.75 \mathrm{E}-93$ & 99.879 \\
\hline Pu-244_56 & & & & & & & & & & 1.44E-96 & 8.598 & 1.67E-97 & 3.35E-96 & 0.121 \\
\hline SOF-chain & & & & & & & & & & & & & $2.76 \mathrm{E}-93$ & $3.63 E+92$ \\
\hline $\mathrm{Cm}-248$ & 0.1 & 0.05 & 4673 & 14 & 15 & 13 & 100 & 1350 & 1350 & 1.92E-76 & 2.1 & $9.16 \mathrm{E}-77$ & 1.83E-75 & 0 \\
\hline Pu-244 & & & & & & & & & & $2.33 \mathrm{E}-48$ & 8.597 & $2.71 \mathrm{E}-49$ & $5.43 E-48$ & 99.952 \\
\hline Pu-244_56 & & & & & & & & & & $1.11 \mathrm{E}-51$ & 8.598 & $1.29 \mathrm{E}-52$ & $2.59 \mathrm{E}-51$ & 0.048 \\
\hline SOF-chain & & & & & & & & & & & & & $5.43 E-48$ & $1.84 \mathrm{E}+47$ \\
\hline $\mathrm{Cm}-248$ & 0.1 & 0.05 & 4671 & 12 & 15 & 13 & 1350 & 10000 & 10000 & 9.92E-29 & 2.1 & $4.72 \mathrm{E}-29$ & $9.45 E-28$ & 0 \\
\hline Pu-244 & & & & & & & & & & 1.23E-08 & 8.597 & 1.43E-09 & $2.86 \mathrm{E}-08$ & 99.963 \\
\hline Pu-244_56 & & & & & & & & & & $4.49 \mathrm{E}-12$ & 8.598 & $5.22 \mathrm{E}-13$ & $1.05 \mathrm{E}-11$ & 0.037 \\
\hline SOF-chain & & & & & & & & & & & & & 2.86E-08 & $3.49 \mathrm{E}+07$ \\
\hline $\mathrm{Cm}-248$ & 1 & 0.5 & 4673 & 14 & 15 & 13 & 0 & 100 & 100 & $4.09 E-119$ & 2.1 & 1.95E-119 & 3.89E-119 & 0 \\
\hline $\mathrm{Pu}-244$ & & & & & & & & & & 1.18E-92 & 8.597 & $1.38 \mathrm{E}-93$ & $2.75 \mathrm{E}-93$ & 99.879 \\
\hline Pu-244_56 & & & & & & & & & & 1.44E-95 & 8.598 & 1.67E-96 & 3.35E-96 & 0.121 \\
\hline SOF-chain & & & & & & & & & & & & & $2.76 \mathrm{E}-93$ & $3.63 E+92$ \\
\hline $\mathrm{Cm}-248$ & 1 & 0.5 & 4673 & 14 & 15 & 13 & 100 & 1350 & 1350 & 1.92E-75 & 2.1 & $9.16 \mathrm{E}-76$ & 1.83E-75 & 0 \\
\hline Pu-244 & & & & & & & & & & $2.29 \mathrm{E}-47$ & 8.597 & $2.66 \mathrm{E}-48$ & 5.33E-48 & 99.952 \\
\hline Pu-244_56 & & & & & & & & & & $1.09 \mathrm{E}-50$ & 8.598 & 1.27E-51 & $2.54 \mathrm{E}-51$ & 0.048 \\
\hline
\end{tabular}




\begin{tabular}{|c|c|c|c|c|c|c|c|c|c|c|c|c|c|c|}
\hline 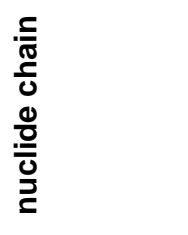 & 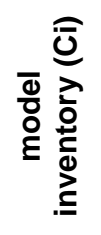 & 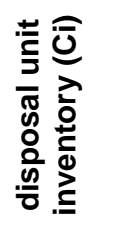 & 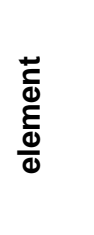 & $\begin{array}{l}\overline{0} \\
\frac{0}{0} \\
\stackrel{\varrho}{\varrho}\end{array}$ & $\begin{array}{l}\mathfrak{0} \\
\frac{0}{0} \\
\stackrel{0}{c}\end{array}$ & 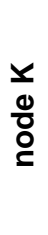 & 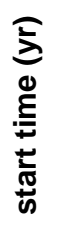 & 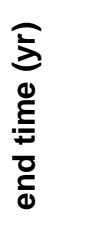 & 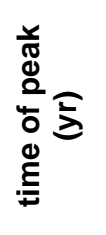 & 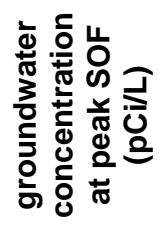 & 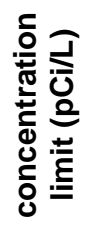 & 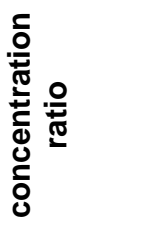 & 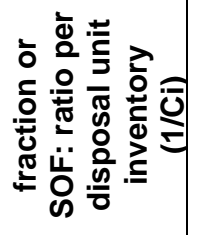 & 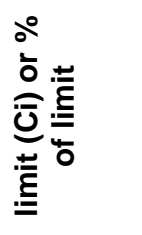 \\
\hline $\mathrm{Pu}-244$ & & & & & & & & & & $4.95 \mathrm{E}-08$ & 8.597 & 5.76E-09 & $1.15 \mathrm{E}-08$ & 99.964 \\
\hline Pu-244_56 & & & & & & & & & & $1.81 \mathrm{E}-11$ & 8.598 & $2.10 \mathrm{E}-12$ & $4.21 \mathrm{E}-12$ & 0.036 \\
\hline SOF-chain & & & & & & & & & & & & & 1.15E-08 & $8.68 E+07$ \\
\hline Cf-249 & 0.001 & 0.0005 & 4673 & 14 & 15 & 13 & 0 & 100 & 100 & 1.52E-94 & 7.4 & 2.05E-95 & 4.11E-92 & 0 \\
\hline $\mathrm{Cm}-245$ & & & & & & & & & & $6.43 \mathrm{E}-99$ & 7.6 & $8.46 \mathrm{E}-100$ & 1.69E-96 & 0 \\
\hline Pu-241 & & & & & & & & & & 7.69E-95 & 300.8 & $2.56 \mathrm{E}-97$ & 5.11E-94 & 0 \\
\hline Pu-241_56 & & & & & & & & & & 2.22E-95 & 54520 & $4.08 \mathrm{E}-100$ & $8.16 \mathrm{E}-97$ & 0 \\
\hline $\mathrm{Am}-241$ & & & & & & & & & & $1.16 \mathrm{E}-97$ & 7.6 & 1.53E-98 & $3.06 \mathrm{E}-95$ & 0 \\
\hline Np-237 & & & & & & & & & & $4.31 \mathrm{E}-48$ & 8.9 & 4.84E-49 & 9.69E-46 & 100 \\
\hline SOF-chain & & & & & & & & & & & & & $9.69 \mathrm{E}-46$ & $1.03 E+45$ \\
\hline Cf-249 & 0.001 & 0.0005 & 4671 & 12 & 15 & 13 & 100 & 1350 & 1350 & 1.69E-52 & 7.4 & $2.28 \mathrm{E}-53$ & $4.57 \mathrm{E}-50$ & 0 \\
\hline Cm-245 & & & & & & & & & & 2.32E-56 & 7.6 & 3.05E-57 & $6.10 \mathrm{E}-54$ & 0 \\
\hline Pu-241_56 & & & & & & & & & & $4.11 \mathrm{E}-55$ & 54520 & 7.54E-60 & 1.51E-56 & 0 \\
\hline Am-241 & & & & & & & & & & $2.53 \mathrm{E}-56$ & 7.6 & 3.33E-57 & 6.65E-54 & 0 \\
\hline $\mathrm{Np}-237$ & & & & & & & & & & $2.37 \mathrm{E}-14$ & 8.9 & $2.66 \mathrm{E}-15$ & $5.32 \mathrm{E}-12$ & 100 \\
\hline SOF-chain & & & & & & & & & & & & & $5.32 \mathrm{E}-12$ & $1.88 \mathrm{E}+11$ \\
\hline Cf-249 & 0.001 & 0.0005 & 3871 & 12 & 15 & 11 & 1350 & 10000 & 10000 & $4.31 \mathrm{E}-19$ & 7.4 & 5.83E-20 & 1.17E-16 & 0 \\
\hline $\mathrm{Cm}-245$ & & & & & & & & & & $7.85 \mathrm{E}-21$ & 7.6 & $1.03 \mathrm{E}-21$ & 2.07E-18 & 0 \\
\hline Pu-241 & & & & & & & & & & 9.07E-20 & 300.8 & $3.02 \mathrm{E}-22$ & 6.03E-19 & 0 \\
\hline Pu-241_56 & & & & & & & & & & $6.01 \mathrm{E}-21$ & 54520 & $1.10 \mathrm{E}-25$ & $2.21 \mathrm{E}-22$ & 0 \\
\hline Am-241 & & & & & & & & & & $1.57 \mathrm{E}-20$ & 7.6 & $2.06 \mathrm{E}-21$ & $4.13 \mathrm{E}-18$ & 0 \\
\hline $\mathrm{Np}-237$ & & & & & & & & & & $2.17 \mathrm{E}-08$ & 8.9 & $2.44 \mathrm{E}-09$ & 4.89E-06 & 100 \\
\hline SOF-chain & & & & & & & & & & & & & 4.89E-06 & $2.05 \mathrm{E}+05$ \\
\hline Cf-249 & 0.01 & 0.005 & 4673 & 14 & 15 & 13 & 0 & 100 & 100 & 1.52E-93 & 7.4 & $2.05 E-94$ & 4.11E-92 & 0 \\
\hline $\mathrm{Cm}-245$ & & & & & & & & & & $6.43 \mathrm{E}-98$ & 7.6 & $8.46 \mathrm{E}-99$ & 1.69E-96 & 0 \\
\hline Pu-241 & & & & & & & & & & 7.69E-94 & 300.8 & $2.56 \mathrm{E}-96$ & $5.11 \mathrm{E}-94$ & 0 \\
\hline Pu-241_56 & & & & & & & & & & $2.22 \mathrm{E}-94$ & 54520 & $4.08 \mathrm{E}-99$ & $8.16 \mathrm{E}-97$ & 0 \\
\hline Am-241 & & & & & & & & & & 1.16E-96 & 7.6 & 1.53E-97 & 3.06E-95 & 0 \\
\hline $\mathrm{Np}-237$ & & & & & & & & & & $4.31 \mathrm{E}-47$ & 8.9 & $4.85 \mathrm{E}-48$ & $9.69 \mathrm{E}-46$ & 100 \\
\hline SOF-chain & & & & & & & & & & & & & $9.69 \mathrm{E}-46$ & $1.03 \mathrm{E}+45$ \\
\hline
\end{tabular}




\begin{tabular}{|c|c|c|c|c|c|c|c|c|c|c|c|c|c|c|}
\hline 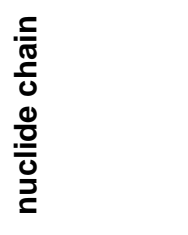 & 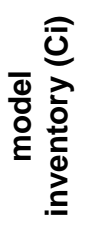 & 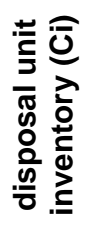 & 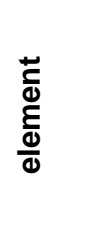 & $\begin{array}{l}\overline{0} \\
\frac{0}{0} \\
\stackrel{\varrho}{\varrho}\end{array}$ & $\begin{array}{l}\mathfrak{0} \\
\frac{0}{0} \\
\stackrel{0}{c}\end{array}$ & 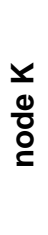 & 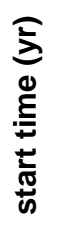 & 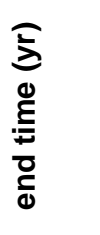 & 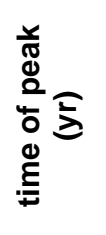 & 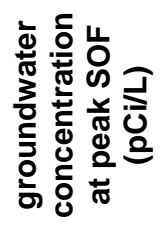 & 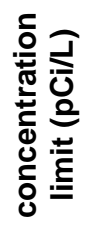 & 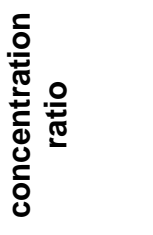 & 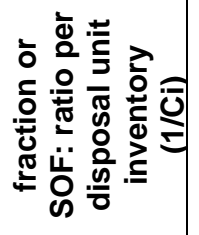 & 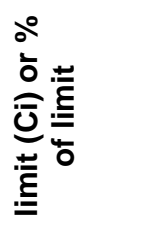 \\
\hline Pu-241 & & & & & & & & & & $4.32 \mathrm{E}-53$ & 300.8 & 1.44E-55 & $2.87 \mathrm{E}-53$ & 0 \\
\hline Pu-241_56 & & & & & & & & & & $4.11 \mathrm{E}-54$ & 54520 & 7.54E-59 & $1.51 \mathrm{E}-56$ & 0 \\
\hline Am-241 & & & & & & & & & & 2.53E-55 & 7.6 & 3.33E-56 & 6.66E-54 & 0 \\
\hline Np-237 & & & & & & & & & & $2.37 \mathrm{E}-13$ & 8.9 & $2.66 \mathrm{E}-14$ & $5.32 \mathrm{E}-12$ & 100 \\
\hline SOF-chain & & & & & & & & & & & & & $5.32 \mathrm{E}-12$ & $1.88 \mathrm{E}+11$ \\
\hline Cf-249 & 0.01 & 0.005 & 3871 & 12 & 15 & 11 & 1350 & 10000 & 10000 & $4.31 \mathrm{E}-18$ & 7.4 & 5.83E-19 & 1.17E-16 & 0 \\
\hline Cm-245 & & & & & & & & & & $7.85 \mathrm{E}-20$ & 7.6 & 1.03E-20 & $2.07 E-18$ & 0 \\
\hline Pu-241 & & & & & & & & & & 9.07E-19 & 300.8 & $3.02 E-21$ & $6.03 E-19$ & 0 \\
\hline Pu-241_56 & & & & & & & & & & $6.01 \mathrm{E}-20$ & 54520 & 1.10E-24 & $2.21 \mathrm{E}-22$ & 0 \\
\hline Am-241 & & & & & & & & & & $1.57 \mathrm{E}-19$ & 7.6 & $2.06 \mathrm{E}-20$ & $4.13 \mathrm{E}-18$ & 0 \\
\hline Np-237 & & & & & & & & & & 2.17E-07 & 8.9 & $2.44 \mathrm{E}-08$ & 4.89E-06 & 100 \\
\hline SOF-chain & & & & & & & & & & & & & 4.89E-06 & $2.05 E+05$ \\
\hline $\mathrm{Cm}-245$ & & & & & & & & & & $6.43 \mathrm{E}-97$ & 7.6 & 8.46E-98 & 1.69E-96 & 0 \\
\hline Pu-241 & & & & & & & & & & 7.69E-93 & 300.8 & 2.56E-95 & 5.11E-94 & 0 \\
\hline Pu-241_56 & & & & & & & & & & $2.22 \mathrm{E}-93$ & 54520 & 4.08E-98 & $8.16 \mathrm{E}-97$ & 0 \\
\hline $\mathrm{Am}-241$ & & & & & & & & & & $1.16 \mathrm{E}-95$ & 7.6 & $1.53 \mathrm{E}-96$ & $3.06 \mathrm{E}-95$ & 0 \\
\hline Np-237 & & & & & & & & & & $4.31 \mathrm{E}-46$ & 8.9 & 4.85E-47 & $9.69 \mathrm{E}-46$ & 100 \\
\hline SOF-chain & & & & & & & & & & & & & $9.69 \mathrm{E}-46$ & $1.03 E+45$ \\
\hline Cf-249 & 0.1 & 0.05 & 4671 & 12 & 15 & 13 & 100 & 1350 & 1350 & 1.69E-50 & 7.4 & $2.28 \mathrm{E}-51$ & $4.57 \mathrm{E}-50$ & 0 \\
\hline $\mathrm{Cm}-245$ & & & & & & & & & & 2.32E-54 & 7.6 & 3.05E-55 & $6.10 \mathrm{E}-54$ & 0 \\
\hline Pu-241 & & & & & & & & & & $4.32 \mathrm{E}-52$ & 300.8 & 1.44E-54 & $2.87 E-53$ & 0 \\
\hline Pu-241_56 & & & & & & & & & & $4.11 \mathrm{E}-53$ & 54520 & 7.54E-58 & $1.51 \mathrm{E}-56$ & 0 \\
\hline Am-241 & & & & & & & & & & $2.53 \mathrm{E}-54$ & 7.6 & 3.33E-55 & 6.66E-54 & 0 \\
\hline Np-237 & & & & & & & & & & $2.37 \mathrm{E}-12$ & 8.9 & $2.66 \mathrm{E}-13$ & $5.32 \mathrm{E}-12$ & 100 \\
\hline SOF-chain & & & & & & & & & & & & & $5.32 \mathrm{E}-12$ & $1.88 \mathrm{E}+11$ \\
\hline Cf-249 & 0.1 & 0.05 & 3871 & 12 & 15 & 11 & 1350 & 10000 & 10000 & $4.31 \mathrm{E}-17$ & 7.4 & $5.83 \mathrm{E}-18$ & 1.17E-16 & 0 \\
\hline $\mathrm{Cm}-245$ & & & & & & & & & & $7.85 \mathrm{E}-19$ & 7.6 & $1.03 \mathrm{E}-19$ & $2.07 \mathrm{E}-18$ & 0 \\
\hline Pu-241 & & & & & & & & & & 9.07E-18 & 300.8 & $3.02 \mathrm{E}-20$ & $6.03 E-19$ & 0 \\
\hline Pu-241_56 & & & & & & & & & & $6.01 \mathrm{E}-19$ & 54520 & 1.10E-23 & $2.21 \mathrm{E}-22$ & 0 \\
\hline $\mathrm{Am}-241$ & & & & & & & & & & $1.57 \mathrm{E}-18$ & 7.6 & $2.06 \mathrm{E}-19$ & $4.13 \mathrm{E}-18$ & 0 \\
\hline
\end{tabular}




\begin{tabular}{|c|c|c|c|c|c|c|c|c|c|c|c|c|c|c|}
\hline $\begin{array}{l}. \frac{1}{\pi} \\
\frac{2}{0} \\
\frac{0}{0} \\
\frac{0}{0} \\
\frac{0}{2}\end{array}$ & 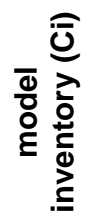 & 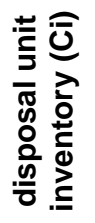 & 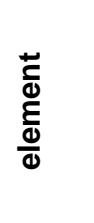 & $\begin{array}{l}\overline{0} \\
\frac{0}{0} \\
\text { 은 }\end{array}$ & $\begin{array}{l}7 \\
\stackrel{0}{0} \\
\stackrel{0}{0}\end{array}$ & 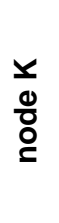 & 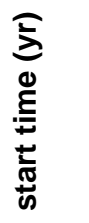 & 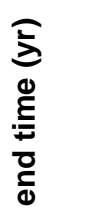 & 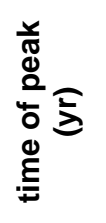 & 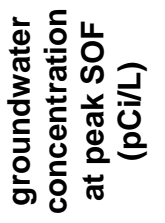 & 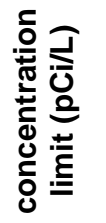 & 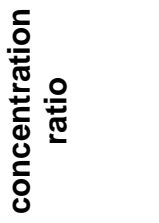 & 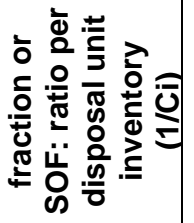 & 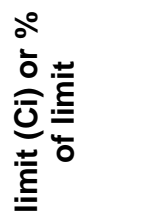 \\
\hline Cf-249 & 1 & 0.5 & 4673 & 14 & 15 & 13 & 0 & 100 & 100 & $1.52 \mathrm{E}-91$ & 7.4 & $2.05 E-92$ & $4.11 \mathrm{E}-92$ & 0 \\
\hline Cm-245 & & & & & & & & & & $6.43 E-96$ & 7.6 & $8.46 \mathrm{E}-97$ & 1.69E-96 & 0 \\
\hline Pu-241 & & & & & & & & & & 7.69E-92 & 300.8 & $2.56 \mathrm{E}-94$ & $5.11 \mathrm{E}-94$ & 0 \\
\hline Pu-241_56 & & & & & & & & & & $2.22 \mathrm{E}-92$ & 54520 & $4.08 \mathrm{E}-97$ & $8.16 \mathrm{E}-97$ & 0 \\
\hline Am-241 & & & & & & & & & & 1.16E-94 & 7.6 & 1.53E-95 & 3.06E-95 & 0 \\
\hline $\mathrm{Np}-237$ & & & & & & & & & & $4.31 \mathrm{E}-45$ & 8.9 & $4.85 \mathrm{E}-46$ & $9.69 \mathrm{E}-46$ & 100 \\
\hline SOF-chain & & & & & & & & & & & & & $9.69 \mathrm{E}-46$ & $1.03 \mathrm{E}+45$ \\
\hline Cf-249 & 1 & 0.5 & 4671 & 12 & 15 & 13 & 100 & 1350 & 1350 & $1.69 \mathrm{E}-49$ & 7.4 & $2.28 \mathrm{E}-50$ & $4.57 \mathrm{E}-50$ & 0 \\
\hline $\mathrm{Cm}-245$ & & & & & & & & & & $2.32 \mathrm{E}-53$ & 7.6 & $3.05 \mathrm{E}-54$ & $6.10 \mathrm{E}-54$ & 0 \\
\hline Pu-241 & & & & & & & & & & $4.32 \mathrm{E}-51$ & 300.8 & $1.44 \mathrm{E}-53$ & $2.87 \mathrm{E}-53$ & 0 \\
\hline Pu-241_56 & & & & & & & & & & $4.11 \mathrm{E}-52$ & 54520 & $7.54 \mathrm{E}-57$ & $1.51 \mathrm{E}-56$ & 0 \\
\hline Am-241 & & & & & & & & & & $2.53 E-53$ & 7.6 & 3.33E-54 & $6.66 \mathrm{E}-54$ & 0 \\
\hline SOF-chain & & & & & & & & & & & & & $5.32 \mathrm{E}-12$ & $1.88 \mathrm{E}+11$ \\
\hline Cf-249 & 1 & 0.5 & 3871 & 12 & 15 & 11 & 1350 & 10000 & 10000 & $4.31 \mathrm{E}-16$ & 7.4 & $5.83 \mathrm{E}-17$ & 1.17E-16 & 0 \\
\hline $\mathrm{Cm}-245$ & & & & & & & & & & $7.85 \mathrm{E}-18$ & 7.6 & $1.03 \mathrm{E}-18$ & $2.07 \mathrm{E}-18$ & 0 \\
\hline $\mathrm{Pu}-241$ & & & & & & & & & & $9.07 \mathrm{E}-17$ & 300.8 & $3.02 \mathrm{E}-19$ & $6.03 \mathrm{E}-19$ & 0 \\
\hline Pu-241 56 & & & & & & & & & & $6.01 \mathrm{E}-18$ & 54520 & 1.10E-22 & $2.21 \mathrm{E}-22$ & 0 \\
\hline Am-241 & & & & & & & & & & $1.57 \mathrm{E}-17$ & 7.6 & $2.06 \mathrm{E}-18$ & $4.13 \mathrm{E}-18$ & 0 \\
\hline $\mathrm{Np}-237$ & & & & & & & & & & $2.17 E-05$ & 8.9 & $2.44 \mathrm{E}-06$ & 4.89E-06 & 100 \\
\hline SOF-chain & & & & & & & & & & & & & 4.89E-06 & $2.05 \mathrm{E}+05$ \\
\hline
\end{tabular}




\section{Plots of peak water table flux and 100 meter well concentration from PORFLOW aquifer transport simulations}

Figures A-8 through A-94 show PORFLOW flux and concentration data in graphical form, with the exception of $\mathrm{Zr}-95$, Nb-95, Po-210, Th-228, which were too short-lived to produce meaningful groundwater concentrations. These figures were automatically generated using computer batch files from a generic template, which introduces minor formatting problems. The labels "C", "C2", etc. refer to sequential members of the chain being modeled. The chain is defined in the figure caption. A key is provided for up to 6 species in the groundwater concentration plots, but typically a smaller number of species is present. Each case plotted is a combination of a nuclide parent and an initial inventory (Ci) for 2 disposal units denoted by the label "parent_inventory". 


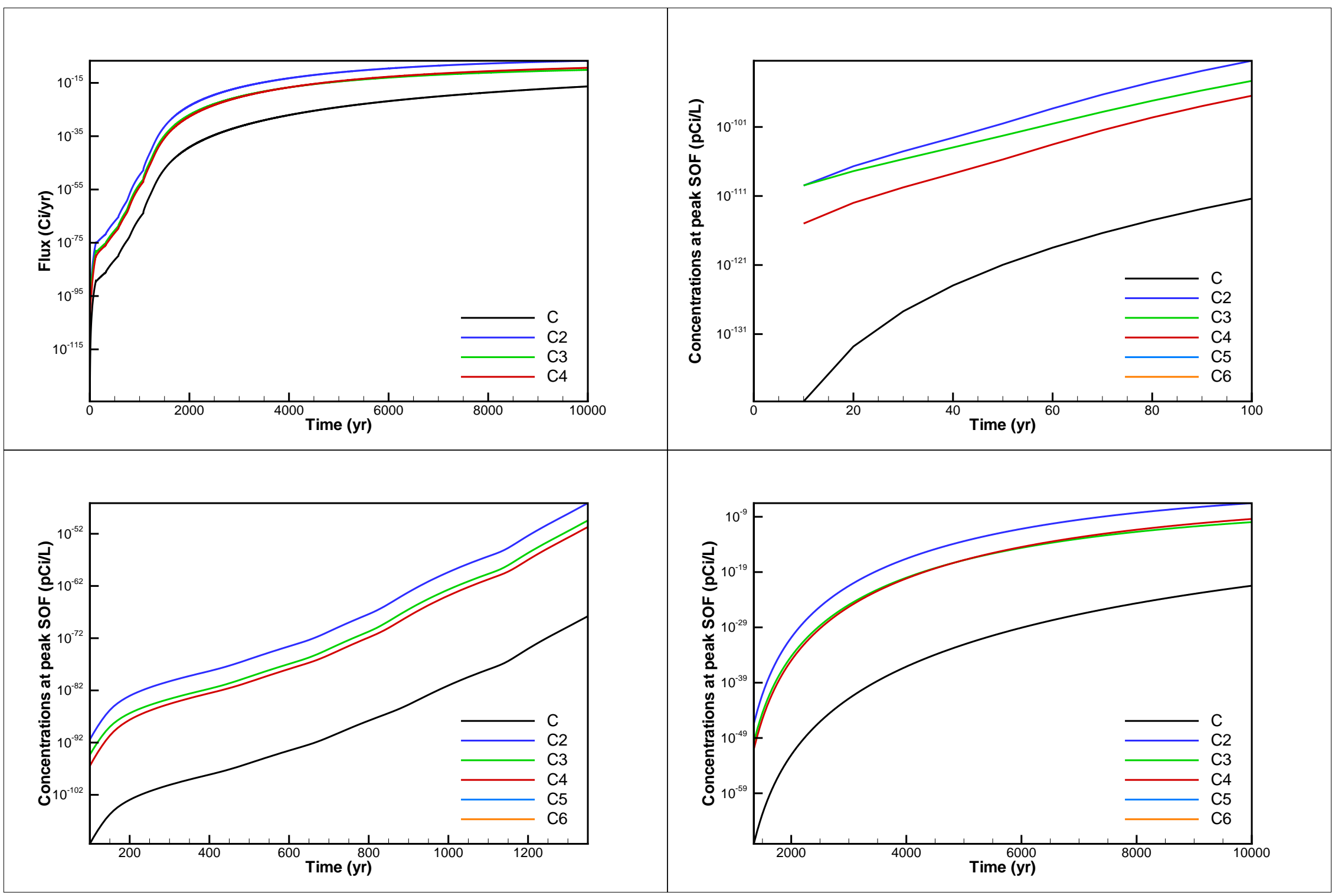

Figure A-8. Flux and concentration results for case Am-243_0.001: Am-243 Pu-239 Pu-239_56 U-234 


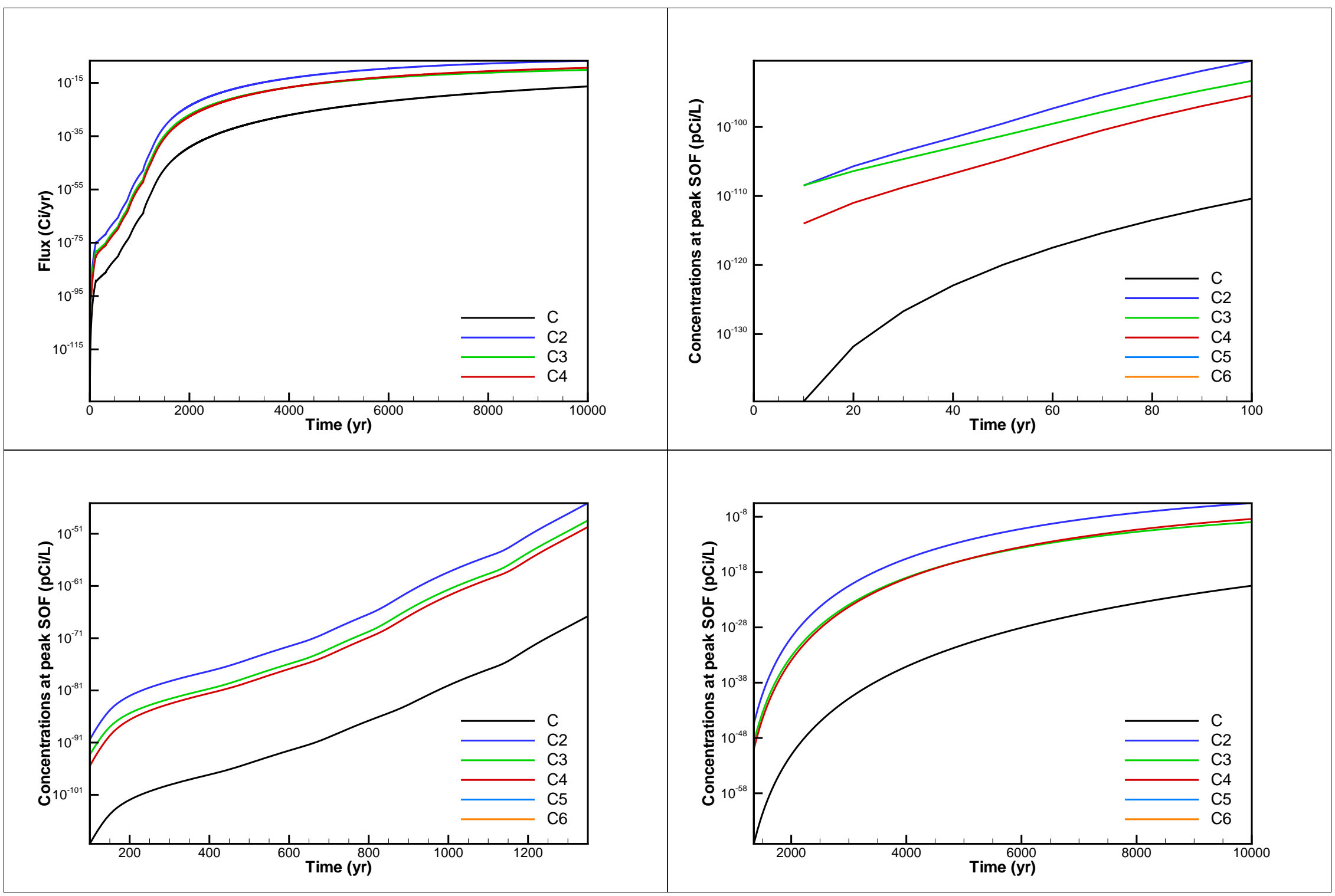

Figure A-9. Flux and concentration results for case Am-243_0.01: Am-243 Pu-239 Pu-239_56 U-234 


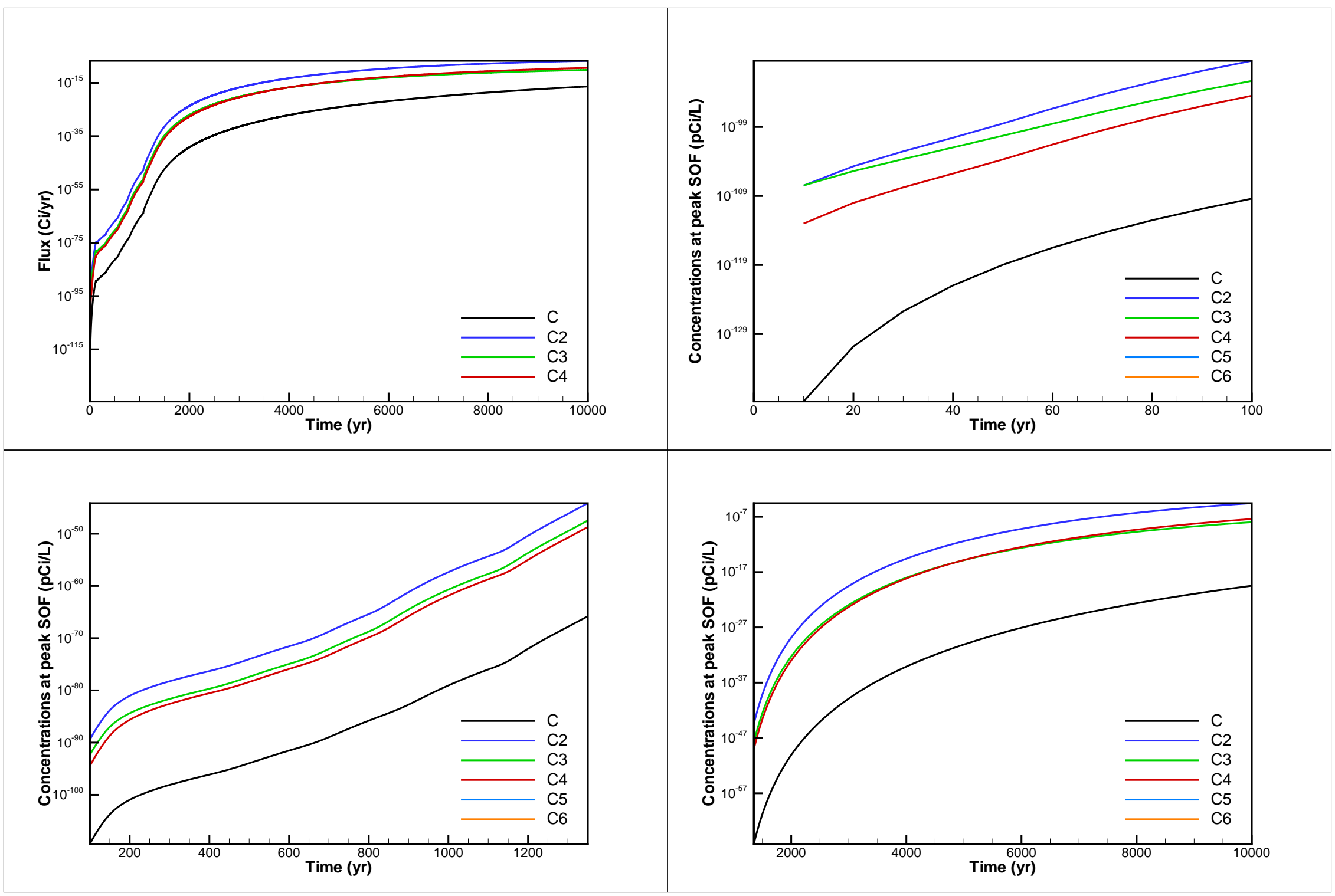

Figure A-10. Flux and concentration results for case Am-243_0.1: Am-243 Pu-239 Pu-239_56 U-234 


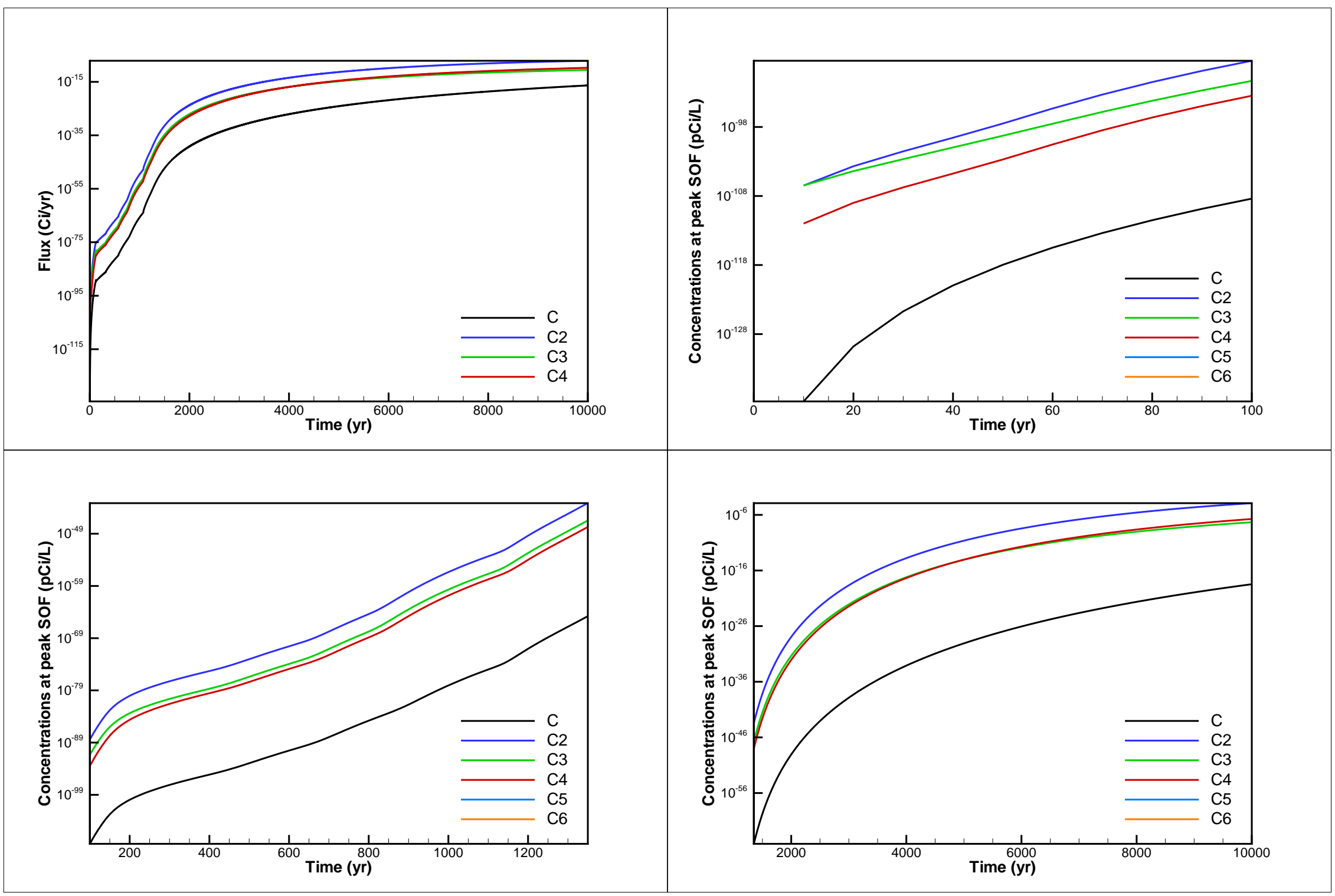

Figure A-11. Flux and concentration results for case Am-243_1: Am-243 Pu-239 Pu-239_56 U-234 


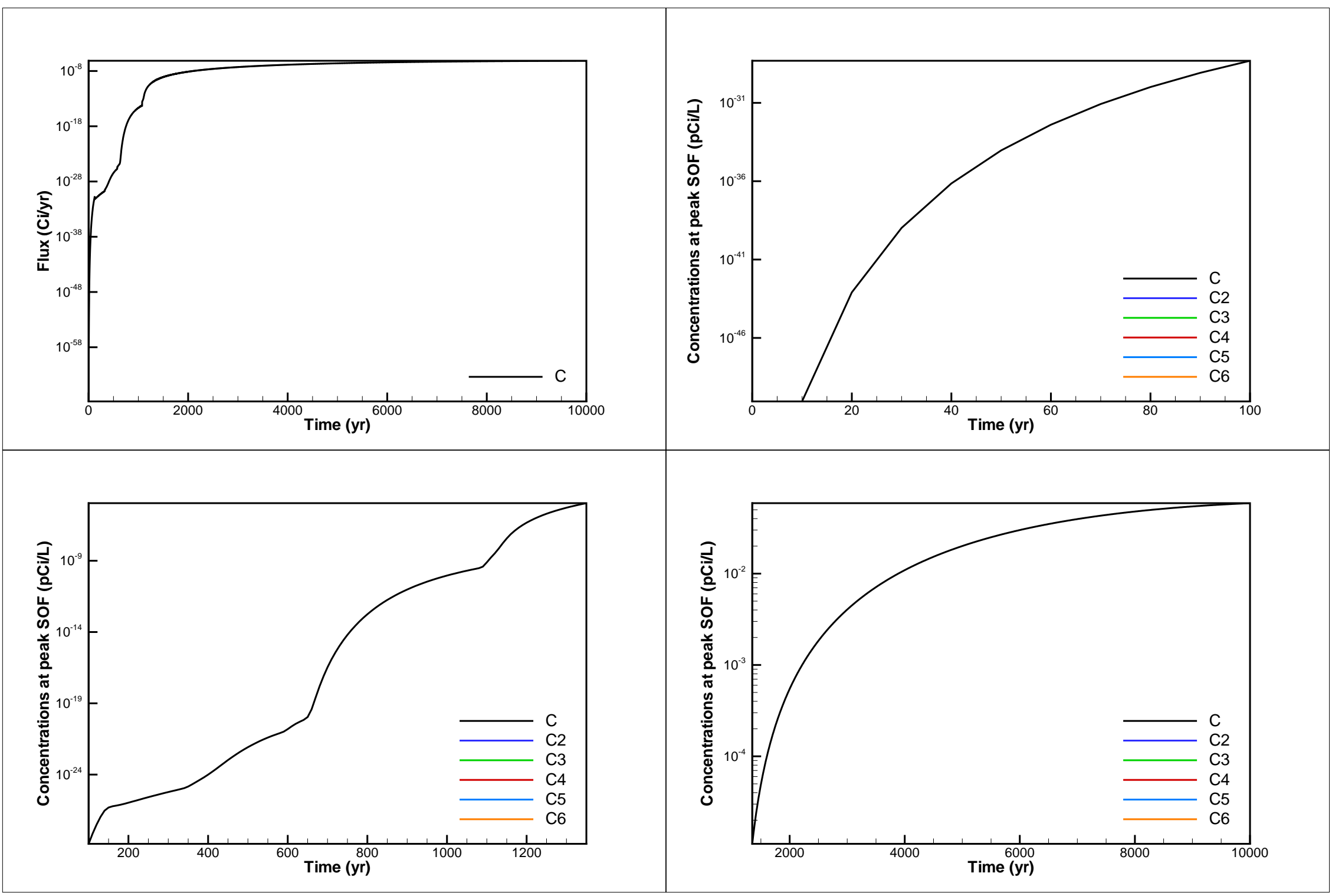

Figure A-12. Flux and concentration results for case C-14_1: C-14 


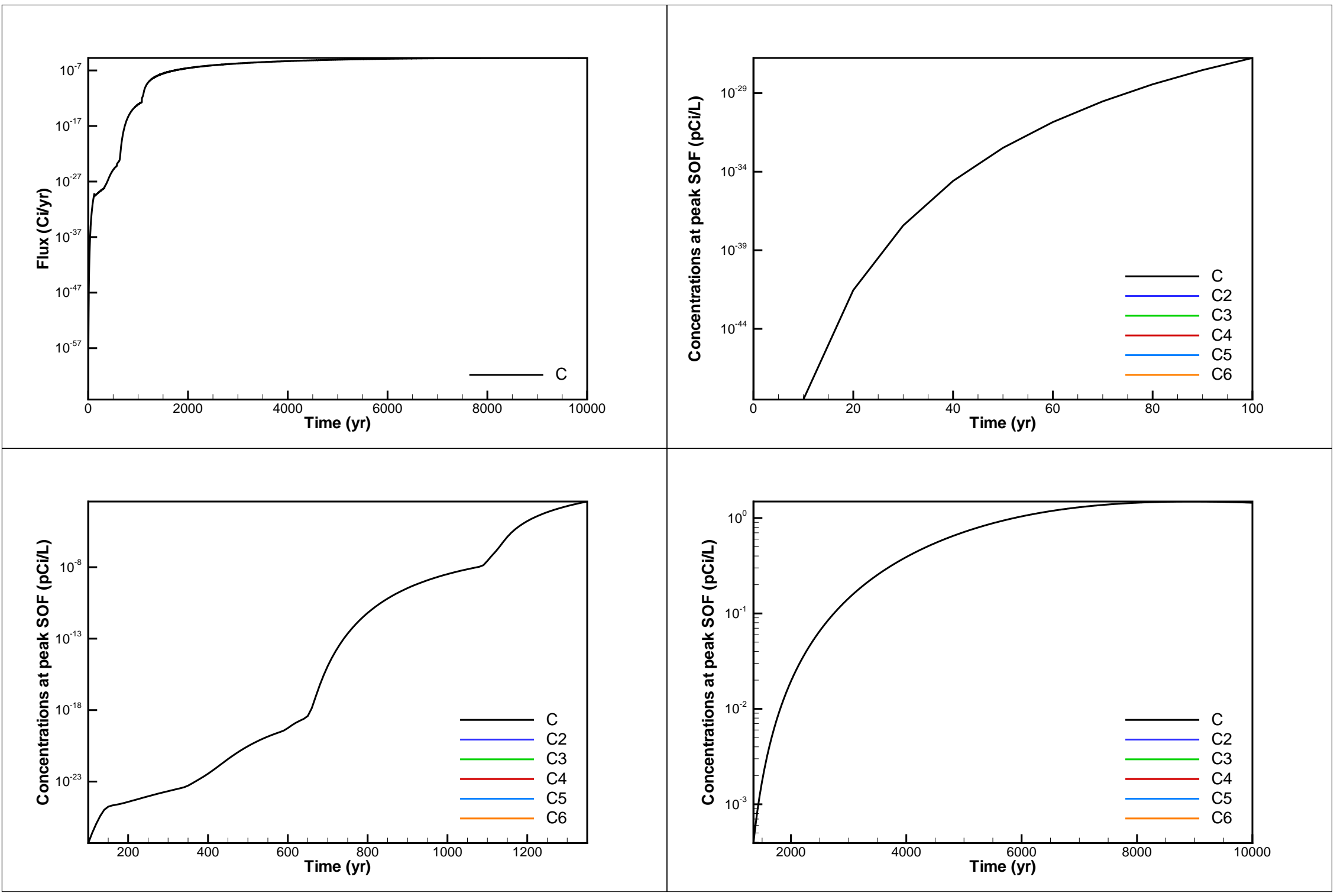

Figure A-13. Flux and concentration results for case C-14_KB_1: C-14_KB 


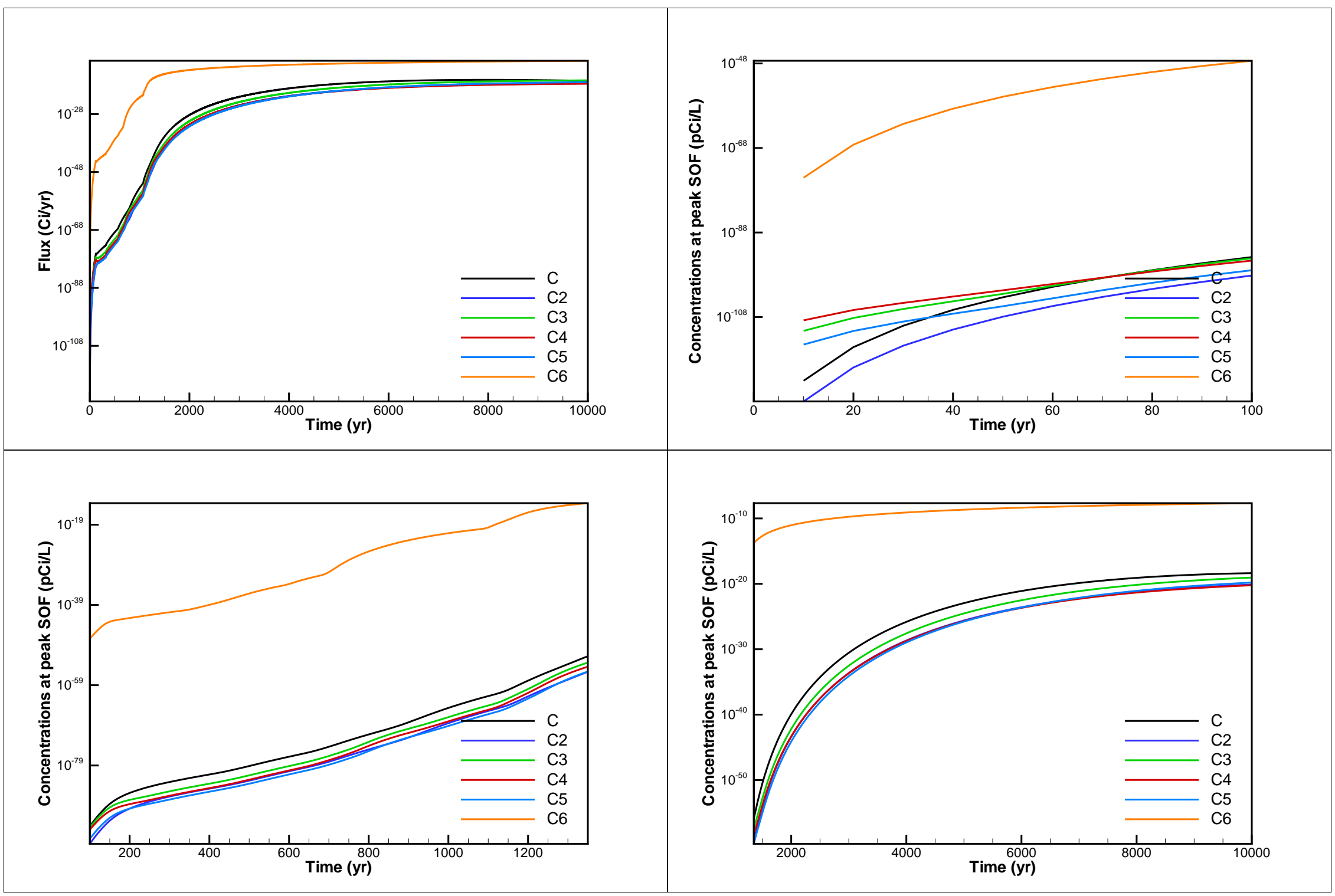

Figure A-14. Flux and concentration results for case Cf-249_0.001: Cf-249 Cm-245 Pu-241 Pu-241_56 Am-241 Np-237 


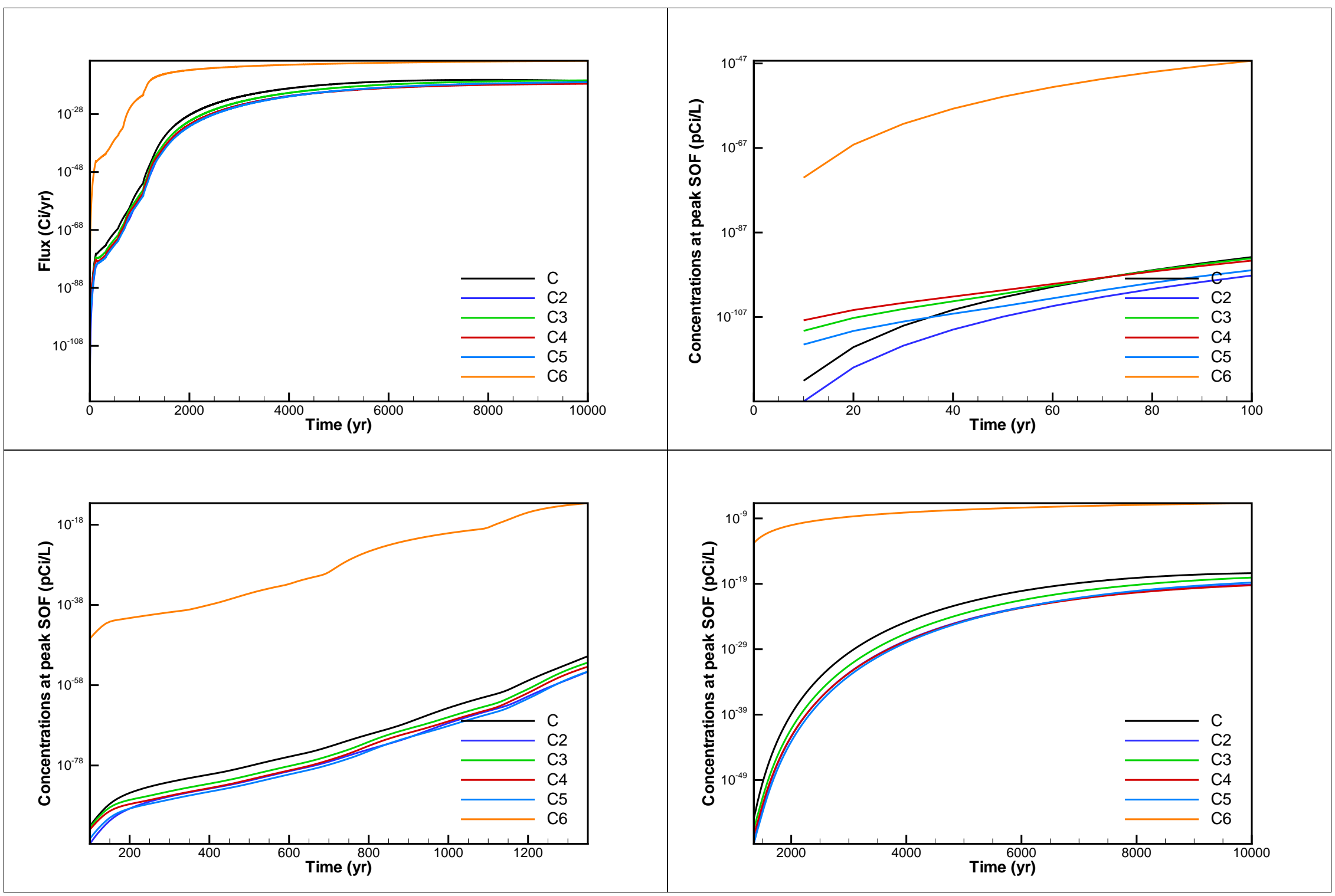

Figure A-15. Flux and concentration results for case Cf-249_0.01: Cf-249 Cm-245 Pu-241 Pu-241_56 Am-241 Np-237 


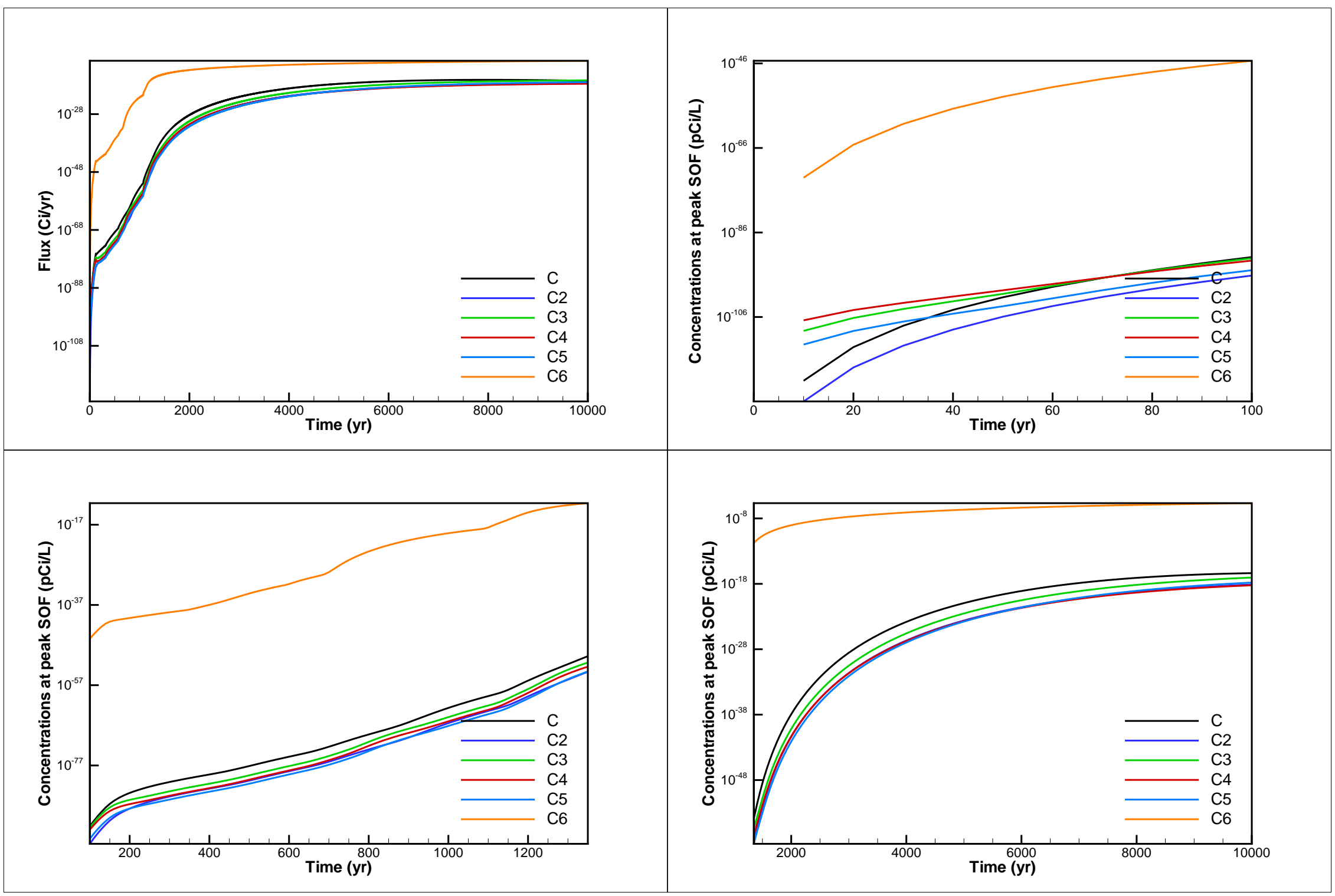

Figure A-16. Flux and concentration results for case Cf-249_0.1: Cf-249 Cm-245 Pu-241 Pu-241_56 Am-241 Np-237 


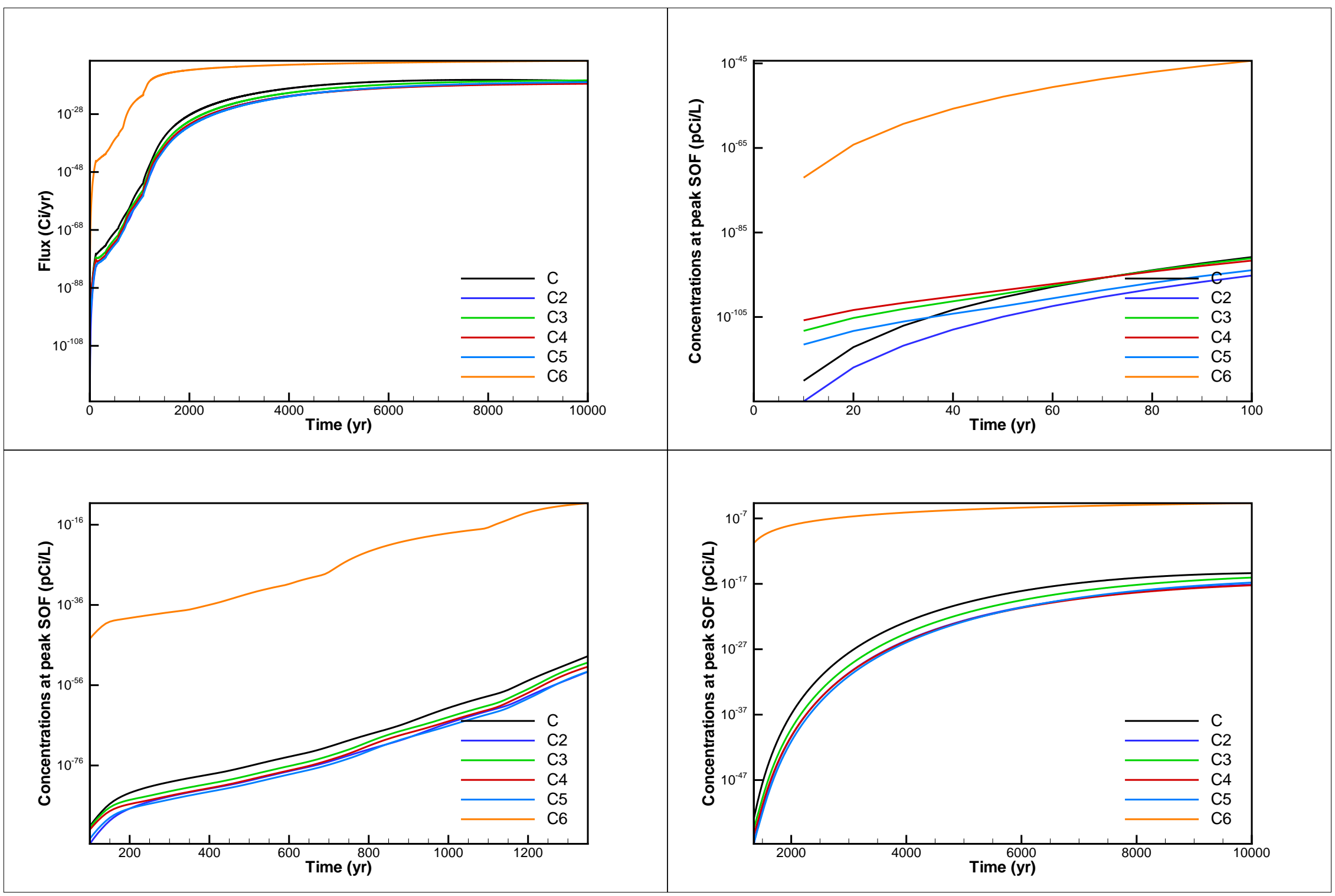

Figure A-17. Flux and concentration results for case Cf-249_1: Cf-249 Cm-245 Pu-241 Pu-241_56 Am-241 Np-237 


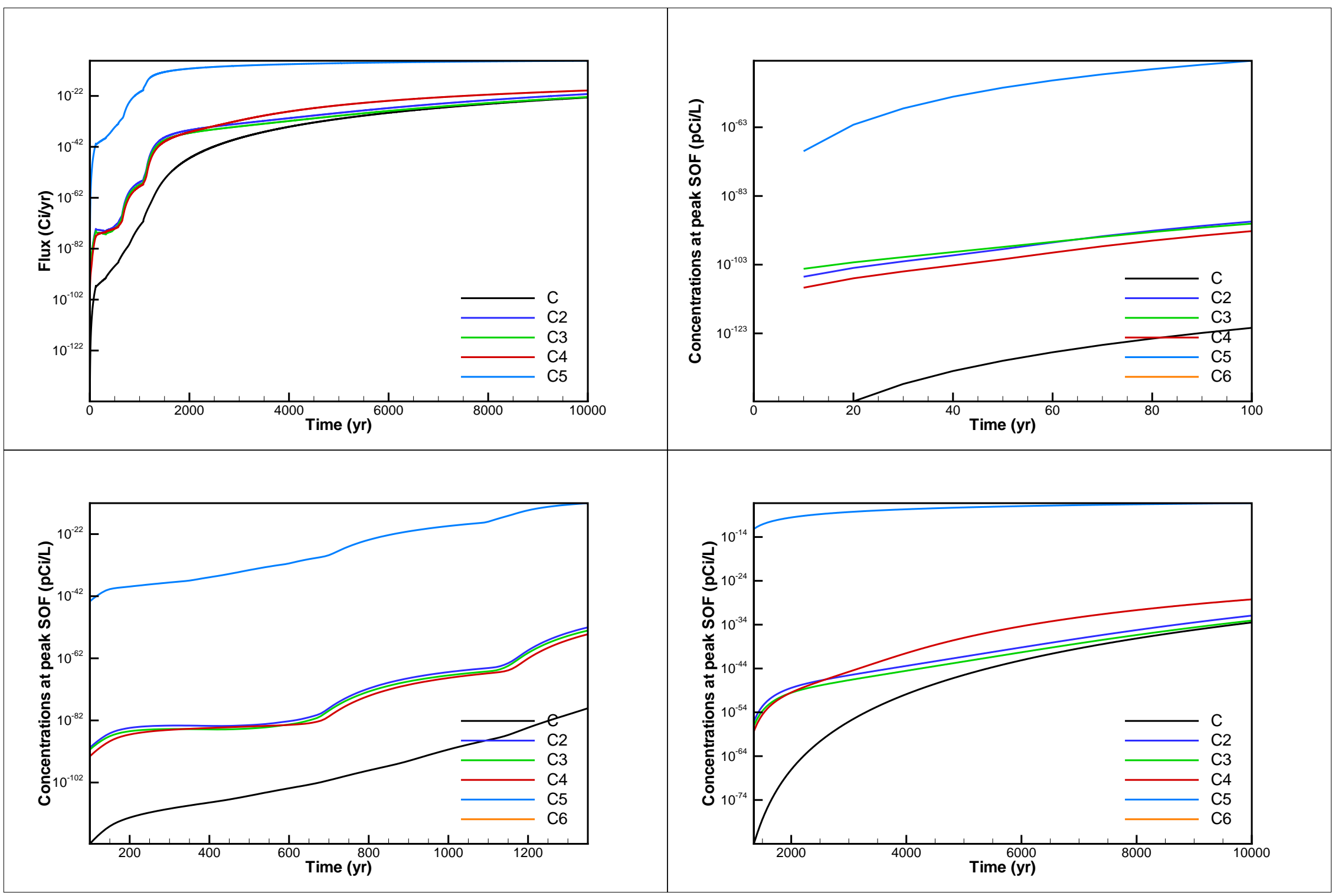

Figure A-18. Flux and concentration results for case Cm-245_0.001: Cm-245 Pu-241 Pu-241_56 Am-241 Np-237 


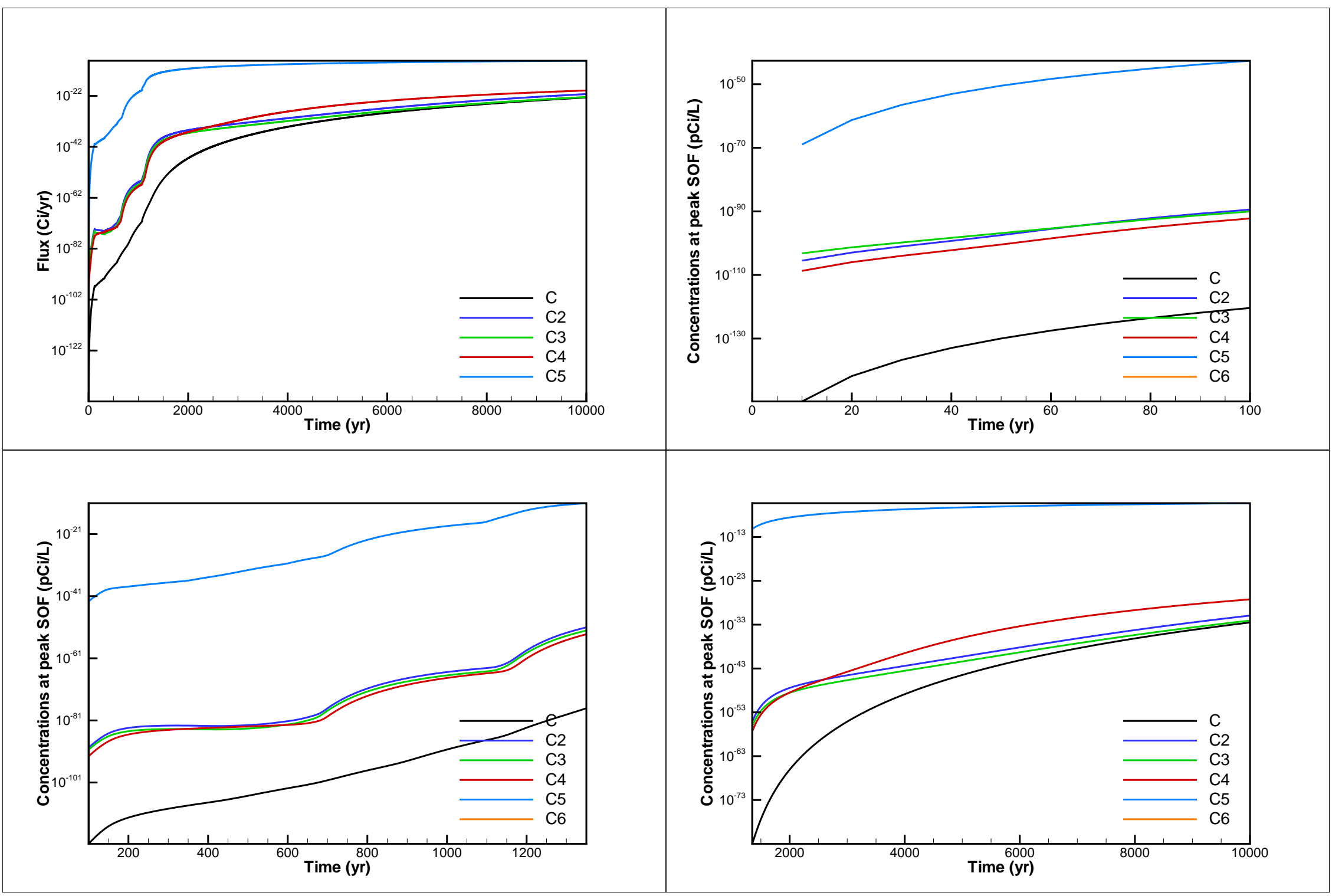

Figure A-19. Flux and concentration results for case Cm-245_0.01: Cm-245 Pu-241 Pu-241_56 Am-241 Np-237 


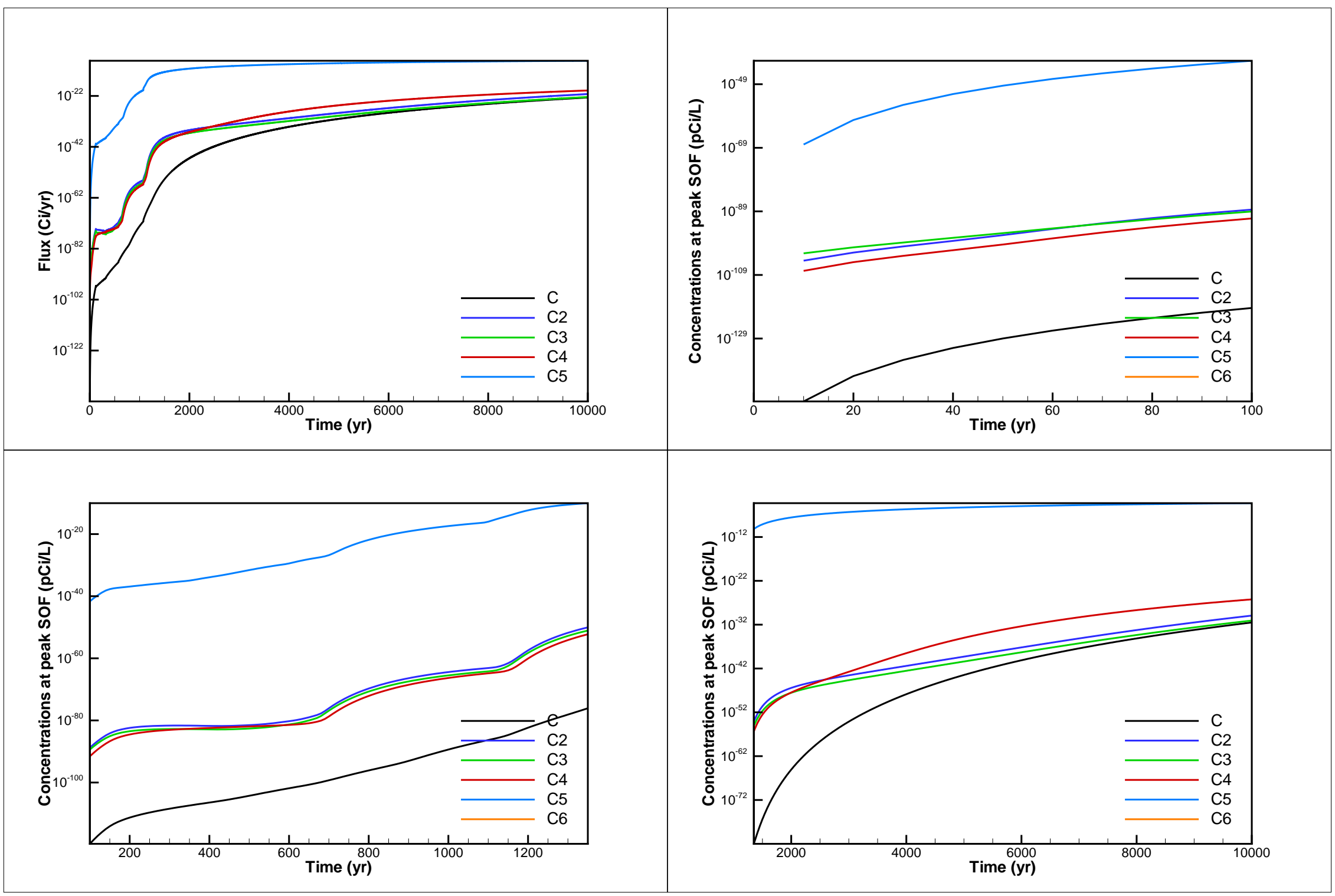

Figure A-20. Flux and concentration results for case Cm-245_0.1: Cm-245 Pu-241 Pu-241_56 Am-241 Np-237 


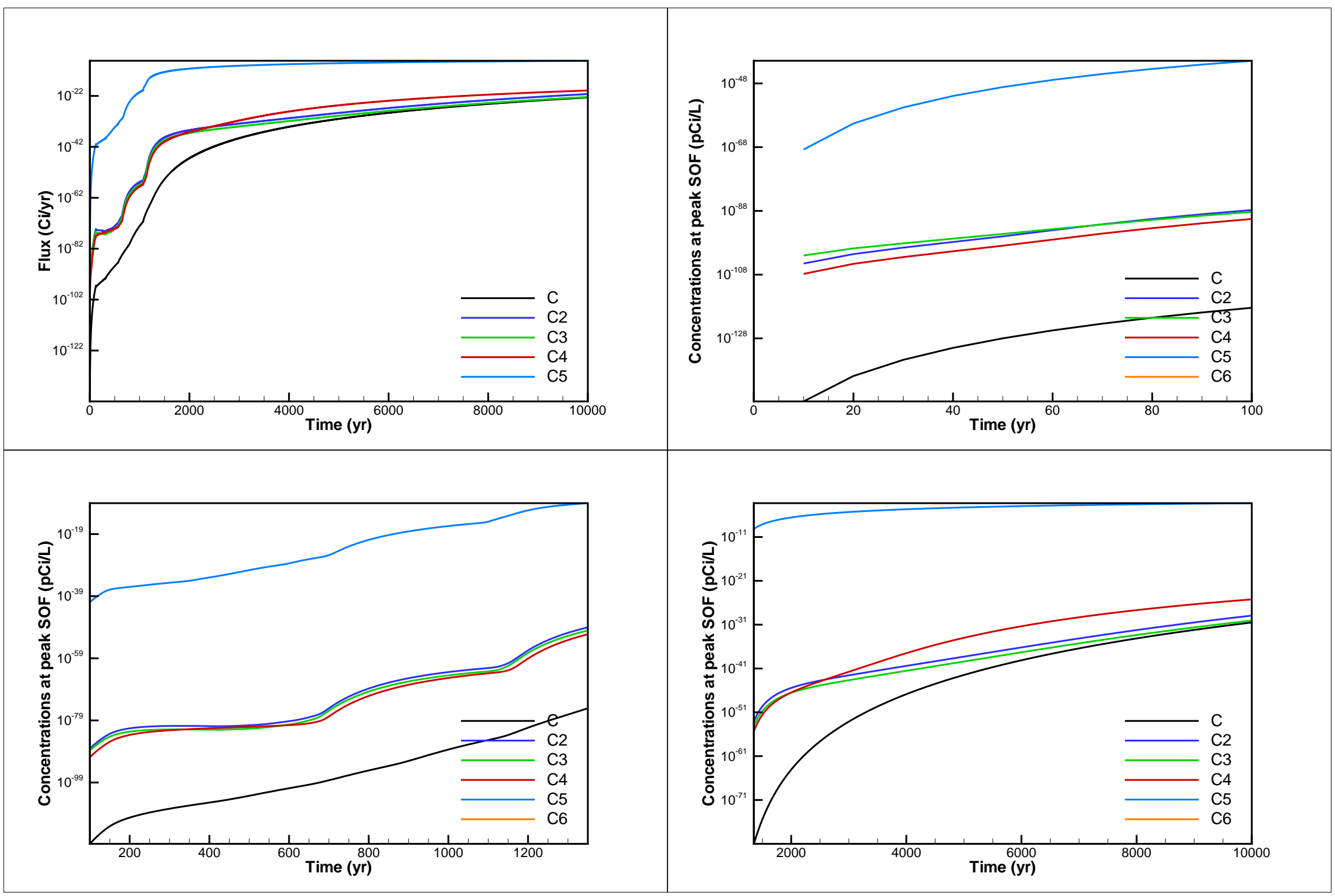

Figure A-21. Flux and concentration results for case Cm-245_1: Cm-245 Pu-241 Pu-241_56 Am-241 Np-237 


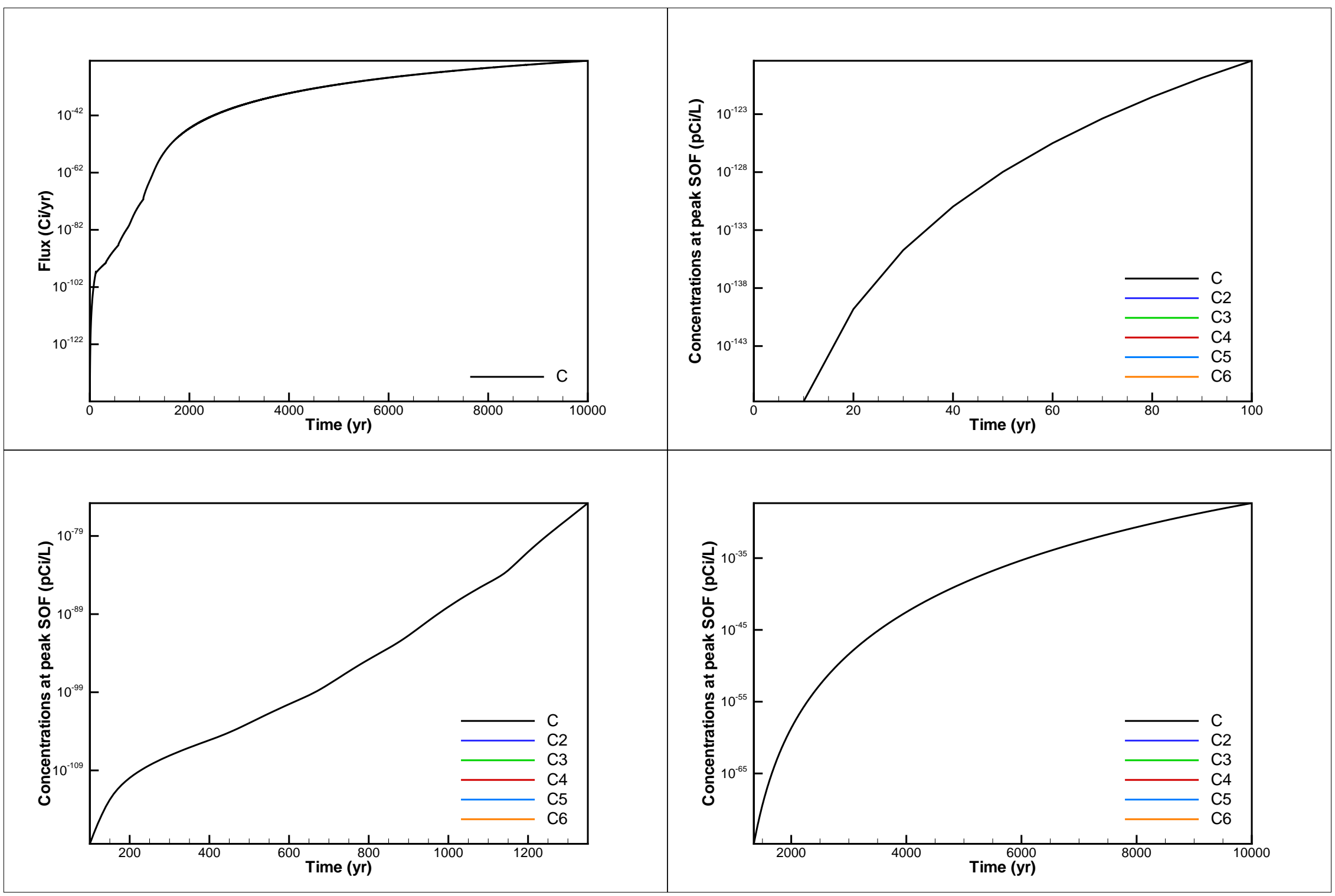

Figure A-22. Flux and concentration results for case Cm-246_1: Cm-246 


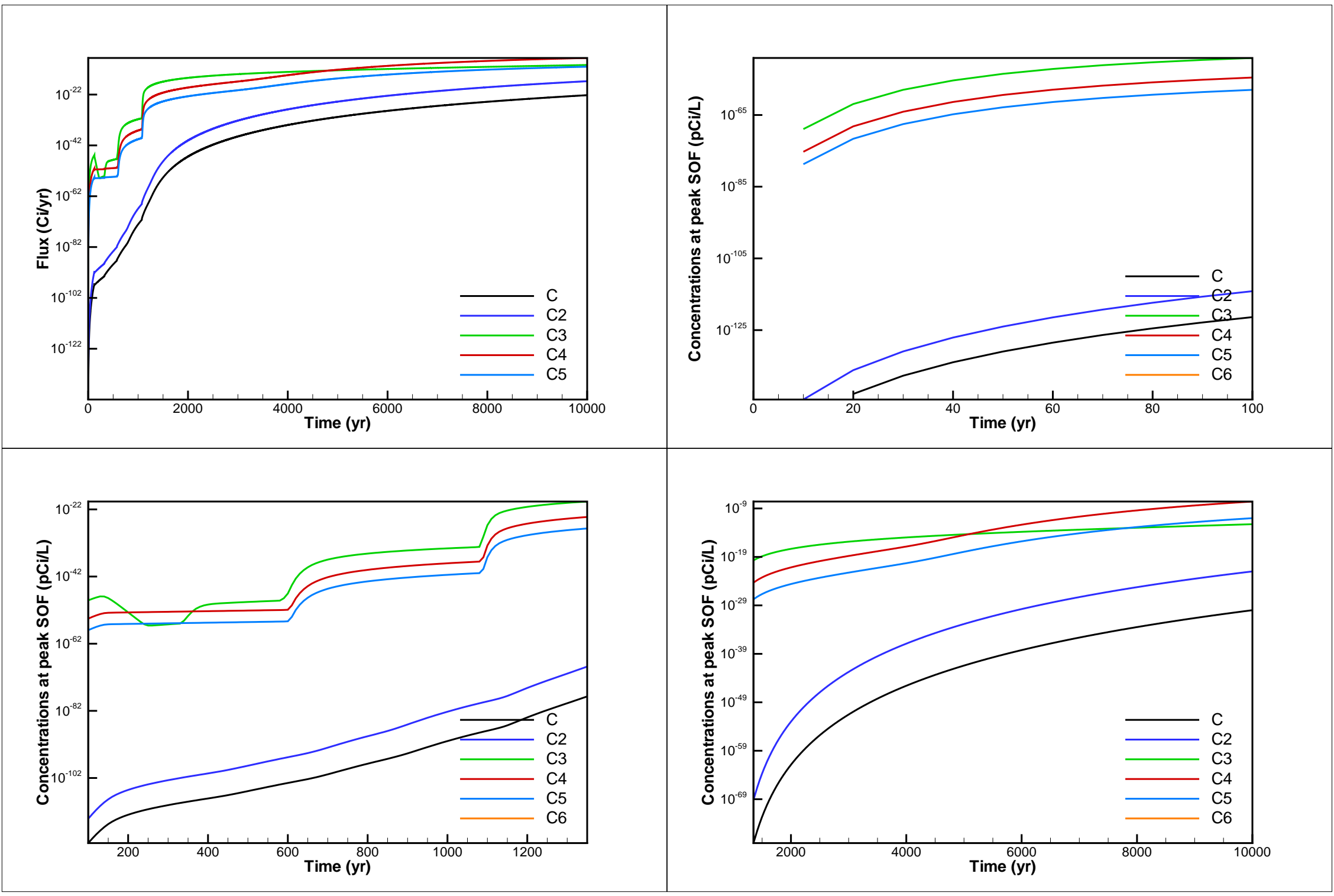

Figure A-23. Flux and concentration results for case Cm-247_0.001: Cm-247 Am-243 Np-239 Pu-239 Pu-239_56 


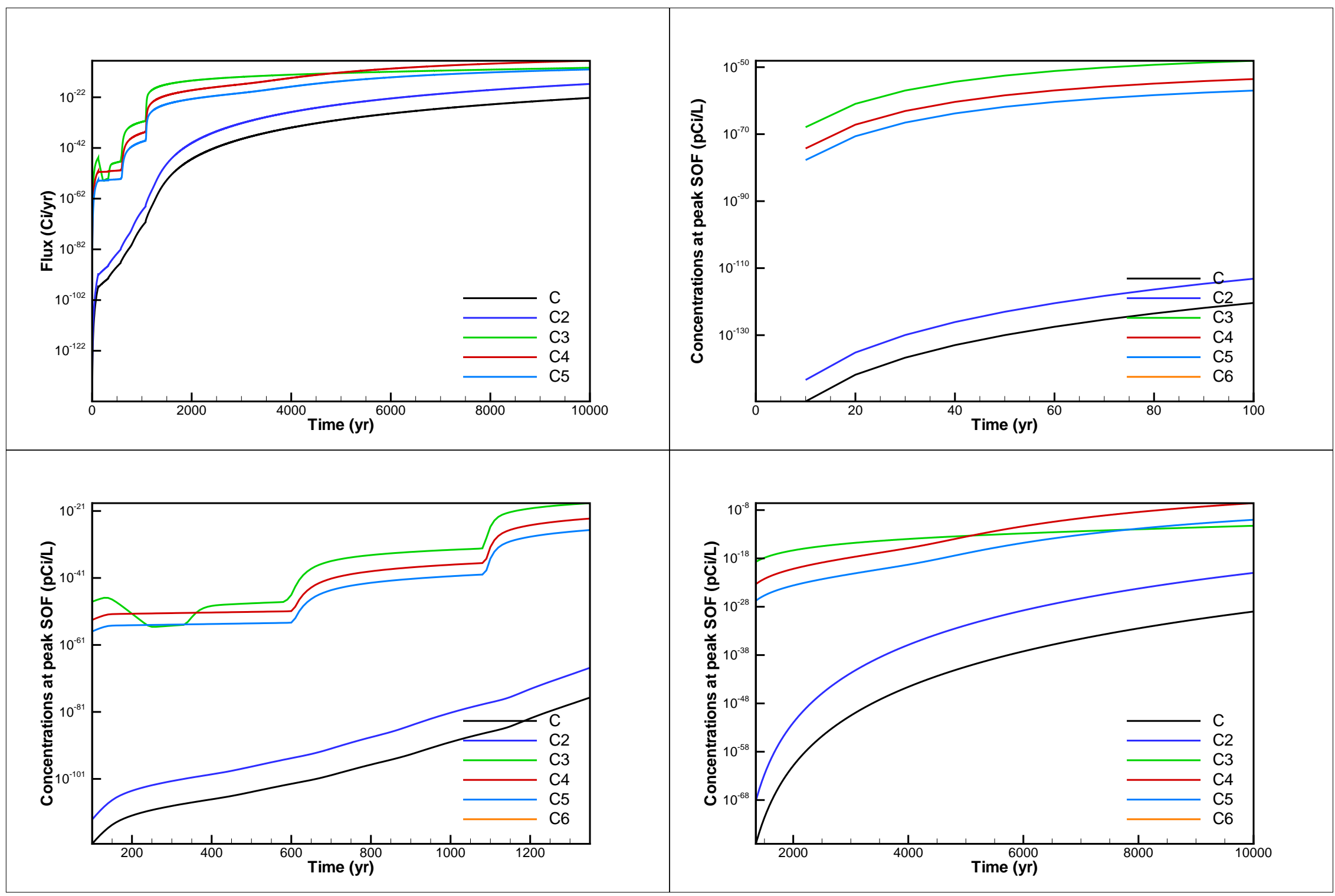

Figure A-24. Flux and concentration results for case Cm-247_0.01: Cm-247 Am-243 Np-239 Pu-239 Pu-239_56 


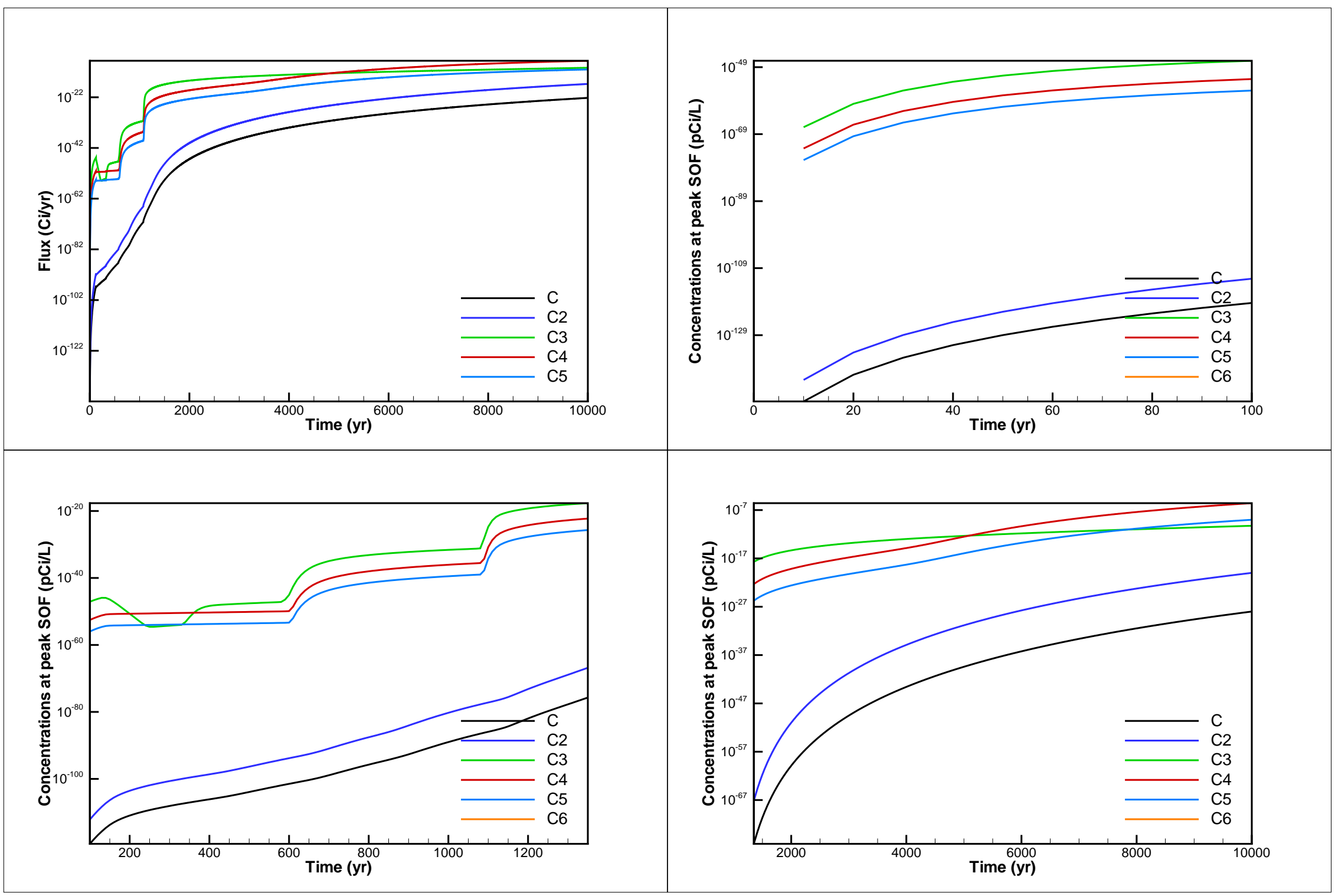

Figure A-25. Flux and concentration results for case Cm-247_0.1: Cm-247 Am-243 Np-239 Pu-239 Pu-239_56 


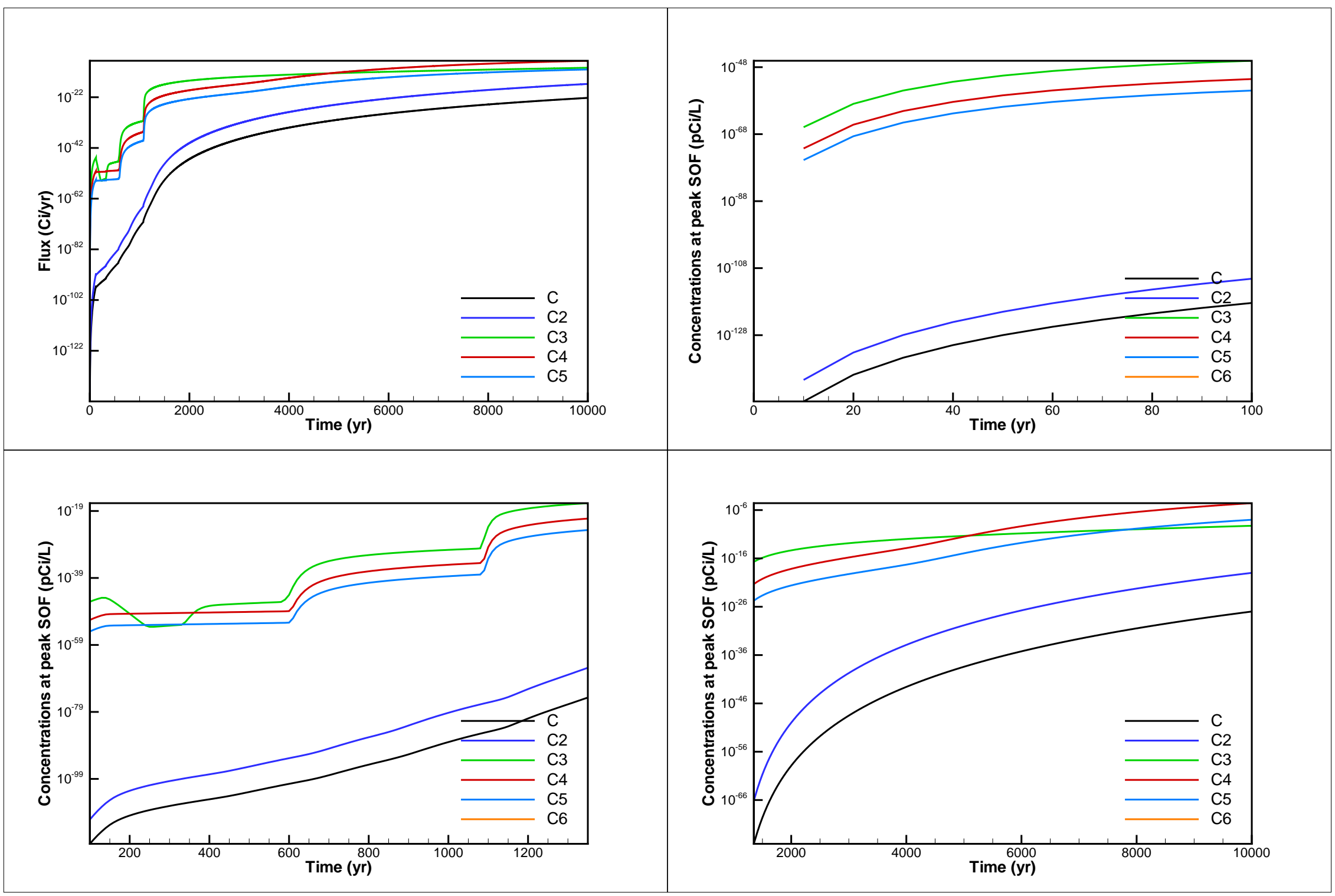

Figure A-26. Flux and concentration results for case Cm-247_1: Cm-247 Am-243 Np-239 Pu-239 Pu-239_56 


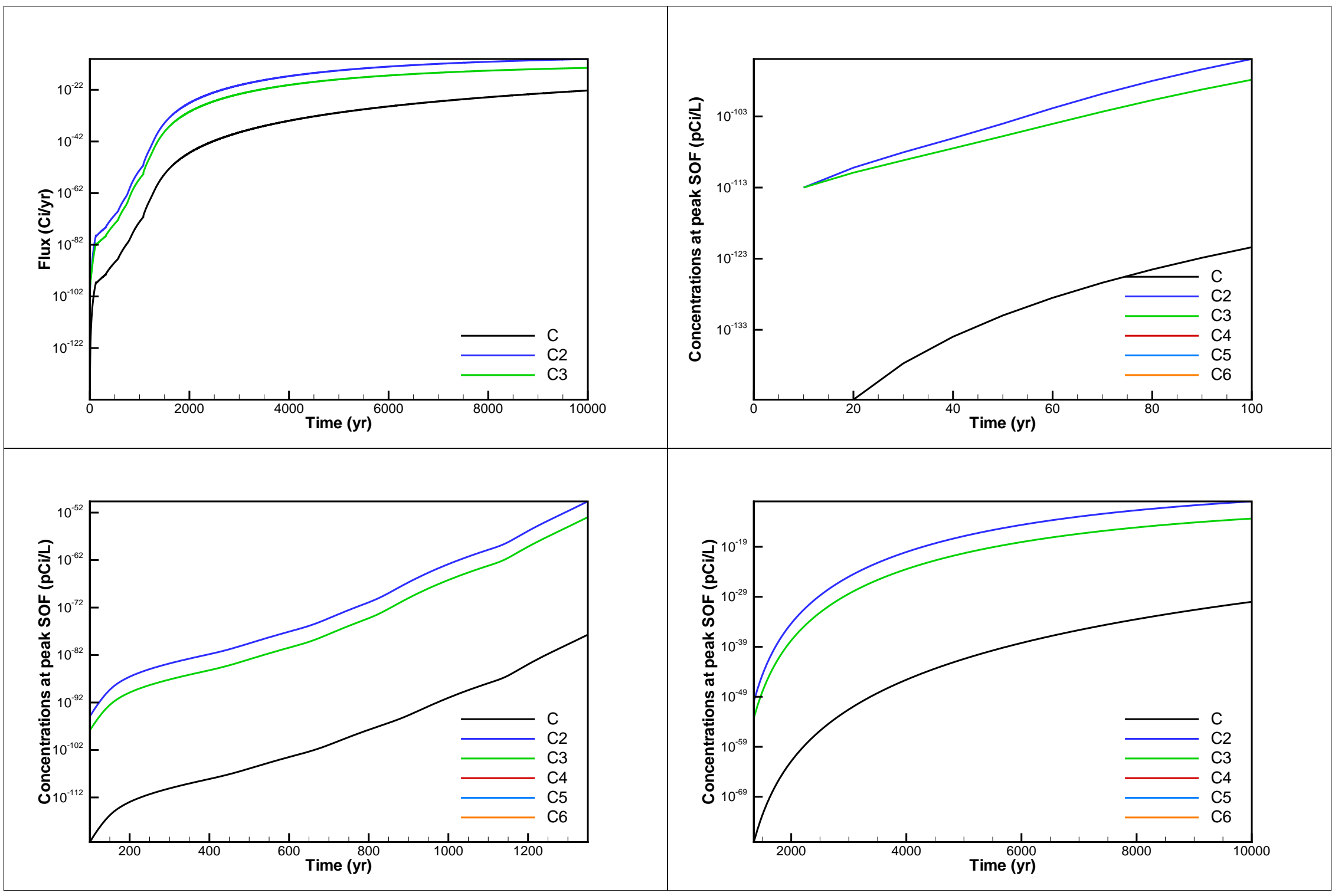

Figure A-27. Flux and concentration results for case Cm-248_0.001: Cm-248 Pu-244 Pu-244_56 


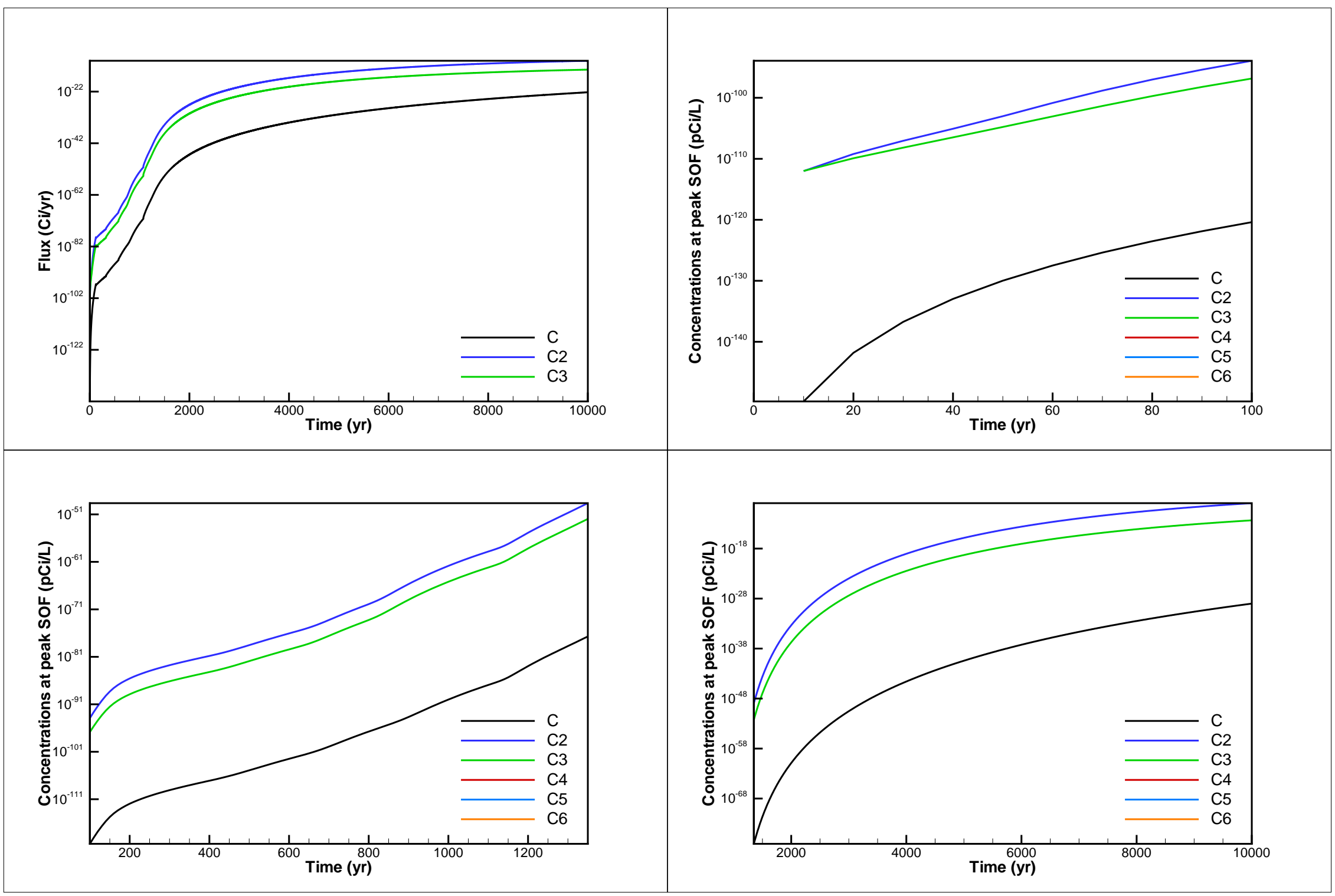

Figure A-28. Flux and concentration results for case Cm-248_0.01: Cm-248 Pu-244 Pu-244_56 


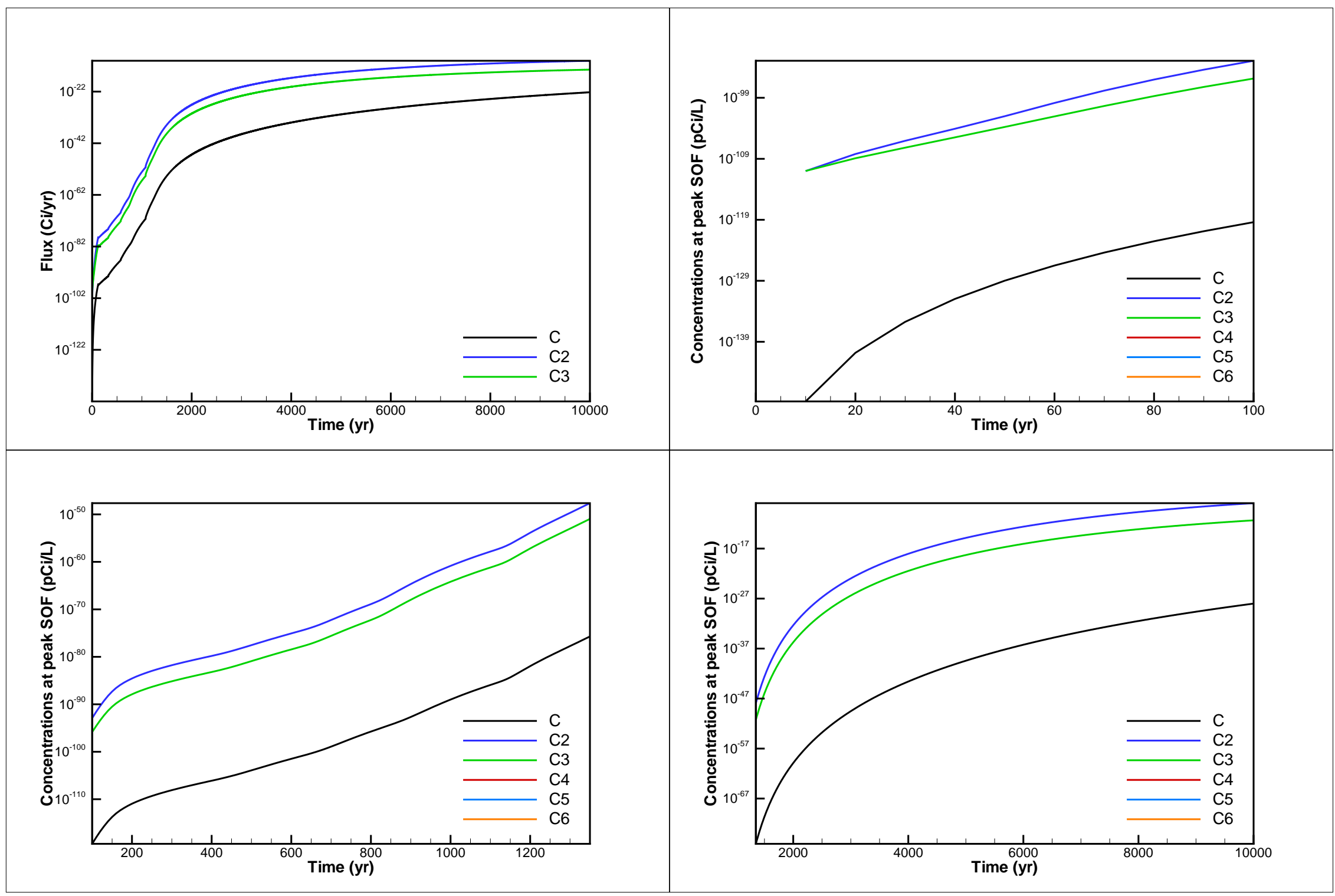

Figure A-29. Flux and concentration results for case Cm-248_0.1: Cm-248 Pu-244 Pu-244_56 


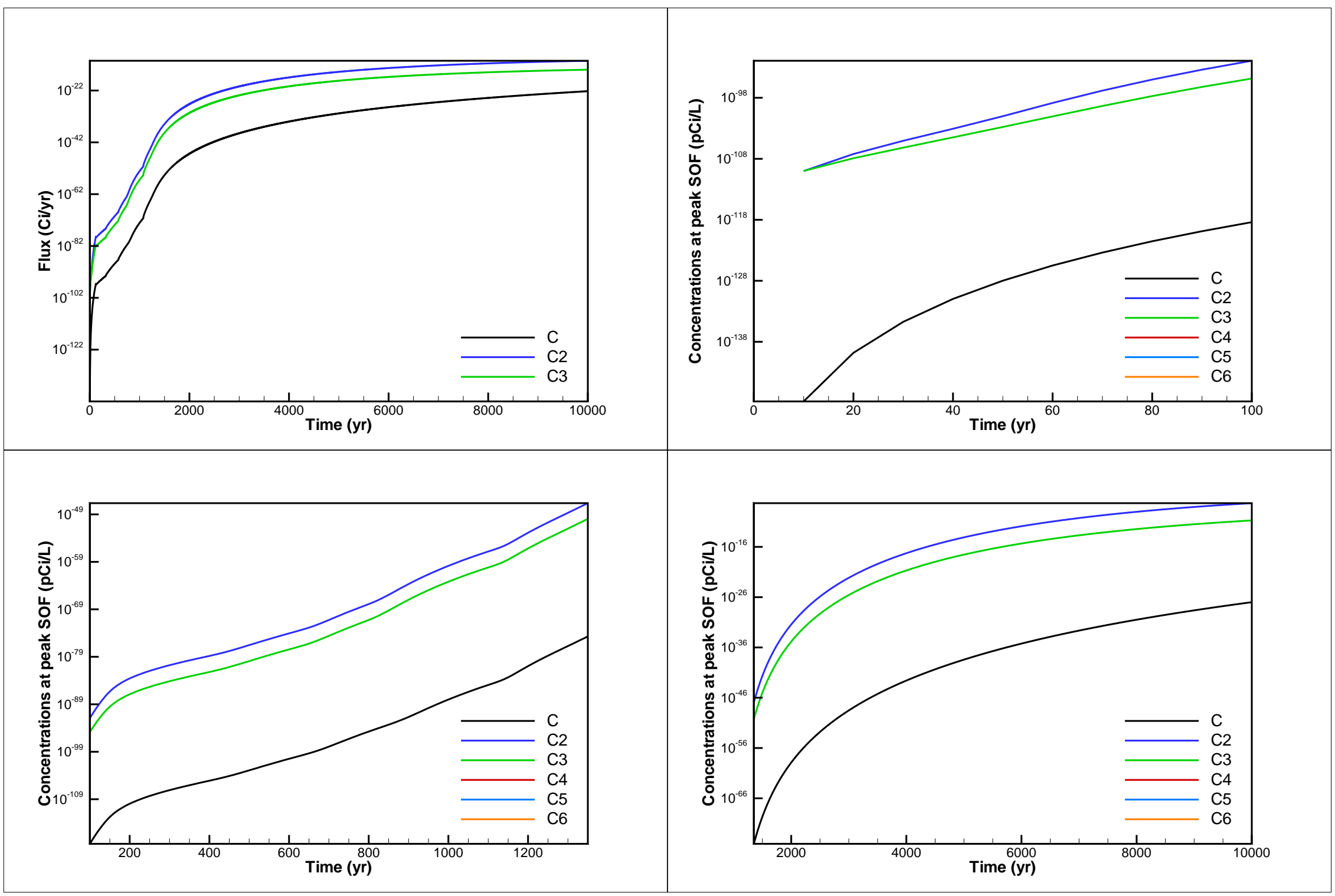

Figure A-30. Flux and concentration results for case Cm-248_1: Cm-248 Pu-244 Pu-244_56 


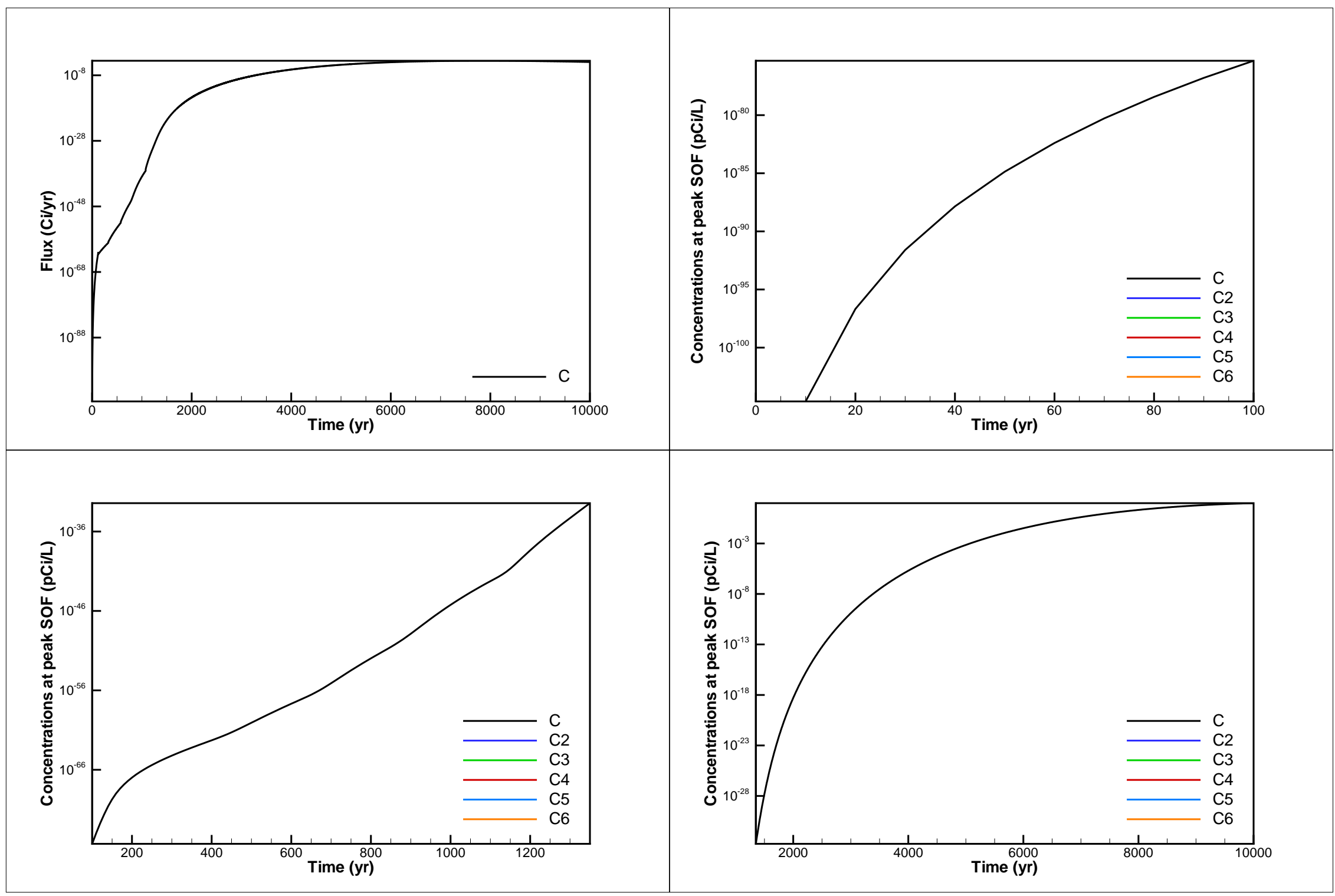

Figure A-31. Flux and concentration results for case Cs-135_1: Cs-135 


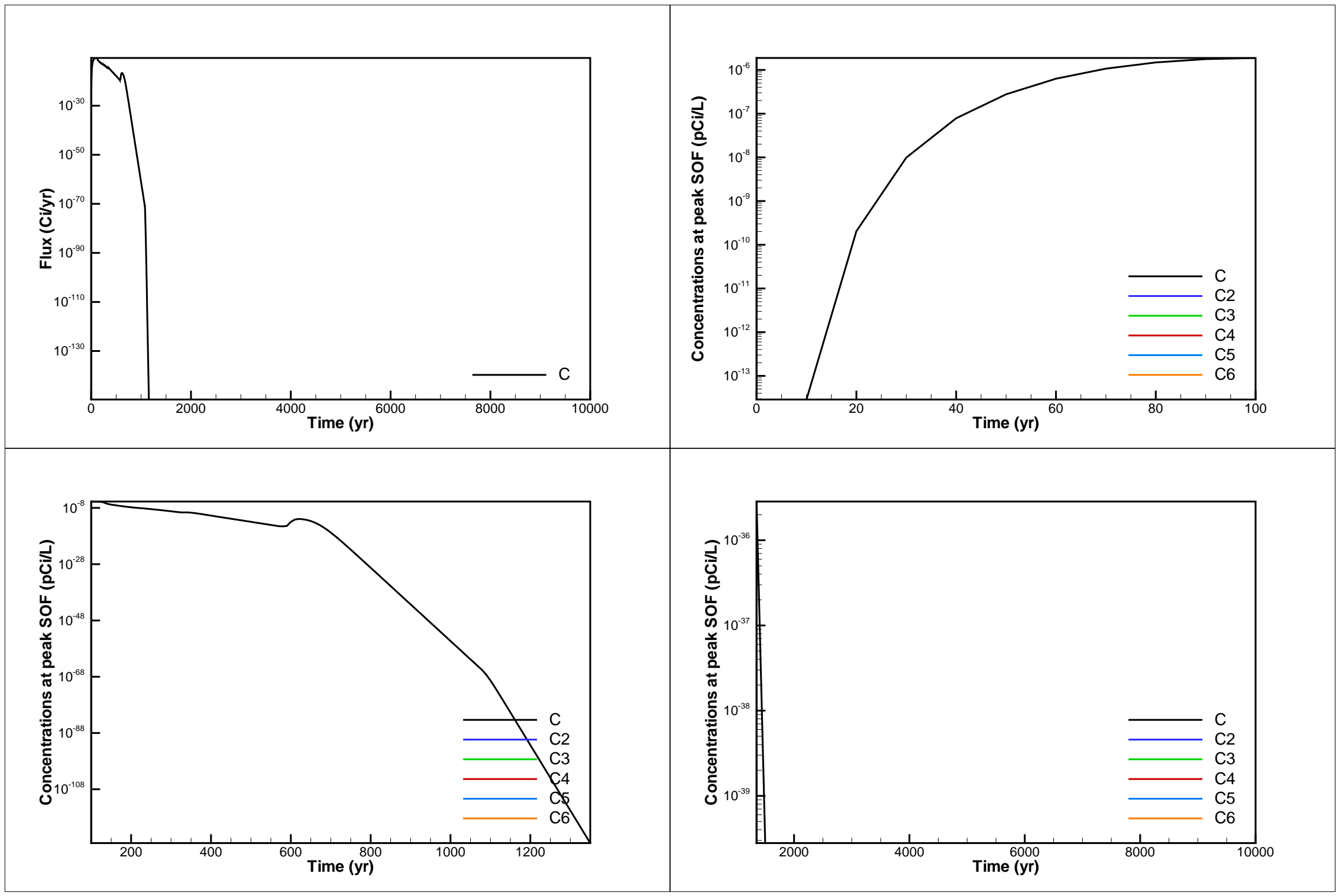

Figure A-32. Flux and concentration results for case H-3_1: H-3 


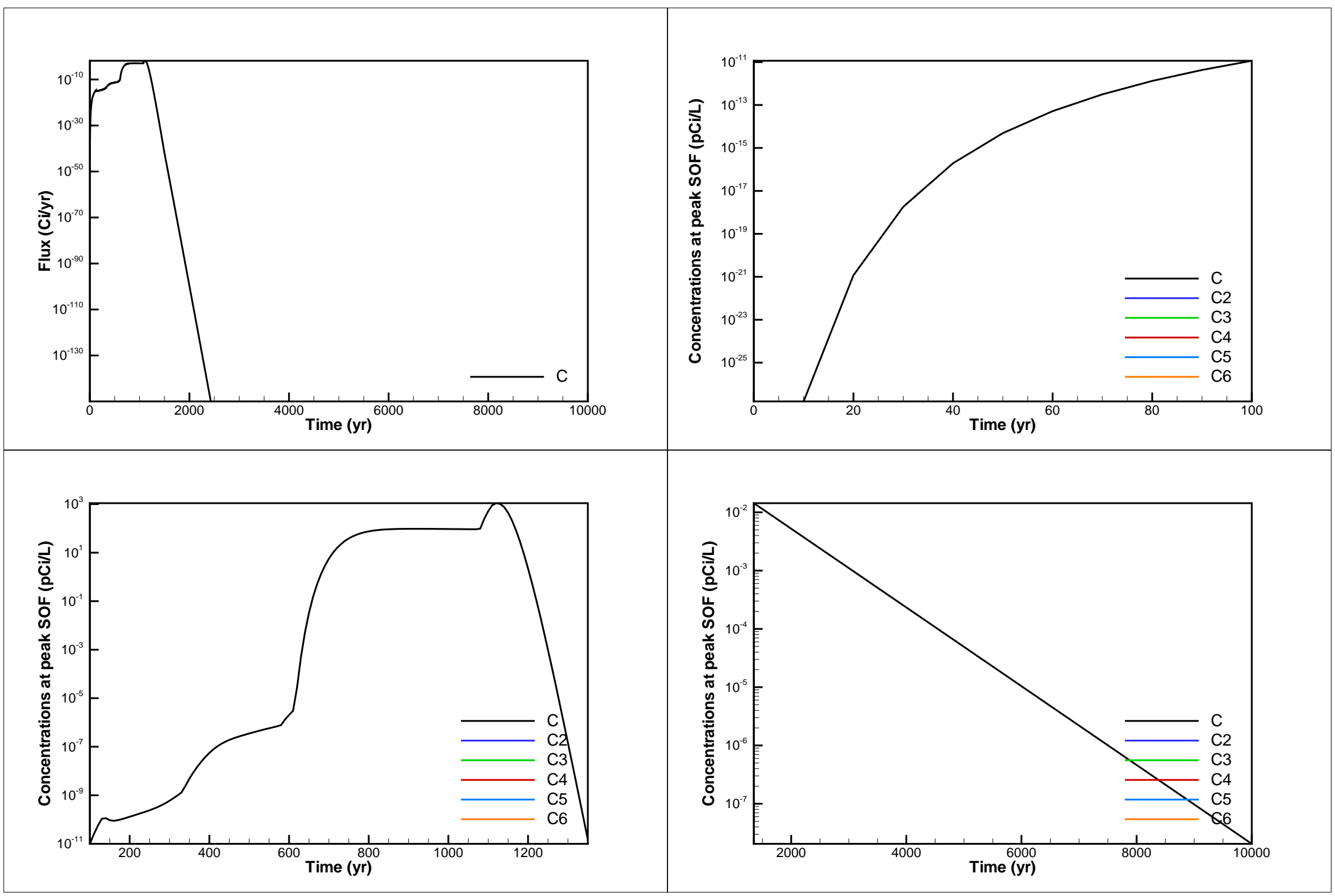

Figure A-33. Flux and concentration results for case I-129_1: I-129 


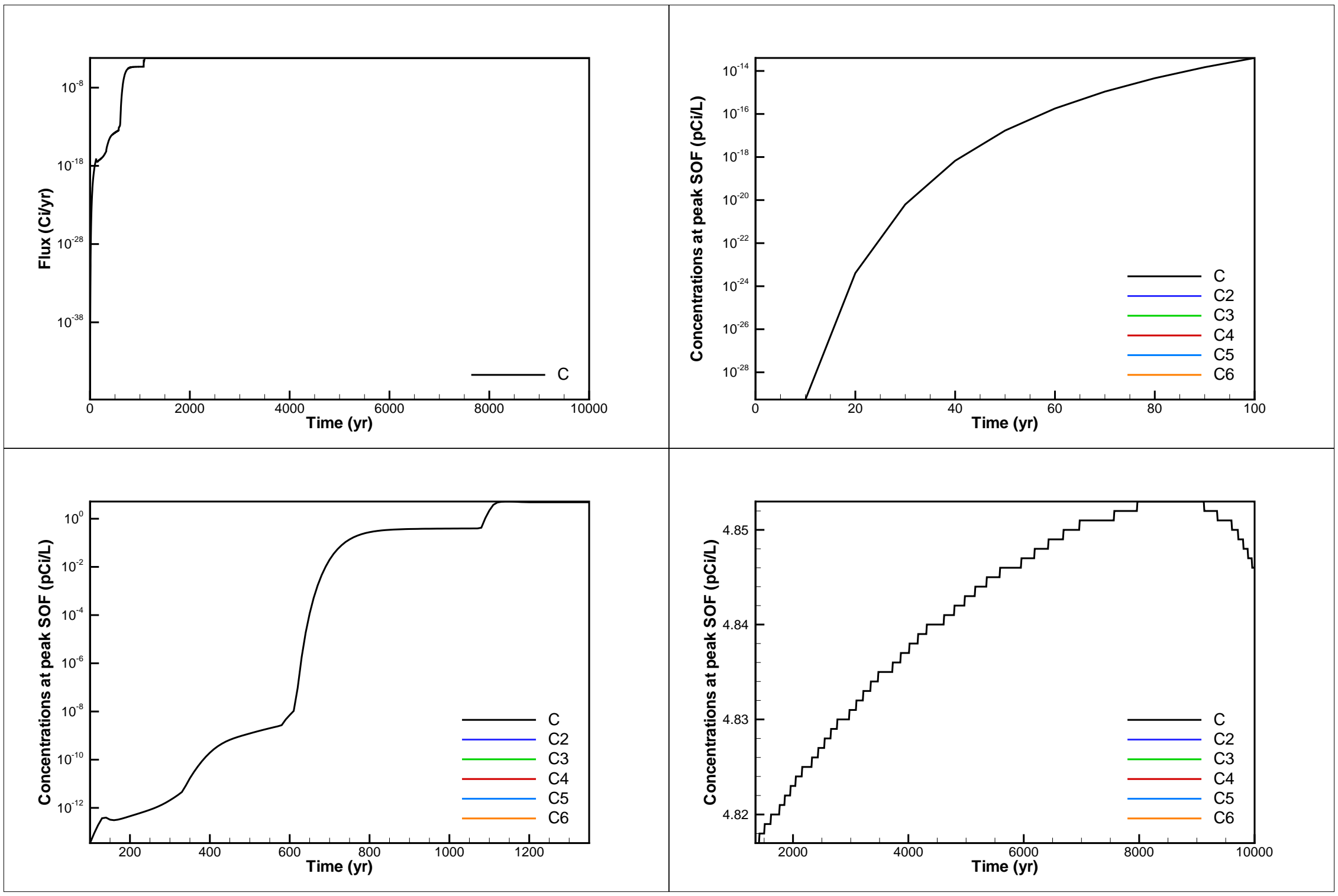

Figure A-34. Flux and concentration results for case I-129_ETF-Carbon_1: I-129_ETF-Carbon 


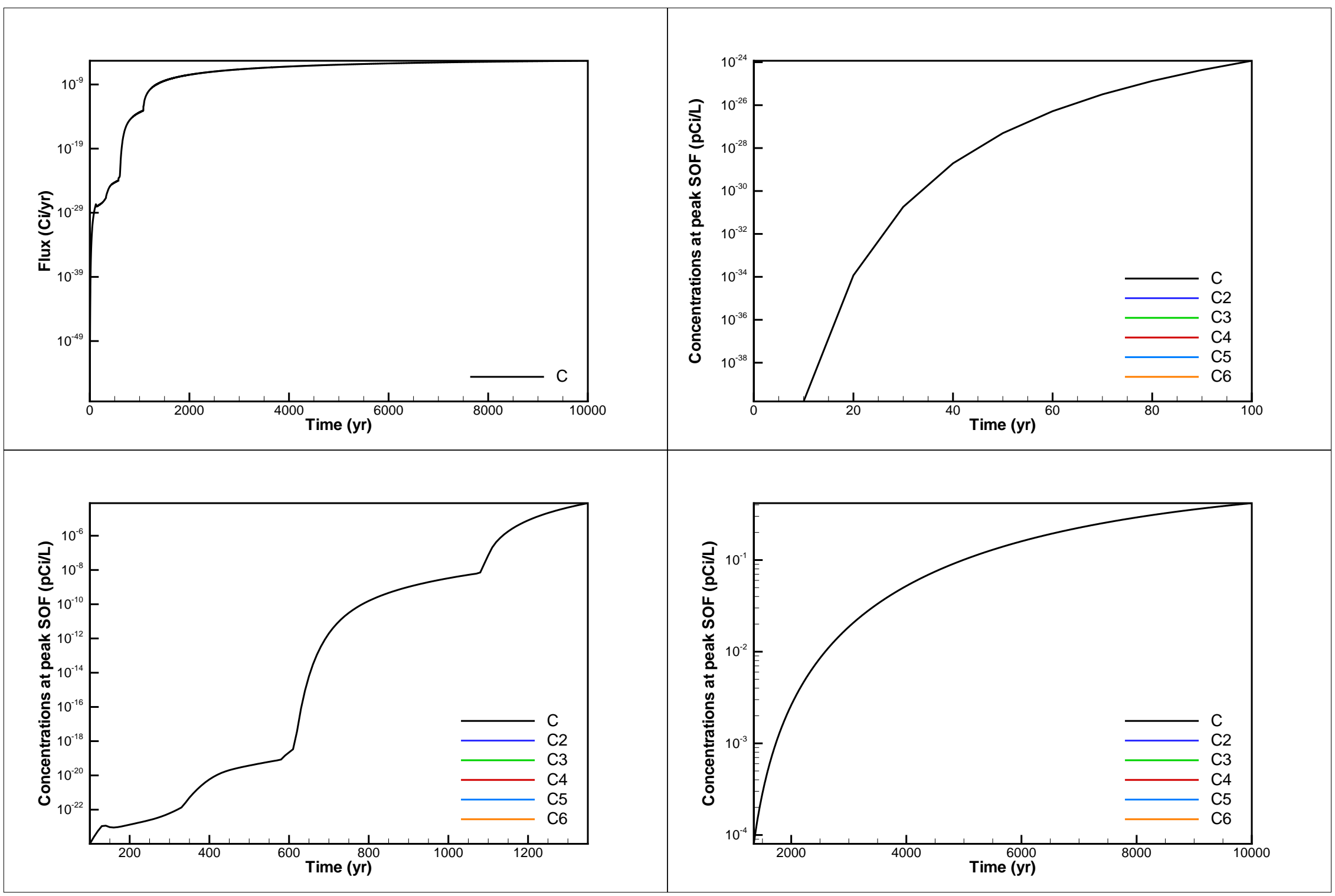

Figure A-35. Flux and concentration results for case I-129_KB_1: I-129_KB 


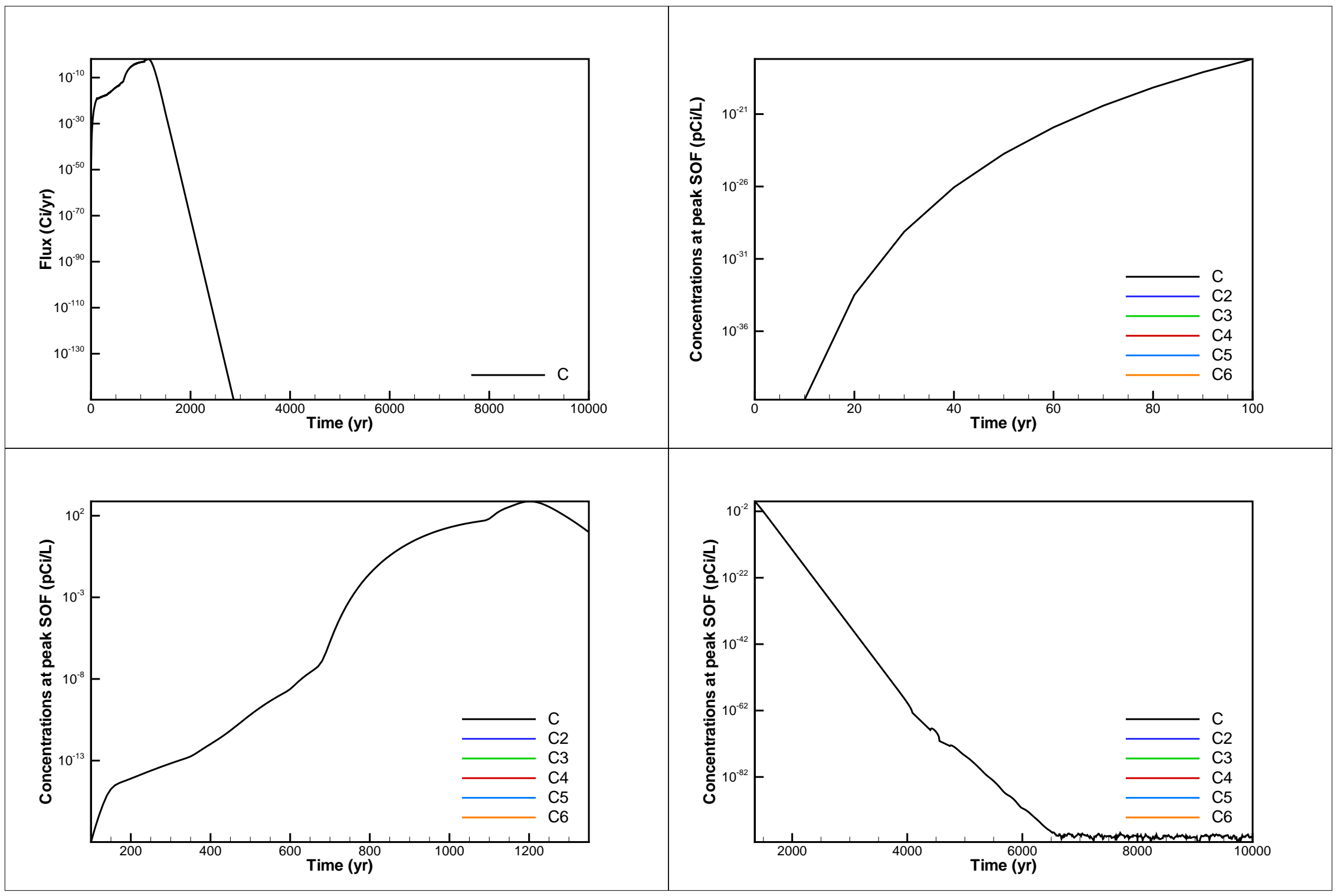

Figure A-36. Flux and concentration results for case K-40_1: K-40 


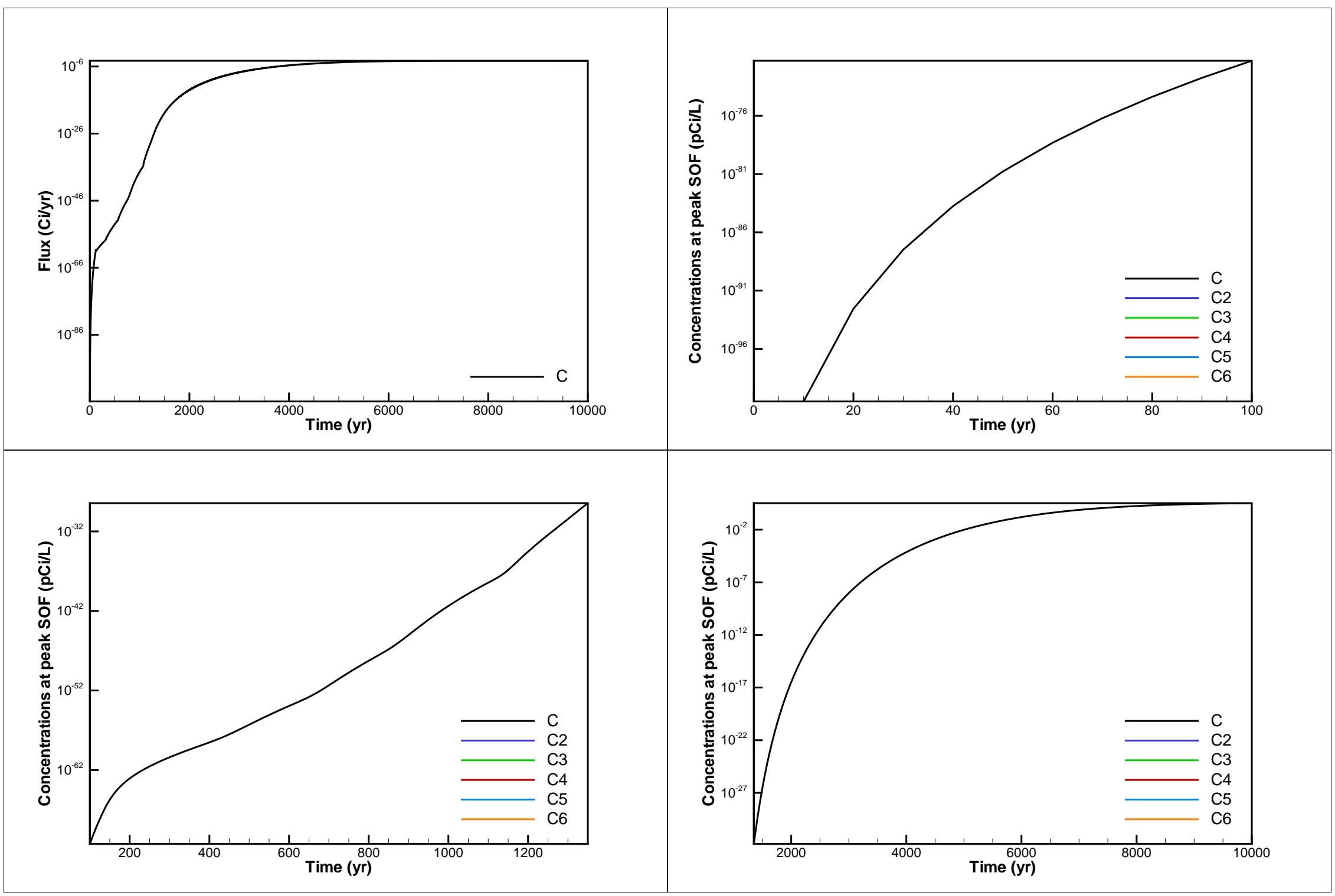

Figure A-37. Flux and concentration results for case Nb-94_1: Nb-94 


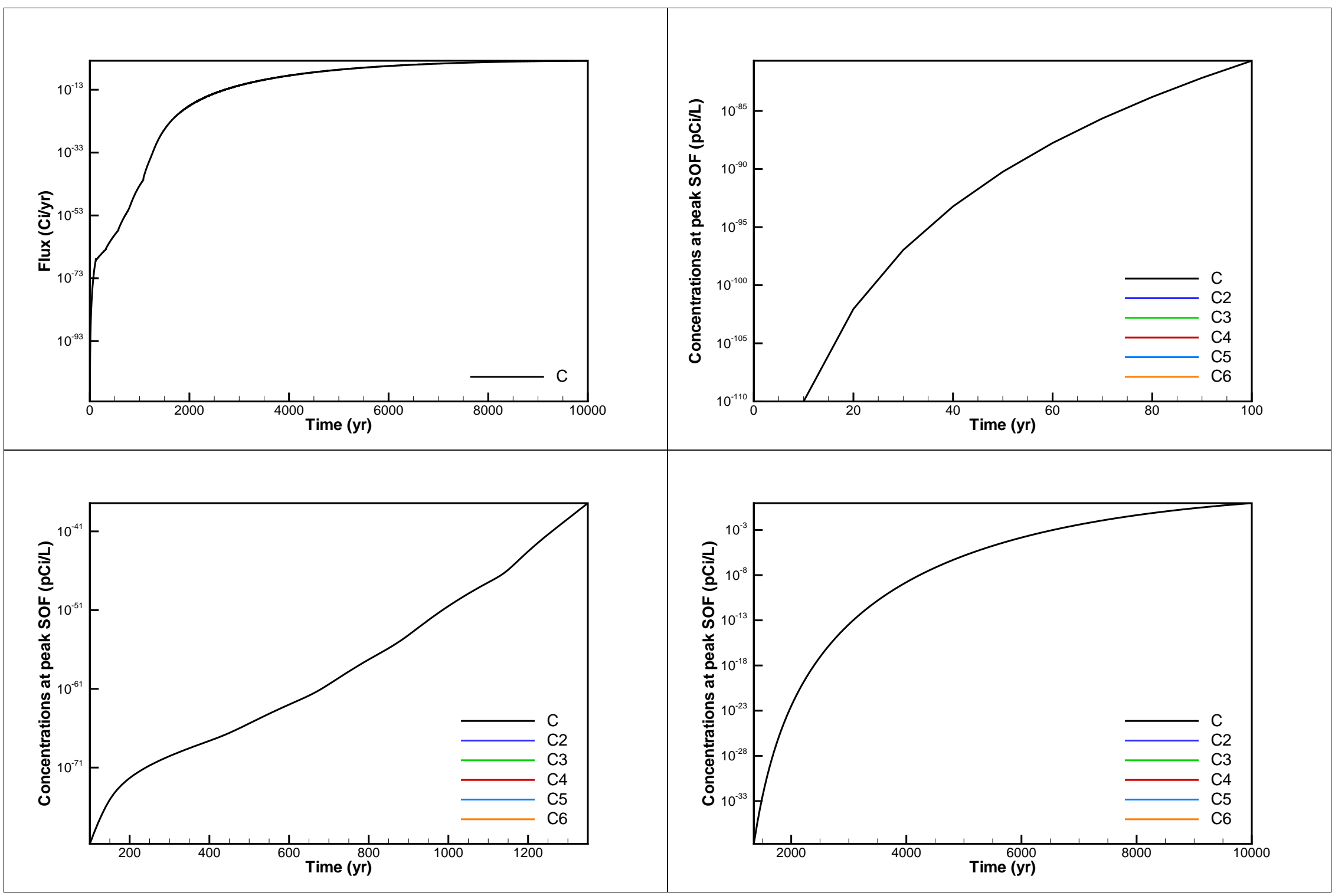

Figure A-38. Flux and concentration results for case Ni-59_1: Ni-59 


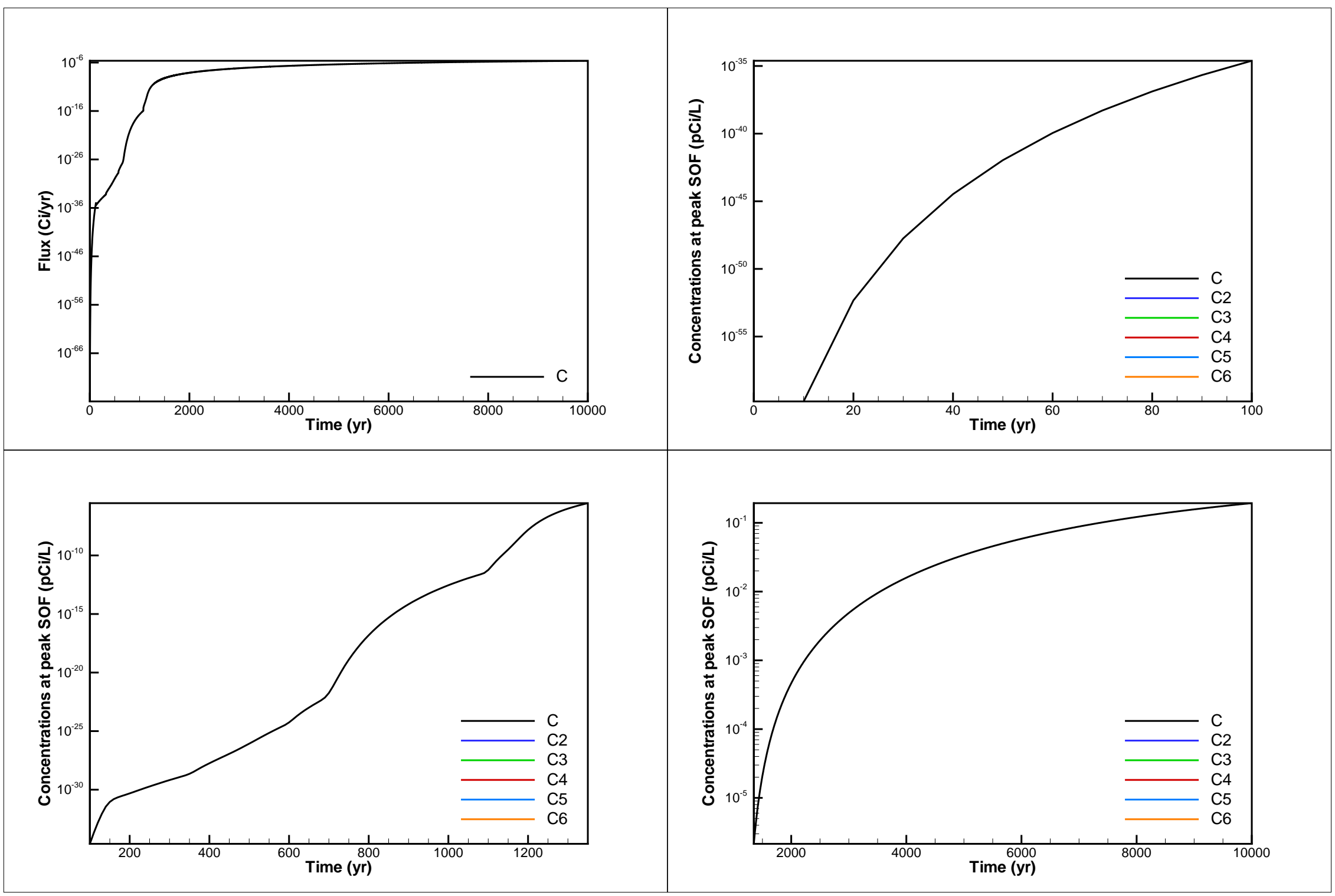

Figure A-39. Flux and concentration results for case Np-237_1: Np-237 


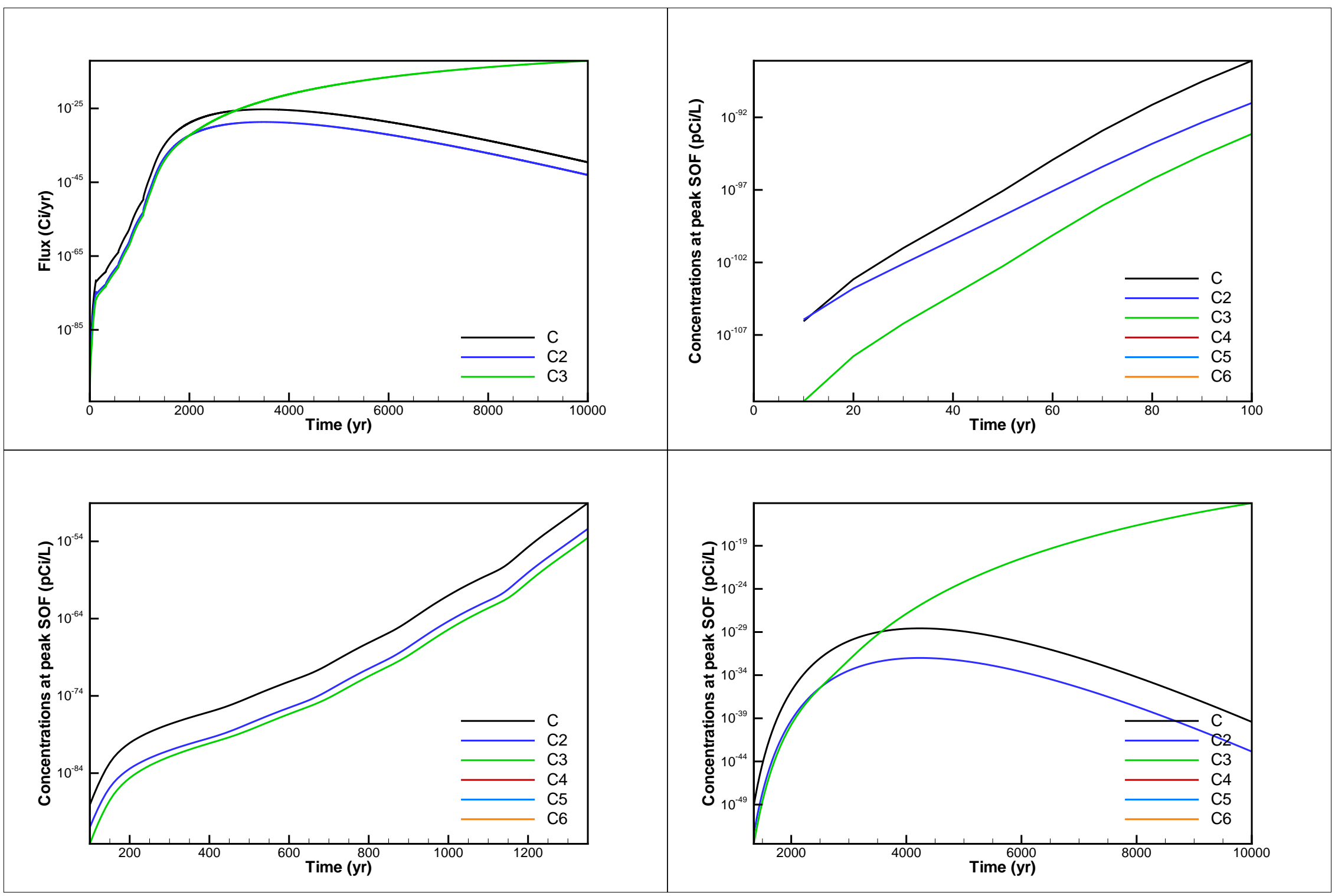

Figure A-40. Flux and concentration results for case Pu-238_0.001: Pu-238 Pu-238_56 U-234 


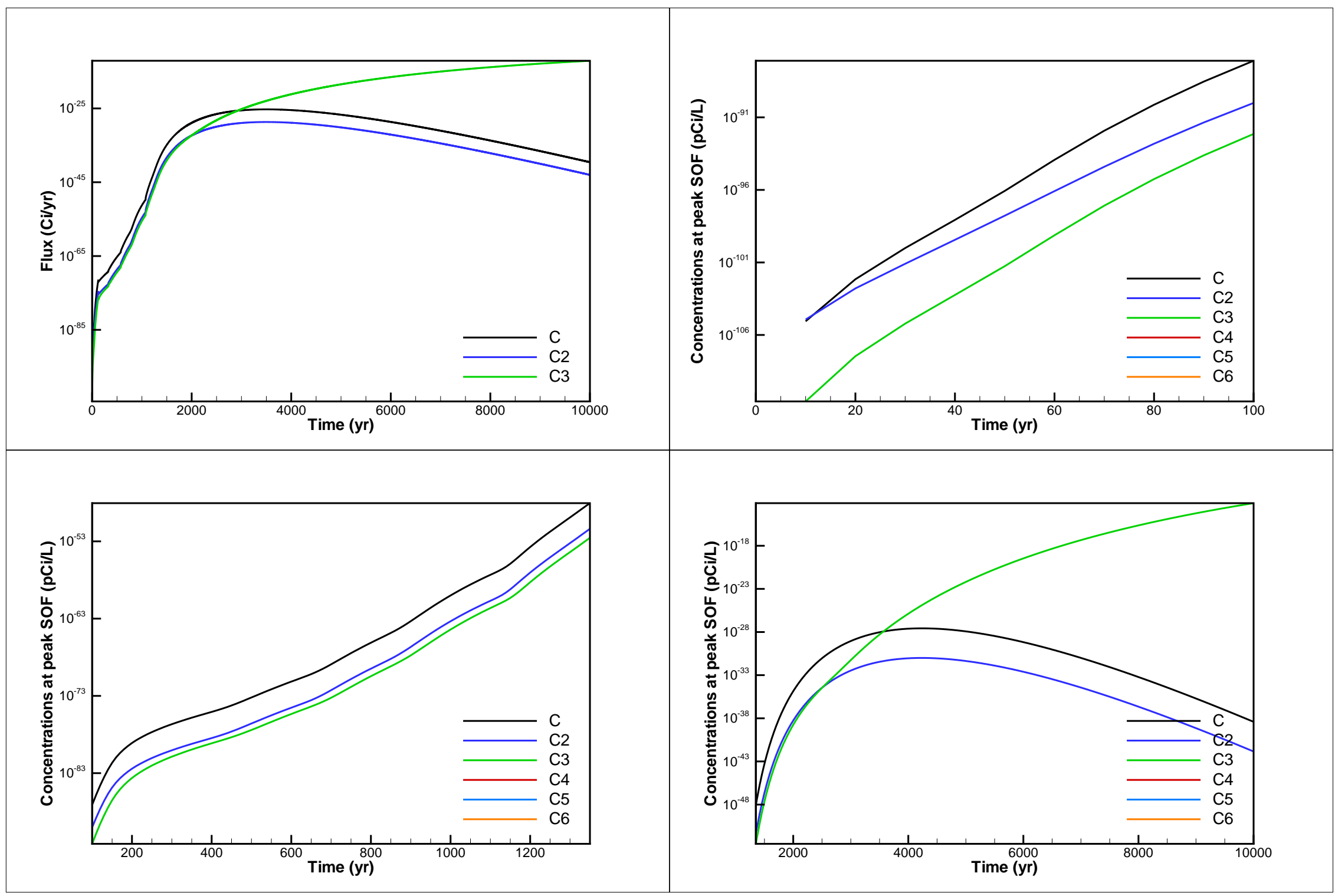

Figure A-41. Flux and concentration results for case Pu-238_0.01: Pu-238 Pu-238_56 U-234 


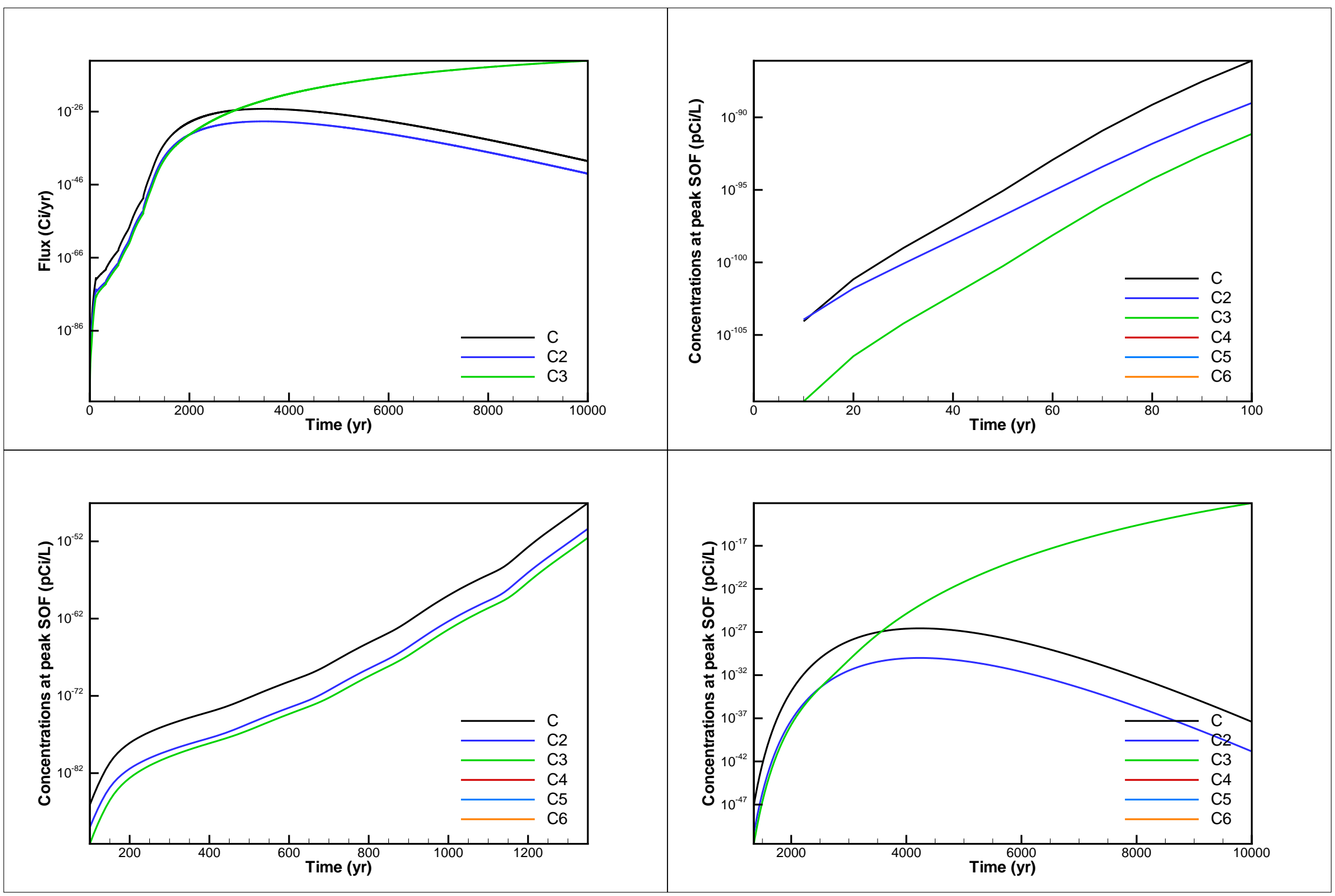

Figure A-42. Flux and concentration results for case Pu-238_0.1: Pu-238 Pu-238_56 U-234 


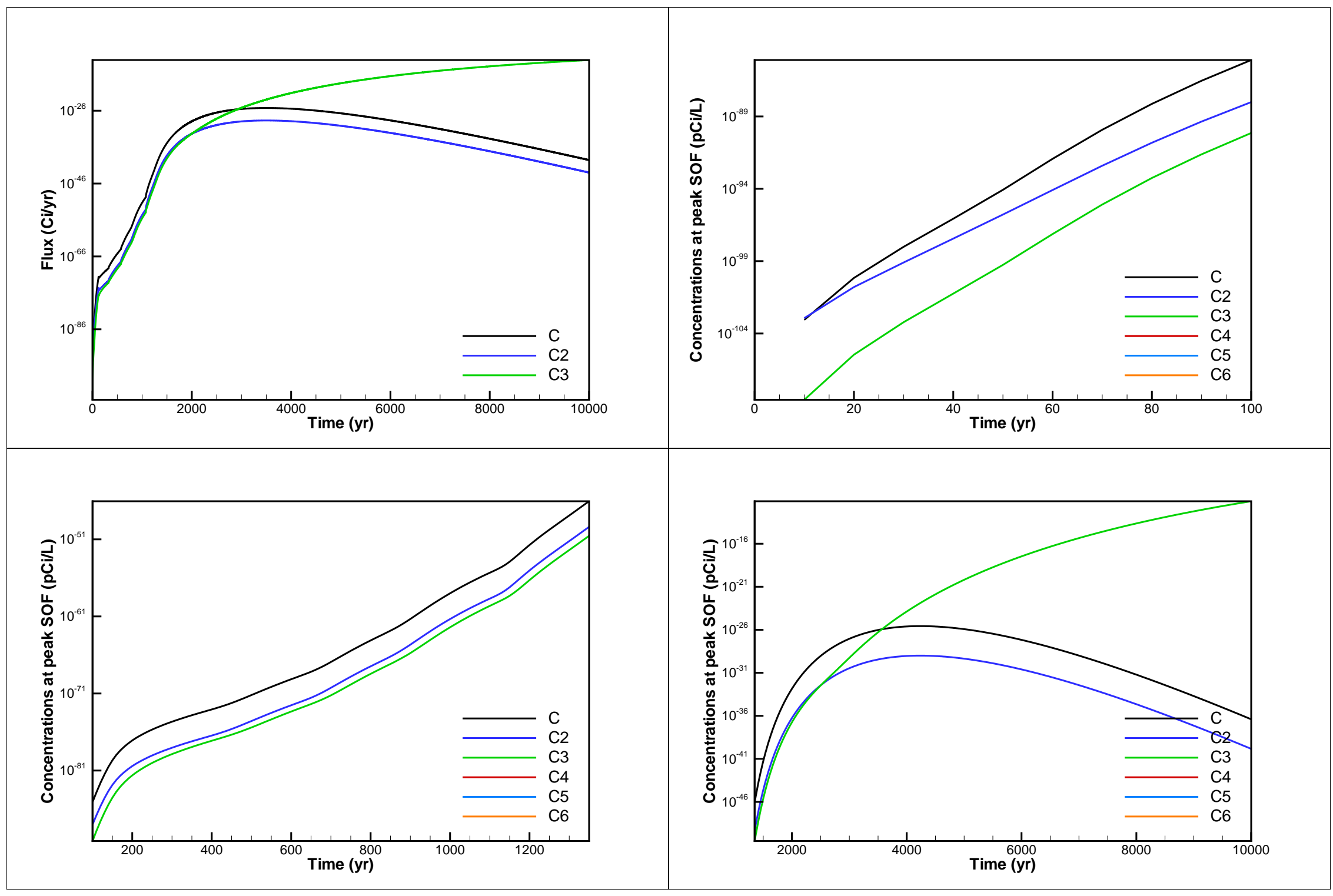

Figure A-43. Flux and concentration results for case Pu-238_1: Pu-238 Pu-238_56 U-234 


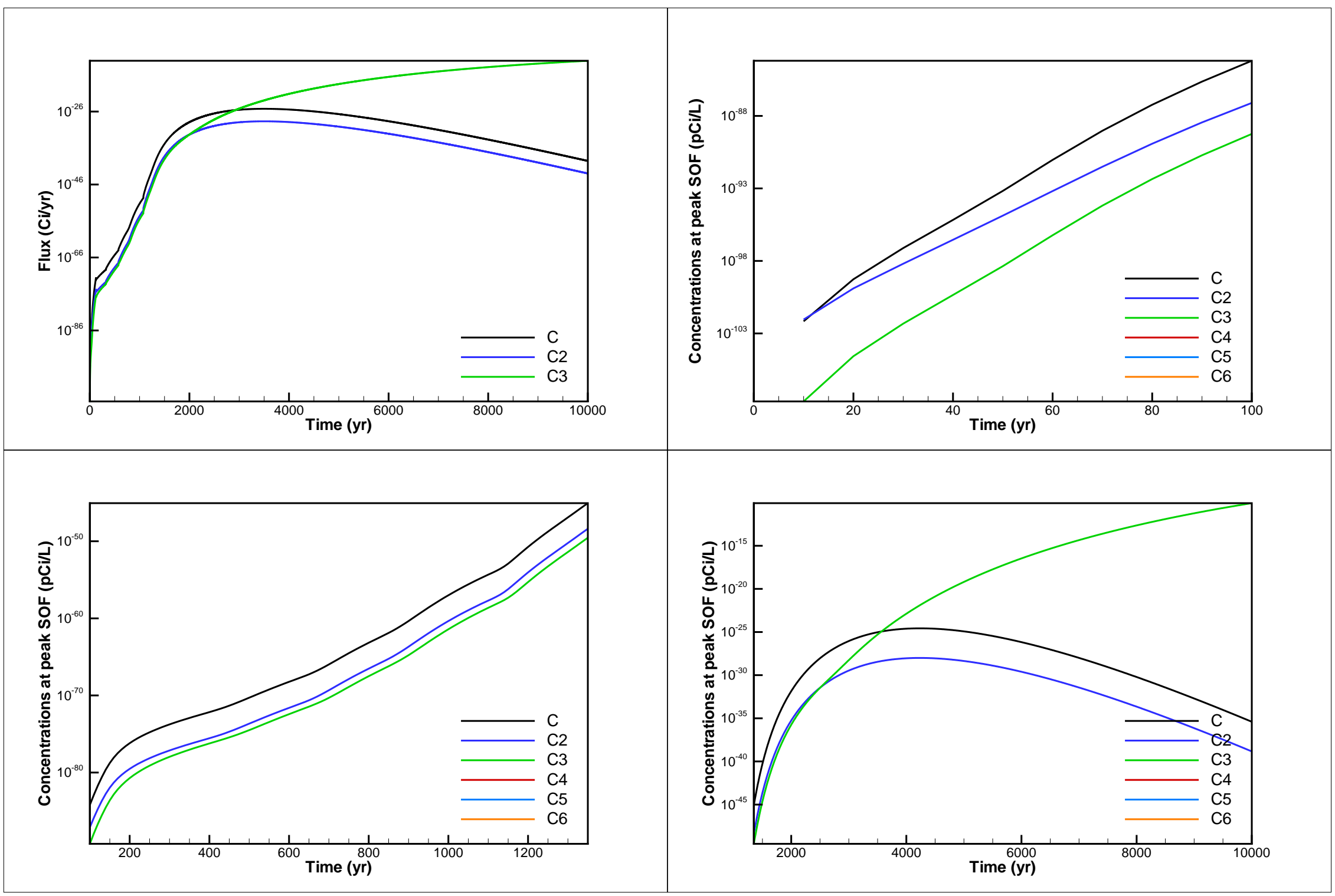

Figure A-44. Flux and concentration results for case Pu-238_10: Pu-238 Pu-238_56 U-234 


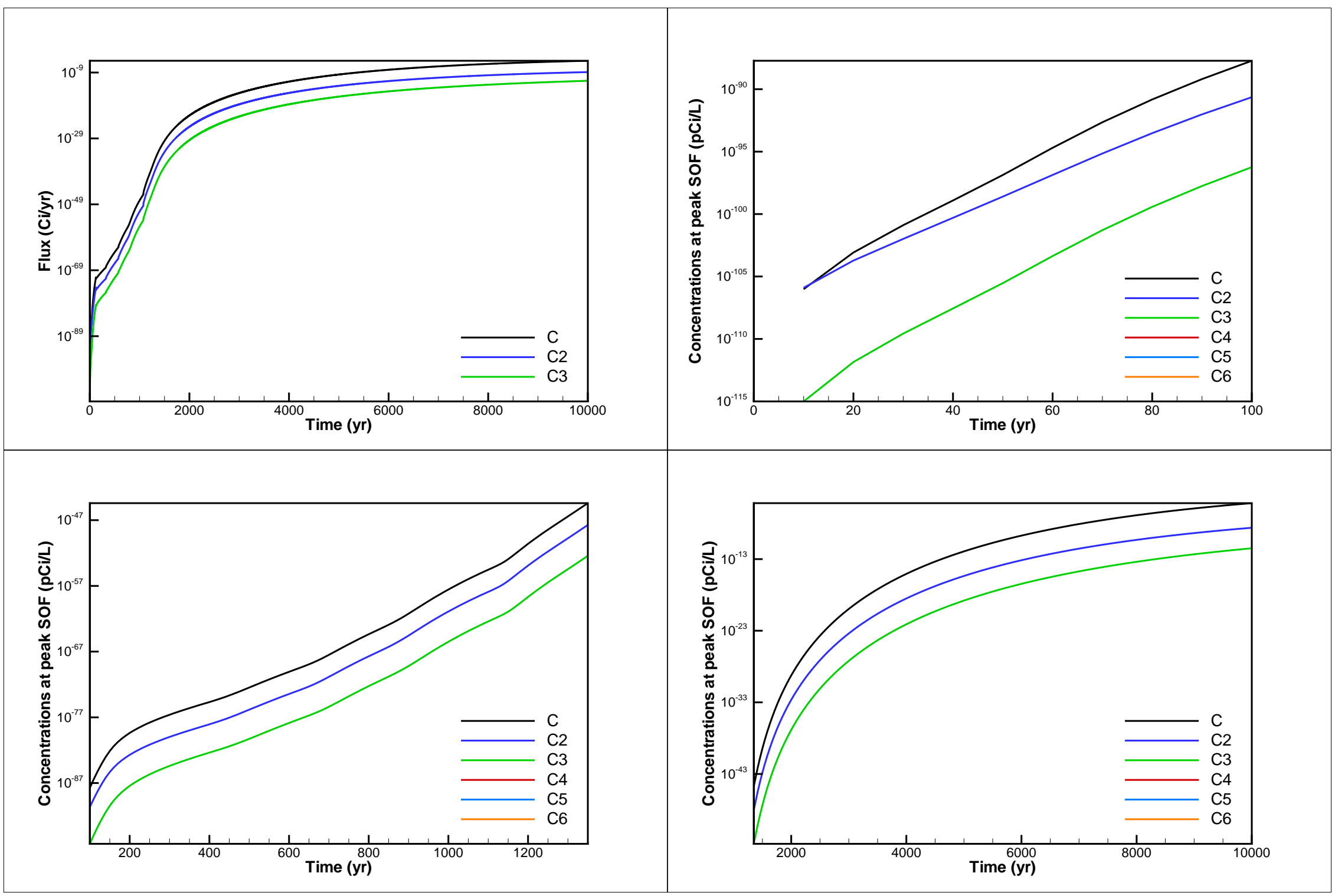

Figure A-45. Flux and concentration results for case Pu-239_0.001: Pu-239 Pu-239_56 U-235 


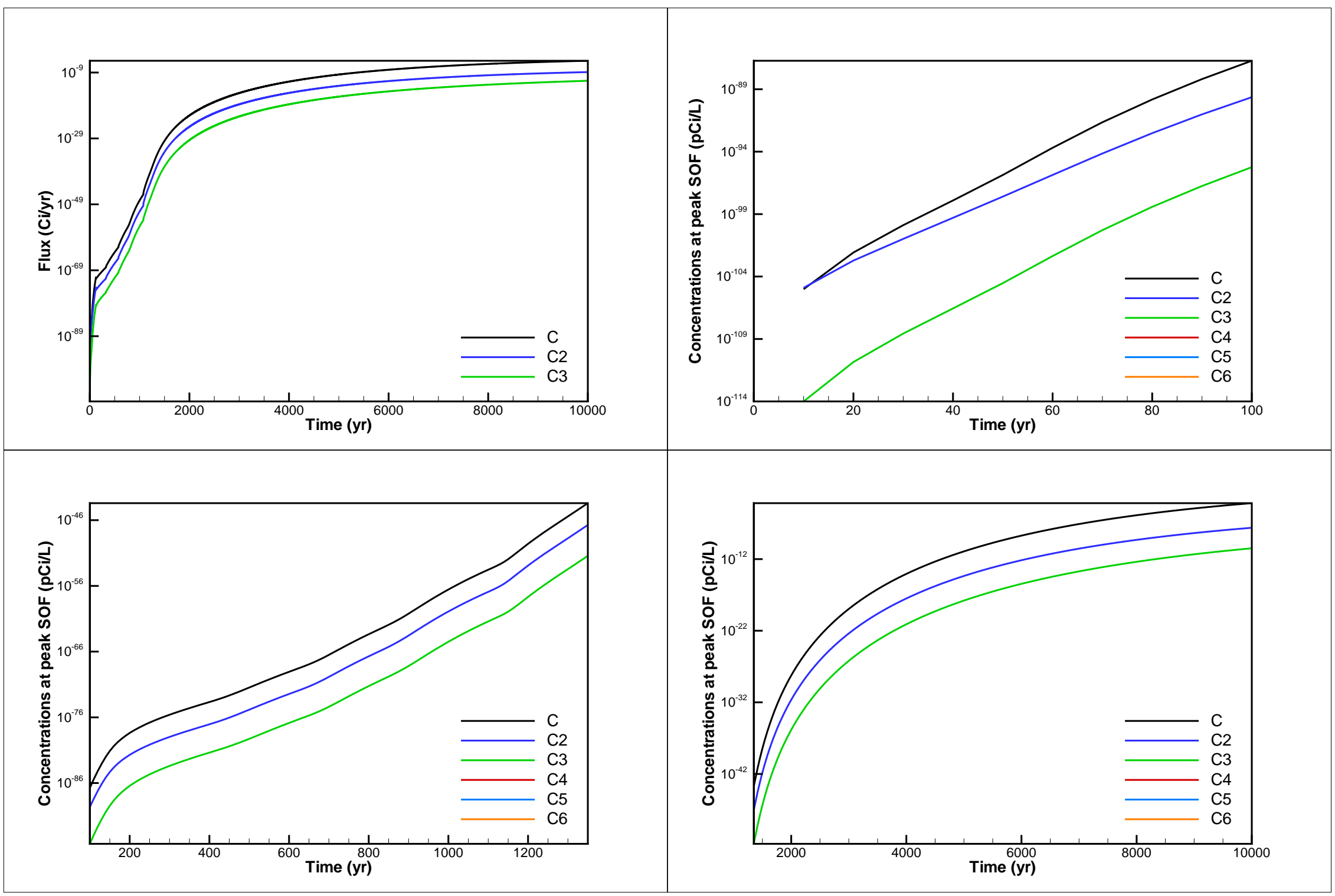

Figure A-46. Flux and concentration results for case Pu-239_0.01: Pu-239 Pu-239_56 U-235 


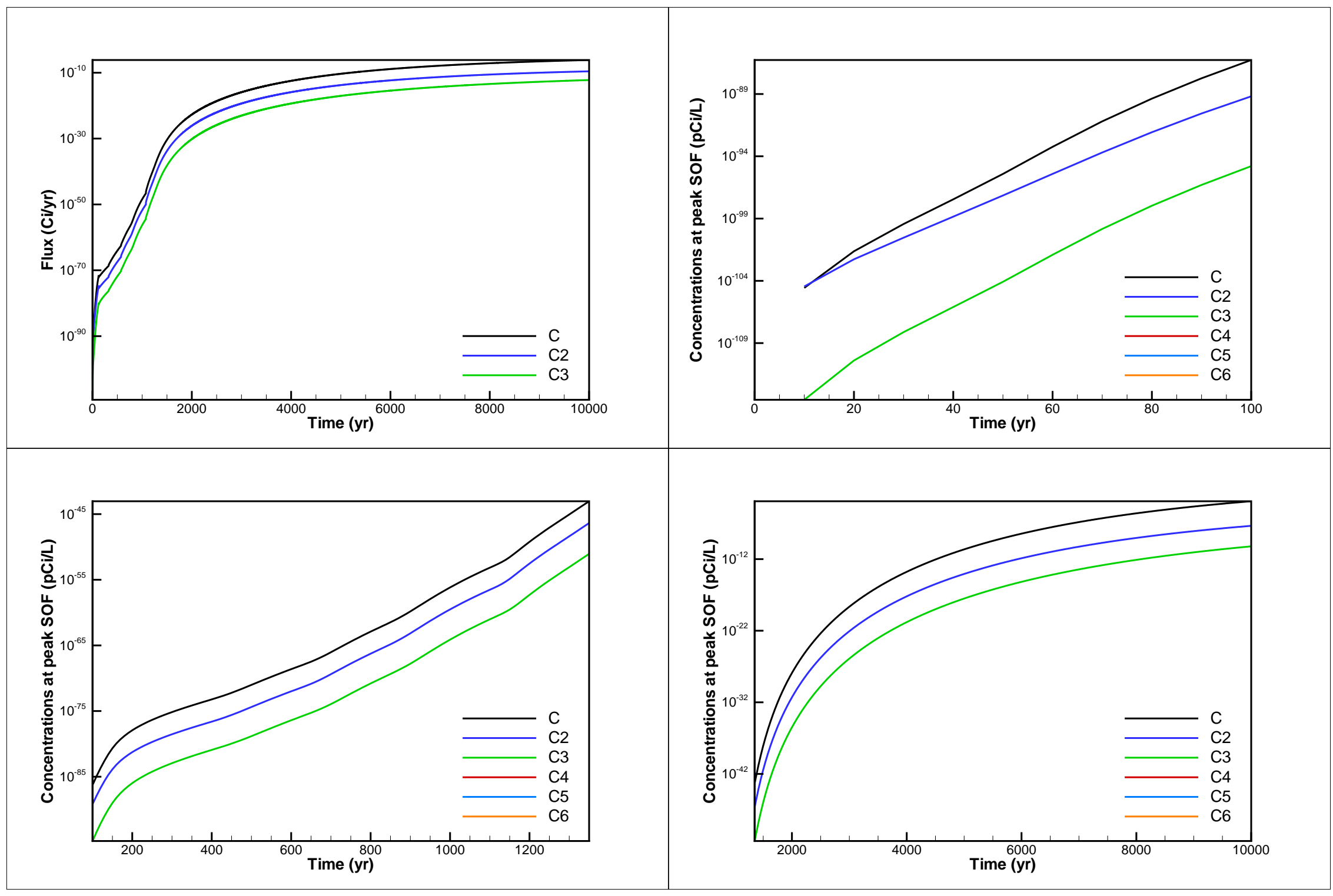

Figure A-47. Flux and concentration results for case Pu-239_0.1: Pu-239 Pu-239_56 U-235 


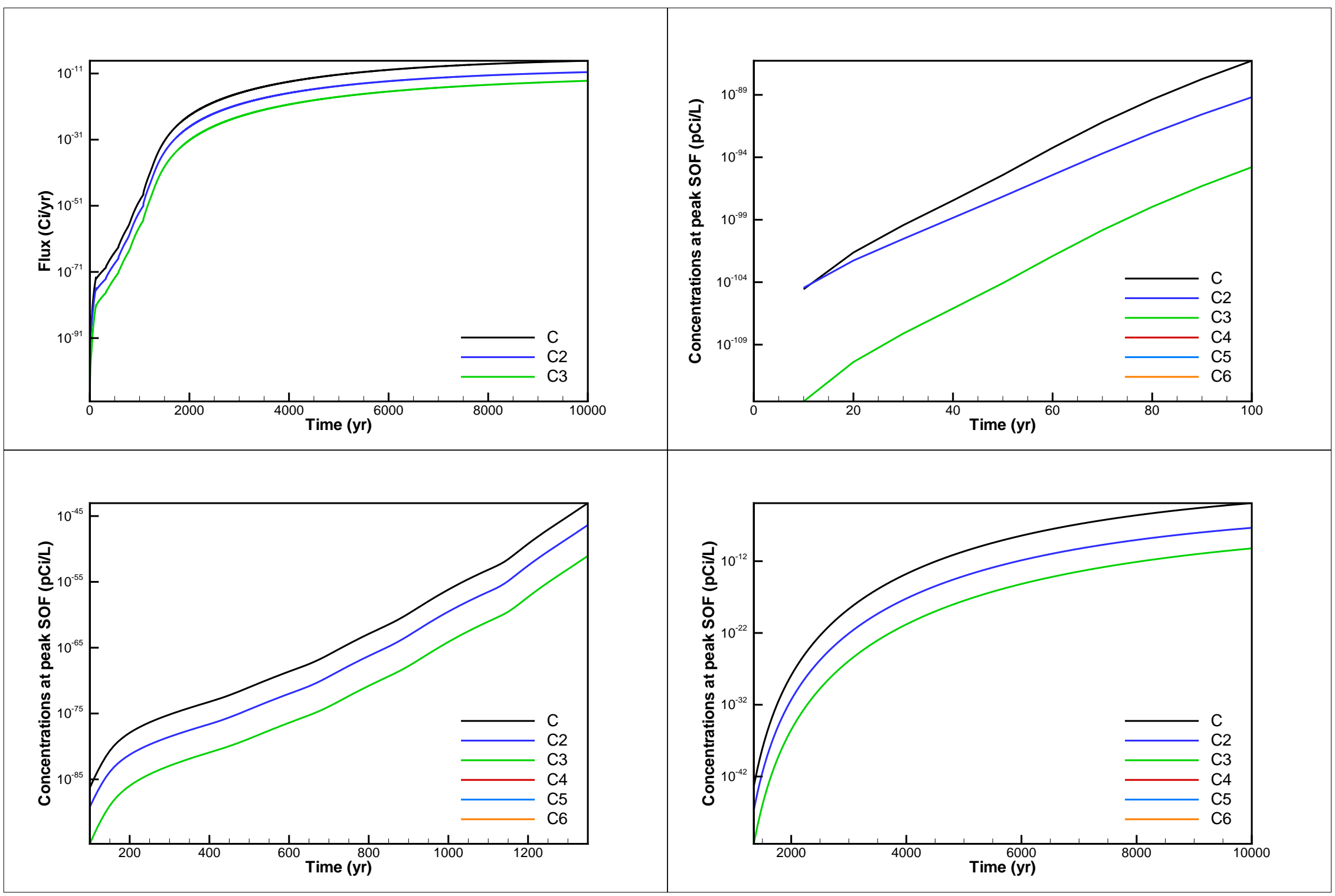

Figure A-48. Flux and concentration results for case Pu-239_1: Pu-239 Pu-239_56 U-235 


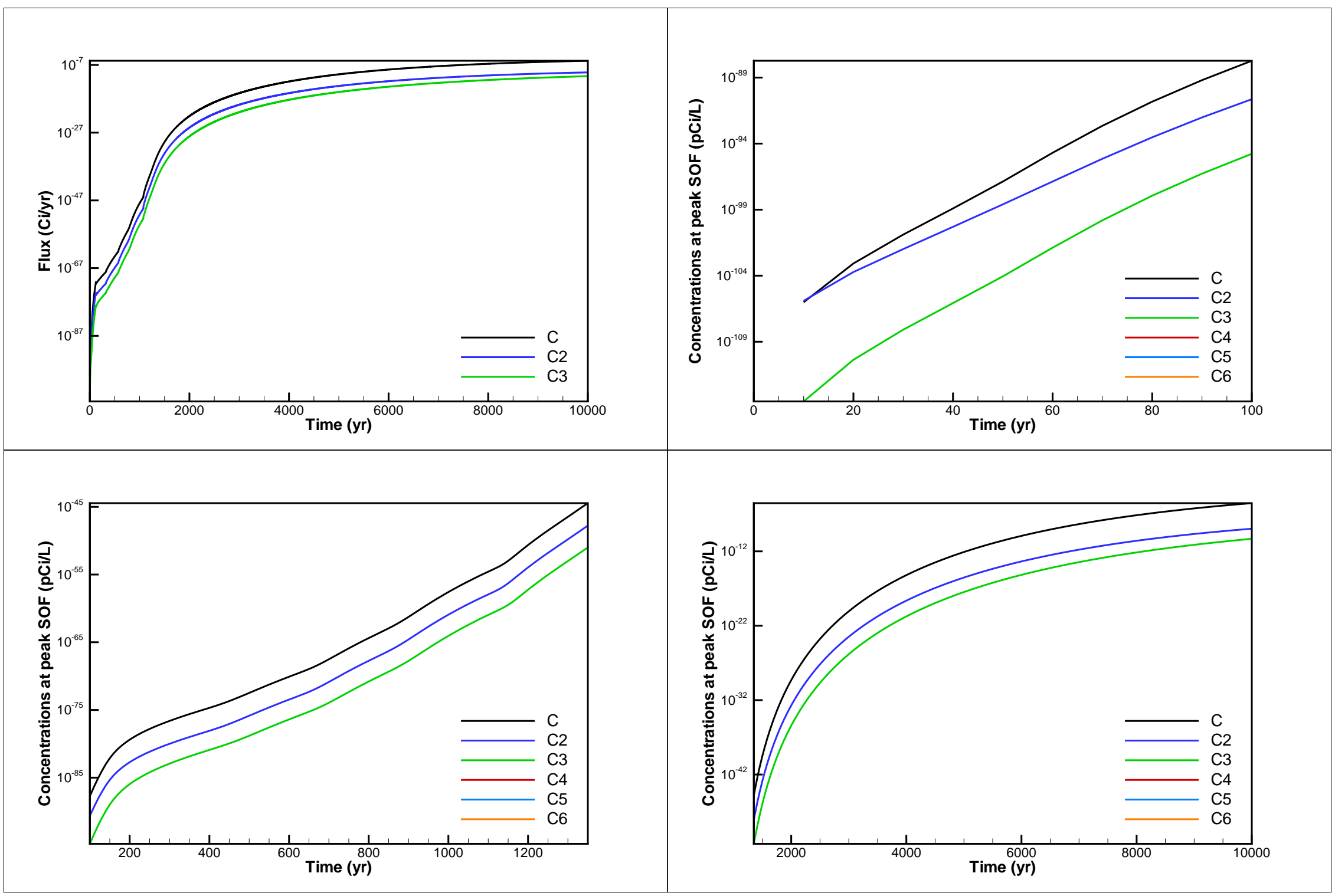

Figure A-49. Flux and concentration results for case Pu-240_0.001: Pu-240 Pu-240_56 U-236 


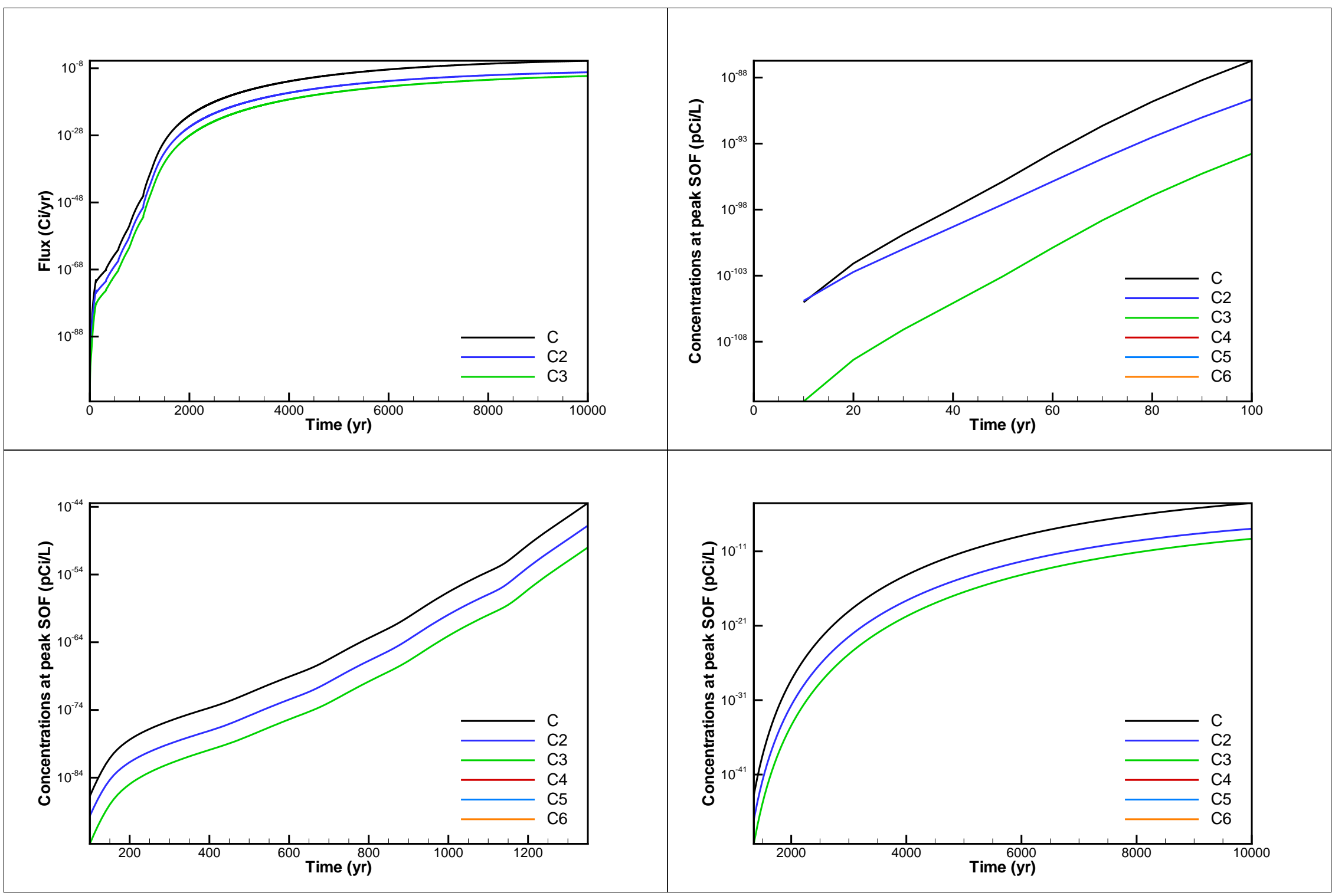

Figure A-50. Flux and concentration results for case Pu-240_0.01: Pu-240 Pu-240_56 U-236 


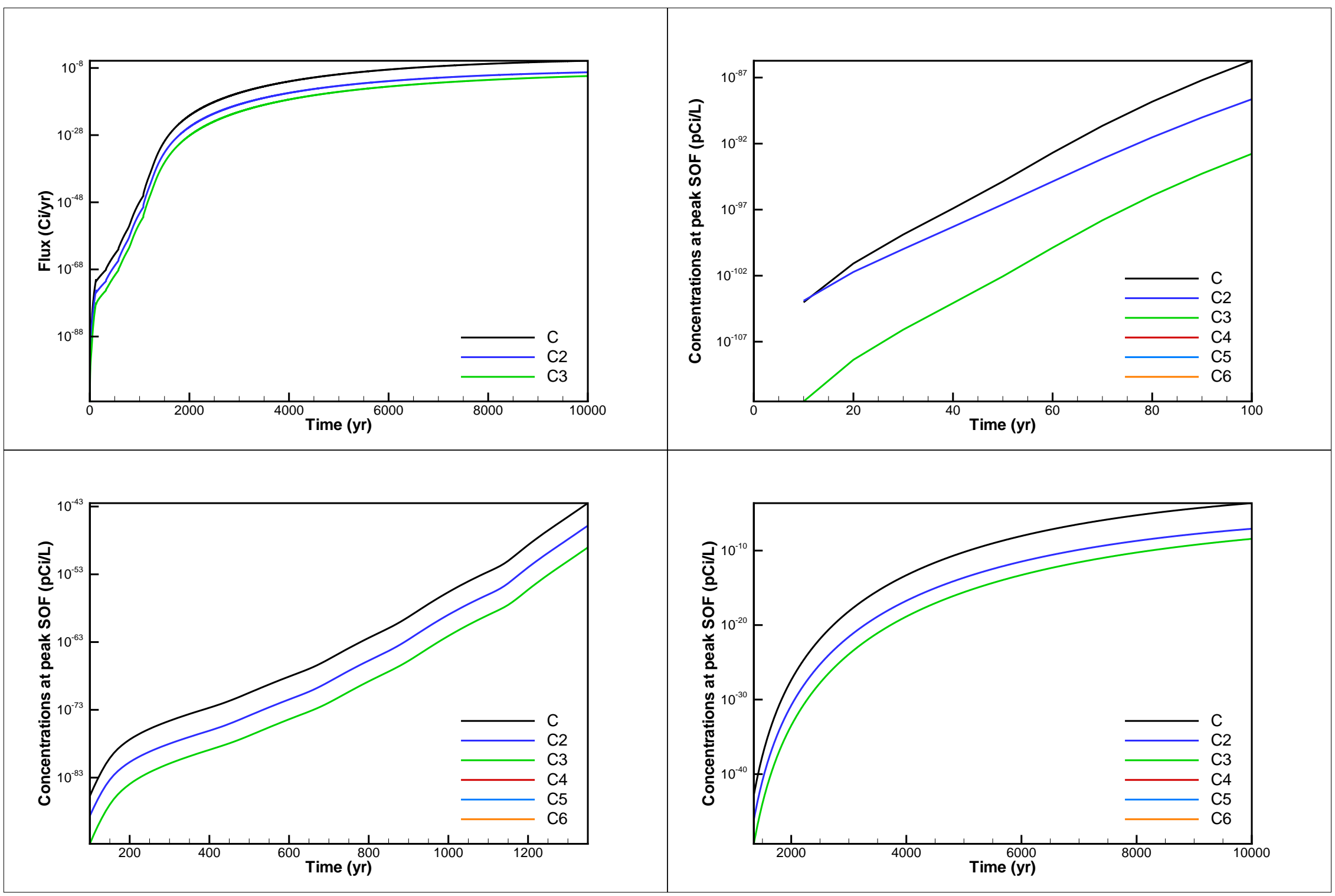

Figure A-51. Flux and concentration results for case Pu-240_0.1: Pu-240 Pu-240_56 U-236 


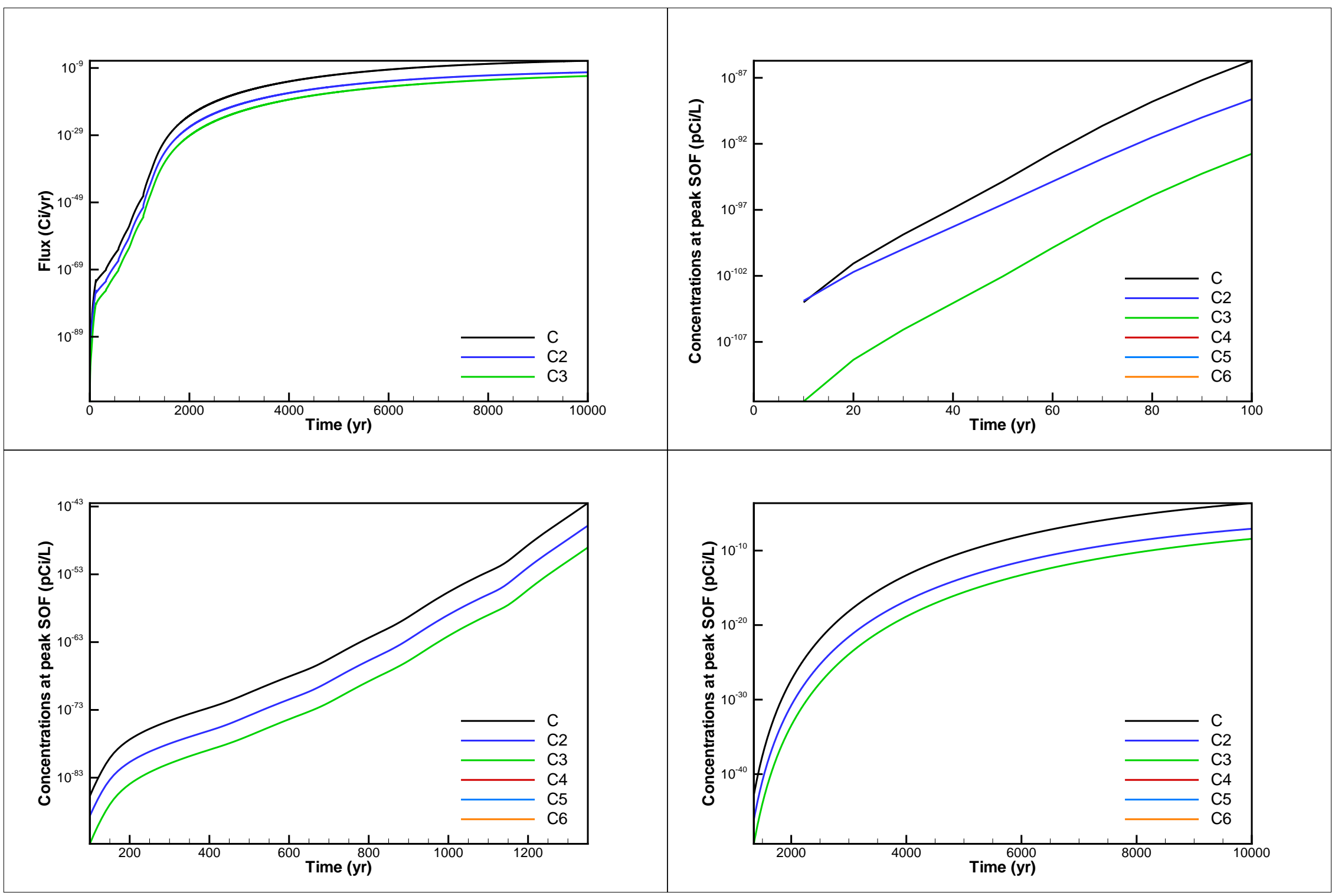

Figure A-52. Flux and concentration results for case Pu-240_1: Pu-240 Pu-240_56 U-236 


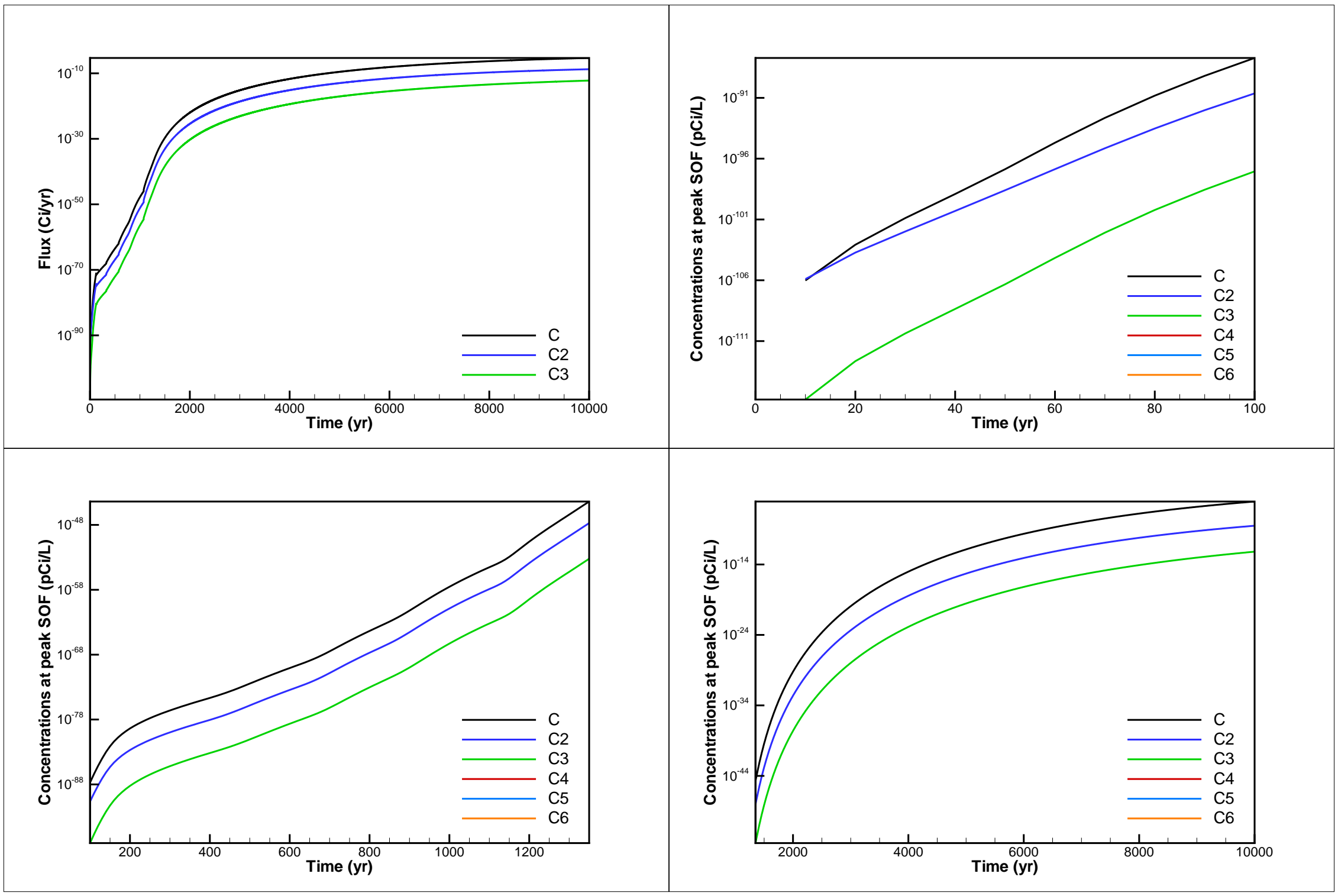

Figure A-53. Flux and concentration results for case Pu-242_0.001: $\mathrm{Pu}-242 \mathrm{Pu}-242 \_56 \mathrm{U}-238$ 


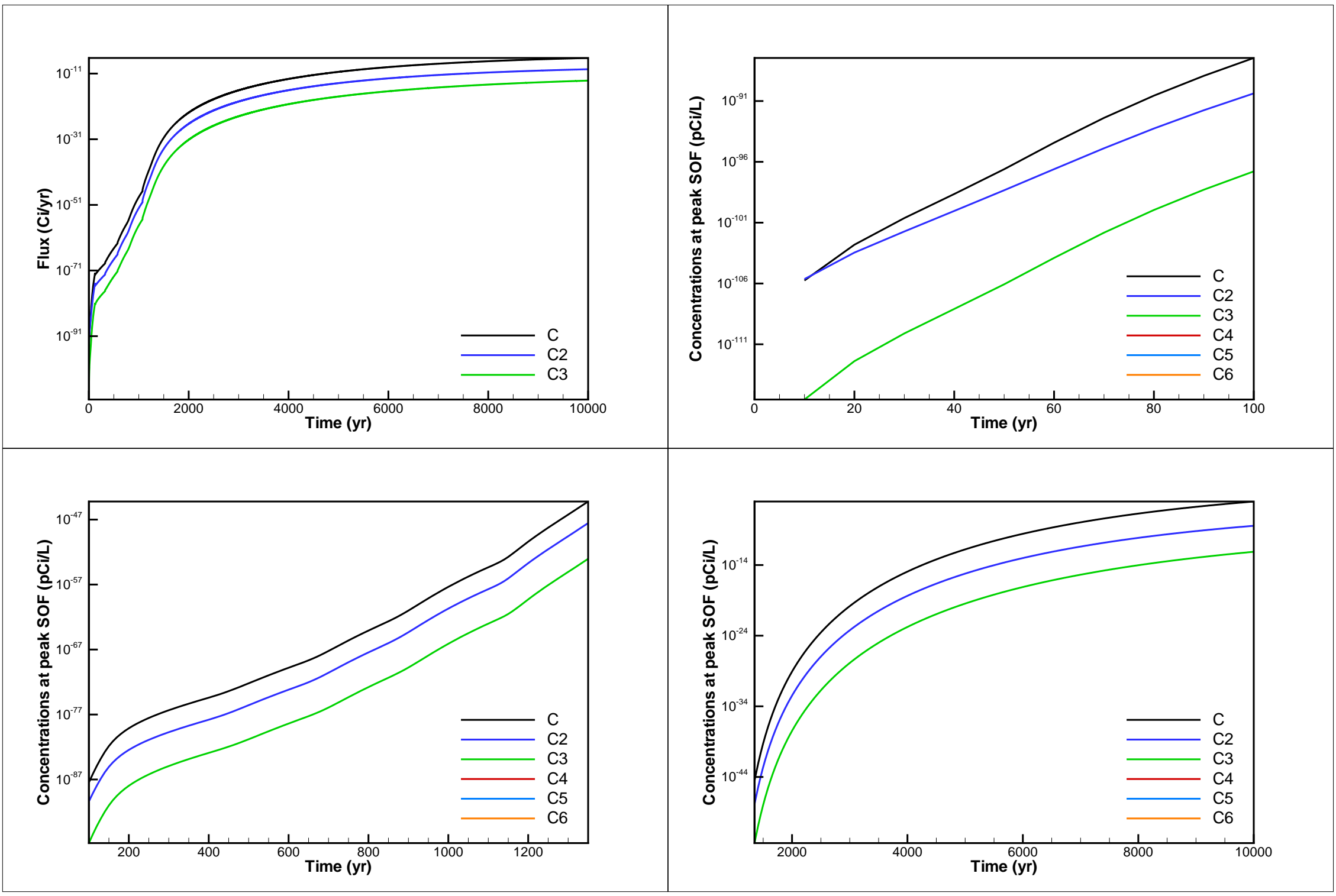

Figure A-54. Flux and concentration results for case Pu-242_0.01: Pu-242 Pu-242_56 U-238 


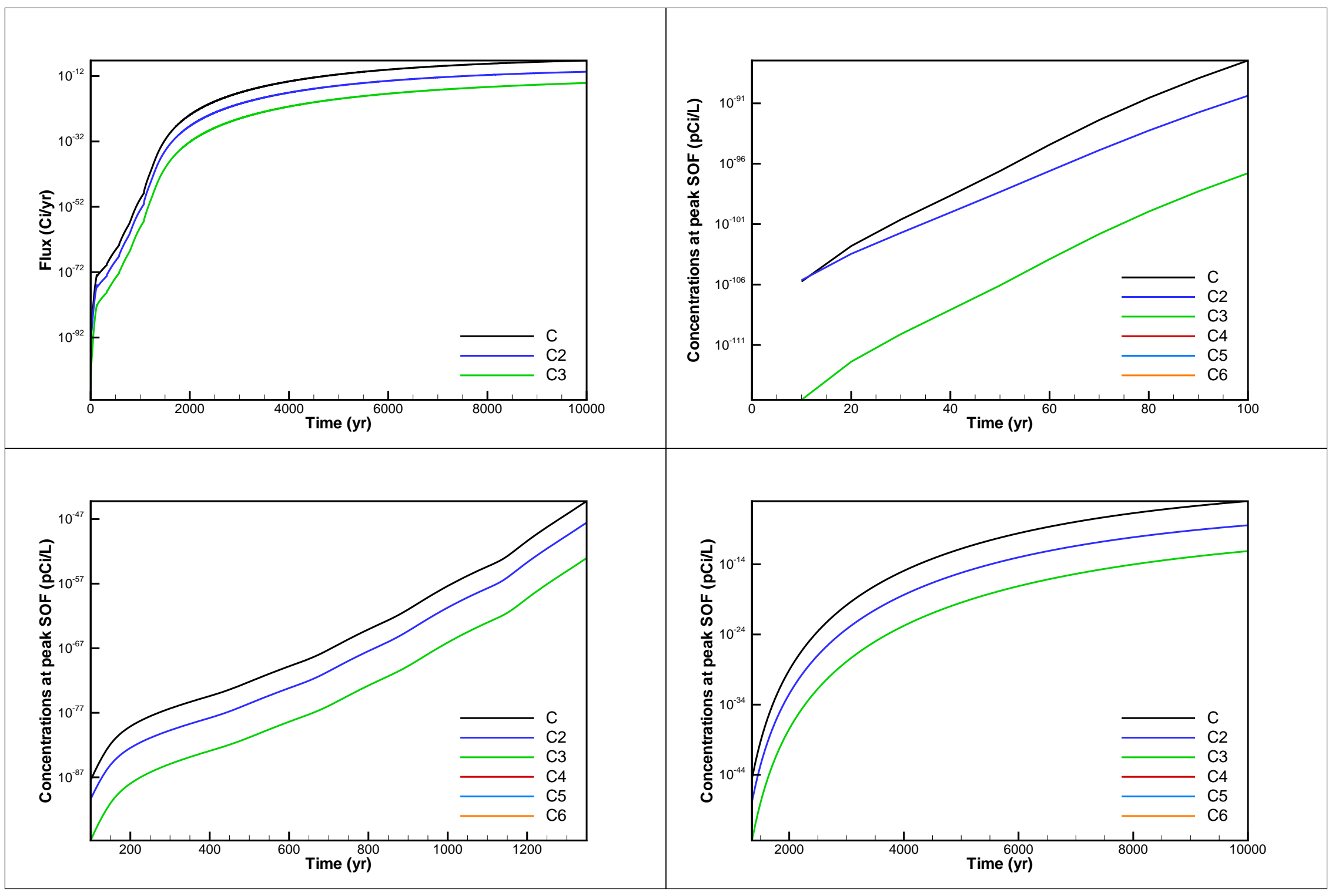

Figure A-55. Flux and concentration results for case Pu-242_0.1: Pu-242 Pu-242_56 U-238 


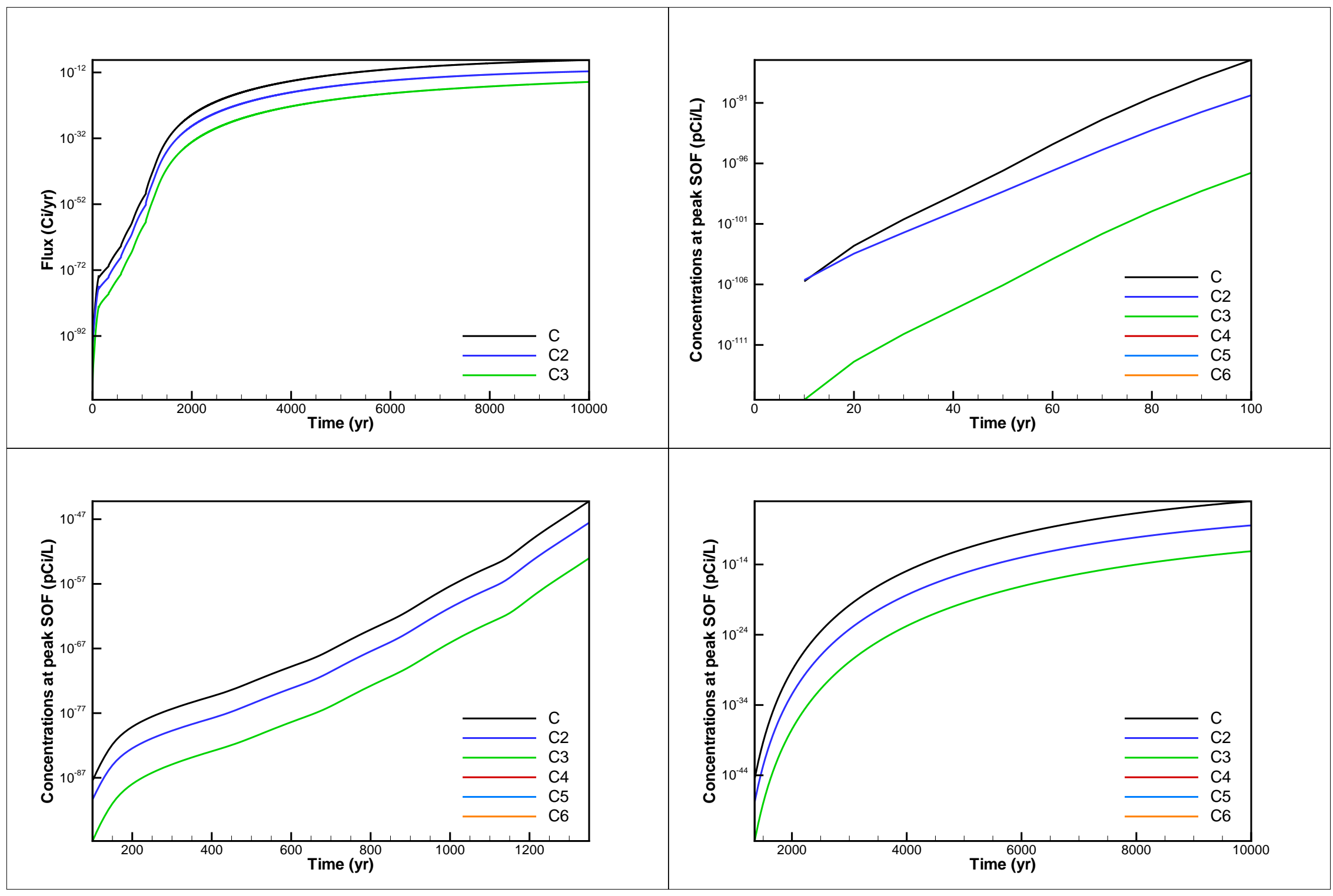

Figure A-56. Flux and concentration results for case Pu-242_1: Pu-242 Pu-242_56 U-238 


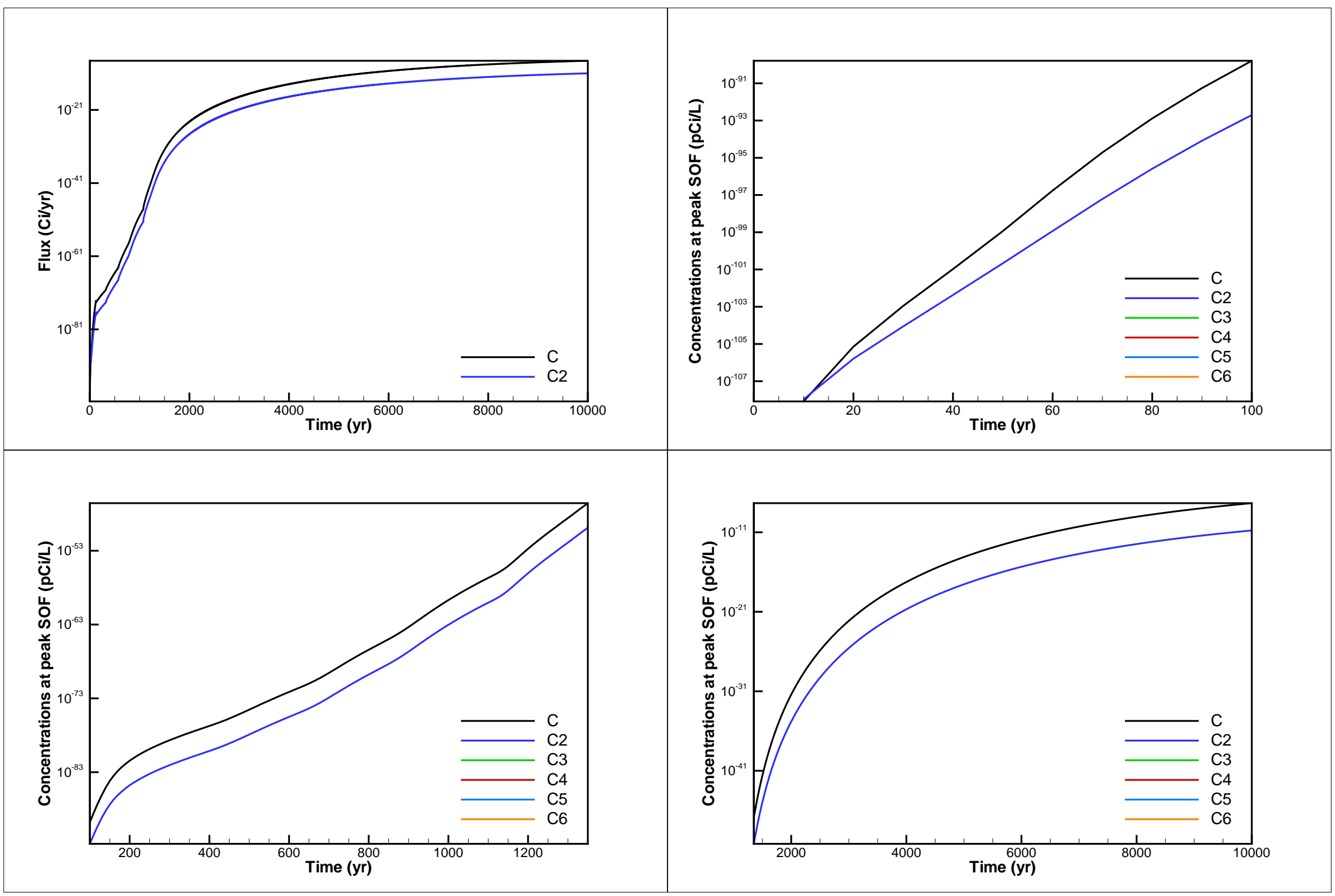

Figure A-57. Flux and concentration results for case Pu-244_0.001: Pu-244 Pu-244_56 


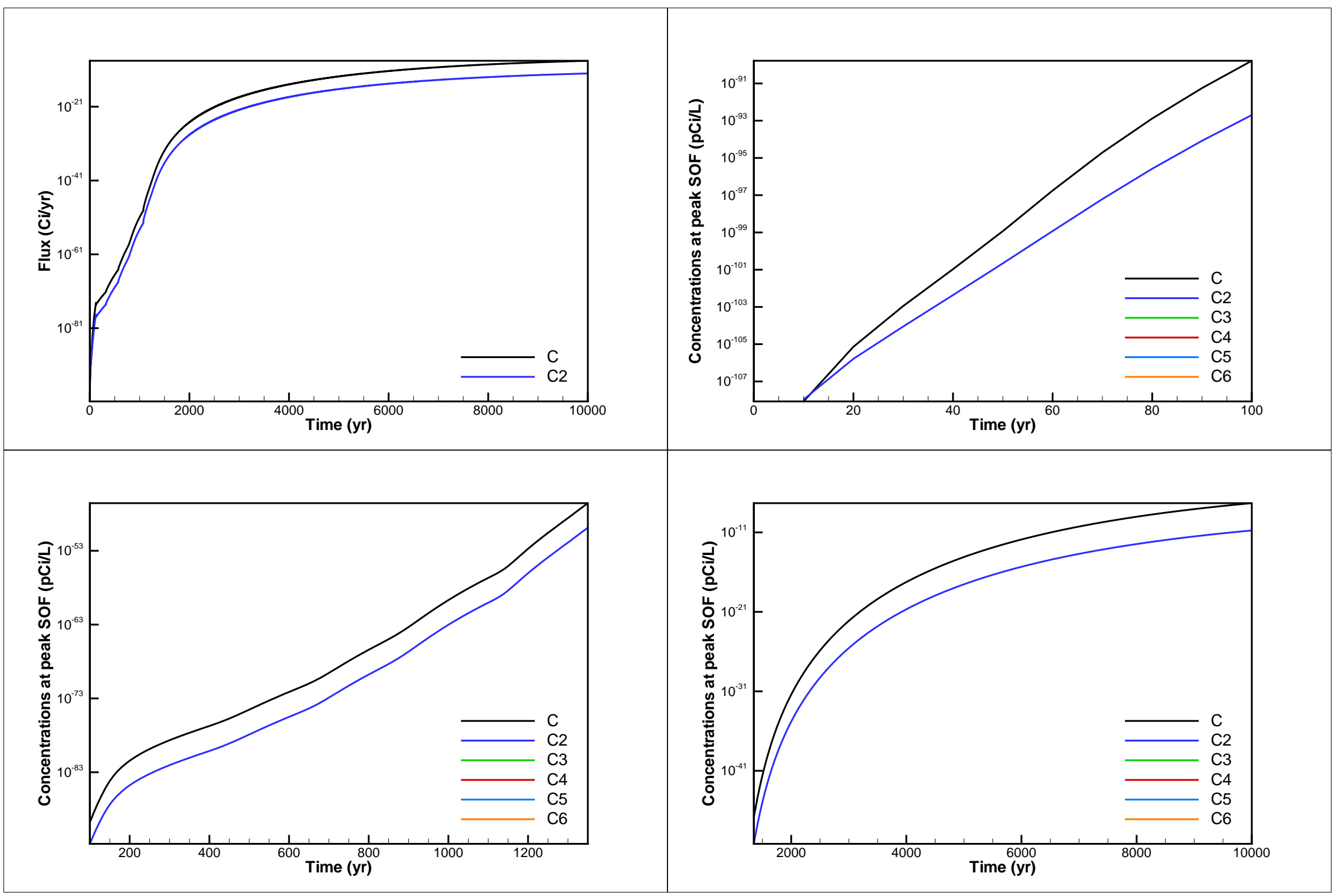

Figure A-58. Flux and concentration results for case Pu-244_0.01: $\mathrm{Pu}-244 \mathrm{Pu}-244 \_56$ 


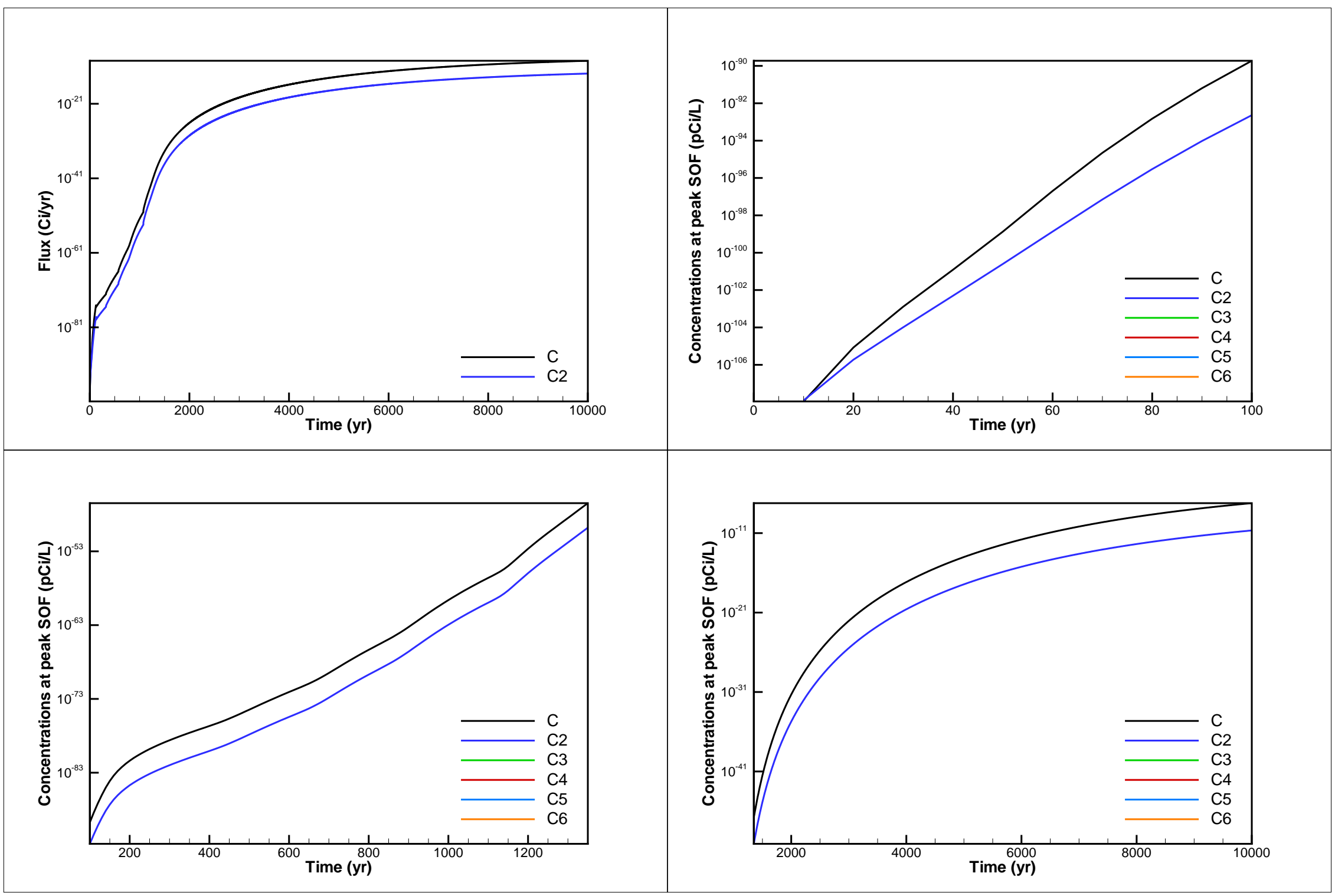

Figure A-59. Flux and concentration results for case Pu-244_0.1: Pu-244 Pu-244_56 


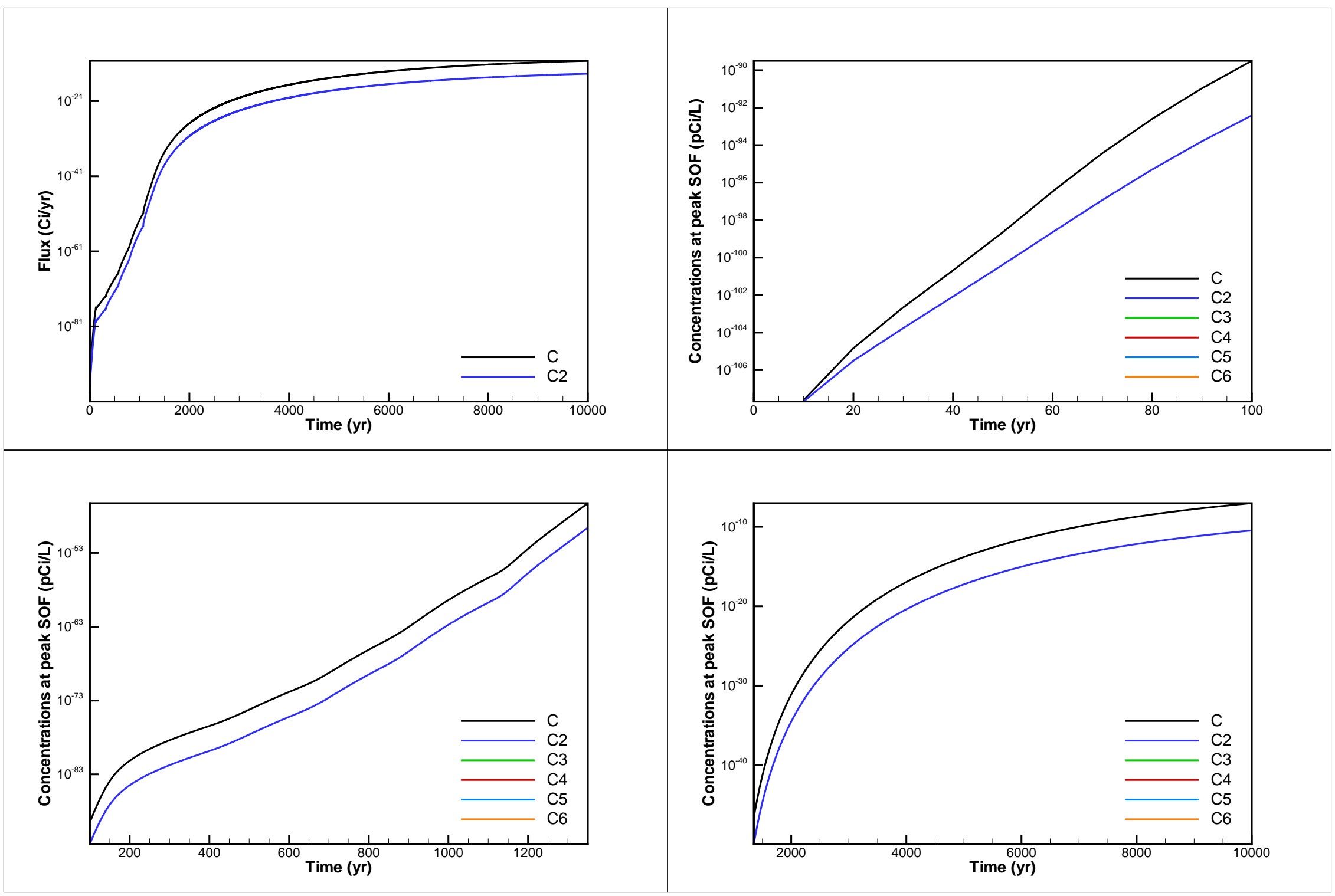

Figure A-60. Flux and concentration results for case Pu-244_1: Pu-244 Pu-244_56 


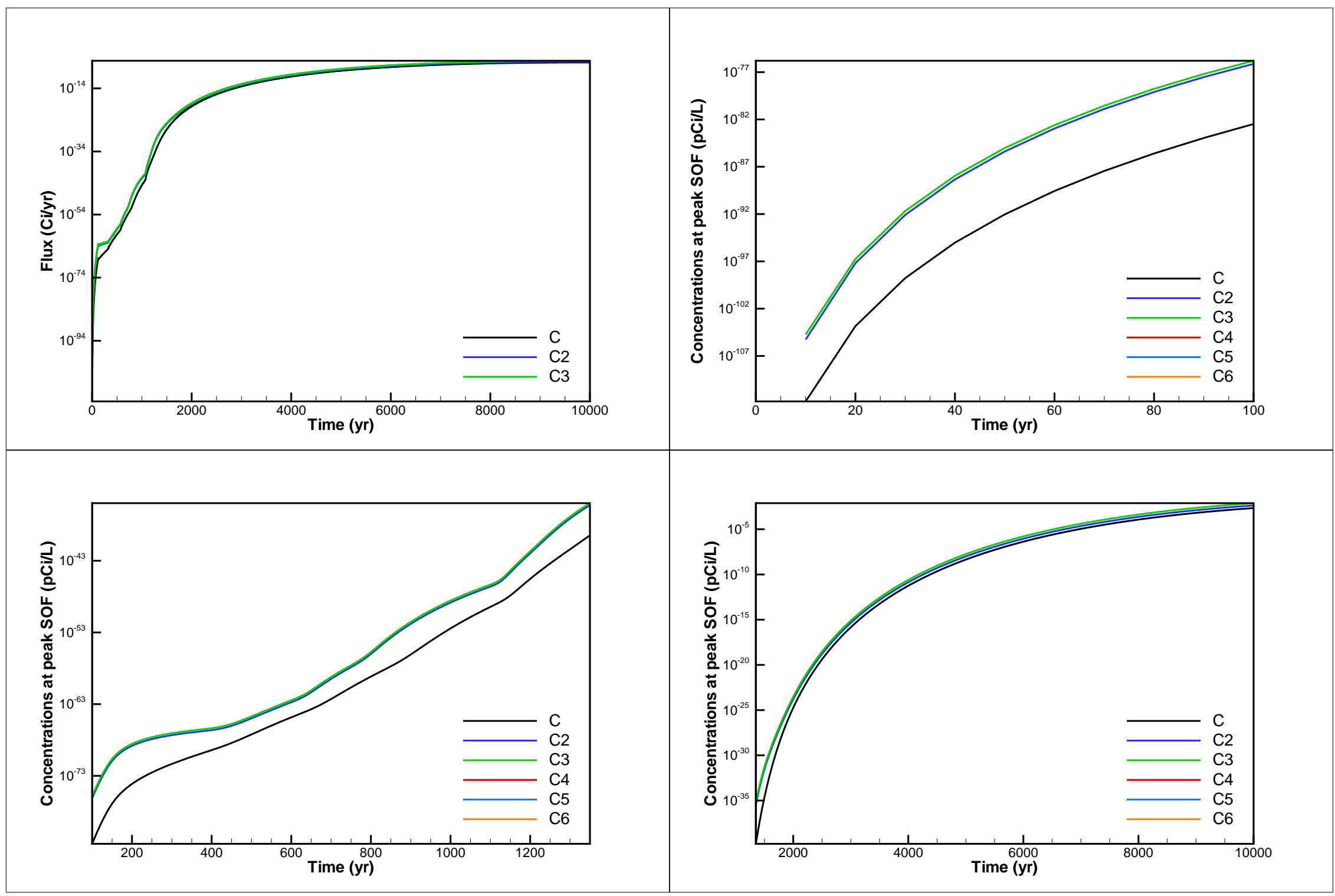

Figure A-61. Flux and concentration results for case Ra-226_1: Ra-226 Pb-210 Po-210 


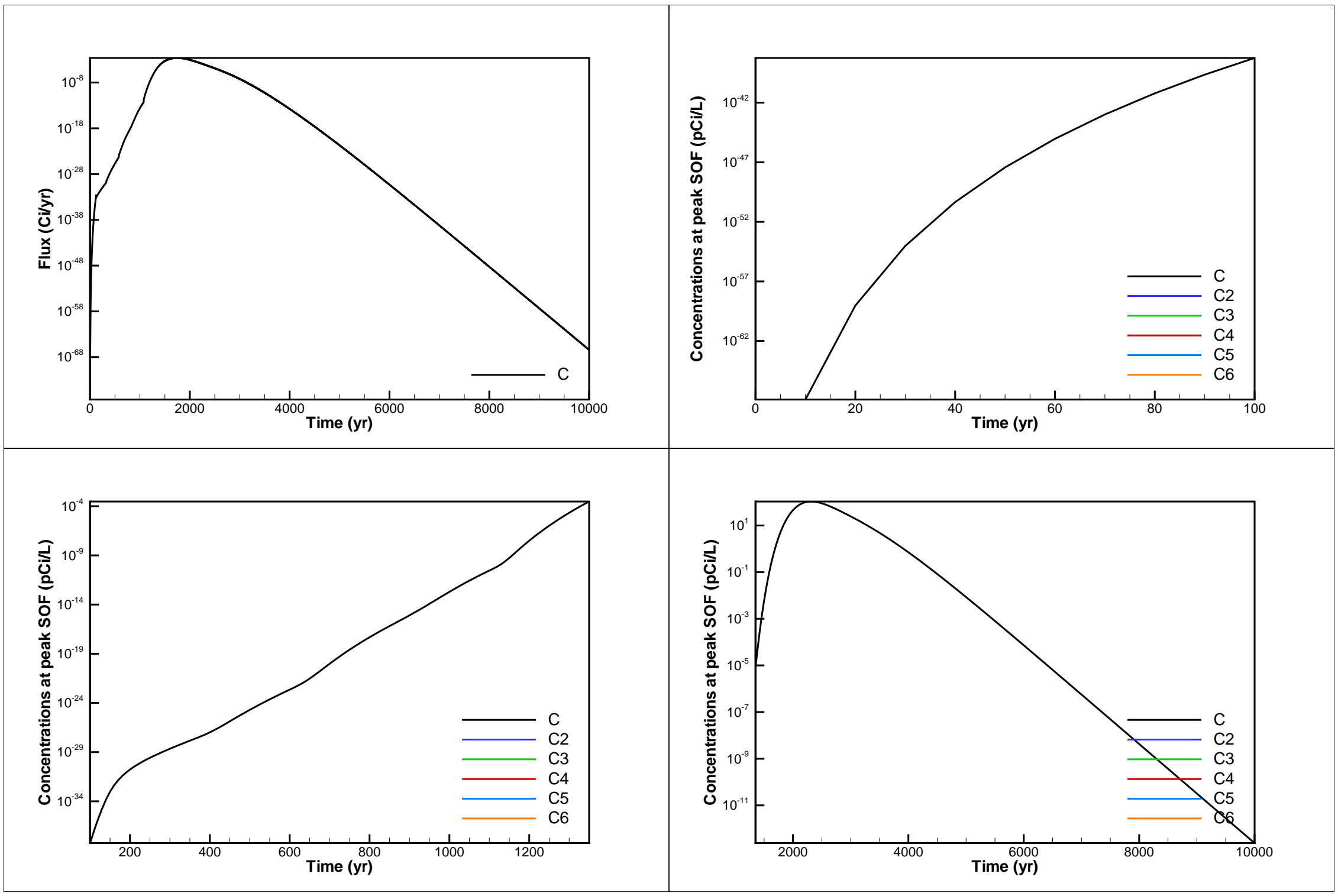

Figure A-62. Flux and concentration results for case Se-79_1: Se-79 


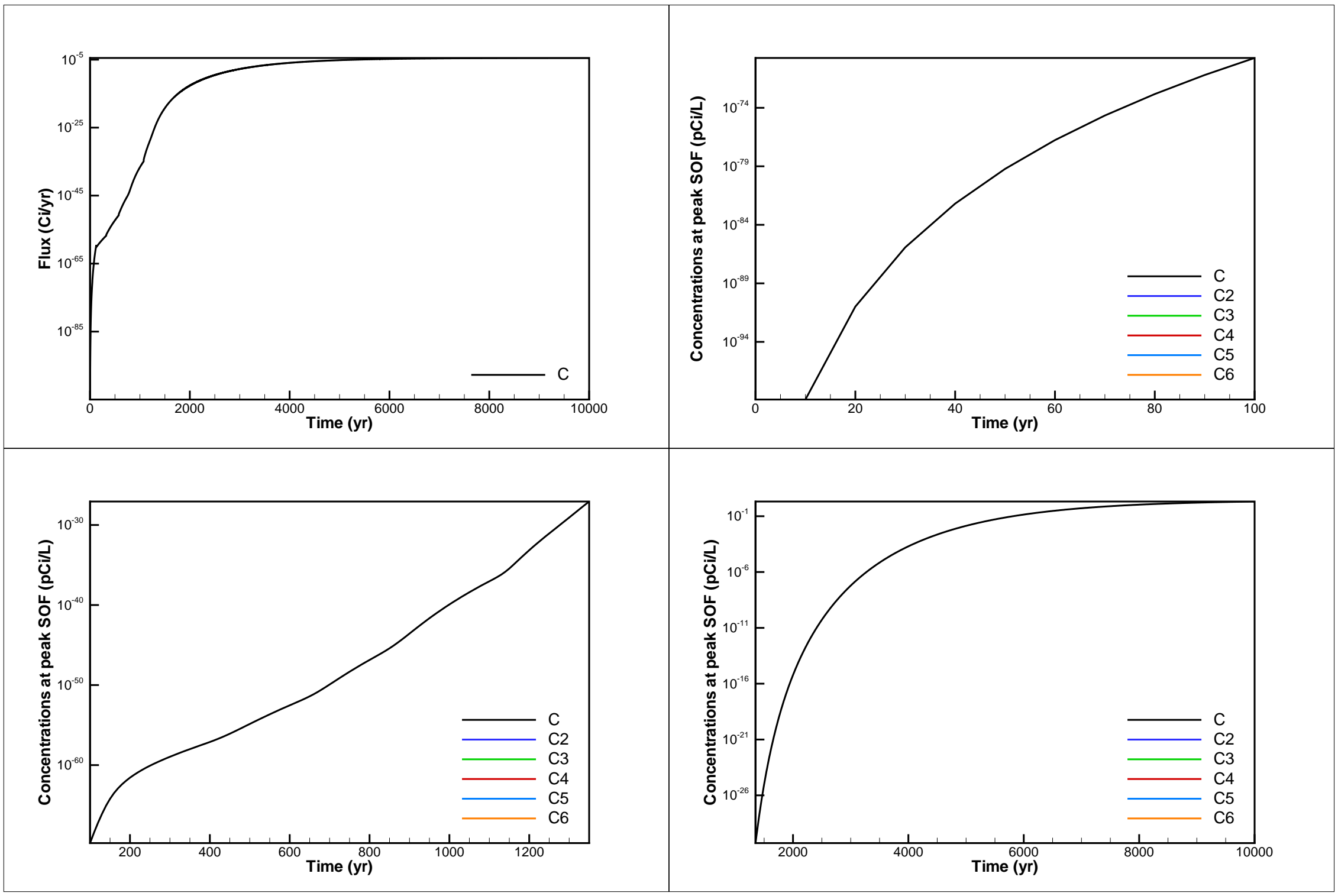

Figure A-63. Flux and concentration results for case Sn-126_1: Sn-126 


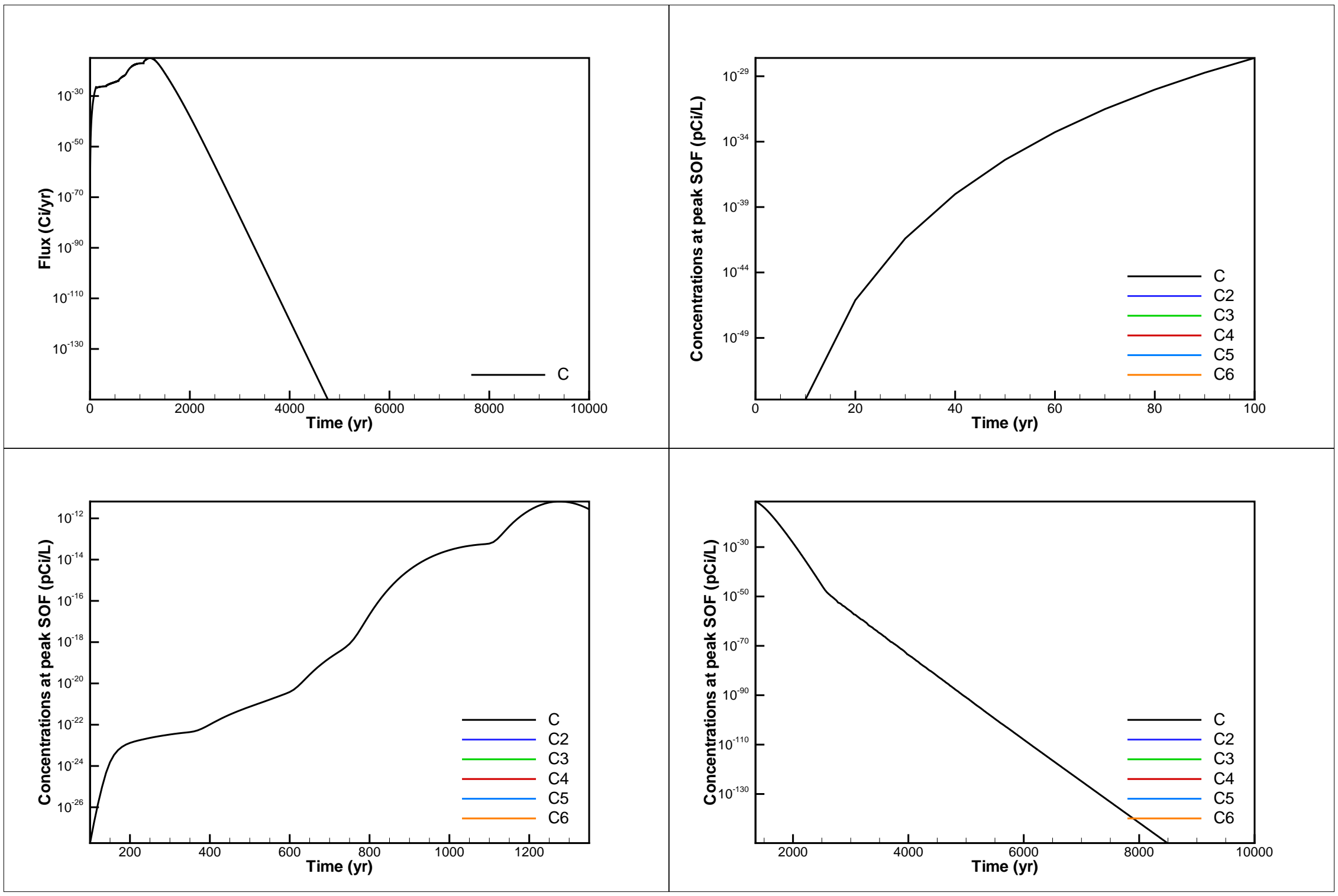

Figure A-64. Flux and concentration results for case Sr-90_1: Sr-90 


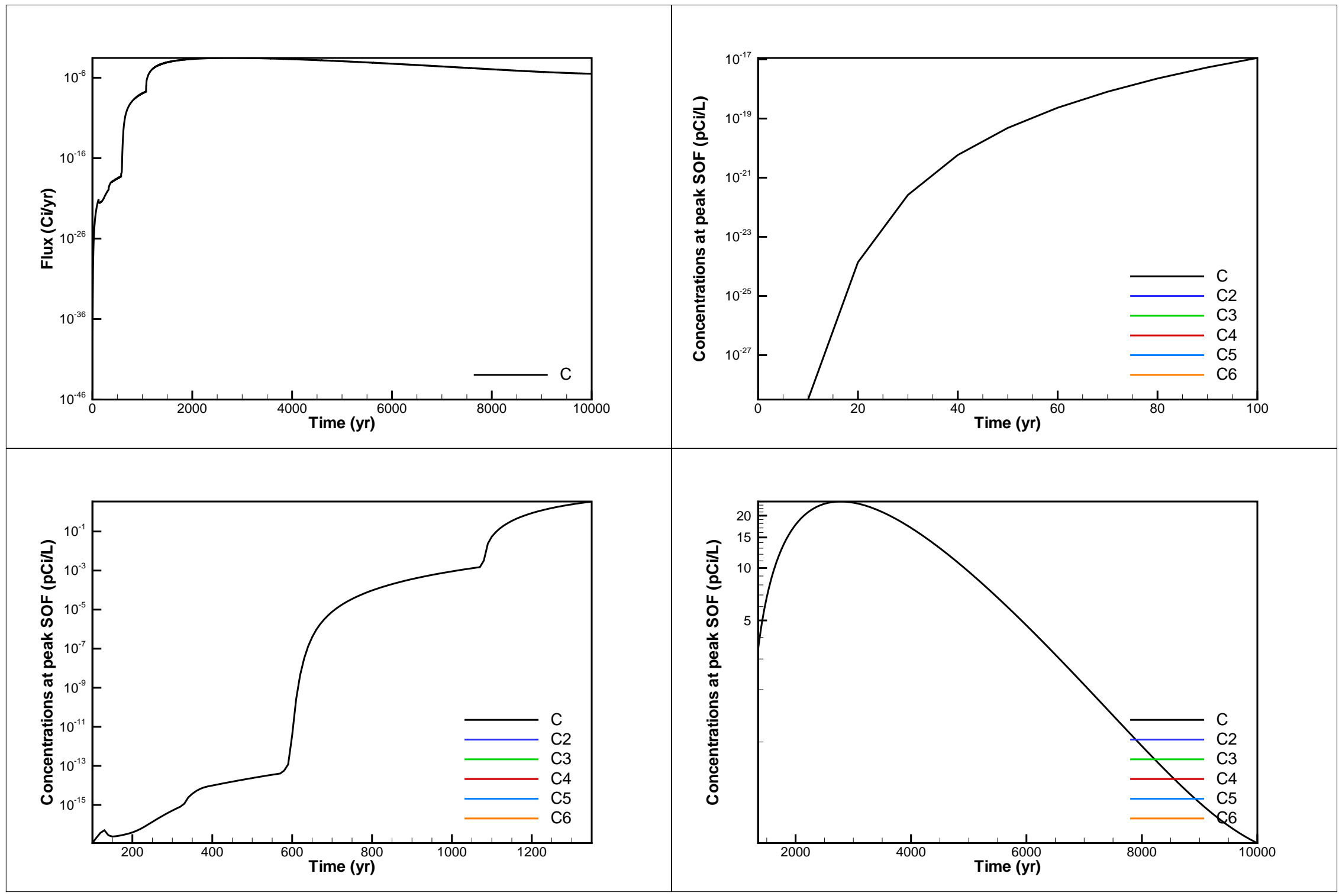

Figure A-65. Flux and concentration results for case Tc-99_1: Tc-99 


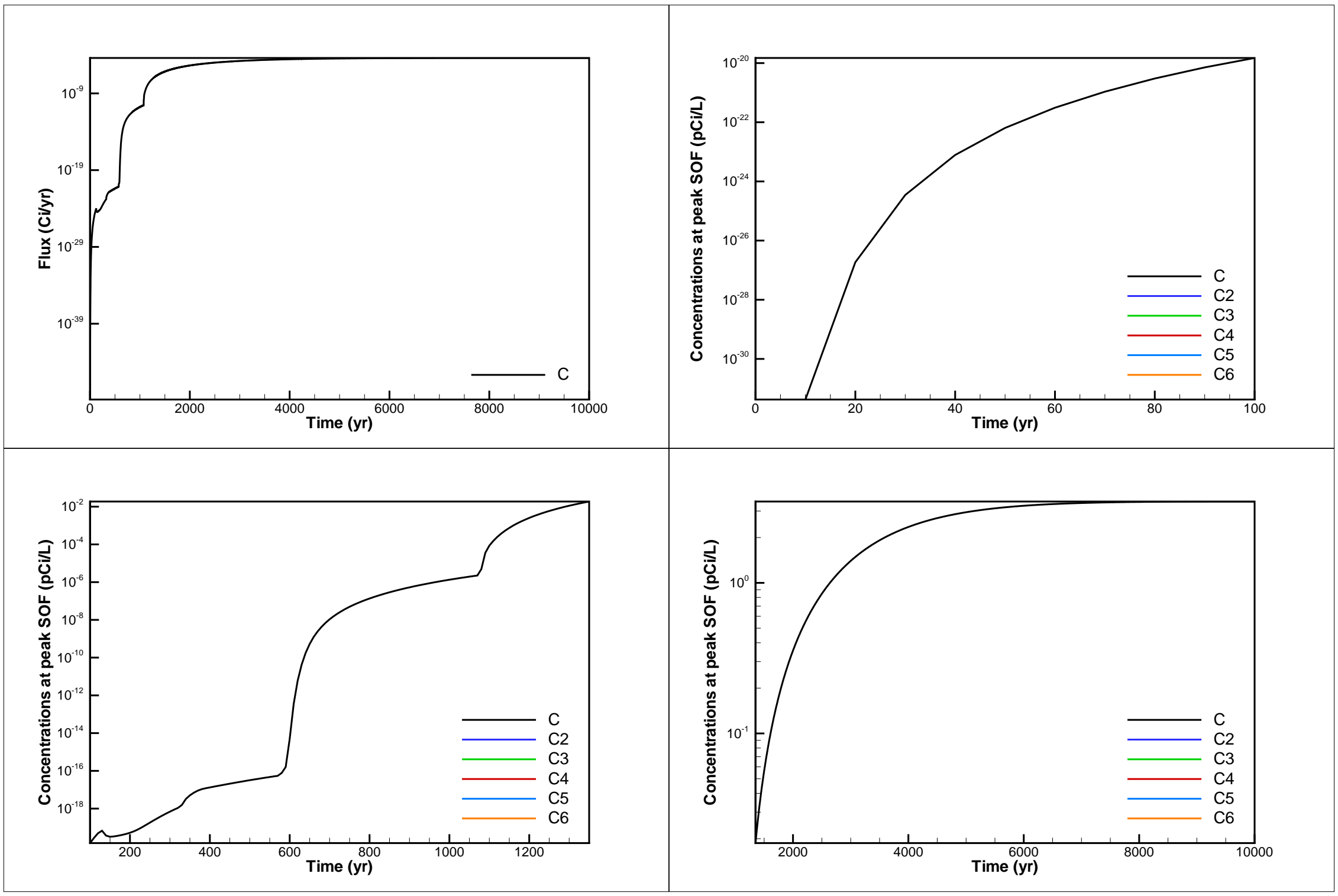

Figure A-66. Flux and concentration results for case Tc-99_KB_1: Tc-99_KB 


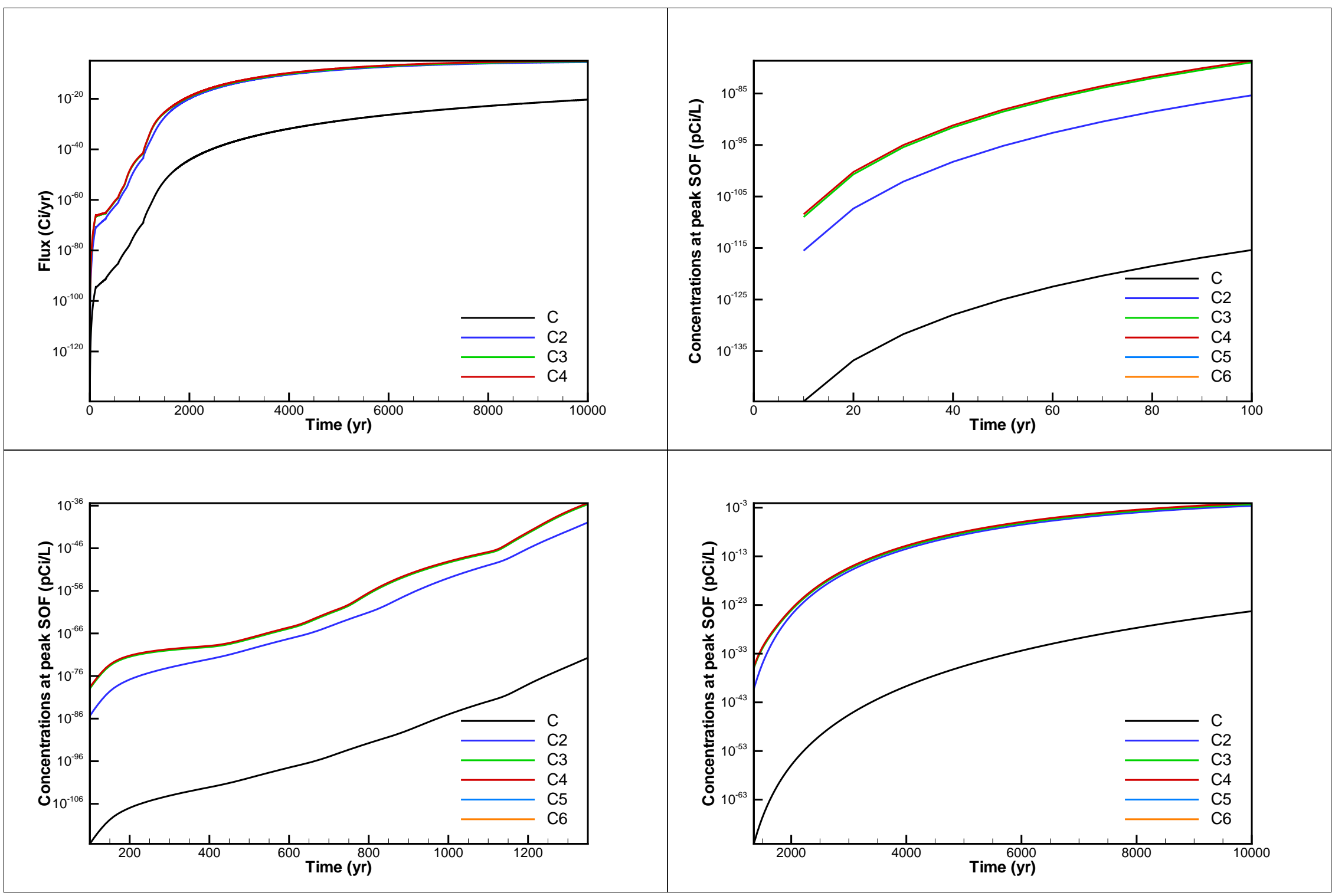

Figure A-67. Flux and concentration results for case Th-230_1: Th-230 Ra-226 Pb-210 Po-210 


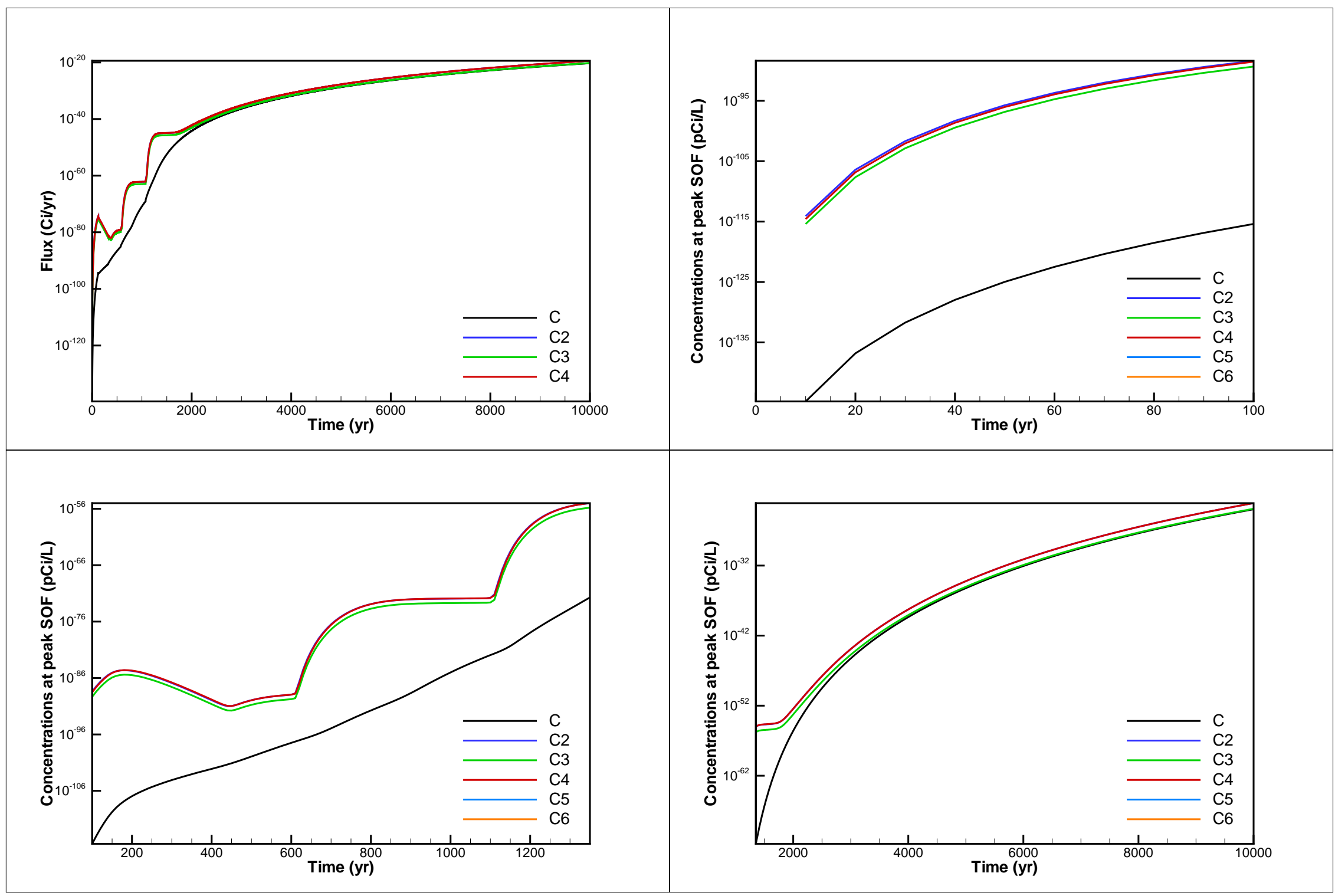

Figure A-68. Flux and concentration results for case Th-232_1: Th-232 Ra-228 Th-228 Ra-224 


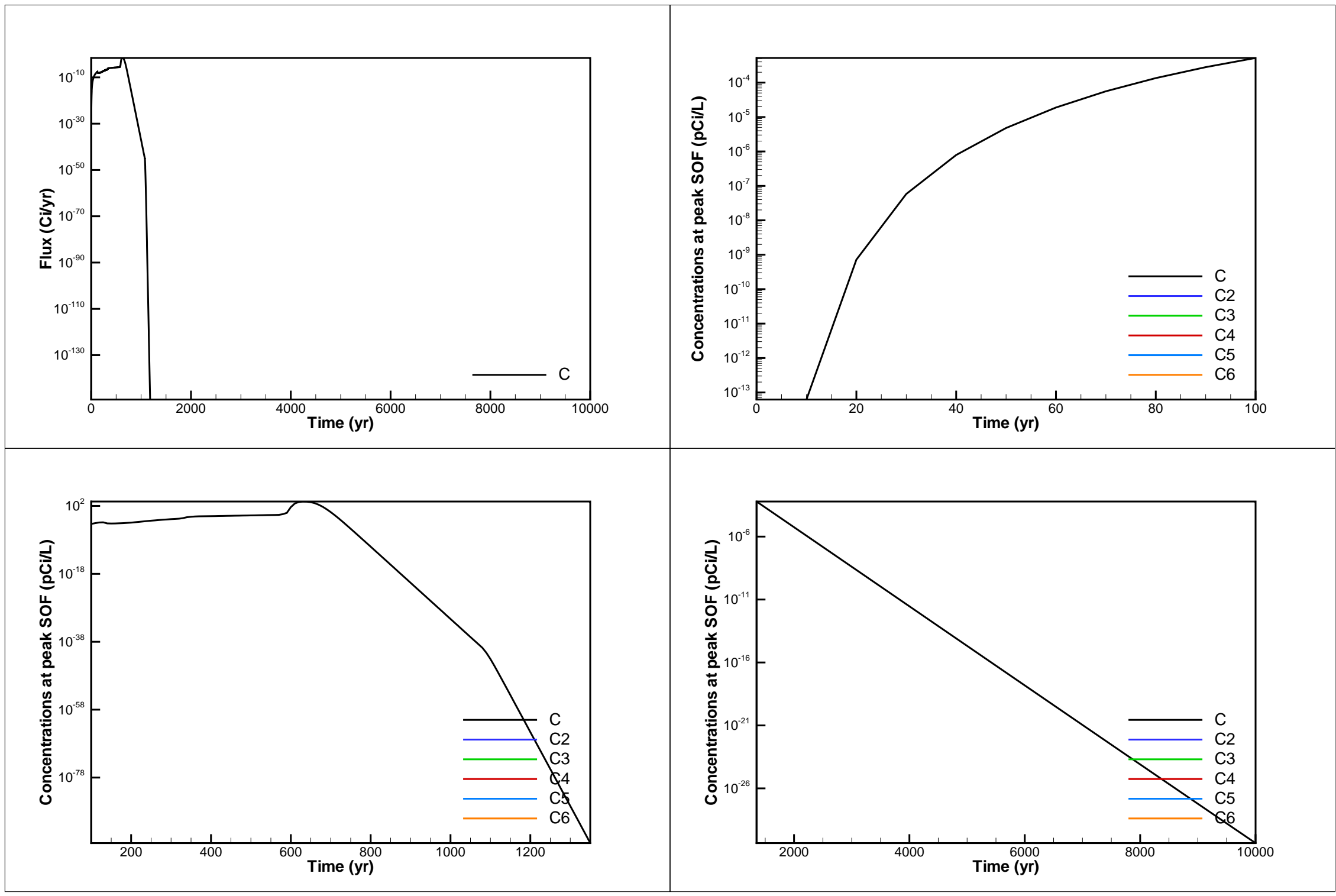

Figure A-69. Flux and concentration results for case Tracer_1: Tracer 


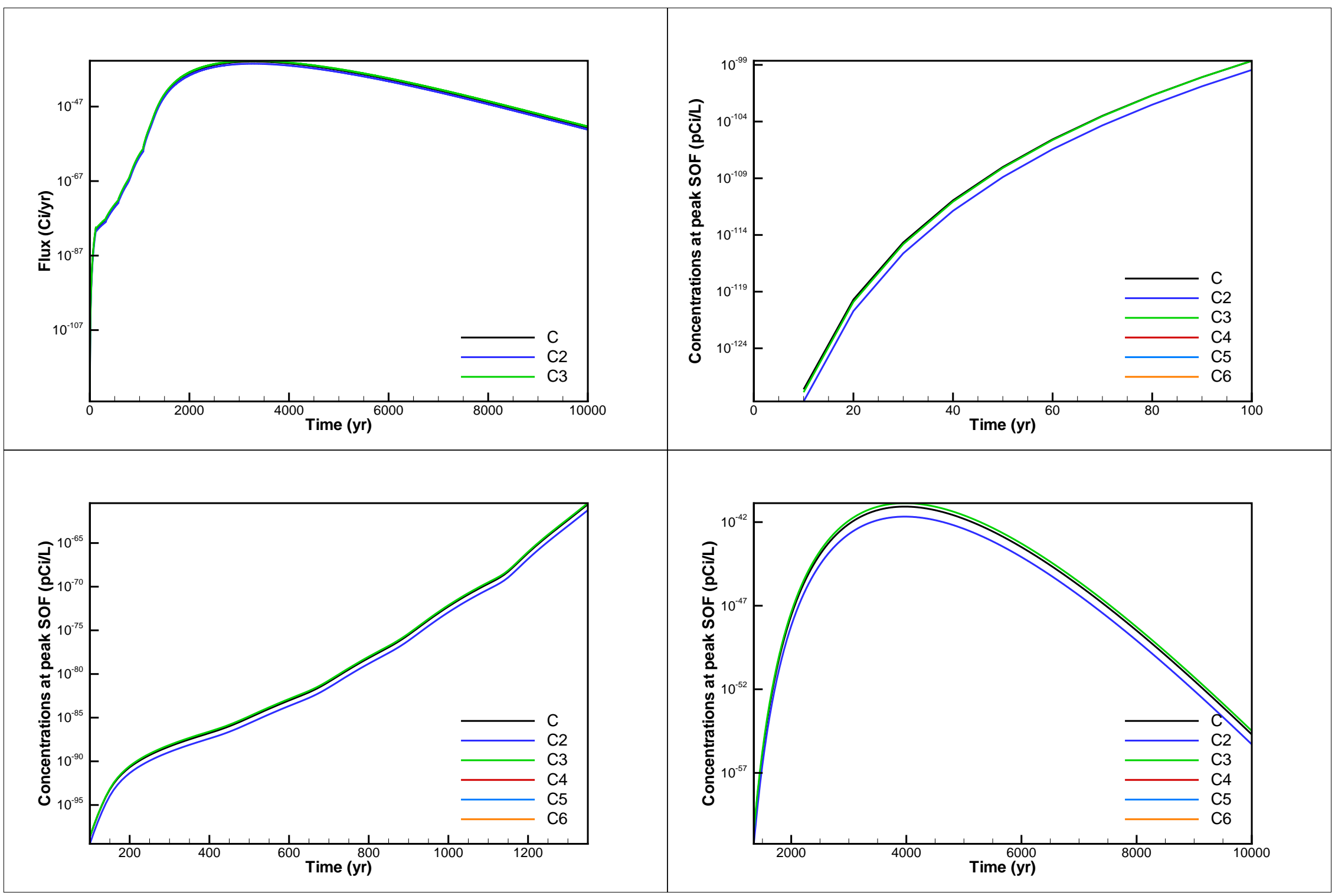

Figure A-70. Flux and concentration results for case U-232_0.001: U-232 Th-228 Ra-224 


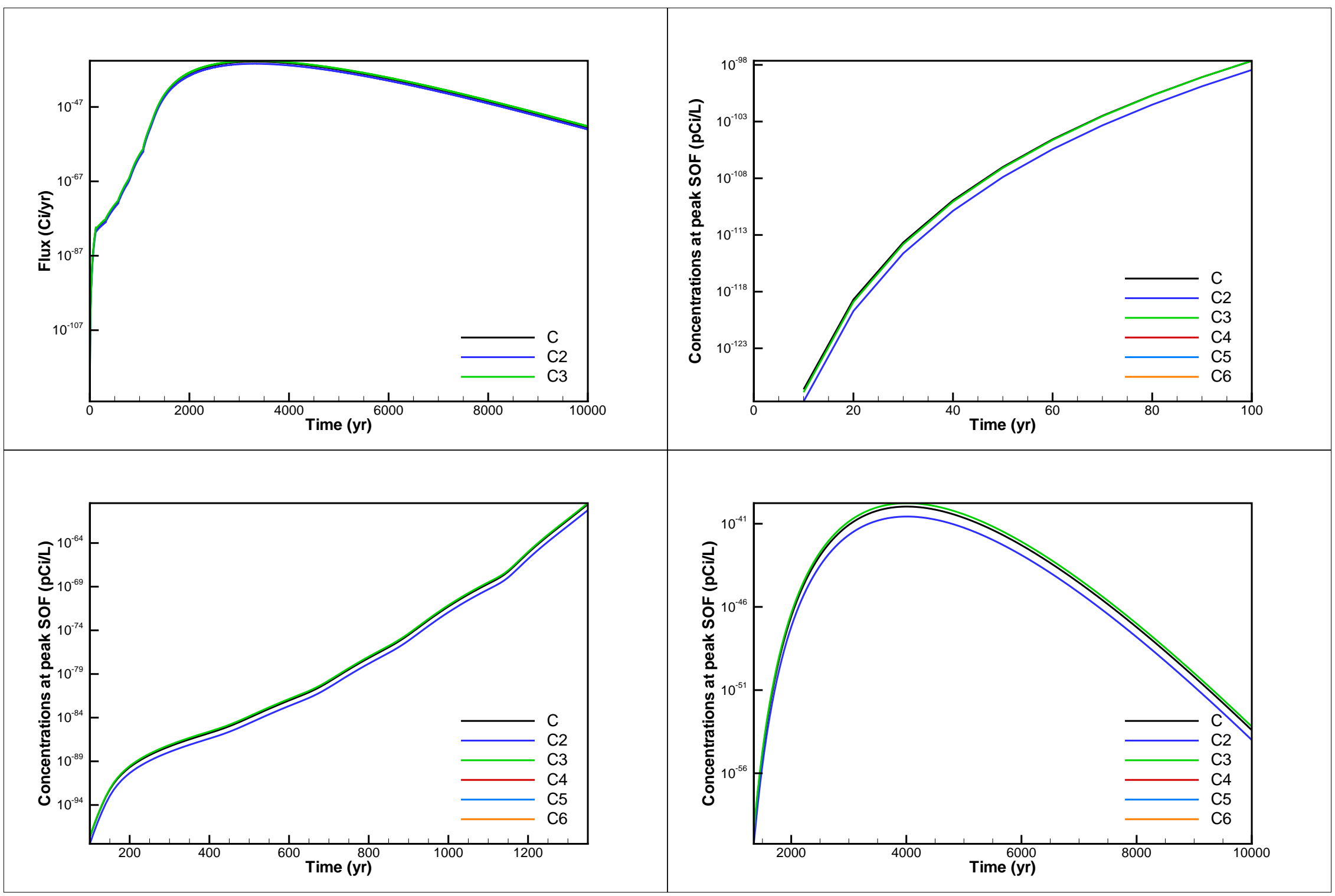

Figure A-71. Flux and concentration results for case U-232_0.01: U-232 Th-228 Ra-224 


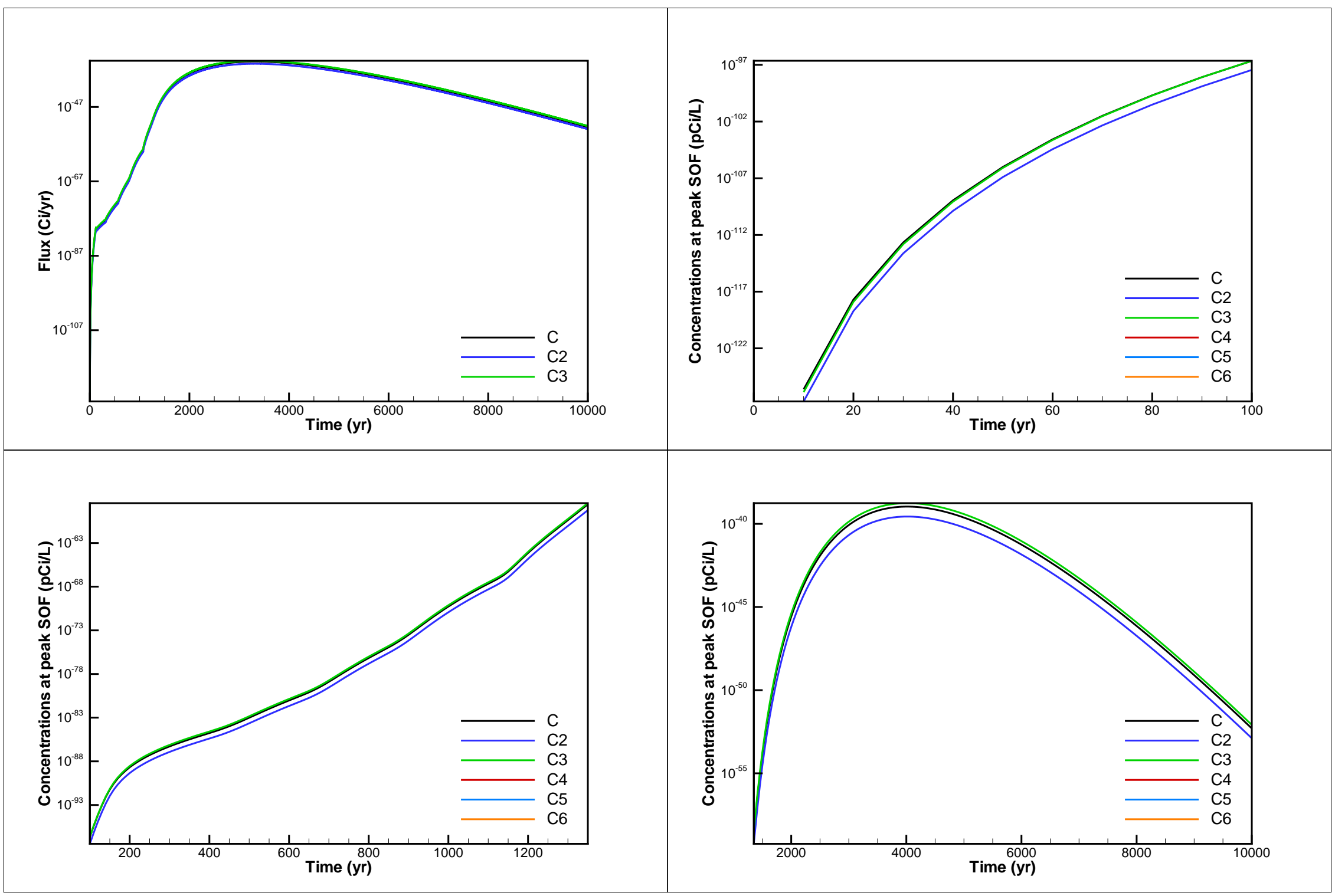

Figure A-72. Flux and concentration results for case U-232_0.1: U-232 Th-228 Ra-224 


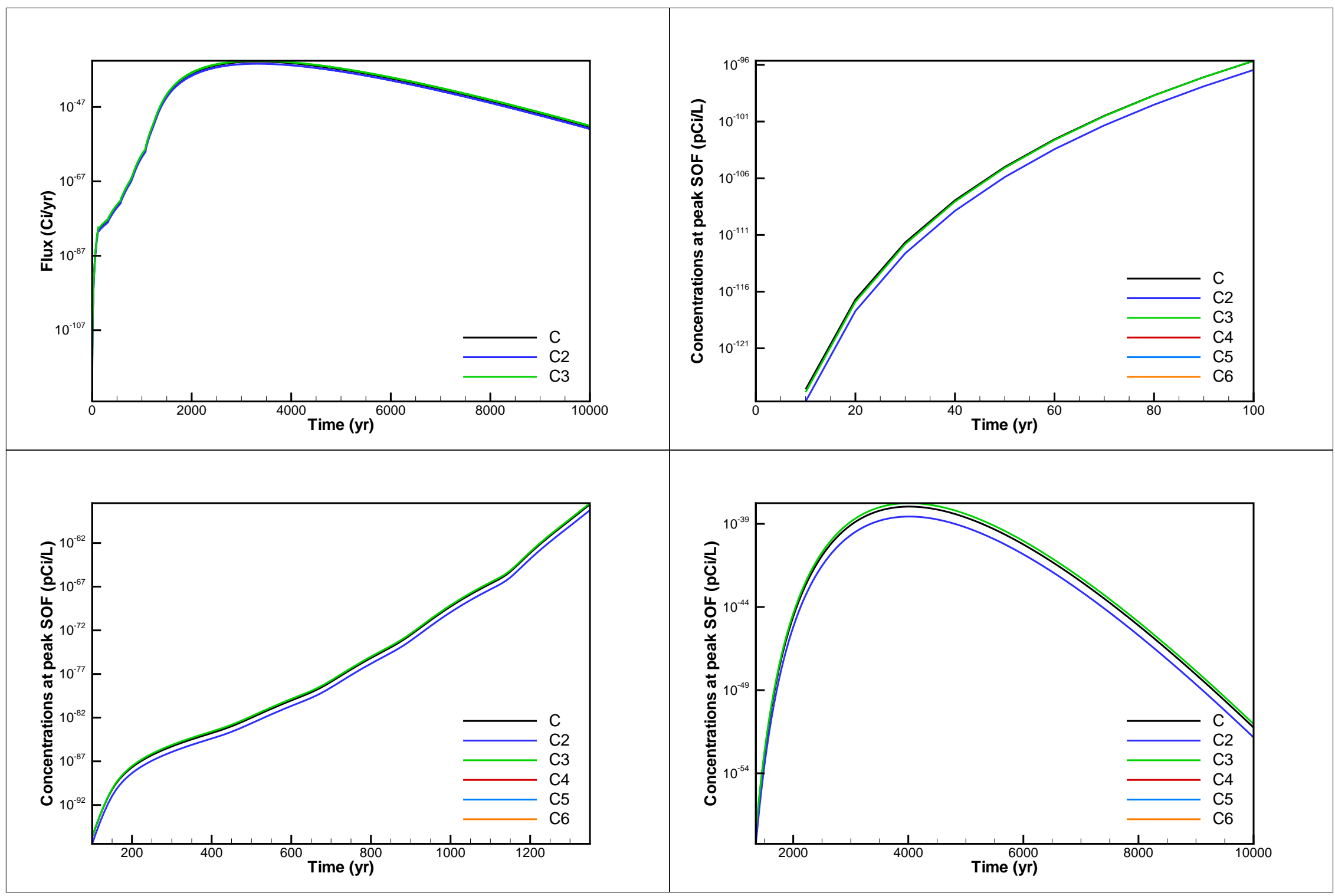

Figure A-73. Flux and concentration results for case U-232_1: U-232 Th-228 Ra-224 


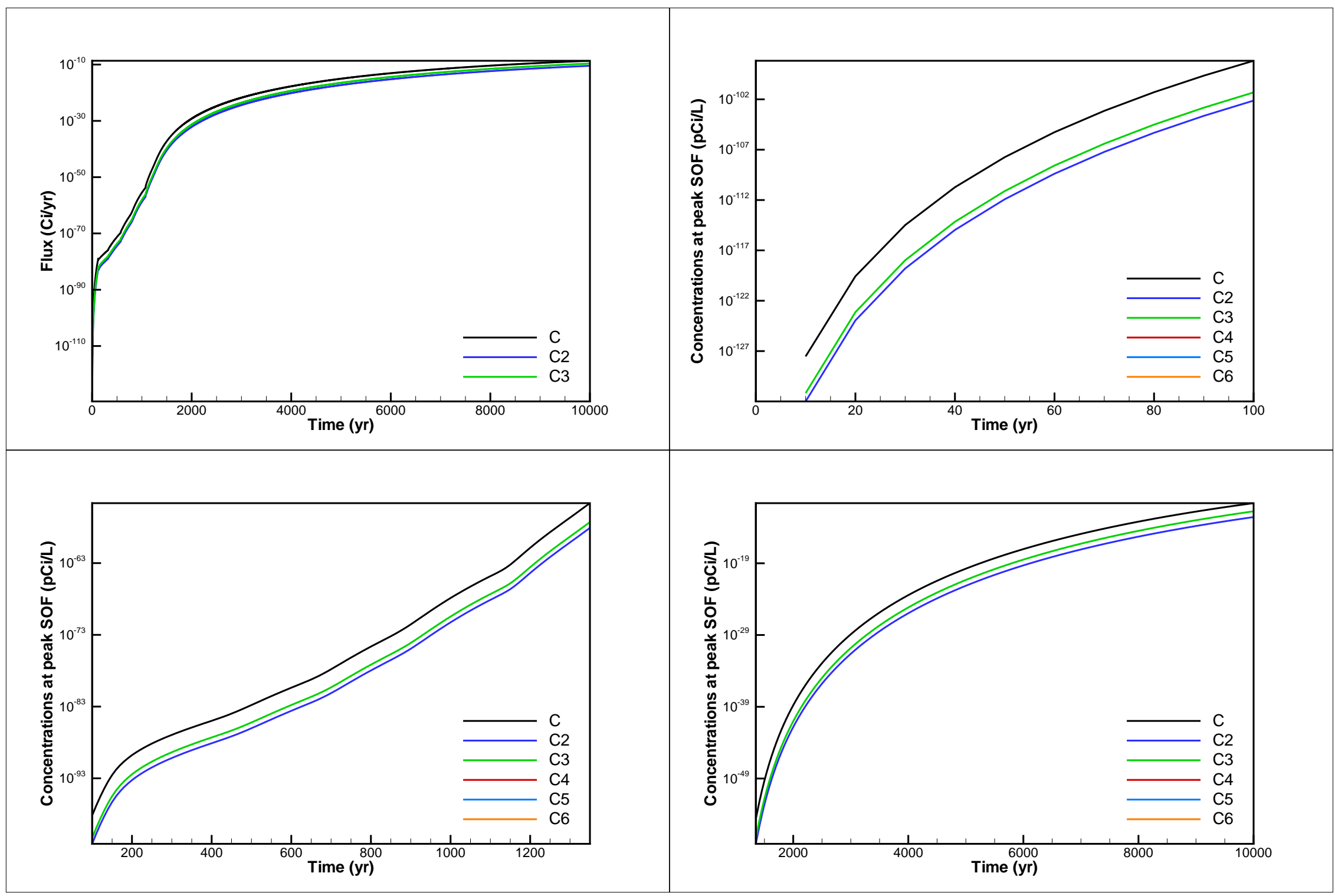

Figure A-74. Flux and concentration results for case U-233_0.001: U-233 Th-229 Ra-225 


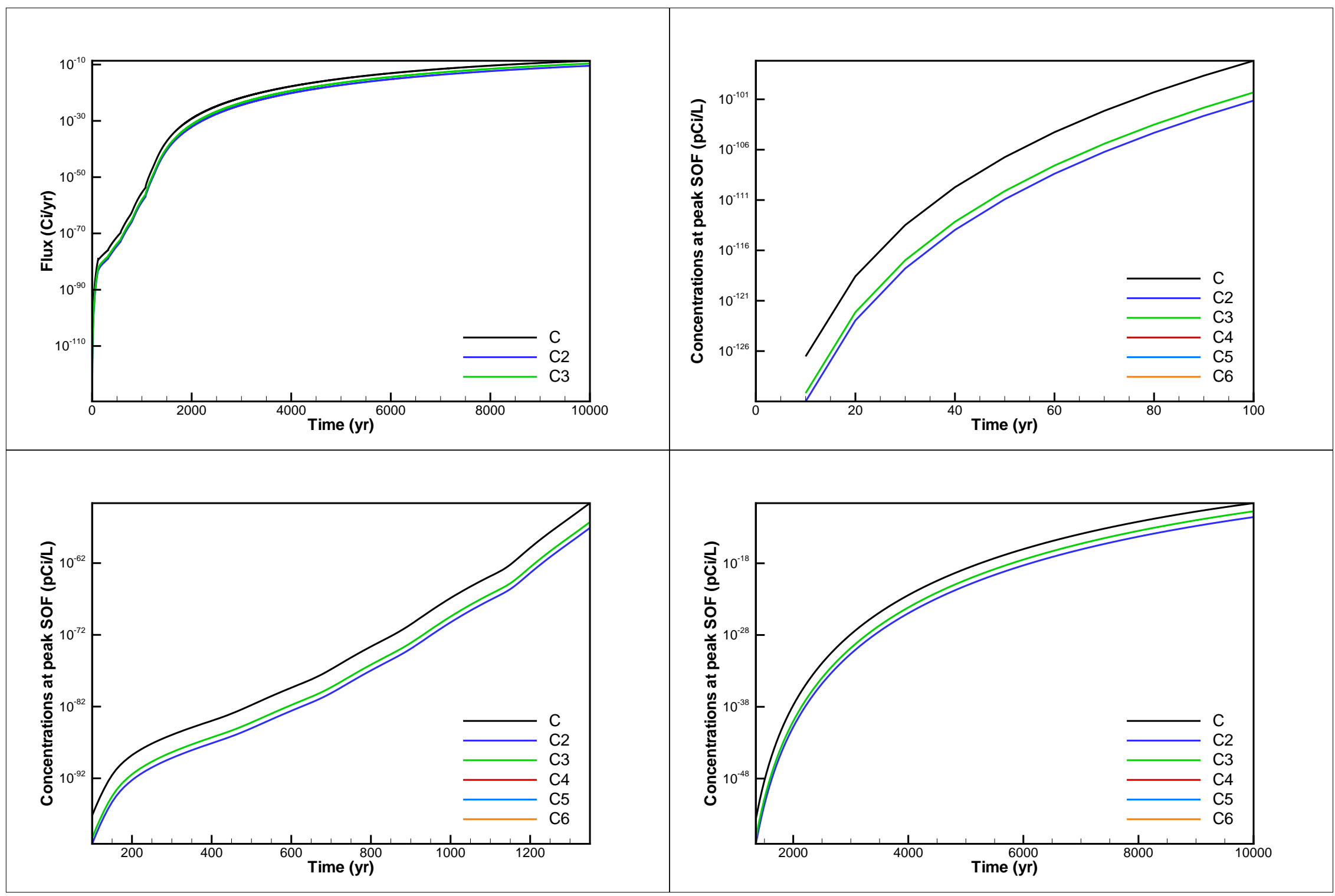

Figure A-75. Flux and concentration results for case U-233_0.01: U-233 Th-229 Ra-225 


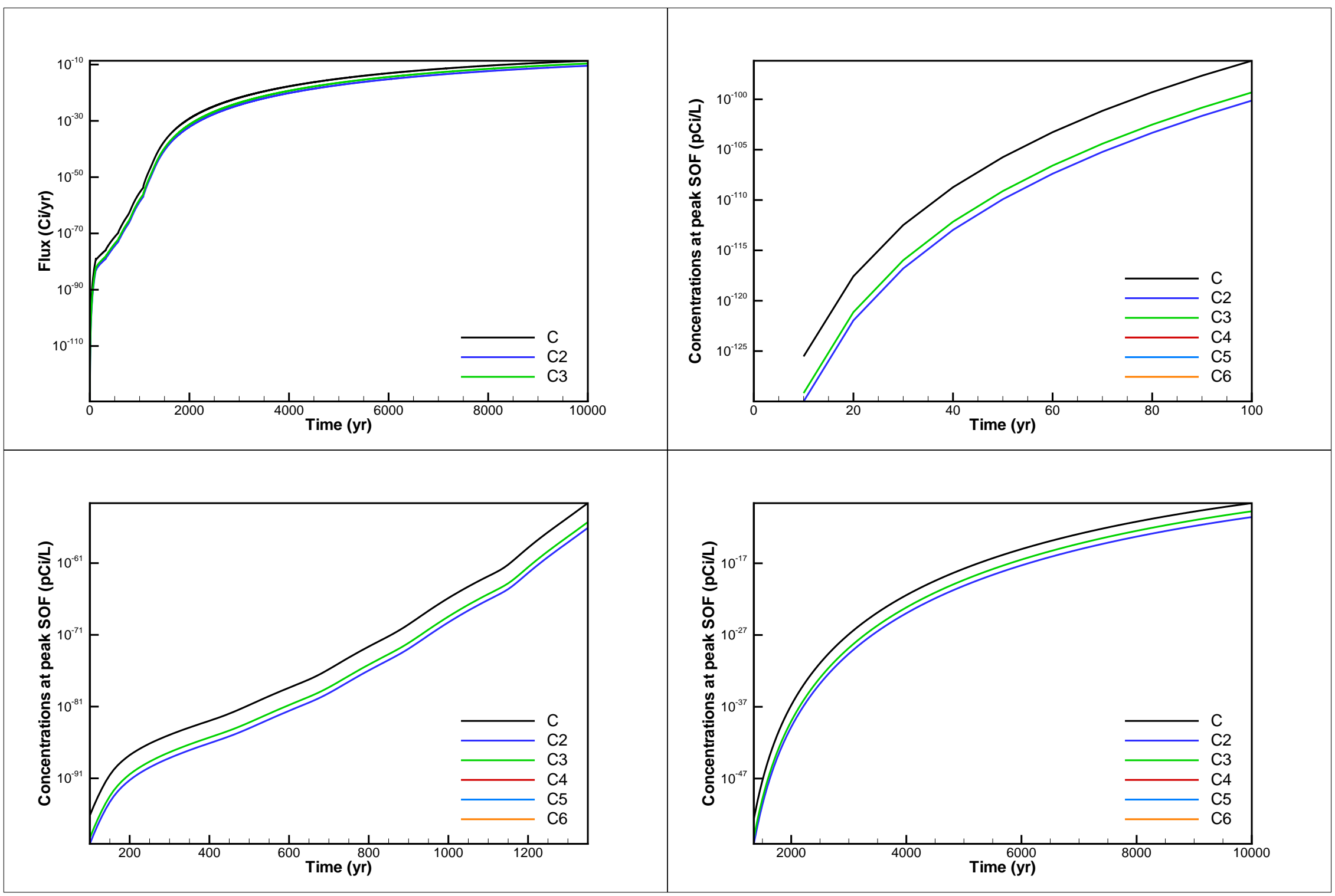

Figure A-76. Flux and concentration results for case U-233_0.1: U-233 Th-229 Ra-225 


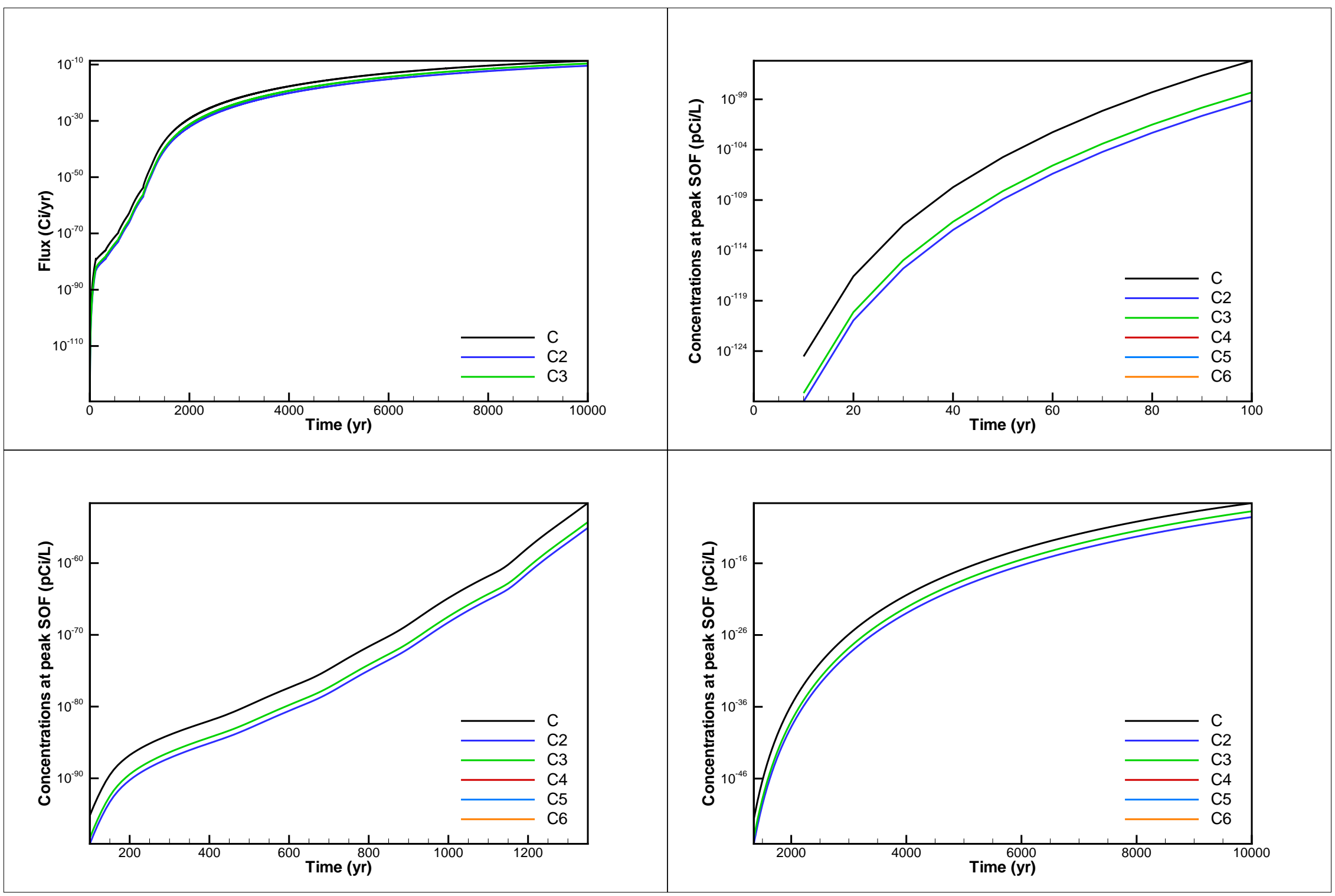

Figure A-77. Flux and concentration results for case U-233_1: U-233 Th-229 Ra-225 


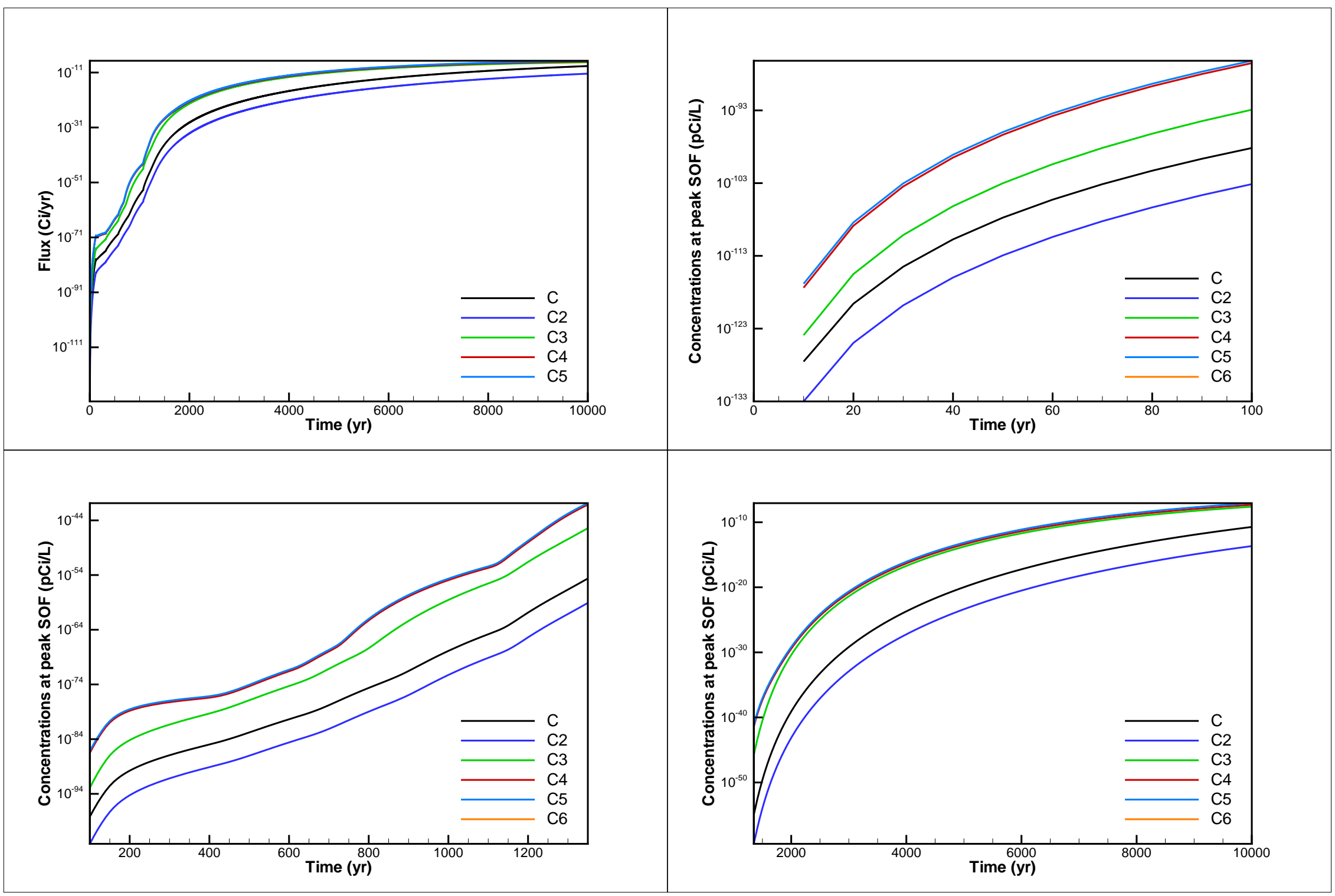

Figure A-78. Flux and concentration results for case U-234_0.001: U-234 Th-230 Ra-226 Pb-210 Po-210 


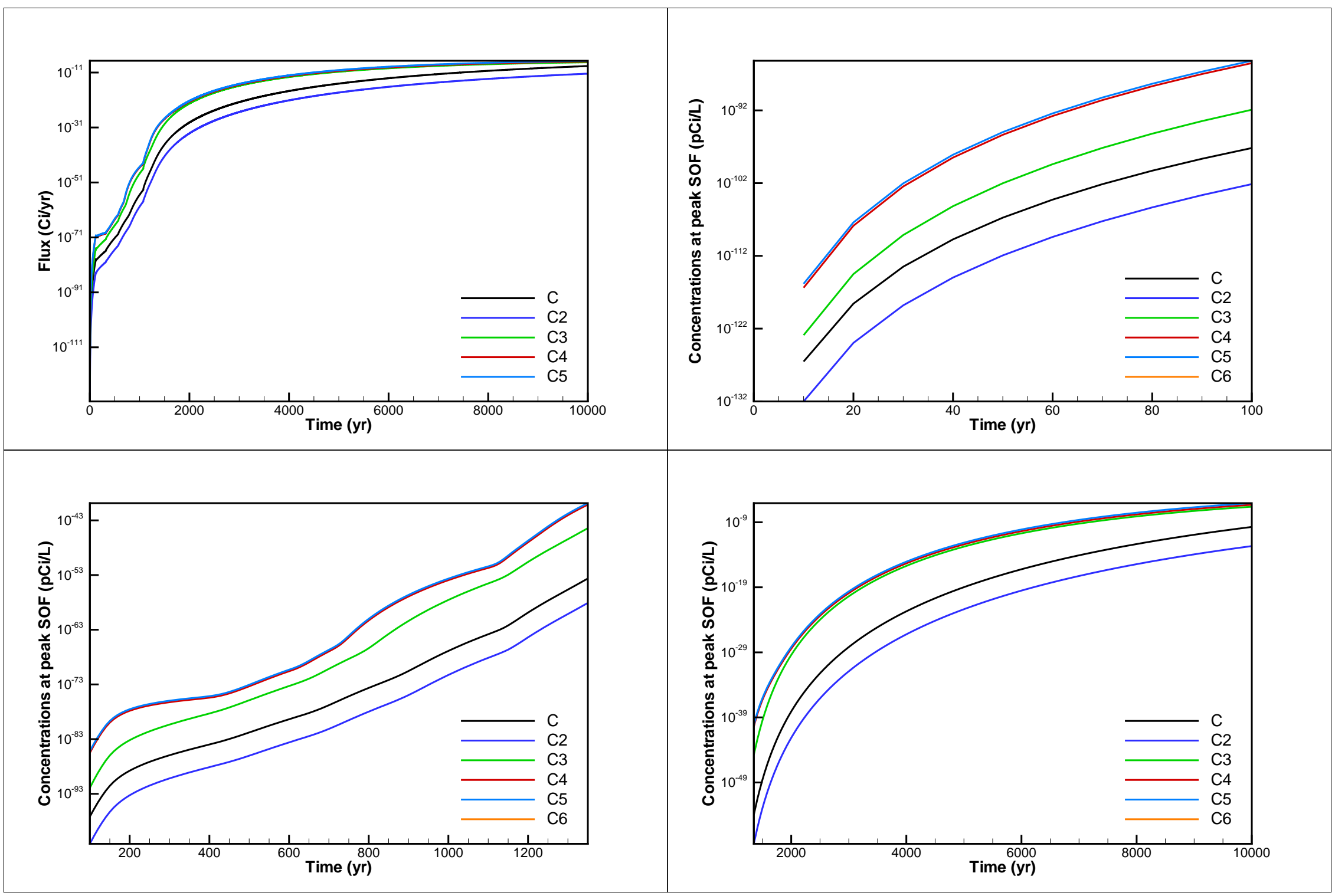

Figure A-79. Flux and concentration results for case U-234_0.01: U-234 Th-230 Ra-226 Pb-210 Po-210 


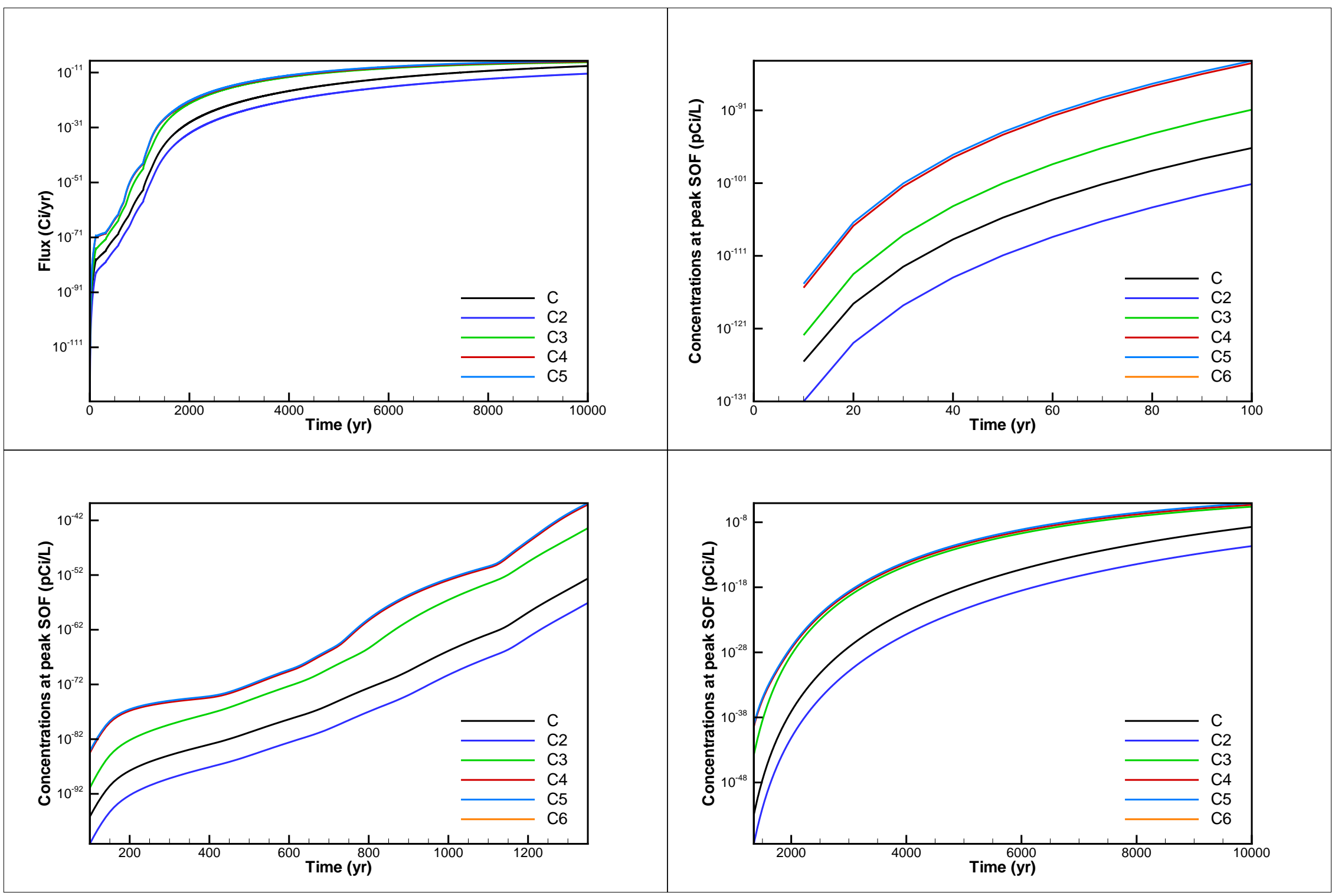

Figure A-80. Flux and concentration results for case U-234_0.1: U-234 Th-230 Ra-226 Pb-210 Po-210 


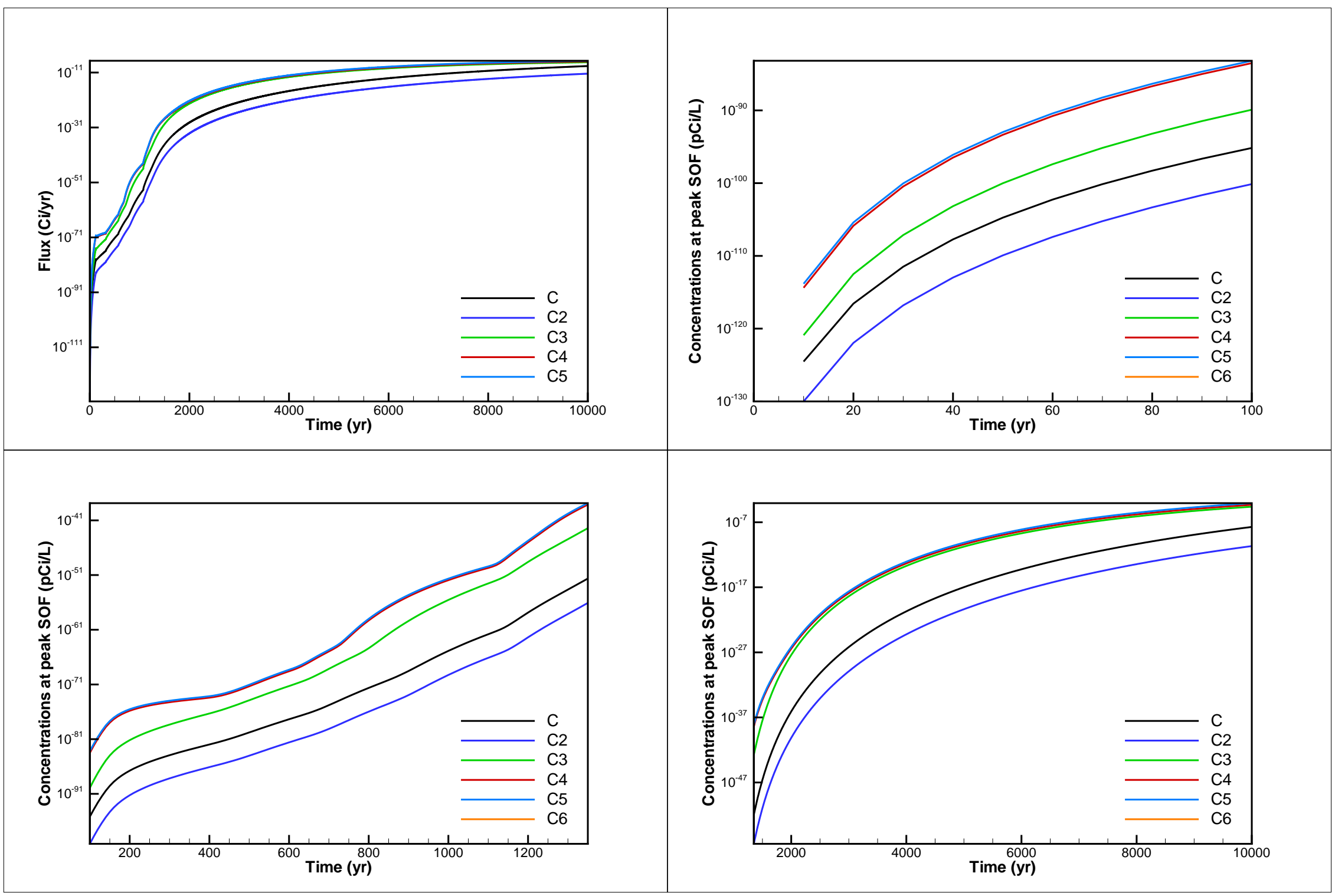

Figure A-81. Flux and concentration results for case U-234_1: U-234 Th-230 Ra-226 Pb-210 Po-210 


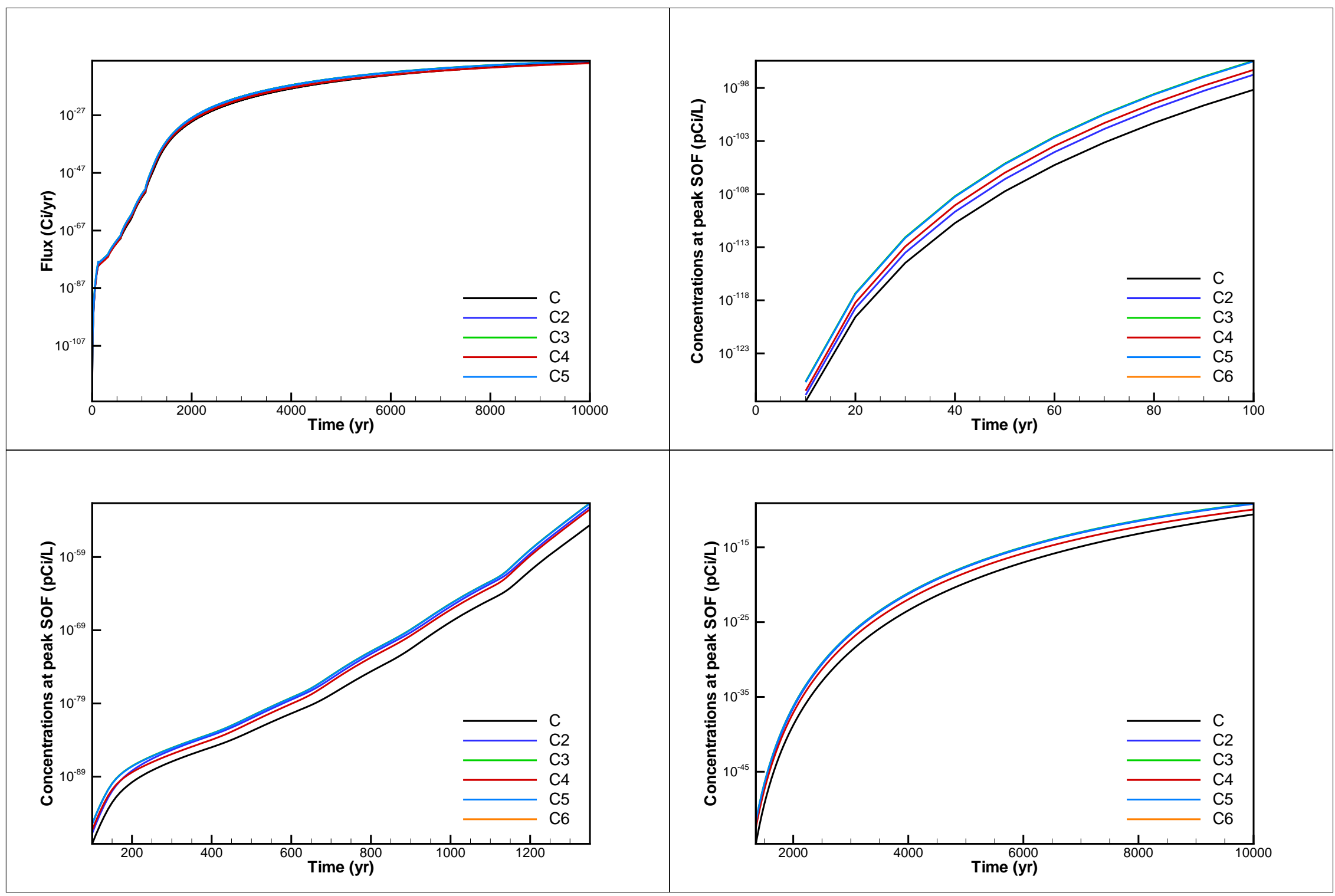

Figure A-82. Flux and concentration results for case U-235_0.001: U-235 Pa-231 Ac-227 Th-227 Ra-223 


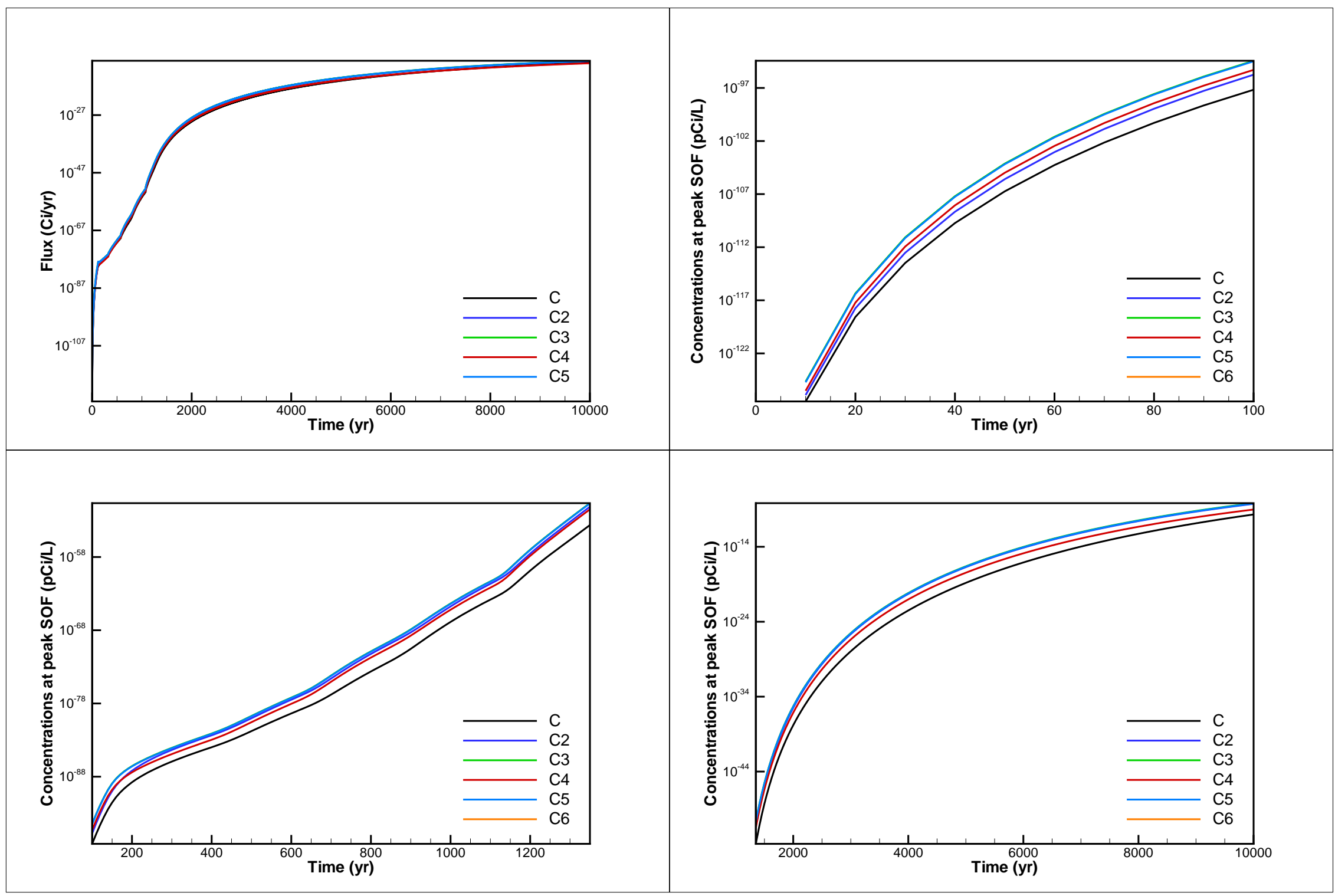

Figure A-83. Flux and concentration results for case U-235_0.01: U-235 Pa-231 Ac-227 Th-227 Ra-223 


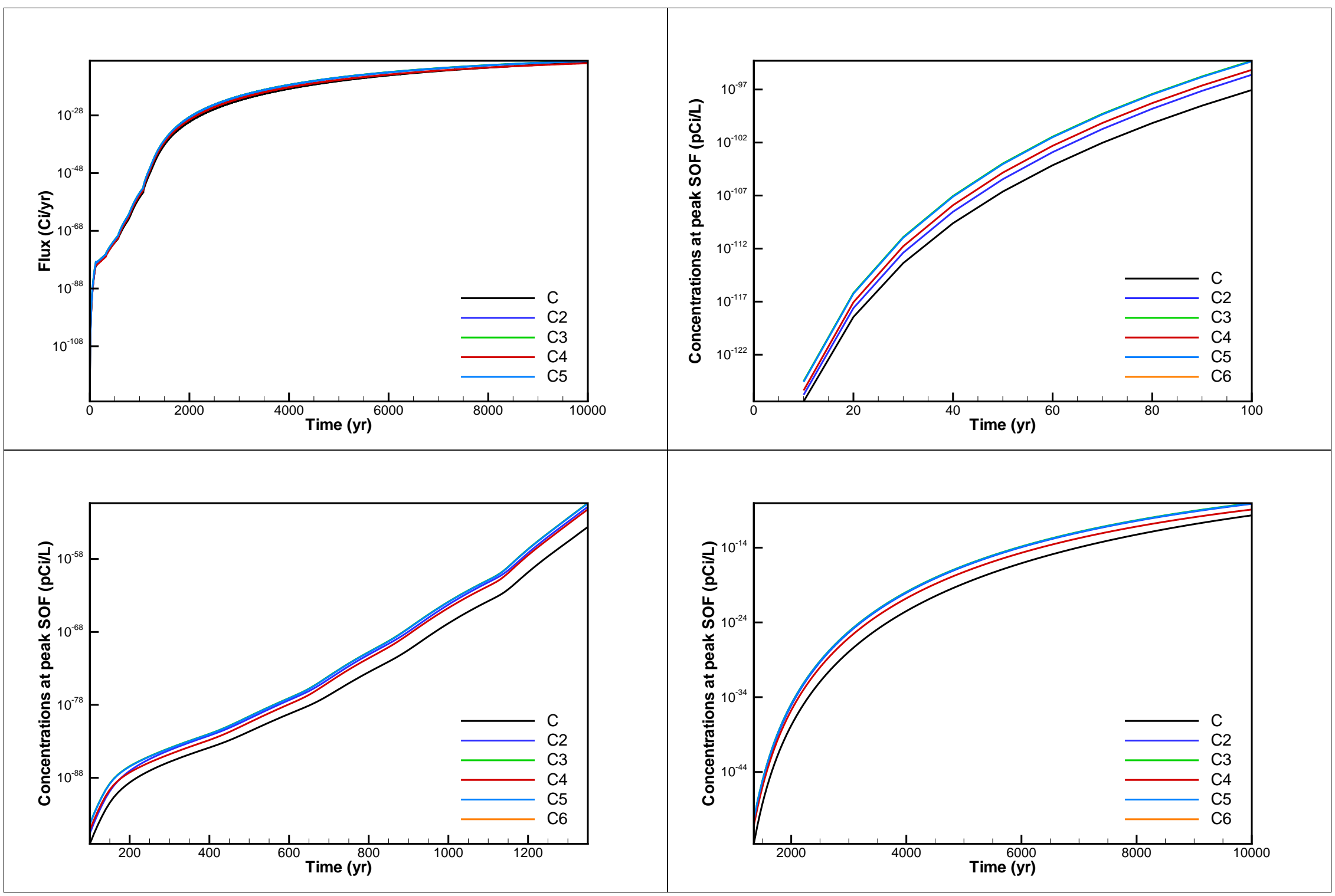

Figure A-84. Flux and concentration results for case U-235_0.1: U-235 Pa-231 Ac-227 Th-227 Ra-223 


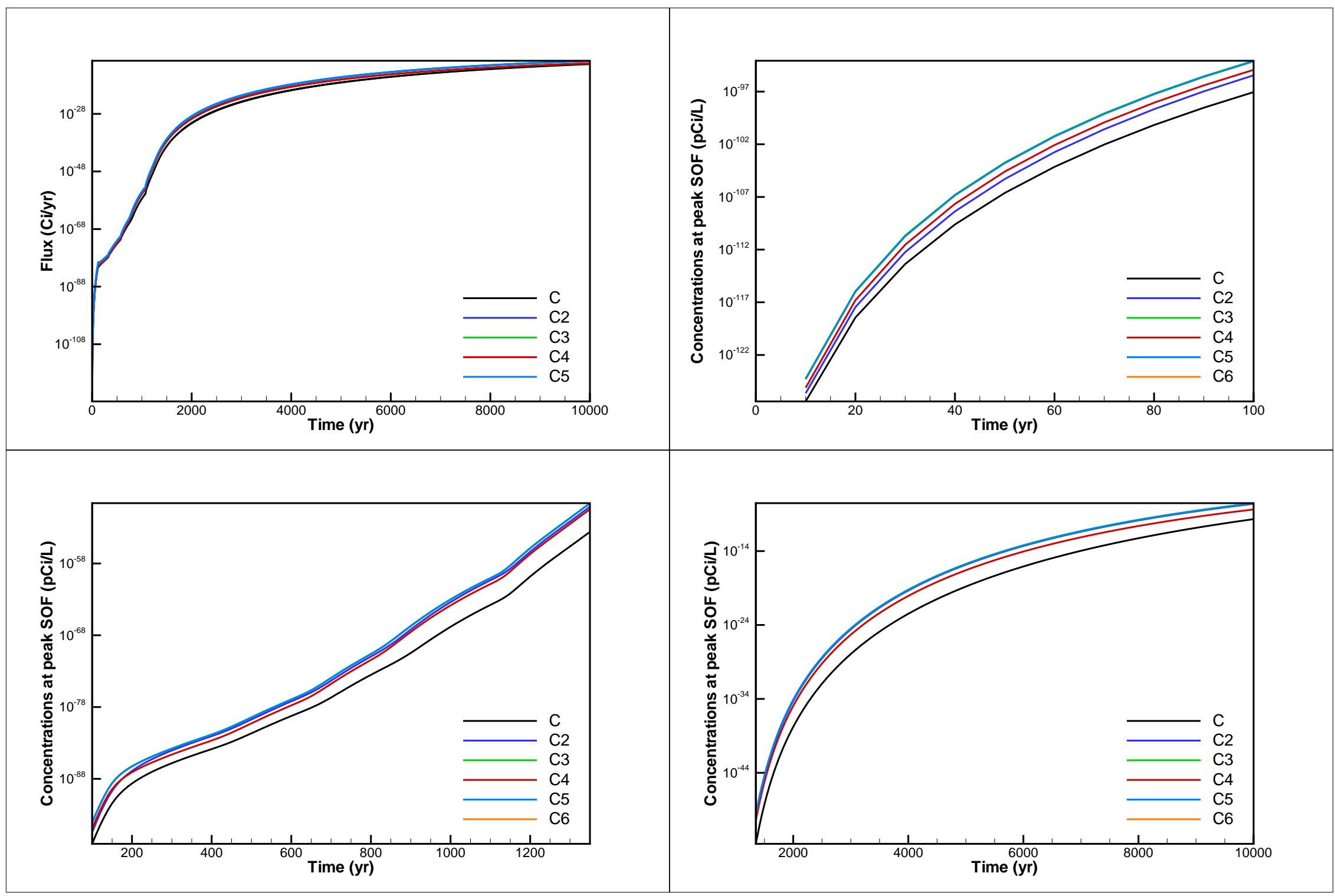

Figure A-85. Flux and concentration results for case U-235_1: U-235 Pa-231 Ac-227 Th-227 Ra-223 


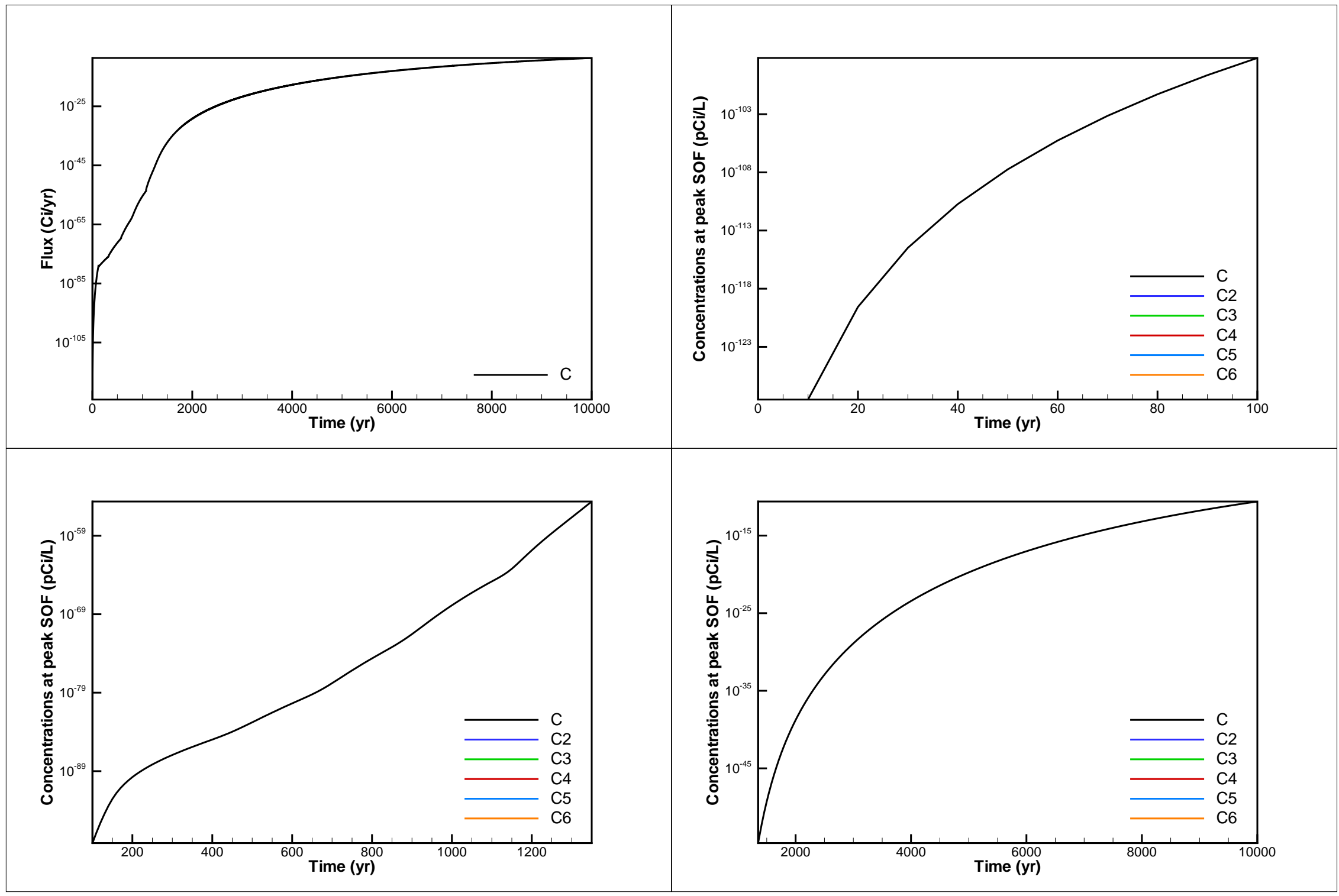

Figure A-86. Flux and concentration results for case U-236_0.001: U-236 


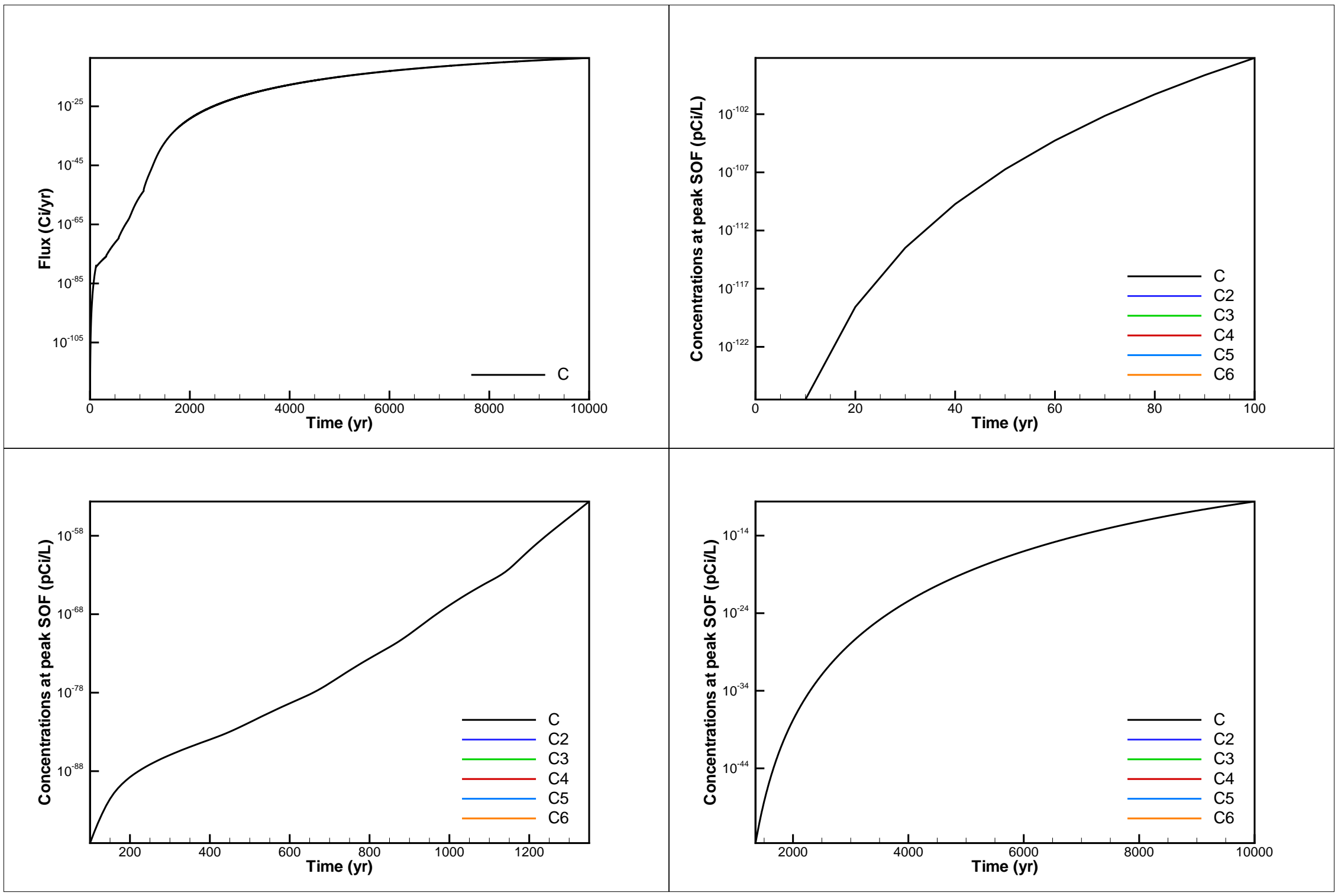

Figure A-87. Flux and concentration results for case U-236_0.01: U-236 


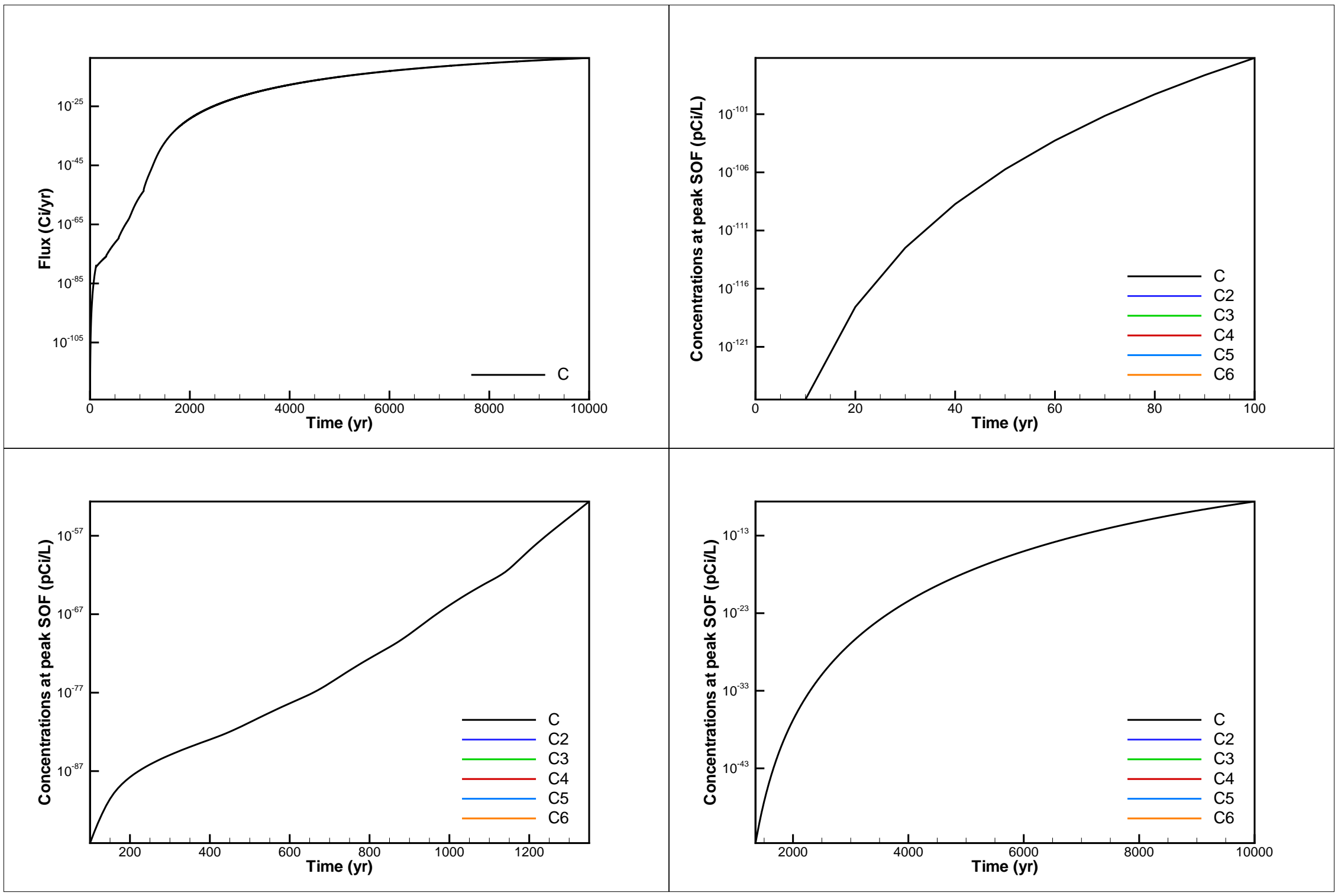

Figure A-88. Flux and concentration results for case U-236_0.1: U-236 


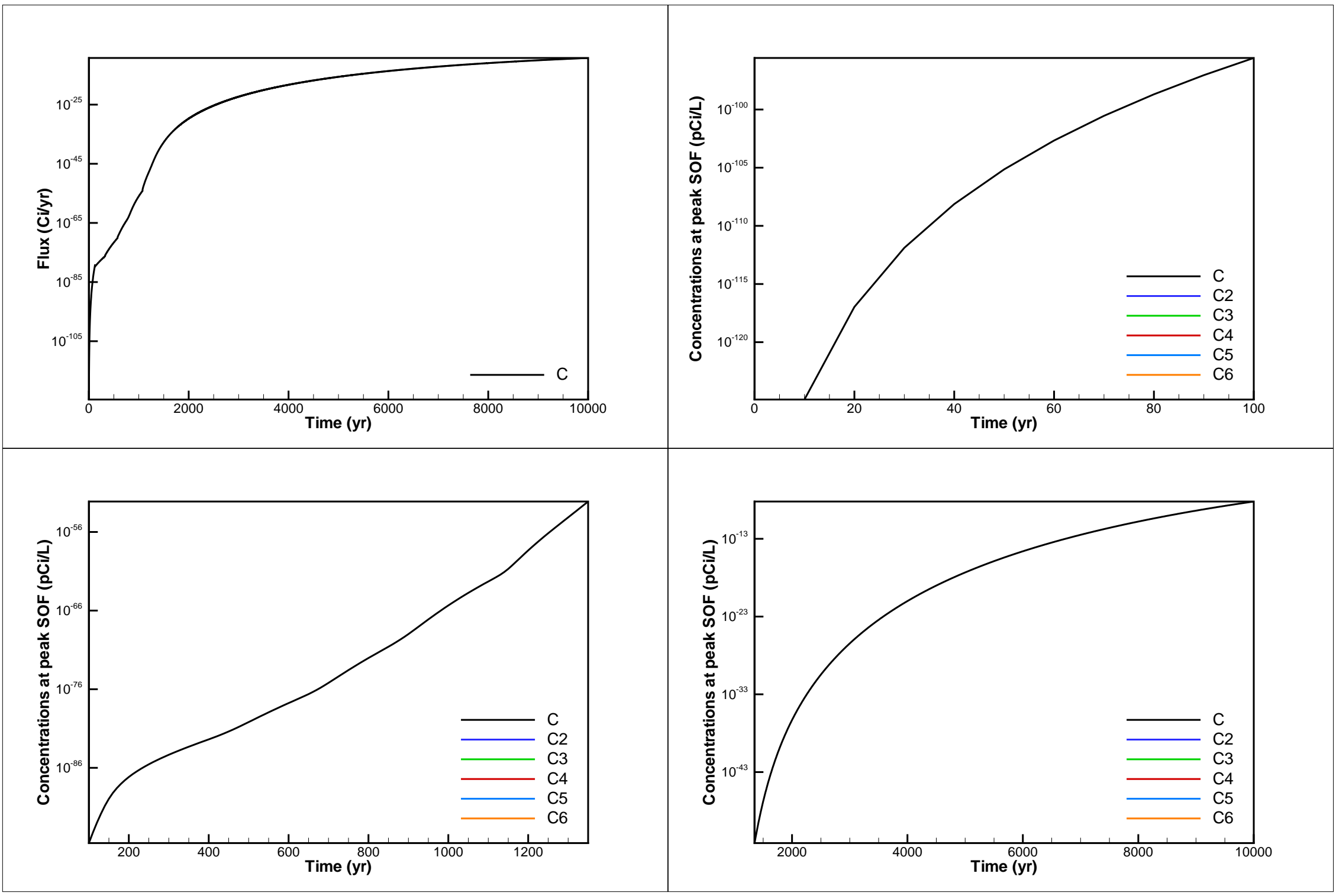

Figure A-89. Flux and concentration results for case U-236_1: U-236 


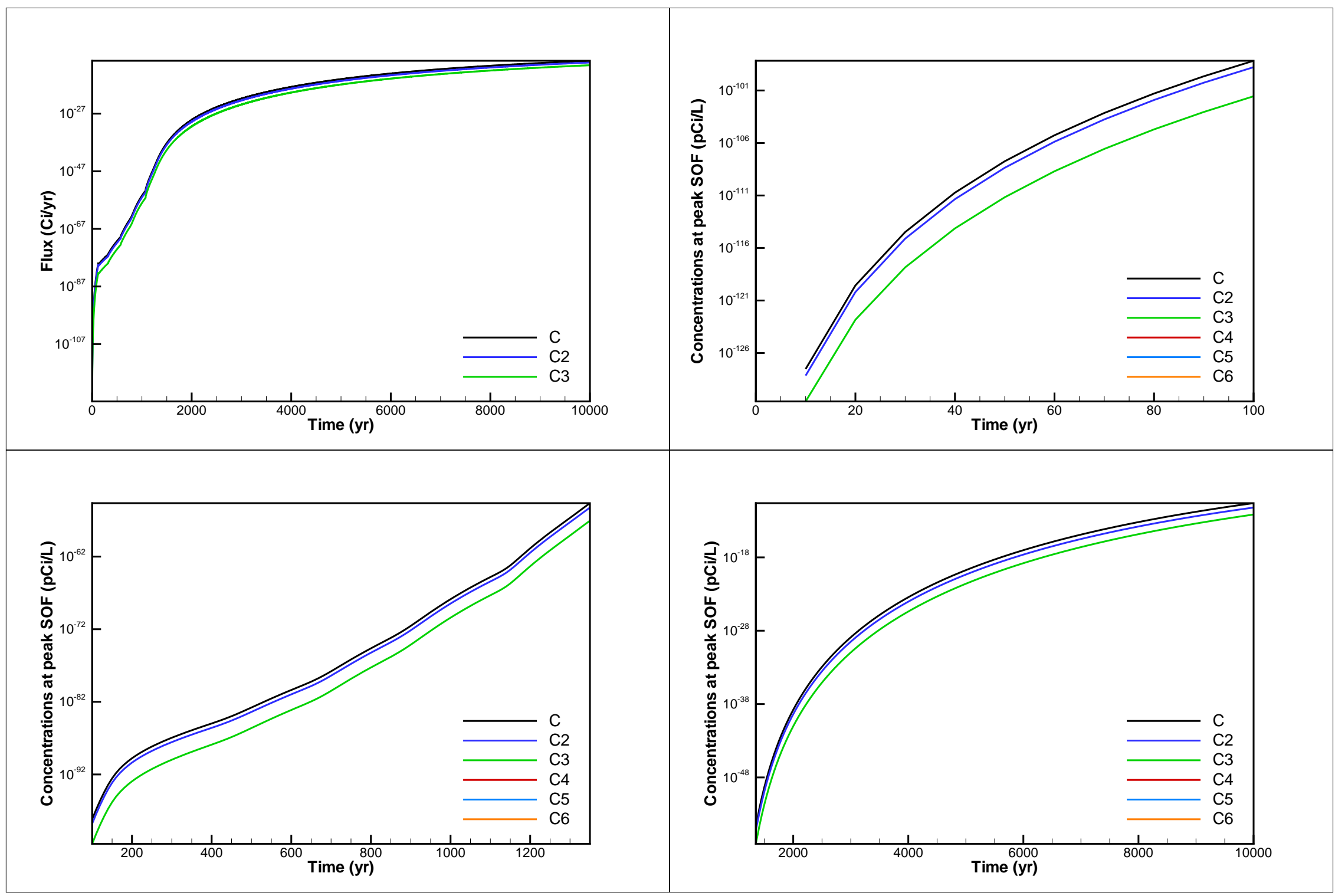

Figure A-90. Flux and concentration results for case U-238_0.001: U-238 Th-234 U-234 


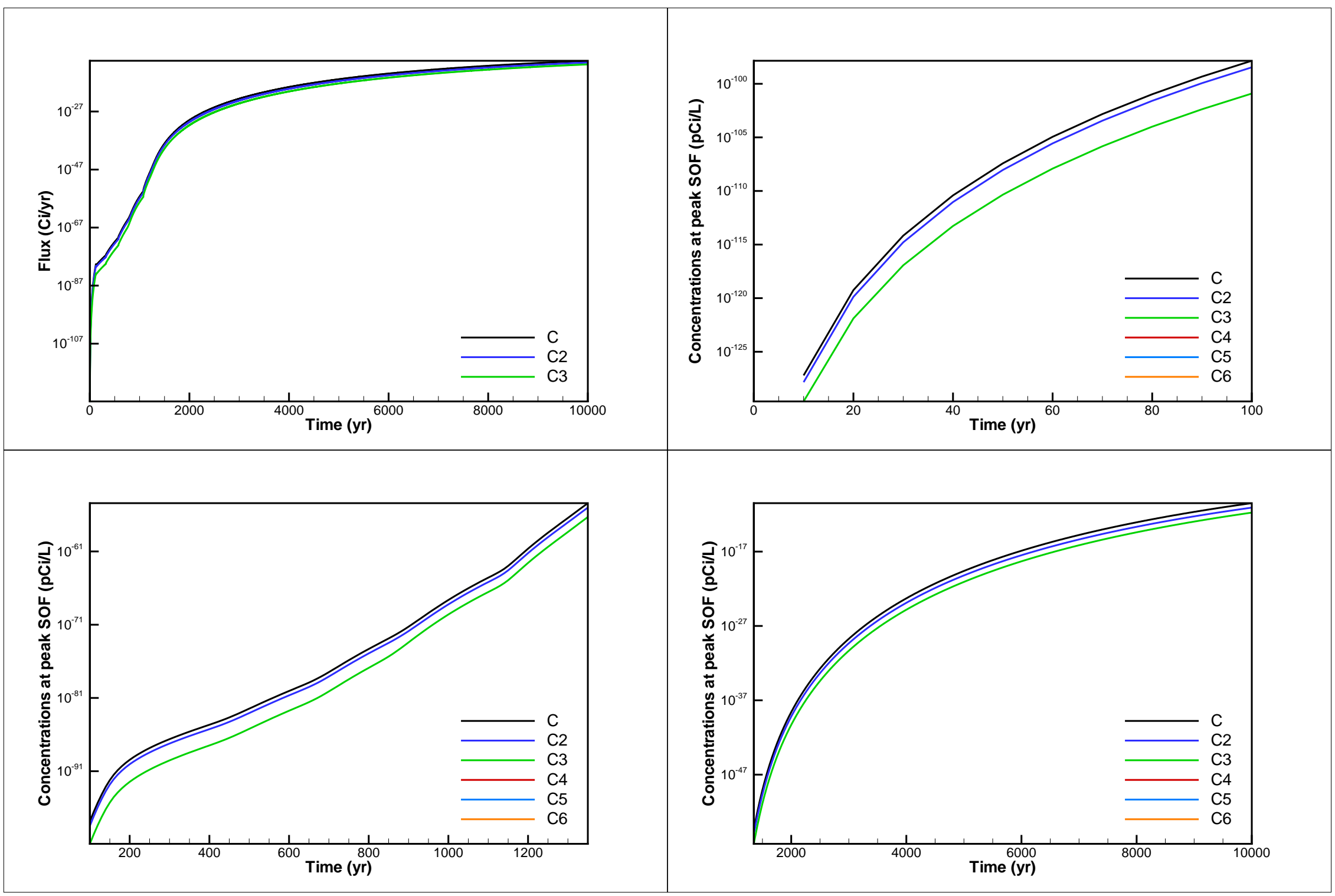

Figure A-91. Flux and concentration results for case U-238_0.01: U-238 Th-234 U-234 


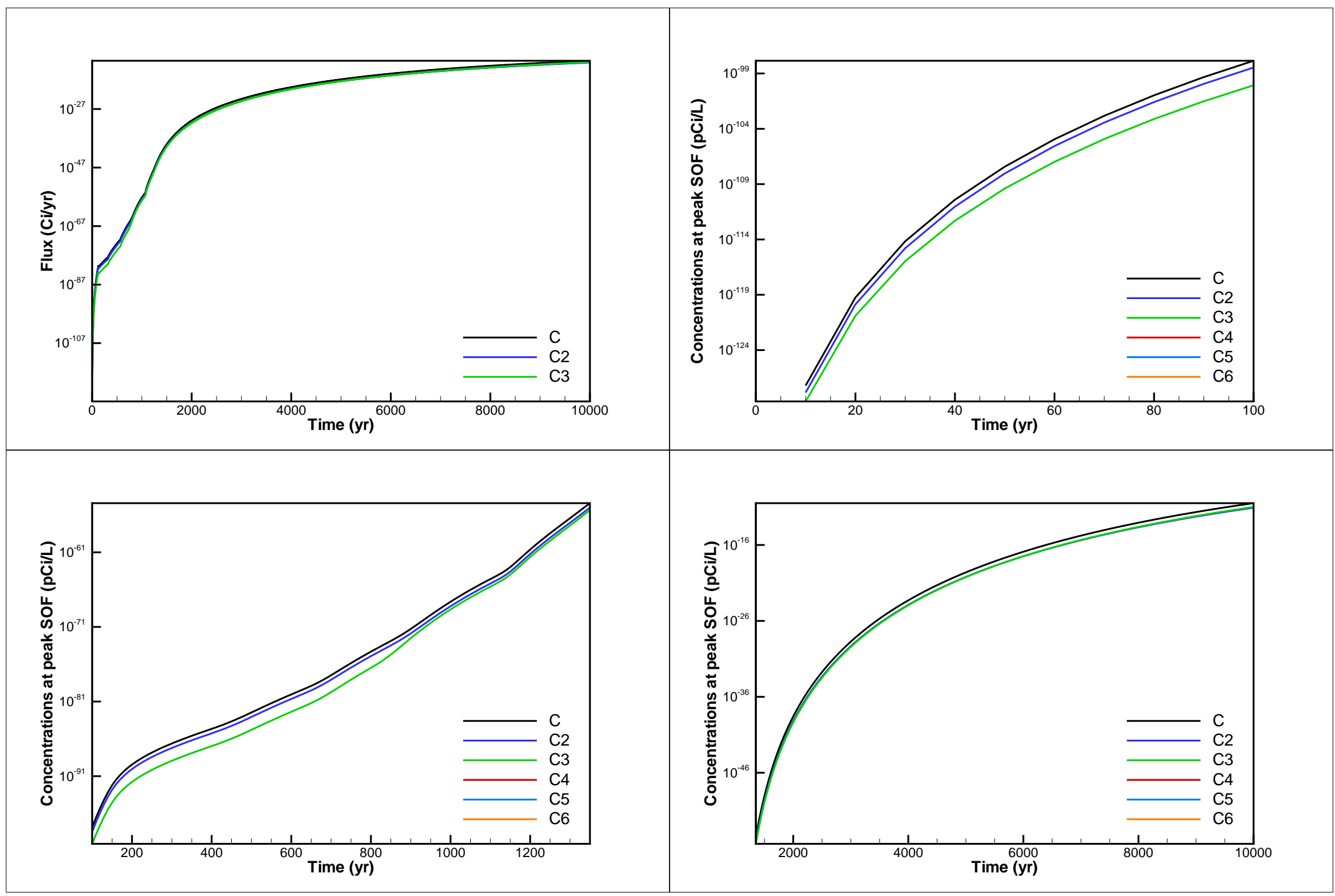

Figure A-92. Flux and concentration results for case U-238_0.1: U-238 Th-234 U-234 


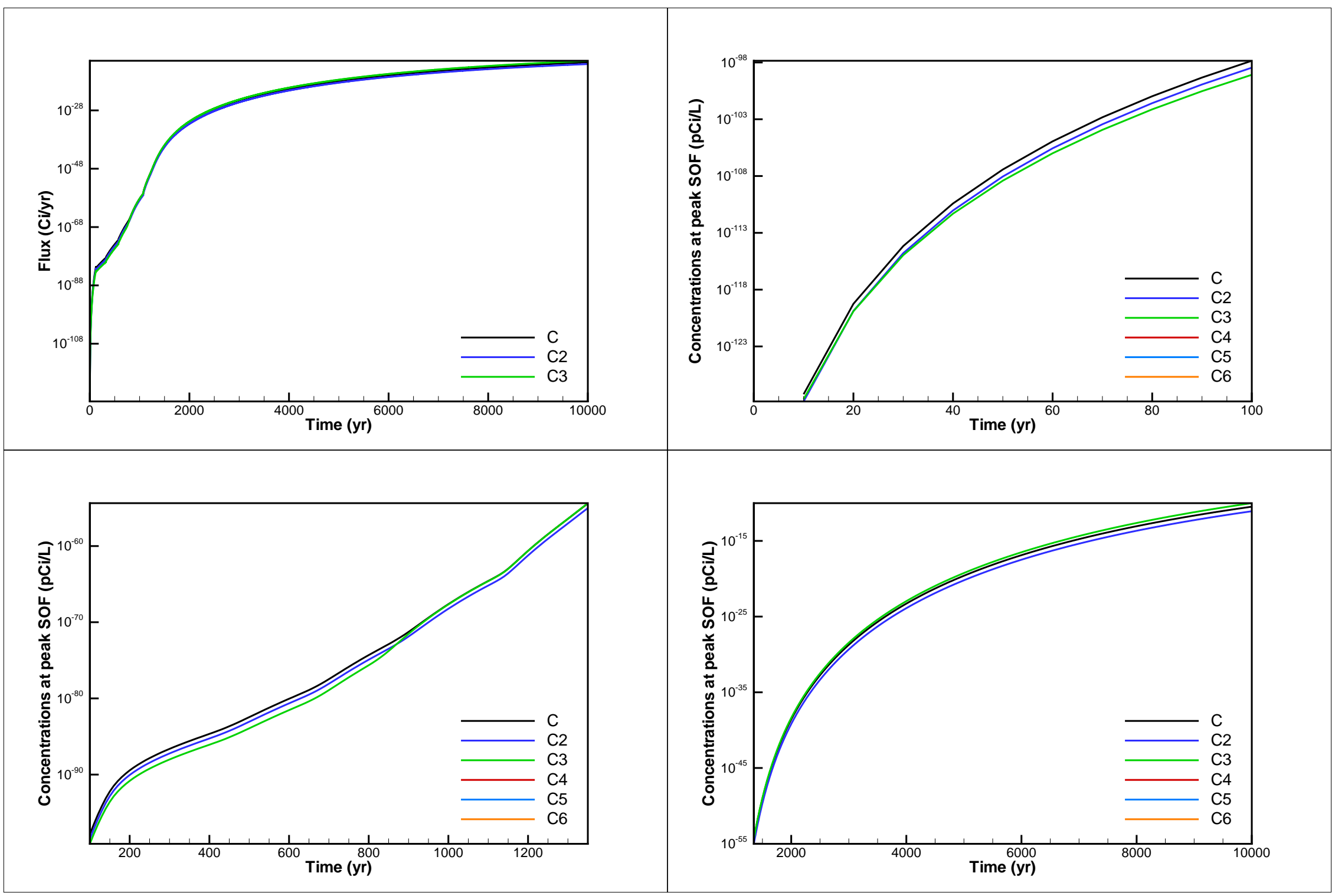

Figure A-93. Flux and concentration results for case U-238_1: U-238 Th-234 U-234 


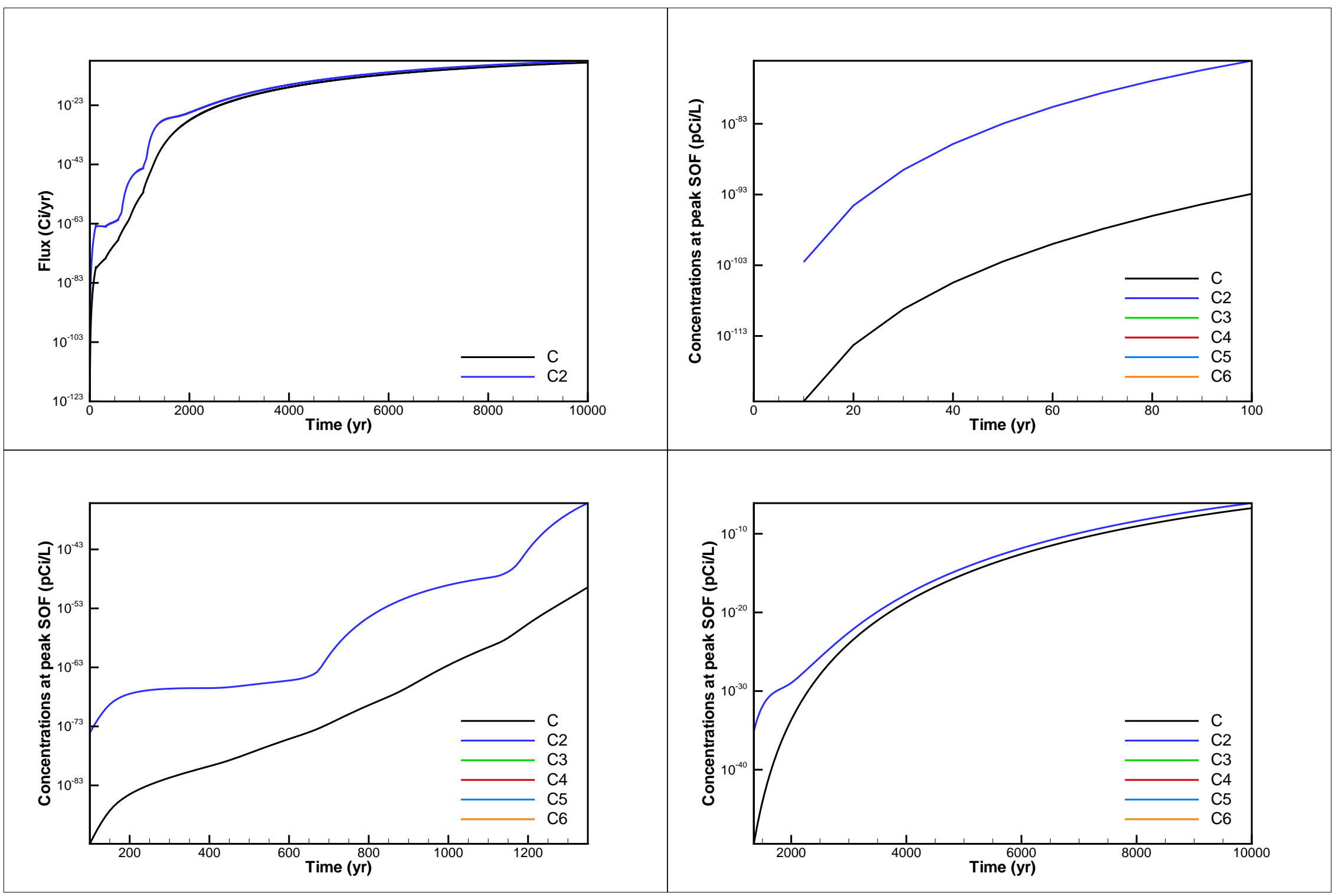

Figure A-94. Flux and concentration results for case Zr-93_1: Zr-93 Nb-93m 


\section{APPENDIX B - ADDITIONAL INFORMATION ON INTRUDER ANALYSIS}

This appendix provides additional detail about the intruder analysis, primarily for design checking and diagnostic purposes. The topics addressed are

- Disposal unit inputs to the automated intruder analysis tool

- Detailed decay chain contributions to the maximum dose for the resident scenario

\section{Disposal unit inputs}

Inputs specific to the disposal unit in the automated intruder analysis are reproduced below

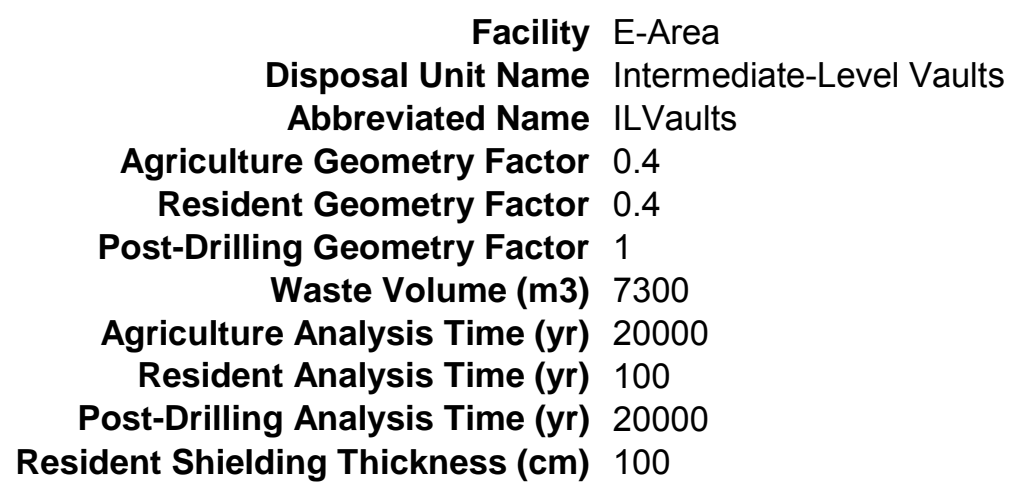

\section{Transient Layer Model (Surface to Top of Waste)}

$\begin{array}{cclcc}\text { Layer } & \text { Thickness }(\mathbf{m}) & \text { Description } & \mathbf{( m m / y r )} & \text { Time }(\mathbf{y r}) \\ 1 & 0.9144 & \text { Soil cover }(36 ") & 1.4 & 0 \\ 2 & 0.3048 & \text { Erosion barrier }(12 ") & 1.00 \mathrm{E}-10 & 0 \\ 3 & 1.6358 & \text { Soil backfill }(64.4 ") & 1.4 & 0 \\ 4 & 1.1176 & \text { Vault roof }\left(27^{\prime \prime}\right)+ & 1.4 & 1000\end{array}$

The geometry factor is based on an IL vault width of $50 \mathrm{ft}$ and a gap of $150 \mathrm{ft}$ between vaults. The latter is consistent with current plans to footprints \#1 and \#5 indicated below (excerpt from drawing W2017860).

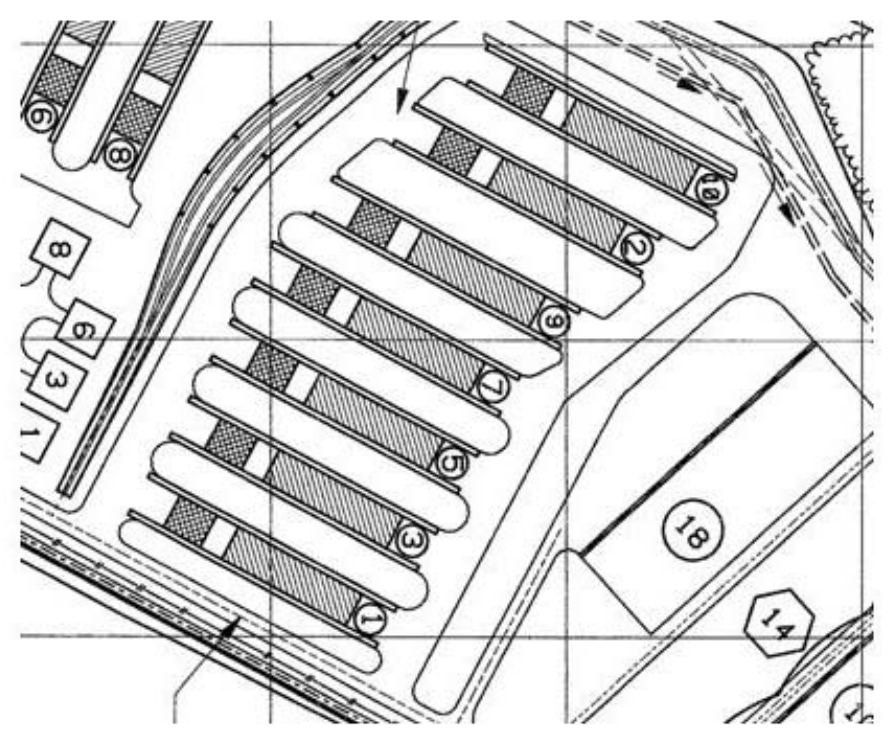


The total width of the combined facilities and gap is $50+150+50=250 \mathrm{ft}$. The width of both facilities excluding the gap is $100 \mathrm{ft}$, producing a geometry factor of $100 \mathrm{ft} / 250 \mathrm{ft}=0.40$.

According to the PA, the combined volume of the ILNT and ILT vaults is $5700+1600 \mathrm{~m}^{3}$ (WSRC-RP-94-218, Revision 1, Section 2.2.2.1).

Cover layers are taken directly from Figure 3.3-2 of Phifer (2004b).

\section{Detailed decay chain contributions}

Table B-1 provides detailed decay chain contributions to the maximum resident scenario inventory limit given in Table 3-1. 
Table B-1 Detailed Decay Chain Contributions to Maximum Intruder-Based Radionuclide Dose Limit for Intermediate-Level Vaults - Resident Scenario with Transient Calculation for 1,000 years

In the following, the bold header line gives the parent radionuclide, the time in years of the maximum dose, and the dose (rem/yr) for $1 \mathrm{Ci}$ of the parent in the waste volume.

Subsequent lines give the decay chain radionuclide, the activity (fraction remaining), and the dose contribution of the radionuclide towards the total.

Zero dose cases, where there is no maximum, are skipped and include the following parent radionuclides: H-3, C-14, S-35, Cl-36, Ar-39, Ca-41, Ni-59, Ni-63, Se-79, Rb-87, Sr-90, Zr-93, Nb93m, Mo-93, Pd-107, Cd-113m, Sn-121m, Cs-135, Sm-151

\begin{tabular}{|c|c|c|}
\hline $\mathrm{Na}-22$ & $100 \mathrm{yr}$ & 9.23E-17 \\
\hline $\mathrm{Na}-22$ & $2.69 \mathrm{E}-12$ & $9.23 \mathrm{E}-17$ \\
\hline Al-26 & $100 \mathrm{yr}$ & 1.51E-04 \\
\hline Al-26 & $1.00 \mathrm{E}+00$ & $1.51 \mathrm{E}-04$ \\
\hline K-40 & $100 \mathrm{yr}$ & 6.53E-06 \\
\hline $\mathrm{K}-40$ & $1.00 \mathrm{E}+00$ & $6.53 \mathrm{E}-06$ \\
\hline Sc-46 & $100 \mathrm{yr}$ & $1.84 \mathrm{E}-136$ \\
\hline Sc-46 & $7.37 \mathrm{E}-132$ & $1.84 \mathrm{E}-136$ \\
\hline Co-60 & $100 \mathrm{yr}$ & 1.25E-10 \\
\hline Co-60 & $1.96 \mathrm{E}-06$ & $1.25 \mathrm{E}-10$ \\
\hline
\end{tabular}

\begin{tabular}{lcc} 
Sb-125 & $\mathbf{1 0 0} \mathbf{y r}$ & $\mathbf{5 . 1 0 E}-18$ \\
\hline $\mathrm{Sb}-125$ & $1.22 \mathrm{E}-11$ & $5.10 \mathrm{E}-18$ \\
$\mathrm{Te}-125 \mathrm{~m}$ & $2.96 \mathrm{E}-12$ & $1.03 \mathrm{E}-26$
\end{tabular}

\begin{tabular}{ccc}
$\mathbf{K r}-\mathbf{8 5}$ & $\mathbf{1 0 0} \mathbf{y r}$ & $\mathbf{2 . 6 4 E}-12$ \\
\hline $\mathrm{Kr}-85$ & $1.61 \mathrm{E}-03$ & $2.64 \mathrm{E}-12$
\end{tabular}

\begin{tabular}{ccc} 
I-129 & $\mathbf{1 0 0}$ yr & $\mathbf{7 . 1 4 E}-42$ \\
\hline $\mathrm{I}-129$ & $1.00 \mathrm{E}+00$ & $7.14 \mathrm{E}-42$
\end{tabular}

\begin{tabular}{lcc} 
Cs-134 & $\mathbf{1 0 0}$ yr & $\mathbf{1 . 7 5 E - 2 0}$ \\
\hline Cs-134 & $2.74 \mathrm{E}-15$ & $1.75 \mathrm{E}-20$ \\
& & \\
Cs-137 & $\mathbf{1 0 0}$ yr & $\mathbf{1 . 2 0 E - 0 7}$ \\
\hline Cs-137 & $9.97 \mathrm{E}-02$ & $0.00 \mathrm{E}+00$ \\
Ba-137m & $9.44 \mathrm{E}-02$ & $1.20 \mathrm{E}-07$
\end{tabular}

\begin{tabular}{ccc} 
Nb-94 & 100 yr & 7.57E-06 \\
\hline $\mathrm{Nb}-94$ & $9.97 \mathrm{E}-01$ & $7.57 \mathrm{E}-06$
\end{tabular}

\begin{tabular}{ccc} 
Tc-99 & $\mathbf{1 0 0} \mathbf{~ y r}$ & $\mathbf{1 . 3 1 E - 1 9}$ \\
\hline Tc-99 & $1.00 \mathrm{E}+00$ & $1.31 \mathrm{E}-19$
\end{tabular}

\begin{tabular}{lcc} 
Ag-108m & $\mathbf{1 0 0}$ yr & 2.56E-06 \\
\hline Ag-108m & $8.47 \mathrm{E}-01$ & $2.56 \mathrm{E}-06$ \\
$\mathrm{Ag}-108$ & $7.54 \mathrm{E}-02$ & $2.03 \mathrm{E}-09$
\end{tabular}

\begin{tabular}{ccc} 
Ba-133 & $\mathbf{1 0 0} \mathbf{y r}$ & $\mathbf{5 . 9 7 E - 1 1}$ \\
\hline Ba-133 & $1.40 \mathrm{E}-03$ & $5.97 \mathrm{E}-11$ \\
& & \\
Eu-152 & $\mathbf{1 0 0} \mathbf{~ y r}$ & $\mathbf{1 . 1 2 E - 0 7}$ \\
\hline Eu-152 & $5.93 \mathrm{E}-03$ & $1.12 \mathrm{E}-07$ \\
Gd-152 & $3.46 \mathrm{E}-14$ & $0.00 \mathrm{E}+00$
\end{tabular}

\begin{tabular}{lcc} 
& & \\
Sn-126 & $\mathbf{1 0 0}$ yr & 4.69E-06 \\
\hline Sn-126 & $9.99 \mathrm{E}-01$ & $6.14 \mathrm{E}-15$ \\
$\mathrm{Sb}-126 \mathrm{~m}$ & $9.99 \mathrm{E}-01$ & $3.55 \mathrm{E}-06$ \\
$\mathrm{Sb}-126$ & $1.40 \mathrm{E}-01$ & $1.14 \mathrm{E}-06$
\end{tabular}

\begin{tabular}{|c|c|c|}
\hline Eu-154 & $100 \mathrm{yr}$ & $6.25 E-09$ \\
\hline Eu-154 & $3.14 \mathrm{E}-04$ & $6.25 \mathrm{E}-09$ \\
\hline Eu-155 & $100 \mathrm{yr}$ & $6.44 \mathrm{E}-20$ \\
\hline Eu-155 & $4.76 \mathrm{E}-07$ & $6.44 \mathrm{E}-20$ \\
\hline W-181 & $100 \mathrm{yr}$ & 1.69E-104 \\
\hline W-181 & $2.20 \mathrm{E}-91$ & $1.69 \mathrm{E}-104$ \\
\hline W-185 & $100 \mathrm{yr}$ & 1.02E-161 \\
\hline W-185 & $4.94 \mathrm{E}-147$ & $1.02 \mathrm{E}-161$ \\
\hline
\end{tabular}


Table B-1 Detailed Decay Chain Contributions to Maximum Intruder-Based Radionuclide Dose Limit for Intermediate-Level Vaults - Resident Scenario with Transient Calculation for 1,000 years

\begin{tabular}{|c|c|c|}
\hline W-188 & $100 \mathrm{yr}$ & $1.08 \mathrm{E}-165$ \\
\hline W-188 & $4.76 \mathrm{E}-159$ & $2.24 \mathrm{E}-169$ \\
\hline Re-188 & $4.81 \mathrm{E}-159$ & $1.08 \mathrm{E}-165$ \\
\hline Pb-210 & $100 \mathrm{yr}$ & 1.83E-12 \\
\hline $\mathrm{Pb}-210$ & $4.47 \mathrm{E}-02$ & $1.72 \mathrm{E}-33$ \\
\hline Bi-210 & 4.47E-02 & $0.00 \mathrm{E}+00$ \\
\hline Рo-210 & $4.55 \mathrm{E}-02$ & $1.83 \mathrm{E}-12$ \\
\hline Bi-207 & $100 \mathrm{yr}$ & 2.33E-06 \\
\hline $\mathrm{Bi}-207$ & $1.11 \mathrm{E}-01$ & $2.33 \mathrm{E}-06$ \\
\hline Ra-226 & $100 \mathrm{yr}$ & 7.05E-05 \\
\hline Ra-226 & $9.58 \mathrm{E}-01$ & $7.14 \mathrm{E}-12$ \\
\hline Rn-222 & $9.58 \mathrm{E}-01$ & $2.68 \mathrm{E}-10$ \\
\hline Ро-218 & $9.58 \mathrm{E}-01$ & $0.00 \mathrm{E}+00$ \\
\hline $\mathrm{Pb}-214$ & $9.57 \mathrm{E}-01$ & 8.89E-08 \\
\hline At-218 & $1.92 \mathrm{E}-04$ & $0.00 \mathrm{E}+00$ \\
\hline $\mathrm{Bi}-214$ & $9.58 \mathrm{E}-01$ & $7.04 \mathrm{E}-05$ \\
\hline Po-214 & $9.57 \mathrm{E}-01$ & $3.66 \mathrm{E}-10$ \\
\hline $\mathrm{Pb}-210$ & $9.26 \mathrm{E}-01$ & $3.56 \mathrm{E}-32$ \\
\hline Bi-210 & $9.26 \mathrm{E}-01$ & $0.00 \mathrm{E}+00$ \\
\hline Po-210 & $9.25 \mathrm{E}-01$ & $3.72 \mathrm{E}-11$ \\
\hline Ra-228 & $100 \mathrm{yr}$ & 1.94E-09 \\
\hline Ra-228 & $5.82 \mathrm{E}-06$ & $0.00 \mathrm{E}+00$ \\
\hline Ac- 228 & $5.82 \mathrm{E}-06$ & $8.02 \mathrm{E}-11$ \\
\hline Th-228 & 8.71E-06 & $1.09 \mathrm{E}-17$ \\
\hline $\mathrm{Ra}-224$ & 8.73E-06 & 7.81E-16 \\
\hline Rn-220 & 8.73E-06 & $4.52 \mathrm{E}-15$ \\
\hline Ро-216 & 8.73E-06 & $6.04 \mathrm{E}-16$ \\
\hline $\mathrm{Pb}-212$ & 8.73E-06 & $9.34 \mathrm{E}-15$ \\
\hline Bi-212 & 8.73E-06 & $3.48 \mathrm{E}-11$ \\
\hline Tl-208 & $3.14 \mathrm{E}-06$ & $1.82 \mathrm{E}-09$ \\
\hline Po-212 & $5.59 \mathrm{E}-06$ & $0.00 \mathrm{E}+00$ \\
\hline
\end{tabular}

\begin{tabular}{lcc} 
Ac-227 & $\mathbf{1 0 0}$ yr & $\mathbf{8 . 2 0 E}-\mathbf{0 9}$ \\
\hline Ac-227 & $4.14 \mathrm{E}-02$ & $1.21 \mathrm{E}-16$ \\
Fr-223 & $5.72 \mathrm{E}-04$ & $9.08 \mathrm{E}-12$ \\
Th-227 & $4.09 \mathrm{E}-02$ & $1.24 \mathrm{E}-10$ \\
Ra-223 & $4.16 \mathrm{E}-02$ & $2.35 \mathrm{E}-10$ \\
Rn-219 & $4.16 \mathrm{E}-02$ & $2.82 \mathrm{E}-10$ \\
Po-215 & $4.16 \mathrm{E}-02$ & $2.09 \mathrm{E}-12$ \\
Pb-211 & $4.16 \mathrm{E}-02$ & $6.62 \mathrm{E}-09$ \\
Bi-211 & $4.16 \mathrm{E}-02$ & $2.70 \mathrm{E}-10$ \\
Tl-207 & $4.15 \mathrm{E}-02$ & $6.54 \mathrm{E}-10$ \\
Po-211 & $1.16 \mathrm{E}-04$ & $4.39 \mathrm{E}-12$ \\
& & \\
Th-228 & $\mathbf{1 0 0 ~ y r}$ & $\mathbf{3 . 8 2 E}-20$ \\
\hline Th-228 & $1.79 \mathrm{E}-16$ & $2.24 \mathrm{E}-28$ \\
Ra-224 & $1.80 \mathrm{E}-16$ & $1.61 \mathrm{E}-26$ \\
Rn-220 & $1.80 \mathrm{E}-16$ & $9.31 \mathrm{E}-26$ \\
Po-216 & $1.80 \mathrm{E}-16$ & $1.24 \mathrm{E}-26$ \\
Pb-212 & $1.80 \mathrm{E}-16$ & $1.92 \mathrm{E}-25$ \\
Bi-212 & $1.80 \mathrm{E}-16$ & $7.16 \mathrm{E}-22$ \\
Tl-208 & $6.46 \mathrm{E}-17$ & $3.75 \mathrm{E}-20$ \\
Po-212 & $1.15 \mathrm{E}-16$ & $0.00 \mathrm{E}+00$ \\
& & \\
Th-229 & $\mathbf{1 0 0 ~ y r}$ & $\mathbf{1 . 9 0 E - 0 6}$ \\
\hline Th-229 & $9.91 \mathrm{E}-01$ & $2.73 \mathrm{E}-11$ \\
Ra-225 & $9.91 \mathrm{E}-01$ & $1.75 \mathrm{E}-40$ \\
Ac-225 & $9.91 \mathrm{E}-01$ & $1.99 \mathrm{E}-10$ \\
Fr-221 & $9.91 \mathrm{E}-01$ & $1.65 \mathrm{E}-10$ \\
At-217 & $9.91 \mathrm{E}-01$ & $3.23 \mathrm{E}-10$ \\
Bi-213 & $9.91 \mathrm{E}-01$ & $1.43 \mathrm{E}-07$ \\
Tl-209 & $2.14 \mathrm{E}-02$ & $1.76 \mathrm{E}-06$ \\
Po-213 & $9.69 \mathrm{E}-01$ & $1.24 \mathrm{E}-10$ \\
Pb-209 & $9.91 \mathrm{E}-01$ & $0.00 \mathrm{E}+00$ \\
& & \\
Th-230 & $\mathbf{1 0 0 0}$ yr & $\mathbf{2 . 5 8 E - 0 5}$ \\
\hline Th-230 & $9.91 \mathrm{E}-01$ & $8.10 \mathrm{E}-14$ \\
Ra-226 & $3.50 \mathrm{E}-01$ & $2.61 \mathrm{E}-12$ \\
Rn-222 & $3.50 \mathrm{E}-01$ & $9.80 \mathrm{E}-11$ \\
Po-218 & $3.50 \mathrm{E}-01$ & $0.00 \mathrm{E}+00$ \\
Pb-214 & $3.50 \mathrm{E}-01$ & $3.25 \mathrm{E}-08$ \\
At-218 & $7.00 \mathrm{E}-05$ & $0.00 \mathrm{E}+00$ \\
Bi-214 & $3.50 \mathrm{E}-01$ & $2.57 \mathrm{E}-05$ \\
Pb-210 & $3.50 \mathrm{E}-01$ & $1.34 \mathrm{E}-10$ \\
& $3.41 \mathrm{E}-01$ & $1.31 \mathrm{E}-32$ \\
$3.41 \mathrm{E}-01$ & $0.00 \mathrm{E}+00$ \\
$3.41 \mathrm{E}-01$ & $1.37 \mathrm{E}-11$ \\
& &
\end{tabular}


Table B-1 Detailed Decay Chain Contributions to Maximum Intruder-Based Radionuclide Dose Limit for Intermediate-Level Vaults - Resident Scenario with Transient Calculation for 1,000 years

\begin{tabular}{lcc} 
Th-232 & 180 yr & 2.26E-04 \\
\hline Th-232 & $1.00 \mathrm{E}+00$ & $3.97 \mathrm{E}-15$ \\
Ra-228 & $1.00 \mathrm{E}+00$ & $0.00 \mathrm{E}+00$ \\
Ac-228 & $1.00 \mathrm{E}+00$ & $1.38 \mathrm{E}-05$ \\
Th-228 & $1.00 \mathrm{E}+00$ & $1.25 \mathrm{E}-12$ \\
Ra-224 & $1.00 \mathrm{E}+00$ & $8.95 \mathrm{E}-11$ \\
Rn-220 & $1.00 \mathrm{E}+00$ & $5.18 \mathrm{E}-10$ \\
Po-216 & $1.00 \mathrm{E}+00$ & $6.92 \mathrm{E}-11$ \\
Pb-212 & $1.00 \mathrm{E}+00$ & $1.07 \mathrm{E}-09$ \\
Bi-212 & $1.00 \mathrm{E}+00$ & $3.98 \mathrm{E}-06$ \\
Tl-208 & $3.59 \mathrm{E}-01$ & $2.09 \mathrm{E}-04$ \\
Po-212 & $6.41 \mathrm{E}-01$ & $0.00 \mathrm{E}+00$ \\
& & \\
Pa-231 & $\mathbf{2 3 0}$ yr & $\mathbf{1 . 9 8 E - 0 7}$ \\
\hline Pa-231 & $9.95 \mathrm{E}-01$ & $1.52 \mathrm{E}-09$ \\
Ac-227 & $9.95 \mathrm{E}-01$ & $2.91 \mathrm{E}-15$ \\
Fr-223 & $1.37 \mathrm{E}-02$ & $2.18 \mathrm{E}-10$ \\
Th-227 & $9.81 \mathrm{E}-01$ & $2.96 \mathrm{E}-09$ \\
Ra-223 & $9.95 \mathrm{E}-01$ & $5.63 \mathrm{E}-09$ \\
Rn-219 & $9.95 \mathrm{E}-01$ & $6.74 \mathrm{E}-09$ \\
Po-215 & $9.95 \mathrm{E}-01$ & $5.00 \mathrm{E}-11$ \\
Pb-211 & $9.95 \mathrm{E}-01$ & $1.58 \mathrm{E}-07$ \\
Bi-211 & $9.95 \mathrm{E}-01$ & $6.45 \mathrm{E}-09$ \\
Tl-207 & $9.92 \mathrm{E}-01$ & $1.56 \mathrm{E}-08$ \\
Po-211 & $2.78 \mathrm{E}-03$ & $1.05 \mathrm{E}-10$ \\
& & \\
U-232 & $\mathbf{1 0 0 ~ y r}$ & $\mathbf{8 . 0 0 E - 0 5}$ \\
\hline $\mathrm{U}-232$ & $3.66 \mathrm{E}-01$ & $1.04 \mathrm{E}-14$ \\
Th-228 & $3.76 \mathrm{E}-01$ & $4.71 \mathrm{E}-13$ \\
Ra-224 & $3.76 \mathrm{E}-01$ & $3.37 \mathrm{E}-11$ \\
Rn-220 & $3.76 \mathrm{E}-01$ & $1.95 \mathrm{E}-10$ \\
Po-216 & $3.76 \mathrm{E}-01$ & $2.60 \mathrm{E}-11$ \\
Pb-212 & $3.76 \mathrm{E}-01$ & $4.02 \mathrm{E}-10$ \\
Bi-212 & $3.76 \mathrm{E}-01$ & $1.50 \mathrm{E}-06$ \\
Tl-208 & $1.35 \mathrm{E}-01$ & $7.85 \mathrm{E}-05$ \\
Po-212 & $2.41 \mathrm{E}-01$ & $0.00 \mathrm{E}+00$ \\
& &
\end{tabular}

\begin{tabular}{|c|c|c|}
\hline U-233 & $1000 \mathrm{yr}$ & 1.73E-07 \\
\hline U-233 & $9.96 \mathrm{E}-01$ & $4.80 \mathrm{E}-15$ \\
\hline Th-229 & 8.99E-02 & $2.48 \mathrm{E}-12$ \\
\hline Ra-225 & 8.99E-02 & $1.59 \mathrm{E}-41$ \\
\hline Ac- 225 & $8.99 \mathrm{E}-02$ & $1.81 \mathrm{E}-11$ \\
\hline Fr-221 & 8.99E-02 & $1.50 \mathrm{E}-11$ \\
\hline At-217 & 8.99E-02 & $2.93 \mathrm{E}-11$ \\
\hline $\mathrm{Bi}-213$ & 8.99E-02 & $1.30 \mathrm{E}-08$ \\
\hline Tl-209 & $1.94 \mathrm{E}-03$ & $1.60 \mathrm{E}-07$ \\
\hline Рo-213 & $8.80 \mathrm{E}-02$ & $1.12 \mathrm{E}-11$ \\
\hline Pb-209 & 8.99E-02 & $0.00 \mathrm{E}+00$ \\
\hline U-234 & $1000 \mathrm{yr}$ & 1.27E-07 \\
\hline U-234 & $9.97 \mathrm{E}-01$ & $2.55 \mathrm{E}-15$ \\
\hline Th-230 & $9.14 \mathrm{E}-03$ & $7.47 \mathrm{E}-16$ \\
\hline $\mathrm{Ra}-226$ & $1.73 \mathrm{E}-03$ & $1.29 \mathrm{E}-14$ \\
\hline $\mathrm{Rn}-222$ & $1.73 \mathrm{E}-03$ & $4.83 \mathrm{E}-13$ \\
\hline Рo-218 & $1.73 \mathrm{E}-03$ & $0.00 \mathrm{E}+00$ \\
\hline $\mathrm{Pb}-214$ & $1.73 \mathrm{E}-03$ & $1.60 \mathrm{E}-10$ \\
\hline At-218 & $3.45 \mathrm{E}-07$ & $0.00 \mathrm{E}+00$ \\
\hline $\mathrm{Bi}-214$ & $1.73 \mathrm{E}-03$ & $1.27 \mathrm{E}-07$ \\
\hline Рo-214 & $1.73 \mathrm{E}-03$ & $6.60 \mathrm{E}-13$ \\
\hline $\mathrm{Pb}-210$ & $1.62 \mathrm{E}-03$ & $6.25 \mathrm{E}-35$ \\
\hline Bi-210 & $1.62 \mathrm{E}-03$ & $0.00 \mathrm{E}+00$ \\
\hline Po-210 & $1.62 \mathrm{E}-03$ & $6.52 \mathrm{E}-14$ \\
\hline U-235 & $1000 \mathrm{yr}$ & 4.15E-09 \\
\hline U-235 & $1.00 \mathrm{E}+00$ & $1.25 \mathrm{E}-10$ \\
\hline Th-231 & $1.00 \mathrm{E}+00$ & $1.78 \mathrm{E}-13$ \\
\hline $\mathrm{Pa}-231$ & $2.09 \mathrm{E}-02$ & $3.20 \mathrm{E}-11$ \\
\hline Ac-227 & $2.03 \mathrm{E}-02$ & $5.93 \mathrm{E}-17$ \\
\hline Fr-223 & $2.80 \mathrm{E}-04$ & $4.44 \mathrm{E}-12$ \\
\hline Th-227 & $2.00 \mathrm{E}-02$ & $6.04 \mathrm{E}-11$ \\
\hline $\mathrm{Ra}-223$ & $2.03 \mathrm{E}-02$ & $1.15 \mathrm{E}-10$ \\
\hline Rn-219 & $2.03 \mathrm{E}-02$ & $1.37 \mathrm{E}-10$ \\
\hline Рo-215 & $2.03 \mathrm{E}-02$ & $1.02 \mathrm{E}-12$ \\
\hline $\mathrm{Pb}-211$ & $2.03 \mathrm{E}-02$ & $3.23 \mathrm{E}-09$ \\
\hline Bi-211 & $2.03 \mathrm{E}-02$ & $1.31 \mathrm{E}-10$ \\
\hline Tl-207 & $2.02 \mathrm{E}-02$ & $3.19 \mathrm{E}-10$ \\
\hline Po-211 & $5.68 \mathrm{E}-05$ & $2.14 \mathrm{E}-12$ \\
\hline
\end{tabular}


Table B-1 Detailed Decay Chain Contributions to Maximum Intruder-Based Radionuclide Dose Limit for Intermediate-Level Vaults - Resident Scenario with Transient Calculation for 1,000 years

\begin{tabular}{|c|c|c|c|c|c|}
\hline U-236 & $1000 \mathrm{yr}$ & $1.10 \mathrm{E}-11$ & Pu-238 & $1000 \mathrm{yr}$ & 3.58E-11 \\
\hline U-236 & $1.00 \mathrm{E}+00$ & $4.37 \mathrm{E}-21$ & $\mathrm{Pu}-238$ & $3.69 \mathrm{E}-04$ & $8.36 \mathrm{E}-30$ \\
\hline Th-232 & $4.93 \mathrm{E}-08$ & $1.96 \mathrm{E}-22$ & U-234 & $3.56 \mathrm{E}-04$ & $9.10 \mathrm{E}-19$ \\
\hline Ra-228 & 4.89E-08 & $0.00 \mathrm{E}+00$ & Th-230 & $2.85 \mathrm{E}-06$ & $2.33 \mathrm{E}-19$ \\
\hline Ac-228 & $4.89 \mathrm{E}-08$ & $6.75 \mathrm{E}-13$ & Ra-226 & $4.87 \mathrm{E}-07$ & $3.63 \mathrm{E}-18$ \\
\hline Th-228 & $4.88 \mathrm{E}-08$ & $6.11 \mathrm{E}-20$ & $\mathrm{Rn}-222$ & $4.87 \mathrm{E}-07$ & $1.36 \mathrm{E}-16$ \\
\hline Ra-224 & 4.88E-08 & 4.37E-18 & Po-218 & 4.87E-07 & $0.00 \mathrm{E}+00$ \\
\hline $\mathrm{Rn}-220$ & $4.88 \mathrm{E}-08$ & $2.53 \mathrm{E}-17$ & $\mathrm{~Pb}-214$ & $4.87 \mathrm{E}-07$ & $4.52 \mathrm{E}-14$ \\
\hline Po-216 & $4.88 \mathrm{E}-08$ & $3.38 \mathrm{E}-18$ & At-218 & $9.73 \mathrm{E}-11$ & $0.00 \mathrm{E}+00$ \\
\hline $\mathrm{Pb}-212$ & $4.88 \mathrm{E}-08$ & $5.22 \mathrm{E}-17$ & Bi-214 & $4.87 \mathrm{E}-07$ & $3.58 \mathrm{E}-11$ \\
\hline Bi-212 & $4.88 \mathrm{E}-08$ & $1.94 \mathrm{E}-13$ & Рo-214 & $4.87 \mathrm{E}-07$ & $1.86 \mathrm{E}-16$ \\
\hline Tl-208 & $1.75 \mathrm{E}-08$ & $1.02 \mathrm{E}-11$ & $\mathrm{~Pb}-210$ & $4.55 \mathrm{E}-07$ & $1.75 \mathrm{E}-38$ \\
\hline \multirow[t]{2}{*}{ Po-212 } & $3.13 \mathrm{E}-08$ & $0.00 \mathrm{E}+00$ & Bi-210 & $4.55 \mathrm{E}-07$ & $0.00 \mathrm{E}+00$ \\
\hline & & & Po-210 & $4.54 \mathrm{E}-07$ & $1.83 \mathrm{E}-17$ \\
\hline U-238 & $1000 \mathrm{yr}$ & 1.29E-07 & & & \\
\hline U-238 & $1.00 \mathrm{E}+00$ & $1.17 \mathrm{E}-21$ & Pu-239 & $1000 \mathrm{yr}$ & 3.01E-15 \\
\hline Th-234 & $1.00 \mathrm{E}+00$ & $7.79 \mathrm{E}-15$ & $\mathrm{Pu}-239$ & $9.72 \mathrm{E}-01$ & $9.78 \mathrm{E}-16$ \\
\hline $\mathrm{Pa}-234 \mathrm{~m}$ & $1.00 \mathrm{E}+00$ & 9.97E-08 & U-235 & $9.71 \mathrm{E}-07$ & $1.21 \mathrm{E}-16$ \\
\hline $\mathrm{Pa}-234$ & $1.30 \mathrm{E}-03$ & $2.94 \mathrm{E}-08$ & Th-231 & $9.71 \mathrm{E}-07$ & $1.72 \mathrm{E}-19$ \\
\hline U-234 & $2.82 \mathrm{E}-03$ & $7.20 \mathrm{E}-18$ & $\mathrm{~Pa}-231$ & $1.02 \mathrm{E}-08$ & $1.57 \mathrm{E}-17$ \\
\hline Th-230 & $1.29 \mathrm{E}-05$ & $1.06 \mathrm{E}-18$ & Ac- 227 & $9.63 \mathrm{E}-09$ & $2.81 \mathrm{E}-23$ \\
\hline Ra-226 & $1.68 \mathrm{E}-06$ & $1.25 \mathrm{E}-17$ & Fr-223 & $1.33 \mathrm{E}-10$ & $2.11 \mathrm{E}-18$ \\
\hline $\mathrm{Rn}-222$ & $1.68 \mathrm{E}-06$ & $4.71 \mathrm{E}-16$ & Th-227 & 9.49E-09 & $2.86 \mathrm{E}-17$ \\
\hline Po-218 & $1.68 \mathrm{E}-06$ & $0.00 \mathrm{E}+00$ & Ra-223 & $9.62 \mathrm{E}-09$ & $5.45 \mathrm{E}-17$ \\
\hline $\mathrm{Pb}-214$ & $1.68 \mathrm{E}-06$ & $1.56 \mathrm{E}-13$ & Rn-219 & $9.62 \mathrm{E}-09$ & $6.52 \mathrm{E}-17$ \\
\hline At-218 & $3.36 \mathrm{E}-10$ & $0.00 \mathrm{E}+00$ & Po-215 & $9.62 \mathrm{E}-09$ & $4.84 \mathrm{E}-19$ \\
\hline Bi-214 & $1.68 \mathrm{E}-06$ & $1.24 \mathrm{E}-10$ & $\mathrm{~Pb}-211$ & $9.62 \mathrm{E}-09$ & $1.53 \mathrm{E}-15$ \\
\hline Po-214 & $1.68 \mathrm{E}-06$ & $6.44 \mathrm{E}-16$ & Bi-211 & $9.62 \mathrm{E}-09$ & $6.24 \mathrm{E}-17$ \\
\hline $\mathrm{Pb}-210$ & $1.53 \mathrm{E}-06$ & $5.90 \mathrm{E}-38$ & Tl-207 & $9.59 \mathrm{E}-09$ & $1.51 \mathrm{E}-16$ \\
\hline Bi-210 & $1.53 \mathrm{E}-06$ & $0.00 \mathrm{E}+00$ & Рo-211 & $2.69 \mathrm{E}-11$ & $1.02 \mathrm{E}-18$ \\
\hline \multirow[t]{2}{*}{ Po-210 } & $1.53 \mathrm{E}-06$ & $6.16 \mathrm{E}-17$ & & & \\
\hline & & & Pu-240 & $1000 \mathrm{yr}$ & $1.56 \mathrm{E}-16$ \\
\hline Np-237 & $1000 \mathrm{yr}$ & 1.55E-08 & $\mathrm{Pu}-240$ & $9.00 \mathrm{E}-01$ & $6.12 \mathrm{E}-27$ \\
\hline Np-237 & $1.00 \mathrm{E}+00$ & $2.56 \mathrm{E}-12$ & U-236 & $2.81 \mathrm{E}-05$ & $1.23 \mathrm{E}-25$ \\
\hline $\mathrm{Pa}-233$ & $1.00 \mathrm{E}+00$ & $1.51 \mathrm{E}-08$ & Th-232 & $7.05 \mathrm{E}-13$ & $2.80 \mathrm{E}-27$ \\
\hline U-233 & $4.34 \mathrm{E}-03$ & $2.09 \mathrm{E}-17$ & Ra-228 & $6.94 \mathrm{E}-13$ & $0.00 \mathrm{E}+00$ \\
\hline Th-229 & $1.99 \mathrm{E}-04$ & $5.49 \mathrm{E}-15$ & Ac- 228 & $6.94 \mathrm{E}-13$ & $9.56 \mathrm{E}-18$ \\
\hline $\mathrm{Ra}-225$ & $1.99 \mathrm{E}-04$ & $3.52 \mathrm{E}-44$ & Th-228 & $6.90 \mathrm{E}-13$ & $8.64 \mathrm{E}-25$ \\
\hline Ac- 225 & $1.99 \mathrm{E}-04$ & $3.99 \mathrm{E}-14$ & Ra-224 & $6.90 \mathrm{E}-13$ & $6.17 \mathrm{E}-23$ \\
\hline Fr-221 & $1.99 \mathrm{E}-04$ & $3.32 \mathrm{E}-14$ & $\mathrm{Rn}-220$ & $6.90 \mathrm{E}-13$ & $3.57 \mathrm{E}-22$ \\
\hline At-217 & $1.99 \mathrm{E}-04$ & $6.49 \mathrm{E}-14$ & Po-216 & $6.90 \mathrm{E}-13$ & 4.77E-23 \\
\hline Bi-213 & $1.99 \mathrm{E}-04$ & $2.87 \mathrm{E}-11$ & $\mathrm{~Pb}-212$ & $6.90 \mathrm{E}-13$ & $7.38 \mathrm{E}-22$ \\
\hline Tl-209 & $4.30 \mathrm{E}-06$ & $3.53 \mathrm{E}-10$ & Bi-212 & $6.90 \mathrm{E}-13$ & $2.75 \mathrm{E}-18$ \\
\hline Po-213 & $1.95 \mathrm{E}-04$ & $2.49 \mathrm{E}-14$ & Tl-208 & $2.48 \mathrm{E}-13$ & $1.44 \mathrm{E}-16$ \\
\hline Pb-209 & $1.99 \mathrm{E}-04$ & $0.00 \mathrm{E}+00$ & Po-212 & $4.42 \mathrm{E}-13$ & $0.00 \mathrm{E}+00$ \\
\hline
\end{tabular}


Table B-1 Detailed Decay Chain Contributions to Maximum Intruder-Based Radionuclide Dose Limit for Intermediate-Level Vaults - Resident Scenario with Transient Calculation for 1,000 years

\begin{tabular}{lcc} 
Pu-241 & 1000 yr & 8.08E-14 \\
\hline Pu-241 & $8.59 \mathrm{E}-22$ & $0.00 \mathrm{E}+00$ \\
Am-241 & $6.88 \mathrm{E}-03$ & $1.04 \mathrm{E}-22$ \\
$\mathrm{~Np}-237$ & $5.28 \mathrm{E}-06$ & $1.35 \mathrm{E}-17$ \\
$\mathrm{~Pa}-233$ & $5.28 \mathrm{E}-06$ & $7.99 \mathrm{E}-14$ \\
$\mathrm{U}-233$ & $1.41 \mathrm{E}-08$ & $6.78 \mathrm{E}-23$ \\
Th-229 & $4.73 \mathrm{E}-10$ & $1.30 \mathrm{E}-20$ \\
$\mathrm{Ra}-225$ & $4.73 \mathrm{E}-10$ & $8.37 \mathrm{E}-50$ \\
$\mathrm{Ac}-225$ & $4.73 \mathrm{E}-10$ & $9.49 \mathrm{E}-20$ \\
$\mathrm{Fr}-221$ & $4.73 \mathrm{E}-10$ & $7.89 \mathrm{E}-20$ \\
$\mathrm{At}-217$ & $4.73 \mathrm{E}-10$ & $1.54 \mathrm{E}-19$ \\
$\mathrm{Bi}-213$ & $4.73 \mathrm{E}-10$ & $6.81 \mathrm{E}-17$ \\
$\mathrm{Tl}-209$ & $1.02 \mathrm{E}-11$ & $8.40 \mathrm{E}-16$ \\
$\mathrm{Po}-213$ & $4.62 \mathrm{E}-10$ & $5.91 \mathrm{E}-20$ \\
$\mathrm{~Pb}-209$ & $4.73 \mathrm{E}-10$ & $0.00 \mathrm{E}+00$ \\
& & \\
Pu-242 & $\mathbf{1 0 0 0}$ yr & $\mathbf{2 . 0 0 E}-14$ \\
\hline $\mathrm{Pu}-242$ & $9.98 \mathrm{E}-01$ & $8.42 \mathrm{E}-26$ \\
$\mathrm{U}-238$ & $1.55 \mathrm{E}-07$ & $1.81 \mathrm{E}-28$ \\
Th-234 & $1.55 \mathrm{E}-07$ & $1.21 \mathrm{E}-21$ \\
$\mathrm{~Pa}-234 \mathrm{~m}$ & $1.55 \mathrm{E}-07$ & $1.54 \mathrm{E}-14$ \\
$\mathrm{~Pa}-234$ & $2.01 \mathrm{E}-10$ & $4.56 \mathrm{E}-15$ \\
$\mathrm{U}-234$ & $2.19 \mathrm{E}-10$ & $5.59 \mathrm{E}-25$ \\
$\mathrm{Th}-230$ & $6.69 \mathrm{E}-13$ & $5.47 \mathrm{E}-26$ \\
$\mathrm{Ra}-226$ & $6.66 \mathrm{E}-14$ & $4.96 \mathrm{E}-25$ \\
$\mathrm{Rn}-222$ & $6.66 \mathrm{E}-14$ & $1.87 \mathrm{E}-23$ \\
$\mathrm{Po}-218$ & $6.66 \mathrm{E}-14$ & $0.00 \mathrm{E}+00$ \\
$\mathrm{~Pb}-214$ & $6.66 \mathrm{E}-14$ & $6.19 \mathrm{E}-21$ \\
$\mathrm{At}-218$ & $1.33 \mathrm{E}-17$ & $0.00 \mathrm{E}+00$ \\
$\mathrm{Bi}-214$ & $6.66 \mathrm{E}-14$ & $4.90 \mathrm{E}-18$ \\
$\mathrm{Po}-214$ & $6.66 \mathrm{E}-14$ & $2.55 \mathrm{E}-23$ \\
$\mathrm{~Pb}-210$ & $5.89 \mathrm{E}-14$ & $2.27 \mathrm{E}-45$ \\
$\mathrm{Bi}-210$ & $5.89 \mathrm{E}-14$ & $0.00 \mathrm{E}+00$ \\
Po-210 & $5.88 \mathrm{E}-14$ & $2.36 \mathrm{E}-24$ \\
& &
\end{tabular}

\begin{tabular}{lcc} 
Pu-244 & 100 yr & 2.74E-06 \\
\hline Pu-244 & $1.00 \mathrm{E}+00$ & $1.36 \mathrm{E}-37$ \\
$\mathrm{U}-240$ & $9.99 \mathrm{E}-01$ & $1.09 \mathrm{E}-35$ \\
Np-240m & $9.99 \mathrm{E}-01$ & $2.74 \mathrm{E}-06$ \\
Pu-240 & $1.05 \mathrm{E}-02$ & $7.13 \mathrm{E}-29$ \\
$\mathrm{U}-236$ & $1.56 \mathrm{E}-08$ & $6.80 \mathrm{E}-29$ \\
Th-232 & $2.55 \mathrm{E}-17$ & $1.01 \mathrm{E}-31$ \\
Ra-228 & $2.00 \mathrm{E}-17$ & $0.00 \mathrm{E}+00$ \\
Ac-228 & $1.78 \mathrm{E}-17$ & $2.45 \mathrm{E}-22$ \\
Th-228 & $1.95 \mathrm{E}-17$ & $2.45 \mathrm{E}-29$ \\
Ra-224 & $1.89 \mathrm{E}-17$ & $1.70 \mathrm{E}-27$ \\
Rn-220 & $1.79 \mathrm{E}-17$ & $9.27 \mathrm{E}-27$ \\
Po-216 & $1.80 \mathrm{E}-17$ & $1.25 \mathrm{E}-27$ \\
Pb-212 & $1.89 \mathrm{E}-17$ & $2.02 \mathrm{E}-26$ \\
$\mathrm{Bi}-212$ & $1.96 \mathrm{E}-17$ & $7.82 \mathrm{E}-23$ \\
Tl-208 & $6.71 \mathrm{E}-18$ & $3.90 \mathrm{E}-21$ \\
Po-212 & $1.15 \mathrm{E}-17$ & $0.00 \mathrm{E}+00$ \\
& & \\
Am-241 & $\mathbf{1 0 0 0}$ yr & $\mathbf{2 . 4 7 E}-12$ \\
\hline Am-241 & $2.01 \mathrm{E}-01$ & $3.05 \mathrm{E}-21$ \\
$\mathrm{~Np}-237$ & $1.61 \mathrm{E}-04$ & $4.13 \mathrm{E}-16$ \\
Pa-233 & $1.61 \mathrm{E}-04$ & $2.44 \mathrm{E}-12$ \\
$\mathrm{U}-233$ & $4.40 \mathrm{E}-07$ & $2.12 \mathrm{E}-21$ \\
Th-229 & $1.51 \mathrm{E}-08$ & $4.16 \mathrm{E}-19$ \\
Ra-225 & $1.51 \mathrm{E}-08$ & $2.67 \mathrm{E}-48$ \\
Ac-225 & $1.51 \mathrm{E}-08$ & $3.03 \mathrm{E}-18$ \\
Fr-221 & $1.51 \mathrm{E}-08$ & $2.52 \mathrm{E}-18$ \\
At-217 & $1.51 \mathrm{E}-08$ & $4.92 \mathrm{E}-18$ \\
$\mathrm{Bi}-213$ & $1.51 \mathrm{E}-08$ & $2.18 \mathrm{E}-15$ \\
Tl-209 & $3.26 \mathrm{E}-10$ & $2.68 \mathrm{E}-14$ \\
Po-213 & $1.48 \mathrm{E}-08$ & $1.89 \mathrm{E}-18$ \\
Pb-209 & $1.51 \mathrm{E}-08$ & $0.00 \mathrm{E}+00$
\end{tabular}


Table B-1 Detailed Decay Chain Contributions to Maximum Intruder-Based Radionuclide Dose Limit for Intermediate-Level Vaults - Resident Scenario with Transient Calculation for 1,000 years

\begin{tabular}{lcc} 
Am-242m & 100 yr & 1.75E-08 \\
\hline Am-242m & $6.12 \mathrm{E}-01$ & $1.06 \mathrm{E}-15$ \\
$\mathrm{~Np}-238$ & $2.91 \mathrm{E}-03$ & $1.75 \mathrm{E}-08$ \\
$\mathrm{Am}-242$ & $6.09 \mathrm{E}-01$ & $8.48 \mathrm{E}-14$ \\
$\mathrm{Pu}-238$ & $3.45 \mathrm{E}-01$ & $7.81 \mathrm{E}-27$ \\
$\mathrm{U}-234$ & $6.04 \mathrm{E}-05$ & $1.54 \mathrm{E}-19$ \\
$\mathrm{Th}-230$ & $2.04 \mathrm{E}-08$ & $1.67 \mathrm{E}-21$ \\
$\mathrm{Ra}-226$ & $2.31 \mathrm{E}-10$ & $1.72 \mathrm{E}-21$ \\
$\mathrm{Rn}-222$ & $2.31 \mathrm{E}-10$ & $6.48 \mathrm{E}-20$ \\
$\mathrm{Po}-218$ & $2.31 \mathrm{E}-10$ & $0.00 \mathrm{E}+00$ \\
$\mathrm{~Pb}-214$ & $2.31 \mathrm{E}-10$ & $2.15 \mathrm{E}-17$ \\
$\mathrm{At}-218$ & $4.63 \mathrm{E}-14$ & $0.00 \mathrm{E}+00$ \\
$\mathrm{Bi}-214$ & $2.31 \mathrm{E}-10$ & $1.70 \mathrm{E}-14$ \\
$\mathrm{Po}-214$ & $2.31 \mathrm{E}-10$ & $8.85 \mathrm{E}-20$ \\
$\mathrm{~Pb}-210$ & $9.53 \mathrm{E}-11$ & $3.67 \mathrm{E}-42$ \\
$\mathrm{Bi}-210$ & $9.52 \mathrm{E}-11$ & $0.00 \mathrm{E}+00$ \\
$\mathrm{Po}-210$ & $9.30 \mathrm{E}-11$ & $3.74 \mathrm{E}-21$ \\
$\mathrm{Pu}-242$ & $2.52 \mathrm{E}-05$ & $2.13 \mathrm{E}-30$ \\
$\mathrm{Cm}-242$ & $5.05 \mathrm{E}-01$ & $6.48 \mathrm{E}-25$ \\
$\mathrm{U}-238$ & $2.12 \mathrm{E}-13$ & $2.48 \mathrm{E}-34$ \\
$\mathrm{Th}-234$ & $2.11 \mathrm{E}-13$ & $1.65 \mathrm{E}-27$ \\
$\mathrm{~Pa}-234 \mathrm{~m}$ & $2.11 \mathrm{E}-13$ & $2.11 \mathrm{E}-20$ \\
$\mathrm{~Pa}-234$ & $2.75 \mathrm{E}-16$ & $6.22 \mathrm{E}-21$ \\
& & \\
$\mathrm{Am}-243$ & $\mathbf{1 0 0}$ yr & $\mathbf{2 . 2 8 E}-\mathbf{0 9}$ \\
\hline $\mathrm{Am}-243$ & $9.91 \mathrm{E}-01$ & $7.14 \mathrm{E}-14$ \\
$\mathrm{~Np}-239$ & $9.91 \mathrm{E}-01$ & $2.28 \mathrm{E}-09$ \\
$\mathrm{Pu}-239$ & $2.86 \mathrm{E}-03$ & $2.88 \mathrm{E}-18$ \\
$\mathrm{U}-235$ & $1.41 \mathrm{E}-10$ & $1.76 \mathrm{E}-20$ \\
$\mathrm{Th}-231$ & $1.41 \mathrm{E}-10$ & $2.50 \mathrm{E}-23$ \\
$\mathrm{~Pa}-231$ & $9.94 \mathrm{E}-14$ & $1.52 \mathrm{E}-22$ \\
$\mathrm{Ac}-227$ & $4.69 \mathrm{E}-14$ & $1.37 \mathrm{E}-28$ \\
$\mathrm{Fr}-223$ & $6.47 \mathrm{E}-16$ & $1.03 \mathrm{E}-23$ \\
$\mathrm{Th}-227$ & $4.61 \mathrm{E}-14$ & $1.39 \mathrm{E}-22$ \\
$\mathrm{Ra}-223$ & $4.67 \mathrm{E}-14$ & $2.64 \mathrm{E}-22$ \\
$\mathrm{Rn}-219$ & $4.67 \mathrm{E}-14$ & $3.16 \mathrm{E}-22$ \\
$\mathrm{Po}-215$ & $4.67 \mathrm{E}-14$ & $2.35 \mathrm{E}-24$ \\
$\mathrm{~Pb}-211$ & $4.67 \mathrm{E}-14$ & $7.43 \mathrm{E}-21$ \\
$\mathrm{Pi}-211$ & $4.67 \mathrm{E}-14$ & $3.03 \mathrm{E}-22$ \\
& $4.65 \mathrm{E}-14$ & $7.34 \mathrm{E}-22$ \\
& $1.31 \mathrm{E}-16$ & $4.93 \mathrm{E}-24$ \\
& &
\end{tabular}

\begin{tabular}{|c|c|c|}
\hline Cm-242 & $1000 \mathrm{yr}$ & 1.82E-13 \\
\hline $\mathrm{Cm}-242$ & $0.00 \mathrm{E}+00$ & $0.00 \mathrm{E}+00$ \\
\hline $\mathrm{Pu}-238$ & $1.89 \mathrm{E}-06$ & $4.27 \mathrm{E}-32$ \\
\hline U-234 & $1.81 \mathrm{E}-06$ & $4.63 \mathrm{E}-21$ \\
\hline Th-230 & $1.45 \mathrm{E}-08$ & $1.19 \mathrm{E}-21$ \\
\hline Ra-226 & $2.47 \mathrm{E}-09$ & $1.84 \mathrm{E}-20$ \\
\hline Rn-222 & $2.47 \mathrm{E}-09$ & $6.92 \mathrm{E}-19$ \\
\hline Po-218 & $2.47 \mathrm{E}-09$ & $0.00 \mathrm{E}+00$ \\
\hline $\mathrm{Pb}-214$ & $2.47 \mathrm{E}-09$ & $2.30 \mathrm{E}-16$ \\
\hline At-218 & $4.94 \mathrm{E}-13$ & $0.00 \mathrm{E}+00$ \\
\hline Bi-214 & $2.47 \mathrm{E}-09$ & $1.82 \mathrm{E}-13$ \\
\hline Po-214 & $2.47 \mathrm{E}-09$ & $9.46 \mathrm{E}-19$ \\
\hline $\mathrm{Pb}-210$ & $2.31 \mathrm{E}-09$ & 8.88E-41 \\
\hline $\mathrm{Bi}-210$ & $2.31 \mathrm{E}-09$ & $0.00 \mathrm{E}+00$ \\
\hline Po-210 & 2.31E-09 & $9.27 \mathrm{E}-20$ \\
\hline Cm-243 & $100 \mathrm{yr}$ & 1.03E-10 \\
\hline $\mathrm{Cm}-243$ & $9.24 \mathrm{E}-02$ & $1.03 \mathrm{E}-10$ \\
\hline $\mathrm{Pu}-239$ & $1.09 \mathrm{E}-03$ & $1.10 \mathrm{E}-18$ \\
\hline Am-243 & $8.55 \mathrm{E}-06$ & $6.16 \mathrm{E}-19$ \\
\hline U-235 & $7.33 \mathrm{E}-11$ & $9.15 \mathrm{E}-21$ \\
\hline Th-231 & $7.33 \mathrm{E}-11$ & $1.30 \mathrm{E}-23$ \\
\hline $\mathrm{Pa}-231$ & $6.02 \mathrm{E}-14$ & $9.20 \mathrm{E}-23$ \\
\hline Ac-227 & $3.02 \mathrm{E}-14$ & $8.81 \mathrm{E}-29$ \\
\hline Fr-223 & 4.16E-16 & $6.60 \mathrm{E}-24$ \\
\hline Th-227 & $2.97 \mathrm{E}-14$ & $8.95 \mathrm{E}-23$ \\
\hline Ra-223 & $3.00 \mathrm{E}-14$ & $1.70 \mathrm{E}-22$ \\
\hline Rn-219 & $3.00 \mathrm{E}-14$ & $2.03 \mathrm{E}-22$ \\
\hline Po-215 & $3.00 \mathrm{E}-14$ & $1.51 \mathrm{E}-24$ \\
\hline $\mathrm{Pb}-211$ & $3.00 \mathrm{E}-14$ & $4.78 \mathrm{E}-21$ \\
\hline $\mathrm{Bi}-211$ & $3.00 \mathrm{E}-14$ & $1.95 \mathrm{E}-22$ \\
\hline Tl-207 & $2.99 \mathrm{E}-14$ & $4.72 \mathrm{E}-22$ \\
\hline Po-211 & $8.41 \mathrm{E}-17$ & $3.17 \mathrm{E}-24$ \\
\hline Np-239 & $8.55 \mathrm{E}-06$ & $1.96 \mathrm{E}-14$ \\
\hline
\end{tabular}


Table B-1 Detailed Decay Chain Contributions to Maximum Intruder-Based Radionuclide Dose Limit for Intermediate-Level Vaults - Resident Scenario with Transient Calculation for 1,000 years

\begin{tabular}{lcc} 
Cm-244 & 1000 yr & 4.09E-19 \\
\hline Cm-244 & $2.34 \mathrm{E}-17$ & $2.00 \mathrm{E}-42$ \\
Pu-240 & $2.49 \mathrm{E}-03$ & $1.69 \mathrm{E}-29$ \\
$\mathrm{U}-236$ & $7.55 \mathrm{E}-08$ & $3.30 \mathrm{E}-28$ \\
Th-232 & $1.85 \mathrm{E}-15$ & $7.34 \mathrm{E}-30$ \\
Ra-228 & $1.82 \mathrm{E}-15$ & $0.00 \mathrm{E}+00$ \\
Ac-228 & $1.82 \mathrm{E}-15$ & $2.50 \mathrm{E}-20$ \\
Th-228 & $1.81 \mathrm{E}-15$ & $2.26 \mathrm{E}-27$ \\
Ra-224 & $1.81 \mathrm{E}-15$ & $1.62 \mathrm{E}-25$ \\
Rn-220 & $1.81 \mathrm{E}-15$ & $9.35 \mathrm{E}-25$ \\
Po-216 & $1.81 \mathrm{E}-15$ & $1.25 \mathrm{E}-25$ \\
Pb-212 & $1.81 \mathrm{E}-15$ & $1.93 \mathrm{E}-24$ \\
Bi-212 & $1.81 \mathrm{E}-15$ & $7.19 \mathrm{E}-21$ \\
Tl-208 & $6.49 \mathrm{E}-16$ & $3.77 \mathrm{E}-19$ \\
Po-212 & $1.16 \mathrm{E}-15$ & $0.00 \mathrm{E}+00$ \\
& & \\
Cm-245 & $\mathbf{1 0 0 0}$ yr & $\mathbf{1 . 2 1 E - 1 1}$ \\
\hline Cm-245 & $9.22 \mathrm{E}-01$ & $9.81 \mathrm{E}-12$ \\
Pu-241 & $9.23 \mathrm{E}-01$ & $0.00 \mathrm{E}+00$ \\
Am-241 & $7.54 \mathrm{E}-01$ & $1.14 \mathrm{E}-20$ \\
Np-237 & $1.52 \mathrm{E}-04$ & $3.91 \mathrm{E}-16$ \\
Pa-233 & $1.52 \mathrm{E}-04$ & $2.31 \mathrm{E}-12$ \\
$\mathrm{U}-233$ & $2.44 \mathrm{E}-07$ & $1.17 \mathrm{E}-21$ \\
Th-229 & $5.94 \mathrm{E}-09$ & $1.64 \mathrm{E}-19$ \\
Ra-225 & $5.94 \mathrm{E}-09$ & $1.05 \mathrm{E}-48$ \\
Ac-225 & $5.94 \mathrm{E}-09$ & $1.19 \mathrm{E}-18$ \\
Fr-221 & $5.94 \mathrm{E}-09$ & $9.91 \mathrm{E}-19$ \\
At-217 & $5.94 \mathrm{E}-09$ & $1.94 \mathrm{E}-18$ \\
Bi-213 & $5.94 \mathrm{E}-09$ & $8.56 \mathrm{E}-16$ \\
Tl-209 & $1.28 \mathrm{E}-10$ & $1.05 \mathrm{E}-14$ \\
Po-213 & $5.81 \mathrm{E}-09$ & $7.42 \mathrm{E}-19$ \\
Pb-209 & $5.94 \mathrm{E}-09$ & $0.00 \mathrm{E}+00$ \\
& &
\end{tabular}

\begin{tabular}{|c|c|c|}
\hline Cm-246 & $1000 \mathrm{yr}$ & $1.77 \mathrm{E}-17$ \\
\hline $\mathrm{Cm}-246$ & $8.64 \mathrm{E}-01$ & $6.45 \mathrm{E}-37$ \\
\hline $\mathrm{Pu}-242$ & $1.73 \mathrm{E}-03$ & $1.46 \mathrm{E}-28$ \\
\hline U-238 & $1.37 \mathrm{E}-10$ & $1.61 \mathrm{E}-31$ \\
\hline Th-234 & $1.37 \mathrm{E}-10$ & $1.07 \mathrm{E}-24$ \\
\hline $\mathrm{Pa}-234 \mathrm{~m}$ & $1.37 \mathrm{E}-10$ & $1.37 \mathrm{E}-17$ \\
\hline $\mathrm{Pa}-234$ & $1.78 \mathrm{E}-13$ & $4.04 \mathrm{E}-18$ \\
\hline U-234 & $1.31 \mathrm{E}-13$ & $3.33 \mathrm{E}-28$ \\
\hline Th-230 & $3.02 \mathrm{E}-16$ & $2.47 \mathrm{E}-29$ \\
\hline Ra-226 & $2.45 \mathrm{E}-17$ & $1.82 \mathrm{E}-28$ \\
\hline $\mathrm{Rn}-222$ & $2.45 \mathrm{E}-17$ & $6.86 \mathrm{E}-27$ \\
\hline Po-218 & $2.45 \mathrm{E}-17$ & $0.00 \mathrm{E}+00$ \\
\hline $\mathrm{Pb}-214$ & $2.45 \mathrm{E}-17$ & $2.27 \mathrm{E}-24$ \\
\hline At-218 & $4.90 \mathrm{E}-21$ & $0.00 \mathrm{E}+00$ \\
\hline Bi-214 & $2.45 \mathrm{E}-17$ & $1.80 \mathrm{E}-21$ \\
\hline Po-214 & $2.45 \mathrm{E}-17$ & $9.37 \mathrm{E}-27$ \\
\hline $\mathrm{Pb}-210$ & $2.11 \mathrm{E}-17$ & $8.10 \mathrm{E}-49$ \\
\hline Bi-210 & $2.11 \mathrm{E}-17$ & $0.00 \mathrm{E}+00$ \\
\hline Po-210 & $2.10 \mathrm{E}-17$ & $8.44 \mathrm{E}-28$ \\
\hline Cm-247 & $1000 \mathrm{yr}$ & 6.99E-08 \\
\hline $\mathrm{Cm}-247$ & $1.00 \mathrm{E}+00$ & $6.92 \mathrm{E}-08$ \\
\hline $\mathrm{Pu}-243$ & $1.00 \mathrm{E}+00$ & $4.81 \mathrm{E}-10$ \\
\hline Am-243 & 8.98E-02 & $6.47 \mathrm{E}-15$ \\
\hline Np-239 & 8.98E-02 & $2.06 \mathrm{E}-10$ \\
\hline Pu-239 & $1.30 \mathrm{E}-03$ & $1.31 \mathrm{E}-18$ \\
\hline U-235 & $4.30 \mathrm{E}-10$ & 5.37E-20 \\
\hline Th-231 & $4.30 \mathrm{E}-10$ & $7.64 \mathrm{E}-23$ \\
\hline $\mathrm{Pa}-231$ & $2.28 \mathrm{E}-12$ & $3.49 \mathrm{E}-21$ \\
\hline Ac-227 & $2.02 \mathrm{E}-12$ & $5.91 \mathrm{E}-27$ \\
\hline Fr-223 & $2.79 \mathrm{E}-14$ & $4.43 \mathrm{E}-22$ \\
\hline Th-227 & $1.99 \mathrm{E}-12$ & $6.01 \mathrm{E}-21$ \\
\hline Ra-223 & $2.02 \mathrm{E}-12$ & $1.14 \mathrm{E}-20$ \\
\hline Rn-219 & $2.02 \mathrm{E}-12$ & $1.37 \mathrm{E}-20$ \\
\hline Po-215 & $2.02 \mathrm{E}-12$ & $1.02 \mathrm{E}-22$ \\
\hline $\mathrm{Pb}-211$ & $2.02 \mathrm{E}-12$ & $3.21 \mathrm{E}-19$ \\
\hline Bi-211 & $2.02 \mathrm{E}-12$ & $1.31 \mathrm{E}-20$ \\
\hline Tl-207 & $2.01 \mathrm{E}-12$ & $3.18 \mathrm{E}-20$ \\
\hline Po-211 & $5.65 \mathrm{E}-15$ & $2.13 \mathrm{E}-22$ \\
\hline
\end{tabular}


Table B-1 Detailed Decay Chain Contributions to Maximum Intruder-Based Radionuclide Dose Limit for Intermediate-Level Vaults - Resident Scenario with Transient Calculation for 1,000 years

\begin{tabular}{|c|c|c|c|c|c|}
\hline Cm-248 & $1000 \mathrm{yr}$ & $2.17 \mathrm{E}-11$ & Cf-249 & $100 \mathrm{yr}$ & 4.81E-08 \\
\hline $\mathrm{Cm}-248$ & $9.98 \mathrm{E}-01$ & $3.93 \mathrm{E}-26$ & Cf-249 & $8.21 \mathrm{E}-01$ & $4.81 \mathrm{E}-08$ \\
\hline $\mathrm{Pu}-244$ & 7.94E-06 & $1.08 \mathrm{E}-42$ & $\mathrm{Cm}-245$ & 7.37E-03 & $7.84 \mathrm{E}-14$ \\
\hline U-240 & 7.93E-06 & 8.64E-41 & $\mathrm{Pu}-241$ & $5.95 \mathrm{E}-03$ & $0.00 \mathrm{E}+00$ \\
\hline $\mathrm{Np}-240 \mathrm{~m}$ & 7.93E-06 & $2.17 \mathrm{E}-11$ & Am-241 & $3.96 \mathrm{E}-04$ & $5.99 \mathrm{E}-24$ \\
\hline $\mathrm{Pu}-240$ & $4.05 \mathrm{E}-07$ & $2.75 \mathrm{E}-33$ & $\mathrm{~Np}-237$ & $3.80 \mathrm{E}-09$ & $9.74 \mathrm{E}-21$ \\
\hline U-236 & $4.03 \mathrm{E}-12$ & $1.76 \mathrm{E}-32$ & $\mathrm{~Pa}-233$ & $3.79 \mathrm{E}-09$ & $5.73 \mathrm{E}-17$ \\
\hline Th-232 & $5.10 \mathrm{E}-20$ & $2.03 \mathrm{E}-34$ & U-233 & $3.71 \mathrm{E}-13$ & $1.79 \mathrm{E}-27$ \\
\hline Ra-228 & $4.70 \mathrm{E}-20$ & $0.00 \mathrm{E}+00$ & Th-229 & $6.34 \mathrm{E}-16$ & $1.75 \mathrm{E}-26$ \\
\hline Ac-228 & $5.34 \mathrm{E}-20$ & $7.36 \mathrm{E}-25$ & $\mathrm{Ra}-225$ & $6.32 \mathrm{E}-16$ & $1.12 \mathrm{E}-55$ \\
\hline Th-228 & $6.35 \mathrm{E}-20$ & $7.96 \mathrm{E}-32$ & Ac- 225 & $6.31 \mathrm{E}-16$ & $1.27 \mathrm{E}-25$ \\
\hline $\mathrm{Ra}-224$ & $6.09 \mathrm{E}-20$ & $5.45 \mathrm{E}-30$ & Fr-221 & $6.31 \mathrm{E}-16$ & $1.05 \mathrm{E}-25$ \\
\hline $\mathrm{Rn}-220$ & $5.86 \mathrm{E}-20$ & $3.03 \mathrm{E}-29$ & At-217 & $6.31 \mathrm{E}-16$ & $2.06 \mathrm{E}-25$ \\
\hline Po-216 & $5.14 \mathrm{E}-20$ & $3.55 \mathrm{E}-30$ & Bi-213 & $6.31 \mathrm{E}-16$ & $9.09 \mathrm{E}-23$ \\
\hline $\mathrm{Pb}-212$ & $5.66 \mathrm{E}-20$ & $6.06 \mathrm{E}-29$ & Tl-209 & $1.36 \mathrm{E}-17$ & $1.12 \mathrm{E}-21$ \\
\hline Bi-212 & $6.13 \mathrm{E}-20$ & $2.44 \mathrm{E}-25$ & Po-213 & $6.17 \mathrm{E}-16$ & $7.88 \mathrm{E}-26$ \\
\hline Tl-208 & $2.07 \mathrm{E}-20$ & $1.20 \mathrm{E}-23$ & $\mathrm{~Pb}-209$ & $6.31 \mathrm{E}-16$ & $0.00 \mathrm{E}+00$ \\
\hline \multirow[t]{2}{*}{ Po-212 } & $3.89 \mathrm{E}-20$ & $0.00 \mathrm{E}+00$ & & & \\
\hline & & & Cf-250 & $1000 \mathrm{yr}$ & 4.69E-20 \\
\hline Bk-249 & $100 \mathrm{yr}$ & $1.24 \mathrm{E}-10$ & Cf-250 & $9.67 \mathrm{E}-24$ & $9.26 \mathrm{E}-43$ \\
\hline Bk-249 & $5.06 \mathrm{E}-34$ & $0.00 \mathrm{E}+00$ & $\mathrm{Cm}-246$ & $2.38 \mathrm{E}-03$ & $1.78 \mathrm{E}-39$ \\
\hline Cf-249 & $2.12 \mathrm{E}-03$ & $1.24 \mathrm{E}-10$ & $\mathrm{Pu}-242$ & $4.66 \mathrm{E}-06$ & $3.93 \mathrm{E}-31$ \\
\hline $\mathrm{Cm}-245$ & $1.88 \mathrm{E}-05$ & $2.00 \mathrm{E}-16$ & U-238 & $3.63 \mathrm{E}-13$ & $4.25 \mathrm{E}-34$ \\
\hline $\mathrm{Pu}-241$ & $1.51 \mathrm{E}-05$ & $0.00 \mathrm{E}+00$ & Th-234 & $3.63 \mathrm{E}-13$ & $2.83 \mathrm{E}-27$ \\
\hline Am-241 & $9.89 \mathrm{E}-07$ & $1.50 \mathrm{E}-26$ & $\mathrm{~Pa}-234 \mathrm{~m}$ & $3.63 \mathrm{E}-13$ & $3.62 \mathrm{E}-20$ \\
\hline $\mathrm{Np}-237$ & $9.37 \mathrm{E}-12$ & $2.40 \mathrm{E}-23$ & $\mathrm{~Pa}-234$ & 4.72E-16 & $1.07 \mathrm{E}-20$ \\
\hline $\mathrm{Pa}-233$ & $9.34 \mathrm{E}-12$ & $1.41 \mathrm{E}-19$ & U-234 & $3.39 \mathrm{E}-16$ & $8.66 \mathrm{E}-31$ \\
\hline U-233 & $9.01 \mathrm{E}-16$ & $4.34 \mathrm{E}-30$ & Th-230 & $7.70 \mathrm{E}-19$ & $6.29 \mathrm{E}-32$ \\
\hline Th-229 & $1.52 \mathrm{E}-18$ & $4.20 \mathrm{E}-29$ & Ra-226 & $6.14 \mathrm{E}-20$ & $4.58 \mathrm{E}-31$ \\
\hline Ra-225 & $1.52 \mathrm{E}-18$ & $2.69 \mathrm{E}-58$ & $\mathrm{Rn}-222$ & $6.14 \mathrm{E}-20$ & $1.72 \mathrm{E}-29$ \\
\hline Ac- 225 & $1.51 \mathrm{E}-18$ & $3.04 \mathrm{E}-28$ & Po-218 & $6.14 \mathrm{E}-20$ & $0.00 \mathrm{E}+00$ \\
\hline Fr-221 & $1.51 \mathrm{E}-18$ & $2.53 \mathrm{E}-28$ & $\mathrm{~Pb}-214$ & $6.14 \mathrm{E}-20$ & $5.71 \mathrm{E}-27$ \\
\hline At-217 & $1.51 \mathrm{E}-18$ & 4.94E-28 & At-218 & $1.23 \mathrm{E}-23$ & $0.00 \mathrm{E}+00$ \\
\hline Bi-213 & $1.51 \mathrm{E}-18$ & $2.18 \mathrm{E}-25$ & Bi-214 & $6.14 \mathrm{E}-20$ & $4.52 \mathrm{E}-24$ \\
\hline Tl-209 & $3.27 \mathrm{E}-20$ & $2.69 \mathrm{E}-24$ & Po-214 & $6.14 \mathrm{E}-20$ & $2.35 \mathrm{E}-29$ \\
\hline Po-213 & $1.48 \mathrm{E}-18$ & $1.89 \mathrm{E}-28$ & $\mathrm{~Pb}-210$ & $5.27 \mathrm{E}-20$ & $2.03 \mathrm{E}-51$ \\
\hline \multirow[t]{2}{*}{$\mathrm{Pb}-209$} & $1.51 \mathrm{E}-18$ & $0.00 \mathrm{E}+00$ & Bi-210 & $5.27 \mathrm{E}-20$ & $0.00 \mathrm{E}+00$ \\
\hline & & & Po-210 & $5.25 \mathrm{E}-20$ & $2.11 \mathrm{E}-30$ \\
\hline
\end{tabular}


Table B-1 Detailed Decay Chain Contributions to Maximum Intruder-Based Radionuclide Dose Limit for Intermediate-Level Vaults - Resident Scenario with Transient Calculation for 1,000 years

\begin{tabular}{lcc} 
Cf-251 & 100 yr & 2.69E-10 \\
\hline Cf-251 & $9.26 \mathrm{E}-01$ & $2.68 \mathrm{E}-10$ \\
$\mathrm{Cm}-247$ & $4.28 \mathrm{E}-06$ & $2.96 \mathrm{E}-13$ \\
$\mathrm{Pu}-243$ & $4.28 \mathrm{E}-06$ & $2.06 \mathrm{E}-15$ \\
$\mathrm{Am}-243$ & $2.03 \mathrm{E}-08$ & $1.46 \mathrm{E}-21$ \\
$\mathrm{~Np}-239$ & $2.03 \mathrm{E}-08$ & $4.67 \mathrm{E}-17$ \\
$\mathrm{Pu}-239$ & $1.96 \mathrm{E}-11$ & $1.97 \mathrm{E}-26$ \\
$\mathrm{U}-235$ & $4.84 \mathrm{E}-19$ & $6.04 \mathrm{E}-29$ \\
$\mathrm{Th}-231$ & $4.84 \mathrm{E}-19$ & $8.59 \mathrm{E}-32$ \\
$\mathrm{~Pa}-231$ & $9.19 \mathrm{E}-24$ & $1.41 \mathrm{E}-32$ \\
$\mathrm{Ac}-227$ & $-6.39 \mathrm{E}-22$ & $-1.87 \mathrm{E}-36$ \\
$\mathrm{Fr}-223$ & $-7.66 \mathrm{E}-24$ & $-1.22 \mathrm{E}-31$ \\
$\mathrm{Th}-227$ & $-1.27 \mathrm{E}-22$ & $-3.83 \mathrm{E}-31$ \\
$\mathrm{Ra}-223$ & $-3.04 \mathrm{E}-22$ & $-1.72 \mathrm{E}-30$ \\
$\mathrm{Rn}-219$ & $-4.46 \mathrm{E}-22$ & $-3.02 \mathrm{E}-30$ \\
$\mathrm{Po}-215$ & $-1.26 \mathrm{E}-22$ & $-6.33 \mathrm{E}-33$ \\
$\mathrm{~Pb}-211$ & $-2.44 \mathrm{E}-22$ & $-3.88 \mathrm{E}-29$ \\
$\mathrm{Bi}-211$ & $-3.85 \mathrm{E}-22$ & $-2.49 \mathrm{E}-30$ \\
$\mathrm{Tl}-207$ & $-6.49 \mathrm{E}-22$ & $-1.02 \mathrm{E}-29$ \\
$\mathrm{Po}-211$ & $-1.20 \mathrm{E}-24$ & $-4.53 \mathrm{E}-32$ \\
& & \\
Cf-252 & $\mathbf{1 0 0 0}$ yr & $\mathbf{1 . 5 9 E}-16$ \\
\hline $\mathrm{Cf}-252$ & $1.55 \mathrm{E}-114$ & $1.60 \mathrm{E}-135$ \\
$\mathrm{Cm}-248$ & $7.35 \mathrm{E}-06$ & $2.90 \mathrm{E}-31$ \\
$\mathrm{Pu}-244$ & $5.83 \mathrm{E}-11$ & $7.90 \mathrm{E}-48$ \\
$\mathrm{U}-240$ & $5.82 \mathrm{E}-11$ & $6.34 \mathrm{E}-46$ \\
$\mathrm{~Np}-240 \mathrm{~m}$ & $5.82 \mathrm{E}-11$ & $1.59 \mathrm{E}-16$ \\
$\mathrm{Pu}-240$ & $2.96 \mathrm{E}-12$ & $2.01 \mathrm{E}-38$ \\
$\mathrm{U}-236$ & $2.93 \mathrm{E}-17$ & $1.28 \mathrm{E}-37$ \\
$\mathrm{Th}-232$ & $4.24 \mathrm{E}-25$ & $1.69 \mathrm{E}-39$ \\
$\mathrm{Ra}-228$ & $4.07 \mathrm{E}-25$ & $0.00 \mathrm{E}+00$ \\
$\mathrm{Ac}-228$ & $4.32 \mathrm{E}-25$ & $5.96 \mathrm{E}-30$ \\
$\mathrm{Th}-228$ & $4.07 \mathrm{E}-25$ & $5.10 \mathrm{E}-37$ \\
$\mathrm{Ra}-224$ & $3.63 \mathrm{E}-25$ & $3.25 \mathrm{E}-35$ \\
$\mathrm{Rn}-220$ & $4.35 \mathrm{E}-25$ & $2.25 \mathrm{E}-34$ \\
$\mathrm{Po}-216$ & $3.92 \mathrm{E}-25$ & $2.71 \mathrm{E}-35$ \\
$\mathrm{~Pb}-212$ & $3.81 \mathrm{E}-25$ & $4.07 \mathrm{E}-34$ \\
$\mathrm{Bi}-212$ & $4.24 \mathrm{E}-25$ & $1.69 \mathrm{E}-30$ \\
$\mathrm{Tl}-208$ & $1.71 \mathrm{E}-25$ & $9.91 \mathrm{E}-29$ \\
Po-212 & $2.81 \mathrm{E}-25$ & $0.00 \mathrm{E}+00$ \\
& &
\end{tabular}




\section{APPENDIX C - ADDITIONAL INFORMATION ON AIR ANALYSIS}

This appendix provides additional detail information on the air limit calculation summarized in Table 4-1.

The dose limit of $10 \mathrm{mrem} / \mathrm{yr}$ is defined in Table 2.5-1 of the PA (McDowell-Boyer et al. 2000). Dose factors are from Simpkins (2004). The release fractions are taken from Table 4.1-1 in the PA (McDowell-Boyer et al. 2000). A 100 year lag time at the 100 meter distance accounts for radioactive decay during the period of institutional control. 


\section{APPENDIX D - ADDITIONAL INFORMATION ON RADON ANALYSIS:}

\section{Evaluation of long-term Radon-222 release from the E-Area Intermediate Level Vault (ILV) Disposal Units}

\section{INTRODUCTION}

This section describes the investigation conducted to evaluate the potential for radon release from the E-Area Intermediate Level Vault (ILV) disposal unit over the 1000-year performance assessment period of interest. The permissible radon flux for DOE facilities is addressed in USDOE 1999a (DOE Order 435.1). This order states that radon, either as a constituent of waste at the time of disposal or produced by radioactive decay following disposal, should not be released from the disposal facility at a rate that would exceed the limit established in 40 CFR Part 61. From this statute, the standard identified for radon release is stated as "No source at a Department of Energy facility shall emit more than $20 \mathrm{pCi} / \mathrm{m}^{2}-\mathrm{s}$ of Rn-222 as an average for the entire source, into the air". No other radon isotopes are identified in this guidance. Therefore, USDOE Order 435.1 indicates that the radon release projected for a specific facility at the land surface should be compared to the $20 \mathrm{pCi} / \mathrm{m}^{2}$-s standard.

This guidance forms the basis for the investigation to evaluate radon flux above an E-Area ILV disposal unit. The scope of the investigation involved defining a decay chain of parent radioisotopes to evaluate with a $1 \mathrm{D}$ vertical numerical model. The model was customized to represent the thickness of the vault waste zones and cover material over the facility. The instantaneous Rn-222 flux at the land surface was evaluated for the Performance Assessment (PA) compliance period of 1000 years. This flux was then compared to the standard identified in 40 CFR Part 61.

The potential parent radioisotopes that can contribute to the creation of $\mathrm{Rn}-222$ are illustrated in Figure D-1. The diagram indicates the specific decay chains that lead to the formation of Rn-222, as well as the half-lives for each radioisotope. The extremely long half-lives of Pu-242 and U$238(375,500$ and 4,500,000 years respectively) cause the other radioisotopes higher up on the chain of parents to be of little concern with regard to their potential to contribute significantly to the Rn-222 flux at the land surface over the period of interest. The only other radon isotope that is mentioned in DOE Order 435.1 is "Rn-220 from Thorium". This decay chain was screened out as a part of this investigation due to its extremely short half-life of 55.6 seconds.

\section{ILV CLOSURE CONSIDERATIONS}

The concepts for closure of the ILV are relevant to the determination of the radon flux at the land surface during the PA evaluation period (1,000 years). ILV construction specifics and closure concept are described in Phifer, 2004, Preliminary E-Area Intermediate Level Vault Closure Cap Infiltration $(U)$ and are summarized below. The current IL Vault is a below grade, reinforced concrete vault. The bottom of the base slab is approximately 26 feet below existing grade and the top of exterior walls are approximately 5.5 feet above existing grade. The exterior vault wall footprint is approximately 279 feet long by 48 feet wide. It contains seven 25 -foot by 44.5 -foot by 28.5 -foot deep bulk waste cells and two 25 -foot by 44.5 -foot by 26 -foot deep cells (interior dimensions). One of the 26-foot deep cells contains 140 vertical silos. The silos are approximately 30 inches in diameter at the top, 20 inches in diameter at the bottom, and 24.83 feet deep (interior dimensions). The other 26-foot deep cell is a bulk waste cell. There is currently one IL Vault in the E-Area LLWF and it is anticipated that two will be required (McDowell-Boyer et al. 2000). 


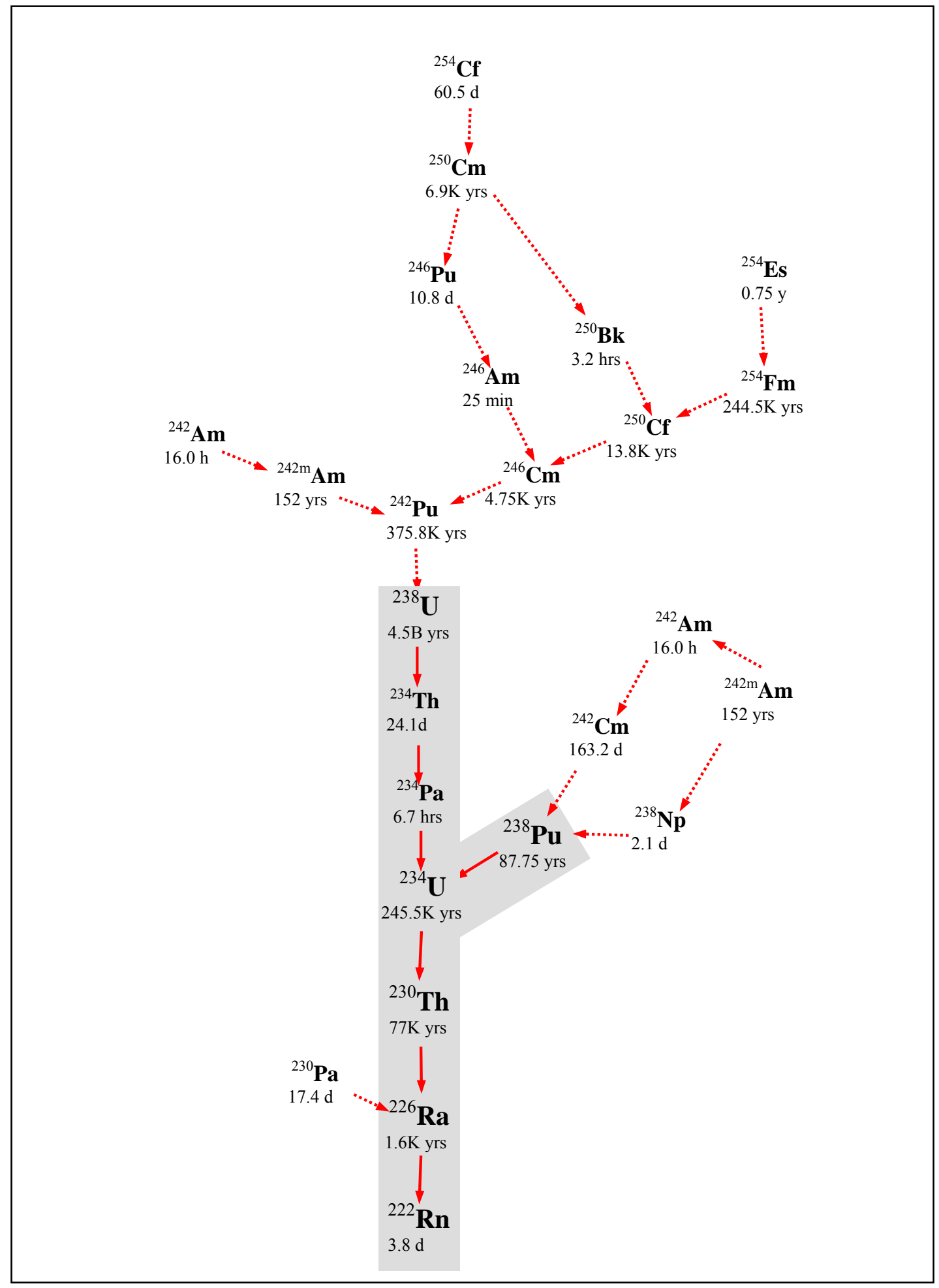

Figure D-1. Decay chain of parent radioisotopes leading to Rn-222 formation.

Rev. 0 
For the purposes of this investigation, it is assumed that there will be a 25 -year operations period during which the unit is loaded with waste. This is followed by a 100-year period of institutional control during which a temporary surface runoff cover will be placed over the ILV and maintained. Following the institutional control period the ILV will be prepared for final closure, as described in Phifer, 2004. Specific information with regard to the construction of the final closure cap is presented below. The final closure cap will exist far into the future and is the configuration that must be considered in evaluating the long-term radon release at the land surface.

\section{$\underline{\text { Closure configuration }}$}

During the operational period, the ILV will be filled with equipment and containerized waste (predominately in drums, B-12 boxes, B-25 boxes, and concrete containers) each placed in the cell in layers. Each layer of waste will be encapsulated in grout, which forms the surface for the placement of the next layer of waste. In this fashion, the full 28.5-foot $(8.7 \mathrm{~m})$ depth of an ILV cell will be filled in, with the upper 17 inches $(0.43 \mathrm{~m})$ being filled in with grout (Phifer, 2004).

As the individual cells are filled they will be grouted shut with the upper 17-inch grout layer. A temporary roof will be maintained over each completed cell until the end of the 25-year operations period. At that point the concrete roof will be constructed and maintained over the 100 -year institutional control period. At the end of institutional control, a permanent closure cap will be constructed to cover the entire ILV (Phifer, 2004).

Table D-1, below, describes the individual components of the closure cap. Materials are indicated with the associated thickness of each component, in inches, $\mathrm{cm}$ and $\mathrm{m}$.

Table D-1. Vertical layer sequence and associated thickness for ILV cover material. (Adapted from Phifer, 2004)

\begin{tabular}{|l|c|c|c|}
\hline \multicolumn{1}{|c|}{ Layer } & $\begin{array}{c}\text { Thickness } \\
\text { (inches) }\end{array}$ & $\begin{array}{c}\text { Thickness } \\
(\mathrm{cm})\end{array}$ & $\begin{array}{c}\text { Thickness } \\
(\mathrm{m})\end{array}$ \\
\hline Topsoil & 6 & 15.2 & 0.15 \\
\hline Upper Compacted Backfill & 30 & 76.2 & 0.76 \\
\hline Erosion Barrier & 12 & 30.5 & 0.30 \\
\hline Geotextile Filter Fabric & 0.1 & 0.25 & 0.003 \\
\hline Middle Compacted Backfill & 12 & 30.5 & 0.30 \\
\hline Geotextile Filter Fabric & 0.1 & 0.25 & 0.003 \\
\hline Drainage Layer & 12 & 30.5 & 0.30 \\
\hline GCL & 0.2 & 0.5 & 0.01 \\
\hline Lower Compacted Backfill & 40 & 102 & 1.02 \\
\hline ILV Roof Slab & 33 (avg.) & 83.8 & 0.84 \\
\hline ILV upper grout layer & 17 & 43.2 & 0.43 \\
\hline Vault Waste Zone & $\sim 345$ & $\sim 877$ & $\sim 8.7$ \\
\hline
\end{tabular}

The components of concern for the long-term radon performance calculation are those that will persist over the 1000-year evaluation period and probably for $>10,000$ years. These components 
are situated below the top of the Erosion Barrier. For the purpose of this investigation, the concrete roof slab is considered to be part of the Soil Cover Zone in the 1D model while the upper grout seal on top of the ILV waste is considered to be part of the ILV Waste Zone. Therefore, the composite thickness of the non-waste material below the top of the Erosion Barrier is 109.4 inches $(2.77 \mathrm{~m})$.

\section{MODEL DEVELOPMENT}

\section{Conceptual Model}

The Rn-222 flux at the land surface above an ILV was evaluated for its specific closure configuration. Rn-222 is generated within the ILV waste zones by radioactive decay of different parent isotopes following along the decay chains that lead to the formation of Rn-222. The decay chains for all possible parent isotopes of Rn-222 are shown in Figure D-1. In this figure the part of the decay chain that was simulated is indicated with the gray shaded area (i.e. beginning with $\mathrm{Pu}-238$ and U-238). Rn-222 generated within the ILV waste zone is in the gaseous phase and diffuses outward from this zone into the air-filled soil pores surrounding the ILV, eventually resulting in some of the radon emanating at the land surface. As such, air is the fluid through which Rn-222 diffuses, although some Rn-222 may dissolve in residual pore water. Advective transport of Rn-222 in air-filled soil pores is not considered to be a significant process when compared to air diffusion. The parent isotopes exist in the solid phase and therefore do not migrate upward through the air-filled pore space, although they could be leached and transported downward from the waste zone by pore water movement. Within the Waste Zone, the assorted waste materials are generally placed in B-12 and B-25 boxes for placement within individual layers. There is a considerable amount of air space that remains inside the boxes when they are surrounded, and covered, with cement.

The time period of interest for which the flux of Rn-222 at the land surface was evaluated is 1000 years, as specified in DOE Order 435.1. An additional 125 years were added to this to account for any possible Rn-222 buildup during the 25-year operational period and 100-year institutional control period.

\section{$\underline{\text { Numerical Model }}$}

The mathematical model utilized in this report is provided by the PORFLOW ${ }^{\mathrm{TM}}$ simulation package. PC-based PORFLOW ${ }^{\mathrm{TM}}$ Version 5.97.0 was used to conduct a series of simulations. PORFLOW ${ }^{\mathrm{TM}}$ is developed and marketed by Analytic \& Computational Research, Inc. to solve problems involving transient and steady-state fluid flow, heat and mass transport in multi-phase, variably saturated, porous or fractured media with dynamic phase change. PORFLOW ${ }^{\mathrm{TM}}$ has been widely used at the SRS and in the DOE complex to address major issues related to the ground water and nuclear waste management.

The governing equation for mass transport of species $k$ in the fluid phase is given by

$$
\frac{\partial C_{k}}{\partial t}+\frac{\partial}{\partial x_{i}}\left(V_{i} C_{k}\right)=\frac{\partial}{\partial x_{i}}\left(D_{i j} \frac{\partial C_{k}}{\partial x_{j}}\right)+\gamma_{k}
$$

where

$\mathrm{C}_{\mathrm{k}} \quad$ concentration of species $k$

$\mathrm{V}_{\mathrm{i}} \quad$ fluid velocity in the $\mathrm{i}^{\text {th }}$ direction 


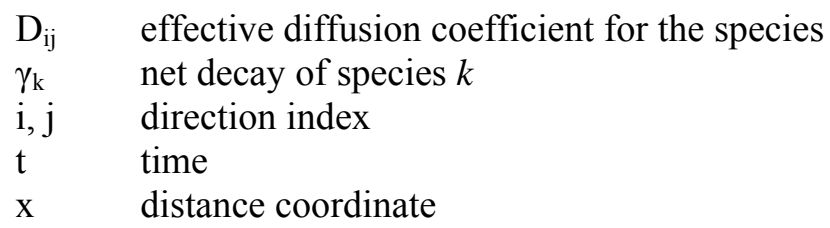

This equation is solved using PORFLOW to evaluate transient $\mathrm{Rn}-222$ transport through the soil cover above the ILV to evaluate Rn-222 flux at the land surface over time.

\section{Model Development and Assumptions}

The numerical representation of the conceptual model is as a 1-dimensional vertical stack of elements configured to represent the thickness of the ILV waste zone and overlying cover material associated with final closure of an ILV. The conditions and assumptions of this model are:

Decay chains evaluated were U-238 $\rightarrow$ Th- $234 \rightarrow$ Pa- $234 \rightarrow$ U-234 $\rightarrow$ Th- $230 \rightarrow$ Ra- $226 \rightarrow \mathrm{Rn}-222$ and $\mathrm{Pu}-238 \rightarrow \mathrm{U}-234 \rightarrow \mathrm{Th}-230 \rightarrow \mathrm{Ra}-226 \rightarrow \mathrm{Rn}-222$. These chains are shaded solid gray in Figure D-1. Each parent in these chains, except Th-234 and Pa-234, were simulated separately as the starting point of the decay chain. Th-234 and Pa-234 have extremely short half-lives compared to the other parent isotopes in these chains. Only a fraction of the Rn-222 generated by the decay of each parent is available for migration away from its source and into open pore space. Since the $\mathrm{Rn}-222$ parent isotopes exist as oxides or in other crystalline forms, only a fraction of Rn-222 generated by decay of Ra-226 has sufficient energy to migrate away from its original location into adjacent pore space before further decay occurs (3.2 day half-life for Rn-222). The fraction of radon escaping its source and migrating into adjacent pore space is approximated by the use of a radon emanation coefficient. This coefficient has been shown to vary between 0.02 and 0.7 in soils but is typically 0.25 (Yu, et. al, 2001). This value is taken as the default factor value for the RESRAD program, developed for the DOE. To account for this effect in this model, an effective source term of $0.25 \mathrm{Ci}$ of parent isotope was utilized as the source term for each $\mathrm{Ci}$ disposed within the facility.

Since Rn-222 exists primarily in the gaseous state, air was taken to be the fluid within which radon transport occurs. Air-diffusion was the only transport mechanism simulated in the model and advective air-transport is assumed to be negligible. Some radon dissolves in pore water but since diffusion proceeds more slowly in that fluid, air-diffusion is the only transport process by which Rn-222 can reach the land surface when it is placed in the ILV. Transport was allowed to proceed only through air-filled pore space and, therefore, residual pore water was treated as if it was part of the solid matrix material within the flow field. No credit was taken for airborne radon dissolving in pore water as it proceeds from the ILV to the land surface although it has been observed to partition between air and water in the ratio of 4 to 1, respectively, at $20 \mathrm{C}$ (Nazaroff and Nero, 1988).

The boundary conditions imposed on the domain included:

- No-flux specified for all parent isotopes at perimeter of the domain

- No-flux specified for Rn-222 along sides and bottom

- Rn-222 concentration set to 0 at land surface. 
Waste is disposed in various forms in the ILV, commonly within B-12 and B-25 boxes. Waste is disposed in individual layers, which are then surrounded and covered by grout. This forms the floor of the next waste layer. Since each waste disposal box may have a considerable percentage of air-filled void space, accurate simulation of air-diffusion of Rn-222 must incorporate these "pockets" in which waste is disposed. The equivalent porosity and residual moisture content of these pockets are much different than the surrounding cement. Within the model, these pockets are assigned a representative air-filled porosity value of 0.5 to reflect the degree of waste compaction. A series of 5 layers, each $0.9 \mathrm{~m}$ tall were configured and separated by 0.9 feet of cement material to approximate the placement of $\mathrm{Rn}-222$ parent isotopes within the waste disposal zone. The source term was assigned in 5 equal components within each of these layers.

Simulations were conducted in transient mode for diffusive transport in air, with results being obtained at 1,125 years and 10,125 years. The rationale for these precise simulation lengths was explained in the conceptual model.

\section{Measures implemented to assure conservative results}

In this analysis, several conditions introduce a significant measure of conservatism into the calculations, these include:

- The use of boundary conditions that force all of the Rn-222 to move upward from the waste disposal zone to the land surface. In reality, some of the Rn-222 diffuses sideways and downward in the air-filled pores surrounding the waste zone, hence ignoring this has the effect of increasing the radon flux at the land surface.

- Not taking credit for the removal of either Rn-222 or of the parent isotopes by pore water moving vertically downward through the model domain. This mechanism would likely carry off some dissolved Rn-222 in addition to the parent isotopes, and therefore its omission has the effect of increasing the estimate of instantaneous Rn-222 flux at the land surface in simulations conducted as a part of this investigation.

- The addition of an extra 125 years to the required 1000-year evaluation period to account for any Rn-222 generated during the operations and institutional control period, thus incrementally increasing the instantaneous Rn-222 flux. The extra time means slightly higher instantaneous fluxes for all parent isotopes except Ra-226.

- Use of the top of the erosion layer in the soil cover as the land surface for the purpose of calculating Rn-222 flux. No credit is taken for the additional distance Rn-222 must migrate above the erosion layer prior to that portion of the Soil Cover Zone eroding away.

- Placement of the source term in fewer layers than will actually be the case as the ILV is filled. With respect to $\mathrm{Rn}-222$ generation, less void space means that $\mathrm{Rn}-222$ concentration gradients can build up sooner to drive Rn-222 diffusion to the land surface through the overlying material.

- Assignment of soil properties to the cement material surrounding the waste packages. This facilitates more rapid diffusion of Rn-222 toward the land surface during the period when intact cement retains properties that would impede air-diffusion. 


\section{Grid Construction}

The model grid was constructed as a node mesh 3 nodes wide by 33 nodes high. This mesh creates the vertical stack of 31 model elements. This node and element configuration is illustrated below in Figure D-2, which also indicates the correspondence of model zones with the revised closure configuration for an ILV structure, as presented in Phifer, 2004. The grid extends upward only as far as the erosion barrier, anticipating that this is the cover thickness that will prevail over the majority of the 1000-year PA evaluation period. A set of consistent units were employed in the simulations for length, mass and time, these being meters, grams and years, respectively. 


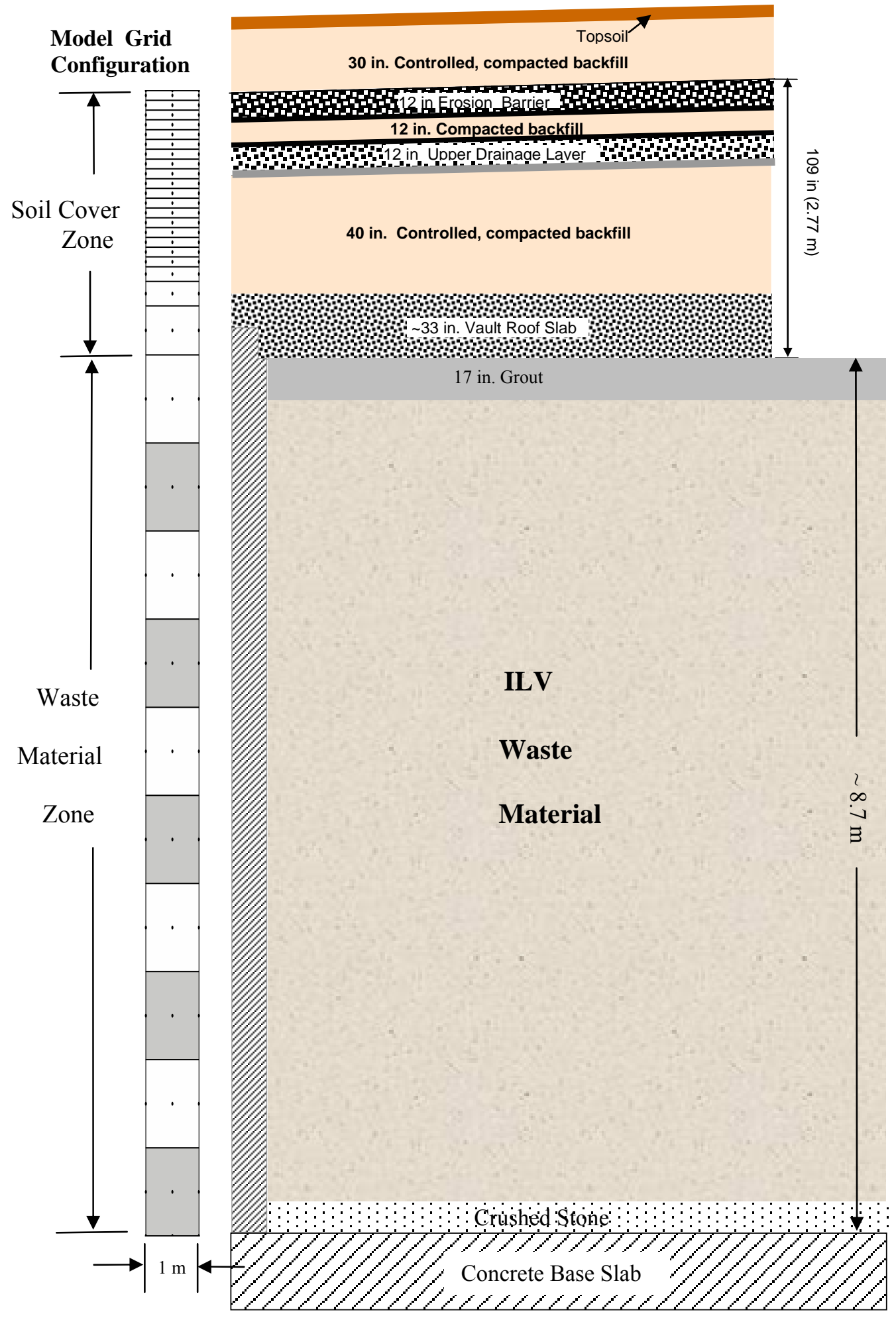

Figure D-2. Model grid and the ILV closure configuration. 


\section{$\underline{\text { Material zones }}$}

The model domain was divided into two primary zones, the ILV Waste Material Zone occupying the lower 345 inches $(8.7 \mathrm{~m})$ of the domain and the Soil Cover Zone, extending 109.4 inches $(\sim 2.78 \mathrm{~m})$ above the Waste Material Zone to the top of the domain. The Soil Cover Zone includes the concrete Vault roof as well as the different closure cap layers. The upper model elements were scaled to correspond to the geometry of the ILV closure cap thickness while the lower model elements were scaled to correspond to the ILV waste zone. The land surface over the PA period of interest is assumed to be the top of the erosion resistant layer, within the closure cap, and no credit is taken for the compacted soil and topsoil above that layer. The darker elements shown in the Waste Material Zone of Figure D-2 represent the individual waste disposal layers. In actuality, the concrete layers separating each waste layer will be thinner, therefore allowing the inclusion of several more waste layers.

\section{Material zone properties and other input parameters}

Material properties utilized within the 1-D numerical model were varied amongst 7 material zones defined within the model domain. Each material zone was assigned values for tortuosity, total porosity, residual saturation, air-filled porosity, matrix density, air density, and diffusivity.

The rock (matrix) density was selected based on the density of quartz, and is regarded to be representative of most soils. Tortuosity was assigned based on the commonly used value associated with a spherical particle tortuosity equation developed by Nielson and Rogers, 1982. Total porosities for soil materials were selected based on the typical values for soil materials found at the SRS. Values utilized for residual moisture in soil material were obtained from the 2D cross-sectional model of the ILV developed for evaluating the groundwater pathway as a part of this investigation. Values utilized were the steady-state values that persist after the degradation of the closure cap. The density of air was obtained from the Bolz, et. al., CRC Handbook of tables for Applied Engineering Science. The Rn-222 diffusivity value was selected based on the value for coefficient of diffusion of Rn-222 in open air, as reported in Rogers, et. al., 1984.

While the Soil Cover and Waste Material zones form a basic distinction in terms of material properties, the Waste Material zone was further differentiated based on the distinction between waste layers and the concrete layers between them. The concrete layers were treated as "degraded" concrete, thus assuming properties similar to soil and were assigned different airfilled porosity values based on the steady-state residual moisture vertical distribution obtained in the groundwater pathway model. Air-filled porosity was calculated by subtracting the residual moisture content from the total porosity. The specific material property zones are listed in Table D-2 along with specific values used to represent matrix and fluid properties. The 7 zones are best described using the model structure illustrated in Figure D-2.

The Soil Cover Zone is treated as a single homogenous zone within this simulation, despite the layering within the closure cap. This simplification is justified for this type conceptual model where radon is simulated as diffusing only through the air filled pores. While the porosities of the separate materials undoubtedly range from a high in the gravel drain layers (up to 0.4) to a low in the compacted soil layers $(<0.1)$ a single value of 0.2 for porosity is regarded to be a representative value for a composite of all the materials.

A typical model input file is presented in the Appendix showing the specific PORFLOW commands used to implement the numerical model. 
Table D-2

Summary of matrix and fluid properties utilized within the numerical model.

\begin{tabular}{|l|c|c|c|c|c|c|c|}
\hline & \multicolumn{7}{|c|}{ Material Type Zones } \\
\hline & 1 & 2 & 3 & 4 & 5 & 6 & 7 \\
\hline Property & $\begin{array}{c}\text { Waste } \\
\text { Zone } \\
\text { Cavities }\end{array}$ & $\begin{array}{c}\text { Concrete } \\
\text { Layer }\end{array}$ & $\begin{array}{c}\text { Concrete } \\
\text { Layer }\end{array}$ & $\begin{array}{c}\text { Concrete } \\
\text { Layer }\end{array}$ & $\begin{array}{c}\text { Concrete } \\
\text { Layer }\end{array}$ & $\begin{array}{c}\text { Concrete } \\
\text { Layer }\end{array}$ & $\begin{array}{c}\text { Soil Cover } \\
\text { Layer }\end{array}$ \\
\hline Tortuosity & 1.0 & 0.66 & 0.66 & 0.66 & 0.66 & 0.66 & 0.66 \\
\hline Total Porosity & 0.5 & 0.3 & 0.3 & 0.3 & 0.3 & 0.3 & 0.3 \\
\hline $\begin{array}{l}\text { Residual } \\
\text { Saturation }\end{array}$ & 0.0 & 0.6 & 0.6 & 0.7 & 0.7 & 0.8 & 0.7 \\
\hline $\begin{array}{l}\text { Air-Filled } \\
\text { Porosity }\end{array}$ & 0.5 & 0.12 & 0.12 & 0.09 & 0.09 & 0.06 & 0.09 \\
\hline $\begin{array}{l}\text { Matrix Density } \\
\left(\mathrm{g} / \mathrm{m}^{3}\right)\end{array}$ & $2.65 \mathrm{E}+06^{\mathrm{a}}$ & $2.65 \mathrm{E}+06^{\mathrm{a}}$ & $2.65 \mathrm{E}+06^{\mathrm{a}}$ & $2.65 \mathrm{E}+06^{\mathrm{a}}$ & $2.65 \mathrm{E}+06^{\mathrm{a}}$ & $2.65 \mathrm{E}+06^{\mathrm{a}}$ & $2.65 \mathrm{E}+06^{\mathrm{a}}$ \\
\hline $\begin{array}{l}\text { Air Density } \\
\left(\mathrm{g} / \mathrm{m}^{3}\right)\end{array}$ & $1.24 \mathrm{E}+03^{\mathrm{b}}$ & $1.24 \mathrm{E}+03^{\mathrm{b}}$ & $1.24 \mathrm{E}+03^{\mathrm{b}}$ & $1.24 \mathrm{E}+03^{\mathrm{b}}$ & $1.24 \mathrm{E}+03^{\mathrm{b}}$ & $1.24 \mathrm{E}+03^{\mathrm{b}}$ & $1.24 \mathrm{E}+03^{\mathrm{b}}$ \\
\hline $\begin{array}{l}\text { Molecular } \\
\text { Diffusivity } \\
\left(\mathrm{m}^{2} / \mathrm{yr}\right)\end{array}$ & $3.47 \mathrm{E}+02^{\mathrm{c}}$ & $3.47 \mathrm{E}+02^{\mathrm{c}}$ & $3.47 \mathrm{E}+02^{\mathrm{c}}$ & $3.47 \mathrm{E}+02^{\mathrm{c}}$ & $3.47 \mathrm{E}+02^{\mathrm{c}}$ & $3.47 \mathrm{E}+02^{\mathrm{c}}$ & $3.47 \mathrm{E}+02^{\mathrm{c}}$ \\
\hline
\end{tabular}

\footnotetext{
a. $\quad$ CRC Handbook of Applied Engineering Science, 2'nd Ed. Table 1-2, pg. 11

b. CRC Handbook of Applied Engineering Science, 2'nd Ed. Table 1-121, pg.192

c. Rogers, et. al., 1984.
}

\section{MODEL RESULTS}

Model simulations were conducted to evaluate the peak instantaneous Rn-222 flux at the land surface over the 1125 year period. This time period includes the 25 -year operations cycle, 100 years of institutional control and 1000-year PA compliance period identified in DOE Order 435.1. Model results were output in $\mathrm{Ci} / \mathrm{m}^{2}-\mathrm{yr}$, consistent with the set of units employed in the model. The results were then converted into $\mathrm{pCi} / \mathrm{m}^{2}-\mathrm{sec}$, which are the units used to define the regulatory flux limit in 40 CFR Part 61, Rev. 4. The results represent the peak Rn-222 flux per square meter at the top of the closure cap erosion barrier and are listed below in Table D-3. The top of the erosion barrier is expected to represent the land surface 1000 years in the future. Also shown in Table D-3 are disposal limits associated with each parent isotope, expressed in $\mathrm{Ci}$ of parent isotope permitted per square meter of surface area of the disposal facility. 
Table D-3. Simulated peak instantaneous Rn-222 flux over 1000-years at the land surface and associated disposal limits for parent isotopes.

\begin{tabular}{|c|c|c|c|}
\hline $\begin{array}{c}\text { Parent Source } \\
(1 \mathrm{Ci})\end{array}$ & $\begin{array}{c}\text { Peak Instantaneous } \\
\text { Rn-222 Flux at land } \\
\text { surface }\end{array}$ & $\begin{array}{c}\text { Unit Disposal Limit } \\
\text { of parent isotope }\end{array}$ & $\begin{array}{c}\text { Disposal Limit of parent } \\
\text { per ILV } \\
\left(75 \mathrm{~m} \mathrm{x} \mathrm{15} \mathrm{m}=1125 \mathrm{~m}^{2}\right)\end{array}$ \\
\hline & $\left(\mathrm{pCi} / \mathrm{m}^{2}-\mathrm{s}\right)$ & $\left(\mathrm{Ci} / \mathrm{m}^{2}\right)$ & $4.18 \mathrm{E}+06$ \\
\hline $\mathrm{Pu}-238$ & $5.38 \mathrm{E}-03$ & $3.72 \mathrm{E}+03$ & $1.1 \mathrm{E}+06$ \\
\hline $\mathrm{U}-238$ & $2.05 \mathrm{E}-02$ & $9.74 \mathrm{E}+02$ & $1.21 \mathrm{E}+03$ \\
\hline $\mathrm{U}-234$ & $1.86 \mathrm{E}+01$ & $1.08 \mathrm{E}+00$ & $6.79 \mathrm{E}+00$ \\
\hline Th-230 & $3.31 \mathrm{E}+03$ & $6.04 \mathrm{E}-03$ & $2.58 \mathrm{E}+00$ \\
\hline Ra-226 & $8.72 \mathrm{E}+03$ & $2.29 \mathrm{E}-03$ & \\
\hline
\end{tabular}

The equation used to convert the simulated Rn-222 activity flux associated with each source isotope to determine the associated disposal limit is:

Disposal Limit (in $\mathrm{Ci} / \mathrm{m}^{2}$ of disposal facility footprint $)=\left(20 \mathrm{pCi} / \mathrm{m}^{2}-\mathrm{sec}\right) /(\mathrm{Rn}-222$ instantaneous flux, in $\left.\mathrm{pCi} / \mathrm{m}^{2}-\mathrm{sec}\right)$.

In this equation the $20 \mathrm{pCi} / \mathrm{m}^{2}-\mathrm{sec}$ is the Rn-222 flux limit applicable to the disposal facility at the land surface 1000 years after facility closure (40 CFR Part 61, Rev4). The Rn-222 instantaneous flux is the simulated flux determined for $1 \mathrm{Ci}$ of each parent isotope evaluated in this investigation.

The instantaneous flux rates at the land surface versus time are shown in Figure D-3 for all 5 parent radioisotopes. The peak instantaneous $\mathrm{Rn}-222$ flux simulated for 4 of the 5 parent isotopes occurs at $\mathrm{t}=1125$ years, or at the end of the PA period of interest. These parent isotopes are Th230, U-234, U-238 and Pu-238. The other parent isotope, Ra-226 has its peak at approximately 64.2 days after disposal and slowly declines after that. 


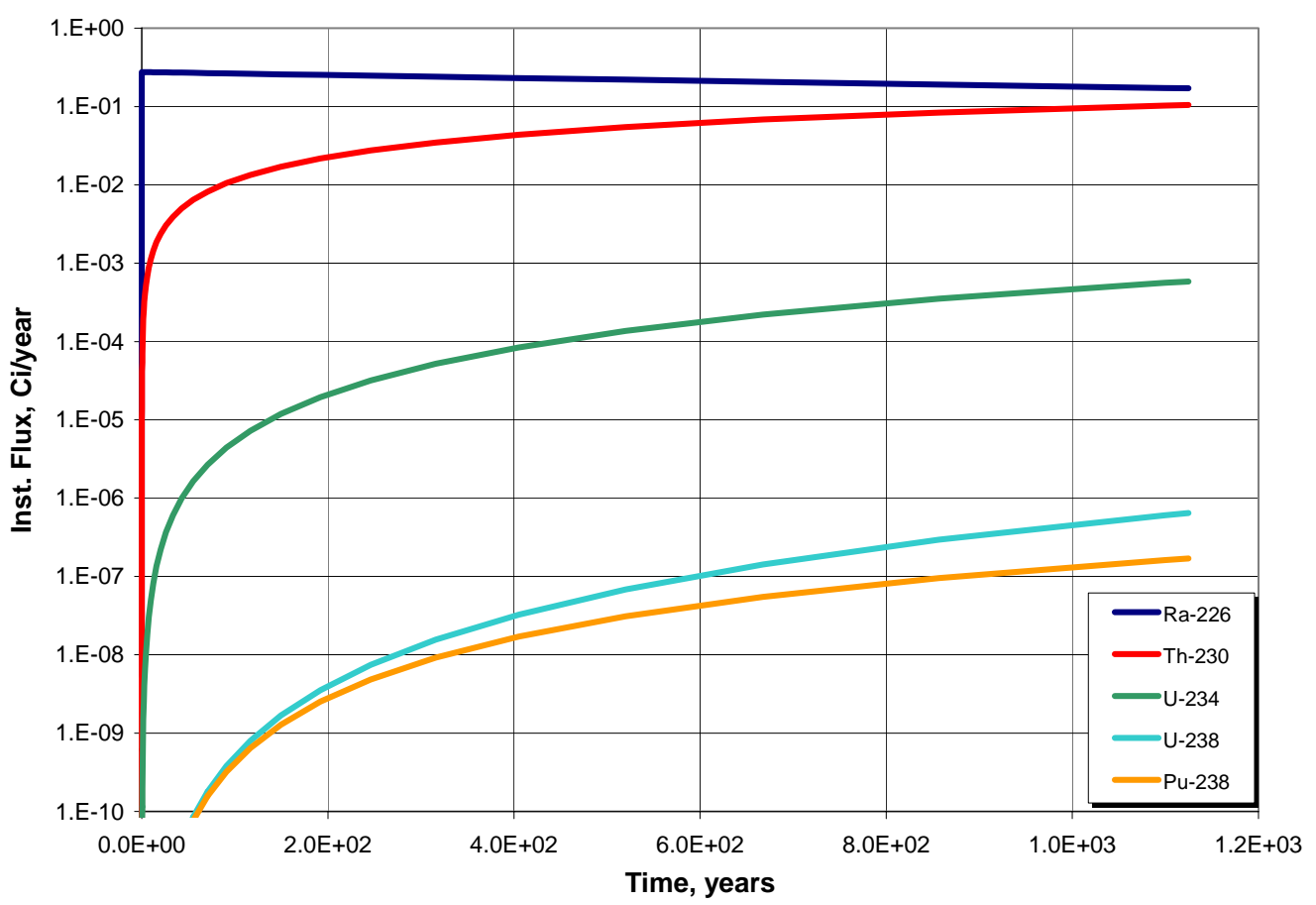

Figure D-3 Simulated instantaneous Rn-222 flux versus time.

\section{DISPOSAL IMPLICATIONS}

To simulate the instantaneous Rn-222 fluxes reported in Table D-3 the parent source isotope was distributed evenly in layers throughout the model waste zone (vault). However, the calculated Rn-222 instantaneous flux at the land surface after 1000 years is quite sensitive to the position of the unit source within the ILV. The instantaneous flux is lower when the parent isotopes are embedded near the base of the Vault and, conversely, is higher if the parent isotopes are embedded near the upper part of the Vault. This relationship is true because Rn-222 has a relatively short half-life of 3.82 days and therefore the flux has a strong negative correlation with the distance over which it must diffuse.

Since the limits calculated in this investigation assume uniform distribution of source isotope in 5 distinct layers throughout the vertical extent of the ILV, a sensitivity analysis was conducted to evaluate the impact of placing the source material within different, vertically differentiated, subzones of the ILV. In this evaluation, the absolute values calculated for disposal limits have no meaning other than to indicate in a relative fashion, how different disposal-modes compare to one another in terms of the resulting disposal limits.

In all sensitivity simulations the source was $1 \mathrm{Ci}$ of $\mathrm{U}-234$ and the time of simulation was 1,125 years. The sub-zones were selected to correspond to the vertical placement of the source as described below in Table D-4. Case 1 is the reference case, being the uniform distribution of the source throughout the full vertical extent of the ILV. In Cases 2-8, the extent of the source zone is expressed in terms of model grid coordinates, in meters from the bottom of the ILV. 
The calculated disposal limit associated with each sensitivity case is graphically illustrated below in Figure D-4, which is a plot of the calculated disposal limit associated with each sensitivity case. Case 4, the situation where the source material is placed exclusively in the lower $1.8 \mathrm{~m}$ of the ILV produces the highest disposal limit, $2.77 \mathrm{E}-01 \mathrm{Ci} / \mathrm{m}^{2}$. The lowest disposal limit, $1.78 \mathrm{E}-02$ $\mathrm{Ci} / \mathrm{m}^{2}$ was realized if the source material was placed in the uppermost $1.8 \mathrm{~m}$ of the ILV. The results of this indicate that with the use of more fine-tuned waste placement practices, higher disposal limits might be achievable.

Table D-4. Description of sensitivity cases and associated disposal limits.

\begin{tabular}{|c|l|c|}
\hline $\begin{array}{c}\text { Sensitivity } \\
\text { Case \# }\end{array}$ & \multicolumn{1}{|c|}{ Description of Source Placement } & $\begin{array}{c}\text { Disposal Limit } \\
\left.\text { (Ci/m }{ }^{2}\right)\end{array}$ \\
\hline Case 1 & Uniformly distributed throughout ILV (base case) & $4.82 \mathrm{E}-02$ \\
\hline Case 2 & Upper half of ILV Waste Zone $(4.5 \mathrm{~m}$ to $9.0 \mathrm{~m})$ & $2.80 \mathrm{E}-02$ \\
\hline Case 3 & Lower half of ILV Waste Zone $(0.0 \mathrm{~m}$ to $4.5 \mathrm{~m})$ & $1.73 \mathrm{E}-01$ \\
\hline Case 4 & Lowermost 2 elements $(0.0 \mathrm{~m}$ to $1.8 \mathrm{~m})$ & $2.77 \mathrm{E}-01$ \\
\hline Case 5 & Next lowermost 2 elements $(1.8 \mathrm{~m}$ to $3.6 \mathrm{~m})$ & $1.69 \mathrm{E}-01$ \\
\hline Case 6 & Next lowermost 2 elements $(3.6 \mathrm{~m}$ to $5.4 \mathrm{~m})$ & $8.31 \mathrm{E}-02$ \\
\hline Case 7 & Next lowermost 2 elements $(5.4 \mathrm{~m}$ to $7.2 \mathrm{~m})$ & $1.78 \mathrm{E}-02$ \\
\hline Case 8 & Uppermost 2 elements $(7.2 \mathrm{~m}$ to $9.0 \mathrm{~m})$ & \\
\hline
\end{tabular}




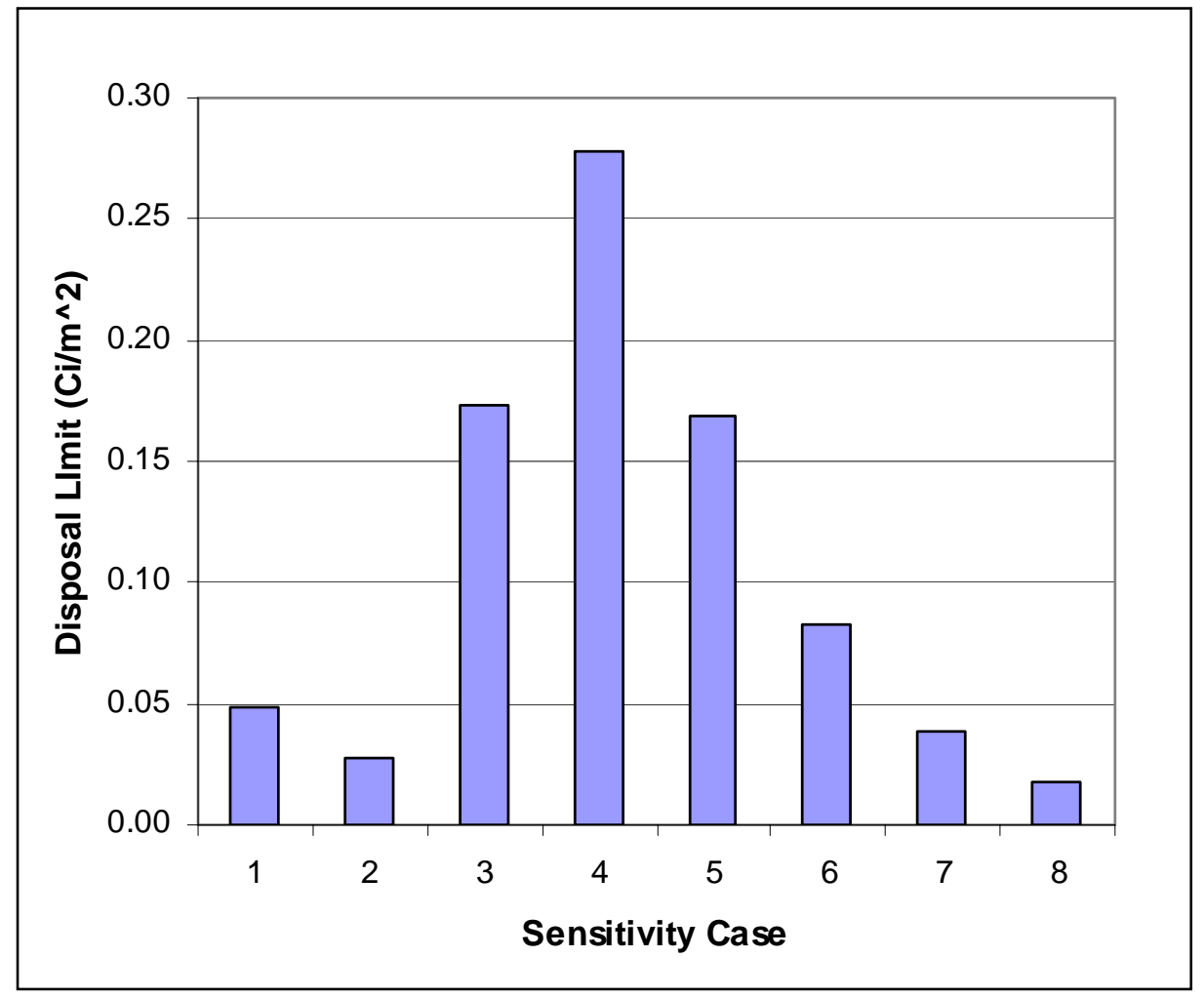

Figure D-4 Source zone sensitivity cases 


\section{REFERENCES}

Bolz, R.E. and G.L. Tuve, (Editors), 1973. Handbook of tables for APPLIED ENGINEERING SCIENCE, 2’nd Edition. CRC Press, 18901 Cranwood Parkway, Cleveland, OH.

McDowell-Boyer, L., A.D. Yu, J.R. Cook, D.C. Kocher, E.L. Wilhite, H. Holmes-Burns, K.E. Young, 1994, Radiological Performance Assessment for the E-Area Low Level Waste Facility. WSRC-RP-94-218, Westinghouse Savannah River Company, Aiken, South Carolina.

McDowell-Boyer et al. 2000. Radiological Performance Assessment for the E-Area Low-Level Waste Facility, Revision 1 (WSRC-RP-94-218). McDowell-Boyer, L., Yu, A. D., Cook, J. R., Kocher, D. C., Wilhite, E. L., Holmes-Burns, H., and Young, K. E. Westinghouse Savannah River Company, Aiken, South Carolina, January 31, 2000.

Nazaroff, W.W., and A.V. Nero (editors), 1998, Radon and its Decay Products in Indoor Air, John Wiley \& Sons, New York, N.Y.

Nielson, K.K., and V.C. Rogers, 1982. A Mathematical model for radon diffusion in earthen materials. NUREG/CR-2765. U.S. Nuclear Regulatory Commission, Washington, D.C.

Phifer, M. A., 2004. Preliminary E-Area Intermediate Level Vault Closure Cap Infiltration (U), WSRC-TR-2004-00280, Rev. 0, Westinghouse Savannah River Company, Aiken, South Carolina, March 12, 2004.

Rogers, V.C., K.K. Nielson, and D.R. Kalkwarf. 1984. Radon Attenuation Handbook for Uranium Mill Tailings Cover Design, prepared for U.S. Nuclear Regulatory Commission, Washington, D.C., NUREG/CR-3533.

USDOE, 1999a. Radioactive Waste Management. USDOE Order 435.1, Office of Environmental Management, United States Department of Energy, Washington, D.C.

40 CFR Part 61 Rev. 4, 1991. National Emission Standards for Hazardous Air Pollutants, Subpart Q, National Emission Standards for Radon Emissions From Department of Energy Facilities.

Yu, C., A.J. Zielen, J.J. Cheng, D.J. LePoire, E. Gnanapragasam, S. Kamboj, J.Arnish, A. Wallo III, W.A. Williams, and H.Peterson, 2001. Users Manual for RESRAD Version 6, Environmental Assessment Division, Argonne National Laboratory. Chicago, Illinois. 


\section{ADDENDUM}

Example input code for ILV simulation

/Title ILV Diffusion for Rn-222

/1-D Vertical Column for disposal of 1 Ci U-234

$/ 2.7 \mathrm{~m}$ of soil on top of $9 \mathrm{~m}$ of waste

/Pockets Created in Waste Zone to place Source Term (5 layers)

/SOURCE TERM: Emanation Factor of 0.25, apportioned to 5 cavities

$/$ R. A. Hiergesell

$/ 7 / 1 / 2004$

/

/DECAY CHAIN is U-234-->Th-230-->Ra226-->Rn222

/SIMULATION UNITS: length $=\mathrm{m}$, mass $=\mathrm{g}$, time $=$ years

$/ * * * * * * * * * * * * * * * * * * * * * * * * * * * * * * * * * * * * * * * * * * * * * * * * * * * * * * * * * * * * * * * * * * * * * * * * * * * *$

GRID 3 by 33

ALLOcate $\mathrm{C} 3$

ALLOcate C4

SCALE 1.0000

COORDINATE X:

$0.0 \quad 1.0$

SCALE 1.0000

COORDINATE Y:

0.00 .91 .82 .73 .64 .55 .46 .37 .28 .1

9.09 .59 .759 .9

$10.010 .1 \quad 10.210 .310 .410 .510 .610 .710 .810 .9$

11.0 11.1 11.2 11.3 11.4 11.5 11.6 11.7 !!! element interfaces

$\mathrm{DATUM}=0.0$.

I

MATErial type 1 from 11 to 32

MATErial type 1 from 14 to 34

MATErial type 1 from 16 to 36

MATErial type 1 from 18 to 38

MATErial type 1 from 110 to 310

MATErial type 2 from 13 to 33

MATErial type 3 from 15 to 35

MATErial type 4 from 17 to 37

MATErial type 5 from 19 to 39

MATErial type 6 from 111 to 311

MATErial type 7 from 112 to 333

FOR 1

DENSity $=1.24 \mathrm{e}+3$

MATErial DENSity $=2.65 \mathrm{e}+6$

MATErial POROsity $=0.5 \quad 0.5 \quad 0.5$

FOR 2

DENSity $=1.24 \mathrm{e}+3$

MATErial DENSity $=2.65 \mathrm{e}+6$

MATErial POROsity $=0.12 \quad 0.12 \quad 0.12$

FOR 3

DENSity $=1.24 \mathrm{e}+3$

MATErial DENSity $=2.65 \mathrm{e}+6$

\$ Waste Package

$\$$ Waste Package

$\$$ Waste Package

$\$$ Waste Package

$\$$ Waste Package

$\$$ Concrete, Rs $=0.6$

$\$$ Concrete, Rs $=0.6$

$\$$ Concrete, Rs $=0.7$

$\$$ Concrete, Rs $=0.7$

$\$$ Concrete, Rs $=0.8$

\$ Soil Cover

\$ fluid density (air) in $\mathrm{g} / \mathrm{m}^{\wedge} 3$

$\$$ Particle density in $\mathrm{g} / \mathrm{m}^{\wedge} 3$

$\$$ Air-filled porosity $(1-0.5)$

$\$$ fluid density (air) in $\mathrm{g} / \mathrm{m}^{\wedge} 3$

$\$$ Particle density in $\mathrm{g} / \mathrm{m}^{\wedge} 3$

$\$$ Air-filled porosity $(.3 \times(1-0.6))$

$\$$ fluid density (air) in $\mathrm{g} / \mathrm{m}^{\wedge} 3$

$\$$ Particle density in $\mathrm{g} / \mathrm{m}^{\wedge} 3$

MATErial POROsity $=0.12 \quad 0.12 \quad 0.12$

FOR 4

DENSity $=1.24 \mathrm{e}+3$

$\$$ Air-filled porosity $(.3 \times(1-0.6))$

$\$$ fluid density (air) in $\mathrm{g} / \mathrm{m}^{\wedge} 3$ 


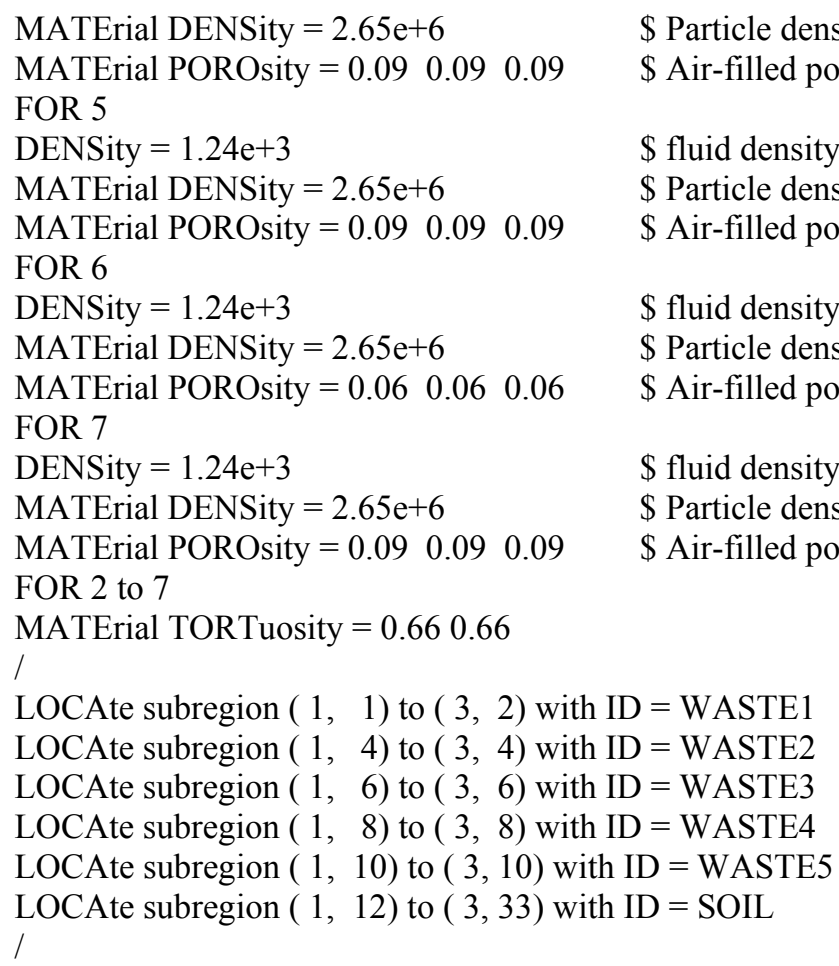

BOUN C2 X- FLUX $=0 . \quad \$$ Th230

BOUN C4 X- FLUX $=0 . \quad$ \$Rn222

\$ Particle density in $\mathrm{g} / \mathrm{m}^{\wedge} 3$

$\$$ Air-filled porosity $(.3 \times(1-0.7))$

\$ fluid density (air) in $\mathrm{g} / \mathrm{m}^{\wedge} 3$

$\$$ Particle density in $\mathrm{g} / \mathrm{m}^{\wedge} 3$

$\$$ Air-filled porosity $(.3 \times(1-0.7))$

\$ fluid density (air) in $\mathrm{g} / \mathrm{m}^{\wedge} 3$

$\$$ Particle density in $\mathrm{g} / \mathrm{m}^{\wedge} 3$

$\$$ Air-filled porosity $(.3 \times(1-0.8))$

$\$$ fluid density (air) in $\mathrm{g} / \mathrm{m}^{\wedge} 3$

$\$$ Particle density in $\mathrm{g} / \mathrm{m}^{\wedge} 3$

$\$$ Air-filled porosity $(.3 \times(1-0.7))$
S R226 


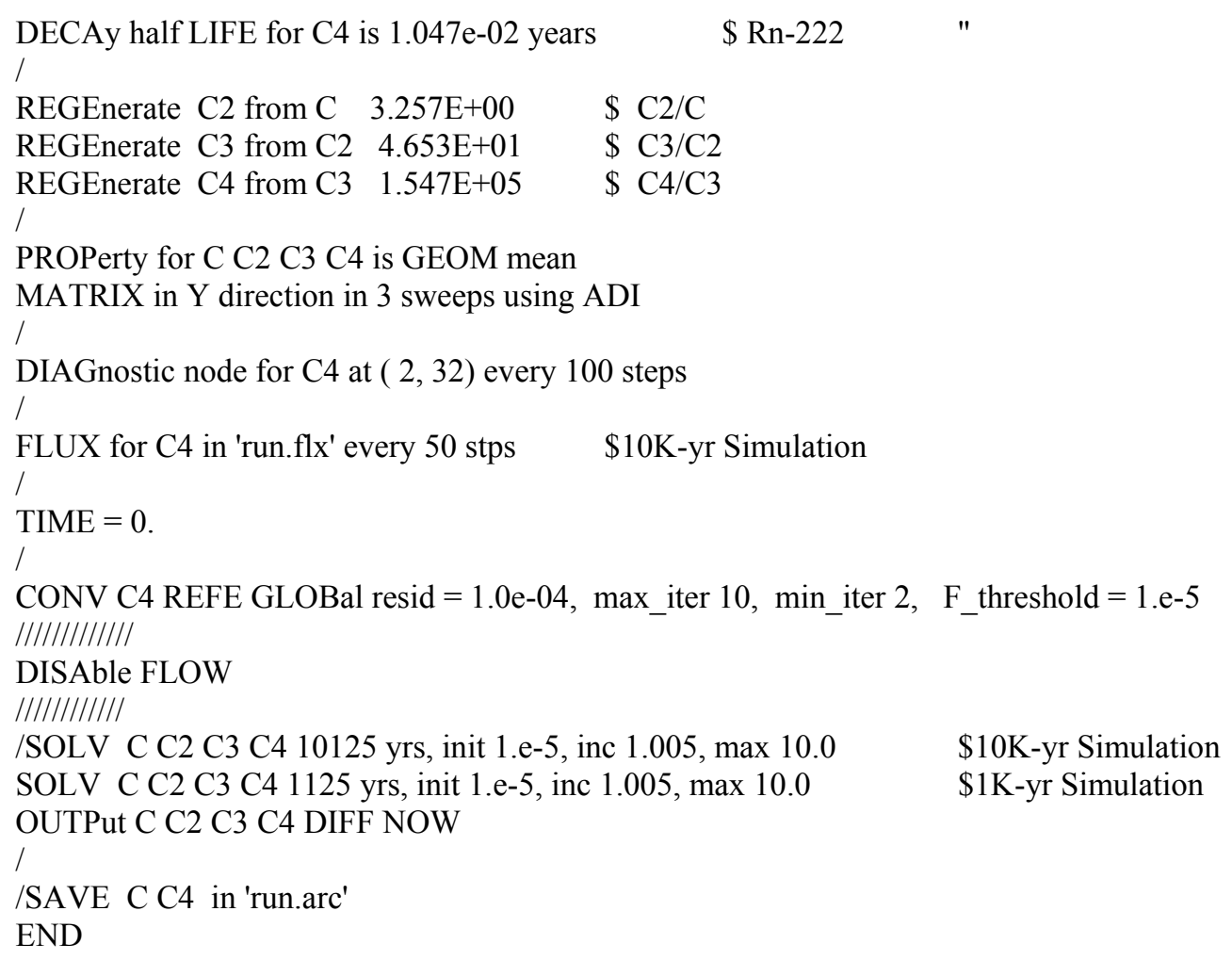




\section{APPENDIX E - ADDITIONAL INFORMATION ON LIMITS CALCULATIONS}

For diagnostic purposes and for comparison to previous limits, Table E-1 provides a more detailed presentation of limits calculated for each radionuclide parent including:

- groundwater pathway limit computed for each inventory modeled in PORFLOW

- previous IL vault limits and limiting pathway from Cook (2002b)

- groundwater pathway results for the 1,000 to 10,000 year time period

If a limit is not given for a nuclide and pathway (blank entry), that combination was previously screened (e.g. Cook 2004). 
Table E-1. Pathway limits, previous limits, and inventory on 7/2/04 for the IL vaults (+++ denotes screened or $>1 . e+20$; blank entry denotes nuclide screened from corresponding pathway analysis).

\begin{tabular}{|c|c|c|c|c|c|c|c|c|c|c|c|c|}
\hline nuclide & $\begin{array}{l}\text { resident } \\
\text { (Ci) }\end{array}$ & $\begin{array}{l}\text { air } \\
\text { (Ci) }\end{array}$ & $\begin{array}{c}\text { radon } \\
\text { (Ci) }\end{array}$ & $\begin{array}{l}\text { GW1 } \\
\text { (Ci) }\end{array}$ & $\begin{array}{c}\text { GW2 } \\
\text { (Ci) }\end{array}$ & $\begin{array}{l}\text { GW3 } \\
\text { (Ci) }\end{array}$ & path & $\begin{array}{c}\text { limit } \\
\text { (Ci) }\end{array}$ & prev path & $\begin{array}{l}\text { prev limit } \\
\text { (Ci) }\end{array}$ & $\begin{array}{c}\text { model } \\
\text { (Ci) }\end{array}$ & $\begin{array}{l}\text { inventory } \\
\text { (Ci) }\end{array}$ \\
\hline Ac-227 & $1.2 \mathrm{E}+07$ & & & & & & resident & $1.2 \mathrm{E}+07$ & & & & \\
\hline$A c-228$ & & & & & & & & +++ & & & & $1.8 \mathrm{E}-05$ \\
\hline $\mathrm{Ag}-108 \mathrm{~m}$ & $3.9 \mathrm{E}+04$ & & & & & & resident & $3.9 \mathrm{E}+04$ & & & & \\
\hline $\mathrm{Ag}-110 \mathrm{~m}$ & & & & & & & & +++ & & & & $3.0 \mathrm{E}-09$ \\
\hline $\mathrm{Al}-26$ & $6.6 \mathrm{E}+02$ & & & & & & resident & $6.6 \mathrm{E}+02$ & & & & \\
\hline Am-241 & & & & +++ & $7.6 \mathrm{E}+09$ & $1.1 \mathrm{E}+05$ & & & & & 0.5 & \\
\hline$:$ & $4.1 \mathrm{E}+10$ & & & +++ & $7.6 \mathrm{E}+09$ & $1.1 \mathrm{E}+05$ & GW2 & $7.6 \mathrm{E}+09$ & resident & $3.0 \mathrm{E}+04$ & & $3.5 \mathrm{E}-01$ \\
\hline Am-242m & $5.7 \mathrm{E}+06$ & & & & & & resident & $5.7 \mathrm{E}+06$ & resident & $2.7 \mathrm{E}+07$ & & $3.2 \mathrm{E}-04$ \\
\hline Am-243 & & & & +++ & +++ & $1.4 \mathrm{E}+04$ & & & & & 0.0005 & \\
\hline$:$ & & & & +++ & +++ & $1.4 \mathrm{E}+04$ & & & & & 0.005 & \\
\hline$:$ & & & & +++ & +++ & $1.4 \mathrm{E}+04$ & & & & & 0.05 & \\
\hline$:$ & & & & +++ & +++ & $3.2 \mathrm{E}+04$ & & & & & 0.5 & \\
\hline$:$ & $4.4 \mathrm{E}+07$ & & & +++ & +++ & $1.4 \mathrm{E}+04$ & resident & $4.4 \mathrm{E}+07$ & resident & $1.9 \mathrm{E}+01$ & & $4.3 \mathrm{E}-04$ \\
\hline $\mathrm{Ba}-133$ & $1.7 \mathrm{E}+09$ & & & & & & resident & $1.7 \mathrm{E}+09$ & & & & \\
\hline $\mathrm{Ba}-137 \mathrm{~m}$ & & & & & & & & +++ & & & & $1.5 \mathrm{E}+02$ \\
\hline $\mathrm{Bi}-207$ & $4.3 \mathrm{E}+04$ & & & & & & resident & $4.3 \mathrm{E}+04$ & & & & \\
\hline $\mathrm{Bi}-210$ & & & & +++ & +++ & +++ & & & & & 0.5 & \\
\hline$:$ & & & & +++ & +++ & +++ & & +++ & & & & $6.1 \mathrm{E}-07$ \\
\hline $\mathrm{Bi}-211$ & & & & & & & & +++ & & & & $3.2 \mathrm{E}-07$ \\
\hline Bi-212 & & & & & & & & +++ & & & & $1.3 \mathrm{E}-04$ \\
\hline $\mathrm{Bi}-214$ & & & & & & & & +++ & & & & 7.7E-01 \\
\hline Bk-249 & $8.1 \mathrm{E}+08$ & & & & & & resident & $8.1 \mathrm{E}+08$ & & & & \\
\hline C-14 & & & & +++ & $9.1 \mathrm{E}+07$ & $1.7 \mathrm{E}+04$ & & & & & 0.5 & \\
\hline 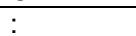 & +++ & $4.2 \mathrm{E}+00$ & & +++ & $9.1 \mathrm{E}+07$ & $1.7 \mathrm{E}+04$ & air & $4.2 \mathrm{E}+00$ & air & $2.7 \mathrm{E}+00$ & & 3.6E-01 \\
\hline C-14_KB & & & & +++ & $2.6 \mathrm{E}+06$ & $6.7 \mathrm{E}+02$ & & & & & 0.5 & \\
\hline$:$ & & $4.2 \mathrm{E}+00$ & & +++ & $2.6 \mathrm{E}+06$ & $6.7 \mathrm{E}+02$ & air & $4.2 \mathrm{E}+00$ & & & & $6.1 \mathrm{E}-02$ \\
\hline Ca-41 & +++ & & & & & & & +++ & & & & \\
\hline Cd-113m & +++ & & & & & & & +++ & & & & \\
\hline Ce-144 & & & & & & & & +++ & & & & $4.2 \mathrm{E}-01$ \\
\hline Cf-249 & & & & +++ & $1.9 \mathrm{E}+11$ & $2.0 \mathrm{E}+05$ & & & & & 0.0005 & \\
\hline : & & & & +++ & $1.9 \mathrm{E}+11$ & $2.0 \mathrm{E}+05$ & & & & & 0.005 & \\
\hline$:$ & & & & +++ & $1.9 \mathrm{E}+11$ & $2.0 \mathrm{E}+05$ & & & & & 0.05 & \\
\hline : & & & & +++ & $1.9 \mathrm{E}+11$ & $2.0 \mathrm{E}+05$ & & & & & 0.5 & \\
\hline : & $2.1 \mathrm{E}+06$ & & & +++ & $1.9 \mathrm{E}+11$ & $2.0 \mathrm{E}+05$ & resident & $2.1 \mathrm{E}+06$ & resident & $8.9 \mathrm{E}+02$ & & 5.6E-07 \\
\hline Cf-250 & $2.1 \mathrm{E}+18$ & & & & & & resident & $2.1 \mathrm{E}+18$ & & & & \\
\hline Cf-251 & $3.7 \mathrm{E}+08$ & & & & & & resident & $3.7 \mathrm{E}+08$ & resident & $2.7 \mathrm{E}+04$ & & 1.7E-06 \\
\hline Cf-252 & & & & +++ & +++ & $4.6 \mathrm{E}+12$ & & & & & 0.0005 & \\
\hline$:$ & & & & +++ & +++ & $4.6 \mathrm{E}+12$ & & & & & 0.005 & \\
\hline$:$ & & & & +++ & +++ & $4.6 \mathrm{E}+12$ & & & & & 0.05 & \\
\hline
\end{tabular}




\begin{tabular}{|c|c|c|c|c|c|c|c|c|c|c|c|c|}
\hline nuclide & $\begin{array}{l}\text { resident } \\
\text { (Ci) }\end{array}$ & $\begin{array}{l}\text { air } \\
(\mathrm{Ci})\end{array}$ & $\begin{array}{l}\text { radon } \\
(\mathrm{Ci})\end{array}$ & $\begin{array}{l}\text { GW1 } \\
(\mathrm{Ci})\end{array}$ & $\begin{array}{l}\text { GW2 } \\
(\mathrm{Ci})\end{array}$ & $\begin{array}{l}\text { GW3 } \\
\text { (Ci) }\end{array}$ & path & $\begin{array}{c}\operatorname{limit} \\
(\mathrm{Ci})\end{array}$ & prev path & $\begin{array}{l}\text { prev limit } \\
\text { (Ci) }\end{array}$ & $\begin{array}{c}\text { model } \\
(\mathrm{Ci})\end{array}$ & $\begin{array}{c}\text { inventory } \\
\text { (Ci) }\end{array}$ \\
\hline : & & & & +++ & +++ & $1.1 \mathrm{E}+13$ & & & & & 0.5 & \\
\hline$:$ & $6.3 \mathrm{E}+14$ & & & ++++ & +++ & $4.6 \mathrm{E}+12$ & resident & $6.3 \mathrm{E}+14$ & resident & $1.0 \mathrm{E}+10$ & & \\
\hline $\mathrm{Cl}-36$ & +++ & & & & & & & +++ & & & & \\
\hline $\mathrm{Cm}-242$ & & & & ++++ & +++ & $1.5 \mathrm{E}+15$ & & & & & 0.0005 & \\
\hline$:$ & & & & +++ & +++ & $1.5 \mathrm{E}+15$ & & & & & 0.005 & \\
\hline$:$ & & & & ++++ & +++ & $1.5 \mathrm{E}+15$ & & & & & 0.05 & \\
\hline$:$ & & & & +++ & +++ & $1.5 \mathrm{E}+15$ & & & & & 0.5 & \\
\hline$:$ & & & & ++++ & +++ & $1.5 \mathrm{E}+15$ & & & & & 5 & \\
\hline : & $5.5 \mathrm{E}+11$ & & & +++ & +++ & $1.5 \mathrm{E}+15$ & resident & $5.5 \mathrm{E}+11$ & & & & 1.4E-05 \\
\hline $\mathrm{Cm}-243$ & $9.7 \mathrm{E}+08$ & & & & & & resident & $9.7 \mathrm{E}+08$ & & & & $7.1 \mathrm{E}-03$ \\
\hline Cm-244 & & & & +++ & +++ & $4.9 \mathrm{E}+05$ & & & & & 0.0005 & \\
\hline : & & & & +++ & +++ & $4.9 \mathrm{E}+05$ & & & & & 0.005 & \\
\hline$:$ & & & & +++ & +++ & $6.3 \mathrm{E}+05$ & & & & & 0.05 & \\
\hline : & & & & ++++ & +++ & $6.3 \mathrm{E}+06$ & & & & & 0.5 & \\
\hline : & $2.5 \mathrm{E}+17$ & & & +++ & +++ & $4.9 \mathrm{E}+05$ & resident & $2.5 \mathrm{E}+17$ & resident & $4.6 \mathrm{E}+07$ & & $5.9 \mathrm{E}-01$ \\
\hline $\mathrm{Cm}-245$ & & & & ++++ & $4.8 \mathrm{E}+09$ & $8.3 E+03$ & & & & & 0.0005 & \\
\hline$:$ & & & & +++ & $4.8 \mathrm{E}+09$ & $8.3 \mathrm{E}+03$ & & & & & 0.005 & \\
\hline$:$ & & & & ++++ & $4.8 \mathrm{E}+09$ & $8.3 \mathrm{E}+03$ & & & & & 0.05 & \\
\hline$:$ & & & & ++++ & $4.8 \mathrm{E}+09$ & $8.3 \mathrm{E}+03$ & & & & & 0.5 & \\
\hline$:$ & $8.2 \mathrm{E}+09$ & & & +++ & $4.8 \mathrm{E}+09$ & $8.3 \mathrm{E}+03$ & GW2 & $4.8 \mathrm{E}+09$ & resident & $3.8 \mathrm{E}+01$ & & $7.0 \mathrm{E}-06$ \\
\hline $\mathrm{Cm}-246$ & & & & +++ & +++ & +++ & & & & & 0.5 & \\
\hline & $5.7 \mathrm{E}+15$ & & & +++ & +++ & +++ & resident & $5.7 \mathrm{E}+15$ & resident & $2.4 \mathrm{E}+05$ & & $8.5 \mathrm{E}-06$ \\
\hline $\mathrm{Cm}-247$ & & & & ++++ & $6.8 \mathrm{E}+18$ & $1.5 \mathrm{E}+05$ & & & & & 0.0005 & \\
\hline$:$ & & & & +++ & $6.8 \mathrm{E}+18$ & $1.5 \mathrm{E}+05$ & & & & & 0.005 & \\
\hline$:$ & & & & ++++ & $6.8 \mathrm{E}+18$ & $1.5 \mathrm{E}+05$ & & & & & 0.05 & \\
\hline$:$ & & & & +++ & $6.8 \mathrm{E}+18$ & $1.5 \mathrm{E}+05$ & & & & & 0.5 & \\
\hline : & $1.4 \mathrm{E}+06$ & & & +++ & $6.8 \mathrm{E}+18$ & $1.5 \mathrm{E}+05$ & resident & $1.4 \mathrm{E}+06$ & resident & $3.4 \mathrm{E}+00$ & & $2.0 \mathrm{E}-10$ \\
\hline $\mathrm{Cm}-248$ & & & & +++ & +++ & $3.5 \mathrm{E}+07$ & & & & & 0.0005 & \\
\hline$:$ & & & & ++++ & +++ & $3.5 \mathrm{E}+07$ & & & & & 0.005 & \\
\hline$:$ & & & & +++ & +++ & $3.5 \mathrm{E}+07$ & & & & & 0.05 & \\
\hline : & & & & +++ & +++ & $8.7 \mathrm{E}+07$ & & & & & 0.5 & \\
\hline$:$ & $4.6 \mathrm{E}+09$ & & & +++ & +++ & $3.5 \mathrm{E}+07$ & resident & $4.6 \mathrm{E}+09$ & resident & $8.1 \mathrm{E}+02$ & & $3.1 \mathrm{E}-15$ \\
\hline Co-57 & & & & & & & & +++ & & & & $5.1 \mathrm{E}-05$ \\
\hline Co-58 & & & & & & & & +++ & & & & $2.3 \mathrm{E}+00$ \\
\hline Co-60 & $8.0 \mathrm{E}+08$ & & & & & & resident & $8.0 \mathrm{E}+08$ & resident & $6.3 \mathrm{E}+08$ & & $7.5 \mathrm{E}+01$ \\
\hline Cr-51 & & & & & & & & +++ & & & & $8.4 \mathrm{E}-03$ \\
\hline Cs-134 & & & & & & & & +++ & & & & 7.7E-01 \\
\hline Cs-135 & & & & +++ & +++ & $4.6 \mathrm{E}+01$ & & & & & 0.5 & \\
\hline $\bar{\sigma}$ & +++ & & & +++ & +++ & $4.6 \mathrm{E}+01$ & & +++ & GW & $1.2 \mathrm{E}+02$ & & \\
\hline Cs-137 & $8.3 E+05$ & & & & & & resident & $8.3 \mathrm{E}+05$ & resident & $6.5 \mathrm{E}+05$ & & $2.2 \mathrm{E}+02$ \\
\hline Eu-152 & $8.9 \mathrm{E}+05$ & & & & & & resident & $8.9 \mathrm{E}+05$ & & & & $4.1 \mathrm{E}-05$ \\
\hline Eu-154 & $1.6 \mathrm{E}+07$ & & & & & & resident & $1.6 \mathrm{E}+07$ & resident & $1.1 \mathrm{E}+07$ & & $1.8 \mathrm{E}-01$ \\
\hline
\end{tabular}




\begin{tabular}{|c|c|c|c|c|c|c|c|c|c|c|c|c|}
\hline nuclide & $\begin{array}{l}\text { resident } \\
\text { (Ci) }\end{array}$ & $\begin{array}{l}\text { air } \\
\text { (Ci) }\end{array}$ & $\begin{array}{l}\text { radon } \\
(\mathrm{Ci})\end{array}$ & $\begin{array}{l}\text { GW1 } \\
(\mathrm{Ci})\end{array}$ & $\begin{array}{l}\text { GW2 } \\
(\mathrm{Ci})\end{array}$ & $\begin{array}{l}\text { GW3 } \\
\text { (Ci) }\end{array}$ & path & $\begin{array}{c}\operatorname{limit} \\
(\mathrm{Ci})\end{array}$ & prev path & $\begin{array}{l}\text { prev limit } \\
\text { (Ci) }\end{array}$ & $\begin{array}{l}\text { model } \\
(\mathrm{Ci})\end{array}$ & $\begin{array}{c}\text { inventory } \\
\text { (Ci) }\end{array}$ \\
\hline Eu-155 & $1.6 \mathrm{E}+18$ & & & & & & resident & $1.6 \mathrm{E}+18$ & & & & $1.2 \mathrm{E}-02$ \\
\hline Fe-55 & & & & & & & & +++ & & & & $8.4 \mathrm{E}+01$ \\
\hline Fe-59 & & & & & & & & +++ & & & & $1.6 \mathrm{E}-02$ \\
\hline $\mathrm{H}-3$ & & & & $5.3 E+09$ & $5.5 E+09$ & +++ & & & & & 0.5 & \\
\hline$:$ & +++ & $1.3 \mathrm{E}+09$ & & $5.3 E+09$ & $5.5 E+09$ & +++ & air & $1.3 \mathrm{E}+09$ & air & $5.5 \mathrm{E}+07$ & & $5.3 \mathrm{E}+05$ \\
\hline $\mathrm{Hf}-175$ & & & & & & & & +++ & & & & $1.9 \mathrm{E}-05$ \\
\hline $\mathrm{Hf}-181$ & & & & & & & & +++ & & & & $6.1 \mathrm{E}-03$ \\
\hline $\mathrm{l}-129$ & & & & $4.3 \mathrm{E}+10$ & $4.6 \mathrm{E}-04$ & $3.5 \mathrm{E}+01$ & & & & & 0.5 & \\
\hline$:$ & +++ & & & $4.3 E+10$ & $4.6 \mathrm{E}-04$ & $3.5 \mathrm{E}+01$ & GW2 & 4.6E-04 & GW & $5.2 \mathrm{E}-04$ & & 5.1E-05 \\
\hline $\begin{array}{l}\text { I- } \\
129 \_E T F- \\
\text { Carbon }\end{array}$ & & & & $1.2 \mathrm{E}+13$ & 9.7E-02 & $1.0 \mathrm{E}-01$ & & & & & 0.5 & \\
\hline$:$ & & & & $1.2 \mathrm{E}+13$ & 9.7E-02 & 1.0E-01 & GW2 & 9.7E-02 & GW & 1.4E-01 & & 2.0E-03 \\
\hline $\mathrm{I}-129$ KB & & & & +++ & $6.2 \mathrm{E}+03$ & $1.2 \mathrm{E}+00$ & & & & & 0.5 & \\
\hline$:$ & & & & +++ & $6.2 \mathrm{E}+03$ & $1.2 \mathrm{E}+00$ & GW2 & $6.2 \mathrm{E}+03$ & & & & $6.0 \mathrm{E}-06$ \\
\hline $\ln -113 \mathrm{~m}$ & & & & & & & & +++ & & & & $5.0 \mathrm{E}-06$ \\
\hline $\mathrm{K}-40$ & & & & $2.3 \mathrm{E}+19$ & $2.0 \mathrm{E}-01$ & $2.3 \mathrm{E}+01$ & & & & & 0.5 & \\
\hline$:$ & $1.5 \mathrm{E}+04$ & & & $2.3 \mathrm{E}+19$ & $2.0 \mathrm{E}-01$ & $2.3 \mathrm{E}+01$ & GW2 & $2.0 \mathrm{E}-01$ & & & & $7.8 \mathrm{E}-07$ \\
\hline $\mathrm{Kr}-85$ & $3.8 \mathrm{E}+10$ & & & & & & resident & $3.8 \mathrm{E}+10$ & & & & $2.0 \mathrm{E}+01$ \\
\hline Mn-54 & & & & & & & & +++ & & & & $4.2 \mathrm{E}-01$ \\
\hline Mo-93 & +++ & & & & & & & +++ & & & & \\
\hline $\mathrm{Na}-22$ & $1.1 \mathrm{E}+15$ & & & & & & resident & $1.1 \mathrm{E}+15$ & & & & \\
\hline Nb-93m & +++ & & & & & & & +++ & & & & $2.7 \mathrm{E}-03$ \\
\hline $\mathrm{Nb}-94$ & & & & +++ & +++ & $1.5 \mathrm{E}+02$ & & & & & 0.5 & \\
\hline$:$ & $1.3 E+04$ & & & +++ & +++ & $1.5 \mathrm{E}+02$ & resident & $1.3 \mathrm{E}+04$ & & & & $1.1 \mathrm{E}-03$ \\
\hline $\mathrm{Nb}-95$ & & & & & & & & +++ & & & & $2.5 \mathrm{E}-02$ \\
\hline $\mathrm{Nb}-95 \mathrm{~m}$ & & & & +++ & +++ & +++ & & & & & 0.5 & \\
\hline$:$ & & & & +++ & +++ & +++ & & +++ & & & & $9.6 \mathrm{E}-05$ \\
\hline $\mathrm{Ni}-59$ & & & & +++ & +++ & $1.6 \mathrm{E}+02$ & & & & & 0.5 & \\
\hline$:$ & +++ & & & +++ & +++ & $1.6 \mathrm{E}+02$ & & +++ & GW & $3.0 \mathrm{E}+02$ & & $5.4 \mathrm{E}-02$ \\
\hline $\mathrm{Ni}-63$ & +++ & & & & & & & +++ & & & & $1.2 \mathrm{E}+01$ \\
\hline $\mathrm{Np}-237$ & & & & +++ & $1.5 \mathrm{E}+06$ & $2.3 E+01$ & & & & & 0.5 & \\
\hline$:$ & $6.4 \mathrm{E}+06$ & & & +++ & $1.5 \mathrm{E}+06$ & $2.3 \mathrm{E}+01$ & GW2 & $1.5 \mathrm{E}+06$ & resident & $6.0 \mathrm{E}+00$ & & $1.9 \mathrm{E}-03$ \\
\hline $\mathrm{Pa}-231$ & $5.1 \mathrm{E}+05$ & & & & & & resident & $5.1 \mathrm{E}+05$ & & & & \\
\hline $\mathrm{Pa}-234$ & & & & & & & & +++ & & & & 1.3E-01 \\
\hline Pa-234m & & & & & & & & +++ & & & & $1.8 \mathrm{E}-01$ \\
\hline $\mathrm{Pb}-210$ & $5.5 \mathrm{E}+10$ & & & & & & resident & $5.5 \mathrm{E}+10$ & & & & 7.7E-01 \\
\hline $\mathrm{Pb}-212$ & & & & & & & & +++ & & & & $1.3 \mathrm{E}-04$ \\
\hline $\mathrm{Pb}-214$ & & & & & & & & +++ & & & & 7.7E-01 \\
\hline Pd-107 & +++ & & & & & & & +++ & & & & \\
\hline $\mathrm{Pm}-147$ & & & & & & & & +++ & & & & $2.8 \mathrm{E}+00$ \\
\hline Po-210 & & & & & & & & +++ & & & & $6.1 \mathrm{E}-07$ \\
\hline
\end{tabular}




\begin{tabular}{|c|c|c|c|c|c|c|c|c|c|c|c|c|}
\hline nuclide & $\begin{array}{l}\text { resident } \\
\text { (Ci) }\end{array}$ & $\begin{array}{l}\text { air } \\
(\mathrm{Ci})\end{array}$ & $\begin{array}{c}\text { radon } \\
(\mathrm{Ci})\end{array}$ & $\begin{array}{l}\text { GW1 } \\
\text { (Ci) }\end{array}$ & $\begin{array}{c}\text { GW2 } \\
\text { (Ci) }\end{array}$ & $\begin{array}{c}\text { GW3 } \\
\text { (Ci) }\end{array}$ & path & $\begin{array}{c}\text { limit } \\
(\mathrm{Ci})\end{array}$ & prev path & $\begin{array}{l}\text { prev limit } \\
\text { (Ci) }\end{array}$ & $\begin{array}{c}\text { model } \\
(\mathrm{Ci})\end{array}$ & $\begin{array}{c}\text { inventory } \\
\text { (Ci) }\end{array}$ \\
\hline Po-212 & & & & & & & & +++ & & & & $5.3 \mathrm{E}-07$ \\
\hline Po-214 & & & & & & & & +++ & & & & 7.7E-01 \\
\hline Po-216 & & & & & & & & +++ & & & & $1.3 \mathrm{E}-04$ \\
\hline Po-218 & & & & & & & & +++ & & & & 7.7E-01 \\
\hline Pr-144 & & & & & & & & +++ & & & & 3.3E-01 \\
\hline $\mathrm{Pr}-144 \mathrm{~m}$ & & & & & & & & ++++ & & & & $3.1 \mathrm{E}-03$ \\
\hline Pu-238 & & & & +++ & +++ & $7.5 \mathrm{E}+12$ & & & & & 0.0005 & \\
\hline$:$ & & & & +++ & ++++ & $7.5 \mathrm{E}+12$ & & & & & 0.005 & \\
\hline : & & & & +++ & +++ & $7.5 \mathrm{E}+12$ & & & & & 0.05 & \\
\hline$:$ & & & & +++ & +++ & $7.5 \mathrm{E}+12$ & & & & & 0.5 & \\
\hline$:$ & & & & +++ & +++ & $7.5 \mathrm{E}+12$ & & & & & 5 & \\
\hline : & $2.8 \mathrm{E}+09$ & & $4.2 \mathrm{E}+06$ & +++ & +++ & $7.5 \mathrm{E}+12$ & radon & $4.2 \mathrm{E}+06$ & GW & $2.2 \mathrm{E}+10$ & & $1.3 \mathrm{E}+00$ \\
\hline Pu-239 & & & & +++ & +++ & $6.3 \mathrm{E}+02$ & & & & & 0.0005 & \\
\hline$:$ & & & & +++ & +++ & $6.3 \mathrm{E}+02$ & & & & & 0.005 & \\
\hline$:$ & & & & +++ & +++ & $3.3 \mathrm{E}+03$ & & & & & 0.05 & \\
\hline$:$ & & & & +++ & ++++ & $3.3 \mathrm{E}+04$ & & & & & 0.5 & \\
\hline$:$ & $3.3 E+13$ & & & +++ & ++++ & $6.3 \mathrm{E}+02$ & resident & $3.3 E+13$ & resident & $2.9 \mathrm{E}+04$ & & $3.6 \mathrm{E}-01$ \\
\hline $\mathrm{Pu}-240$ & & & & +++ & +++ & $1.4 \mathrm{E}+03$ & & & & & 0.0005 & \\
\hline$:$ & & & & +++ & +++ & $1.4 \mathrm{E}+03$ & & & & & 0.005 & \\
\hline$:$ & & & & +++ & +++ & 1.7E+03 & & & & & 0.05 & \\
\hline : & & & & +++ & +++ & $1.7 \mathrm{E}+04$ & & & & & 0.5 & \\
\hline$:$ & $6.4 \mathrm{E}+14$ & & & +++ & +++ & $1.4 \mathrm{E}+03$ & resident & $6.4 \mathrm{E}+14$ & resident & $1.3 \mathrm{E}+05$ & & $4.5 \mathrm{E}-02$ \\
\hline Pu-241 & & & & +++ & $2.3 \mathrm{E}+11$ & $3.5 \mathrm{E}+06$ & & & & & 0.5 & \\
\hline$:$ & $1.2 \mathrm{E}+12$ & & & +++ & $2.3 \mathrm{E}+11$ & $3.5 \mathrm{E}+06$ & GW2 & $2.3 \mathrm{E}+11$ & resident & $8.9 E+05$ & & $1.5 \mathrm{E}+00$ \\
\hline Pu-242 & & & & +++ & +++ & $4.9 \mathrm{E}+02$ & & & & & 0.0005 & \\
\hline$:$ & & & & +++ & +++ & $4.2 \mathrm{E}+03$ & & & & & 0.005 & \\
\hline$:$ & & & & +++ & +++ & $4.2 \mathrm{E}+04$ & & & & & 0.05 & \\
\hline$:$ & & & & +++ & +++ & $4.2 \mathrm{E}+05$ & & & & & 0.5 & \\
\hline$:$ & $5.0 \mathrm{E}+12$ & & & +++ & +++ & $4.9 \mathrm{E}+02$ & resident & $5.0 \mathrm{E}+12$ & resident & $5.2 \mathrm{E}+04$ & & $3.0 \mathrm{E}-03$ \\
\hline Pu-244 & & & & +++ & +++ & $9.2 \mathrm{E}+04$ & & & & & 0.0005 & \\
\hline$:$ & & & & +++ & +++ & $9.0 \mathrm{E}+05$ & & & & & 0.005 & \\
\hline$:$ & & & & +++ & +++ & $7.2 \mathrm{E}+06$ & & & & & 0.05 & \\
\hline$:$ & & & & +++ & +++ & $4.6 \mathrm{E}+07$ & & & & & 0.5 & \\
\hline : & $3.7 \mathrm{E}+04$ & & & +++ & +++ & $9.2 \mathrm{E}+04$ & resident & $3.7 \mathrm{E}+04$ & resident & $3.1 \mathrm{E}+00$ & & 1.2E-02 \\
\hline Ra-224 & & & & & & & & +++ & & & & $1.3 \mathrm{E}-04$ \\
\hline Ra-226 & & & & +++ & +++ & $9.6 \mathrm{E}+01$ & & & & & 0.5 & \\
\hline$:$ & $1.4 \mathrm{E}+03$ & & $2.6 \mathrm{E}+00$ & +++ & +++ & $9.6 \mathrm{E}+01$ & radon & $2.6 \mathrm{E}+00$ & & & & 7.7E-01 \\
\hline Ra-228 & $5.2 \mathrm{E}+07$ & & & & & & resident & $5.2 \mathrm{E}+07$ & & & & $1.8 \mathrm{E}-05$ \\
\hline $\mathrm{Rb}-87$ & +++ & & & & & & & +++ & & & & \\
\hline Rh-103m & & & & & & & & +++ & & & & 6.9E-05 \\
\hline Rh-106 & & & & & & & & +++ & & & & 4.1E-02 \\
\hline Rn-220 & & & & & & & & +++ & & & & $1.3 \mathrm{E}-04$ \\
\hline
\end{tabular}




\begin{tabular}{|c|c|c|c|c|c|c|c|c|c|c|c|c|}
\hline nuclide & $\begin{array}{l}\text { resident } \\
\text { (Ci) }\end{array}$ & $\begin{array}{l}\text { air } \\
(\mathrm{Ci})\end{array}$ & $\begin{array}{l}\text { radon } \\
\text { (Ci) }\end{array}$ & $\begin{array}{l}\text { GW1 } \\
\text { (Ci) }\end{array}$ & $\begin{array}{l}\text { GW2 } \\
\text { (Ci) }\end{array}$ & $\begin{array}{l}\text { GW3 } \\
\text { (Ci) }\end{array}$ & path & $\begin{array}{c}\operatorname{limit} \\
(\mathrm{Ci})\end{array}$ & prev path & $\begin{array}{l}\text { prev limit } \\
\text { (Ci) }\end{array}$ & $\begin{array}{l}\text { model } \\
(\mathrm{Ci})\end{array}$ & $\begin{array}{l}\text { inventory } \\
\text { (Ci) }\end{array}$ \\
\hline Rn-222 & & & & & & & & +++ & & & & 7.7E-01 \\
\hline Ru-103 & & & & & & & & +++ & & & & $6.9 \mathrm{E}-05$ \\
\hline Ru-106 & & & & & & & & +++ & & & & $1.1 \mathrm{E}-01$ \\
\hline Sb-125 & $2.0 \mathrm{E}+16$ & & & & & & resident & $2.0 \mathrm{E}+16$ & & & & $1.0 \mathrm{E}-01$ \\
\hline Se-79 & & & & +++ & $1.2 \mathrm{E}+06$ & $3.3 \mathrm{E}+00$ & & & & & 0.5 & \\
\hline & +++ & & & +++ & $1.2 \mathrm{E}+06$ & $3.3 \mathrm{E}+00$ & GW2 & $1.2 \mathrm{E}+06$ & GW & $2.9 \mathrm{E}+01$ & & $1.0 \mathrm{E}-04$ \\
\hline Sm-151 & +++ & & & & & & & +++ & & & & \\
\hline Sn-113 & & & & & & & & +++ & & & & $5.0 \mathrm{E}-06$ \\
\hline Sn-121m & +++ & & & & & & & +++ & & & & \\
\hline Sn-126 & & & & +++ & +++ & $7.3 \mathrm{E}+01$ & & & & & 0.5 & \\
\hline$:$ & $2.1 \mathrm{E}+04$ & & & +++ & +++ & $7.3 \mathrm{E}+01$ & resident & $2.1 \mathrm{E}+04$ & resident & 7.5E-01 & & $4.9 \mathrm{E}-04$ \\
\hline Sr-90 & & & & +++ & $6.1 \mathrm{E}+11$ & $1.8 \mathrm{E}+12$ & & & & & 0.5 & \\
\hline- & +++ & & & +++ & $6.1 \mathrm{E}+11$ & $1.8 \mathrm{E}+12$ & GW2 & $6.1 \mathrm{E}+11$ & GW & $1.8 \mathrm{E}+10$ & & $2.1 \mathrm{E}+01$ \\
\hline Ta-182 & & & & & & & & +++ & & & & 3.4E-05 \\
\hline Tc-99 & & & & $4.0 \mathrm{E}+19$ & $1.3 \mathrm{E}+02$ & $1.9 \mathrm{E}+01$ & & & & & 0.5 & \\
\hline$:$ & $7.7 \mathrm{E}+17$ & & & $4.0 \mathrm{E}+19$ & $1.3 \mathrm{E}+02$ & $1.9 \mathrm{E}+01$ & GW2 & $1.3 \mathrm{E}+02$ & GW & $2.5 \mathrm{E}+01$ & & $6.1 \mathrm{E}-02$ \\
\hline Tc-99 KB & & & & +++ & $2.4 \mathrm{E}+04$ & $1.3 \mathrm{E}+02$ & & & & & 0.5 & \\
\hline$:$ & $7.7 \mathrm{E}+17$ & & & +++ & $2.4 \mathrm{E}+04$ & $1.3 \mathrm{E}+02$ & GW2 & $2.4 \mathrm{E}+04$ & & & & $2.0 \mathrm{E}-02$ \\
\hline Te-125m & & & & & & & & +++ & & & & $3.0 \mathrm{E}-03$ \\
\hline Th-228 & & & & +++ & +++ & +++ & & & & & 0.5 & \\
\hline 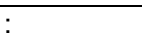 & $2.6 \mathrm{E}+18$ & & & +++ & +++ & +++ & resident & $2.6 \mathrm{E}+18$ & & & & $1.3 \mathrm{E}-04$ \\
\hline Th-229 & $5.3 \mathrm{E}+04$ & & & & & & resident & $5.3 E+04$ & & & & \\
\hline Th-230 & & & & +++ & +++ & $8.8 \mathrm{E}+01$ & & & & & 0.5 & \\
\hline$:$ & $3.9 \mathrm{E}+03$ & & $6.8 \mathrm{E}+00$ & +++ & +++ & $8.8 \mathrm{E}+01$ & radon & $6.8 \mathrm{E}+00$ & & & & $1.8 \mathrm{E}-05$ \\
\hline Th-231 & & & & & & & & +++ & & & & $1.9 \mathrm{E}-03$ \\
\hline Th-232 & & & & +++ & +++ & +++ & & & & & 0.5 & \\
\hline : & $4.4 \mathrm{E}+02$ & & & +++ & +++ & +++ & resident & $4.4 \mathrm{E}+02$ & resident & $4.1 \mathrm{E}-01$ & & $4.9 \mathrm{E}-05$ \\
\hline Th-234 & & & & & & & & +++ & & & & $3.1 \mathrm{E}-01$ \\
\hline TI-208 & & & & & & & & +++ & & & & $1.2 \mathrm{E}-07$ \\
\hline U-232 & & & & +++ & +++ & +++ & & & & & 0.0005 & \\
\hline- & & & & +++ & +++ & +++ & & & & & 0.005 & \\
\hline$:$ & & & & +++ & +++ & +++ & & & & & 0.05 & \\
\hline : & & & & +++ & +++ & +++ & & & & & 0.5 & \\
\hline : & $1.3 \mathrm{E}+03$ & & & +++ & +++ & +++ & resident & $1.3 \mathrm{E}+03$ & resident & $9.4 \mathrm{E}+02$ & & $1.1 \mathrm{E}-04$ \\
\hline U-233 & & & & +++ & +++ & $1.7 \mathrm{E}+09$ & & & & & 0.0005 & \\
\hline$:$ & & & & +++ & +++ & $1.7 \mathrm{E}+09$ & & & & & 0.005 & \\
\hline : & & & & +++ & +++ & $1.7 \mathrm{E}+09$ & & & & & 0.05 & \\
\hline$:$ & & & & +++ & +++ & $1.7 \mathrm{E}+09$ & & & & & 0.5 & \\
\hline : & $5.8 \mathrm{E}+05$ & & & +++ & +++ & $1.7 E+09$ & resident & $5.8 \mathrm{E}+05$ & resident & $7.0 \mathrm{E}+00$ & & 1.1E-02 \\
\hline U-233D & & & & +++ & +++ & $1.7 \mathrm{E}+09$ & & & & & 0.0005 & \\
\hline$:$ & & & & +++ & +++ & $1.7 \mathrm{E}+09$ & & & & & 0.005 & \\
\hline$:$ & & & & +++ & +++ & $1.7 \mathrm{E}+09$ & & & & & 0.05 & \\
\hline
\end{tabular}

Rev. 0 


\begin{tabular}{|c|c|c|c|c|c|c|c|c|c|c|c|c|}
\hline nuclide & $\begin{array}{c}\text { resident } \\
\text { (Ci) }\end{array}$ & $\begin{array}{l}\text { air } \\
\text { (Ci) }\end{array}$ & $\begin{array}{c}\text { radon } \\
\text { (Ci) }\end{array}$ & $\begin{array}{l}\text { GW1 } \\
\text { (Ci) }\end{array}$ & $\begin{array}{l}\text { GW2 } \\
\text { (Ci) }\end{array}$ & $\begin{array}{l}\text { GW3 } \\
\text { (Ci) }\end{array}$ & path & $\begin{array}{c}\text { limit } \\
(\mathrm{Ci})\end{array}$ & prev path & $\begin{array}{l}\text { prev limit } \\
\text { (Ci) }\end{array}$ & $\begin{array}{c}\text { model } \\
(\mathrm{Ci})\end{array}$ & $\begin{array}{c}\text { inventory } \\
\text { (Ci) }\end{array}$ \\
\hline : & & & & +++ & +++ & 1.7E+09 & & & & & 0.5 & \\
\hline : & $5.8 \mathrm{E}+05$ & & & +++ & +++ & $1.7 \mathrm{E}+09$ & resident & $5.8 \mathrm{E}+05$ & & & & $1.1 \mathrm{E}-01$ \\
\hline U-234 & & & & +++ & +++ & $8.5 \mathrm{E}+03$ & & & & & 0.0005 & \\
\hline$:$ & & & & +++ & +++ & $8.5 \mathrm{E}+03$ & & & & & 0.005 & \\
\hline$:$ & & & & +++ & ++++ & $8.5 \mathrm{E}+03$ & & & & & 0.05 & \\
\hline$:$ & & & & +++ & +++ & $8.5 \mathrm{E}+03$ & & & & & 0.5 & \\
\hline$:$ & $7.9 \mathrm{E}+05$ & & $1.2 \mathrm{E}+03$ & +++ & +++ & $8.5 \mathrm{E}+03$ & radon & $1.2 \mathrm{E}+03$ & radon & $1.5 \mathrm{E}+01$ & & $2.4 \mathrm{E}-01$ \\
\hline U-235 & & & & +++ & +++ & $4.7 \mathrm{E}+05$ & & & & & 0.0005 & \\
\hline : & & & & +++ & +++ & $5.6 \mathrm{E}+05$ & & & & & 0.005 & \\
\hline : & & & & +++ & ++++ & $4.2 \mathrm{E}+06$ & & & & & 0.05 & \\
\hline : & & & & +++ & +++ & $1.2 \mathrm{E}+07$ & & & & & 0.5 & \\
\hline$:$ & $2.4 \mathrm{E}+07$ & & & +++ & +++ & $4.7 \mathrm{E}+05$ & resident & $2.4 \mathrm{E}+07$ & resident & $6.0 \mathrm{E}+00$ & & $2.4 \mathrm{E}-03$ \\
\hline$U-235 D$ & & & & +++ & +++ & $4.7 \mathrm{E}+05$ & & & & & 0.0005 & \\
\hline : & & & & +++ & +++ & $5.6 \mathrm{E}+05$ & & & & & 0.005 & \\
\hline$:$ & & & & +++ & +++ & $4.2 \mathrm{E}+06$ & & & & & 0.05 & \\
\hline$:$ & & & & +++ & +++ & $1.2 \mathrm{E}+07$ & & & & & 0.5 & \\
\hline : & $2.4 \mathrm{E}+07$ & & & +++ & +++ & $4.7 \mathrm{E}+05$ & resident & $2.4 \mathrm{E}+07$ & & & & $6.1 \mathrm{E}-03$ \\
\hline U-236 & & & & +++ & +++ & $2.8 \mathrm{E}+09$ & & & & & 0.0005 & \\
\hline$:$ & & & & +++ & ++++ & $2.8 \mathrm{E}+09$ & & & & & 0.005 & \\
\hline : & & & & +++ & +++ & $2.8 \mathrm{E}+09$ & & & & & 0.05 & \\
\hline : & & & & +++ & +++ & $1.1 \mathrm{E}+10$ & & & & & 0.5 & \\
\hline : & $9.1 \mathrm{E}+09$ & & & +++ & +++ & $2.8 \mathrm{E}+09$ & resident & $9.1 \mathrm{E}+09$ & resident & $3.1 \mathrm{E}+04$ & & 1.7E-03 \\
\hline U-238 & & & & +++ & +++ & $2.0 \mathrm{E}+08$ & & & & & 0.0005 & \\
\hline : & & & & +++ & +++ & $1.5 \mathrm{E}+09$ & & & & & 0.005 & \\
\hline$:$ & & & & +++ & +++ & $1.5 \mathrm{E}+10$ & & & & & 0.05 & \\
\hline : & & & & +++ & +++ & $1.2 \mathrm{E}+11$ & & & & & 0.5 & \\
\hline : & $7.7 \mathrm{E}+05$ & & $1.1 \mathrm{E}+06$ & +++ & +++ & $2.0 \mathrm{E}+08$ & resident & $7.7 \mathrm{E}+05$ & resident & $4.9 \mathrm{E}+01$ & & $5.8 \mathrm{E}-01$ \\
\hline W-188 & +++ & & & & & & & +++ & & & & \\
\hline $\mathrm{Y}-90$ & & & & & & & & +++ & & & & $1.6 \mathrm{E}+01$ \\
\hline Zn-65 & & & & & & & & +++ & & & & 3.6E-01 \\
\hline Zr-93 & & & & +++ & +++ & $5.7 \mathrm{E}+08$ & & & & & 0.5 & \\
\hline 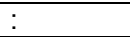 & +++ & & & +++ & ++++ & $5.7 \mathrm{E}+08$ & & +++ & GW & $9.8 \mathrm{E}+05$ & & $6.4 \mathrm{E}-06$ \\
\hline Zr-95 & & & & +++ & +++ & +++ & & & & & 0.5 & \\
\hline : & & & & +++ & +++ & +++ & & +++ & & & & 1.1E-02 \\
\hline
\end{tabular}




\section{APPENDIX F - DESIGN CHECKING}

Design checking of the IL vault Special Analysis was performed by the following technical professionals

- S. Aleman groundwater analysis

- L. Koffman intruder analysis

- T. Hang radon analysis

- E. Wilhite air analysis and overal analysis

Design checking instructions, comments and resolutions are provided below 


\section{Design checking instructions for the groundwater, intruder, air and overall analyses}

\section{Design Check Instructions for IL Vault Special Analysis (WSRC-TR-2004-00346)}

Requirements for performing reviews of technical reports are defined in Procedure Manual E-7, 2.40, "Design Verification and Checking", and the complementary manual WSRC-IM-2002-00011 Rev. 1 "Technical Report Design Check Guidelines" provides additional guidance. General lines of inquiry are defined in Table 1 of the latter. The purpose of these instructions is to define specific lines of inquiry appropriate for the IL Vault Special Analysis. The specific instructions given below are intended to supplement the general lines of inquiry, rather than constrain the scope of design checking.

\section{Groundwater Analysis}

Following a general inspection of the groundwater pathway analysis and associated PORFLOW input and output files, the following specific checks are requested. A spot check of one radionuclide and inventory will typically be a sufficient check that pre- and post-processing algorithms/software are working correctly.

general transport

- verify that each Kd value and solubility limit from Table A-1 used in PORFLOW runs can be traced to the PA or a superceding approved document

- verify that each concentration limit from Table A-1 used in the groundwater pathway analysis is correct based on published values for MCLs and $25 \mathrm{mrem} / \mathrm{yr}$ concentration values

- verify that first-order transformation parameters have been correctly defined in PORFLOW input files (DECAy and REGEneration keywords)

vadose zone flow

- verify that the infiltration transient proposed by Phifer (2004b) has been appropriately implemented in the flow model

- verify that other aspects of the VZ conceptual model have been preserved with respect to the PA

vadose zone transport

- verify that the initial inventory is correct for the $2 \mathrm{D}$ slice being modeled

- verify that solubility limits for $\mathrm{U}$ and $\mathrm{Pu}$ isotopes have been adequately implemented in the waste zone in PORFLOW input files

aquifer flow 
- verify that the volumetric flowrate (FC) and saturation (S) fields computed for the refined zoom-in grid are consistent with those from the GSA_PORFLOW flow model

\section{$\underline{\text { aquifer transport }}$}

- verify that the contaminant flux transient assigned to aquifer source nodes has been correctly computed from vadose zone simulation output

- verify that the aquifer source nodes have been correctly defined

- verify that nodes representing a potential 100 meter well have been adequately identified

- verify that peak sum-of-fraction and limits post-processing steps have been correctly performed

\section{Intruder Analysis}

Nearly all of the intruder analysis calculations were performed within a recently developed software tool, which has been design checked (Koffman 2004). The present design check should focus on verifying that inputs to the automated intruder analysis tool are appropriate, and that software results have been transferred to technical report tables (e.g. Tables 3-1 and 6-1) without error. Specific inputs that should be checked include

- the volume of the disposal unit

- the geometry factor for the Resident scenario

- the thickness of the soil/cover layers overlying the disposal unit (Phifer 2004a)

The basis for these inputs is described in Appendix B of the report.

\section{Air Analysis}

The primary change to the air analysis with respect to the PA is use of updated dose factors, reflecting average meteorological conditions for the period 1997-2001 measured near H-Area. The PA calculation method was retained for the Special Analysis. The design check should focus on verifying that

- the revised dose factors have been correctly calculated, and transcribed to report Table 4-1

- the air limit calculation summarized in Table 4-1 uses the method and other inputs described in the PA

- limits from the air analysis have been correctly transferred to Table 6-1. 


\section{Radon Analysis}

Provided in separate document.

\section{Overall Analysis and Technical Report}

A high-level review of the overall analysis and technical report should be conducted to ensure that

- the conceptual approach is acceptable

- critical assumptions have been adequately justified and/or referenced

- the proposed limits address all nuclides for which limits are needed

- report text, tables, and appendices are consistent

- adequate background and introductory discussion is provided

- conclusions and recommendations are supported by preceding report discussion 


\section{Design checking comments and resolution for groundwater analysis}

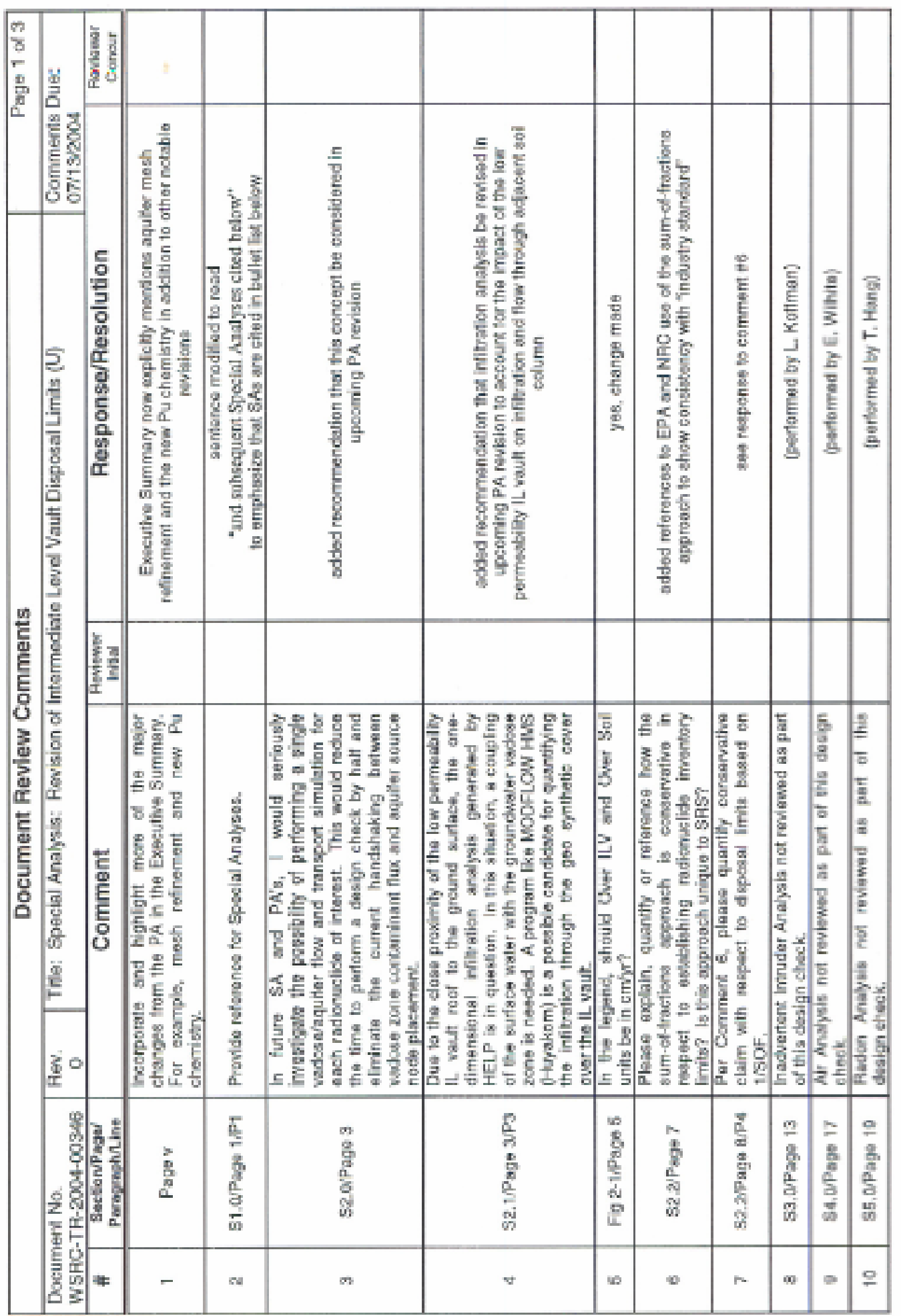




\begin{tabular}{|c|c|c|c|c|c|c|c|c|}
\hline 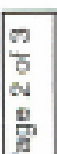 & 过 & 는 & & & & & & \\
\hline & 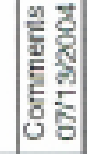 & & & 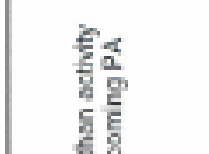 & 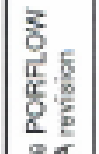 & 8 & & 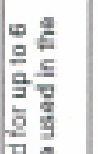 \\
\hline & 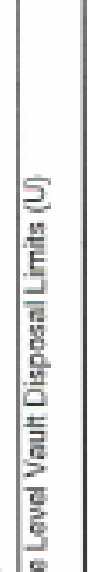 & 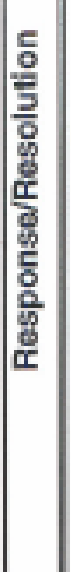 & 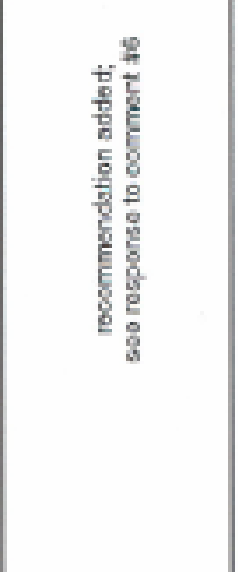 & 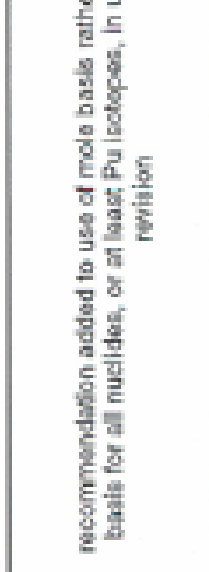 & 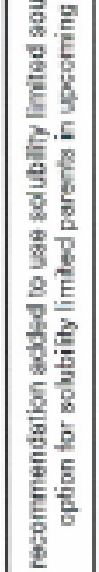 & 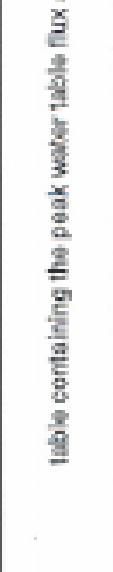 & 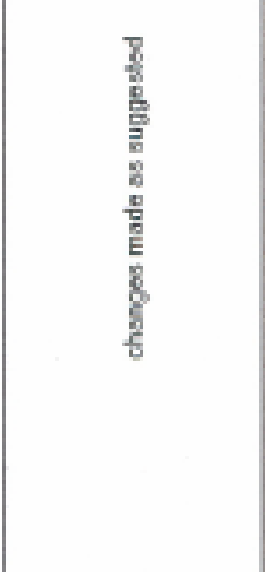 & 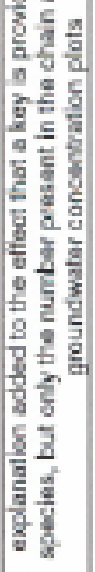 \\
\hline है & 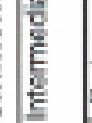 & 䢣 & & & & & & \\
\hline 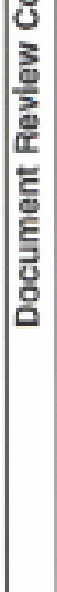 & 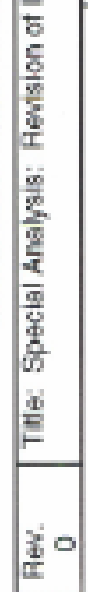 & 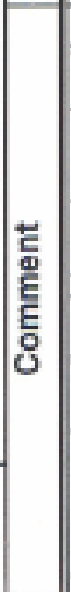 & 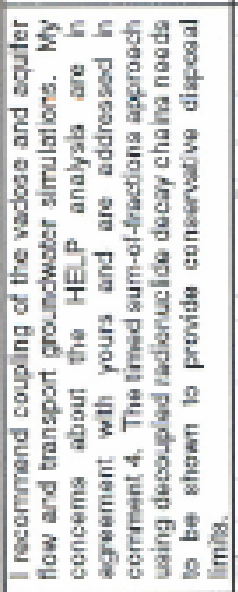 & 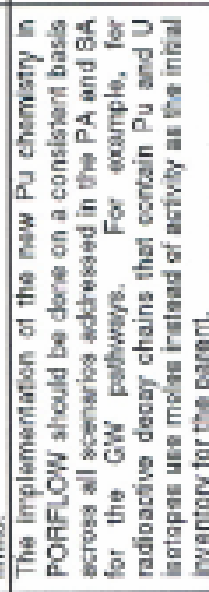 & 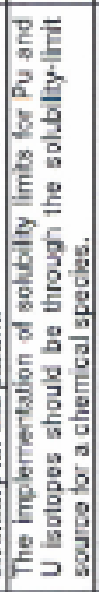 & 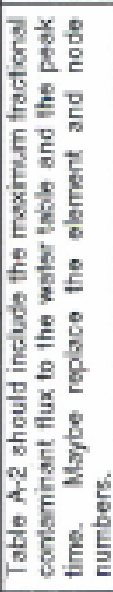 & 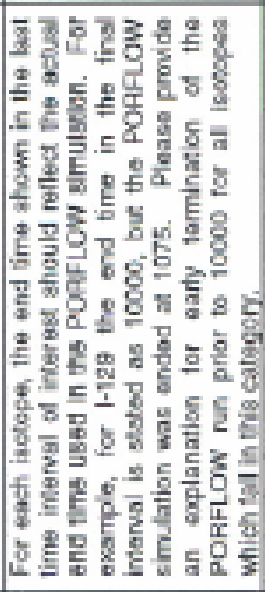 & 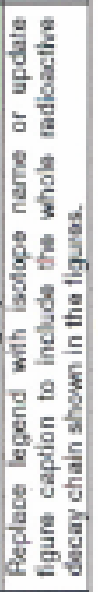 \\
\hline & 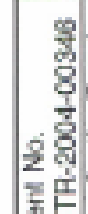 & 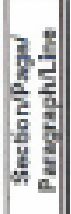 & 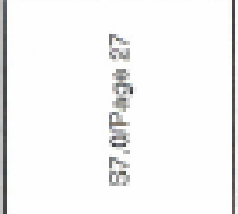 & 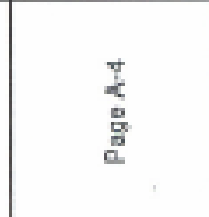 & s & 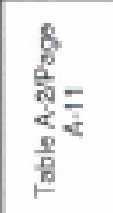 & 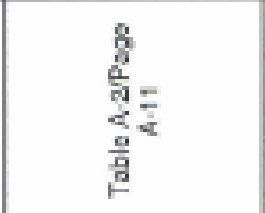 & 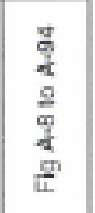 \\
\hline & 步要 & 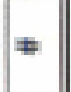 & $=$ & $\underline{9}$ & 끄 & $\nexists$ & $\stackrel{\oplus}{q}$ & $\stackrel{\oplus}{\circ}$ \\
\hline
\end{tabular}




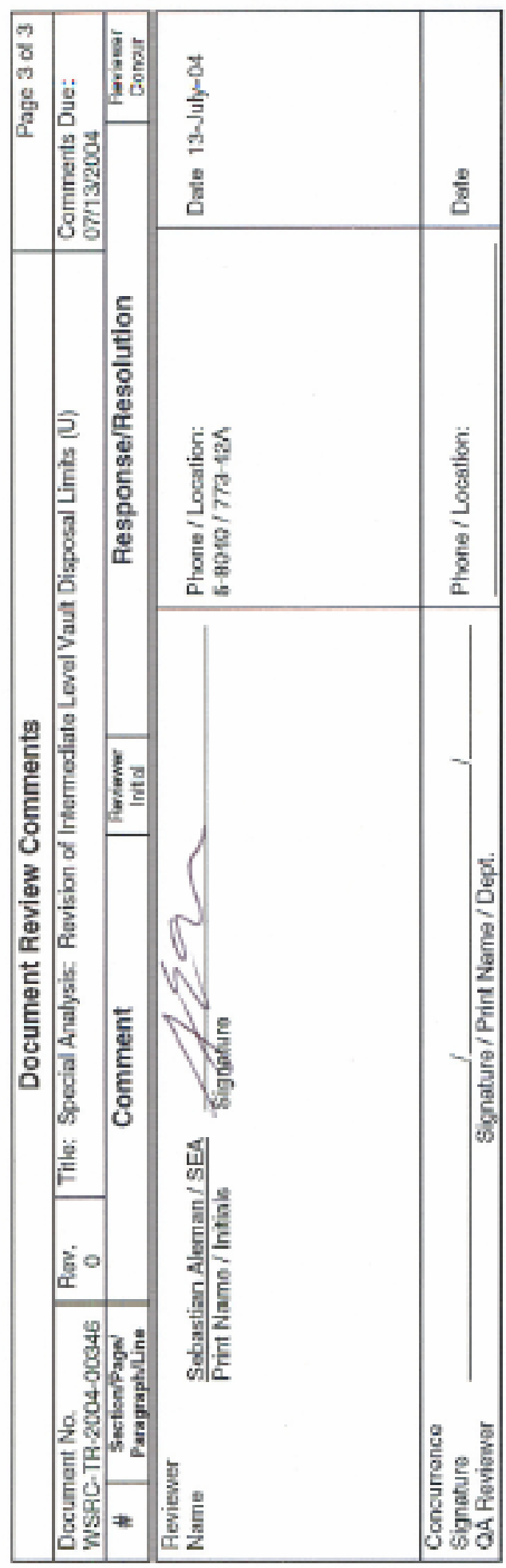

Rev. 0 


\section{Design checking comments and resolution for intruder analysis}

Larry Koffman/WSRC/Srs
07/12/2004 03:54 PM

\author{
To Gregory Flach/WSRC/Srs@srs \\ $\mathrm{cc}$ \\ bcc \\ Subject Design Check of ILV Intruder Analysis [
}

I have completed a design check of the ILV Intruder Analysis contained in your report WSRC-TR-2004-00346 rev0 draftB.pdf sent on 7/7/04. I reviewed Section 3.0 (pp 13-16), Section 6.0 (pp 21-25), and Appendix B (pp B-1 to B-11).

I have one required change: the units for Concentration Limit in Table 3-1 should be microCuries/m3, not Curies/m3. This correction needs to be made in the header on pages 14,15 , and 16 . The units for Inventory Limit are correct.

I found one typo: the first line of Appendix B has "primary", which should be "primarily".

I have one suggestion that is not required. In Section 3.1 in the middle of page 13 at the end of the third paragraph, the sentence ends as "and then relies on the computer model to perform pathways calculations at a time increment of 10 years." The time increment is a user input and is not fixed, so I suggest that you end with "calculations at a specified time increment that is nominally 10 years."

In reviewing Appendix $\mathrm{B}, \mathrm{I}$ checked that your calculation of geometry factor is correct, that the revised volume is consistent with the description in the PA, and that the thickness of the soilcover layers is consistent with Phifer's document.

I checked that Table 3.1 has the correct values for the given inputs and that Table B- 1 is the corresponding detailed table. I also checked that the resident limit column in Table 6-1 has the same values as Table 3.1 when rounded to one decimal place.

\section{Design checking comments and resolution for air analysis}

Elmer Wilhite/WSRC/Srs

07/13/2004 04:36 PM

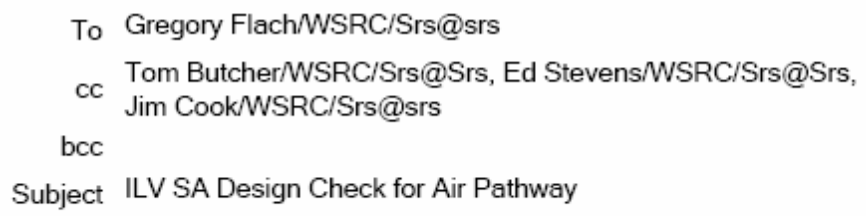

Greg:

I've completed the design check for the air pathway. I confirmed that you had extracted the correct numbers from the EAV PA for the dose limit and the release fractions for tritium and $\mathrm{C}-14$. I also confirmed that you had used the correct dose factors from Simpkins (2004).

Then, I confirmed that your calculations of the dose fractions and the inventory limits were correctly done.

Elmer

\section{Design checking comments and resolution for overall analysis}

Comments provided and resolved through hand-marked paper copies of report drafts. 


\section{Design checking instructions, comments and resolution for radon analysis}

$\underline{\text { Round } 1}$

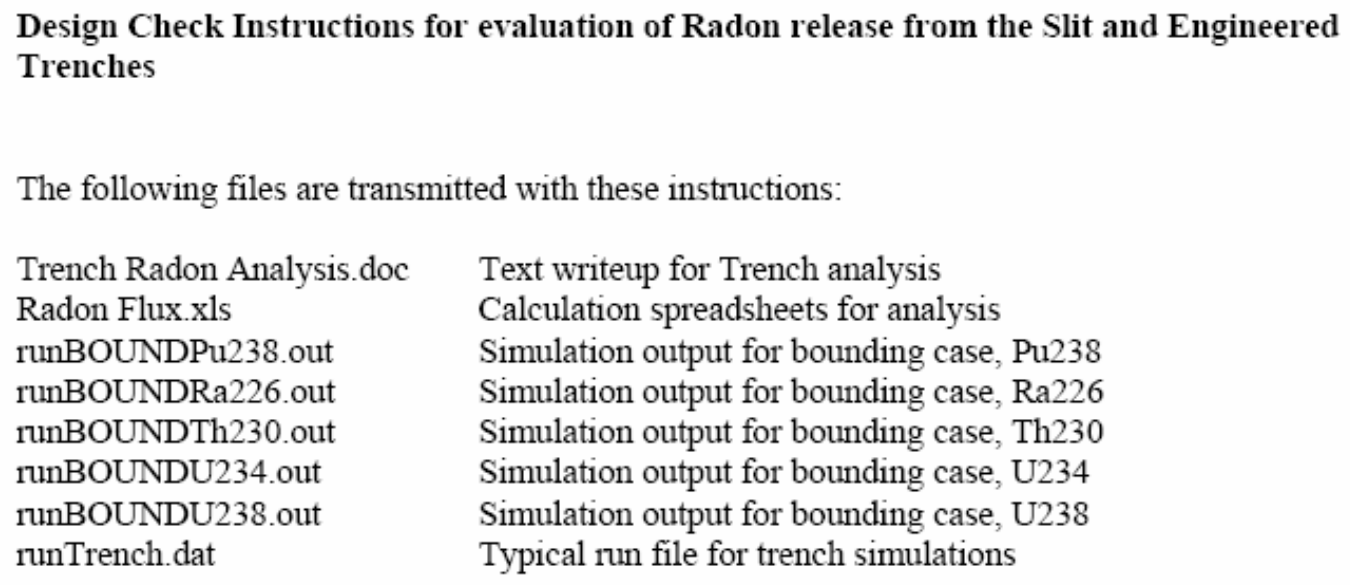

The following files are transmitted with these instructions:

Trench Radon Analysis.doc

Radon Flux.xls

Text writeup for Trench analysis

runBOUNDPu238. out

Calculation spreadsheets for analysis

runBOUNDRa226.out

Simulation output for bounding case, Pu238

runBOUNDTh230.out

Simulation output for bounding case, Ra226

runBOUNDU234.out

Simulation output for bounding case, Th230

runBOUNDU238.out

Simulation output for bounding case, U234

runTrench.dat

Simulation output for bounding case, U238

Typical run file for trench simulations

1. Check the input parameters identified in Table 2 of "Trench Radon Analysis.doc" for being reasonable, noting the units used in the model: meters, grams, and years.

A single value of 0.2 was used for porosity in this work. The previous PA (WSRC-RP_94-218, Rev. 1) used different porosity values for different materials, e.g., 0.42 for soil and waste, 0.51 for backfill, 0.38 for drainage, 0.18 for barrier. Some explanation is needed to justify the use of a single porosity.

Text will be added.

2. Examine the "half-life" worksheet in Excel Spreadsheet "RadonFlux.xls" to verify that the appropriate half-lives were used for each isotope that was simulated.

The half-life of 7.538E+04 yrs was used for Th-230 in this report compared to $7.70 \mathrm{E}+4$ yrs given in the PA.

The 7.538E+04 value comes from the Nuclear Wallet Cards, BNL and is regarded as more accurate than other estimates.

3. Spot check the output files for each isotope to verify that the input parameters are correctly represented.

Input parameters are correctly used in the output files for each isotope (i.e.,

runBOUNDPu238.out, runBOUNDRa226.out, runBOUNDTh230.out, runBOUNDU234.out, runBOUNDU238.out).

$O K$

4. Examine the typical run file to verify that the logic of the simulation makes sense.

A typical run file (runTrench.dat) was examined. Overall the logic of the simulation makes sense. The following comments result from further examination of all input files for 'noncompaction of waste' case and the input file shown in the Appendix for the 'dynamic compaction of waste' case: 
a. Since three nodes are specified in $\mathrm{X}$ direction, only two $\mathrm{X}$ coordinate values need be specified at cell faces as follows:

COORdinate $\mathrm{X}$ :

$0.0 \quad 1.0$

The presence of the additional coordinate does not make a difference in the simulated results, however any future simulations will be changed in accordance with the comment.

b. Rather than from 0 to $7.8 \mathrm{~m}, \mathrm{Y}$ coordinates values should range from 0 to $7.88 \mathrm{~m}$ as shown in Table 1 and Figure 2 (i.e., compacted waste zone plus soil cover zone excluding topsoil and upper backfill).

The use of a slightly shorter stack was used to round off the numbers when the grid coordinates were generated. The small difference ( $8 \mathrm{~cm}$ or 3.1 inches) is a conservative change. Fluxes will be larger by a very small amount because of the difference in dimensions. The incremental increase in accuracy to be obtained by changing the grid dimensions is not regarded as sufficient justification to spend the time needed implement that change now.

c. For the 'non-compaction of waste' case, the waste zone thickness is $4.9 \mathrm{~m}$. Hence the specifications for material zones should be revised as follows:

\section{MATErial type 1 from 11 to 324 \$ WASTE}

MATErial type 2 from 125 to 338 \$ SOIL

The use of $Y=22$ instead of $Y=24$ to define the Waste Zone thickness in the Bounding Case (no compaction of waste material) makes a small, less conservative, difference for the Bounding Case. Precise adherence to the thickness of the compacted soil thickness is not absolutely necessary since it only impacts how conservative the conservative Bounding Case is. However, since the changes are fairly easy to make, I've made them, recalculated the fluxes and the associated limits and incorporated them into the appropriate tables.

5. Check the Rn-222 instantaneous flux from the output file for each parent isotope and compare to value in the "Trench and ILV" worksheet in the file "Radon Flux.xls" to verify that the correct numbers were utilized in calculating the disposal limits for each of the 4 configuration scenarios. (1000-year case) - actually 1125 years simulated. Note, the flux numbers used in the Bounding case calculations are found in column $L$ for the 1125-year simulations.

Output files (i.e., runBOUNDPu238.out, runBOUNDRa226.out, runBOUNDTh230.out, runBOUNDU234.out, runBOUNDU238. out) for the non-compacted case were examined and the following instantaneous flux values at 1125 yrs are obtained:

\begin{tabular}{|c|c|}
\hline $\begin{array}{l}\text { Parent } \\
\text { Isotope }\end{array}$ & $\begin{array}{c}\text { Instantaneous Rn-222 Flux at Land Surface } \\
\text { @ } 1125 \mathrm{yr} \text { s } \\
\left(\mathrm{Ci} / \mathrm{m}^{2}-\mathrm{yr}\right)\end{array}$ \\
\hline Pu-238 & $4.811168 \mathrm{E}-6$ \\
\hline U-238 & $1.83563 \mathrm{E}-5$ \\
\hline
\end{tabular}




\begin{tabular}{|c|c|}
\hline U-234 & $1.65922 \mathrm{E}-2$ \\
\hline Th-230 & 2.961 \\
\hline Ra-226 & 4.81993 \\
\hline
\end{tabular}

These values are correctly used in cells L21 to L25 in the 'Trench and ILV Flux' worksheet of the file 'Radon Flux.xls' However, the purpose of this work is to determine the disposal limit for each isotope. Hence, rather than the flux at $1125 \mathrm{yrs}$, the peak flux values must be used. Since no flux files are provided for this design check, it is not clear if the instantaneous flux values at 1125 yrs are also peak values. Only instantaneous flux values for the non-compacted case (i.e., the bounding case) were checked, since no output files for the compacted case are available. It would be strongly recommended that the report include plots of instantaneous fluxes for each parent isotope for both compacted and non-compacted cases.

Agreed. The table shows only Ra-226 with a peak prior to 1125 years. The others peak at the 1125-year point but that is not apparent to the reader. The suggested changes have been incorporated.

Further examination of the 'Trench and ILV Flux' worksheet reveals that the conversion factor (i.e., $1 \mathrm{E}+12 / 31557600$ ) used in columns $\mathrm{G}$ and $\mathrm{M}$ to convert ' $\mathrm{Ci} / \mathrm{m}^{2}-\mathrm{yr}$ ' to ' $\mathrm{pCi} / \mathrm{m}^{2}-\mathrm{s}$ ' is incorrect. The correct factor should be $1 \mathrm{E}+12 / 31536000$. The value 31536000 that is the product of ' $365 \mathrm{x}$ $24 \times 3600^{\prime}$ is to convert yr into s.

The value used represents the conversion from years to seconds assuming there are 365.25 days in a year, to account for leap years. I believe this to be more accurate for the purposes of this investigation.

\section{Spot check calculations made in the Trench and ILV worksheet to determine the disposal unit limits for each of the 4 Scenarios.}

The disposal areas per unit ( $\mathrm{m}^{2} /$ disposal unit) are correctly calculated in cells O18, Q18, S18 and U18. The disposal limits for each of the four scenarios are also correctly determined from the instantaneous flux values in cells M21 to M25.

$O K$

7. Check the numbers in Tables 3 and 4 to be sure the numbers have been correctly transcribed from the "Trench and ILV" worksheet in the file "Radon Flux.xls".

The values shown in Tables 3 and 4 have been correctly transcribed from the 'Trench and ILV' worksheet. In Table 3, the values for Ra- 226 have been revised to represent the peak flux that occurs at 64.3 days after source (see comments in item 5 above). The values for Ra- 226 in Table 4 need be corrected accordingly.

It has been updated as were the other new values that resulted from re-definition of the Waste Zone from $Y=1$ to 22 to $Y=1$ to 24 (non-compacted BOUNDING case). 
$\underline{\text { Round } 2}$

\section{Design Check Instructions for evaluation of Radon release from the ILV}

The following files are transmitted with these instructions:

ILV Radon Analysis3.doc Text writeup for Trench analysis

PeakFluxSummary.xls Spreadsheets of results

\section{Read through the main text - ILV Radon Analysis3.doc, parts that have changed are indicated with blue text.}

The author is provided with a copy of the text that includes all marked suggested editorial changes for revision.

The comments provided have been incorporated in their entirety.

2. Check the input parameters identified in Table 2 of "ILV Radon Analysis3.doc" to be sure they correspond correctly with values listed in the example input file located in the Appendix.

Input parameters given in Table 2 have been correctly used in the PorFlow input file shown in the Appendix. In regard to the matrix density, the (solid) quartz was selected. Since the modeled matrix includes both solid and water (saturation), its density should combine both quartz and water density.

A test case was run to evaluate the impact of using a composite matrix density reflecting both the density of quartz and the density of water for the water filled volume. Both densities were incorporated using a weighting factor based on their respective volume percentages. The results were identical to the previous simulations that utilized a matrix density of quartz and therefore no action is required.

3. Compare the results in PeakFluxSummary.xls to be sure they've been correctly transcribed into ILV Radon Analysis3.doc from PeakFluxSummary.xls.

The results have been correctly transcribed from PeakFluxSummary.xls into the 'ILV Radon Analysis3.doc' report.

$O K$ 